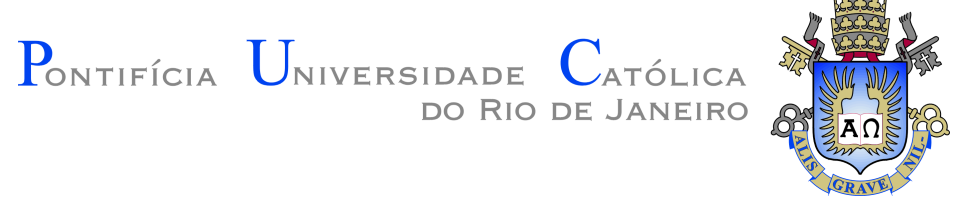

Guilherme Simon da Rosa

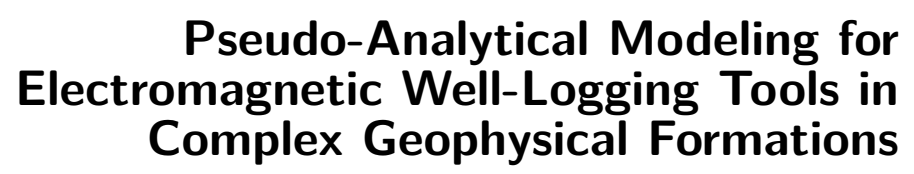

\begin{abstract}
Tese de Doutorado
Thesis presented to the Programa de Pós-graduação em Engenharia Elétrica of PUC-Rio in partial fulfilment of the requirements for the degree of Doutor em Engenharia Elétrica.
\end{abstract}

Advisor : Prof. José Ricardo Bergmann

Co-advisor: Prof. Fernando Lisboa Teixeira 

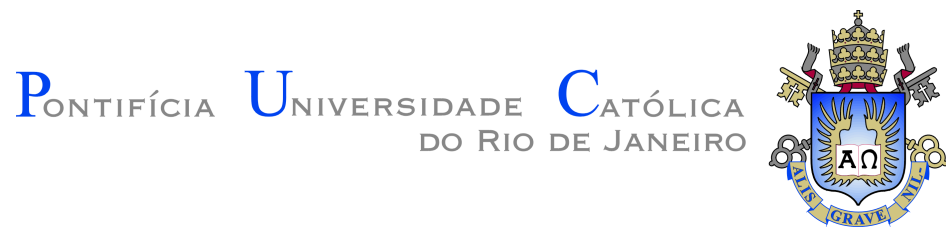

Guilherme Simon da Rosa

\section{Pseudo-Analytical Modeling for Electromagnetic Well-Logging Tools in Complex Geophysical Formations}

Thesis presented to the Programa de Pós-graduação em Engenharia Elétrica of PUC-Rio in partial fulfilment of the requirements for the degree of Doutor em Engenharia Elétrica. Approved by the undersigned Examination Committee.

Prof. José Ricardo Bergmann

Advisor

Departamento de Engenharia Elétrica - PUC-Rio

Prof. Fernando Lisboa Teixeira

Co-advisor

ElectroScience Laboratory - The Ohio State University

Prof. Flavio José Vieira Hasselmann

Departamento de Engenharia Elétrica - PUC-Rio

Prof. Adaildo Gomes D’Assunção

Departamento de Engenharia Elétrica - UFRN

Prof. Cássio Gonçalves do Rego

Departamento de Engenharia Eletrônica - UFMG

Prof. Sandro Rogério Zang

Departamento das Engenharias de Telecomunicações e

Mecatrônica - UFSJ

Prof. Marcio da Silveira Carvalho

Coordenador Setorial do Centro Técnico Científico - PUC-Rio 
All rights reserved.

\section{Guilherme Simon da Rosa}

Received the B.S. degree in electrical engineering from the Federal University of Santa Maria, Santa Maria, Brazil, in 2011, and the M.S. degree in electrical engineering from the Pontifical Catholic University of Rio de Janeiro, Rio de Janeiro, Brazil, in 2013. His research interests include pseudoanalytical techniques for wave scattering modeling in complex media.

Bibliographic data

Simon da Rosa, Guilherme

Pseudo-Analytical Modeling for Electromagnetic WellLogging Tools in Complex Geophysical Formations / Guilherme Simon da Rosa; advisor: José Ricardo Bergmann; co-advisor: Fernando Lisboa Teixeira. - Rio de Janeiro: PUCRio, Departamento de Engenharia Elétrica, 2017.

v., 299 f.: il. ; $29,7 \mathrm{~cm}$

Tese (doutorado) - Pontifícia Universidade Católica do Rio de Janeiro, Departamento de Engenharia Elétrica.

Inclui referências bibliográficas.

1. Engenharia Elétrica - Tese. 2. Engenharia Elétrica Tese. 3. Sensores de prospecção de petróleo. 4. Métodos de casamento de modos. 5. Meios anisotrópicos. 6. Meios estratificados. I. Bergmann, José Ricardo. II. Lisboa Teixeira, Fernando. III. Pontifícia Universidade Católica do Rio de Janeiro. Departamento de Engenharia Elétrica. IV. Título. 


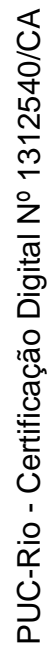

This work is dedicated to the memory of Carmelina Argenta Simon. 


\section{Acknowledgements}

First, I would like to thank my advisor and mentor, Professor José Ricardo Bergmann, for the support, encouragement and decisive guidance he has provided me in the last years at PUC-Rio. His philosophia vitæ will reverberates on many aspects of my future academic career and personality.

I am grateful to my co-advisor, Professor Fernando Lisboa Teixeira, who provided insight and expertise that greatly assisted me during this work.

I would like to thank to the members of my doctoral committee, Professors Flavio José Vieira Hasselmann, Adaildo Gomes D'Assunção, Cássio Gonçalves do Rego, Sandro Rogério Zang and Emanoel Paiva de Oliveira Costa for their helpful discussions and suggestions.

I also like to thank all professors who taught me the fascinating aspects of the Electromagnetic Theory.

Special thanks to colleagues at PUC-Rio and OSU for their friendship and many valuable technical discussions.

I thank to PUC-Rio for the exemption of school fees offered by the Programa de Pós-Graduação em Engenharia Elétrica.

Finally, I wish to thank my family for endless support.

This work was supported in part by the Brazilian Agency CNPq under Grants 140236/2013-9 and 141847/2016-6, and in part by the Brazilian Agency CAPES under Grant 000316/2015-06. 


\section{Abstract}

Simon da Rosa, Guilherme; Bergmann, José Ricardo (Advisor); Lisboa Teixeira, Fernando (Co-Advisor). Pseudo-Analytical Modeling for Electromagnetic Well-Logging Tools in Complex Geophysical Formations. Rio de Janeiro, 2017. 299p. Tese de Doutorado - Departamento de Engenharia Elétrica, Pontifícia Universidade Católica do Rio de Janeiro.

This research presents a study on numerical techniques to model the electromagnetic propagation in geophysical formations commonly encountered in oil well drilling. The employment of electromagnetic sensors surrounding the drill bit allows inferring the constitutive parameters of the soil around the well. In recent years, advances in electromagnetic logging technology have enabled the real-time modeling of this problem. In this way, the drilling direction can be guided in order to maximize the exploitation of oil, gas, and other fossil hydrocarbons. The complex geophysical formations that are prevalent in this type of problem can be effectively handled using brute-force numerical techniques such as finite-differences, finite-elements and finite-volumes. However, these techniques suffer from relatively high cost in terms of both computer memory and CPU time. The advancement of real-time logging technology demands approaches that are more efficient than purely numerical methods. In this work, we employ the mode-matching technique combining attractive features of the well-known pseudo-analytical approaches to obtain a new technique for analyzing directional well-logging tools in anisotropic formations with both radial and axial stratifications. The proposed technique allows to model problems not yet explored, but with a strong technological motivation, such as electromagnetic propagation along curved wells and drilling along inclined layers. We present a series of validation results showing that the novel technique introduced in this study can model accurately and efficiently electromagnetic logging sensors used in oil and gas exploration.

\section{Keywords}

Well-logging sensors; Mode matching methods; Anisotropic media; Multilayered media. 


\section{Resumo}

Simon da Rosa, Guilherme; Bergmann, José Ricardo; Lisboa Teixeira, Fernando. Modelagem Pseudoanalítica para Ferramentas de Perfilagem Eletromagnética em Formações Geofísicas Complexas. Rio de Janeiro, 2017. 299p. Tese de Doutorado - Departamento de Engenharia Elétrica, Pontifícia Universidade Católica do Rio de Janeiro.

Esta tese apresenta um estudo sobre técnicas de modelagem numérica utilizadas na análise da propagação eletromagnética em formações geofísicas comumente encontradas na perfuração de poços de petróleo. O emprego de sensores eletromagnéticos adjacentes à broca de perfuração permite a inferência dos parâmetros constitutivos do solo ao redor do poço. Nos últimos anos, os avanços da tecnologia de perfilagem eletromagnética permitiram a modelagem em tempo real do problema, possibilitando direcionar a perfuração do poço a fim de maximizar a exploração de petróleo, gás, e outros hidrocarbonetos fósseis. Formações geofísicas complexas são predominantes neste tipo de problema, e geralmente são modeladas usando técnicas numéricas de força bruta como os métodos de diferenças finitas, dos elementos finitos ou dos volumes finitos. No entanto, estas técnicas têm um custo computacional relativamente alto em termos de memória e tempo de processamento. $\mathrm{O}$ avanço da tecnologia de perfilagem em tempo real requer abordagens mais eficientes. Neste trabalho nós empregamos o método do casamento de modos combinado com uma série de características positivas dos métodos pseudoanalíticos conhecidos na literatura para obter uma técnica inédita que permite analisar poços direcionais com estratificações radiais e longitudinais em formações geofísicas anisotrópicas. A técnica proposta permite modelar problemas ainda não explorados, mas com motivação tecnológica iminente, como a propagação eletromagnética ao longo de poços curvados e a perfuração em camadas inclinadas em relação ao eixo axial do poço. Nós apresentamos uma série de resultados de validação que demonstram que a técnica introduzida neste trabalho pode modelar de forma acurada e eficiente sensores de perfilagem eletromagnética usados na exploração de petróleo e gás.

\section{Palavras-chave}

Sensores de prospecção de petróleo; Métodos de casamento de modos; Meios anisotrópicos; Meios estratificados. 


\section{Table of contents}

1 Introduction $\quad 23$

1.1 General Introduction 23

1.2 Organization of the Dissertation 24

2 Well-Logging Tools and its Environment $\quad 26$

2.1 Introduction 26

2.2 Electromagnetic Well-Logging Sensors 27

2.3 Directional Wells 29

2.4 On the Problem Statement and the Proposed Solution 31

3 Analysis of Well-Logging Tools in Vertical Wells 36

3.1 Introduction 36

3.2 Electromagnetic Fields in Cylindrical Structures 39

3.3 Fields Along Radial Stratifications $\quad 45$

3.4 Fields Along Axial Stratifications 65

3.5 Mode Excitation from Sources Inside a Cylindrical Waveguide $\quad 75$

3.6 Flowchart for the Proposed Technique $\quad 89$

3.7 Numerical Results and Validation 90

3.8 Comparison of the Proposed Method Versus the R-NMM 127

3.9 Modeling of LWD Sensors Inside Mandrel Grooves 135

3.10 Some Investigations on Wireless Telemetry for Oilfield Applications 144

$\begin{array}{ll}3.11 \text { Preliminary Conclusion } & 153\end{array}$

4 Analysis of Well-Logging Tools in Directional Wells 154

$\begin{array}{lll}4.1 & \text { Introduction } & 154\end{array}$

4.2 Electromagnetic Fields in Toroidal Coordinates 157

4.3 Fields Along Radial Stratifications 193

$\begin{array}{lll}4.4 & \text { Fields Along Axial Stratifications } & 199\end{array}$

4.5 Mode Excitation from Sources Inside a Toroidal Waveguide 205

$\begin{array}{lll}4.6 & \text { Numerical Results and Validation } & 215\end{array}$

$\begin{array}{lll}4.7 & \text { Preliminary Conclusion } & 247\end{array}$

5 Dipping-Bed Boundaries: A Mathematical Description 248

$\begin{array}{ll}5.1 \text { Introduction } & 248\end{array}$

5.2 Mode-Matching along Oblique Surfaces 248

$\begin{array}{ll}5.3 \text { Preliminary Conclusion } & 257\end{array}$

6 Conclusions and Suggestions for Future Research 258

$\begin{array}{ll}\text { Bibliography } & 261\end{array}$

A Wave Equation in Cylindrical Coordinates $\quad 275$

B Modal Amplitudes from the Transmitter to the Receiver 278

B.1 Fields Matching Approach 278 
B.2 Geometrical Optics Approach 287

B.3 Comparison of Formulations 295

C Local Toroidal Coordinate System $\quad 297$

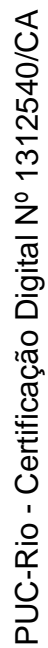




\section{List of figures}

2.1 Geometry of a triaxial TCA well-logging tool (antennas TX, RX and $\mathrm{RX}_{2}$ ) within a stratified geophysical formation.

2.2 PeriScope 15 from Schlumberger. This sensor operates at $2 \mathrm{MHz}$ and is comprised by 6 horizontal-coil transmitter antennas and 4 receiver antennas ( 2 horizontal-coils and 2 tilted-coils) that provide the azimuthal sensibility. Image obtained in [1, Fig. 3].

2.3 ADR sensor from Halliburton. This is a multi-frequency sensor that operates at $125 \mathrm{kHz}, 500 \mathrm{kHz}$ and $2 \mathrm{MHz}$, and is comprised by 6 horizontal-coil transmitter antennas and 3 tilted-coil receiver antennas that provide the azimuthal sensibility. Image obtained in [2, Fig. 3].

2.4 Several wells drilled from the same platform.

2.6 Long-, medium- and short-radius directional wells classified according its drilling build rates. This image is based on an illustration shown in [3, p. 24].

2.7 A typical planed well hole profile with directional drilling bending in both horizontal and vertical directions. This image is based on an illustration shown in [4, Fig. 7].

2.8 Junction between a straight and a bent structure (a), and the attempts to define a R-NMM in (b) and an A-NMM in (c).

2.9 Radially-stratified and axially-layered structures with constant axial curvature.

3.1 Geometry of a triaxial TCA well-logging tool within a stratified geophysical formation.

3.2 Longitudinal and cross-sectional views of a radially-stratified waveguide. Hard walls are depicted by the diagonal stripes.

3.3 Integration paths on the complex plane $k_{z}$.

3.5 Longitudinal view of the junction between two semi-infinitely-long and radially-stratified waveguides.

3.6 Geometry of a stratified cylindrical structure. The cross-section of each region is formed by an arbitrary number of layers. A transmitting TCA is placed at $z=z_{T}$, in region $m$, and the desired receiver antenna is located at $z=z_{R}$, in region $n$.

3.7 Sources in an axially-infinitely-long and radially-stratified waveguide. 76

3.8 Transmitting tilted-coil antenna with current density $\mathbf{J}_{T}$.

3.9 Orthogonal views of a transmitting tilted-coil antenna crossing the junction at $z=z_{1}$. In order to simplify the graphical representation, we use $z_{T}=0$ and $\phi_{T}=0$.

3.10 Flowchart for the algorithm of the proposed technique.

3.11 Geometry of a triaxial well-logging tool within a stratified geophysical formation. 
3.12 Voltage amplitude and phase received by antenna $\mathrm{RX}_{1}$. The response for $r_{N}=r_{N-1}+\alpha \delta_{N, \max }, \alpha=0.25$ (dashed-line) to $0.75 \mathrm{in}$, with steps of $0.125 \mathrm{in}$. Vertical arrows indicate the convergence sense.

3.13 Voltage amplitude and phase received by antenna $\mathrm{RX}_{2}$. The responses for $r_{N}=r_{N-1}+\alpha \delta_{N, \max }, \alpha=0.25$ (dashed-line) to 0.75 , with steps of 0.125 are shown. Vertical arrows indicate the convergence sense.

3.14 Relative amplitude voltage received by two antennas moving with one transmitter antenna across a bed layer ( $z=0$ to $z=60$ in). The responses for $r_{N}=r_{N-1}+\alpha \delta_{N, \max }, \alpha=0.25$ (dashed-line) to 0.75 , with steps of 0.125 are shown. Vertical arrows indicate the convergence sense.

3.15 Relative phase voltage received by two antennas moving with one transmitter antenna across a bed layer ( $z=0$ to $z=60$ in). The responses for $r_{N}=r_{N-1}+\alpha \delta_{N, \max }, \alpha=0.25$ (dashed-line) to 0.75 , with steps of 0.125 are shown. Vertical arrows indicate the convergence sense.

3.16 Voltage ratio for a triaxial LWD logging tool operating at $500 \mathrm{kHz}$ and $2 \mathrm{MHz}$. The results from the present method are shown by solid lines. The small circles are FDTD results from $[5,6]$.

3.17 Convergence of the voltage amplitude ratio for $A_{d B}$ at $\Delta z=5$ in. The response for five values of $A_{d B}$ (from $-10 \mathrm{~dB}$ to $-30 \mathrm{~dB}$, with steps of $5 \mathrm{~dB}$ ) are presented. Vertical arrows indicate the convergence sense.

3.18 Voltage ratio and phase difference for a triaxial LWD logging tool traversing a bed layer with and without invasion. The results from the present algorithm are indicated by solid lines. The small circles and crosses are FV results from [7].

3.19 Geometry of a triaxial well-logging tool within a stratified geophysical formation. A 10-in-invasion zone is present in formation 2.

3.20 Geometry of a triaxial TCA well-logging tool within a stratified geophysical formation.

3.21 Voltage at TCA receiver $\mathrm{RX}_{1}$ with $\theta_{R}=10^{\circ}$ due to a unit current excitation transmitting TCA with $\theta_{T}=45^{\circ}$. The results from the present algorithm are indicated by solid lines. The small circles and dots are FDTD and R-NMM results, respectively, from [6, pp. 106107].

3.22 Voltage at TCA receiver $\mathrm{RX}_{2}$ with $\theta_{R}=10^{\circ}$ due to a unit current excitation transmitting TCA with $\theta_{T}=45^{\circ}$. The results from the present algorithm are indicated by solid lines. The small circles and dots are FDTD and R-NMM results, respectively, from [6, pp. 106107]. 
3.23 Voltage at TCA receiver $\mathrm{RX}_{1}$ with $\theta_{R}=20^{\circ}$ due to a unit current excitation transmitting TCA with $\theta_{T}=45^{\circ}$. The results from the present algorithm are indicated by solid lines. The small circles and dots are FDTD and R-NMM results, respectively, from [6, pp. 106107].

3.24 Voltage at TCA receiver $\mathrm{RX}_{2}$ with $\theta_{R}=20^{\circ}$ due to a unit current excitation transmitting TCA with $\theta_{T}=45^{\circ}$. The results from the present algorithm are indicated by solid lines. The small circles and dots are FDTD and R-NMM results, respectively, from [6, pp. 106107].

3.25 Voltage at TCA receiver $\mathrm{RX}_{1}$ with $\theta_{R}=30^{\circ}$ due to a unit current excitation transmitting TCA with $\theta_{T}=45^{\circ}$. The results from the present algorithm are indicated by solid lines. The small circles and dots are FDTD and R-NMM results, respectively, from [6, pp. 106107].

3.26 Voltage at TCA receiver $\mathrm{RX}_{2}$ with $\theta_{R}=30^{\circ}$ due to a unit current excitation transmitting TCA with $\theta_{T}=45^{\circ}$. The results from the present algorithm are indicated by solid lines. The small circles and dots are FDTD and R-NMM results, respectively, from [6, pp. 106107].

3.27 Voltage at TCA receiver $\mathrm{RX}_{1}$ with $\theta_{R}=40^{\circ}$ due to a unit current excitation transmitting TCA with $\theta_{T}=45^{\circ}$. The results from the present algorithm are indicated by solid lines. The small circles and dots are FDTD and R-NMM results, respectively, from [6, pp. 106107].

3.28 Voltage at TCA receiver $\mathrm{RX}_{2}$ with $\theta_{R}=40^{\circ}$ due to a unit current excitation transmitting TCA with $\theta_{T}=45^{\circ}$. The results from the present algorithm are indicated by solid lines. The small circles and dots are FDTD and R-NMM results, respectively, from [6, pp. 106107].

3.29 Amplitude of the received voltages $V_{R 1}$ and $V_{R 2}$ for $\theta_{R}=10^{\circ}$ in terms of its azimuthal contributions. The total voltages are shown in dashed lines.

3.30 Amplitude of the received voltages $V_{R 1}$ and $V_{R 2}$ for $\theta_{R}=20^{\circ}$ in terms of its azimuthal contributions. The total voltages are shown in dashed lines.

3.31 Amplitude of the received voltages $V_{R 1}$ and $V_{R 2}$ for $\theta_{R}=30^{\circ}$ in terms of its azimuthal contributions. The total voltages are shown in dashed lines.

3.32 Amplitude of the received voltages $V_{R 1}$ and $V_{R 2}$ for $\theta_{R}=40^{\circ}$ in terms of its azimuthal contributions. The total voltages are shown in dashed lines.

3.33 Convergence of the voltage amplitude and phase received by antenna $\operatorname{RX}_{2}\left(\theta_{R}=40^{\circ}\right)$ for $A_{d B}$ at $\Delta z=5$ in. The response from six values of $A_{d B}$ (from $-10 \mathrm{~dB}$ to $-60 \mathrm{~dB}$, with steps of $10 \mathrm{~dB}$ ) are presented. 
3.34 Convergence of the voltage amplitude and phase received by antenna $\operatorname{RX}_{2}\left(\theta_{R}=40^{\circ}\right)$ for $A_{d B}$ at $\Delta z=5$ in. The response for five values of $A_{d B}$ (from $-10 \mathrm{~dB}$ to $-20 \mathrm{~dB}$, with steps of $2.5 \mathrm{~dB}$ ) are presented. Vertical arrows indicate the convergence sense.

3.35 Geometry of a triaxial TCA well-logging tool within an anisotropic stratified geophysical formation.

3.36 Voltage at TCA receiver $\mathrm{RX}_{1}$ with $\theta_{R}=15^{\circ}$ due to a unit current excitation transmitting TCA with $\theta_{T}=45^{\circ}$. The results from the present algorithm are indicated by solid lines. The small circles and dots are FDTD and R-NMM results, respectively, from [6, pp. 108109], [8].

3.37 Voltage at TCA receiver $\mathrm{RX}_{2}$ with $\theta_{R}=15^{\circ}$ due to a unit current excitation transmitting TCA with $\theta_{T}=45^{\circ}$. The results from the present algorithm are indicated by solid lines. The small circles and dots are FDTD and R-NMM results, respectively, from [6, pp. 108109], [8].

3.38 Voltage at TCA receiver $\mathrm{RX}_{1}$ with $\theta_{R}=25^{\circ}$ due to a unit current excitation transmitting TCA with $\theta_{T}=45^{\circ}$. The results from the present algorithm are indicated by solid lines. The small circles and dots are FDTD and R-NMM results, respectively, from [6, pp. 108109], [8].

3.39 Voltage at TCA receiver $\mathrm{RX}_{2}$ with $\theta_{R}=25^{\circ}$ due to a unit current excitation transmitting TCA with $\theta_{T}=45^{\circ}$. The results from the present algorithm are indicated by solid lines. The small circles and dots are FDTD and R-NMM results, respectively, from [6, pp. 108109], [8].

3.40 Voltage at TCA receiver $\mathrm{RX}_{1}$ with $\theta_{R}=35^{\circ}$ due to a unit current excitation transmitting TCA with $\theta_{T}=45^{\circ}$. The results from the present algorithm are indicated by solid lines. The small circles and dots are FDTD and R-NMM results, respectively, from [6, pp. 108109], [8].

3.41 Voltage at TCA receiver $\mathrm{RX}_{2}$ with $\theta_{R}=35^{\circ}$ due to a unit current excitation transmitting TCA with $\theta_{T}=45^{\circ}$. The results from the present algorithm are indicated by solid lines. The small circles and dots are FDTD and R-NMM results, respectively, from [6, pp. 108109], [8].

3.42 Voltage at TCA receiver $\mathrm{RX}_{1}$ with $\theta_{R}=45^{\circ}$ due to a unit current excitation transmitting TCA with $\theta_{T}=45^{\circ}$. The results from the present algorithm are indicated by solid lines. The small circles and dots are FDTD and R-NMM results, respectively, from [6, pp. 108109], [8].

3.43 Voltage at TCA receiver $\mathrm{RX}_{2}$ with $\theta_{R}=45^{\circ}$ due to a unit current excitation transmitting TCA with $\theta_{T}=45^{\circ}$. The results from the present algorithm are indicated by solid lines. The small circles and dots are FDTD and R-NMM results, respectively, from [6, pp. 108109], [8]. 
3.44 Voltage amplitude in decibel, $20 \log _{10}\left(\operatorname{abs}\left(V_{R 2}\right)\right)$, at TCA receiver $\mathrm{RX}_{2}$ due a transmitter TCA with tilt angle of $45^{\circ}$. The contribution of the azimuthal harmonics $n=0,1,2$ and 3 are depicted.

3.45 Geometry of a triaxial LWD tool (antennas TX, RX 1 and $\mathrm{RX}_{2}$ ) within a stratified geophysical formation.

3.46 Geometry of a triaxial well-logging tool within the geophysical formation for the case 1 .

3.47 Voltage amplitude ratio and phase difference for the triaxial LWD tool depicted in case 1 (see Fig. 3.46) as a function of the invasion thickness $d$. The results from our (A-NMM-based) algorithm and from the R-NMM [9] are indicated by solid lines and small circles, respectively.

3.48 Geometry of a triaxial well-logging tool within the geophysical formation for the case 2.

3.49 Voltage amplitude ratio and phase difference for a triaxial LWD tool traversing the bed layers depicted in case 2 (see Fig. 3.48) for several bed layer thickness $h$. The results from our (A-NMM-based) algorithm and from the R-NMM [9] are indicated by solid lines and small circles, respectively.

3.50 Geometry of a triaxial well-logging tool within the geophysical formation for the case 3 .

3.51 Voltage amplitude ratio and phase difference for a triaxial LWD tool traversing the bed layers depicted in case 3 (see Fig. 3.50). The results from our (A-NMM-based) algorithm and from the RNMM [9] are indicated by solid lines and small circles, respectively.

3.52 Side view of a coil antenna (depicted by $\otimes$ ) placed within a groove on the metallic mandrel.

3.53 LWD tool for different groove heights.

3.54 Convergence of the voltage amplitude ratio received by a LWD tool whose antennas are placed inside grooves $(d=h=1$ in) on the conductor mandrel for $A_{d B}$ at $\Delta z=0.5 \mathrm{in}$. The radial domain is truncated at $r_{N}=r_{N-1}+\alpha \delta_{N}, \alpha=2,3,4$ and 5 .

3.55 Convergence of the voltage phase difference received by a LWD tool whose antennas are placed inside grooves $(d=h=1$ in) on the conductor mandrel for $A_{d B}$ at $\Delta z=0.5 \mathrm{in}$. The radial domain is truncated at $r_{N}=r_{N-1}+\alpha \delta_{N}, \alpha=2,3,4$ and 5 .

3.56 Voltage amplitude ratio and phase difference received by a LWD tool whose antennas are placed inside grooves on the conductor mandrel. Horizontal dashed lines indicate two relevant groove height: $h=6$ in and 24 in.

3.57 Model employed to simulate the mandrel indentations. The details of the antennas positions and the dimensions of grooves on mandrel are shown on the right, in which the TCAs have $\rho_{T}=\rho_{R 1}=\rho_{R 2}=$ 3 in.

3.58 Voltage at TCA receiver $\mathrm{RX}_{1}$ for various values of $\theta_{T}$ and $\theta_{R 1}$.

3.59 Voltage at TCA receiver $\mathrm{RX}_{2}$ for various values of $\theta_{T}$ and $\theta_{R 2}$.

3.60 Amplitude ratio and phase difference response for a transmitter TCA with $\theta_{T}=0$ in respect to the tilt angle of receivers. 
3.61 Amplitude ratio and phase difference response for a transmitter TCA with $\theta_{T}=15^{\circ}$ in respect to the tilt angle of receivers.

3.62 Amplitude ratio and phase difference response for a transmitter TCA with $\theta_{T}=30^{\circ}$ in respect to the tilt angle of receivers.

3.63 Amplitude ratio and phase difference response for a transmitter TCA with $\theta_{T}=45^{\circ}$ in respect to the tilt angle of receivers.

3.64 Sectional view of an oil well using wireless telemetry.

3.65 Influence of the oil-based mud conductivities $(\sigma$, given in $\mathrm{S} / \mathrm{m})$ in the attenuation of the received signal along the axial distance from the source.

3.66 Transversal magnetic field $H_{\phi}$ of the modes $A$ and $B$. The axial attenuation constant $\alpha=\Im m\left(k_{z}\right)$ of each mode is presented. Metallic pipe layers are shaded.

3.67 Influence of the soil formations conductivities $\left(\sigma_{\# 1}, \sigma_{\# 2}\right)$ in the attenuation of the received signal along the axial distance from the source. The annulus has high losses $(\sigma=10 \mathrm{~S} / \mathrm{m})$.

3.68 Relative voltage received by two antennas moving with one transmitter antenna across a bed boundary. The results of our approach are depicted by the solid (for amplitude) and dashed (for phase) lines. The small dots (• for amplitude and $\circ$ for phase) are the results from [10] (from graphical reading).

3.69 Relative voltage received by two antennas moving with one transmitter antenna across a bed boundary. The results of our approach are depicted by the solid lines. The small dots are the results from [10].

3.70 Normalized modal $E_{\phi}$ fields for $25 \mathrm{MHz}$ (dashed line) and for $200 \mathrm{MHz}$ (solid line). The value of $\Im m\left(k_{z, 0 p}\right)$ is shown for each field.

3.71 Eigenvalues $k_{1 \rho}^{2}$ for $1 \mathrm{GHz}$ hybrid $\mathrm{HE}_{1 p}$ and $\mathrm{EH}_{1 p}$ modes for a 2-mradius tunnel, with $\epsilon_{1\{s, z\}}=\epsilon_{0}$ and $\epsilon_{2\{s, z\}}=(10+i) \epsilon_{0}$. There is branch-cut associated to the unbounded problem, and it is depicted by the horizontal dashed-line.

3.72 Axial electric field of the mode $A\left(k_{1 \rho}=-1.1968+i 0.0523\right)$ for the unbounded $\left(r_{N} \rightarrow \infty\right)$ waveguide, and for the bounded PML $1\left(\tilde{r}_{N}=3+i 0.03 \mathrm{~m}\right)$ and PML $2\left(\tilde{r}_{N}=3+i 0.3 \mathrm{~m}\right)$.

3.73 Comparison of the electric field along the axial distance. The results of our approach are depicted by the solid line. The small dots are the results from [11]. The free-space impedance is $Z_{0}$.

3.74 Comparison of the electric field along the axial distance. The results of our approach are depicted by the solid line. The small dots are the results from [12].

4.1 Geometry of vertical and directional wells.

4.2 Geometry of a well-logging sensor within a directional well. An auxiliary coordinate system is presented.

4.3 Cylindrical $(\rho, \phi, z)$ and toroidal $(\rho, \phi, \zeta)$ coordinate systems.

4.4 Toroidal coordinates $(\eta, \theta, \psi)$ [13, p. 113, Fig. 4.04]. The coordinate surfaces are toroids ( $\eta=$ const.), spherical bowls ( $\theta=$ const.) and half-planes ( $\psi=$ const.). 
4.5 Junction between two semi-infinitely-long waveguides.

4.6 Sources inside a radially-stratified and axially-toroidal waveguide.

4.7 Transmitting coil antenna with current density $\mathbf{J}_{T}$ placed at a plane of constant $\zeta$.

4.8 Axial propagation constants $k_{z, n p}$ for a cylindrical waveguide with $r_{0}=4$-in, $r_{1}=5$-in and $\tilde{r}_{N}=60$-in $+i 20$-in, where layer 1 has $\sigma_{1}=5 \times 10^{-4} \mathrm{~S} / \mathrm{m}$ and layer 2 has $\sigma_{2}=1 \mathrm{~S} / \mathrm{m}$.

4.9 Voltage amplitude received by a coil antenna located $\zeta_{R}-\zeta_{T}$ away from the source and inside a bent borehole with curvature $R^{-1}$.

4.10 Voltage phase received by a coil antenna located $\zeta_{R}-\zeta_{T}$ away from the source and inside a bent borehole with curvature $R^{-1}$.

4.11 Voltages at $\mathrm{RX}_{1}$. Zeroth-order results are shown in dashed-line. The combination of the zeroth- and first-order perturbation corrections are indicated by solid lines. The small circles (for $R=400$ in) and crosses (for $R=200$ in) are FDTD results.

4.12 Voltages at $\mathrm{RX}_{2}$. Zeroth-order results are shown in dashed-line. The combination of the zeroth- and first-order perturbation corrections are indicated by solid lines. The small circles (for $R=400$ in) and crosses (for $R=200$ in) are FDTD results.

4.13 Schematic used in the CST modeling in the case $R=200 \mathrm{in}$. The spacial domain is limited by a parallelepiped with dimension $\Delta X \times$ $\Delta Y \times \Delta Z$ on the global Cartesian coordinate system $(X, Y, Z)$ using a PEC boundary condition. To improve the visualization of small details, the aspect ratio of coordinates $X / Y$ and $Z / Y$ is 2 by 1 . The center of curvature points to the negative $X$-axis.

4.14 Voltage at $\mathrm{RX}_{2}$ for $R=200$ in. Zeroth-order results are shown in dashed-lines. The combination of the zeroth- and 1st-order perturbation corrections are indicated by solid lines. The small dots, crosses, and triangles are FDTD results for simulations 12, 13, and 14 , respectively.

4.15 Schematics used in the CST modeling. The aspect ratio of horizontal $(X)$ and vertical $(Y)$ coordinates is 1 by 1 . In all cases, the center of curvature points to the left side of the page.

4.16 Voltages at $\mathrm{RX}_{2}$ for $R=200$ in.

4.17 Voltages at $\mathrm{RX}_{2}$ for $R=200 \mathrm{in}$; Zoom near $1.75 \mathrm{MHz}$.

5.1 Illustration of a LWD tool in a dipping bed. (a) Geometry of the triaxial coil LWD tool. (b) A simplified model of the tool using magnetic dipole sources in the absence of mandrel and borehole.

5.2 Waveguide junctions at oblique surfaces.

6.1 Geometry of LWD sensor inside a misaligned borehole.

B.1 The axially-stratified structure under consideration.

B.2 Description of the algorithm used to calculate the amplitudes of the forward and backward waves at the observation point.

B.3 Algorithm used to calculate $\hat{\bar{C}}_{m}$ and $\hat{\bar{D}}_{m}$. 
B.4 Algorithm used to calculate the amplitudes of the forward and backward waves at $z=z_{R}$ (in region $n$ ) due to a source at $z=z_{T}$ (in region $m$ ), for $m<n$.

B.5 Algorithm used to calculate the amplitudes of the forward and backward waves at $z=z_{R}$ (in region $n$ ) due to a source at $z=z_{T}$ (in region $m$ ), for $m>n$.

B.6 Algorithm used to calculate the amplitudes of the forward and backward waves at $z=z_{R}$ due to a source at $z=z_{T}$ when these positions are in region $m=n$.

B.7 The source and the observation point in the region $m=n$.

B.8 The contribution of the forward source amplitudes to the received fields in the forward direction.

B.9 The contribution of the backward source amplitudes to the received fields in the forward direction.

B.10 The contribution of the forward source amplitudes to the received fields in the backward direction.

B.11 The contribution of the backward source amplitudes to the received fields in the backward direction.

C.1 Geometry of a Torus and its coordinate systems. 


\section{List of tables}

2.1 Horizontal-well classifications (data from [3, p. 24]) 31

3.1 Number of modes for $A_{d B}$ at 5 in 93

3.2 Number of modes for $A_{d B}$ at 5 in 116

3.3 Dimensions and parameters of the cased oil well. 146

4.1 Number of boundary conditions $d$

4.2 Axial field patterns for the mode \#1 (for $n=0$ ) in a toroidal waveguide with $\tilde{r}_{N}=60$-in $+i 20$-in as a function of the radius of curvature $R$. The PML layer is place at $\rho>40$-in and its inner boundary is indicated by the dashed-circles. The center of curvature points to positive $x$-axis.

4.3 Axial field patterns for the mode \#2 (for $n=0$ ) in a toroidal waveguide with $\tilde{r}_{N}=60$-in $+i 20$-in as a function of the radius of curvature $R$. The PML layer is place at $\rho>40$-in and its inner boundary is indicated by the dashed-circles. The center of curvature points to positive $x$-axis.

4.4 Axial field patterns for the mode \#3 (for $n=0$ ) in a toroidal waveguide with $\tilde{r}_{N}=60$-in $+i 20$-in as a function of the radius of curvature $R$. The PML layer is place at $\rho>40$-in and its inner boundary is indicated by the dashed-circles. The center of curvature points to positive $x$-axis.

4.5 Axial field patterns for the mode $\# 4$ (for $n=0$ ) in a toroidal waveguide with $\tilde{r}_{N}=60$-in $+i 20$-in as a function of the radius of curvature $R$. The PML layer is place at $\rho>40$-in and its inner boundary is indicated by the dashed-circles. The center of curvature points to positive $x$-axis.

4.6 Axial field patterns for the mode $\# 5$ (for $n=0$ ) in a toroidal waveguide with $\tilde{r}_{N}=60$-in $+i 20$-in as a function of the radius of curvature $R$. The PML layer is place at $\rho>40$-in and its inner boundary is indicated by the dashed-circles. The center of curvature points to positive $x$-axis.

4.7 Axial field patterns for the mode \#6 (for $n=+1$ ) in a toroidal waveguide with $\tilde{r}_{N}=60$-in $+i 20$-in as a function of the radius of curvature $R$. The PML layer is place at $\rho>40$-in and its inner boundary is indicated by the dashed-circles. The center of curvature points to positive $x$-axis.

4.8 Axial field patterns for the mode $\# 7$ (for $n=+1$ ) in a toroidal waveguide with $\tilde{r}_{N}=60$-in $+i 20$-in as a function of the radius of curvature $R$. The PML layer is place at $\rho>40$-in and its inner boundary is indicated by the dashed-circles. The center of curvature points to positive $x$-axis. 
4.9 Axial field patterns for the mode \#8 (for $n=+1$ ) in a toroidal waveguide with $\tilde{r}_{N}=60$-in $+i 20$-in as a function of the radius of curvature $R$. The PML layer is place at $\rho>40$-in and its inner boundary is indicated by the dashed-circles. The center of curvature points to positive $x$-axis.

4.10 Axial field patterns for the mode \#9 (for $n=+1$ ) in a toroidal waveguide with $\tilde{r}_{N}=60$-in $+i 20$-in as a function of the radius of curvature $R$. The PML layer is place at $\rho>40$-in and its inner boundary is indicated by the dashed-circles. The center of curvature points to positive $x$-axis.

4.11 Axial field patterns for the mode $\# 10$ (for $n=+1$ ) in a toroidal waveguide with $\tilde{r}_{N}=60$-in $+i 20$-in as a function of the radius of curvature $R$. The PML layer is place at $\rho>40$-in and its inner boundary is indicated by the dashed-circles. The center of curvature points to positive $x$-axis.

4.12 Azimuthal electric field patterns for the mode \#1 (for $n=0$ ) in a toroidal waveguide with $\tilde{r}_{N}=60$-in $+i 20$-in as a function of the radius of curvature $R$. The borehole-to-formation and the PML inner boundaries are indicated by the dashed-circles. The center of curvature points to positive $x$-axis.

4.13 Azimuthal electric field patterns for the mode \#2 (for $n=0$ ) in a toroidal waveguide with $\tilde{r}_{N}=60$-in $+i 20$-in as a function of the radius of curvature $R$. The borehole-to-formation and the PML inner boundaries are indicated by the dashed-circles. The center of curvature points to positive $x$-axis.

4.14 Azimuthal electric field patterns for the mode \#3 (for $n=0$ ) in a toroidal waveguide with $\tilde{r}_{N}=60$-in $+i 20$-in as a function of the radius of curvature $R$. The borehole-to-formation and the PML inner boundaries are indicated by the dashed-circles. The center of curvature points to positive $x$-axis.

4.15 Azimuthal electric field patterns for the mode \#4 (for $n=0$ ) in a toroidal waveguide with $\tilde{r}_{N}=60$-in $+i 20$-in as a function of the radius of curvature $R$. The borehole-to-formation and the PML inner boundaries are indicated by the dashed-circles. The center of curvature points to positive $x$-axis.

4.16 Azimuthal electric field patterns for the mode \#5 (for $n=0$ ) in a toroidal waveguide with $\tilde{r}_{N}=60$-in $+i 20$-in as a function of the radius of curvature $R$. The borehole-to-formation and the PML inner boundaries are indicated by the dashed-circles. The center of curvature points to positive $x$-axis.

4.17 Azimuthal electric field patterns for the mode \#6 (for $n=+1$ ) in a toroidal waveguide with $\tilde{r}_{N}=60$-in $+i 20$-in as a function of the radius of curvature $R$. The borehole-to-formation and the PML inner boundaries are indicated by the dashed-circles. The center of curvature points to positive $x$-axis. 
4.18 Azimuthal electric field patterns for the mode $\# 7$ (for $n=+1$ ) in a toroidal waveguide with $\tilde{r}_{N}=60$-in $+i 20$-in as a function of the radius of curvature $R$. The borehole-to-formation and the PML inner boundaries are indicated by the dashed-circles. The center of curvature points to positive $x$-axis.

4.19 Azimuthal electric field patterns for the mode \#8 (for $n=+1$ ) in a toroidal waveguide with $\tilde{r}_{N}=60$-in $+i 20$-in as a function of the radius of curvature $R$. The borehole-to-formation and the PML inner boundaries are indicated by the dashed-circles. The center of curvature points to positive $x$-axis.

4.20 Azimuthal electric field patterns for the mode \#9 (for $n=+1$ ) in a toroidal waveguide with $\tilde{r}_{N}=60$-in $+i 20$-in as a function of the radius of curvature $R$. The borehole-to-formation and the PML inner boundaries are indicated by the dashed-circles. The center of curvature points to positive $x$-axis.

4.21 Azimuthal electric field patterns for the mode $\# 10$ (for $n=+1$ ) in a toroidal waveguide with $\tilde{r}_{N}=60$-in $+i 20$-in as a function of the radius of curvature $R$. The borehole-to-formation and the PML inner boundaries are indicated by the dashed-circles. The center of curvature points to positive $x$-axis. 


\section{List of Principal Symbols and Acronyms}

The following is a list of the principal symbols and the notation used throughout the dissertation. Other symbols are also defined within the text.

$2 \mathrm{D}$ and $3 \mathrm{D}$

The two- and three-dimensional spaces, respectively.

A-NMM and R-NMM Axial and radial versions of the Numerical ModeMatching technique, respectively.

$\mathrm{AR}$ and $\mathrm{PD}$

Amplitude ratio and phase difference of the voltages received by a pair of antennas, respectively.

$\hat{\alpha}$

A unit vector pointing to direction $\alpha$.

$\exp (-i \omega t)$

Time-harmonic dependence for the electromagnetic vector phasors. The time and angular frequency are depicted by $t$ and $\omega$, respectively.

FDs, FEs and FVs

Brute-force numerical techniques: finite differences, finite elements and finite volumes, respectively.

FDTD

Finite-difference time-domain technique.

G, $G_{\alpha}$

GSM

A vector field and its component pointing to direction $\alpha$, respectively.

Generalized scattering matrix.

$i, j$

When not used as subscripts, they are the complex and the bi-complex imaginary unities, respectively, such that $i=j=(-1)^{1 / 2}$, but $i j \neq-1$.

LWD and MWD Logging-while-drilling and measurement-whiledrilling, respectively.

$\stackrel{\bar{M}}{\bar{V}}$

A matrix $M$ and a column-vector $V$, respectively.

$\overline{\bar{M}}^{t}, \overline{\bar{M}}^{-1}$

The transpose and the inverse of matrix $M$, respectively. 
NMM

PEC and PMC

PML

$R$

RHS and LHS

$\overline{\bar{R}}_{j, j \pm 1}^{(\rho)}, \overline{\bar{T}}_{j, j \pm 1}^{(\rho)}$

$\tilde{\overline{\bar{R}}}_{j, j \pm 1}^{(\rho)}, \tilde{\overline{\bar{T}}}_{j, j \pm 1}^{(\rho)}$

$\overline{\bar{R}}_{j, j \pm 1}^{(\zeta)}, \overline{\bar{T}}_{j, j \pm 1}^{(\zeta)}$

$\tilde{\overline{\bar{R}}}_{j, j \pm 1}^{(\zeta)}, \tilde{\overline{\bar{T}}}_{j, j \pm 1}^{(\zeta)}$

TCA

$\mathrm{TE}_{n p}^{z}, \mathrm{TM}_{n p}^{z}$ and $\mathrm{TEM}^{z}$

$\mathrm{TX}$ and $\mathrm{RX}_{q}$

$X_{i(n p), j\left(n^{\prime} p^{\prime}\right)}$
Numerical mode-matching.

Perfect electric and perfect magnetic conductors, respectively.

Perfectly matched layer.

Radius of curvature for bent waveguides in toroidal coordinates.

The right- and left-hand side of an equation.

Local reflection and transmission matrix for radial layer $j$ to $j \pm 1$, respectively.

Generalized reflection and transmission matrix for radial layer $j$ to $j \pm 1$, respectively.

Local reflection and transmission matrix for axial region $j$ to $j \pm 1$, respectively. In cylindrical coordinates $\zeta \rightarrow z$.

Generalized reflection and transmission matrix for axial region $j$ to $j \pm 1$, respectively. In cylindrical coordinates $\zeta \rightarrow z$.

Tilted-coil antenna.

The npth modal field transversal electric, magnetic and electromagnetic with respect to axis $z$, respectively.

The transmitting antenna and the $q$ th receiving antenna, respectively.

The Reaction of the $n p$ th modal field in region $i$ to the $n^{\prime} p^{\prime}$ th modal field in region $j$. 


\section{Introduction}

\section{1}

\section{General Introduction}

Various numerical techniques have been used over the years for modeling logging-while-drilling (LWD) and measurement-while-drilling (MWD) tools used in hydrocarbon exploration. Analytical techniques were used in [14-16] to solve the problem of LWD sensors in layered formations. These techniques are numerically efficient, but appear restrict to the modeling of two-dimensional (2D) geophysical formations.

The complex formations that are prevalent in this type of problem can be handled using brute-force three-dimensional (3D) numerical techniques based on the spatial discretization such as finite-differences (FDs), finite-elements (FEs) and finite-volumes (FVs) [6,7,17-20].

The finite-difference time-domain (FDTD) or Yee's method [21] is a very popular technique for modeling the electromagnetic propagation in complex environments. Modern FDTD implementations use very effective absorbing boundary conditions to simulate an infinite unbounded computational domain, and this technique have been receiving a lot of attention for the modeling of electromagnetic LWD sensors. However, as all others brute-force approaches, FDTD suffer from relatively high cost in terms of both computer memory and CPU time.

An alternative way to solve 3D problems are the numerical modematching-based techniques, which can provide a middle-ground in terms of computational costs between brute-force and pseudo-analytical techniques [6, $8,10,15,22-32]$. Typically, these methods combine a 1D-FE solution in one coordinate direction with an analytical field expansion in the others transversal directions.

In typical computational electromagnetics (CEM) applications, we know the sources and the propagation media, such the direct problem can be promptly solved by means of Maxwell's equations. As the soil formation surrounding the sensor is unknown, the modeling of a LWD tool requires the solution of the inverse problem: we know the sources and by measurements of 
radiated fields, we can estimate the constitutive parameters of the media. In this process, the key point to success are fast and accurate CEM methods.

Since the frequency of operation and physical parameters (e.g. the resistivity of the Earth formation) in many well logging problems can vary by several orders of magnitude, it is important to ensure that the method of analysis is sufficiently robust to yield accurate solutions under a wide range of input parameters. In some circumstances, the computational cost required by brute-force techniques can become prohibitive. To handle this problem, we need to explore new semi-analytical approaches that are more efficient than purely numerical ones. With that in mind, the objectives of this research are: the development of new CEM techniques to accurately model typical scenarios of oil and gas exploration, including the modeling of complex directional wells. In addition, we want a robust algorithm to address the problem based on geometrical and physical interpretations in order to reveal directions for the inverse problem.

In this dissertation, we explore novel concepts of the axial mode-matching combining attractive features of the pseudo-analytical techniques, to obtain a new flexible and computationally efficient technique for analyzing directional well-logging tools in anisotropic formations which can be easily extended to modeling wells with curvature.

\section{2 \\ Organization of the Dissertation}

This dissertation is organized as follows. In Chapter 2, we present a brief description of well-logging sensors and the typical electromagnetic environment encountered in practical drilling prospecting. We present the state-of-art of electromagnetic LWD technologies provided by the top two world's largest oilfield service companies. The engineering solution of these sensors; such as the antennas geometry, operating frequencies, among others; will be explored in details. We also present several features and specifications of commercial resistivity LWD sensors employed in modern directional drilling. At the end of this chapter, we present the solution strategy we proposed to model the problem at hand.

In Chapter 3, we present an efficient mode-matching technique to analyze tilted-coil antennas (TCAs) in anisotropic geophysical formations. In this problem, a number of coil antennas with arbitrary relative tilt angle with respect to the symmetry axis are used to radiate electromagnetic fields in a cylindrically layered medium comprised of a metallic mandrel, a borehole, and a surrounding layered Earth formation. This configuration corresponds 
to that of directional well-logging tools used in oil and gas exploration. Our technique combines closed-form solutions for the Maxwell's equations in uniaxially anisotropic and radially-stratified cylindrical coordinates with the generalized scattering matrix (GSM) at each axial discontinuity based on the mode-matching technique. The field radiated by a transmitter TCA source is represented by a set of modal coefficients which, after computation using GSM matrices, are used to compute the transimpedances. We present validation results which show that our technique can efficiently model directional welllogging tools used for oil and gas exploration.

In Chapter 4, we study the well-logging tools response along directional wells. We present a novel pseudo-analytical formulation to handle complex 3D oriented wells. The problem is approximated by using junctions of segments of toroidal-sector radially-stratified waveguides. We present a procedure to decouple the axial fields in local toroidal coordinates, as well as a perturbation series solution in terms of the torus curvature. We present numerical results which demonstrate that the presented method can accurately model the electromagnetic propagation inside curved boreholes. The computational cost of the novel technique is just a fraction of that required by an FDTD simulation.

In Chapter 5, we explore the mode-matching technique as an alternative to modeling dipping-bed boundaries. A preliminary mathematical description of the problem is presented.

Finally, Chapter 6 summarizes the most important results showed in this dissertation. Proposed activities for future works based on the generalized directional mode-matching technique are also pointed out. 


\section{2}

\section{Well-Logging Tools and its Environment}

\section{1}

\section{Introduction}

The process of prospecting of hydrocarbons reservoirs in the Earth's formation is a topic of great interest to engineers and scientists involved in oilfield exploration. Well-logging evaluation is a process in which sensors are placed inside a drilled borehole in order to acquire information of the soil formation properties as the porosity and the electrical resistivity.

Over the last decades, the conventional wireline logging technology was a dominant procedure, where electrical cables carry the sensor tool down to the borehole after the drilling completion. More recently, measurement-whiledrilling (MWD) or logging-while-drilling (LWD) sensor tools have allowed the execution of the logging and drilling processes at same time of the borehole placement. This type of well-logging sensor will referred as real-time LWD tool along this dissertation. The focus of the dissertation is on the study and development of mathematical techniques to modeling electromagnetic LWD sensors due the economical importance of this technology for the oil and gas industry.

Typical formation scenario present in oilfield prospecting includes a series of fractured soil formations whose the electromagnetic constitutive parameters (permittivity and permeability) can be often assumed uniform within a given layer. These formation layers are often anisotropic and may display a high electrical conductivity.

In most drilling systems, the drill collar rotates and transmits weight to the drill bit, which grinds and crushes rocks into small particles; the drilling cuttings. To help the drilling process, the borehole is filled with mud to cooling and lubricating the bit. The mud is pumped down through the drill pipe and wash away the cuttings at the bit and then flows back to the surface of the well. The drilling mud can either presents high conductivity (waterbased mud) or low conductivity (oil-based mud), and depending on the soil porosity along the well, the mud can infiltrate into the formation. This invasion zone is formed by a transition media between the mud and the soil formation, 
whose electrical properties are intermediate to the inner-borehole mud and the outside-surrounding formation.

In this chapter we will describe some aspects of LWD sensors, as their geometries and electromagnetic characteristics. Additionally, we present typical specifications for directional wells placement. After a brief review on usual computational electromagnetics (CEM) algorithms, we finally present a novel solution strategy to modeling LWD tools in complex geophysical formations.

\section{2}

\section{Electromagnetic Well-Logging Sensors}

The ability of a portion of the soil to conduct electricity is directly related to the amount of water present in the geophysical formation. By understanding bed resistivity, it is possible to determine the hydrocarbon saturation in a formation and its relation with the porosity of the soil [33, Ch. 2]. LWD tools are typically comprised of an array of coil antennas wrapped around a metallic mandrel, which is located inside a borehole, and placed close to the drill bit, as illustrated in Fig. 2.1. The operating frequency for this tools range from $100 \mathrm{kHz}$ to about $1 \mathrm{GHz}$; in which $2-\mathrm{MHz}$ sensors are the most common. As the operating wavelength is very large $(150 \mathrm{~m}$ at $2 \mathrm{MHz})$ compared to the borehole diameter (about 6 to $30 \mathrm{in}$, where 1 in $=2.54 \times 10^{-2} \mathrm{~m}$ ), and as typically the media is non-magnetic and lossy, the conduction currents are very large compared to the displacement currents. In real-world applications, precise measurements of only the electromagnetic conductivity (or its corresponding inverse, the resistivity) are allowed ${ }^{1}$. By this reason, electromagnetic LWD tools are known as resistivity sensors.

Several drilling contractors provide today electromagnetic tools for LWD solutions, among them we highlight the sensors: PeriScope 15 (provide by Schlumberger) and Azimuthal Deep Resistivity Sensor (ADR) (provided by Halliburton). In each of these sensors different engineering solutions are employed to explore the formation resistivity of the soil around the borehole. A common feature presence in all of these LWD tools is an antenna array wrapped around the metallic mandrel, as illustrated in Fig. 2.2 and Fig. 2.3. Some solutions employ various transmitters and receivers antennas, disposed at various spacing, and operating at a variety of frequencies. Typically, horizontalcoil antennas are employed, but some LWD tools use tilted-coil antennas in order to achieve azimuthal sensibility.

\footnotetext{
${ }^{1}$ Empirical relationships between relative dielectric constant and resistivity can be found in $[34,35]$.
} 
In geophysical prospecting using LWD tools, the parameters of interest are the amplitude ratio (AR) and phase difference (PD) between the voltages received by the sensor antennas. The relation of the measured voltages and the resistivity of the Earth formation can be established by means of inversion algorithms based on the electromagnetic simulations of several drilling scenarios. Real-time logging technology demands accurate and numerically efficient

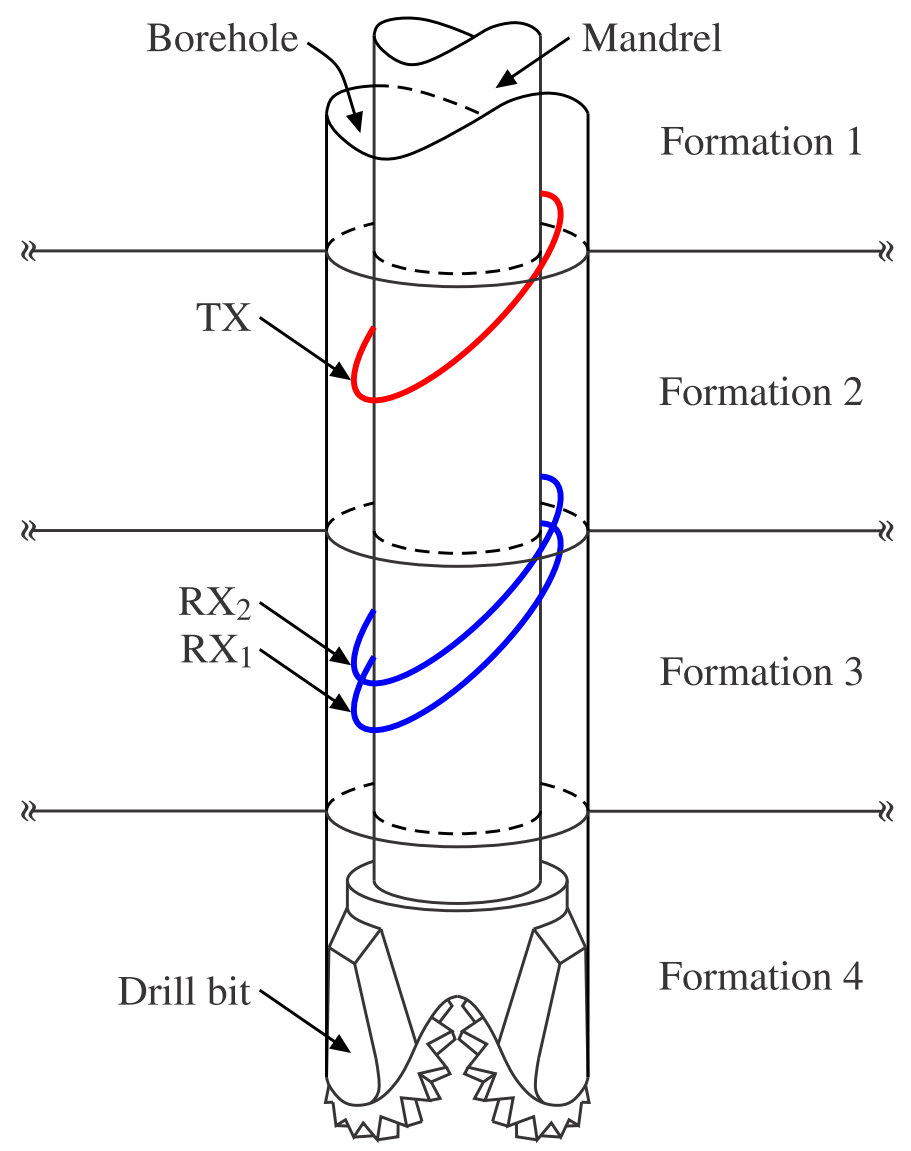

Figure 2.1: Geometry of a triaxial TCA well-logging tool (antennas TX, RX and $\mathrm{RX}_{2}$ ) within a stratified geophysical formation.

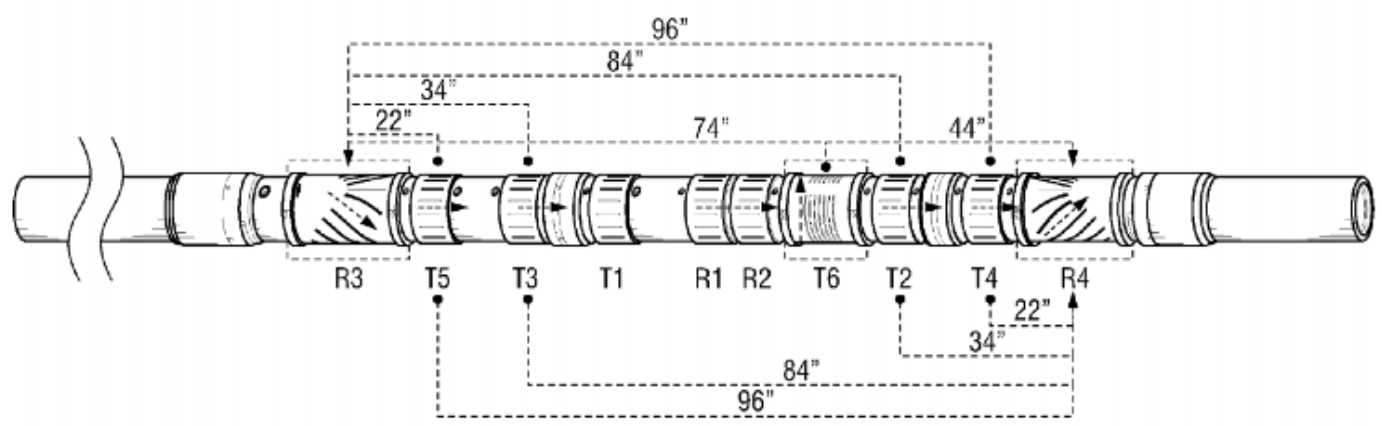

Figure 2.2: PeriScope 15 from Schlumberger. This sensor operates at $2 \mathrm{MHz}$ and is comprised by 6 horizontal-coil transmitter antennas and 4 receiver antennas ( 2 horizontal-coils and 2 tilted-coils) that provide the azimuthal sensibility. Image obtained in [1, Fig. 3]. 


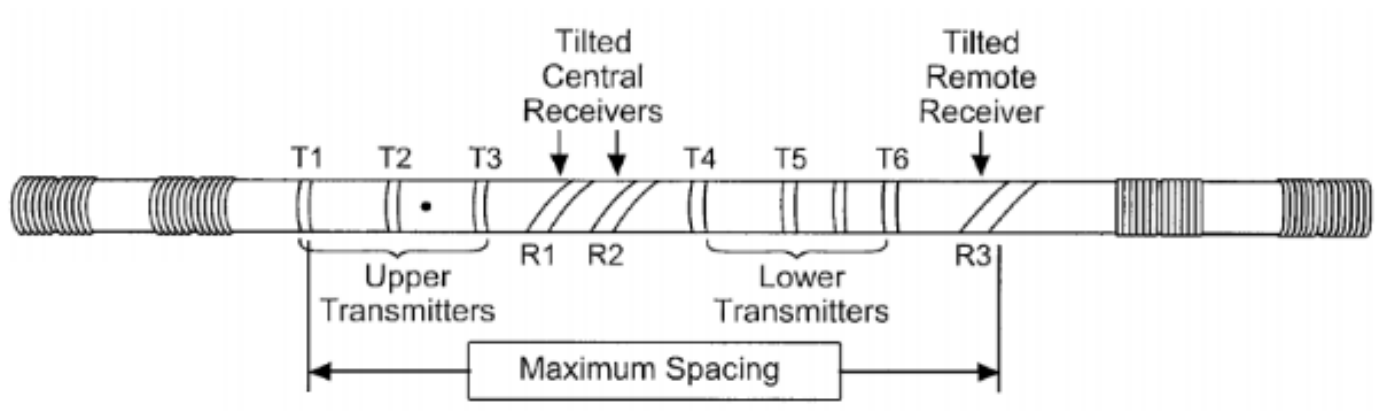

Figure 2.3: ADR sensor from Halliburton. This is a multi-frequency sensor that operates at $125 \mathrm{kHz}, 500 \mathrm{kHz}$ and $2 \mathrm{MHz}$, and is comprised by 6 horizontalcoil transmitter antennas and 3 tilted-coil receiver antennas that provide the azimuthal sensibility. Image obtained in [2, Fig. 3].

simulation algorithms in order to proper estimate the formation outside the borehole at the same time that it is drilled.

\section{3}

\section{Directional Wells}

Recently, the directional drilling has become increasingly prevalent in new oilfields exploration in order to minimize the operational cost. Compared to convention (i.e. strictly vertical) drilling, directional drilling yield several advantages for oil and gas exploration as oilfield productivity can be increased by deploying multiple wells drilled from a single platform, as depicted in Fig. 2.4. Additional benefits of directional drilling include [33, Ch. 7]: (1) drilling of a new well to intersect and recover an uncontrolled well, (2) sidetrack an old well to prospect new reservoirs, (3) horizontal wells placement, among others.

The main geometrical parameter that characterize a directional well is the deviation angle, viz. $\theta_{d}$, (in relation to the vertical axis) of the planed borehole trajectory. The change in inclination over a normalized length of the well is known in technical literature as built rate (and often termed as dogleg severity); denoted here as $q$ and usually expressed in degrees per feet (ft), where $1 \mathrm{ft}=12 \mathrm{in}$. With help of the directional well depicted in Fig. 2.5, we can easily find trigonometric relations between the desired deviated angle $\theta_{d}$ and the equipment built rate $q$ with other geometrical quantities. The radius of curvature of the bent stretch is given by $R=180 /(\pi q)$, and consequently, the length of this stretch is $L=\pi R \theta_{d} / 180=\theta_{d} / q$, both expressed in feet.

Directional wells are normally classified into three groups according the rate in which its inclination changes in the vertical plane [3], [36, pp. 111120], [37], [38]: short-, medium- and long-radius wells (see the comparison of 
horizontal well radii depicted in Fig. 2.6, where feasible horizontal stretches are also presented). The drilling profile needed to a horizontal well placement dictates the build rate and the drilling technology required. Drill contractors typically classify horizontal wells according the specification parameters shown in Table 2.1.

Long-radius wells have a relatively low curvature and it make use of conventional completion techniques [37]. Medium-radius lateral was developed to allow conventional directional drilling equipment and logging techniques to drive horizontal drainage wells $[3,37]$. Many manufacturers offer drilling assemblies that are capable of drilling medium-radius wells by means of a near-bit bent motor used to adjust the degree of bending [36]. Short-radius horizontal wells have build rates of $1.5^{\circ}$ to $3^{\circ}$ per ft, which equates to radii greater than $20 \mathrm{ft}$. These wells are drilled with very small diameter boreholes

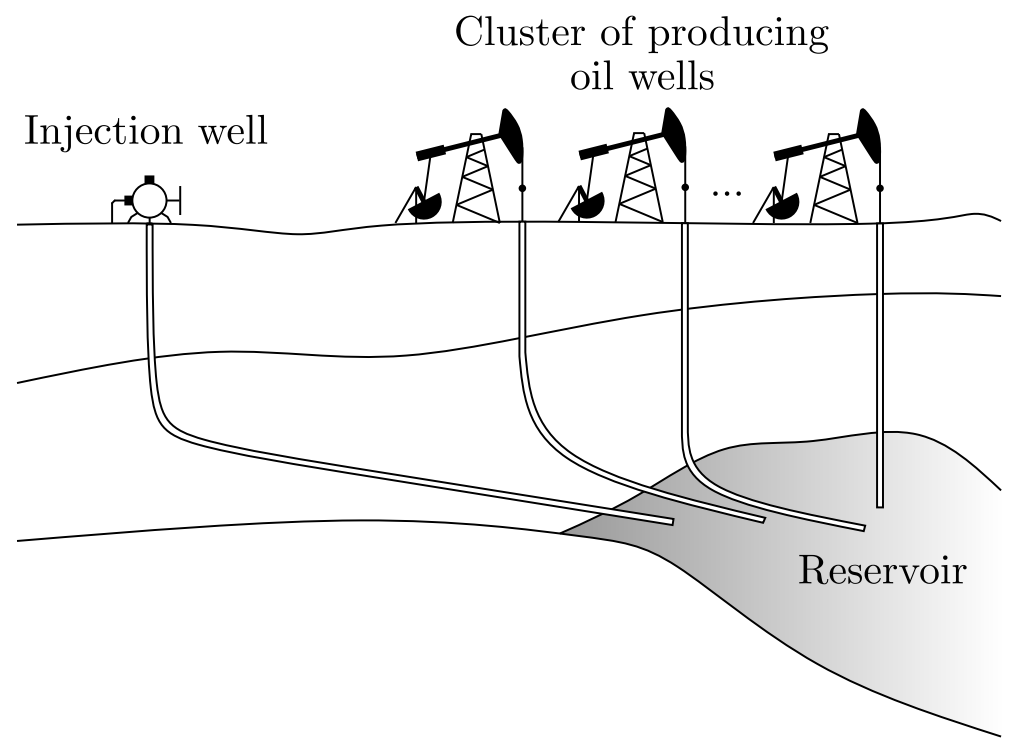

Figure 2.4: Several wells drilled from the same platform.

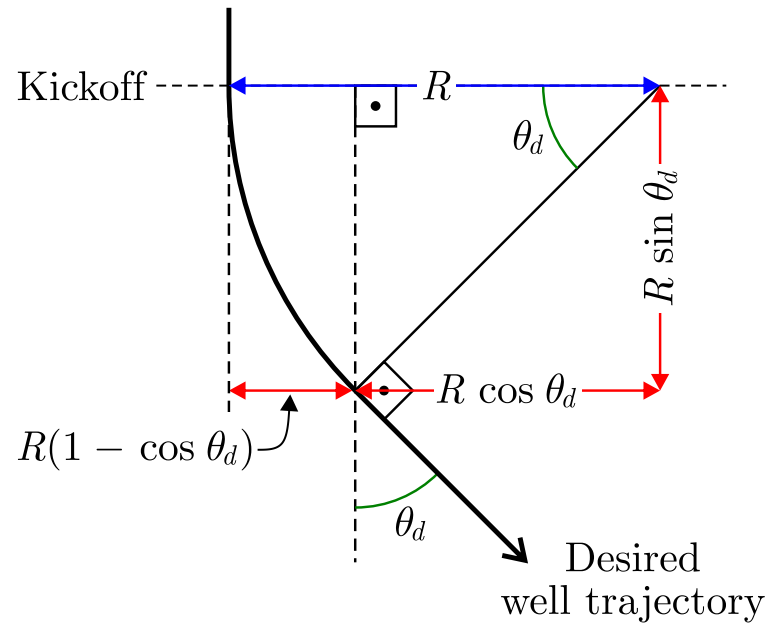

Figure 2.5: Simple directional well with its main geometrical parameters. 
Table 2.1: Horizontal-well classifications (data from [3, p. 24])

\begin{tabular}{crc}
\hline Well type & Built rate & Radius of curvature \\
\hline Long & $2^{\circ}$ to $6^{\circ}$ per $100 \mathrm{ft}$ & 3000 to $1000 \mathrm{ft}$ \\
Medium & $8^{\circ}$ to $50^{\circ}$ per $100 \mathrm{ft}$ & 700 to $125 \mathrm{ft}$ \\
Short & $150^{\circ}$ to $300^{\circ}$ per $100 \mathrm{ft}$ & 40 to $20 \mathrm{ft}$ \\
\hline
\end{tabular}

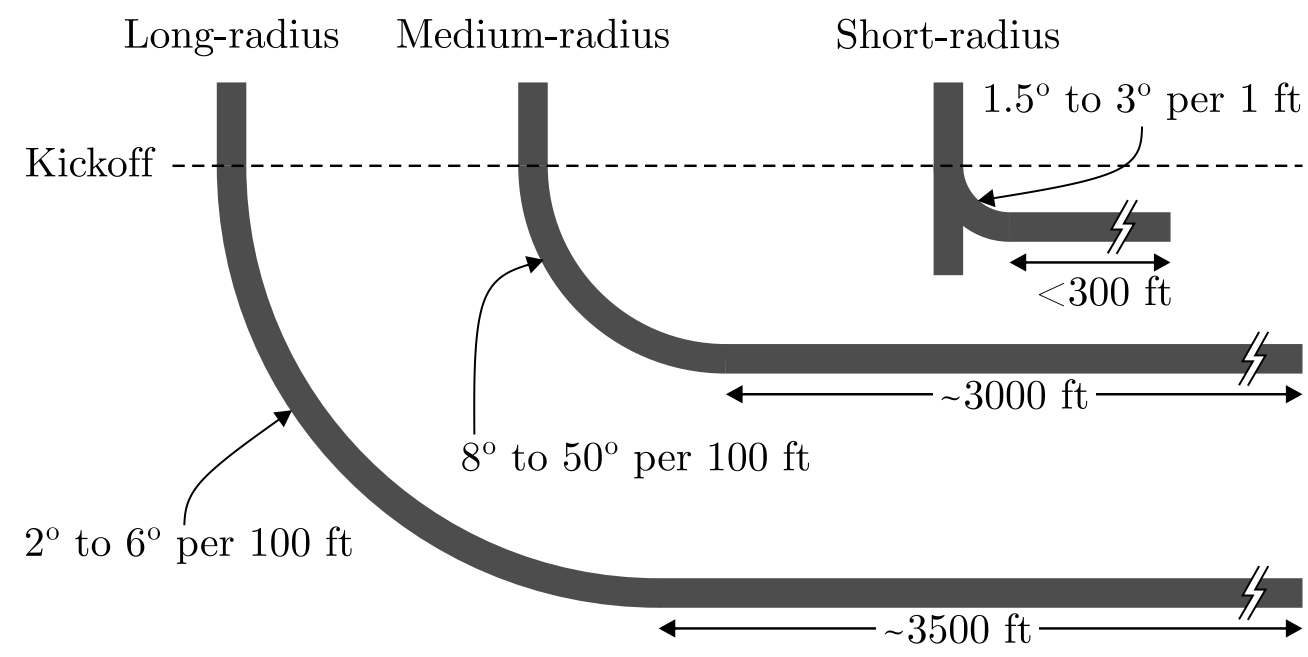

Figure 2.6: Long-, medium- and short-radius directional wells classified according its drilling build rates. This image is based on an illustration shown in $[3$, p. 24].

and are most commonly employed as a re-entry from an old well, which of course requires specialized drilling tools and techniques [37].

Although the modern drilling techniques handle short-radius directional wells, commercial resistivity LWD sensor such as the ADR sensor [39] and the PeriScope 15 [40] only allow real-time logging with built rates lower than $30^{\circ}$ per $100 \mathrm{ft}$, with a radius of curvature greater than $191 \mathrm{ft}$.

To increase the oil recovery, the reservoir can be exposed by lateral wellbores coming from an initial horizontal well, as depicted in Fig. 2.7. In this case, the production well can now presets bending in both horizontal and vertical directions, by using a three-dimensional (3D) geosteering.

\section{4}

\section{On the Problem Statement and the Proposed Solution}

The electromagnetic modeling of LWD tools inside complex geophysical formations is a challenging task to CEM. Several complications arrive due the non-conformal boundaries involved in the problem. Looking for computationally efficient CEM, in this work we combined several analytical solutions for well-known problems with new techniques developed herein. 


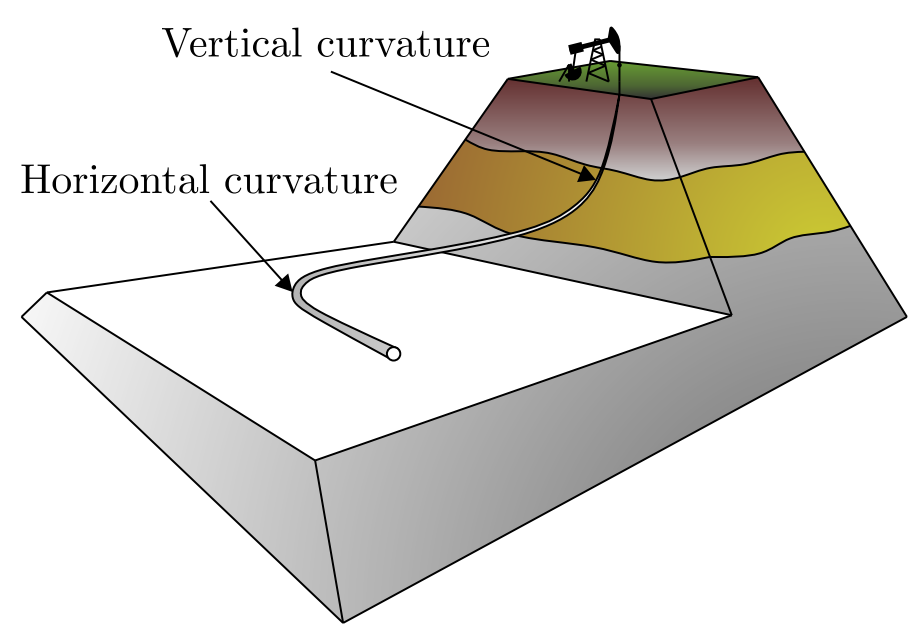

Figure 2.7: A typical planed well hole profile with directional drilling bending in both horizontal and vertical directions. This image is based on an illustration shown in [4, Fig. 7].

The formulation we proposed herein is based on a vector modal eigenfield expansion of Maxwell's equations in cylindrical coordinates. This result will be employed to find eigenfields in toroidal coordinates. The choice of this coordinate system was motivated because we can approximate a directional well path using sections of toroidal structures, each one with a constant bending curvature $^{2}$ as illustrated in Fig. 2.8(a). Next, to satisfy the boundary conditions at each junction, we have employed the Lorentz reciprocity theorem, which allow us to derive a new generalization to the mode-matching approach based on the conservation of the reaction [41], [42, pp. 399-400].

Logging-while-drilling (LWD) tools, as that illustrated in Fig. 2.1, are commonly modeled using a mode-matching-based formulation [10, 31, 43], whereby a finite-element (FE) discretization is used along each horizontal layer of a cylindrical stratified medium. In the vertical direction, an analytical modematching procedure is then used to match the fields between each horizontal layer. This approach is known as numerical mode-matching (NMM), but will be denoted here in a more descriptive form: axial NMM (A-NMM). This approach is computationally much more efficient than 3D brute-force CEM techniques such as finite-differences (FDs), FEs and finite-volumes (FVs) [5, 17, 20, 44, 45], and, as such, it is more suited for integration into reconstruction (inversion) algorithms.

A typical LWD tool moves with the downhole drilling so that, at each new tool position along the well, it is necessary to recalculate several matrices

\footnotetext{
${ }^{2}$ The assumption of a constant curvature will, of course, greatly simplify the mathematical analysis for the problem at hand. Regardless of this analytical convenience, the current directional drilling technology also bore wells with constant bending angles along a stretch of the well, as described above in Fig. 2.6 and in Table 2.1.
} 


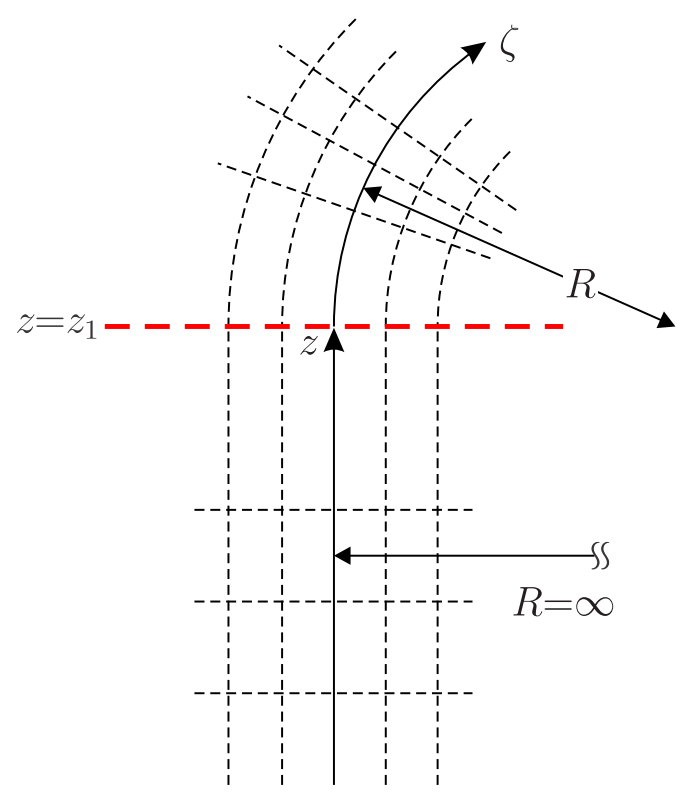

(a) Straight to bent junction

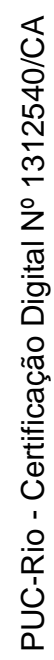

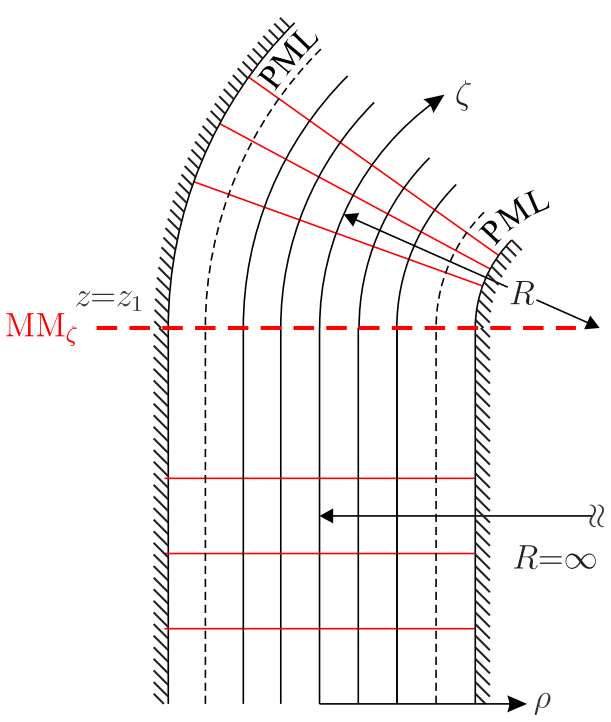

PML

तIIIIIIIIIIIIIIII $\rightarrow \rho$

(b) R-NMM approach

(c) A-NMM approach

Figure 2.8: Junction between a straight and a bent structure (a), and the attempts to define a R-NMM in (b) and an A-NMM in (c). 
incorporating the reflections from the horizontal layers above and below the source and the receiving antennas. This is a drawback of the A-NMM, as pointed out in [46]. In $[8,27,28,46]$, an alternative NMM approach was proposed to circumvent this issue in LWD applications. As both transmitting and receiving LWD antennas are placed around a metallic mandrel inside the borehole and typically have the same radius, they are located in the same radial layer regardless of the axial position of the tool in the logging well. Therefore, it becomes more efficient to first perform a mode expansion of the fields along vertical direction and then a mode-matching along each radial layer. This approach will be denoted here as R-NMM. Some antennas of the LWD sensor have a non-zero span along the axial direction, such the vertical mode expansion could be seen as more appropriate to this type of problem in the case of straight wells.

Although suitable to model TCA tools in straight wells, the approach used in [8] cannot be easily generalized to curved wells, where axial bending is present. In order to clarify possible issues, consider a radially-stratified and axially-layered structure as those shown in Fig. 2.9(a) and Fig. 2.9(c). Both the A-NMM and R-NMM approaches can be used in this case. Consider now that this structure becomes axially bent, with a constant curvature, $R^{-1}$, as illustrated in Fig. 2.9(b) and Fig. 2.9(d). Notably, we can continue to using both A-NMM and R-NMM to proper model the structure: the R-NMM will be enforced at each radial interface and the A-NMM will be used to match fields along axial boundaries. However, in case of the junction of a straight to bent well, as shown in Fig. 2.8, our attempts to model this problem using the R-NMM have failed because we will always need a A-NMM as highlighted in Fig. 2.8(b). In this case, the A-NMM (depicted in Fig. 2.8(c)) becomes a more natural choice.

In next chapters, novel concepts for electromagnetic field simulations are introduced via pseudo-analytical enforcements to provide a flexible technique to analyzing LWD tools in complex geophysical formations. The presented technique does not rely on spatial discretization as former well-known CEM methods, resulting in a robust and numerical efficient algorithm and a more practical forward engine for integration into inversion algorithms designed to estimate the Earth formation properties given the LWD tool response. 


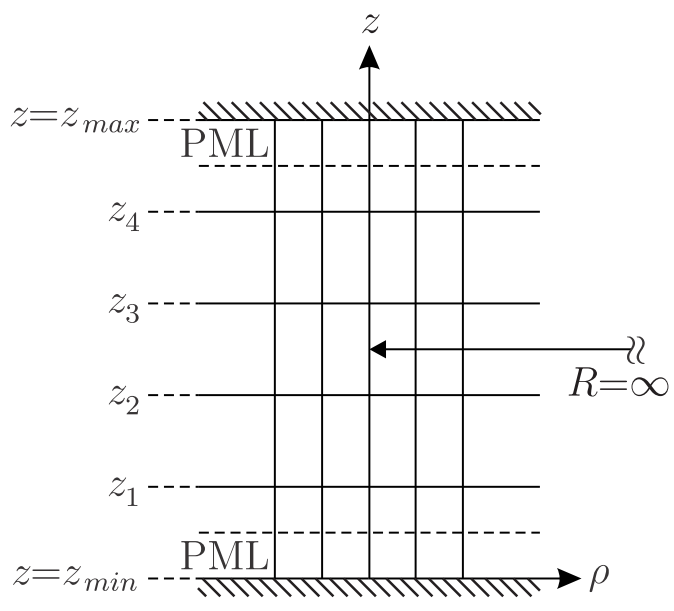

(a) Straight structure for R-NMM

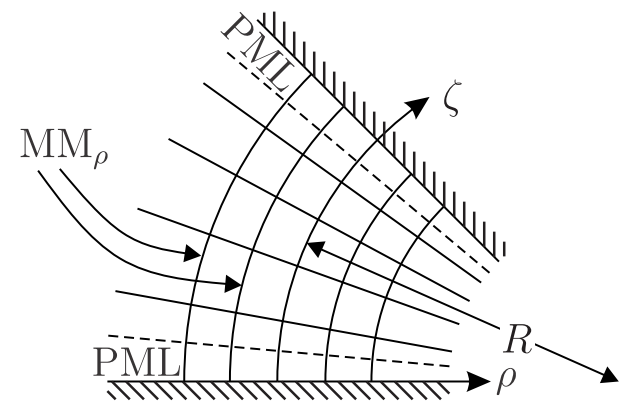

(b) Bent structure for R-NMM

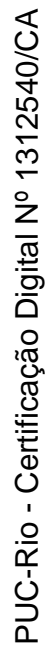

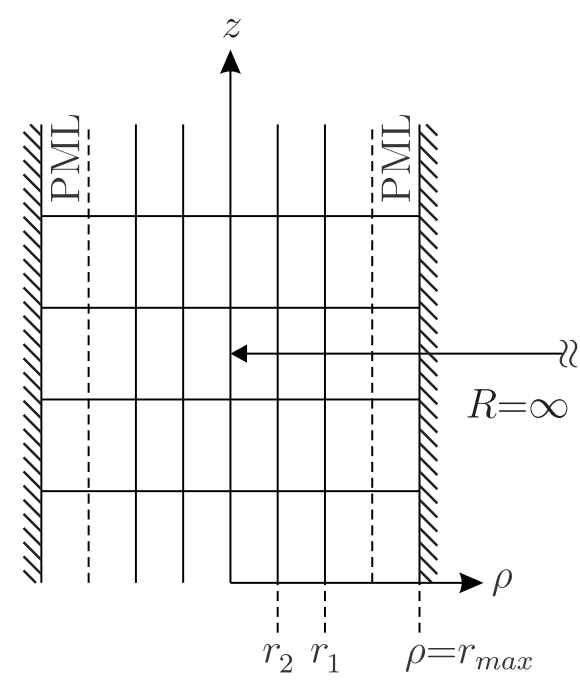

(c) Straight structure for A-NMM

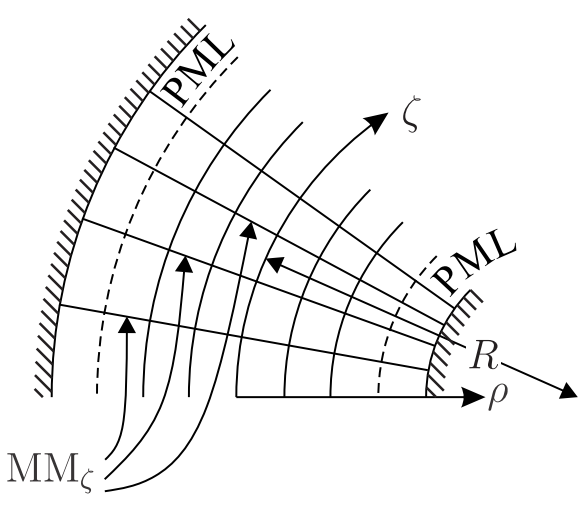

(d) Bent structure for A-NMM

Figure 2.9: Radially-stratified and axially-layered structures with constant axial curvature. 


\section{3}

\section{Analysis of Well-Logging Tools in Vertical Wells}

\section{1}

\section{Introduction}

Over the years, various techniques have been used to properly model logging-while-drilling (LWD) tools used in hydrocarbon exploration $[8,14,15$, $26,47,48]$. The complex geophysical formations that are prevalent in this type of problem can be handled using brute-force numerical techniques such as finitedifferences (FDs), finite-elements (FEs) and finite-volumes (FVs) [6, 7, 17-20]. However, these techniques suffer from relatively high cost in terms of both computer memory and CPU time. LWD tools are typically comprised of coil antennas wrapped around a metallic mandrel which is located inside a borehole, as illustrated in Fig. 3.1. This geometry can be well approximated as a cylindrically stratified medium $[10,14]$. In this case, pseudo-analytical methods $[10,14,29,47-50]$ are a good alternative to brute-force methods as the former provide more efficient algorithms and a more practical forward engine for integration into inversion algorithms designed to estimate the Earth formation properties given the tool response [26, 48, 49].

In [14] and [15, Ch. 3] a pseudo-analytical technique was used to model small dipoles and coil antennas in a radially-layered structure using closed-form $2 \times 2$ reflection and transmission matrices. This technique was generalized to model tilted-coil antennas (TCAs) in a recent work presented in [47]. Another pseudo-analytical technique was used in [15, Ch. 2], [16], but now to model point sources in an axially-layered structure. In all these approaches, to solve radial or axial stratifications, the pseudo-analytical methods shown the field solutions represented by an inverse Fourier transform in $z$-direction over a sum of azimuthal harmonics. It is well-known that the inverse Fourier transform present numerical convergence issues when radial position of the observation point is the same, or very near, the radial position of the source. In order to model typical logging-while-drilling (LWD) tools, generally we consider that both transmitter and receiver antennas share the same radius, and to correctly solve the inverse transform we need to extract the direct field from the integration and then combine its contribution to calculate the final 


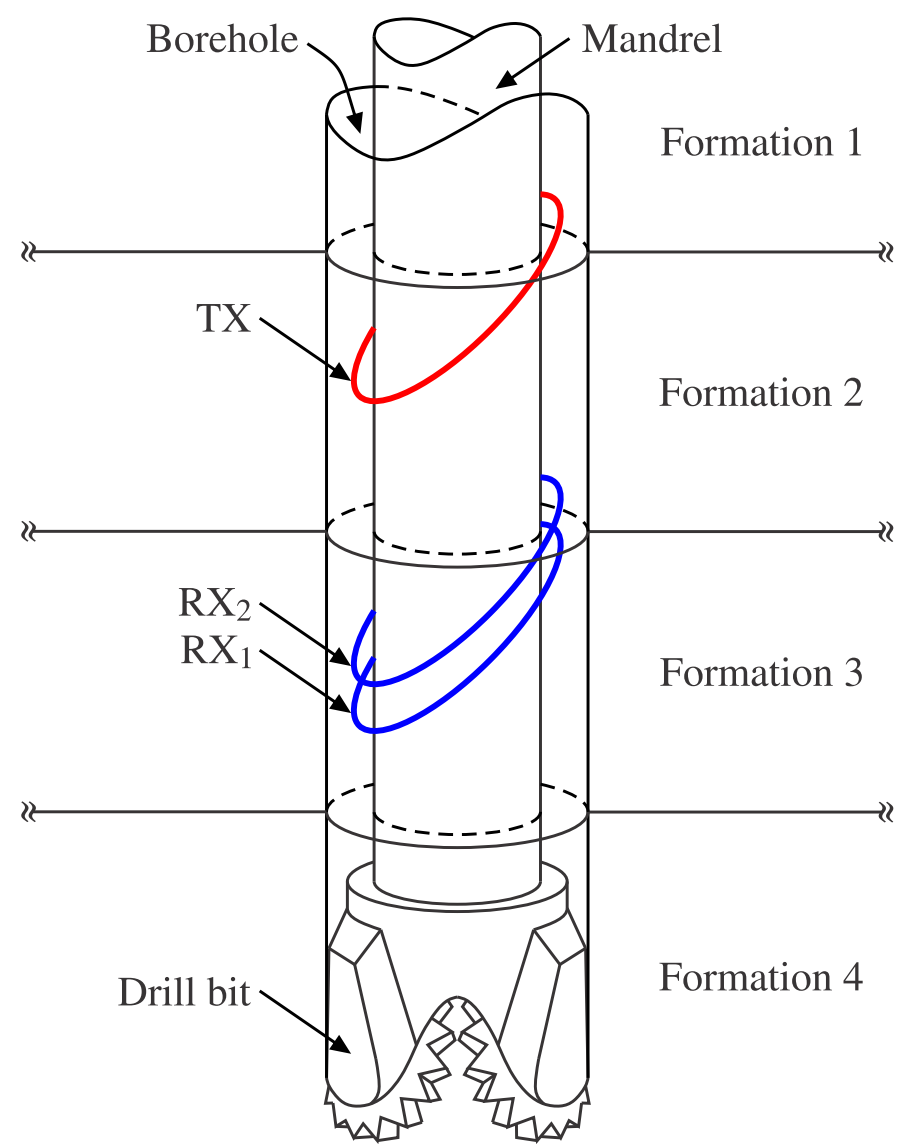

Figure 3.1: Geometry of a triaxial TCA well-logging tool within a stratified geophysical formation.

solution $[6,14,47,49]$.

To the best of our knowledge, the pseudo-analytical modeling of LWD tools appear restrict to two-dimensional (2D) structures with only axial stratifications, or with only radial stratifications. An alternative way to solve three-dimensional (3D) problems is to employ the numerical mode-matching (NMM) techniques, which provides a middle-ground in terms of computational costs between brute-force and pseudo-analytical techniques [6, 8, 10, 15, 22-32]. Typically, the NMM combines the FD or FE method in one coordinate direction with an analytical (modal) expansion in the others transversal directions. The NMM is very accurate and still flexible enough to account for inhomogeneities in the Earth formation along both radial and axial directions.

Tilted-coil antennas (TCAs) are routinely utilized in well-logging tools $[18,47-49]$ to provide directional response (azimuthal sensitivity). Welllogging tools employing TCAs are also often called triaxial tools. In [8], the NMM was extended to model triaxial tools in vertical wells with both radial and axial stratifications using a vertical mode expansion in conjunction with a mode-matching along the radial discontinuities. This approach will be denoted 
here as R-NMM. The NMM can be also formulated in an alternate fashion, i.e., with a horizontal mode expansion $[15,26,29]$ combined with a mode-matching across the stratifications in the axial direction. This approach will be denoted here as A-NMM.

Most prior A-NMM formulations approximate the TCAs used in triaxial tools as (infinitesimal) magnetic dipoles. This is done to facilitate the source expansion and subsequent analysis. Here, we do not make this approximation and instead incorporate the actual TCA source geometry into the modematching formulation. Since a TCA has a non-zero span along the axial direction, the vertical mode expansion could be seen as more appropriate to this type of problem in the case of straight wells. Although suitable to model TCA tools in straight wells, the approach used in [8] cannot be easily generalized to curved wells, where axial bending is present. This type of well geometry occurs in deviated drilling. In this case, the A-NMM becomes a more natural choice to be combined with the perturbation theory used to model bent waveguides presented in [51]. Regardless of the choice for the modal expansion, since the frequency of operation and physical parameters (in particular the resistivity of the mud fluid and the surrounding Earth formation) in typical well-logging problems can vary by several orders of magnitude, it is important to ensure that the method of analysis is sufficiently robust to yield accurate solutions under a wide range of input parameters $[5,50,52,53]$. In this chapter, we introduce a new axial mode-matching formulation to provide a flexible technique for analyzing directional well-logging tools in anisotropic formations. The proposed formulation is based on an analytical eigenmode expansion that does not rely on spatial discretization. The advantage of this kind of approach is that the fields can be represented by a sum of a relatively small number of eigenmodes compared to the number of grid points or discrete mesh required in another techniques.

The remaining of this chapter is organized as follows. Section 3.2Section 3.6 discuss the main ingredients adopted to increase the robustness of the proposed algorithm, among them: (1) a radial perfectly matched layer to mimic the open boundary problem based on a bounded domain with a discrete set of modes and obviating the need to account for radiation modes, (2) an efficient deflationary process to remove the poles of the characteristic equation enabling a robust computation of the eigensolutions of the characteristic equation, (3) the use of Lorentz reciprocity theorem to expand the source in terms of modal fields propagating in the axial direction, thus facilitating the modematching at axial discontinuities, (4) a stationary formula based on conservation of reaction to obtain the generalized scattering matrices at regions with 
axial stratification, and (5) a Jacobi-Anger expansion to obtain fast-converging expressions for the transimpedances (receiver voltages due to a unit current excitation) when the TCAs traverse an axial discontinuity. Section 3.7 presents numerical results to validate the technique and demonstrate its usefulness in predicting the response of triaxial tools in complex anisotropic geophysical formations. Section 3.8 presents a comparison between the proposed technique versus an improved R-NMM approach recently reported in the literature. Section 3.9 presents a comprehensive analysis of well-logging sensors housed inside grooves within the metallic mandrel. Section 3.10 presents some considerations for the modeling of wireless telemetry systems for oilfield applications. Finally, Section 3.11 provides some concluding remarks.

\section{2}

\section{Electromagnetic Fields in Cylindrical Structures}

The geometry shown in Fig. 3.1 is used to model the geophysical formation around a typical well-logging tool. We assume the sensor is placed sufficiently far from the drill bit, so that we can approximate the vertical formation as an unbounded, axially-stratified media. In the radial direction, each region is modeled as a bounded, radially-stratified waveguide. Field solutions for Maxwell's equations in uniaxially anisotropic multilayered cylindrical structures are well-known, and to avoid repetition, we adopt here a notation similar to that in $[6,8,15,47,48,50]$ by assuming a time-harmonic dependence in the form $\exp (-i \omega t)$. It should be observed that the notation adopted here despite being very similar to that in [48] and [15], differs slightly in a few places. A list of principal symbols employed in this dissertation is presented in page 21 .

The Maxwell's equations in a homogeneous anisotropic medium are

$$
\begin{gathered}
\nabla \times \mathbf{E}=i \omega \overline{\bar{\mu}} \cdot \mathbf{H}, \\
\nabla \times \mathbf{H}=-i \omega \overline{\bar{\epsilon}} \cdot \mathbf{E}+\mathbf{J}, \\
\nabla \cdot(\overline{\bar{\epsilon}} \cdot \mathbf{E})=\varrho, \\
\nabla \cdot(\overline{\bar{\mu}} \cdot \mathbf{H})=0,
\end{gathered}
$$

where $\mathbf{E}$ and $\mathbf{H}$ are the electric and the magnetic fields, respectively, due to the impressed electric current and charge densities $\mathbf{J}$ and $\varrho$.

Our media is characterized by the complex permeability

$$
\overline{\bar{\mu}}=\operatorname{diag}\left(\mu_{s}, \mu_{s}, \mu_{z}\right), \text { with } \mu_{\{s, z\}}=\mu_{0} \mu_{\{s, z\}}
$$

and permittivity 


$$
\overline{\bar{\epsilon}}=\operatorname{diag}\left(\epsilon_{s}, \epsilon_{s}, \epsilon_{z}\right) \text {, with } \epsilon_{\{s, z\}}=\epsilon_{0} \epsilon_{r\{s, z\}}+i \sigma_{\{s, z\}} / \omega
$$

tensors, represented in cylindrical coordinates. We have decomposed the tensors into axial (along $z$ ) and transverse components, with subscripts $z$ and $s$, respectively. We have also expressed the constitutive parameters in terms of the usual vacuum constants $\epsilon_{0}$ and $\mu_{0}$.

We next consider the fields in a source-free region and decompose the problem into axial and transversal components as

$$
\mathbf{G}=\mathbf{G}_{s}+\hat{z} G_{z}
$$

in which $\mathbf{G}=\mathbf{E}$ or $\mathbf{H}, G_{z}=E_{z}$ or $H_{z}$, and

$$
\boldsymbol{\nabla}=\boldsymbol{\nabla}_{s}+\hat{z} \frac{\partial}{\partial z}
$$

where $\nabla_{s}$ denotes the transverse two-dimensional nabla operator given by

$$
\nabla_{s}=\hat{\rho} \frac{\partial}{\partial \rho}+\hat{\phi} \frac{1}{\rho} \frac{\partial}{\partial \rho}
$$

Taking the curl of the equation (3-1a) and projecting the resulting vector onto $\hat{z}$, we can find

$$
\hat{z} \cdot[\nabla \times(\nabla \times \mathbf{E})]=i \omega \hat{z} \cdot[\nabla \times(\overline{\bar{\mu}} \cdot \mathbf{H})] .
$$

We will show that the above scalar wave equation can be represented in a more familiar form.

As the problem at hand deal with homogeneous media, the right-hand side (RHS) of (3-7) can be simplified by using

$$
\hat{z} \cdot[\boldsymbol{\nabla} \times(\overline{\bar{\mu}} \cdot \mathbf{H})]=\mu_{s} \hat{z} \cdot(\boldsymbol{\nabla} \times \mathbf{H}) .
$$

Noting that $\hat{z} \cdot(\boldsymbol{\nabla} \times \mathbf{H})=-i \omega \epsilon_{z} E_{z}$, we can derive

$$
\hat{z} \cdot[\nabla \times(\overline{\bar{\mu}} \cdot \mathbf{H})]=-i \omega \mu_{s} \epsilon_{z} E_{z} .
$$

Consequently, (3-7) becomes

$$
\hat{z} \cdot[\nabla \times(\nabla \times \mathbf{E})]=\omega^{2} \mu_{s} \epsilon_{z} E_{z} .
$$

The left-hand side (LHS) of (3-10) can be simplified after using the vector identity

$$
\boldsymbol{\nabla} \times \boldsymbol{\nabla} \times \mathbf{E}=\boldsymbol{\nabla}(\boldsymbol{\nabla} \cdot \mathbf{E})-(\boldsymbol{\nabla} \cdot \boldsymbol{\nabla}) \mathbf{E} .
$$

The divergence of the electric and magnetic fields do not vanish anymore as in the isotropic media $[15,54]$. The Gauss's law for magnetism (3-1d) can 
be decomposed as

$$
\begin{aligned}
\boldsymbol{\nabla} \cdot(\overline{\bar{\mu}} \cdot \mathbf{H}) & =\left(\boldsymbol{\nabla}+\hat{z} \frac{\partial}{\partial z}\right) \cdot\left(\mu_{s} \mathbf{H}_{s}+\hat{z} H_{z}\right) \\
& =\mu_{s} \boldsymbol{\nabla}_{s} \cdot \mathbf{H}_{s}+\mu_{z} \frac{\partial H_{z}}{\partial z} \\
& =\mu_{s} \boldsymbol{\nabla} \cdot \mathbf{H}-\mu_{s}\left(1-\frac{\mu_{z}}{\mu_{s}}\right) \frac{\partial H_{z}}{\partial z}=0
\end{aligned}
$$

and a dual equation can be easily established for the electric field, such we can derive

$$
\begin{gathered}
\boldsymbol{\nabla} \cdot \mathbf{E}=\left(1-\frac{\epsilon_{z}}{\epsilon_{s}}\right) \frac{\partial E_{z}}{\partial z}, \text { and } \\
\boldsymbol{\nabla} \cdot \mathbf{H}=\left(1-\frac{\mu_{z}}{\mu_{s}}\right) \frac{\partial H_{z}}{\partial z} .
\end{gathered}
$$

As expected, $\boldsymbol{\nabla} \cdot \mathbf{E}$ and $\boldsymbol{\nabla} \cdot \mathbf{H}$ vanish for the isotropic limits $\epsilon_{s}=\epsilon_{z}$ and $\mu_{s}=\mu_{z}$.

Combining the above results when we take the projection of equation (3-11) onto $\hat{z}$ allow us to find the LHS of (3-10):

$$
\begin{aligned}
\hat{z} \cdot[\boldsymbol{\nabla} \times(\boldsymbol{\nabla} \times \mathbf{E})] & =\hat{z} \cdot \boldsymbol{\nabla}(\boldsymbol{\nabla} \cdot \mathbf{E})-\hat{z} \cdot(\boldsymbol{\nabla} \cdot \boldsymbol{\nabla}) \mathbf{E} \\
& =\left(1-\frac{\epsilon_{z}}{\epsilon_{s}}\right) \frac{\partial^{2} E_{z}}{\partial z^{2}}-\nabla^{2} E_{z},
\end{aligned}
$$

where $\nabla^{2}$ is the scalar Laplacian in cylindrical coordinates given by

$$
\nabla^{2}=\frac{1}{\rho} \frac{\partial}{\partial \rho}\left(\rho \frac{\partial}{\partial \rho}\right)+\frac{1}{\rho^{2}} \frac{\partial^{2}}{\partial \phi^{2}}+\frac{\partial^{2}}{\partial z^{2}} .
$$

Finally, after substituting (3-15) into (3-10), one obtains

$$
\nabla_{s}^{2} E_{z}+\frac{\epsilon_{z}}{\epsilon_{s}} \frac{\partial^{2} E_{z}}{\partial z^{2}}+\omega^{2} \mu_{s} \epsilon_{z} E_{z}=0
$$

in which the two-dimensional Laplacian operator is defined by

$$
\nabla_{s}^{2}=\nabla^{2}-\frac{\partial^{2}}{\partial z^{2}}
$$

A wave equation dual to (3-18) can be derived for the axial magnetic field using a procedure analogous to that shown above. It follows that

$$
\nabla_{s}^{2} H_{z}+\frac{\mu_{z}}{\mu_{s}} \frac{\partial^{2} H_{z}}{\partial z^{2}}+\omega^{2} \mu_{z} \epsilon_{s} H_{z}=0
$$

It is clear that (3-18) and (3-20) are homogeneous Helmholtz wave equations for $E_{z}$ and $H_{z}$ whose solutions can be constructed by the method of separation of variables [55, Ch. 5]. The Helmholtz equation in cylindrical coordinates 


$$
\frac{1}{\rho} \frac{\partial}{\partial \rho}\left(\rho \frac{\partial \psi}{\partial \rho}\right)+\frac{1}{\rho^{2}} \frac{\partial^{2} \psi}{\partial \phi^{2}}+\frac{p_{z}}{p_{s}} \frac{\partial^{2} \psi}{\partial z^{2}}+\omega^{2} p_{z} \tilde{p}_{s} \psi=0,
$$

where $\psi=\left\{E_{z}, H_{z}\right\}, p=\{\epsilon, \mu\}$ and $\tilde{p}=\{\mu, \epsilon\}$, can be solved by the elementary wave functions of the form

$$
R_{n}\left(\sqrt{\frac{p_{z}}{p_{s}}} k_{\rho} \rho\right) \Phi(n \phi) Z\left(k_{z} z\right)
$$

where $\Phi(n \phi)$ and $Z\left(k_{z} z\right)$ are harmonic functions and $R_{n}(\cdot)$ is a general solution for the Bessel equation of integer order $n$. The detailed solution for (3-21) is presented in Appendix A.

Linear combination of the elementary wave functions are also a solution to (3-21) [55, p. 200], such that the general solution is in the shape of

$$
\psi=\sum_{n} \sum_{k_{z}} C_{n, k_{z}} R_{n}\left(\sqrt{\frac{p_{z}}{p_{s}}} k_{\rho} \rho\right) \Phi(n \phi) Z\left(k_{z} z\right),
$$

where the double summation is over all possible values of $n$ and $k_{z}$, with appropriated constants $C_{n, k_{z}}$ given by a source boundary condition. The inclusion of a source implies in a non-homogeneous Helmholtz equation. The Lorentz reciprocity theorem will be employed in Section 3.5 to find the modal source amplitudes by using the source-free fields derived here.

\subsection{1}

\section{Axial Fields}

The forward-propagating axial fields can be written in a compact fashion as

$$
\left[\begin{array}{l}
E_{z} \\
H_{z}
\end{array}\right]=\sum_{n=-\infty}^{\infty} \sum_{p=1}^{\infty}\left[\begin{array}{l}
e_{z, n p}(\rho) \\
h_{z, n p}(\rho)
\end{array}\right] e^{i n \phi+i k_{z, n p} z} .
$$

Notice that the sum over the azimuthal indices is taken through the positive and negative integer values of $n$ due to the choice of the exponential form $\exp (i n \phi)$. Another commonly used form is a linear combination of sine and cosine functions as stated in Appendix A, but in this case, the sum could be restricted over the positive integer values of $n$ due the parity of the harmonic sinusoidal functions in relation to its arguments. Also, as we intend to express the sources contribution into the axial direction, the form $\exp \left(i k_{z, n p} z\right)$ appears appropriated indeed. Thus, we prefer the form shown in (3-24) because the fields can be represented in a compacted shape, and, as will shown in Section 3.3.5, a series of symmetric relations applies when we use this representation.

The modal propagation constant in the $z$-direction is $k_{z, n p}$, and the radial propagation constant $k_{\rho, n p}$ satisfies 


$$
k_{\rho, n p}^{2}=k_{s}^{2}-k_{z, n p}^{2}, \quad k_{s}^{2}=\omega^{2} \mu_{s} \epsilon_{s} .
$$

The azimuthal dependence of the fields is associated to index $n$, while $p$ refers to its radial dependency. Due to phase matching, the fields assume the $\exp \left(i k_{z, n p} z\right)$ dependence throughout all layers over the transverse cross-section.

In order to simplify the notation, we will temporarily drop the modal subscript $n p$ and also the argument of $e_{z, n p}$ and $h_{z, n p}$, restoring them later as needed. The radially-dependent coefficients in (3-24) are given by a linear combination of first-kind Hankel and Bessel function of integer order $n$. In a compact notation, we can write them as

$$
\left[\begin{array}{c}
e_{z, n p} \\
h_{z, n p}
\end{array}\right]=\overline{\bar{H}}_{z n}\left(k_{\rho} \rho\right) \bar{a}+\overline{\bar{J}}_{z n}\left(k_{\rho} \rho\right) \bar{b},
$$

where

$$
\overline{\bar{G}}_{z n}\left(k_{\rho} \rho\right)=\left[\begin{array}{cc}
G_{n}\left(k_{\rho}^{e} \rho\right) & 0 \\
0 & G_{n}\left(k_{\rho}^{h} \rho\right)
\end{array}\right],
$$

with $G_{n}$ representing $H_{n}^{(1)}$ or $J_{n}$. The field amplitudes $\bar{a}$ and $\bar{b}$ are vectors $2 \times 1$ to be determined from the boundary conditions, and are given by

$$
\bar{a}=\left[\begin{array}{l}
a_{n p}^{e} \\
a_{n p}^{h}
\end{array}\right], \quad \bar{b}=\left[\begin{array}{l}
b_{n p}^{e} \\
b_{n p}^{h}
\end{array}\right] .
$$

For simplicity, we have introduced $k_{\rho}^{e, h}=\alpha^{e, h} k_{\rho}$ in which the anisotropic coefficients $\alpha^{e, h}$ are given by

$$
\alpha^{e}=\sqrt{\frac{\epsilon_{z}}{\epsilon_{s}}}, \quad \alpha^{h}=\sqrt{\frac{\mu_{z}}{\mu_{s}}} .
$$

Under some conditions, the formulas in (3-26) suffer from the numerical overflow of the Bessel and Hankel functions due extreme arguments and/or orders. Recent studies have shown that this problem can be circumvented through suitable rescaling of various expressions involved in the computational chain $[50,52]$, as well as expressing (3-26) by the ratios of Bessel or Hankel functions [53]. The LWD tools modeled in this dissertation do not required high orders cylindrical functions, but for extreme conductivity media the techniques reported in $[50,52,53]$ need to be used.

\subsection{2}

\section{Transversal Fields}

The transversal fields components can be expressed as a combinations of the axial ones, as will be shown in the following. The Maxwell's curl equations (3-1a) and (3-1b) in a source-free region can now be rewritten as 


$$
\begin{gathered}
\nabla_{s} \times\left(\hat{z} E_{z}\right) \pm i k_{z} \hat{z} \times \mathbf{E}_{s}=i \omega \mu_{s} \mathbf{H}_{s} \\
\nabla_{s} \times\left(\hat{z} H_{z}\right) \pm i k_{z} \hat{z} \times \mathbf{H}_{s}=-i \omega \epsilon_{s} \mathbf{E}_{s} \\
\nabla_{s} \times \mathbf{E}_{s}=i \omega \mu_{z} \hat{z} H_{z} \\
\nabla_{s} \times \mathbf{H}_{s}=-i \omega \epsilon_{z} \hat{z} E_{z}
\end{gathered}
$$

where we have assumed that the axial dependence of all field vectors match with those in $(3-24)$, i.e., $\exp \left(i k_{z} z\right)$. In the above derivation we have replaced $\partial / \partial z$ by $i k_{z}$.

Initially, by the vector multiplication of $\hat{z}$ on both sides of the equations in (3-30a) and (3-30b), and using the identities [54, p. 165]

$$
\begin{gathered}
\hat{z} \times\left(\nabla_{s} \times \hat{z} G_{z}\right)=\nabla_{s} G_{z}, \text { and } \\
\hat{z} \times\left(\hat{z} \times \mathbf{G}_{s}\right)=-\mathbf{G}_{s},
\end{gathered}
$$

and after a few manipulations, we can express the transversal fields as a combinations of the axial ones as

$$
\begin{aligned}
& \mathbf{E}_{s}=\frac{1}{k_{s}^{2}}\left[i k_{z} \boldsymbol{\nabla}_{s} E_{z}+i \omega \mu \boldsymbol{\nabla}_{s} \times\left(\hat{z} H_{z}\right)\right], \\
& \mathbf{H}_{s}=\frac{1}{k_{s}^{2}}\left[i k_{z} \boldsymbol{\nabla}_{s} H_{z}-i \omega \epsilon \boldsymbol{\nabla}_{s} \times\left(\hat{z} E_{z}\right)\right] .
\end{aligned}
$$

In a compact shape, the $\rho$-dependent transversal fields can be written as

$$
\begin{aligned}
& {\left[\begin{array}{l}
e_{\phi, n p} \\
h_{\phi, n p}
\end{array}\right]=\overline{\bar{H}}_{\phi n}\left(k_{\rho} \rho\right) \bar{a}+\overline{\bar{J}}_{\phi n}\left(k_{\rho} \rho\right) \bar{b},} \\
& {\left[\begin{array}{l}
e_{\rho, n p} \\
h_{\rho, n p}
\end{array}\right]=\overline{\bar{H}}_{\rho n}\left(k_{\rho} \rho\right) \bar{a}+\overline{\bar{J}}_{\rho n}\left(k_{\rho} \rho\right) \bar{b},}
\end{aligned}
$$

where

$$
\begin{gathered}
\overline{\bar{G}}_{\phi n}\left(k_{\rho} \rho\right)=\frac{1}{k_{\rho}^{2} \rho}\left[\begin{array}{cc}
-n k_{z} G_{n}\left(k_{\rho}^{e} \rho\right) & -i \omega \mu_{s} k_{\rho}^{h} \rho G_{n}^{\prime}\left(k_{\rho}^{h} \rho\right) \\
i \omega \epsilon_{s} k_{\rho}^{e} \rho G_{n}^{\prime}\left(k_{\rho}^{e} \rho\right) & -n k_{z} G_{n}\left(k_{\rho}^{h} \rho\right)
\end{array}\right], \\
\overline{\bar{G}}_{\rho n}\left(k_{\rho} \rho\right)=\frac{1}{k_{\rho}^{2} \rho}\left[\begin{array}{cc}
i k_{z} k_{\rho}^{e} \rho G_{n}^{\prime}\left(k_{\rho}^{e} \rho\right) & -n \omega \mu_{s} G_{n}\left(k_{\rho}^{h} \rho\right) \\
n \omega \epsilon_{s} G_{n}\left(k_{\rho}^{e} \rho\right) & i k_{z} k_{\rho}^{h} \rho G_{n}^{\prime}\left(k_{\rho}^{h} \rho\right)
\end{array}\right],
\end{gathered}
$$

where the ' represents the first derivative with respect to argument of the cylindrical functions. Similarly to previous definitions, $G_{n}$ stands for $H_{n}^{(1)}$ in the matrices associated to $\bar{a}$. In addition, $G_{n}$ stands for $J_{n}$ in the matrices associated to $\bar{b}$. The field components in (3-26) and (3-36) reduces to ones 
found in [48] and $[29]^{1}$ for $\alpha^{h}=1$, and reduces to the usual isotropic solution found in $[15, \mathrm{ch} .3]$ for isotropic media $\left(\alpha^{e, h}=1\right)$. Note that in the above expressions for the transverse fields, the $\phi$ and $z$ dependent exponential factor has been omitted for simplicity. Also, the full expression involves the sums over $n$ and $p$ indices, as expressed in (3-24).

\section{3}

\section{Fields Along Radial Stratifications}

Consider the radially-stratified waveguide shown in Fig. 3.2, composed by $N$ layers. Each layer is formed by an uniaxially anisotropic medium over $r_{j-1}<\rho<r_{j}, j=1,2, \ldots, N$, and for $0<\phi<2 \pi$.

Due to the linearly independence of the modal fields with $\exp (i n \phi)$ dependence, we can enforce our boundary conditions for each $n$th harmonic one at a time. The fields at each layer $j$ can be written, in a shorthand notation introduced in [15, Ch. 3], as

$$
\begin{gathered}
\bar{g}_{j \alpha}=\left[\overline{\bar{H}}_{\alpha n}\left(k_{j \rho} \rho\right)+\overline{\bar{J}}_{\alpha n}\left(k_{j \rho} \rho\right) \tilde{\overline{\bar{R}}}_{j, j+1}^{(\rho)}\right] \bar{a}_{j}, \text { or } \\
\bar{g}_{j \alpha}=\left[\overline{\bar{H}}_{\alpha n}\left(k_{j \rho} \rho\right) \tilde{\overline{\bar{R}}}_{j, j-1}^{(\rho)}+\overline{\bar{J}}_{\alpha n}\left(k_{j \rho} \rho\right)\right] \bar{b}_{j},
\end{gathered}
$$

where $\bar{g}_{j \alpha}=\left[e_{j \alpha} h_{j \alpha}\right]^{t}$ is a $2 \times 1$ column vector with the electric and magnetic field components in direction $\alpha=\{\rho, \phi, z\}$. In order to enforce the appropriated boundary conditions at $\rho=r_{j}$, we employ $2 \times 2$ generalized reflection matrices $\tilde{\overline{\bar{R}}}_{j, j \pm 1}^{(\rho)}$ given by $[15$, Ch. 3$]$
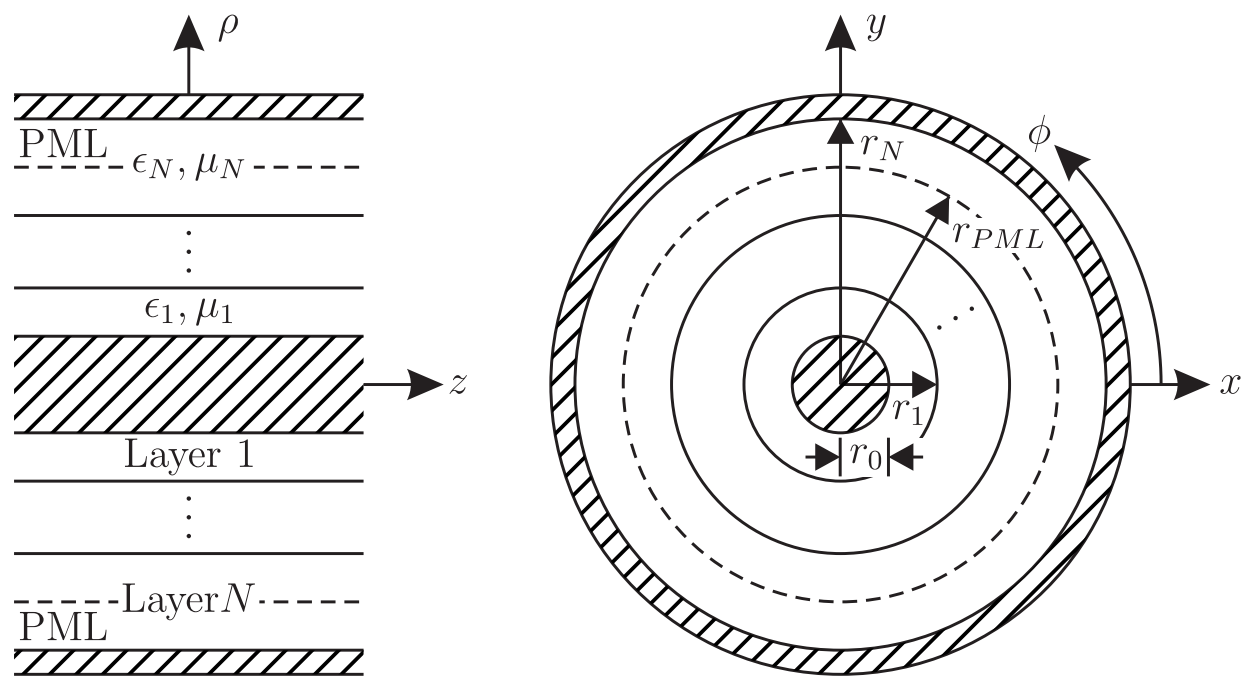

Figure 3.2: Longitudinal and cross-sectional views of a radially-stratified waveguide. Hard walls are depicted by the diagonal stripes.

${ }^{1}$ We found a misprint in the equation for the azimuthal electric field in [29, eq. 3]: the contribution of the axial electric field should be multiplied by $i k_{z}$. 


$$
\tilde{\overline{\bar{R}}}_{j, j \pm 1}^{(\rho)}=\overline{\bar{R}}_{j, j \pm 1}^{(\rho)}+\overline{\bar{T}}_{j \pm 1, j}^{(\rho)} \tilde{\overline{\bar{R}}}_{j \pm 1, j \pm 2}^{(\rho)}\left(\overline{\bar{I}}-\overline{\bar{R}}_{j \pm 1, j}^{(\rho)} \tilde{\overline{\bar{R}}}_{j \pm 1, j \pm 2}^{(\rho)}\right)^{-1} \overline{\bar{T}}_{j, j \pm 1}^{(\rho)}
$$

where the local reflection and transmission matrices can be succinctly written as

$$
\begin{gathered}
\overline{\bar{R}}_{j, j+1}^{(\rho)}=\overline{\bar{D}}_{j a}^{-1}\left[\overline{\bar{H}}_{\phi j+1, j} \overline{\bar{H}}_{z j, j}-\overline{\bar{H}}_{\phi j+1, j} \overline{\bar{H}}_{z j+1, j} \overline{\bar{H}}_{\phi j+1, j}^{-1} \overline{\bar{H}}_{\phi j, j}\right], \\
\overline{\bar{R}}_{j+1, j}^{(\rho)}=\overline{\bar{D}}_{j b}^{-1}\left[\overline{\bar{J}}_{\phi j, j} \overline{\bar{J}}_{z j, j} \overline{\bar{J}}_{\phi j, j}^{-1} \overline{\bar{J}}_{\phi j+1, j}-\overline{\bar{J}}_{\phi j, j} \overline{\bar{J}}_{z j+1, j}\right], \\
\overline{\bar{T}}_{j, j+1}^{(\rho)}=\overline{\bar{D}}_{j b}^{-1}\left[\overline{\bar{J}}_{\phi j, j} \overline{\bar{H}}_{z j, j}-\overline{\bar{J}}_{\phi j, j} \overline{\bar{J}}_{z j, j} \overline{\bar{J}}_{\phi j, j}^{-1} \overline{\bar{H}}_{\phi j, j}\right], \\
\overline{\bar{T}}_{j+1, j}^{(\rho)}=\overline{\bar{D}}_{j a}^{-1}\left[\overline{\bar{H}}_{\phi j+1, j} \overline{\bar{H}}_{z j+1, j} \overline{\bar{H}}_{\phi j+1, j}^{-1} \overline{\bar{J}}_{\phi j+1, j}-\overline{\bar{H}}_{\phi j+1, j} \overline{\bar{J}}_{z j+1, j}\right],
\end{gathered}
$$

where $j=1,2, \ldots, N-1$ and

$$
\begin{aligned}
\overline{\bar{D}}_{j a} & =\overline{\bar{H}}_{\phi j+1, j} \overline{\bar{H}}_{z j+1, j} \overline{\bar{H}}_{\phi j+1, j}^{-1} \overline{\bar{J}}_{\phi j, j}-\overline{\bar{H}}_{\phi j+1, j} \overline{\bar{J}}_{z j, j}, \\
\overline{\bar{D}}_{j b} & =\overline{\bar{J}}_{\phi j, j} \overline{\bar{H}}_{z j+1, j}-\overline{\bar{J}}_{\phi j, j} \overline{\bar{J}}_{z j, j} \overline{\bar{J}}_{\phi j, j}^{-1} \overline{\bar{H}}_{\phi j+1, j} .
\end{aligned}
$$

In the above we have employed a shorthand notation similar to that suggested in [56], such that

$$
\overline{\bar{B}}_{\alpha i, j}=\overline{\bar{B}}_{\alpha n}\left(k_{i \rho} r_{j}\right),
$$

where $\overline{\bar{B}}_{\alpha n}=\overline{\bar{H}}_{\alpha n}$ or $\overline{\bar{J}}_{\alpha n}$ and $\alpha=\{\phi, z\}$.

The notation employed here is very similar to that introduced in [15]. However, we employ the extra superscript $(\rho)$ in all scattering matrices relating the radial discontinuities. This is because in Section 3.4.1 we use the superscript $(z)$ in the GSM matrices that associate fields across axial discontinuities. In this manner, we have a clear identification of the distinct $R$ and $T$ matrices used to incorporate the radial and the axial discontinuities.

The matrices $\overline{\bar{R}}^{(\rho)}$ and $\overline{\bar{T}}^{(\rho)}$ in (3-43) can be reduced exactly to those shown in [56, eq. 31]. Despite the fact that the latter expressions are more compact, we adopt the forms shown above because, as it will be shown later, poles stemming from matrix inversions can be more easily addressed based on $\overline{\bar{D}}_{j a}$ and $\overline{\bar{D}}_{j b}$. Also note that for isotropic media $\overline{\bar{H}}_{z i, j}$ and $\overline{\bar{J}}_{z i, j}$ become diagonal matrices, and (3-43) reduces to the well-know expressions for isotropic media [15, Ch. 3], as expected.

\subsection{1}

\section{Guidance Condition}

On comparing (3-40) with (3-41), we can see that

$$
\bar{a}_{j}=\tilde{\overline{\bar{R}}}_{j, j-1}^{(\rho)} \bar{b}_{j}, \quad \text { and } \quad \bar{b}_{j}=\tilde{\overline{\bar{R}}}_{j, j+1}^{(\rho)} \bar{a}_{j}
$$


or, alternatively we can write

$$
\left(\overline{\bar{I}}-\tilde{\overline{\bar{R}}}_{j, j+1}^{(\rho)} \tilde{\overline{\bar{R}}}_{j, j-1}^{(\rho)}\right) \bar{b}_{j}=\left(\overline{\bar{I}}-\tilde{\overline{\bar{R}}}_{j, j-1}^{(\rho)} \tilde{\overline{\bar{R}}}_{j, j+1}^{(\rho)}\right) \bar{a}_{j}=\overline{0},
$$

which allow us to find a general guidance condition for the modes as

$$
\operatorname{det}\left(\overline{\bar{I}}-\tilde{\overline{\bar{R}}}_{j, j+1}^{(\rho)} \tilde{\overline{\bar{R}}}_{j, j-1}^{(\rho)}\right)=0 .
$$

This condition holds for all $j=1,2, \ldots, N$, but the corresponding dispersion relations share the same set of eigenmode solutions. If we select $j=N$ in (3-48), since $\tilde{\overline{\bar{R}}}_{N, N+1}^{(\rho)}=\overline{\bar{R}}_{N, N+1}^{(\rho)}[15]$, the solutions of characteristic equation

$$
f\left(k_{z}\right)=\operatorname{det}\left(\overline{\bar{I}}-\overline{\bar{R}}_{N, N+1}^{(\rho)} \tilde{\overline{\bar{R}}}_{N, N-1}^{(\rho)}\right)=0
$$

are the discrete values of $k_{z}$ that contribute to our modal solution. We can track solutions for (3-49) into the complex plane by means of the technique presented in [57], but there is no guarantee that all desired eigenmodes will be found. We first need to know the number of solutions to be found.

We can find all desired eigensolutions of (3-49) in a given region of the complex plane $k_{z}$ (or $k_{j \rho}$ ) using the Argument Principle [58], [59, pp. 281-288] and the technique shown in [60], but we first need a dispersion relation free of singularities.

\subsection{2}

\section{Radial Perfectly Matched Layer}

The radial direction of the problem at hand is truncated by a perfectly matched layer (PML) [61-63] to mimic an unbounded medium. The PML extends over $r_{P M L}<\rho<r_{N}$, as illustrated in Fig. 3.2. We use the complex coordinate stretching formulation of the PML because it allow us to reuse all close-form eigenmode formulas shown above. To this end, we first define a one-to-one mapping of the radial coordinate $\rho$ to a stretched $\tilde{\rho}[62,64]$ as

$$
\rho \rightarrow \tilde{\rho}=\int_{0}^{\rho} s_{\rho}\left(\rho^{\prime}\right) d \rho^{\prime}
$$

The use of an integral in the above ensures that $\tilde{\rho}$ varies smoothly, even if $s_{\rho}$ as discontinuities. Typically, the complex stretching variable $s_{\rho}$ employ a polynomial profile defined by

$$
s_{\rho}(\rho)= \begin{cases}1, & \text { for } \rho<r_{P M L} \\ 1+i \alpha_{P M L}\left(\frac{\rho-r_{P M L}}{r_{N}-r_{P M L}}\right)^{q}, & \text { for } r_{P M L} \leq \rho \leq r_{N}\end{cases}
$$

which result in the mapping 


$$
\tilde{\rho}= \begin{cases}\rho, & \text { for } \rho<r_{P M L} \\ \rho+i \alpha_{P M L} \frac{\left(\rho-r_{P M L}\right)^{q+1}}{(q+1)\left(r_{N}-r_{P M L}\right)^{q}}, & \text { for } r_{P M L} \leq \rho \leq r_{N}\end{cases}
$$

The coordinate stretching does not formally change the appearance of Maxwell's equations $[62,64]$. As a result, we can reuse the well-known closedform solutions by mapping the original (real) radial space into a complex coordinate system. Note that in our approach the analytical expressions for the field solutions are independent of the PML profile, and we just need to assume an appropriated complex-value for the outermost radius of the waveguide [57,62-64], i.e.,

$$
r_{N} \rightarrow \tilde{r}_{N}=r_{N}^{\prime}+i r_{N}^{\prime \prime}
$$

We now need to proper set the values $r_{N}^{\prime}$ and $r_{N}^{\prime \prime}$. The real value of the domain truncation $r_{N}^{\prime}$ must be large enough to allow the attenuation of evanescent fields. In contrast, the complex domain of the radial direction $r_{N}^{\prime \prime}$ must be truncated using an appropriated value in order to absorbs the outgoing waves (propagating modes) before they reach the boundaries of the simulated domain.

Formations typically encountered in well drilling environment present conductivity losses, such as the eigenmodes are complex and naturally suffer attenuation before being reflected by an truncation hard-wall. In this case, even a real-valued domain truncation can properly simulate the problem of interest. To reduce the reflection by the truncating boundary, typical, we can truncate radial domain as larger as few skin depths [6,61,63,65-68]. Thus, we can define $r_{N}^{\prime}$ as

$$
r_{N}^{\prime}=\delta_{N} \alpha_{s d}
$$

where $\delta_{N}$ is the skin depth in the outermost layer and $\alpha_{s d}$ is a constant.

A desired attenuation for the propagating waves dictates the value of the imaginary radial boundary $r_{N}^{\prime \prime}$. For $\operatorname{abs}\left(\tilde{r}_{N}\right) \gg 1$, we can approximate the cylindrical wave at the boundary truncation by a local plane wave. In this case, the amplitude of the reflected wave due to a hard-wall placed at $\tilde{r}_{N}$ can be approximated by

$$
R \sim e^{i 2 k_{N \rho} \tilde{r}_{N}} .
$$

Further, it is convenient to write the modal radial wavenumber as $k_{N \rho}=$ $k_{N} \cos \theta_{i}$, where $\theta_{i}$ can be interpreted as the local plane wave incident angle with respect to $\hat{n}=-\hat{\rho}$. For a normal incidence, viz., $\theta_{i}=0$, we can estimate the value of $r_{N}^{\prime \prime}$ using the desired reflection $R(0)$ at the radial domain boundary using

$$
r_{N}^{\prime \prime}=-\frac{1}{k_{N}^{\prime}}\left[\frac{\log (R(0))}{2}+k_{N}^{\prime \prime} r_{N}^{\prime}\right]
$$


Notice that for a loss-less media, $k_{N}^{\prime \prime}=0$, the real part of the complex radius $\tilde{r}_{N}$ has no effect to attenuate an outgoing wave. In this case, the complex stretching dictates the fields attenuation. In contrast, for lossy media, the real space attenuates a propagating wave even without the complex stretching in radial direction. In other words, a large real domain $r_{N}^{\prime}$ sometimes can provide a reflection smaller than $R(0)$. In the typical well-logging environment, at low frequency, the medium are lossy, and a severe attenuation can be anticipated.

For the problem of interest, in which $\sigma / \omega \gg \epsilon_{0} \epsilon_{r}$, non-magnetic media $\left(\mu_{r}=1\right)$ presents wavenumber given by $k_{N} \approx(1+i) / \delta_{N}$. This means that both $k_{N}^{\prime}$ and $k_{N}^{\prime \prime}$ are proportional to $\omega^{1 / 2}$. In this way, using the definition in (3-54), and assuming $R(0)<1$, we can derive

$$
r_{N}^{\prime \prime}=\delta_{N}\left[\frac{|\log (R(0))|}{2}-\alpha_{s d}\right] .
$$

Case the value of $r_{N}^{\prime \prime}$ given by the above is negative, we do not need to employ the complex stretching to bound the radial domain because the real domain thickness provide a large attenuation and a corresponding back-reflection smaller then $R(0)$.

As an alternative, the PML could be interpreted as radially dependent biaxial anisotropic layer $[69,70]$. This approach is very popular and can easily implemented in FD and FE codes. Nevertheless, to the best of our knowledge, the proper solution for the electromagnetic fields requires radial eigenfunctions that satisfy the Bessel equation with complex orders in addition the azimuthal and axial harmonics $e^{i n \phi}$ and $e^{ \pm i k_{z, n p} z}[71-73]$. Besides the difficulty to dealing with Bessel functions of complex orders, case we had used this approach, the PML region should be discretized in a series of sublayers to account the radial dependence of the media inside the absorbing space. Then, to avoid this staircase approximation to the PML profile (and the associated reflections), our algorithm employ the aforementioned complex stretched coordinate interpretation of the PML.

\subsection{3}

\section{Matching the Radial Boundary Conditions}

\subsubsection{1}

\section{Surface Impedance Boundary Condition for Truncate an Open Radial Domain}

The second order (in terms of the wavenumber $k$ ) approximated impedance boundary condition for a medium described by $Z=(\mu / \epsilon)^{1 / 2}$ and covering $\rho \leq r_{0}, \hat{n}=+\hat{\rho}$, can be written as $[74,75]$ 


$$
\begin{aligned}
& E_{\phi}=-Z\left\{\left[1+\frac{1}{2 i k \rho}+\frac{1}{2(2 k \rho)^{2}}-\frac{1}{2 k^{2}}\left(\frac{n^{2}}{\rho^{2}}-k_{z}^{2}\right)\right] H_{z}+\frac{n k_{z}}{k^{2} \rho} H_{\phi}\right\} \\
& E_{z}=Z\left\{\left[1-\frac{1}{2 i k \rho}-\frac{3}{2(2 k \rho)^{2}}+\frac{1}{2 k^{2}}\left(\frac{n^{2}}{\rho^{2}}-k_{z}^{2}\right)\right] H_{\phi}+\frac{n k_{z}}{k^{2} \rho} H_{z} \cdot\right\}
\end{aligned}
$$

For an impedance wall placed at $\rho=r_{N}$, covering $\rho \geq r_{N}, \hat{n}=-\hat{\rho}$, the boundary conditions to be imposed are similar to the above, but we need to change the sign of $E_{\phi}$ and $E_{z}$.

We can combine (3-58) and (3-59) into a matrix form as

$$
\left[\begin{array}{cc}
0 & Z_{\phi z} \\
\frac{1}{Z_{z \phi}} & -\frac{Z_{z z}}{Z_{z \phi}}
\end{array}\right]\left[\begin{array}{c}
E_{z} \\
H_{z}
\end{array}\right]+\left[\begin{array}{cc}
0 & Z_{\phi \phi} \\
0 & 0
\end{array}\right]\left[\begin{array}{c}
E_{\phi} \\
H_{\phi}
\end{array}\right]=\left[\begin{array}{c}
E_{\phi} \\
H_{\phi}
\end{array}\right],
$$

or in a more compact form:

$$
\overline{\bar{M}} \bar{G}_{z}=(\overline{\bar{I}}-\overline{\bar{N}}) \bar{G}_{\phi}
$$

By using (3-41), the condition (3-61) for an impedance wall at $\rho=r_{0}$ can be written as

$$
\begin{aligned}
& \overline{\bar{M}}_{0}\left[\overline{\bar{H}}_{z n}\left(k_{1 \rho} r_{0}\right) \overline{\bar{R}}_{1,0}^{(\rho)}+\overline{\bar{J}}_{z n}\left(k_{1 \rho} r_{0}\right)\right] \bar{b}_{1}= \\
&\left(\overline{\bar{I}}-\overline{\bar{N}}_{0}\right)\left[\overline{\bar{H}}_{\phi n}\left(k_{1 \rho} r_{0}\right) \overline{\bar{R}}_{1,0}^{(\rho)}+\overline{\bar{J}}_{\phi n}\left(k_{1 \rho} r_{0}\right)\right] \bar{b}_{1},
\end{aligned}
$$

Therefore, the local reflection matrix $\overline{\bar{R}}_{1,0}^{(\rho)}$ is given by

$$
\begin{aligned}
\overline{\bar{R}}_{1,0}^{(\rho)}=\left[\left(\overline{\bar{I}}-\overline{\bar{N}}_{0}\right) \overline{\bar{H}}_{\phi n}\left(k_{1 \rho} r_{0}\right)\right. & \left.-\overline{\bar{M}}_{0} \overline{\bar{H}}_{z n}\left(k_{1 \rho} r_{0}\right)\right]^{-1} \\
& \times\left[\overline{\bar{M}}_{0} \overline{\bar{J}}_{z n}\left(k_{1 \rho} r_{0}\right)-\left(\overline{\bar{I}}-\overline{\bar{N}}_{0}\right) \overline{\bar{J}}_{\phi n}\left(k_{1 \rho} r_{0}\right)\right]
\end{aligned}
$$

For an impedance wall placed ar $\rho=r_{N}$, we can proper combine (3-40) and (3-61) such as the local reflection matrix $\overline{\bar{R}}_{N, N+1}^{(\rho)}$ assumes the form

$$
\begin{aligned}
\overline{\bar{R}}_{N, N+1}^{(\rho)}=-[(\overline{\bar{I}}+ & \left.\left.\overline{\bar{N}}_{N+1}\right) \overline{\bar{J}}_{\phi n}\left(k_{N \rho} r_{N}\right)+\overline{\bar{M}}_{N+1} \overline{\bar{J}}_{z n}\left(k_{N \rho} r_{N}\right)\right]^{-1} \\
& \times\left[\overline{\bar{M}}_{N+1} \overline{\bar{H}}_{z n}\left(k_{N \rho} r_{N}\right)-\left(\overline{\bar{I}}+\overline{\bar{N}}_{N+1}\right) \overline{\bar{H}}_{\phi n}\left(k_{N \rho} r_{N}\right)\right] .
\end{aligned}
$$

The matrices $\overline{\bar{M}}_{j}$ and $\overline{\bar{N}}_{j}$ employed above in (3-63) and (3-64) are defined as

where

$$
\begin{gathered}
\overline{\bar{M}}_{j}\left[\begin{array}{cc}
0 & Z_{j, \phi z} \\
\frac{1}{Z_{j, z \phi}} & -\frac{Z_{j, z z}}{Z_{j, z \phi}}
\end{array}\right], \text { and } \\
\overline{\bar{N}}_{j}\left[\begin{array}{cc}
0 & Z_{j, \phi \phi} \\
0 & 0
\end{array}\right],
\end{gathered}
$$




$$
\begin{gathered}
Z_{j, \phi z}=-\sqrt{\frac{\mu_{j}}{\epsilon_{j}}}\left[1+\frac{1}{2 i k_{j} r_{j}}+\frac{1}{2\left(2 k_{j} r_{j}\right)^{2}}-\frac{1}{2 k_{j}^{2}}\left(\frac{n^{2}}{r_{j}^{2}}-k_{z}^{2}\right)\right], \\
Z_{j, z \phi}=\sqrt{\frac{\mu_{j}}{\epsilon_{j}}}\left[1-\frac{1}{2 i k_{j} r_{j}}-\frac{3}{2\left(2 k_{j} r_{j}\right)^{2}}+\frac{1}{2 k_{j}^{2}}\left(\frac{n^{2}}{r_{j}^{2}}-k_{z}^{2}\right)\right], \\
Z_{j, \phi \phi}=-Z_{j, z z}=-\sqrt{\frac{\mu_{j}}{\epsilon_{j}}} \frac{n k_{z}}{k_{j}^{2} r_{j}} .
\end{gathered}
$$

Case the electrical conductivity of the impedance wall goes to infinity, after same simplifications, we can reduce (3-63) and (3-64) to

$$
\begin{gathered}
\overline{\bar{R}}_{1,0}^{(\rho)}=\left[\begin{array}{cc}
-\frac{J_{n}\left(k_{1 \rho}^{e} r_{0}\right)}{H_{n}\left(k_{1 \rho}^{e} r_{0}\right)} & 0 \\
0 & -\frac{J_{n}^{\prime}\left(k_{1 \rho}^{h} r_{0}\right)}{H_{n}^{\prime}\left(k_{1 \rho}^{h} r_{0}\right)}
\end{array}\right], \text { and } \\
\overline{\bar{R}}_{N, N+1}^{(\rho)}=\left[\begin{array}{cc}
-\frac{H_{n}\left(k_{N \rho}^{e} r_{N}\right)}{J_{n}\left(k_{N \rho}^{e} r_{N}\right)} & 0 \\
0 & -\frac{H_{n}^{\prime}\left(k_{N \rho}^{h} r_{N}\right)}{J_{n}^{\prime}\left(k_{N \rho}^{h} r_{N}\right)}
\end{array}\right],
\end{gathered}
$$

which are the well-know [15, p. 352], [48, eq. 27] reflection matrices for a perfect electric conductor (PEC).

Case the magnetic permeability of the impedance wall goes to infinity, after same simplifications, we can reduce (3-63) and (3-64) to

$$
\begin{gathered}
\overline{\bar{R}}_{1,0}^{(\rho)}=\left[\begin{array}{cc}
-\frac{J_{n}^{\prime}\left(k_{1 \rho}^{e} r_{0}\right)}{H_{n}^{\prime}\left(k_{1 \rho}^{e} r_{0}\right)} & 0 \\
0 & -\frac{J_{n}\left(k_{1 \rho}^{h} r_{0}\right)}{H_{n}\left(k_{1 \rho}^{h} r_{0}\right)}
\end{array}\right], \text { and } \\
\overline{\bar{R}}_{N, N+1}^{(\rho)}=\left[\begin{array}{cc}
-\frac{H_{n}^{\prime}\left(k_{N \rho}^{e} r_{N}\right)}{J_{n}^{\prime}\left(k_{N \rho}^{e} r_{N}\right)} & 0 \\
0 & -\frac{H_{n}\left(k_{N \rho}^{h} r_{N}\right)}{J_{n}\left(k_{N \rho}^{h} r_{N}\right)}
\end{array}\right],
\end{gathered}
$$

which can be recognized as the reflection matrices for a perfect magnetic conductor (PMC).

\subsubsection{2}

\section{Solving the Characteristic Equation}

Various zero-finding techniques have been proposed over the years to solve the roots of a nonlinear characteristic equation $f(\gamma)=0$. The problem of finding complex propagation constants requires a two-dimensional zero search, and several works employ quasi-static approximations to solve a simplified transcendental equation $[11,12,57,66,76-79]$. As a second step, these initial approximations $\left(\gamma_{n}\right)$ can be refined by means of some Newton-Raphson iterations, i.e.,

$$
\gamma_{n+1}=\gamma_{n}-\frac{f\left(\gamma_{n}\right)}{f^{\prime}\left(\gamma_{n}\right)},
$$

where $f^{\prime}$ is the derivative of $f$ in respect to $\gamma$.

A proper choice of an initial guess $\gamma_{0}$ is critical for the convergence of (3-74). By this reason, the solution of the guided modes in lossy structures 
require special care. In $[11,12]$, the eigenvalues of a circular waveguide embedded in lossy surroundings were determined from the initial guess given by the high-frequency asymptotic analysis of the problem. The root-finding strategy employed in $[57,80]$ starts out from the lossless eigenvalues of the structure. These eigenvalues lie on the coordinate axis and can be calculated with relatively little numerical effort [57] by using a root search along real (for propagating modes) and the imaginary axis (for evanescent modes). In a second step, we need to gradually increase the losses in the structure and track the modes as they move from the coordinate axis into the complex plane $[57,80]$. The techniques used in these works proved to be numerically efficient in many cases. However, these approaches fail when two eigenvalues are very close or the structure supports complex modes [81-83].

The Newton-Raphson is a very popular technique, however, should be observed that if $f^{\prime}\left(\gamma_{n}\right)$ is too small, the $n$th correction term may diverge. This weak point is particularly serious when the value of the initial guess $\left(\gamma_{0}\right)$ is far from root $\gamma$ [84]. The restriction placed on $\gamma_{0}$ to lie in a region sufficiently small around the solution can be relaxed of we reduce the size of the correction $\Delta \gamma_{n}=\gamma_{n+1}-\gamma_{n}$ by introducing a small positive factor $\epsilon(0 \leq \epsilon \leq 1)$ in (3-74) such as

$$
\Delta \gamma_{n}=-\frac{f\left(\gamma_{n}\right)}{f^{\prime}\left(\gamma_{n}\right)} \epsilon
$$

The essential feature of the above equation is avoid the failure to convergence: note that even when $f^{\prime}\left(\gamma_{n}\right)$ becomes small, the amount $\epsilon$ weighs the RHS of the above equation so that the correction term $\Delta \gamma_{n}$ does not diverge.

By taking limit of both sides of (3-75) when $\epsilon \rightarrow 0$, the $n$th correction term $\Delta \gamma_{n}$ and the factor $\epsilon$ change into $d \gamma$ and $d s$, respectively. Thus, (3-75) can be rearranged as

$$
\frac{d \gamma}{d s}=-\frac{f\left(\gamma_{n}\right)}{f^{\prime}\left(\gamma_{n}\right)}=-\frac{d \gamma}{d(\log (f))}
$$

The above is known as the scalar Davidenko differential equation [85, p. 162], [84,86-88], whose solution can be written as

$$
f(\gamma)=C e^{-s}
$$

where $C$ is an integration constant. Now, it is clear that for $s \rightarrow \infty$, we recover our initial characteristic equation $f(\gamma)=0$. We can further simplify equation (3-76) as follows. Decomposing the $\gamma$ into its real and imaginary parts as $\gamma=\gamma^{\prime}+i \gamma^{\prime \prime}$ and using the Cauchy-Riemann relations, the Davidenko's expressions of a two-coupled first-order ordinary differential equation with an 
independent scalar dummy variable $s$ are obtained as $[84,88]$

$$
\left\{\begin{array}{l}
\frac{\gamma^{\prime}}{d s}=-\frac{1}{\left|f^{\prime}\right|^{2}}\left[\Re e(f) \Re e\left(f^{\prime}\right)+\Im m(f) \Im m\left(f^{\prime}\right)\right] \\
\frac{\gamma^{\prime \prime}}{d s}=+\frac{1}{\left|f^{\prime}\right|^{2}}\left[\Re e(f) \Im m\left(f^{\prime}\right)-\Im m(f) \Re e\left(f^{\prime}\right)\right]
\end{array}\right.
$$

We can solve $\gamma^{\prime}$ and $\gamma^{\prime \prime}$ with the help of the Runge-Kutta algorithm [84], by observing the convergence of both real and imaginary parts of $\gamma$ as $s$ becomes large [84, 86, 88-90].

The advantage of Davidenko's method is that it relaxes the restriction on the choice of the initial guess for the complex root [89] and it can be used when other methods like Newton-Raphson fail to converge. However, one disadvantage of the Davidenko equation is that the error in solving it inevitably accumulate as one marches in $s$ [85], Also, the Davidenko equation, as well as the Newton's method, is singular at limit points or bifurcation points or wherever $f^{\prime}(\gamma)=0[85]$.

Sometimes the analytical derivative of the characteristic equation is very complicated and requires a large computational effort. In these cases, a linear interpolation of $f(\gamma)$ allows to approximate $f^{\prime}$ by means of a finite-difference version of the Newton's method known the secant method [87]. Starting with initial guesses $\gamma_{0}$ and $\gamma_{1}$, the secant method is defined by the recurrence relation [87]

$$
\gamma_{n+1}=\gamma_{n}-\frac{\gamma_{n}-\gamma_{n-1}}{f\left(\gamma_{n}\right)-f\left(\gamma_{n-1}\right)} f\left(\gamma_{n}\right)
$$

which is an approximation for (3-74).

Another widely used root-finding technique is the Muller's method. This method uses parabolic interpolation to approximate $f(\gamma)$ and converges faster than the secant method and is specially preferable to find complex roots [87]. In Muller's method, we start with three points, viz., $\gamma_{0}, \gamma_{1}$ and $\gamma_{2}$, and then we can approximate the zeros of $f(\gamma)$ by the recurrence relation [87], [91, p. 466]

$$
\gamma_{n+1}=\gamma_{n}-\left(\gamma_{n}-\gamma_{n-1}\right) \frac{2 c}{b \pm \sqrt{b^{2}-4 a c}},
$$

where

$$
\begin{aligned}
& a=q f\left(\gamma_{n}\right)-q(1+q) f\left(\gamma_{n-1}\right)+q^{2} f\left(\gamma_{n-2}\right), \\
& b=(2 q+1) f\left(\gamma_{n}\right)-(1+q)^{2} f\left(\gamma_{n-1}\right)+q^{2} f\left(\gamma_{n-2}\right), \\
& c=(1+q) f\left(\gamma_{n}\right),
\end{aligned}
$$

and

$$
q=\frac{\gamma_{n}-\gamma_{n-1}}{\gamma_{n-1}-\gamma_{n-2}}
$$


In (3-80) we must choose one of the two roots. The sign in the denominator is chosen to make its absolute value as large as possible [91, p. 466]. Notice that the square root in the denominator can give complex roots even when the starting values are all real. This feature makes the method very useful for solving dispersions equation for the guided modes in lossless structures supporting complex backwards waves. In this situation, $f(\gamma)$ is a real valued function, but some of their solutions can assume complex values. If a purely real or a purely imaginary initial guess is used in Newton's or in the secant method, the iterations will never converge to complex values. By contrast, in Muller's approach, the same guess will converge to the complex-valued solution, as illustrated in the works on corrugated as well as dielectric loaded waveguides shown in [92]. The Muller's method also was successful to find a few low-order complex eigenvalues in lossy stratified cylindrical waveguides [11,12,93-96].

By using the above described root-finding methods there is no guarantee that all zeros are found. Thus, a critical point in the complex root finding is to establish how many zeros lie within a given region of a complex plane. A widely used procedure $[58,60,97-110]$ to ascertain the number of roots to be found in a given search region is the argument principle ${ }^{2}$. According to [59, pp. 281288], the winding number of a complex function $f(\gamma)$, which is analytic and nonzero on a closed contour $C$, is given by

$$
N_{0}-N_{p}=\frac{1}{2 \pi i} \oint_{C} \frac{f^{\prime}(\gamma)}{f(\gamma)} d \gamma
$$

where $N_{0}$ is the number of zeros and $N_{p}$ is the number of poles of $f$ that lie inside $C$, and the partial derivative of $f$ in respect to $\gamma$ is given by $f^{\prime}$. The integral over the contour is taken in counterclockwise sense. According to the previous definition, the winding number for one zero is +1 and for one singularity is -1 . Case the characteristic equation $f(\gamma)=0$ does not presents poles; i.e., $N_{p}=0$; the value given by (3-85) is an integer equal to the total number of zeros of $f$ inside $C$.

The technique proposed in [58] interpolates the function $f(\gamma)$ by a monic polynomial of degree $N_{0}$, whose zeros coincide with the zeros of $f(\gamma)$ in $C$. In this way, we can reduce our initial problem into the easier computation of the zeros of a polynomial. Recently, a new view on this technique as presented in [100]. The approach shown in [100] has generalized the former formulation from [58] into the simple eigenvalue problem given by

$$
\overline{\bar{H}}<\bar{x}=\lambda \overline{\bar{H}} \bar{x}
$$

${ }^{2}$ Also known as winding number [59, pp. 281-288], method of contour integration [105], Cauchy integration method [102] or Delves-Lyness method (due the pioneering work shown in [58]). 
where $\overline{\bar{H}}$ and $\overline{\bar{H}}<$ are Hankel matrices defined by

$$
\overline{\bar{H}}=\left[\begin{array}{cccc}
s_{0} & s_{1} & \ldots & s_{k-1} \\
s_{1} & \ddots & & \vdots \\
\vdots & & \ddots & \vdots \\
s_{k-1} & s_{k} & \ldots & s_{2 k-2}
\end{array}\right], \quad \text { and } \quad \overline{\bar{H}}^{<}=\left[\begin{array}{cccc}
s_{1} & s_{2} & \ldots & s_{k} \\
s_{2} & \ddots & & \vdots \\
\vdots & & \ddots & \vdots \\
s_{k} & s_{k+1} & \ldots & s_{2 k-1}
\end{array}\right]
$$

for $k=N_{0}$. The entries of the above matrices are the Newton sums of the unknown zeros, given by ${ }^{3}$

$$
s_{p}=\frac{1}{2 \pi i} \oint_{C} \gamma^{p} \frac{f^{\prime}(\gamma)}{f(\gamma)} d \gamma
$$

The solution for the characteristic equation $f(\gamma)$ inside $C$ are the $N_{0}$ eigenvalues $\lambda$ that satisfies (3-86).

Note that the Hankel matrices $\overline{\bar{H}}$ and $\overline{\bar{H}}^{<}$require $2 N_{0}$ Newton sums to be constructed. If $f$ has many zeros inside $C$, then the associated polynomial is of high degree and could be very ill-conditioned $[58,100]$, and thus we need to calculate the integrals $s_{p}$ very accurately. In order to avoid the use of high precision arithmetic, the work in [58] suggests the choice of contours $C$ with no more than 5 zeros inside. In algorithms reported in $[99,102,105]$ a more conservative criteria was employed: the region of search is divided into subcontours, containing not more than four roots.

Appears clear that a large number of roots inside the contour strongly affect the accuracy of the numerical evaluation of the roots, and for this reason, in several applications $[104,107]$ the complete computational domain of interest is partitioned into small sub-domains in such a way that only one root (with multiplicity equal to one) is enclosed by time. Well-isolated simple zeros are determined fast and with high accuracy by just using the integrals $s_{0}$ and $s_{1}$. In this case, we first compute $s_{0}$ in a given contours. Next, if $s_{0}=0$ this domain is discarded. Otherwise, case $s_{0}>1$ we must continue subdividing the domain until $s_{0}=1$. Finally, for each sub-domain $C_{j}$ in which $s_{0}=1$ we can find our required root by means of ${ }^{4}$

$$
\gamma_{j}=\frac{1}{2 \pi i} \oint_{C_{j}} \gamma \frac{f^{\prime}(\gamma)}{f(\gamma)} d \gamma
$$

\footnotetext{
${ }^{3}$ In a similar formulation shown in [101], a derivative-free algorithm was presented for computing zeros of analytic function. In this approach, $f^{\prime}(\gamma)$ is replaced by 1 into (3-88). As a consequence, in this new approach the multiplicities of the zeros cannot be computed explicitly.
}

${ }^{4} \mathrm{An}$ alternative to (3-90) is the derivative-free form given by $[101,111]$

$$
\gamma_{j}=\left(\oint_{C_{j}} \frac{1}{f(\gamma)} d \gamma\right)^{-1} \oint_{C_{j}} \frac{\gamma}{f(\gamma)} d \gamma
$$


It should be observed that the above equation is equivalent to $\gamma_{j}=s_{1} / s_{0}$, satisfying (3-86). This is a very interesting technique because we have the guaranty that inside $C_{j}$ there only one zero to be found. In this case, we can even employ the Newton-Raphson iterations (3-74) using a guess inside $C_{j}$ instead of (3-90). As said before, the polynomial approximation must be of low degree to avoid high magnitude coefficients $s_{p}, p>0$. However, since the exact result $s_{0}$ is known to be an integer, the accuracy required in the numerical integration is low $[58,100]$. Nevertheless, this approach appear restrict to find roots with multiplicity one (or we need the knowledge of multiplicity for each root) and requires a lot of time-consuming two-dimensional bisections, as observed in $[104,107]$.

To our experience, the argument principle can be used to find all roots of a transcendental equation in a given region of the complex plane without requiring initial guesses. However, the numerical difficulty in the calculation of parameters $s_{p}$ makes the method computationally demanding. Also, multiple zeros and very close zeros leads to severe ill-conditioning. Besides that, the choice of the contour $C$ is critical to the calculation of $s_{p}$ : even when just only one zero is inside $C, s_{p}$ can diverge if the contour pass too close the zero inside or close to the zeros outside $C$. This problem is particularly worrying when we partitioned our region of search in several sub-contours. Another equally serious problem is the presence of singularities in our characteristic equation. As mentioned before, each pole cancel out the contribution of a proper zero. In $[99,102,103,108]$ the location of the poles in characteristic equations are estimated and then a (assumed) safe contour is generated to avoid the poles. However, sometimes the contour needs to pass very close to zeros e poles.

The problem of a circular waveguide structure embedded in lossy surroundings as analyzed in [103]. In order to not embed the singularities of the characteristic equation, the integration path suggested in [103] requires to pass close to singularities, demanding special numerical techniques to avoid poor numerical results for $s_{0}$. To minimize the effects of the poles, the work in [108] modifies the winding number approach by working with a contour in the complex plane $\gamma^{2}$, where the positions of the poles and zeros are not too close. However, the trouble with identification and removal of singularities still persists. It is important to remind that the presence of non-recognized singularities (poles) leads to a mistake in the counting of propagation constants, and by this reason, in [110] we proposed a solution for the issues reported in the problem analyzed in $[103,108]$ by redefining the modal characteristic equation in order to be free of singularities. A modified version of the characteristic equation of a lossy circular waveguide was introduced in order to simplify the calculation 
of $s_{0}$. In [110], we have described modifications in classical formulation to find the propagation constants of the guided modes that exclude the singularities of the traditional characteristic equation, making unnecessary the task of locating the poles of this equation and avoid the trial-and-error procedure involved in the definition of the winding number contour.

By combining some of the interesting features of the early described techniques to solve the roots of characteristic equation, in next sections we will generalize the formulation shown in $[60,109,110]$ to proper remove the singularities of the dispersion relation for the radially-stratified cylindrical waveguide in (3-49) and derive guidelines to efficiently application of the argument principle in order to find all desired eigensolutions.

\subsubsection{3}

\section{Pole-Free Characteristic Equation}

As said before, the discrete eigenvalues $k_{z}$ that contributes to our modal solution satisfy

$$
f\left(k_{z}\right)=\operatorname{det}\left(\overline{\bar{I}}-\overline{\bar{R}}_{N, N+1}^{(\rho)} \tilde{\overline{\bar{R}}}_{N, N-1}^{(\rho)}\right)=0 .
$$

Unfortunately, the above characteristic equation has a set of poles that impair the correct location of the eigenvalues in a desired region of complex plane. The poles of this equation can be removed using a deflationary process in which a new characteristic equation is defined as $f_{p f}\left(k_{z}\right)=f\left(k_{z}\right) f_{p}\left(k_{z}\right)$. The function $f_{p}$ inserts zeros in the positions of the poles of $f$, so that $f_{p f}$ preserves the same zeros of $f$, but is free of singularities.

Noting that all required matrices in (3-91) involve $2 \times 2$ matrix operations, we can rewrite the determinant as

$$
\operatorname{det}(\overline{\bar{I}}-\overline{\bar{A}})=1+\operatorname{det}(\overline{\bar{A}})-\operatorname{tr}(\overline{\bar{A}})
$$

where $\operatorname{tr}(\overline{\bar{A}})$ is the trace of the matrix $\overline{\bar{A}}=\overline{\bar{R}}_{N, N+1}^{(\rho)} \tilde{\overline{\bar{R}}}_{N, N-1}^{(\rho)}$.

From last equation, we can see that the poles arise due the matrix inversion operations of $\overline{\bar{D}}_{j a}$ and $\overline{\bar{D}}_{j b}$ in (3-43) and also due to $\left(\overline{\bar{I}}-\overline{\bar{R}}_{j-1, j}^{(\rho)} \tilde{\overline{\bar{R}}}_{j-1, j-2}^{(\rho)}\right)$ in (3-42). For further analysis, it is appropriate to write the inverse of a matrix $\overline{\bar{B}}$ as

$$
\overline{\bar{B}}^{-1}=\frac{\operatorname{adj}(\overline{\bar{B}})}{\operatorname{det}(\overline{\bar{B}})},
$$

where $\operatorname{adj}(\overline{\bar{B}})$ is the transposed of the matrix of cofactors of $\overline{\bar{B}}$.

Besides the matrix inversions, the singularities of Hankel functions in the numerator of the matrices $\overline{\bar{R}}^{(\rho)}$ and $\overline{\bar{T}}^{(\rho)}$ that occur when $k_{j \rho}=0$ or $k_{z}= \pm k_{j}$ also insert poles in the characteristic equation. The points at which $k_{z}= \pm k_{j}$ 
will be called here as critical points because the ramifications of Hankel functions emanating from these points can lead to further issues. However, multicylindrical bounded medium are absence of branch-points singularities at $k_{z}= \pm k_{j}$ [112]. Despite the equation (3-91) be free from branch-points, it may have poles (or zeros) at these critical points. Thus, to facilitate obtaining $f_{p}$, we can define

$$
f_{p}\left(k_{z}\right)=f_{i}\left(k_{z}\right) f_{b p}\left(k_{z}\right)
$$

where $f_{i}$ is a function that removes the poles due matrix inversion and $f_{b p}$ remove the poles due to the critical points.

Noting the denominators of (3-63) and (3-64), it is appropriate to define

$$
\begin{array}{r}
\overline{\bar{D}}_{0 b}=\left[\left(\overline{\bar{I}}-\overline{\bar{N}}_{0}\right) \overline{\bar{H}}_{\phi n}\left(k_{1 \rho} r_{0}\right)-\overline{\bar{M}}_{0} \overline{\bar{H}}_{z n}\left(k_{1 \rho} r_{0}\right)\right], \\
\overline{\bar{D}}_{N a}=\left[\left(\overline{\bar{I}}+\overline{\bar{N}}_{N+1}\right) \overline{\bar{J}}_{\phi n}\left(k_{N \rho} r_{N}\right)+\overline{\bar{M}}_{N+1} \overline{\bar{J}}_{z n}\left(k_{N \rho} r_{N}\right)\right] .
\end{array}
$$

The following specializations apply to perfect electric or perfect magnetic walls:

$$
\begin{gathered}
\overline{\bar{D}}_{0 b}=\left[\begin{array}{cc}
H_{n}\left(k_{1 \rho}^{e} r_{0}\right) & 0 \\
0 & H_{n}^{\prime}\left(k_{1 \rho}^{h} r_{0}\right)
\end{array}\right] \text { for a PEC wall at } \rho=r_{0}, \\
\overline{\bar{D}}_{N a}=\left[\begin{array}{cc}
J_{n}\left(k_{N \rho}^{e} r_{N}\right) & 0 \\
0 & J_{n}^{\prime}\left(k_{N \rho}^{h} r_{N}\right)
\end{array}\right] \text { for a PEC wall at } \rho=r_{N}, \\
\overline{\bar{D}}_{0 b}=\left[\begin{array}{cc}
H_{n}^{\prime}\left(k_{1 \rho}^{e} r_{0}\right) & 0 \\
0 & H_{n}\left(k_{1 \rho}^{h} r_{0}\right)
\end{array}\right] \text { for a PMC wall at } \rho=r_{0}, \\
\overline{\bar{D}}_{N a}=\left[\begin{array}{cc}
J_{n}^{\prime}\left(k_{N \rho}^{e} r_{N}\right) & 0 \\
0 & J_{n}\left(k_{N \rho}^{h} r_{N}\right)
\end{array}\right] \text { for a PMC wall at } \rho=r_{N} .
\end{gathered}
$$

It should be noted that we have $\overline{\bar{R}}_{1,0}^{(\rho)}=\overline{\overline{0}}$ and $\overline{\bar{D}}_{0 b}=\overline{\bar{I}}$ if there is no hard wall placed at $\rho=r_{0}$.

Using the above definitions, after some manipulations, we can show that the zeros of

$$
f_{i}\left(k_{z}\right)=\operatorname{det}\left(\overline{\bar{D}}_{N a}\right) \operatorname{det}\left(\overline{\bar{D}}_{0 b}\right) \prod_{j=1}^{N-1}\left[\operatorname{det}\left(\overline{\bar{D}}_{j b}\right) \operatorname{det}\left(\overline{\bar{I}}-\overline{\bar{R}}_{j, j+1}^{(\rho)} \tilde{\overline{\bar{R}}}_{j, j-1}^{(\rho)}\right)\right]
$$

are poles of $f\left(k_{z}\right)$.

In order to determine $f_{b p}\left(k_{z}\right)$, we must evaluate $f\left(k_{z}\right) f_{i}\left(k_{z}\right)$ for $k_{j \rho} \rightarrow 0$. We can verify that

$$
f_{b p}\left(k_{z}\right)=\prod_{j=1}^{N}\left(k_{j}^{2}-k_{z}^{2}\right)^{m(j)}
$$

where the multiplicity $m(j)$ of the critical point $k_{z}= \pm k_{j}$ is given by 


$$
m(j)= \begin{cases}\frac{3}{2}-\delta_{1, N}\left[\frac{1}{2}+\delta_{0, n}-\frac{u(0)}{2}\right] & \\ \quad-\delta_{0, r_{0}}\left[\frac{1}{2}+n+\delta_{0, n}\left(1-\delta_{1, N}\right)\right]+\frac{u(0)}{2}, & \text { if } j=1, \\ \frac{3}{2}+\frac{u(N)}{2}, & \text { if } j=N \text { and } N>1, \\ 0, & \text { if } j=N+1, \\ 2, & \text { otherwise. }\end{cases}
$$

In the above, the Kronecker delta $\delta_{i, j}$ is equal to 1 if $i=j$ and is equal to 0 otherwise. The presence of a hard wall placed at $\rho=r_{0}$ is indicated by $r_{0}>0$. In contrast, $r_{0}=0$ means that there is no truncation in the inner radial domain. The function $u(j)$ is an indicator for finite and non-zero impedance boundary condition truncating the layer $j$ such as

$$
\begin{gathered}
u(0)=\left(1-\delta_{0, r_{0}}\right)\left(1-\delta_{\infty,\left|Z_{0}\right|}\right)\left(1-\delta_{0, Z_{0}}\right), \text { and } \\
u(N)=\left(1-\delta_{\infty,\left|Z_{N}\right|}\right)\left(1-\delta_{0, Z_{N}}\right) .
\end{gathered}
$$

For a PEC or PMC we have, of course, $u(j)=0$.

By combining (3-91), (3-101) and (3-102), we can write a pole free characteristic equation as

$$
\begin{aligned}
f_{p f}\left(k_{z}\right)=\operatorname{det} & \left(\overline{\bar{I}}-\overline{\bar{R}}_{N, N+1}^{(\rho)} \tilde{\overline{\bar{R}}}_{N, N-1}^{(\rho)}\right)\left(k_{j}^{2}-k_{z}^{2}\right)^{m(N)} \operatorname{det}\left(\overline{\bar{D}}_{0 b}\right) \operatorname{det}\left(\overline{\bar{D}}_{N a}\right) \\
& \times \prod_{j=1}^{N-1}\left[\left(k_{j}^{2}-k_{z}^{2}\right)^{m(j)} \operatorname{det}\left(\overline{\bar{D}}_{j b}\right) \operatorname{det}\left(\overline{\bar{I}}-\overline{\bar{R}}_{j, j+1}^{(\rho)} \tilde{\overline{\bar{R}}}_{j, j-1}^{(\rho)}\right)\right] .
\end{aligned}
$$

Introducing

$$
\overline{\bar{D}}_{j}= \begin{cases}\overline{\bar{D}}_{j a}, & \text { if } j=N \\ \overline{\bar{D}}_{j b}, & \text { if } j<N\end{cases}
$$

and assuming that $\tilde{\overline{\bar{R}}}_{0,-1}^{(\rho)}=\overline{\overline{0}}$, we can derive a compact shape for $f_{p f}\left(k_{z}\right)$ as

$$
f_{p f}\left(k_{z}\right)=\prod_{j=1}^{N+1} \operatorname{det}\left[\left(k_{j}^{2}-k_{z}^{2}\right)^{m(j) / 2} \overline{\bar{D}}_{j-1}\left(\overline{\bar{I}}-\overline{\bar{R}}_{j-1, j}^{(\rho)} \tilde{\bar{R}}_{j-1, j-2}^{(\rho)}\right)\right],
$$

or, alternatively:

$$
f_{p f}\left(k_{z}\right)=\operatorname{det} \prod_{j=1}^{N+1}\left[\left(k_{j}^{2}-k_{z}^{2}\right)^{m(j) / 2} \overline{\bar{D}}_{j-1}\left(\overline{\bar{I}}-\overline{\bar{R}}_{j-1, j}^{(\rho)} \tilde{\overline{\bar{R}}}_{j-1, j-2}^{(\rho)}\right)\right] .
$$

Notice that we do not need to proper define $k_{N+1}$ because $m(N+1)=0$.

Now that we have a pole-free characteristic equation, we can determine the number of eigensolutions that lie inside a given contour $C$ by mean of 


$$
N_{0}=\frac{1}{i 2 \pi} \int_{C}\left[\frac{f_{p f}^{\prime}\left(k_{z}\right)}{f_{p f}\left(k_{z}\right)}\right] d k_{z} .
$$

According to our experience, an efficient numerical algorithm can be constructed calculating $N_{0}$ just one time, avoiding the time-consuming bisection sub-partitioning early reported. We can proper define a region of search in complex plane $k_{z}$ that lead to field solutions with attenuation less than a desired attenuation at a given axial distance. In this way, it is quite prudent assess the asymptotic behavior of $f_{p f}$ before define $C$.

\subsubsection{4}

\section{Eigensolution Approximations and Zero Finding}

The complex coordinate stretching described in (3-53) maps the outermost radius into the complex-valued quantity $\tilde{r}_{N}$. A typical well-posed and highly absorbing PML has $\operatorname{abs}\left(\tilde{r}_{N}\right) \gg r_{N-1}$. This condition allow us to establish an approximation for the axial wavenumber corresponding to modal fields concentrated inside the PML as

$$
\begin{aligned}
& k_{z}^{e} \sim \pm \sqrt{k_{N}^{2}-\left[\frac{p \pi}{\alpha_{N}^{e}\left(\tilde{r}_{N}-r_{N-1}\right)}\right]^{2}}, \\
& k_{z}^{h} \sim \pm \sqrt{k_{N}^{2}-\left[\frac{p \pi}{\alpha_{N}^{h}\left(\tilde{r}_{N}-r_{N-1}\right)}\right]^{2}},
\end{aligned}
$$

for $p=1,2,3, \ldots$. In the above, the square root sign is chosen so that $\Im m\left(k_{z}^{e, h}\right) \geq 0$. The above estimates for $k_{z}^{e}$ and $k_{z}^{h}$ correspond to asymptotic approximations for large $k_{z}$ and $k_{j \rho}$ for quasi-TM and quasi-TE fields, respectively, and are independent of the azimuthal index. By using the above approximate eigenvalues, we can accelerate finding the location of most eigenvalues; however, for typical LWD applications, the arguments of the cylindrical functions $k_{j \rho} r_{j}, j=1, \ldots, N-1$, are small and asymptotic approximations are inappropriate to find the eigenvalues corresponding to modal fields concentrated outside the PML layer. These remaining eigenvalues can be found using random-generated guesses for the Muller's method [91, p. 466].

\subsubsection{5}

\section{Region of Search}

Usually, the region of search for forward traveling waves is defined to cover the first quadrant of the complex $k_{z}\left(=k_{z}^{\prime}+i k_{z}^{\prime \prime}\right)$ plane $[78,103]$, i.e., $k_{z}^{\prime}, k_{z}^{\prime \prime}>0$ as shown in Fig. 3.3(a). However, cylindrically layered waveguides can support solutions with $k_{z}^{\prime}<0$. These so-called backward-waves can appears in closed inhomogeneous waveguides [81,82], [42, pp. 684-685]. Thus, a region 
of search covering $\Im m\left(k_{z}\right)>0$ is adopted. The bounding contour $C^{*}$ is defined as shown in Fig. 3.3(b), consisting of sub-paths $C_{i}^{*}, i=1, \ldots, 4$. The value $k_{z, \text { max }}^{\prime \prime}$ leading to field solutions with attenuation less than $A_{d B}$ (in decibels) at an axial distance $\Delta z$ is given by

$$
k_{z, \text { max }}^{\prime \prime}=-\frac{\log (10)}{20} \frac{A_{d B}}{\Delta z} .
$$

The value of $k_{z, \text { max }}^{\prime}$ can be estimated based on the maximum expected variation of the axial wavenumber $k_{z}$.

Typically, the critical points $k_{j \rho}, j=1,2,3, \ldots$, lead to the large value that $\Re e\left(k_{z}\right)$ can assumes. Thus, a conservative estimation for $k_{z, \max }^{\prime}$ is the largest value of the real part of the wavenumber $k_{j}$. If the critical point associate with $k_{z}=k_{j}$ presents an imaginary part larger than $k_{z, \max }^{\prime \prime}$, the contribution of eigensolutions to the fields is negligible, and this critical point is not relevant to determine $k_{z, \max }^{\prime}$. Consequently, we can define $k_{z, \max }^{\prime}$ by means of

$$
\xi \max \left[\Re e\left(k_{j, r e l}\right)\right],
$$

where $k_{j, r e l}$ is a relevant wavenumber of the layer $j$ whose $\Im m\left(k_{j}\right)<k_{z, \text { max }}^{\prime \prime}$. As mentioned in [50], it is desirable to include a multiplicative factor $\xi$ to avoid numerical issues for modal solutions near critical points where $k_{z}=k_{j}$. Numerical simulation and experimental verifications show that $\xi=1.1$ gives good results.

To our experience, the expression in (3-114) is not adequate to proper estimate $k_{z, \max }^{\prime}$ when the waveguide outermost layer is bounded by a PML. A more accurate estimative can be derived by the intersection of $k_{z}^{e, h}$ (in (3-111) and (3-112)) with the line $i k_{z, \text { max }}^{\prime \prime}$, i.e.,

$$
\Re e\left(k_{z}^{e, h}\right)=\frac{\Re e\left(k_{z}^{e, h}\right)}{\Im m\left(k_{z}^{e, h}\right)} k_{z, \max }^{\prime \prime}
$$

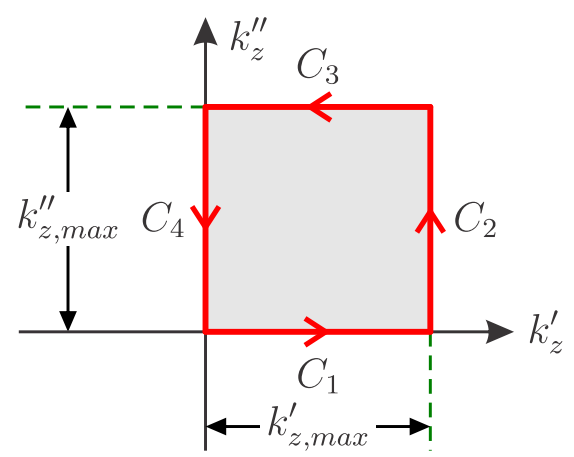

(a) Slow convergence

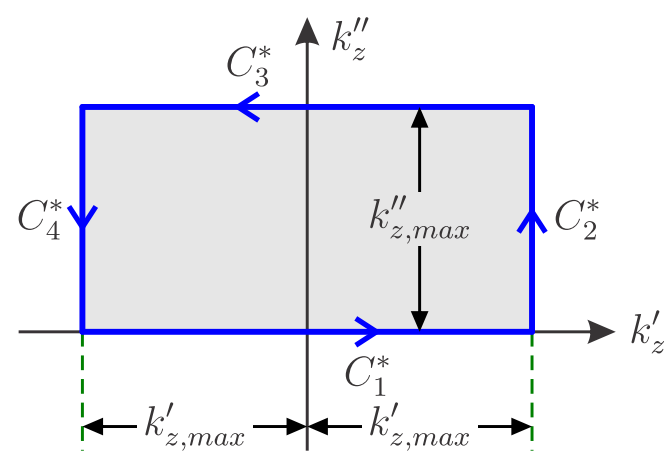

(b) Fast convergence (avoiding $C_{1}^{*}$ )

Figure 3.3: Integration paths on the complex plane $k_{z}$. 
The complex stretching of the PML in (3-53) will modify the spectral distribution of the eigensolution as estimated in (3-111) and (3-112): the eigenvalues will be deflected towards the real axis according the values of $\alpha_{N}^{e, h}$, $\tilde{r}_{N}$ and $r_{N}$.

For LWD applications wherein the anisotropic parameters $\alpha_{N}^{e, h}$ have small imaginary parts, the quasi-static approximation for the high order modes results in

$$
\Re e\left(k_{z}^{e, h}\right)=\frac{\alpha_{P M L}}{r_{N}-r_{N-1}} k_{z, \max }^{\prime \prime} .
$$

Now, we can combine the above with (3-114) in order to properly determine $k_{z, \max }^{\prime}$ using

$$
k_{z, \text { max }}^{\prime}=\max \left[1.1 \times \Re e\left(k_{j, r e l}\right), \frac{\alpha_{P M L}}{r_{N}-r_{N-1}} k_{z, \text { max }}^{\prime \prime}\right] .
$$

If the contour passes very close to roots, a slow-down in the convergence of the numerical line integral in (3-110) results. In particular, the integral over $C_{1}^{*}$ has poor convergence if $\omega \sigma \ll 1$ or $\omega \sigma \gg 1[108,110]$. Based on the symmetric behavior of the characteristic (3-108), $f_{p f}\left(-k_{z}\right)=f_{p f}\left(k_{z}\right)$, and using the results from [60], we can show that the sub-contour $C_{1}^{*}$ does not contribute to the winding number integral, and we can thus express the total number of eigensolutions to be found inside the region in $C^{*}$ as

$$
N_{0}=\frac{1}{i 2 \pi}\left\{\int_{C_{2}^{*}}[\cdot] d k_{z}+\int_{C_{3}^{*}}[\cdot] d k_{z}+\int_{C_{4}^{*}}[\cdot] d k_{z}\right\},
$$

where $[\cdot]$ refers to the expression within brackets in (3-110).

\subsubsection{6 \\ Deflation}

The winding number determined by (3-110) takes into account the number of roots of a function including its multiplicity, e.g., function $f(\gamma)=\gamma^{2}$ has two roots at the origin. Although degenerated modes are not expected in radially-stratified cylindrical structures, the location of complex eigenvalues may be very close to each other, causing convergence issues. To prevent this problem, once one of the roots is determined, we can remove it by performing deflation. In this way, we eliminate the eigenvalues already found and perform a new zero-search on the updated characteristic equation given by

$$
f_{\text {upd }}\left(k_{z}\right)=f_{p f}\left(k_{z}\right) \prod_{j=1}^{N_{\text {in }}}\left(k_{z}^{2}-\gamma_{j, \text { in }}^{2}\right)^{-1} \prod_{j=1}^{N_{\text {out }}}\left(k_{z}^{2}-\gamma_{j, \text { out }}^{2}\right)^{-1} .
$$

In the above, we consider two sets of already known solutions: a set of $N_{\text {in }}$ zeros inside $C^{*}$ and given by $\gamma_{j, i n}$, and a set of $N_{\text {out }}$ zeros outside $C^{*}$ and defined as $\gamma_{j, \text { out }}$. The square function allow us to deflate the eigensolution associated to 
the mirror mode, in which $k_{z}^{\prime \prime}<0$. It is important to note that an initial guess inside $C^{*}$ can sometimes lead to a root outside $C^{*}$. Even though eigensolutions external to $C^{*}$ are not of interest, we should properly deflated our updated characteristic equation using $\gamma_{j, \text { out }}$ in order to avoid convergence problems for roots near the boundaries of $C^{*}$. We update (3-119) for each new solution found until the number of roots found in $C^{*}$ is equal to $N_{0}$. This allows the determining the location of roots very close to each other and also solves problems involving high-order roots.

\subsection{4}

\section{Modal Eigenfield Amplitudes}

A common procedure for finding modal amplitudes is to match the source jump [113, p. 62] condition using (3-40) and (3-41) for propagation in forward and backward radial directions $[6,8,14,47-49]$. If the source is placed at layer $j$, we can find the amplitudes of adjacent layers using

$$
\bar{a}_{j+1}=\overline{\bar{S}}_{j, j+1} \bar{a}_{j}, \quad \text { and } \quad \bar{b}_{j-1}=\overline{\bar{S}}_{j, j-1} \bar{b}_{j},
$$

where $\overline{\bar{S}}_{j, j \pm 1}$ is defined in $[15$, Ch. 3] as

$$
\overline{\bar{S}}_{j, j \pm 1}=\left(\overline{\bar{I}}-\overline{\bar{R}}_{j \pm 1, j}^{(\rho)} \tilde{\overline{\bar{R}}}_{j \pm 1, j \pm 2}^{(\rho)}\right)^{-1} \overline{\bar{T}}_{j, j \pm 1}^{(\rho)} .
$$

This method appear restrict to model sources with discrete radial thickness, such point sources. The modeling of more complex excitation geometries is not a straightforward task.

As an alternative, we can employ the Lorentz reciprocity theorem to expand the source in terms of the modal fields propagating to axial direction. This choice allow us to handle arbitrary sources and culminates in a series of simplification which will be shown in Section 3.5. For this purpose, we can define

$$
\overline{\bar{M}}_{N}=\left(\overline{\bar{I}}-\overline{\bar{R}}_{N, N+1}^{(\rho)} \tilde{\overline{\bar{R}}}_{N, N-1}^{(\rho)}\right) .
$$

From (3-47), the solutions to the homogeneous linear system $\overline{\bar{M}}_{N} \bar{b}_{N}=\overline{0}$ correspond to the null space of $\overline{\bar{M}}_{N}$, i.e., $\bar{b}_{N}=\operatorname{null}\left(\overline{\bar{M}}_{N}\right)$.

Noting that $\overline{\bar{M}}_{N}$ is a $2 \times 2$ matrix, we can write

$$
\overline{\bar{M}}_{N}=\left[\begin{array}{ll}
m_{11} & m_{12} \\
m_{21} & m_{22}
\end{array}\right] \text {. }
$$

For $\operatorname{abs}\left(m_{11}\right)>\operatorname{abs}\left(m_{22}\right)$ we find

$$
\bar{b}_{N}=\operatorname{null}\left(\overline{\bar{M}}_{N}\right)=c\left[\begin{array}{c}
-m_{12} \\
m_{11}
\end{array}\right],
$$


and for $\operatorname{abs}\left(m_{22}\right)>\operatorname{abs}\left(m_{11}\right)$ we find

$$
\bar{b}_{N}=\operatorname{null}\left(\overline{\bar{M}}_{N}\right)=c\left[\begin{array}{c}
m_{22} \\
-m_{21}
\end{array}\right],
$$

where $c$ is an arbitrary constant ${ }^{5}$. Finally, the fields in all layers can be derived using (3-120) and (3-41).

\subsection{5}

\section{Mirror Modes and Fields Symmetry}

If the fields associated with $\left(k_{z}, \mathbf{E}_{s}, E_{z}, \mathbf{H}_{s}, H_{z}\right)$ are a solution for (3-30), the mirror mode [42, p. 861] $\left(-k_{z}, \mathbf{E}_{s},-E_{z},-\mathbf{H}_{s}, H_{z}\right)$ is also a solution of Maxwell's equations. In this manner, we can decompose the $(n p)$ th modal fields as

$$
\begin{aligned}
\mathbf{E}_{n p}^{ \pm} & =\left(\mathbf{e}_{s, n p}^{ \pm}+\hat{z} e_{z, n p}^{ \pm}\right) e^{i n \phi \pm i k_{z, n p} z} \\
& =\left(\mathbf{e}_{s, n p}^{+} \pm \hat{z} e_{z, n p}^{+}\right) e^{i n \phi \pm i k_{z, n p} z}, \text { and }
\end{aligned}
$$

$$
\begin{aligned}
\mathbf{H}_{n p}^{ \pm} & =\left(\mathbf{h}_{s, n p}^{ \pm}+\hat{z} h_{z, n p}^{ \pm}\right) e^{i n \phi \pm i k_{z, n p} z} \\
& =\left( \pm \mathbf{h}_{s, n p}^{+}+\hat{z} h_{z, n p}^{+}\right) e^{i n \phi \pm i k_{z, n p} z}
\end{aligned}
$$

In the above, $\mathbf{e}_{s}^{ \pm}, \mathbf{h}_{s}^{ \pm}, e_{z}^{ \pm}$and $h_{z}^{ \pm}$are the non-harmonic portion of the fields propagating to $\pm z$.

By employing (3-126) and (3-127) we can find the backward propagating fields just by changing the sign of $k_{z, n p}$ and correcting the axial and transversal fields as indicated. The sign of the square root of $k_{\rho, n p}$ in (3-25) must remains the same.

We could further simplify our analysis using a series of symmetric relations derived from (3-37), (3-36) and (3-26), as follows. First, we pick the radial and axial wavenumbers to be symmetric over $n: k_{\rho,-n p}=k_{\rho, n p}$ $k_{z,-n p}=k_{z, n p}$.

In relation to $z$-axis (or $k_{z, n p}$ ), we can write:

$$
\begin{array}{ll}
e_{\rho, n p}^{-}=e_{\rho, n p}^{+} & h_{\rho, n p}^{-}=-h_{\rho, n p}^{+} \\
e_{\phi, n p}^{-}=e_{\phi, n p}^{+} & h_{\phi, n p}^{-}=-h_{\phi, n p}^{+} \\
e_{z, n p}^{-}=-e_{z, n p}^{+} & h_{z, n p}^{-}=h_{z, n p}^{+}
\end{array}
$$

${ }^{5}$ Note that if $\bar{b}_{N}$ is a solution, any constant multiplied by $\bar{b}_{N}$ is also a solution. As we expand the source in terms of the modal fields, the appropriated amplitude of each mode is selected, and this arbitrary constant is removed, as will be clear in (3-228). 
In relation to $\phi$-axis (or $n$, for $n \geq 1$ ), we can write:

$$
\begin{array}{ll}
e_{\rho,-n p}^{+}=-(-1)^{n} e_{\rho, n p}^{+} & h_{\rho,-n p}^{+}=(-1)^{n} h_{\rho, n p}^{+} \\
e_{\phi,-n p}^{+}=(-1)^{n} e_{\phi, n p}^{+} & h_{\phi,-n p}^{+}=-(-1)^{n} h_{\phi, n p}^{+} \\
e_{z,-n p}^{+}=-(-1)^{n} e_{z, n p}^{+} & h_{z,-n p}^{+}=(-1)^{n} h_{z, n p}^{+}
\end{array}
$$

Combining both (3-128) and (3-129), we can derive the fields symmetry in relation to $\phi z$-plane (or $n k_{z, n p}$, for $n \geq 1$ ):

$$
\begin{array}{ll}
e_{\rho,-n p}^{-}=-(-1)^{n} e_{\rho, n p}^{+} & h_{\rho,-n p}^{-}=-(-1)^{n} h_{\rho, n p}^{+} \\
e_{\phi,-n p}^{-}=(-1)^{n} e_{\phi, n p}^{+} & h_{\phi,-n p}^{-}=(-1)^{n} h_{\phi, n p}^{+} \\
e_{z,-n p}^{-}=(-1)^{n} e_{z, n p}^{+} & h_{z,-n p}^{-}=(-1)^{n} h_{z, n p}^{+}
\end{array}
$$

The symmetric field relations above are useful to derive our solution as will show in next sections. We anticipate that only the forward propagation fields associated with positive azimuthal index $n$ need to be calculated such that the remaining term shall be a linear combination of the first ones.

\section{4}

\section{Fields Along Axial Stratifications}

\subsection{1}

\section{Axial Mode-Matching}

Consider the waveguide discontinuity problem illustrated in Fig. 3.4. Two semi-infinity-long guides are connected at $z=z_{1}$ by the common aperture over $S_{a}$. In our analysis the cross-sections of the guides 1 and 2 (in regions 1 and 2) are denoted by $S_{1}$ and $S_{2}$, respectively. We assume that the $S_{1}=S_{a}$ and $S_{2}=S_{a}+S_{w}$, as shown in Fig. 3.4. Notice we have assumed that region 2 is larger than region 1 and also $S_{1} \in S_{2}$. The fields transversal to $z$ can be written as

$$
\begin{aligned}
\mathbf{E}_{j s} & =\sum_{n=-\infty}^{\infty} \sum_{p=1}^{\infty}\left(a_{j, n p}^{+} e^{i k_{z, n p} z}+a_{j, n p}^{-} e^{-i k_{z, n p} z}\right) \mathbf{e}_{j s, n p}(\rho) e^{i n \phi}, \text { and } \\
\mathbf{H}_{j s} & =\sum_{n^{\prime}=-\infty}^{\infty} \sum_{p^{\prime}=1}^{\infty}\left(a_{j, n^{\prime} p^{\prime}}^{+} e^{i k_{z, n^{\prime} p^{\prime}} z}-a_{j, n^{\prime} p^{\prime}}^{-} e^{-i k_{z, n^{\prime} p^{\prime}} z}\right) \mathbf{h}_{j s, n^{\prime} p^{\prime}}(\rho) e^{i n^{\prime} \phi}
\end{aligned}
$$

where $j=1$ or 2 . The fields above consider proper boundary conditions bounding the domain of regions 1 and 2, and that there no fields outside the cross-sections $S_{1}$ and $S_{2}$. The wall $S_{w}$ is characterized by the surface impedance $Z_{2 w}$. 

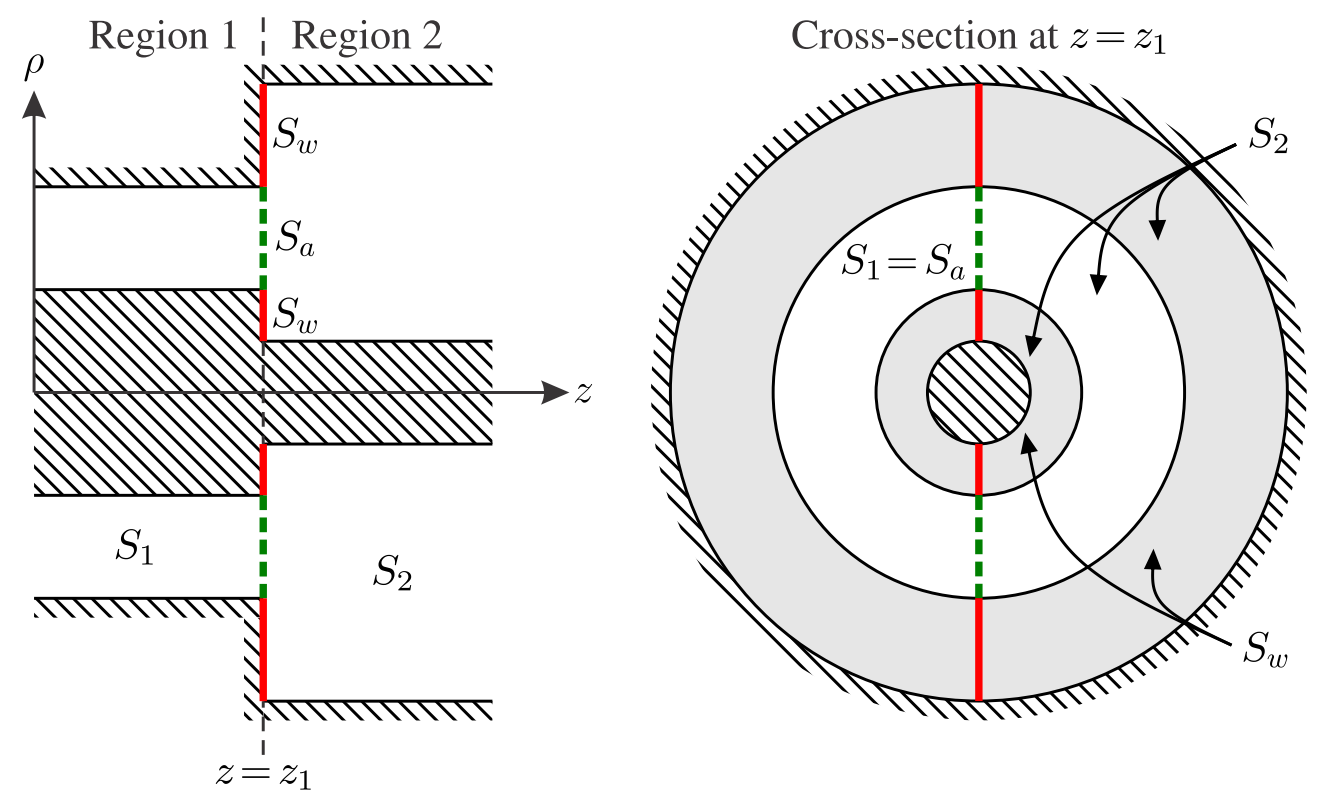

Figure 3.4: Junction between two semi-infinitely-long waveguides.

The continuity of the transversal fields at the junction $\left(z=z_{1}\right)$ must satisfy $[114]$

$$
\begin{gathered}
\hat{z} \times \mathbf{E}_{2 s}=\left\{\begin{array}{c}
\hat{z} \times \mathbf{E}_{1 s}, \text { inside } S_{a}, \\
Z_{2 w} \hat{z} \times\left(\hat{z} \times \mathbf{H}_{2 s}\right), \text { inside } S_{w},
\end{array}\right. \\
\hat{z} \times \mathbf{H}_{2 s}=\hat{z} \times \mathbf{H}_{1 s} \text { inside } S_{a} .
\end{gathered}
$$

In order to simplify the notation, we will rewrite the double sum in our transversal fields in (3-131) and (3-131) through a single sum as

$$
\begin{gathered}
\mathbf{E}_{j s}=\sum_{m}^{\infty}\left(A_{j, m}^{+}+A_{j, m}^{-}\right) \mathbf{E}_{j s, m}, \\
\mathbf{H}_{j s}=\sum_{m^{\prime}}^{\infty}\left(A_{j, m^{\prime}}^{+}-A_{j, m^{\prime}}^{-}\right) \mathbf{H}_{j s, m^{\prime}},
\end{gathered}
$$

where $A_{j, m}^{ \pm}=a_{j, m}^{ \pm} \exp \left( \pm i k_{z, m} z_{1}\right)$ are the forward/backward modal amplitudes at $z=z_{1}$, and the $m$ th transversal field is $\mathbf{G}_{j s, m}=\mathbf{G}_{j s, m}(\rho, \phi), \mathbf{G}=\mathbf{E}$ or $\mathbf{H}$.

Considering $M$ modes in the region 1 and $N$ modes in the region 2 , from the continuity of the transversal electric fields at the junction we can obtain

$$
\begin{aligned}
& \sum_{n=1}^{N}\left(A_{2, n}^{+}+A_{2, n}^{-}\right) \hat{z} \times \mathbf{E}_{2 s, n}=\sum_{m=1}^{M}\left(A_{1, m}^{+}+A_{1, m}^{-}\right) \hat{z} \times \mathbf{E}_{1 s, m}, \text { inside } S_{a}, \\
& \sum_{n=1}^{N}\left(A_{2, n}^{+}+A_{2, n}^{-}\right) \hat{z} \times \mathbf{E}_{2 s, n}=-Z_{2 w} \sum_{n=1}^{N}\left(A_{2, n}^{+}-A_{2, n}^{-}\right) \mathbf{H}_{2 s, n}, \text { inside } S_{w},
\end{aligned}
$$

where we have used the vectorial identity $\mathbf{H}_{2 s}=-\hat{z} \times\left(\hat{z} \times \mathbf{H}_{2 s}\right)$.

Taking the dot product with $\mathbf{H}_{2 s, n^{\prime}}\left(n^{\prime}=1,2, \ldots, N\right)$ in equations (3-137) and (3-138) and integrating over the cross-section $S_{2}$, we can obtain a set of 
$N$ equations given by

$$
\begin{gathered}
\sum_{n=1}^{N}\left(A_{2, n}^{+}+A_{2, n}^{-}\right) \iint_{S_{2}}\left(\mathbf{E}_{2 s, n} \times \mathbf{H}_{2 s, n^{\prime}}\right) \cdot \hat{z} d S= \\
\sum_{m=1}^{M}\left(A_{1, m}^{+}+A_{1, m}^{-}\right) \iint_{S_{1}}\left(\mathbf{E}_{1 s, m} \times \mathbf{H}_{2 s, n^{\prime}}\right) \cdot \hat{z} d S \\
-Z_{2 w} \sum_{n=1}^{N}\left(A_{2, n}^{+}-A_{2, n}^{-}\right) \iint_{S_{w}}\left(\mathbf{H}_{2 s, n} \cdot \mathbf{H}_{2 s, n^{\prime}}\right) d S, \text { for } n^{\prime}=1,2, \ldots, N,
\end{gathered}
$$

where we have employed the vector identity $(\hat{z} \times \mathbf{E}) \cdot \mathbf{H}=(\mathbf{E} \times \mathbf{H}) \cdot \hat{z}$. The above set of equations can be written in a matrix form as

$$
\overline{\bar{Q}}_{2}\left(\bar{A}_{2}^{+}+\bar{A}_{2}^{-}\right)=\overline{\bar{X}}_{12}\left(\bar{A}_{1}^{+}+\bar{A}_{1}^{-}\right)+\overline{\bar{L}}_{2}\left(\bar{A}_{2}^{+}-\bar{A}_{2}^{-}\right),
$$

where the entries of the matrices $\overline{\bar{X}}_{12}, \overline{\bar{Q}}_{2}$ and $\overline{\bar{L}}_{2}$ are given by

$$
\begin{gathered}
\left.\overline{\bar{X}}_{12}\right|_{n, m}=X_{1 m, 2 n}, \\
\left.\overline{\bar{Q}}_{2}\right|_{n, m}=X_{2 m, 2 n}, \\
\left.\overline{\bar{L}}_{2}\right|_{n, m}=L_{2 m, 2 n} .
\end{gathered}
$$

The reaction $[41,115]$ of $m$ th mode in region $i$ to the $n$th mode in region $j$ is defined by the integral

$$
X_{i m, j n}=\left\langle\mathbf{E}_{i s, m}, \mathbf{H}_{j s, n}\right\rangle=\iint_{S_{i}}\left(\mathbf{E}_{i s, m} \times \mathbf{H}_{j s, n}\right) \cdot \hat{z} \rho d \rho d \phi,
$$

and $L_{j m, j n}$ is such as

$$
L_{j m, j n}=-Z_{j w} \iint_{S_{j w}}\left(\mathbf{H}_{j s, m} \cdot \mathbf{H}_{j s, n}\right) \rho d \rho d \phi .
$$

Similarly, following the above steps for the continuity of the magnetic field at $z=z_{1}$, we can write

$$
\sum_{n=1}^{N}\left(A_{2, n}^{+}-A_{2, n}^{-}\right) \hat{z} \times \mathbf{H}_{2 s, n}=\sum_{m=1}^{M}\left(A_{1, m}^{+}-A_{1, m}^{-}\right) \hat{z} \times \mathbf{H}_{1 s, m} \text {, inside } S_{a} .
$$

Taking the dot product with $\mathbf{E}_{1 s, m^{\prime}}\left(m^{\prime}=1,2, \ldots, M\right)$ in equations (3-146) and integrating over the cross-section $S_{1}$, we can obtain a set of $M$ equations given by

$$
\begin{aligned}
& \sum_{n=1}^{N}\left(A_{2, n}^{+}-A_{2, n}^{-}\right) \iint_{S_{1}}\left(\mathbf{E}_{1 s, m^{\prime}} \times \mathbf{H}_{2 s, n}\right) \cdot \hat{z} d S= \\
& \sum_{m=1}^{M}\left(A_{1, m}^{+}-A_{1, m}^{-}\right) \iint_{S_{1}}\left(\mathbf{E}_{1 s, m^{\prime}} \times \mathbf{H}_{2 s, m}\right) \cdot \hat{z} d S, \text { for } m^{\prime}=1,2, \ldots, M,
\end{aligned}
$$


which can be written in a matrix form as

$$
\overline{\bar{X}}_{12}^{t}\left(\bar{A}_{2}^{+}-\bar{A}_{2}^{-}\right)=\overline{\bar{Q}}_{1}\left(\bar{A}_{1}^{+}-\bar{A}_{1}^{-}\right)
$$

where $\overline{\bar{Q}}_{1}$ is given by

$$
\left.\overline{\bar{Q}}_{1}\right|_{n, m}=X_{1 m, 1 n} .
$$

Combining (3-140) and (3-148), we can relate the forward and backward modal amplitudes at the junction $z=z_{1}$ as

$$
\left[\begin{array}{c}
\bar{A}_{1}^{-} \\
\bar{A}_{2}^{+}
\end{array}\right]=\left[\begin{array}{cc}
\overline{\bar{R}}_{12}^{(z)} & \overline{\bar{T}}_{21}^{(z)} \\
\overline{\bar{T}}_{12}^{(z)} & \overline{\bar{R}}_{21}^{(z)}
\end{array}\right]\left[\begin{array}{c}
\bar{A}_{1}^{+} \\
\bar{A}_{2}^{-}
\end{array}\right],
$$

where scattering sub-matrices are given by

$$
\begin{aligned}
& \overline{\bar{R}}_{12}^{(z)}=\left[\overline{\bar{Q}}_{1}+\overline{\bar{X}}_{12}^{t}\left(\overline{\bar{Q}}_{2}-\overline{\bar{L}}_{2}\right)^{-1} \overline{\bar{X}}_{12}\right]^{-1}\left[\overline{\bar{Q}}_{1}-\overline{\bar{X}}_{12}^{t}\left(\overline{\bar{Q}}_{2}-\overline{\bar{L}}_{2}\right)^{-1} \overline{\bar{X}}_{12}\right] \\
& \overline{\bar{T}}_{21}^{(z)}=2\left[\overline{\bar{Q}}_{1}+\overline{\bar{X}}_{12}^{t}\left(\overline{\bar{Q}}_{2}-\overline{\bar{L}}_{2}\right)^{-1} \overline{\bar{X}}_{12}\right]^{-1} \overline{\bar{X}}_{12}^{t}\left[\overline{\bar{I}}+\left(\overline{\bar{Q}}_{2}-\overline{\bar{L}}_{2}\right)^{-1} \overline{\bar{L}}_{2}\right] \\
& \overline{\bar{T}}_{12}^{(z)}=2\left(\overline{\bar{Q}}_{2}-\overline{\bar{L}}_{2}+\overline{\bar{X}}_{12} \overline{\bar{Q}}_{1}^{-1} \overline{\bar{X}}_{12}^{t}\right)^{-1} \overline{\bar{X}}_{12} \text {, and } \\
& \overline{\bar{R}}_{21}^{(z)}=-\left(\overline{\bar{Q}}_{2}-\overline{\bar{L}}_{2}+\overline{\bar{X}}_{12} \overline{\bar{Q}}_{1}^{-1} \overline{\bar{X}}_{12}^{t}\right)^{-1}\left(\overline{\bar{Q}}_{2}+\overline{\bar{L}}_{2}-\overline{\bar{X}}_{12} \overline{\bar{Q}}_{1}^{-1} \overline{\bar{X}}_{12}^{t}\right)
\end{aligned}
$$

\subsubsection{1}

\section{Reaction Integrals}

The modal fields in the above formulation where expressed as a single index mode as in (3-135) and (3-136). Now, it is convenient to recover the double index summation as in (3-131) and (3-132). Then, the reaction of the $(n p)$ th modal fields in region $i$ to the $\left(n^{\prime} p^{\prime}\right)$ th modal fields in region $j$ can be defined as

$$
X_{i(n p), j\left(n^{\prime} p^{\prime}\right)}=\iint_{S_{i}}\left(\mathbf{E}_{i s, n p} \times \mathbf{H}_{j s, n^{\prime} p^{\prime}}\right) \cdot \hat{z} \rho d \rho d \phi,
$$

or

$$
X_{i(n p), j\left(n^{\prime} p^{\prime}\right)}=\iint_{S_{i}}\left[\mathbf{e}_{i s, n p}(\rho) \times \mathbf{h}_{j s, n^{\prime} p^{\prime}}(\rho)\right] \cdot \hat{z} e^{i\left(n+n^{\prime}\right) \phi} \rho d \rho d \phi .
$$

Taking into account the azimuthal orthogonality of the fields, and using the relations in (3-129), the above becomes

$$
\begin{aligned}
X_{i(n p), j\left(n^{\prime} p^{\prime}\right)} & =\delta_{n,-n^{\prime}} \iint_{S_{i}}\left[\mathbf{e}_{i s, n p}(\rho) \times \mathbf{h}_{j s, n^{\prime} p^{\prime}}(\rho)\right] \cdot \hat{z} e^{i\left(n+n^{\prime}\right) \phi} \rho d \rho d \phi \\
& =-\delta_{n,-n^{\prime}} 2 \pi(-1)^{n} \int_{\rho_{i}}\left(s_{n} e_{i \rho, n p} h_{j \phi, n p^{\prime}}+e_{i \phi, n p} h_{j \rho, n p^{\prime}}\right) \rho d \rho,
\end{aligned}
$$

where $s_{n}=1-2 \delta_{0, n}$ and the Kronecker delta is such as $\delta_{n, n^{\prime}}=1$ for $n=n^{\prime}$, and is zero otherwise. Notice that our initial surface integral reduces to a line integral along the radial domain of region $i$. Note that in the above definition 
all the fields propagate to the positive $z$-direction, and the superscript + of the non-harmonic portion of the fields and its radial dependence have been omitted to simplify the notation.

For the special case in which $i=j$, we can take into account the orthogonality of cylindrical function [116, p. 485] and express the self-reaction of the mode $n p$ to mode $n^{\prime} p^{\prime}$ as

$$
X_{j(n p), j\left(n^{\prime} p^{\prime}\right)}=-\delta_{n,-n^{\prime}} \delta_{p, p^{\prime}} 2 \pi(-1)^{n} \int_{\rho_{j}}\left(s_{n} e_{i \rho, n p} h_{j \phi, n p}+e_{i \phi, n p} h_{j \rho, n p}\right) \rho d \rho .
$$

From the above, it is clear that $\overline{\bar{Q}}_{j}$ is a diagonal matrix, and we can properly define the self-reaction associated with the mode $n p$ as

$$
\begin{aligned}
Q_{j, n p} & =X_{j(n p), j(-n p)} \\
& =-2 \pi(-1)^{n} \int_{\rho_{j}}\left(s_{n} e_{j \rho, n p} h_{j \phi, n p}+e_{j \phi, n p} h_{j \rho, n p}\right) \rho d \rho .
\end{aligned}
$$

A consequence of the azimuthal orthogonality of the fields is that we can solve the GSM matrix for each azimuthal index individuality.

From (3-36) and (3-37), the transversal fields of the regions 1 and 2 can be written as

$$
\begin{aligned}
e_{1 \rho} & =\frac{1}{k_{1 \rho}^{2} \rho}\left(i k_{1 z} \alpha_{1}^{e} k_{1 \rho} \rho B_{n}^{\prime e 1}-n \omega \mu_{1 s} B_{n}^{h 1}\right), \\
h_{2 \phi} & =\frac{1}{k_{2 \rho}^{2} \rho}\left(i \omega \epsilon_{2 s} \alpha_{2}^{e} k_{2 \rho} \rho B_{n}^{\prime e 2}-n k_{2 z} B_{n}^{h 2}\right), \\
e_{1 \phi} & =\frac{1}{k_{1 \rho}^{2} \rho}\left(-n k_{1 z} B_{n}^{e 1}-i \omega \mu_{1 s} \alpha_{1}^{h} k_{1 \rho} \rho B_{n}^{\prime h 1}\right), \\
h_{2 \rho} & =\frac{1}{k_{2 \rho}^{2} \rho}\left(n \omega \epsilon_{2 s} B_{n}^{e 2}+i k_{2 z} \alpha_{2}^{h} k_{2 \rho} \rho B_{n}^{\prime h 2}\right),
\end{aligned}
$$

where we drop the modal index $n p$ to shorten notation. In above, $B_{n}^{e, h j}$ are combinations of cylinder functions, which represent the axial fields such as

$$
\begin{gathered}
B_{n}^{e j}=B_{n}^{e j}\left(\alpha^{e} k_{j \rho} \rho\right)=e_{j z}(\rho), \text { and } \\
B_{n}^{h j}=B_{n}^{h j}\left(\alpha^{h} k_{j \rho} \rho\right)=h_{j z}(\rho) .
\end{gathered}
$$

As said before, the prime $\left({ }^{\prime}\right)$ in the cylinder functions means the derivative in respect to the argument.

Substituting the fields in (3-162) into (3-158), we can write the reaction integral

$$
X_{1(n p), 2\left(-n p^{\prime}\right)}=-2 \pi(-1)^{n} \int_{\rho_{1}}\left(s_{n} e_{1 \rho, n p} h_{2 \phi, n p^{\prime}}+e_{1 \phi, n p} h_{2 \rho, n p^{\prime}}\right) \rho d \rho
$$


using a shorthand notation:

$$
\begin{aligned}
X_{1,2}=-2 \pi( & -1)^{n} \int_{\rho_{1}} \frac{1}{k_{1 \rho}^{2} k_{2 \rho}^{2} \rho^{2}} \\
\times & \left\{-\omega \epsilon_{2 s} k_{1 z}\left(s_{n} \alpha_{1}^{e} k_{1 \rho} \rho B_{n}^{\prime e 1} \alpha_{2}^{e} k_{2 \rho} \rho B_{n}^{\prime e 2}+n^{2} B_{n}^{e 1} B_{n}^{e 2}\right)\right. \\
& +\omega \mu_{1 s} k_{2 z}\left(\alpha_{1}^{h} k_{1 \rho} \rho B_{n}^{\prime h 1} \alpha_{2}^{h} k_{2 \rho} \rho B_{n}^{\prime h 2}+n^{2} B_{n}^{h 1} B_{n}^{h 2}\right) \\
& -i k_{1 z} k_{2 z} n\left(\alpha_{1}^{e} k_{1 \rho} \rho B_{n}^{\prime e 1} B_{n}^{h 2}+B_{n}^{e 1} \alpha_{2}^{h} k_{2 \rho} \rho B_{n}^{\prime h 2}\right) \\
& \left.-i \omega^{2} \mu_{1 s} \epsilon_{2 s} n\left(B_{n}^{h 1} \alpha_{2}^{e} k_{2 \rho} \rho B_{n}^{\prime e 2}+\alpha_{1}^{h} k_{1 \rho} \rho B_{n}^{\prime h 1} B_{n}^{e 2}\right)\right\} \rho d \rho,
\end{aligned}
$$

where the modal subscript $n p$ and $-n p^{\prime}$ was properly dropped.

We can simplify (3-166) using the following relations involving the product of cylinder functions $C_{n}(x)$ and $D_{n}(y)$ :

$$
x C_{n}^{\prime}(x) y D_{n}^{\prime}(y)+n^{2} C_{n}(x) D_{n}(y)=\frac{x y}{2}\left[C_{n-1}(x) D_{n-1}(y)+C_{n+1}(x) D_{n+1}(y)\right],
$$

$$
x C_{n}^{\prime}(x) D_{n}+C_{n}(x) y D_{n}^{\prime}(y)=\frac{x y}{2 n}\left[C_{n-1}(x) D_{n-1}(y)-C_{n+1}(x) D_{n+1}(y)\right] .
$$

Using the relations above in (3-166), after a few simplifications we can obtain

$$
\begin{aligned}
X_{1,2}= & -\frac{(-1)^{n} \pi}{k_{1 \rho} k_{2 \rho}}\left\{-s_{n} \omega \epsilon_{2 s} k_{1 z} \alpha_{1}^{e} \alpha_{2}^{e} \mathfrak{L}_{n}^{+}\left(B_{n}^{e 1}, B_{n}^{e 2}\right)+\omega \mu_{1 s} k_{2 z} \alpha_{1}^{h} \alpha_{2}^{h} \mathfrak{L}_{n}^{+}\left(B_{n}^{h 1}, B_{n}^{h 2}\right)\right. \\
& \left.-i k_{1 z} k_{2 z} \alpha_{1}^{e} \alpha_{2}^{h} \mathfrak{L}_{n}^{-}\left(B_{n}^{e 1}, B_{n}^{h 2}\right)-i \omega^{2} \epsilon_{2 s} \mu_{1 s} \alpha_{2}^{e} \alpha_{1}^{h} \mathfrak{L}_{n}^{-}\left(B_{n}^{h 1}, B_{n}^{e 2}\right)\right\}, \quad(3-169)
\end{aligned}
$$

which is valid for $n \geq 0$. The coupling integrals $\mathfrak{L}_{n}^{ \pm}\left(C_{n}, D_{n}\right)$ are defined by

$$
\mathfrak{L}_{n}^{ \pm}\left(C_{n}, D_{n}\right)=\mathfrak{L}_{n-1}\left(C_{n-1}, D_{n-1}\right) \pm \mathfrak{L}_{n+1}\left(C_{n+1}, D_{n+1}\right),
$$

where the Lommel integral is given by

$$
\mathfrak{L}_{m}\left(B_{m}^{1}, B_{m}^{2}\right)=\int_{\rho_{\text {min }}}^{\rho_{\max }} B_{m}^{1}\left(\alpha_{1} k_{1 \rho} \rho\right) B_{m}^{2}\left(\alpha_{2} k_{2 \rho} \rho\right) \rho d \rho,
$$

which is a well-know analytical integral [116, p. 484]. It is interesting to note that reaction $X_{1,2}$ in (3-169) can be broken down into four contribution parts:

$$
X_{1,2}=X_{1,2}^{e e}+X_{1,2}^{h h}+X_{1,2}^{e h}+X_{1,2}^{h e},
$$

which allow us to make a clear distinction of the coupling between the fields arising from $E_{z}$ and $H_{z}$. 
Case $\alpha_{1} k_{1 \rho} \neq \alpha_{2} k_{2 \rho}$, we know that [116, p. 484]

$$
\begin{aligned}
& \int_{\rho_{\min }}^{\rho_{\max }} B_{m}^{1}\left(\alpha_{1} k_{1 \rho} \rho\right) B_{m}^{2}(\left.\alpha_{2} k_{2 \rho} \rho\right) \rho d \rho=\left\{\frac{\rho}{\left(\alpha_{1} k_{1 \rho}\right)^{2}-\left(\alpha_{2} k_{2 \rho}\right)^{2}}\right. \\
&\left.\times\left(\alpha_{1} k_{1 \rho} B_{m+1}^{1} B_{m}^{2}-\alpha_{2} k_{2 \rho} B_{m}^{1} B_{m+1}^{2}\right)\right\}_{\rho_{\min }}^{\rho_{\max }}
\end{aligned}
$$

Thus, we can obtain

$$
\begin{array}{r}
\mathfrak{L}_{m}^{ \pm}\left(B_{m}^{1}, B_{m}^{2}\right)=\left\{\frac { \rho } { ( \alpha _ { 1 } k _ { 1 \rho } ) ^ { 2 } - ( \alpha _ { 2 } k _ { 2 \rho } ) ^ { 2 } } \left\{\alpha_{1} k_{1 \rho} B_{m}^{1} B_{m-1}^{2}-\alpha_{2} k_{2 \rho} B_{m-1}^{1} B_{m}^{2}\right.\right. \\
\left.\left. \pm\left[\alpha_{1} k_{1 \rho} B_{m+2}^{1} B_{m+1}^{2}-\alpha_{2} k_{2 \rho} B_{m+1}^{1} B_{m+2}^{2}\right]\right\}\right\}_{\rho_{\text {min }}}^{\rho_{\text {max }}}
\end{array}
$$

that can be simplified to

$$
\begin{aligned}
\mathfrak{L}_{n}^{ \pm}\left(B_{n}^{1}, B_{n}^{2}\right)= & {\left[\mp \frac{2 n}{\alpha_{1} k_{\rho 1} \alpha_{2} k_{\rho 2}} B_{n}^{1} B_{n}^{2}\right.} \\
& \left.+\frac{(1 \pm 1) \rho}{\left(\alpha_{1} k_{1 \rho}\right)^{2}-\left(\alpha_{2} k_{2 \rho}\right)^{2}}\left(\alpha_{1} k_{1 \rho} B_{n}^{1} B_{n-1}^{2}-\alpha_{2} k_{2 \rho} B_{n-1}^{1} B_{n}^{2}\right)\right]_{\rho_{\text {min }}}^{\rho_{\text {max }}}
\end{aligned}
$$

Case $\alpha_{1} k_{1 \rho} \approx \alpha_{2} k_{2 \rho}$, the above expression for $\mathfrak{L}_{n}^{+}$becomes nearly singular, and further specializations are needed to improve the numerical computations. The application of L'Hospital's rule into (3-173), for $\alpha_{1} k_{1 \rho} \rightarrow \alpha_{2} k_{2 \rho}$, leads to $[117$, p. 134$]$

$$
\begin{aligned}
& \int_{\rho_{\min }}^{\rho_{\max }} B_{m}^{1}\left(\alpha_{1} k_{1 \rho} \rho\right) B_{m}^{2}\left(\alpha_{1} k_{1 \rho} \rho\right) \rho d \rho=\left\{\frac { \rho ^ { 2 } } { 4 } \left[2 B_{m}^{1}\left(\alpha_{1} k_{1 \rho} \rho\right) B_{m}^{2}\left(\alpha_{1} k_{1 \rho} \rho\right)\right.\right. \\
& \left.\left.-B_{m-1}^{1}\left(\alpha_{1} k_{1 \rho} \rho\right) B_{m+1}^{2}\left(\alpha_{1} k_{1 \rho} \rho\right)-B_{m+1}^{1}\left(\alpha_{1} k_{1 \rho} \rho\right) B_{m-1}^{2}\left(\alpha_{1} k_{1 \rho} \rho\right)\right]\right\}_{\rho_{\min }}^{\rho_{\max }}
\end{aligned}
$$

which allow us to derive

$$
\begin{aligned}
\mathfrak{L}_{n}^{ \pm}\left(B_{n}^{1}, B_{n}^{2}\right)=\left\{\frac { \rho ^ { 2 } } { 4 } \left[2 B_{n-1}^{1} B_{n-1}^{2} \pm 2 B_{n+1}^{1} B_{n+1}^{2}\right.\right. & \\
& \left.\left.-B_{n}^{1}\left(B_{n-2}^{2} \pm B_{n+2}^{2}\right)-B_{n}^{2}\left(B_{n-2}^{1} \pm B_{n+2}^{1}\right)\right]\right\}_{\rho_{\min }}^{\rho_{\text {max }}},
\end{aligned}
$$

which in turn can be used to further simplify $\mathfrak{L}_{n}^{-}$as

$$
\mathfrak{L}_{n}^{-}\left(B_{n}^{1}, B_{n}^{2}\right)=\frac{2 n}{\left(\alpha_{1} k_{1 \rho}\right)^{2}}\left[\rho^{2} B_{n}^{1} B_{n}^{2}\right]_{\rho_{\min }}^{\rho_{\max }} .
$$

Notice that the above is valid for $\alpha_{1} k_{1 \rho} \rightarrow \alpha_{2} k_{2 \rho}$ and it is precisely the same result as that of the non-singular parcel of the equation (3-175). 
Another useful special case, that can simplify the self-reaction integrals, comes up when $B_{m}^{1}=B_{m}^{2}$ and $\alpha_{1} k_{1 \rho}=\alpha_{2} k_{2 \rho}$. In this case, we can simplify (3-176) show that [117, p. 135]

$$
\int_{\rho_{\min }}^{\rho_{\max }}\left[B_{m}^{1}\left(\alpha_{1} k_{1 \rho} \rho\right)\right]^{2} \rho d \rho=\left\{\frac{\rho^{2}}{2}\left\{\left[B_{m}^{1}\left(\alpha_{1} k_{1 \rho} \rho\right)\right]^{2}-B_{m-1}^{1} B_{m+1}^{1}\right\}\right\}_{\rho_{\min }}^{\rho_{\max }}
$$

and then

$$
\mathfrak{L}_{n}^{ \pm}\left(B_{n}^{1}, B_{n}^{1}\right)=\left\{\frac{\rho^{2}}{2}\left[\left(B_{n-1}^{1}\right)^{2} \pm\left(B_{n+1}^{1}\right)^{2}-\left(B_{n-2}^{1} B_{n}^{1} \pm B_{n}^{1} B_{n+2}^{1}\right)\right]\right\}_{\rho_{\min }}^{\rho_{\max }} .
$$

As in the previous simplifications, we can further reduce $\mathfrak{L}_{n}^{-}$to

$$
\mathfrak{L}_{n}^{-}\left(B_{n}^{1}, B_{n}^{1}\right)=\frac{2 n}{\left(\alpha_{1} k_{1 \rho}\right)^{2}}\left[\left(\rho B_{n}^{1}\right)^{2}\right]_{\rho_{\min }}^{\rho_{\max }},
$$

which is a special result of (3-178).

\subsubsection{2}

\section{Multiple Radial Layers}

Consider the waveguide junction illustrated in Fig. 3.5. We can write set of radius that define the layers in regions 1 and 2 as

$$
\begin{gathered}
r_{1}=\left\{r_{1,0}, r_{1,1}, r_{1,2}, \ldots, r_{1, N_{1}}\right\}, \text { and } \\
r_{2}=\left\{r_{2,0}, r_{2,1}, r_{2,2}, \ldots, r_{2, N_{2}}\right\},
\end{gathered}
$$

respectively, and also the set of radius in region 2 that intercept the coupling aperture $S_{a}$ as

$$
r_{12}=\left\{r_{2, d+1}, r_{2, d+2}, r_{2, d+3}, \ldots, r_{2, N_{2}-u-1}\right\} .
$$

Consider now the set $r_{a}$, defined by the union of the sets $r_{1}$ and $r_{12}$, and sorted in ascending order, i.e.,

$$
r_{a}=\operatorname{sort}\left(r_{1} \cup r_{12}\right) .
$$

The reaction integral between two generic radially-stratified waveguides in regions 1 and 2 can be written as

$$
\begin{aligned}
& X_{1(n p), 2\left(-n p^{\prime}\right)}=-2 \pi(-1)^{n} \\
& \quad \times \sum_{j=0}^{\operatorname{dim}\left(r_{a}\right)} \int_{r_{a, j}}^{r_{a, j+1}}\left[s_{n} e_{1 \rho, n p}(\rho) h_{2 \phi, n p^{\prime}}(\rho)+e_{1 \phi, n p}(\rho) h_{2 \rho, n p^{\prime}}(\rho)\right] \rho d \rho,
\end{aligned}
$$

and the self-reaction of the region $i=1,2$ can be written as 


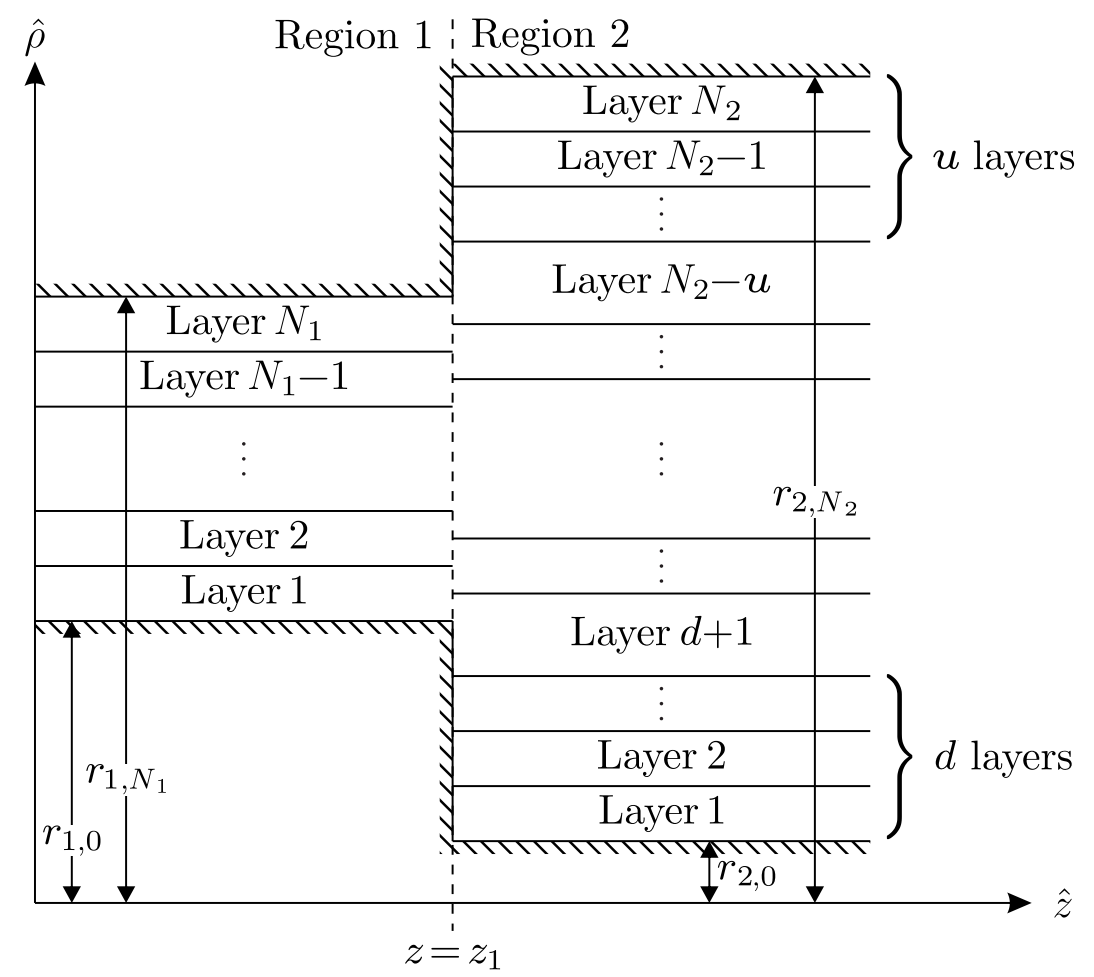

Figure 3.5: Longitudinal view of the junction between two semi-infinitely-long and radially-stratified waveguides.

$$
Q_{i, n p}=-2 \pi(-1)^{n} \sum_{j=0}^{\operatorname{dim}\left(r_{i}\right)} \int_{r_{i, j}}^{r_{i, j+1}}\left[s_{n} e_{i \rho, n p}(\rho) h_{i \phi, n p}(\rho)+e_{i \phi, n p}(\rho) h_{i \rho, n p}(\rho)\right] \rho d \rho .
$$

Notice the above solutions are expresses as a sum of coupling integrals over cross-section segments associated with constant constitutive parameters. Accordingly, all these integrals can be solved analytically using (3-169) by select the proper values of the constitutive parameters, wave numbers, etc., based on the radial position $\rho$.

The axial mode-matching described above allow us to model the junctions between waveguides with an arbitrary number of radial layers in each region. Also, the radial position of the layers can be independent defined in each waveguide. In contrast others formulations, the radial mode-matching approach presented in $[6,8,27,46]$ requires the number of layer and its radial positions shall be equal for all axial regions.

\subsubsection{3}

\section{PML Implications for Mode-Matching}

In Section 3.3.2 we verify that the analytical continuation of the radial variable into the complex plane does not change the well-known solutions for a radially-stratified waveguide. By the same reasons, we can assert that the PML's stretching profile is completely irrelevant in our axial mode-matching 
formulation. Under the mapping $\rho \rightarrow \tilde{\rho}$, we can use all above closed-form coupling integrals, just allowing the outermost radius to assume a complex value, i.e., $r_{N} \rightarrow \tilde{r}_{N}$. In this way, we just need to replace the integration endpoint into the analytical integrals.

If we want to calculate these integrals numerically, it is more appropriated to employ a parametrization into a complex-valued path $L$ such as

$$
\int_{L(\tilde{\rho})} f(\tilde{\rho}) d \tilde{\rho}=\int_{r_{0}}^{r_{N}} f(\tilde{\rho}(\rho)) s_{\rho}(\rho) d \rho
$$

where $s_{\rho}(\rho)=d \tilde{\rho} / d \rho$. Then, the line integral of the complex-valued function $f$ along the path $L$ can be written as

$$
\int_{L}(\tilde{\rho}) f(\tilde{\rho}) d \tilde{\rho}=\int_{r_{0}}^{r_{N}} \Re e\left[f(\tilde{\rho}(\rho)) s_{\rho}(\rho)\right] d \rho+i \int_{r_{0}}^{r_{N}} \Im m\left[f(\tilde{\rho}(\rho)) s_{\rho}(\rho)\right] d \rho .
$$

The above form is suitable to be solved by means of the most common algorithms for numerical integration, i.e., integration of real-valued functions over the real line ${ }^{6}$.

\subsection{2}

\section{Fields at an Observation Point}

By using the GSM in (3-150) we can find the scattering matrices between each pair of waveguide junction. In a waveguide composed by $N$ axial layers, we can use the above formulation to derive the local GSMs for all the waveguide junctions. In Appendix B, we show how to proper transfer the modal amplitudes generated by a source placed at $z=z_{T}$, in region $m$, to an observation point at $z=z_{R}$, in region $n$ (see Fig. 3.6), by means of the equations (B-51) and (B-52). The field components at an arbitrary observation point $\left(\rho, \phi, z=z_{R}\right)$ can then be expressed as

$$
\begin{aligned}
\mathbf{E}_{s}\left(\rho, \phi, z_{R}\right) & =\sum_{n} \sum_{p}\left(A_{R, n p}^{+}+A_{R, n p}^{-}\right) \mathbf{e}_{s, n p}(\rho) e^{i n \phi}, \\
E_{z}\left(\rho, \phi, z_{R}\right) & =\sum_{n} \sum_{p}\left(A_{R, n p}^{+}-A_{R, n p}^{-}\right) e_{z, n p}(\rho) e^{i n \phi}, \\
\mathbf{H}_{s}\left(\rho, \phi, z_{R}\right) & =\sum_{n} \sum_{p}\left(A_{R, n p}^{+}-A_{R, n p}^{-}\right) \mathbf{h}_{s, n p}(\rho) e^{i n \phi}, \\
H_{z}\left(\rho, \phi, z_{R}\right) & =\sum_{n} \sum_{p}\left(A_{R, n p}^{+}+A_{R, n p}^{-}\right) h_{z, n p}(\rho) e^{i n \phi} .
\end{aligned}
$$

More details are presented in Appendix B.

\footnotetext{
${ }^{6}$ We have employed the form shown in (3-189) in our numerical algorithm to verify the correct implementation of the analytical integrals derived in Section 3.4.1.1.
} 


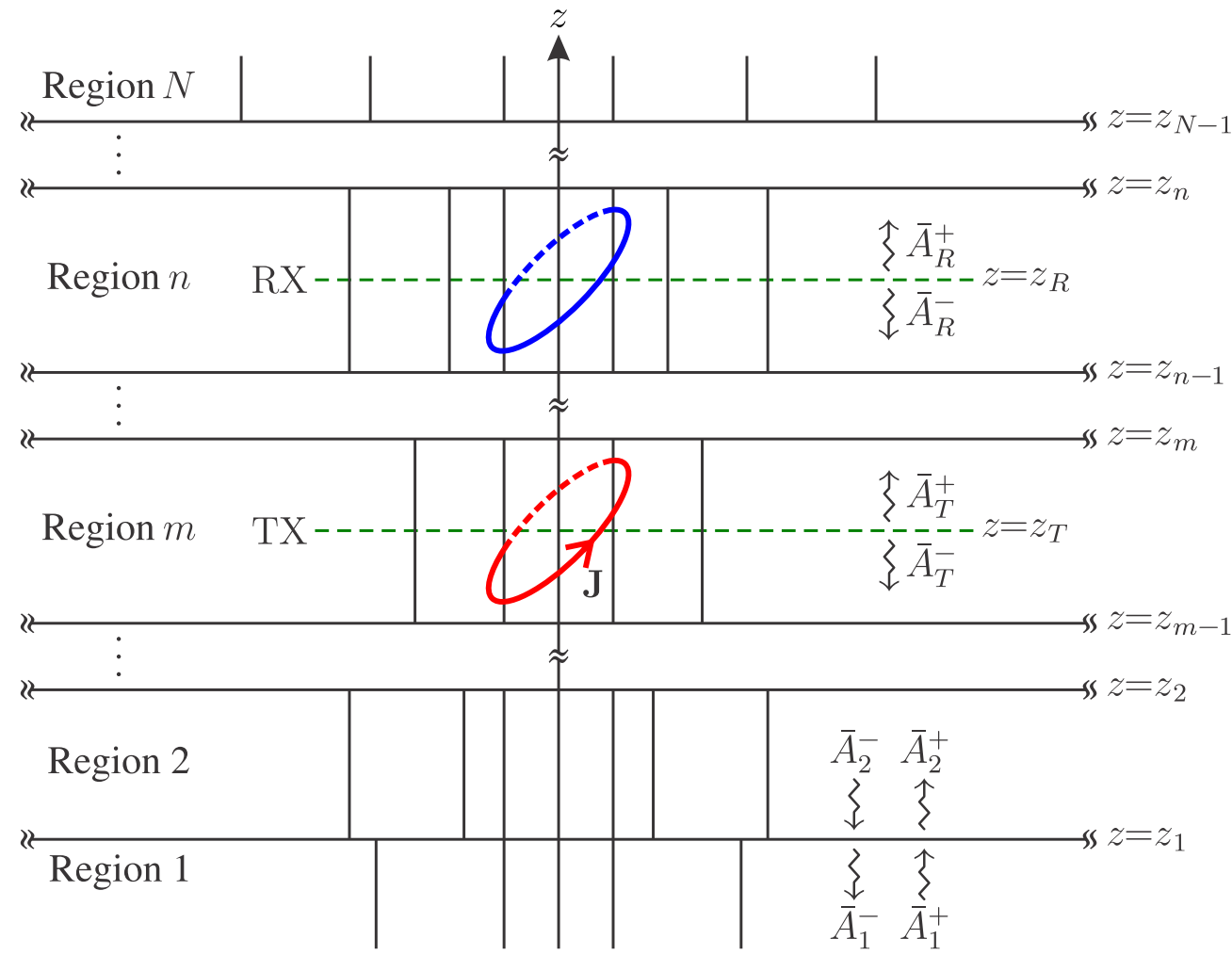

Figure 3.6: Geometry of a stratified cylindrical structure. The cross-section of each region is formed by an arbitrary number of layers. A transmitting TCA is placed at $z=z_{T}$, in region $m$, and the desired receiver antenna is located at $z=z_{R}$, in region $n$.

\section{5}

\section{Mode Excitation from Sources Inside a Cylindrical Waveguide}

We now consider the excitation of the waveguide modes by a generic source, located between two planes at $z=z_{T}^{ \pm}=z_{T} \pm \delta$ as shown in Fig. 3.7. This source generates the fields $\mathbf{E}^{+}$and $\mathbf{H}^{+}$traveling in the positive $z$-direction, and the fields $\mathbf{E}^{-}$and $\mathbf{H}^{-}$traveling to the opposite direction. The electromagnetic fields generated by this source can be expressed in terms of the waveguide modes as

$$
\begin{gathered}
\mathbf{E}^{ \pm}=\sum_{n=-\infty}^{\infty} \sum_{p=1}^{\infty} A_{n p}^{ \pm}\left[\mathbf{e}_{s, n p}^{ \pm}(\rho)+\hat{z} e_{z, n p}^{ \pm}(\rho)\right] e^{ \pm i k_{z, n p}\left(z-z_{T}\right)+i n \phi}, \text { and } \\
\mathbf{H}^{ \pm}=\sum_{n=-\infty}^{\infty} \sum_{p=1}^{\infty} A_{n p}^{ \pm}\left[\mathbf{h}_{s, n p}^{ \pm}(\rho)+\hat{z} h_{z, n p}^{ \pm}(\rho)\right] e^{ \pm i k_{z, n p}\left(z-z_{T}\right)+i n \phi}
\end{gathered}
$$

or, in the view of the symmetry relation in (3-128):

$$
\begin{aligned}
\mathbf{E}^{ \pm} & =\sum_{n=-\infty}^{\infty} \sum_{p=1}^{\infty} A_{n p}^{ \pm}\left[\mathbf{e}_{s, n p}(\rho) \pm \hat{z} e_{z, n p}(\rho)\right] e^{ \pm i k_{z, n p}\left(z-z_{T}\right)+i n \phi}, \text { and } \\
\mathbf{H}^{ \pm} & =\sum_{n=-\infty}^{\infty} \sum_{p=1}^{\infty} A_{n p}^{ \pm}\left[ \pm \mathbf{h}_{s, n p}(\rho)+\hat{z} h_{z, n p}(\rho)\right] e^{ \pm i k_{z, n p}\left(z-z_{T}\right)+i n \phi}
\end{aligned}
$$




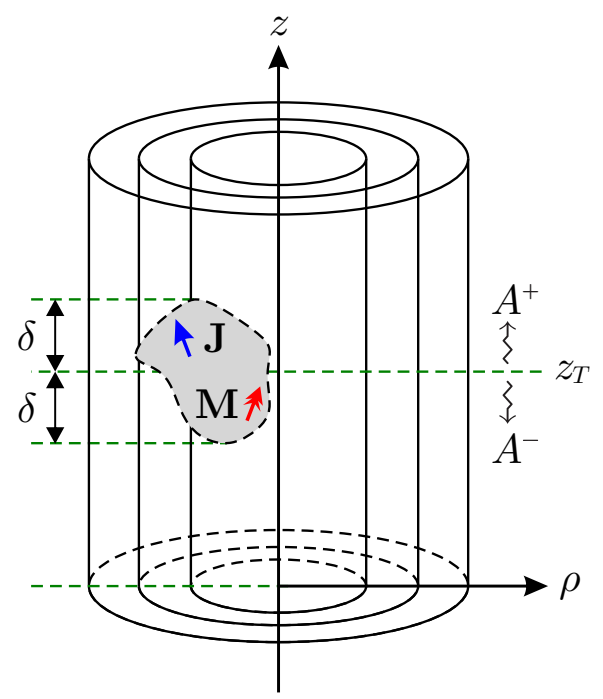

Figure 3.7: Sources in an axially-infinitely-long and radially-stratified waveguide.

for $z \gtrless z_{T}^{ \pm}$.

We can determine the unknown amplitude $A_{n p}^{ \pm}$by using the Lorentz reciprocity theorem. Considering the two sets of sources $\mathbf{J}_{1}, \mathbf{M}_{1}$ and $\mathbf{J}_{2}, \mathbf{M}_{2}$, which generate the fields $\mathbf{E}_{1}, \mathbf{H}_{1}$ and $\mathbf{E}_{1}, \mathbf{H}_{1}$, respectively, in the volume $V$ enclosed by the closed surface $S$, we can derive [118, p. 41]

$\int_{V} \boldsymbol{\nabla} \cdot\left(\mathbf{E}_{1} \times \mathbf{H}_{2}-\mathbf{E}_{2} \times \mathbf{H}_{1}\right) d v=\int_{V}\left(\mathbf{E}_{2} \cdot \mathbf{J}_{1}-\mathbf{E}_{1} \cdot \mathbf{J}_{2}+\mathbf{H}_{1} \cdot \mathbf{M}_{2}-\mathbf{H}_{2} \cdot \mathbf{M}_{1}\right) d v$,

and applying the divergence theorem, we can obtain

$$
\oint_{S}\left(\mathbf{E}_{1} \times \mathbf{H}_{2}-\mathbf{E}_{2} \times \mathbf{H}_{1}\right) \cdot d \mathbf{s}=\int_{V}\left(\mathbf{E}_{2} \cdot \mathbf{J}_{1}-\mathbf{E}_{1} \cdot \mathbf{J}_{2}+\mathbf{H}_{1} \cdot \mathbf{M}_{2}-\mathbf{H}_{2} \cdot \mathbf{M}_{1}\right) d v,
$$

where $d \mathbf{s}$ is an outward pointing vector, normal to the boundary $S$.

For a radially bounded waveguide terminated by a perfectly conductive wall (electric or magnetic), the portion of the surface integral over the waveguide walls vanishes because the tangential fields is zero there; i.e. $\mathbf{E} \times \mathbf{H} \cdot \hat{z}=$ $\mathbf{H} \cdot(\hat{z} \times \mathbf{E})=0$ for a PEC or $\mathbf{E} \times \mathbf{H} \cdot \hat{z}=\mathbf{E} \cdot(\mathbf{H} \times \hat{z})=0$ for a PMC on the walls. This reduces the integration to be just over the guide cross-section $S_{0}^{ \pm}$ at the planes $z=z_{T}^{ \pm}$, i.e.,

$$
\int_{S_{0}^{ \pm}}\left(\mathbf{E}_{1} \times \mathbf{H}_{2}-\mathbf{E}_{2} \times \mathbf{H}_{1}\right) \cdot d \mathbf{s}=\int_{V}\left(\mathbf{E}_{2} \cdot \mathbf{J}_{1}-\mathbf{E}_{1} \cdot \mathbf{J}_{2}+\mathbf{H}_{1} \cdot \mathbf{M}_{2}-\mathbf{H}_{2} \cdot \mathbf{M}_{1}\right) d v
$$

Since the modes are orthogonal over the guide cross-section, we can derive a further simplification:

$$
\int_{S} \mathbf{E}_{n p}^{ \pm} \times \mathbf{H}_{n^{\prime} p^{\prime}}^{ \pm} \cdot \hat{z} d s=\int_{S} \mathbf{E}_{n p}^{ \pm} \times \mathbf{H}_{n^{\prime} p^{\prime}}^{\mp} \cdot \hat{z} d s=0, \text { for } n \neq-n^{\prime} \text { and } p \neq p^{\prime}
$$


Under theses conditions, we can find $A_{n p}^{+}$selecting $\mathbf{E}_{1}$ and $\mathbf{H}_{1}$ as the fields radiated by the sources. In other words, $\mathbf{E}_{1}=\mathbf{E}^{ \pm}$and $\mathbf{H}_{1}=\mathbf{H}^{ \pm}$, depending on whether $z \gtrless z_{T}^{ \pm}$. Also, let $\mathbf{E}_{2}$ and $\mathbf{H}_{2}$ be the $(-n, p)$ th waveguide mode traveling in the negative $z$-direction:

$$
\begin{aligned}
\mathbf{E}_{2} & =\mathbf{E}_{-n p}^{-}, \\
\mathbf{H}_{2} & =\mathbf{H}_{-n p}^{-} .
\end{aligned}
$$

Substituting the above into (3-200) and using $\mathbf{J}_{1}=\mathbf{J}, \mathbf{M}_{1}=\mathbf{M}$, and $\mathbf{J}_{2}=\mathbf{M}_{2}=\mathbf{0}$, we can obtain

$$
\begin{aligned}
& A_{n p}^{+} \int_{S_{0}^{+}}\left(\mathbf{E}_{n p}^{+} \times \mathbf{H}_{-n p}^{-}-\mathbf{E}_{-n p}^{-} \times \mathbf{H}_{n p}^{+}\right) \cdot \hat{z} d s \\
&-A_{n p}^{-} \int_{S_{0}^{-}}\left(\mathbf{E}_{n p}^{-} \times \mathbf{H}_{-n p}^{-}-\mathbf{E}_{-n p}^{-} \times \mathbf{H}_{n p}^{-}\right) \cdot \hat{z} d s= \\
& \int_{V}\left(\mathbf{E}_{-n p}^{-} \cdot \mathbf{J}-\mathbf{H}_{-n p}^{-} \cdot \mathbf{M}\right) d v,
\end{aligned}
$$

where we have used the orthogonality relations in (3-201). We should observed that $d \mathbf{s}$ associated to the fields at $z=z_{T}^{-}$point to the negative $z$-direction, justifying the negative sign in the second integral on LHS of the above equation.

For $n=0$, we can verify that the second surface integral in (3-204) vanish. For $n \neq 0$, we can obtain

$$
\begin{aligned}
& \int_{S_{0}^{-}}\left(\mathbf{E}_{n p}^{-} \times \mathbf{H}_{-n p}^{-}-\mathbf{E}_{-n p}^{-} \times \mathbf{H}_{n p}^{-}\right) \cdot \hat{z} d s= \\
& \iint\left[e_{\rho, n p}^{-} h_{\phi,-n p}^{-}-e_{\phi, n p}^{-} h_{\rho,-n p}^{-}-\left(e_{\rho,-n p}^{-} h_{\phi, n p}^{-}-e_{\phi,-n p}^{-} h_{\rho, n p}^{-}\right)\right] e^{-2 i k_{z, n p} z_{T}^{-}} \rho d \phi d \rho .
\end{aligned}
$$

Using the field relations in (3-128) and (3-130), we can easily verify that the above integral vanish. Then, for any $n$, we can simplify (3-204) in order to get $A_{n p}^{+}$as

where

$$
A_{n p}^{+}=\frac{S_{n p}^{+}}{N_{n p}^{+}},
$$

$$
\begin{gathered}
N_{n p}^{+}=\int_{S_{0}}\left(\mathbf{E}_{n p}^{+} \times \mathbf{H}_{-n p}^{-}-\mathbf{E}_{-n p}^{-} \times \mathbf{H}_{n p}^{+}\right) \cdot \hat{z} d s, \\
S_{n p}^{+}=\int_{V}\left(\mathbf{E}_{-n p}^{-} \cdot \mathbf{J}-\mathbf{H}_{-n p}^{-} \cdot \mathbf{M}\right) d v .
\end{gathered}
$$

For $n=0$, we can obtain

$$
N_{0 p}^{+}=-4 \pi \int\left(e_{\rho, 0 p} h_{\phi, 0 p}-e_{\phi, 0 p} h_{\rho, 0 p}\right) \rho d \rho
$$




$$
\begin{array}{r}
S_{0 p}^{+}=\int_{V}\left[\left(e_{\rho, 0 p} \hat{\rho}+e_{\phi, 0 p} \hat{\phi}-e_{z, 0 p} \hat{z}\right) \cdot \mathbf{J}+\left(h_{\rho, 0 p} \hat{\rho}+h_{\phi, 0 p} \hat{\phi}-h_{z, 0 p} \hat{z}\right) \cdot \mathbf{M}\right] \\
\times e^{-i k_{z, 0 p} z} d v .
\end{array}
$$

In order to simplify the notation, the superscript + as omitted in the transversal fields; i.e., $g_{\alpha, n p}=g_{\alpha, n p}^{+}$, where $g=e$ or $h$ and $\alpha=\rho$ or $\phi$.

For $n \neq 0$, we can obtain

$$
\begin{gathered}
N_{n p}^{+}=4 \pi(-1)^{n} \int\left(e_{\rho, n p} h_{\phi, n p}+e_{\phi, n p} h_{\rho, n p}\right) \rho d \rho, \\
S_{n p}^{+}=(-1)^{n} \int_{V}\left[\left(-e_{\rho, n p} \hat{\rho}+e_{\phi, n p} \hat{\phi}+e_{z, n p} \hat{z}\right) \cdot \mathbf{J}\right. \\
\left.-\left(-h_{\rho, n p} \hat{\rho}+h_{\phi, n p} \hat{\phi}+h_{z, n p} \hat{z}\right) \cdot \mathbf{M}\right] e^{-i n \phi} e^{-i k_{z, n p} z} d v .
\end{gathered}
$$

By repeating the above procedure with $\mathbf{E}_{2}=\mathbf{E}_{-n p}^{+}$and $\mathbf{H}_{2}=\mathbf{H}_{-n p}^{+}$, we can find an expression similar to (3-204) given by

$$
\begin{aligned}
& A_{n p}^{+} \int_{S_{0}^{+}}\left(\mathbf{E}_{n p}^{+} \times \mathbf{H}_{-n p}^{+}-\mathbf{E}_{-n p}^{+} \times \mathbf{H}_{n p}^{+}\right) \cdot \hat{z} d s \\
&-A_{n p}^{-} \int_{S_{0}^{-}}\left(\mathbf{E}_{n p}^{-} \times \mathbf{H}_{-n p}^{+}-\mathbf{E}_{-n p}^{+} \times \mathbf{H}_{n p}^{-}\right) \cdot \hat{z} d s= \\
& \int_{V}\left(\mathbf{E}_{-n p}^{+} \cdot \mathbf{J}-\mathbf{H}_{-n p}^{+} \cdot \mathbf{M}\right) d v .
\end{aligned}
$$

For $n=0$, we clear see that the first surface integral in (3-213) vanish. Using the field relations in (3-129), we can easily verify that the above integral vanish for $n \neq 0$. Then, for any $n$, we can simplify (3-213) in order to get $A_{n p}^{-}$as

$$
A_{n p}^{-}=\frac{S_{n p}^{-}}{N_{n p}^{-}},
$$

where

$$
\begin{gathered}
N_{n p}^{-}=-\int_{S_{0}}\left(\mathbf{E}_{n p}^{-} \times \mathbf{H}_{-n p}^{+}-\mathbf{E}_{-n p}^{+} \times \mathbf{H}_{n p}^{-}\right) \cdot \hat{z} d s \\
S_{n p}^{-}=\int_{V}\left(\mathbf{E}_{-n p}^{+} \cdot \mathbf{J}-\mathbf{H}_{-n p}^{+} \cdot \mathbf{M}\right) d v .
\end{gathered}
$$

For $n=0$, we can obtain

$$
\begin{gathered}
N_{0 p}^{-}=-4 \pi \int\left(e_{\rho, 0 p} h_{\phi, 0 p}-e_{\phi, 0 p} h_{\rho, 0 p}\right) \rho d \rho \\
S_{0 p}^{-}=\int_{V}\left[\left(e_{\rho, 0 p} \hat{\rho}+e_{\phi, 0 p} \hat{\phi}+e_{z, 0 p} \hat{z}\right) \cdot \mathbf{J}-\left(h_{\rho, 0 p} \hat{\rho}+h_{\phi, 0 p} \hat{\phi}+h_{z, 0 p} \hat{z}\right) \cdot \mathbf{M}\right] \\
\times e^{i k_{z, 0 p} z} d v .
\end{gathered}
$$


For $n \neq 0$, we can obtain

$$
\begin{gathered}
N_{n p}^{-}=4 \pi(-1)^{n} \int\left(e_{\rho, n p} h_{\phi, n p}+e_{\phi, n p} h_{\rho, n p}\right) \rho d \rho, \\
S_{n p}^{-}=(-1)^{n} \int_{V}\left[\left(-e_{\rho, n p} \hat{\rho}+e_{\phi, n p} \hat{\phi}-e_{z, n p} \hat{z}\right) \cdot \mathbf{J}\right. \\
\left.-\left(+h_{\rho, n p} \hat{\rho}-h_{\phi, n p} \hat{\phi}+h_{z, n p} \hat{z}\right) \cdot \mathbf{M}\right] e^{-i n \phi} e^{i k_{z, n p} z} d v .
\end{gathered}
$$

Then, for $n=0$ we can write

$$
\begin{array}{r}
S_{0 p}^{ \pm}=\int_{V}\left[\left(e_{\rho, 0 p} \hat{\rho}+e_{\phi, 0 p} \hat{\phi} \mp e_{z, 0 p} \hat{z}\right) \cdot \mathbf{J}-\left(\mp h_{\rho, 0 p} \hat{\rho} \mp h_{\phi, 0 p} \hat{\phi}+h_{z, 0 p} \hat{z}\right) \cdot \mathbf{M}\right] \\
\times e^{\mp i k_{z, 0 p} z} d v, \quad(3-22
\end{array}
$$

or

$$
\begin{array}{r}
S_{0 p}^{ \pm}=\int_{V}\left[\left(e_{\rho, 0 p} \hat{\rho}+e_{\phi, 0 p} \hat{\phi} \mp e_{z, 0 p} \hat{z}\right) \cdot \mathbf{J} \pm\left(h_{\rho, 0 p} \hat{\rho}+h_{\phi, 0 p} \hat{\phi} \mp h_{z, 0 p} \hat{z}\right) \cdot \mathbf{M}\right] \\
\times e^{\mp i k_{z, 0 p} z} d v .
\end{array}
$$

Then, for $n \neq 0$ we can write

$$
\begin{aligned}
S_{n p}^{ \pm}=(-1)^{n} \int_{V} & {\left[\left(-e_{\rho, n p} \hat{\rho}+e_{\phi, n p} \hat{\phi} \pm e_{z, n p} \hat{z}\right) \cdot \mathbf{J}\right.} \\
& \left.-\left(\mp h_{\rho, n p} \hat{\rho} \pm h_{\phi, n p} \hat{\phi}+h_{z, n p} \hat{z}\right) \cdot \mathbf{M}\right] e^{-i n \phi} e^{\mp i k_{z, n p} z} d v
\end{aligned}
$$

or

$$
\begin{aligned}
S_{n p}^{ \pm}=(-1)^{n} \int_{V} & {\left[\left(-e_{\rho, n p} \hat{\rho}+e_{\phi, n p} \hat{\phi} \pm e_{z, n p} \hat{z}\right) \cdot \mathbf{J}\right.} \\
& \left.\mp\left(-h_{\rho, n p} \hat{\rho}+h_{\phi, n p} \hat{\phi} \pm h_{z, n p} \hat{z}\right) \cdot \mathbf{M}\right] e^{-i n \phi} e^{\mp i k_{z, n p} z} d v .
\end{aligned}
$$

Note that $N_{n p}^{-}=N_{n p}^{+}$for all $n$. Furthermore, using the symmetry relations in (3-129), we can also verify that $N_{-n p}^{ \pm}=N_{n p}^{ \pm}$. For this reason, from this point, the modal normalization will be denoted merely by $N_{n p}$.

Introducing $s_{n}=1-2 \delta_{0, n}$, we can obtain expressions for $n=$ $0, \pm 1, \pm 2, \ldots$ given by

$$
\begin{aligned}
S_{n p}^{ \pm}=(-1)^{n} \int_{V} & {\left[\left(-s_{n} e_{\rho, n p} \hat{\rho}+e_{\phi, n p} \hat{\phi} \pm s_{n} e_{z, n p} \hat{z}\right) \cdot \mathbf{J}\right.} \\
& \left. \pm\left(h_{\rho, n p} \hat{\rho}-s_{n} h_{\phi, n p} \hat{\phi} \mp h_{z, n p} \hat{z}\right) \cdot \mathbf{M}\right] e^{-i n \phi} e^{\mp i k_{z, n p} z} d v
\end{aligned}
$$




$$
\begin{aligned}
N_{n p} & =4 \pi(-1)^{n} \int\left(s_{n} e_{\rho, n p} h_{\phi, n p}+e_{\phi, n p} h_{\rho, n p}\right) \rho d \rho \\
& =-2 Q_{n p},
\end{aligned}
$$

where $Q_{n p}$ is the self-reaction of the $(n p)$ th mode, and can be determined by using (3-187).

The definition of $A_{n p}^{ \pm}$is the ratio of $S_{n p}^{ \pm}$by $N_{n p}$. Noting that both $S_{n p}^{ \pm}$ and $N_{n p}$ have the common term $(-1)^{n}$, we can redefine $A_{n p}^{ \pm}$so that

$$
A_{n p}^{ \pm}=\frac{\hat{S}_{n p}^{ \pm}}{\hat{N}_{n p}}
$$

where it is clear that $\hat{S}_{n p}^{ \pm}=(-1)^{n} S_{n p}^{ \pm}$and $\hat{N}_{n p}=(-1)^{n} N_{n p}$.

The wavelength typically employed in geophysical sensing is very large than the wire antenna thickness of the LWD tools. Hence, we can model the sensor tool antennas as filament-like source densities carrying constant currents.

\subsection{1}

\section{Hertzian Electric Dipole}

Consider a Hertzian electric dipole source that points at an angle $\theta_{T}$ from the axial $z$-direction, defined by the current density

$$
\mathbf{J}=j_{T} \boldsymbol{\delta}_{T}
$$

where $j_{T}=I_{T} l_{T}$ is the current dipole moment for a current-carrying $I_{T}$ over an infinitesimal length $l_{T}$, and

$$
\begin{aligned}
\boldsymbol{\delta}_{T} & =\delta\left(\rho-\rho_{T}\right) \frac{\delta\left(\phi-\phi_{T}\right)}{\rho} \delta\left(z-z_{T}\right)\left(\sin \theta_{T} \hat{\rho}+\cos \theta_{T} \hat{z}\right) \\
& =\frac{1}{2 \pi \rho} \sum_{n=-\infty}^{\infty} e^{i n\left(\phi-\phi_{T}\right)} \delta\left(\rho-\rho_{T}\right) \delta\left(z-z_{T}\right)\left(\sin \theta_{T} \hat{\rho}+\cos \theta_{T} \hat{z}\right)
\end{aligned}
$$

The modal amplitude due to $\mathbf{J}$ is given by (3-228), such we can obtain

$$
\begin{aligned}
& S_{n p}^{ \pm}=(-1)^{n} j_{T} \frac{1}{2 \pi} e^{\mp i k_{z, n p} z_{T}} \\
& \times \int_{-\pi}^{\pi}\left\{\sum_{n^{\prime}=-\infty}^{\infty} e^{i n^{\prime}\left(\phi-\phi_{T}\right)}\left[-s_{n} e_{\rho, n p}\left(\rho_{T}\right) \sin \theta_{T} \pm s_{n} e_{z, n p}\left(\rho_{T}\right) \cos \theta_{T}\right]\right\} e^{-i n \phi} d \phi .
\end{aligned}
$$

Using the orthogonality relation

$$
\int_{-\pi}^{\pi} e^{i n^{\prime}\left(\phi-\phi_{T}\right)} e^{-i n \phi} d \phi=\delta_{n^{\prime} n} 2 \pi e^{-i n \phi_{T}},
$$


we obtain

$$
S_{n p}^{ \pm}=(-1)^{n} s_{n} j_{T} e^{-i n \phi_{T}} e^{\mp i k_{z, n p} z_{T}}\left[-e_{\rho, n p}\left(\rho_{T}\right) \sin \theta_{T} \pm e_{z, n p}\left(\rho_{T}\right) \cos \theta_{T}\right] .
$$

\subsection{2}

\section{Hertzian Magnetic Dipole}

Consider now a Hertzian magnetic dipole source that points at an angle $\theta_{T}$ from the axial $z$-direction, defined by the current density

$$
\mathbf{M}=m_{T} \boldsymbol{\delta}_{T},
$$

where $m_{T}=I_{T}^{m} l_{T}$ is the magnetic dipole moment for a magnetic current $I_{T}^{m}$ over the infinitesimal length $l_{T}$, and $\boldsymbol{\delta}_{T}$ is defined in (3-231). This magnetic dipole can model a small current loop with radius $a_{T}$ by means of the principle of duality [55, p. 100], [15, p. 74], [54, p. 234]. We can verify that a coil with $N_{T}$ turns carrying a current $I_{T}$ radiates the same fields as $m_{T}=-i \omega \mu I_{T} \pi a_{T}^{2}$.

The modal amplitude due to $\mathbf{M}$ is given by (3-228), where we can obtain

$$
\begin{aligned}
S_{n p}^{ \pm} & =(-1)^{n} m_{T} \frac{1}{2 \pi} e^{\mp i k_{z, n p} z_{T}} \\
& \times \int_{-\pi}^{\pi}\left\{\sum_{n^{\prime}=-\infty}^{\infty} e^{i n^{\prime}\left(\phi-\phi_{T}\right)}\left[ \pm h_{\rho, n p}\left(\rho_{T}\right) \sin \theta_{T}-h_{z, n p}\left(\rho_{T}\right) \cos \theta_{T}\right]\right\} e^{-i n \phi} d \phi .
\end{aligned}
$$

Using the orthogonality relation (3-233), we obtain

$$
S_{n p}^{ \pm}=(-1)^{n} m_{T} e^{-i n \phi_{T}} e^{\mp i k_{z, n p} z_{T}}\left[ \pm h_{\rho, n p}\left(\rho_{T}\right) \sin \theta_{T}-h_{z, n p}\left(\rho_{T}\right) \cos \theta_{T}\right] .
$$

\subsection{3}

\section{Magnetic Coil Antenna Source}

Consider the excitation of a magnetic loop placed at $z=z_{T}$ and defined by the surface current density

$$
\mathbf{M}=m_{T} \boldsymbol{\delta}_{T},
$$

where $m_{T}=m_{0} \rho_{T}$ is the magnetic moment for a coil with radius $\rho_{T}$, and

$$
\boldsymbol{\delta}_{T}=\frac{\delta\left(\rho-\rho_{T}\right)}{\rho} \delta\left(z-z_{T}\right) \hat{\phi}
$$

The modal amplitude due to $\mathbf{M}$ is given by (3-228), such we can obtain

$$
S_{n p}^{ \pm}= \pm \delta_{0, n} 2 \pi m_{T} h_{\phi, n p}\left(\rho_{T}\right) e^{\mp i k_{z, n p} z_{T}}
$$


Note that the fields in a radially stratified waveguide in general are hybrid modes, but the source defined in (3-238) excites only $\mathrm{TM}_{0 p}^{z}$ modes with azimuthal dependence $n=0$.

\subsection{4}

\section{Tilted-Coil Antenna Source}

Consider a coil antenna source with the coordinates

$$
\boldsymbol{r}_{T}=\hat{\rho} \rho_{T}+\hat{z}\left[z_{T}-\rho_{T} \tan \theta_{T} \cos \left(\phi-\phi_{T}\right)\right],
$$

where $\rho_{T}$ and $z_{T}$ are the radial and the axial position of the center of the coil, and $\phi_{T}$ and $\theta_{T}$ are the azimuthal and elevation tilt angles [47] as shown in Fig. 3.8.

The electrical current density of the coil is given by

$$
\mathbf{J}=I_{T} \boldsymbol{\delta}_{T}
$$

where

$$
\boldsymbol{\delta}_{T}=\delta\left(\rho-\rho_{T}\right) \delta\left(z-z_{T}+\rho_{T} \tan \theta_{T} \cos \left(\phi-\phi_{T}\right)\right)\left(\hat{\phi}+\hat{z} \tan \theta_{T} \sin \left(\phi-\phi_{T}\right)\right) .
$$

The modal amplitude due to $\mathbf{J}$ is given by (3-228), where

$$
\begin{array}{r}
S_{n p}^{ \pm}=(-1)^{n} I_{T} \rho_{T} e^{\mp i k_{z, n p} z_{T}} \int_{-\pi}^{\pi}\left[e_{\phi, n p}\left(\rho_{T}\right) \pm s_{n} e_{z, n p}\left(\rho_{T}\right) \tan \theta_{T} \sin \left(\phi-\phi_{T}\right)\right] \\
\times e^{-i n \phi} e^{ \pm i k_{z, n p} \rho_{T} \tan \theta_{T} \cos \left(\phi-\phi_{T}\right)} d \phi .
\end{array}
$$

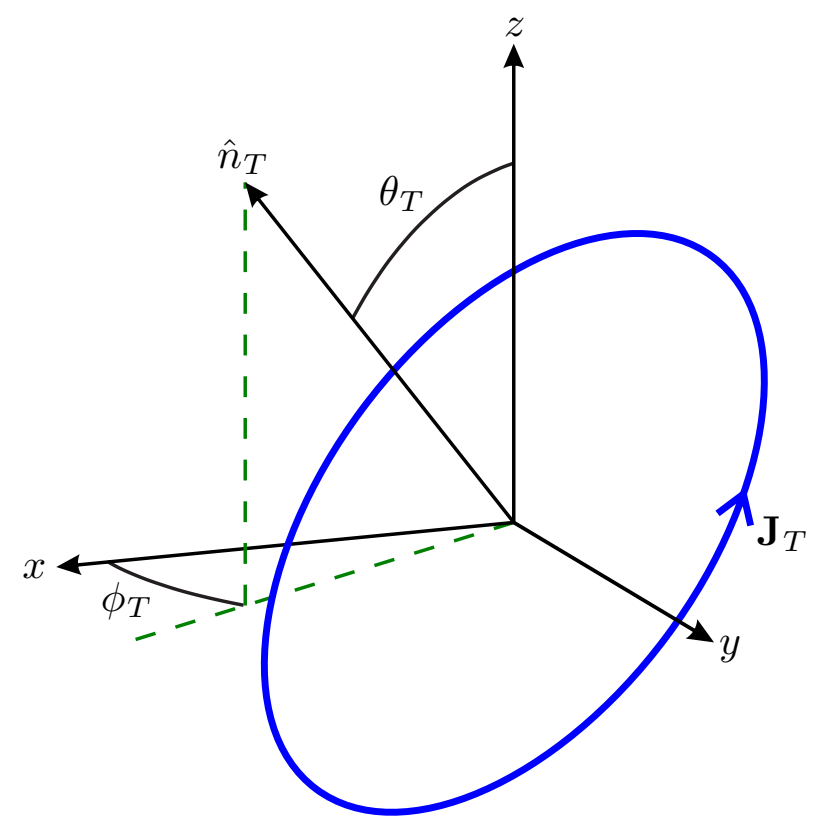

Figure 3.8: Transmitting tilted-coil antenna with current density $\mathbf{J}_{T}$. 
Here is expedite to define

$$
\kappa_{T}=k_{z, n p} \rho_{T} \tan \theta_{T}
$$

and also perform the change of variable $\tilde{\phi}=\phi-\phi_{T}, d \phi / d \tilde{\phi}=1$, such we can now write

$$
\begin{array}{r}
S_{n p}^{ \pm}=(-1)^{n} I_{T} \rho_{T} e^{-i n \phi_{T}} e^{\mp i k_{z, n p} z_{T}} \int_{-\pi-\phi_{T}}^{\pi-\phi_{T}}\left[e_{\phi, n p}\left(\rho_{T}\right) \pm s_{n} e_{z, n p}\left(\rho_{T}\right) \tan \theta_{T} \sin \tilde{\phi}\right] \\
\times e^{-i n \tilde{\phi}} e^{ \pm i \kappa_{T} \cos \tilde{\phi}} d \tilde{\phi} .
\end{array}
$$

Noting that the integrand is periodic in terms of $\phi$ and the integration is over a period interval $(2 \pi)$, we can perform the integration over the symmetric interval $-\pi$ to $\pi$. Thus, unless the multiplicative constants, we need to determine the following integrals:

$$
\begin{aligned}
& I_{\# 1}=\int_{-\pi}^{\pi} e^{-i n \phi \pm i \kappa_{T} \cos \phi} d \phi, \text { and } \\
& I_{\# 2}=\int_{-\pi}^{\pi} \sin \phi e^{-i n \phi \pm i \kappa_{T} \cos \phi} d \phi .
\end{aligned}
$$

The integrand exponential $\exp \left( \pm i \kappa_{T} \cos \phi\right)$ is an even function in terms of $\phi$. Therefore, using the identity $\exp (-i n \phi)=\cos n \phi-i \sin n \phi$, we can verify that only the function in terms of $\cos n \phi$ contributes for the integration in $I_{\# 1}$. Accordingly, we can write

$$
I_{\# 1}=2 \int_{0}^{\pi} \cos n \phi e^{ \pm i \kappa_{T} \cos \phi} d \phi .
$$

Using the identity [116, p. 360]

$$
J_{n}(r)=\frac{i^{-n}}{\pi} \int_{0}^{\pi} \cos n \phi e^{i r \cos \phi} d \phi
$$

we can find

$$
I_{\# 1}=2 \pi i^{n} J_{n}\left( \pm \kappa_{T}\right) .
$$

By an analogous procedure, the integral $I_{\# 2}$ can write as

$$
I_{\# 2}=-2 i \int_{0}^{\pi} \sin \phi \sin n \phi e^{ \pm i \kappa_{T} \cos \phi} d \phi
$$

Noting that $\sin \phi \sin n \phi=\frac{1}{2}[\cos (n-1) \phi-\cos (n+1) \phi]$, we can find

$$
I_{\# 2}=-i\left\{\int_{0}^{\pi} \cos (n-1) \phi e^{ \pm i \kappa_{T} \cos \phi} d \phi-\int_{0}^{\pi} \cos (n+1) \phi e^{ \pm i \kappa_{T} \cos \phi} d \phi\right\},
$$


which can be simplified by using (3-250), resulting in

$$
\begin{aligned}
I_{\# 2} & =-i\left[\pi i^{n-1} J_{n-1}\left( \pm \kappa_{T}\right)-\pi i^{n+1} J_{n+1}\left( \pm \kappa_{T}\right)\right] \\
& =-i \pi i^{n}(-i)\left[J_{n-1}\left( \pm \kappa_{T}\right)+J_{n+1}\left( \pm \kappa_{T}\right)\right] \\
& =-\pi i^{n} \frac{2 n J_{n}\left( \pm \kappa_{T}\right)}{ \pm \kappa_{T}} \\
& =-2 \pi i^{n} \frac{n}{ \pm k_{z, n p} \rho_{T} \tan \theta_{T}} J_{n}\left( \pm \kappa_{T}\right) .
\end{aligned}
$$

By combining the previous results we can obtain

$$
S_{n p}^{ \pm}=(-1)^{n} I_{T} \rho_{T} 2 \pi i^{n} J_{n}\left( \pm \kappa_{T}\right)\left[e_{\phi, n p}\left(\rho_{T}\right)-\frac{n}{k_{z, n p} \rho_{T}} e_{z, n p}\left(\rho_{T}\right)\right] e^{-i n \phi_{T}} e^{\mp i k_{z, n p} z_{T}} .
$$

Note we have used $s_{n} n=n$. Furthermore, note that $S_{-n p}^{ \pm} \exp \left(-i n \phi_{T}\right)=$ $S_{n p}^{ \pm} \exp \left(i n \phi_{T}\right)$, which allow us to write the sums in terms of the azimuthal index $n$ in (3-196) and (3-197) as a cosine series of $n\left(\phi-\phi_{T}\right)$, for $n=0$ to $\infty$.

By combining the previous results we can obtain the modal amplitudes for the transmitting tilted-coil antenna as

$$
A_{t, n p}^{ \pm}=A_{n p}^{ \pm}=\frac{S_{n p}^{ \pm}}{N_{n p}}
$$

From the equations above, it is clear that the transformation

$$
A_{t, n p}^{ \pm}\left(\phi_{T}, z_{T}\right) \rightarrow A_{t, n p}^{ \pm}\left(\phi_{0}, z_{0}\right) e^{-i n \phi_{T} \mp i k_{z, n p}\left(z_{T}-z_{0}\right)}
$$

translates the source from $z=z_{0}$ to $z=z_{T}$ and azimuthally rotate the source by the angle $\phi_{T}$. A similar result was obtained in [47].

\subsection{5}

\section{Received Voltage}

The voltage induced on a tilted-coil antenna is given by

$$
V_{R}=-\int_{V}\left(\mathbf{E}^{+}+\mathbf{E}^{-}\right) \cdot \boldsymbol{\delta}_{R} d v
$$

where $\boldsymbol{\delta}_{R}$ is defined similarly to (3-243), but using the subscript substitution $T \rightarrow R$.

Based on the shape of the received fields shown in Section 3.4.2, we can decouple the forward and the backward contributions for the received voltage as

$$
V_{R}=\sum_{n=-\infty}^{\infty} \sum_{p=1}^{\infty}\left(V_{R, n p}^{+}+V_{R, n p}^{-}\right)
$$

We can express the forward and backward voltages as a function of the 
modal field amplitudes at the receiver position, $A_{r, n p}^{ \pm}$, such as

$$
\begin{array}{r}
V_{R, n p}^{ \pm}=-A_{r, n p}^{ \pm} \rho_{R} e^{ \pm i k_{z, n p} z_{R}} \int_{-\pi}^{\pi}\left[e_{\phi, n p}\left(\rho_{R}\right) \pm e_{z, n p}\left(\rho_{R}\right) \tan \theta_{R} \sin \left(\phi-\phi_{R}\right)\right] \\
\times e^{i n \phi} e^{\mp i k_{z, n p} \rho_{R} \tan \theta_{R} \cos \left(\phi-\phi_{R}\right)} d \phi
\end{array}
$$

Defining

$$
\kappa_{R}=k_{z, n p} \rho_{R} \tan \theta_{R}
$$

and also performing the change of variable $\tilde{\phi}=\phi-\phi_{R}, d \phi / d \tilde{\phi}=1$, we can write

$$
\begin{aligned}
V_{R, n p}^{ \pm}=-A_{r, n p}^{ \pm} \rho_{R} e^{i n \phi_{R}} e^{ \pm i k_{z, n p} z_{R}} \int_{-\pi-\phi_{R}}^{\pi-\phi_{R}}\left[e_{\phi, n p}\left(\rho_{R}\right)\right. & \left. \pm e_{z, n p}\left(\rho_{R}\right) \tan \theta_{R} \sin \tilde{\phi}\right] \\
& \times e^{i n \tilde{\phi}} e^{\mp i \kappa_{R} \cos \tilde{\phi}} d \tilde{\phi}
\end{aligned}
$$

Thus, unless the multiplicative constants, we need to determine the following integrals:

$$
\begin{aligned}
I_{\# 3} & =\int_{-\pi}^{\pi} e^{i n \phi \mp i \kappa_{T} \cos \phi} d \phi, \text { and } \\
I_{\# 4} & =\int_{-\pi}^{\pi} \sin \phi e^{i n \phi \mp i \kappa_{T} \cos \phi} d \phi .
\end{aligned}
$$

The procedure to solve the above integrals is analogous to one used in $I_{\# 1}$ and $I_{\# 2}$, and after few manipulations, we can obtain

$$
\begin{gathered}
I_{\# 3}=2 \pi i^{n} J_{n}\left(\mp \kappa_{R}\right), \text { and } \\
I_{\# 4}=2 \pi i^{n} \frac{n}{\mp k_{z, n p} \rho_{R} \tan \theta_{R}} J_{n}\left(\mp \kappa_{R}\right) .
\end{gathered}
$$

By using the above results, we can then write the voltages in (3-265) as

$$
V_{R, n p}^{ \pm}=-A_{r, n p}^{ \pm} \rho_{R} 2 \pi i^{n} J_{n}\left(\mp \kappa_{R}\right)\left[e_{\phi, n p}\left(\rho_{R}\right)-\frac{n}{k_{z, n p} \rho_{R}} e_{z, n p}\left(\rho_{R}\right)\right] e^{i n \phi_{R}} e^{ \pm i k_{z, n p} z_{R}}
$$

In general, the transmitting and the receiving antennas are not in the same axial region. Then, consider that these antennas are at the regions $t$ and $r$. After finding the source amplitudes at the region $t$, we need to transfer the corresponding modal amplitudes to the observation region $r$. Accordingly, we can redefine the modal amplitudes for both transmitting and receiving antennas at $z_{T, R}=0$, and at $\phi_{T, R}=0$ such as

$$
\begin{aligned}
& A_{T, n p}^{ \pm} \rightarrow A_{t, n p}^{ \pm}\left(z_{T}\right)=A_{t, n p}^{ \pm} e^{\mp i k_{z, n p} z_{T}}, \\
& A_{R, n p}^{ \pm} \rightarrow A_{r, n p}^{ \pm}\left(z_{R}\right)=A_{r, n p}^{ \pm} e^{ \pm i k_{z, n p} z_{R}},
\end{aligned}
$$

and using the following rotating function 


$$
R_{n}\left(\phi^{\prime}, \phi\right)=e^{i n\left(\phi-\phi^{\prime}\right)}
$$

we can obtain

$$
V_{R, n p}^{ \pm}=-A_{R, n p}^{ \pm} \rho_{R} 2 \pi i^{n} J_{n}\left(\mp \kappa_{R}\right)\left[e_{\phi, n p}\left(\rho_{R}\right)-\frac{n}{k_{z, n p} \rho_{R}} e_{z, n p}\left(\rho_{R}\right)\right] R_{n}\left(\phi_{T}, \phi_{R}\right) .
$$

Notice that the axial positions of transmitter and receiver are embedded into $A_{R, n p}^{ \pm}$. The procedure required to transfer the modal amplitudes $A_{T, n p}^{ \pm}$at $z=z_{T}$ to $A_{R, n p}^{ \pm}$at $z=z_{R}$ are presented in Appendix B.

A special case is of interest: consider that both receiver and transmitter antennas are at the same axial region, and there no axial discontinuities. This case consider just radial stratifications in an axial-uniform waveguide. Then, for $z_{R}>z_{T}$ we must calculate the received voltage as $V_{R}=\sum_{n, p} V_{R, n p}^{+}$. For $z_{R}<z_{T}$, the received voltage is $V_{R}=\sum_{n, p} V_{R, n p}^{-}$. Noting that $V_{R, n p}^{ \pm}$is even in terms of $n$, we can obtain

$$
V_{R}^{ \pm}=\sum_{n=0}^{\infty} \sum_{p=1}^{\infty} \epsilon_{n} F_{n p}^{ \pm}\left(\rho_{T}, \rho_{R}\right) \cos n\left(\phi_{R}-\phi_{T}\right) e^{ \pm i k_{z, n p}\left(z_{R}-z_{T}\right)} .
$$

where $\epsilon_{n}=2-\delta_{0, n}$, and

$$
\begin{array}{r}
F_{n p}^{ \pm}\left(\rho_{T}, \rho_{R}\right)=-\frac{I_{T}}{\hat{N}_{n p}} 4 \pi^{2} \rho_{R} \rho_{T} J_{n}\left(\kappa_{R}\right) J_{n}\left(\kappa_{T}\right)\left[e_{\phi, n p}\left(\rho_{R}\right)-\frac{n}{k_{z, n p} \rho_{R}} e_{z, n p}\left(\rho_{R}\right)\right] \\
\times\left[e_{\phi, n p}\left(\rho_{T}\right)-\frac{n}{k_{z, n p} \rho_{T}} e_{z, n p}\left(\rho_{T}\right)\right] . \quad(3-276)
\end{array}
$$

Note that $F_{n p}^{+}\left(\rho_{T}, \rho_{R}\right)=F_{n p}^{-}\left(\rho_{T}, \rho_{R}\right)$, which allow us to concluded that $V_{R}^{+}=V_{R}^{-}$.

A further specialization (of special interest for LWD applications) considers that the radius of the antennas are the same; i.e., $\rho_{R}=\rho_{T}=\rho_{0}$; allowing us to simplify the $F_{n p}^{ \pm}\left(\rho_{T}, \rho_{R}\right)$ as

$$
F_{n p}^{ \pm}\left(\rho_{0}, \rho_{0}\right)=-\frac{I_{T}}{\hat{N}_{n p}} 4 \pi^{2} \rho_{0}^{2} J_{n}\left(\kappa_{R}\right) J_{n}\left(\kappa_{T}\right)\left[e_{\phi, n p}\left(\rho_{0}\right)-\frac{n}{k_{z, n p} \rho_{0}} e_{z, n p}\left(\rho_{0}\right)\right]^{2} .
$$

\subsection{6}

\section{TCA Antenna Along two Axial Regions}

If the transmitter TCA traverses two distinct axial regions, we need to split the coil antenna region in two segments ('sub-antennas'), as illustrated in Fig. 3.9. In this case, instead of (3-247) and (3-248), we should use new integrals over $\phi$. In Fig. 3.9 we consider a TCA located at $z_{T}$, between the regions 1 and 2, and the two resulting sub-antennas are delineated in terms of 

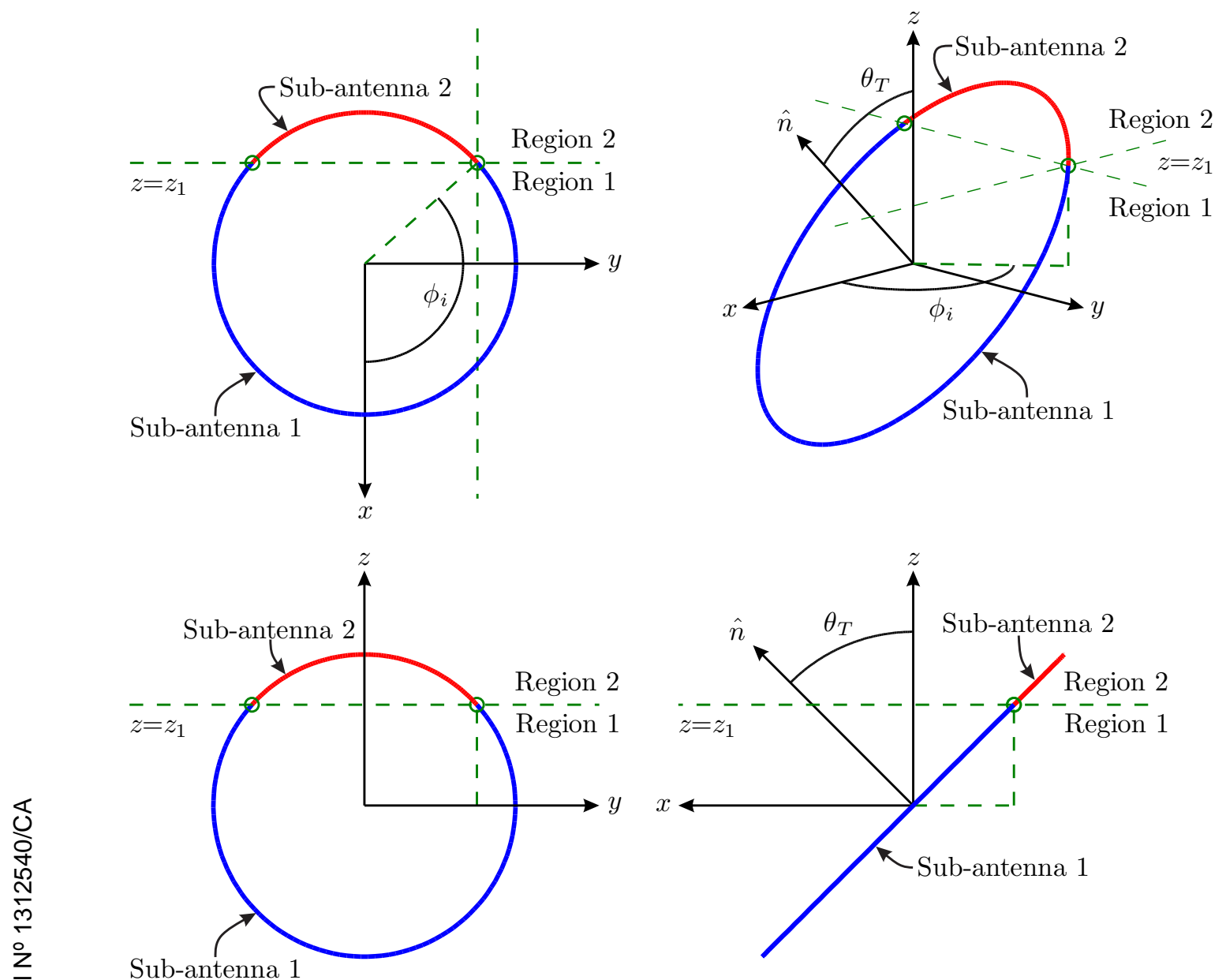

Figure 3.9: Orthogonal views of a transmitting tilted-coil antenna crossing the junction at $z=z_{1}$. In order to simplify the graphical representation, we use $z_{T}=0$ and $\phi_{T}=0$.

the angle $\phi_{i}$, given by

$$
\phi_{i}=\arccos \left(\frac{z_{T}-z_{1}}{\rho_{T} \tan \theta_{T}}\right)+\phi_{T},
$$

Here is expedite to define the integral

$$
I_{1}\left(n, \phi_{i n i}, \phi_{\text {end }}, \kappa\right)=2 \int_{\phi_{i n i}}^{\phi_{\text {end }}} \cos n \phi e^{i \kappa \cos \phi} d \phi,
$$

where, in accordance with the previous expressions for $I_{\# 1}$ and $I_{\# 2}$, we must employ $\phi \in\left[\phi_{s}, \phi_{e}\right]$ with $\phi_{s}=0$ and $\phi_{e}=\phi_{i}$ for sub-antenna 1 , and $\phi_{s}=\phi_{i}$ and $\phi_{e}=\pi$ for sub-antenna 2. Note that the modal excitations (3-244) corresponding to each sub-antenna should use the appropriated value of $\kappa_{T}$ associated to that region.

To the best of our knowledge, the integral in (3-279) does not admit a closed-form expression, but we found a suitable fast convergent series representation as will be described bellow. 
The Jacobi-Anger expansion [116, p. 361]

$$
e^{i \kappa \cos \phi}=\sum_{n^{\prime}=0}^{\infty} \epsilon_{n^{\prime}} i^{n^{\prime}} J_{n^{\prime}}(\kappa) \cos n^{\prime} \phi
$$

allow us to rewrite (3-279) as

$$
I_{1}\left(n, \phi_{i n i}, \phi_{\text {end }}, \kappa\right)=2 \sum_{n^{\prime}=0}^{\infty} \epsilon_{n^{\prime}} i^{n^{\prime}} J_{n^{\prime}}(\kappa) \int_{\phi_{i n i}}^{\phi_{e n d}} \cos n \phi \cos n^{\prime} \phi d \phi .
$$

At this point, the following manipulations are pertinent:

$$
\begin{aligned}
I_{1}\left(n, \phi_{i n i}, \phi_{\text {end }}, \kappa\right) & =2 \sum_{n^{\prime}=0}^{\infty} \epsilon_{n^{\prime}} i^{n^{\prime}} J_{n^{\prime}}(\kappa) \int_{\phi_{i n i}}^{\phi_{\text {end }}} \cos n \phi \cos n^{\prime} \phi d \phi \\
& =\left.2 \sum_{n^{\prime}=0}^{\infty} \epsilon_{n^{\prime}} i^{n^{\prime}} J_{n^{\prime}}(\kappa) \frac{1}{2}\left[\frac{\sin \left(n+n^{\prime}\right) \phi}{n+n^{\prime}}+\frac{\sin \left(n-n^{\prime}\right) \phi}{n-n^{\prime}}\right]\right|_{\phi=\phi_{i n i}} ^{\phi_{\text {end }}} \\
& =\left.\sum_{n^{\prime}=0}^{\infty} \epsilon_{n^{\prime}} \phi i^{n^{\prime}} J_{n^{\prime}}(\kappa)\left\{\operatorname{sinc}\left[\left(n+n^{\prime}\right) \phi\right]+\operatorname{sinc}\left[\left(n-n^{\prime}\right) \phi\right]\right\}\right|_{\phi=\phi_{\text {ini }}} ^{\phi_{\text {end }}}
\end{aligned}
$$

where the function $\operatorname{sinc}(x)=\sin (x) / x$. We can now express $I_{1}$ as

$$
\begin{aligned}
& I_{1}\left(n, \phi_{\text {ini }}, \phi_{\text {end }}, \kappa\right)= \\
& \quad\left\{\phi \sum_{n^{\prime}=0}^{\infty} \epsilon_{n^{\prime}} i^{n^{\prime}} J_{n^{\prime}}(\kappa)\left[\operatorname{sinc}\left(\left(n+n^{\prime}\right) \phi\right)+\operatorname{sinc}\left(\left(n-n^{\prime}\right) \phi\right)\right]\right\}_{\phi=\phi_{\text {ini }}}^{\phi_{\text {end }}} .
\end{aligned}
$$

Another useful integral is

$$
I_{2}\left(n, \phi_{i n i}, \phi_{e n d}, \kappa\right)=-2 i \int_{\phi_{i n i}}^{\phi_{\text {end }}} \sin \phi \sin n \phi e^{i \kappa \cos \phi} d \phi
$$

which reduces to the form of $I_{\# 2}$ in (3-252) for $\phi_{\text {ini }}=0$ and $\phi_{\text {end }}=\pi$.

As far as we know, there is no analytical solution for $I_{2}$, so that we proceed in the same way as for $I_{1}$. The derivative of (3-280) in respect to $\phi$ results in

$$
e^{i \kappa \cos \phi}=\frac{1}{i \kappa \sin \phi} \sum_{n^{\prime}=0}^{\infty} \epsilon_{n^{\prime}} i^{n^{\prime}} J_{n^{\prime}}(\kappa) n^{\prime} \sin n^{\prime} \phi,
$$

which after a few simplifications allow to write $I_{2}$ as

$$
\begin{aligned}
& I_{2}\left(n, \phi_{i n i}, \phi_{\text {end }}, \kappa\right)= \\
& \quad\left\{\frac{\phi}{\kappa} \sum_{n^{\prime}=0}^{\infty} n^{\prime} \epsilon_{n^{\prime}} i^{n^{\prime}} J_{n^{\prime}}(\kappa)\left[\operatorname{sinc}\left(\left(n+n^{\prime}\right) \phi\right)-\operatorname{sinc}\left(\left(n-n^{\prime}\right) \phi\right)\right]\right\}_{\phi=\phi_{i n i}}^{\phi_{\text {end }}} .
\end{aligned}
$$

Using the above definitions for $I_{1}$ and $I_{2}$, the modal amplitude of the 
sub-antennas 1 and 2 should be obtained using

$$
\begin{aligned}
& I_{\# 1}=I_{1}\left(n, \phi_{i n i}, \phi_{e n d}, \pm \kappa_{T}\right), \text { and } \\
& I_{\# 2}=I_{2}\left(n, \phi_{i n i}, \phi_{e n d}, \pm \kappa_{T}\right)
\end{aligned}
$$

for each sub-antenna. Note that for a configuration similar to that shown in Fig. 3.9, we must employ $\phi_{i n i}=0$ and $\phi_{\text {end }}=\phi_{i}$ for sub-antenna 1 , and $\phi_{i n i}=\phi_{i}$ and $\phi_{\text {end }}=\pi$ for sub-antenna 2 . For each segment, we should also select the appropriated value of $\kappa_{T}$ in view of the region of the sub-source.

Case the receiver antenna is between two axial regions, we must proceed like shown above splinting the antennas and considering the contribution of both receiving sub-antennas. Instead of the integrals in (3-266) and (3-267), in this case we must employ

$$
\begin{aligned}
& I_{\# 3}=I_{1}\left(n, \phi_{i n i}, \phi_{\text {end }}, \mp \kappa_{R}\right), \\
& I_{\# 4}=-I_{2}\left(n, \phi_{i n i}, \phi_{\text {end }}, \mp \kappa_{R}\right) .
\end{aligned}
$$

In a compact shape, we can write

$$
\begin{gathered}
I_{1}\left(n, \phi_{s}, \phi_{e}, \kappa\right)=\left[\phi \sum_{n^{\prime}=0}^{\infty} \epsilon_{n^{\prime}} i^{n^{\prime}} J_{n^{\prime}}(\kappa) F_{n n^{\prime}}^{+}(\phi)\right]_{\phi=\phi_{s}}^{\phi_{e}}, \\
I_{2}\left(n, \phi_{s}, \phi_{e}, \kappa\right)=\left[\frac{\phi}{\kappa} \sum_{n^{\prime}=0}^{\infty} n^{\prime} \epsilon_{n^{\prime}} i^{n^{\prime}} J_{n^{\prime}}(\kappa) F_{n n^{\prime}}^{-}(\phi)\right]_{\phi=\phi_{s}}^{\phi_{e}}, \\
F_{n n^{\prime}}^{ \pm}(\phi)=\operatorname{sinc}\left(\left(n+n^{\prime}\right) \phi\right) \pm \operatorname{sinc}\left(\left(n-n^{\prime}\right) \phi\right) .
\end{gathered}
$$

If the receiver TCA traverses two axial regions, we can likewise proceed as above, i.e., by splitting the TCA into two segments and computing the e.m.f. on each segment separately, before adding them up. If necessary, we can also employ the above strategy to model antennas spanning three or more regions by segmenting the TCA according to each region and selecting proper values for $\phi_{s}$ and $\phi_{e}$ in (3-291) and (3-292) for each sub-antenna.

\section{6}

\section{Flowchart for the Proposed Technique}

Based on the above formulation, we now present a complete description of the algorithm employed for numerical calculations as the flowchart depicted in Fig. 3.10. We consider that the series in (3-274) is truncated up to $N_{\phi}$ azimuthal harmonics, and the geophysical formation under analysis is formed by $N_{z}$ axial regions with $N_{\rho}$ radial layers. 


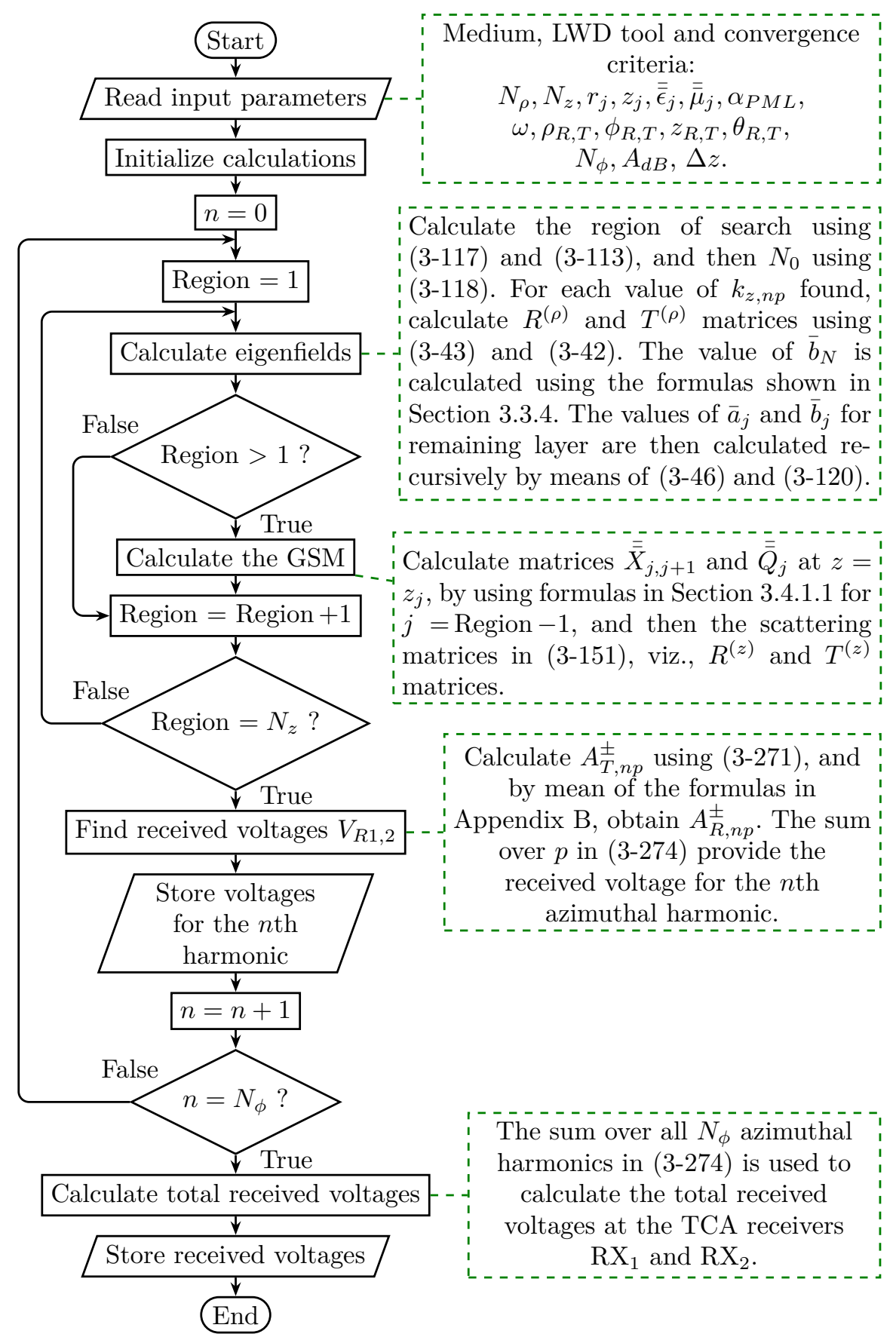

Figure 3.10: Flowchart for the algorithm of the proposed technique.

\section{7}

\section{Numerical Results and Validation}

The proposed technique was successful to reproduces a series of results for the electromagnetic propagation along cylindrically-layered structures as we reported in $[60,109,110,119,120]$. Then, we assume here that our eigenvalue solver algorithm was proper implemented; now we only need to check the axial mode-matching and the complex source enforcements.

To illustrate the application of the proposed method, we present simula- 
tions of a triaxial logging tool consisting of one transmitter and two receivers TCAs in a vertical-well borehole traversing different Earth formation. In all cases, the axial positions of the transmitter, and receivers $\mathrm{RX}_{2}$ and $\mathrm{RX}_{1}$ are $z_{T}$, $z_{T}+24$ in and $z_{T}+30$ in, respectively, where 1 in $=2.54 \times 10^{-2} \mathrm{~m}$. The TCAs have $\rho_{T, R}=4.5$ in and are wrapped around a 4 -in-radius metallic mandrel and inside a 5-in-radius borehole. We assume a homogeneous Dirichlet boundary condition for the tangential electric field at the surface of the metallic mandrel. This geometry was considered before in [5-8] and is used here to validate our formulation. In all simulations below, we consider only non-magnetic media. Also, as typical for modeling of low-frequency tools in geophysical formations, the real part of the complex permittivity everywhere is assumed equal to the vacuum permittivity. The radial domain is truncated at $r_{N}$ by using homogeneous Dirichlet boundary condition. The results are obtained using a double-precision Fortran code running on a PC with a 2.93-GHz Intel Xeon W3540 processor.

\subsection{1}

\section{Horizontal-Coil Antennas LWD Tools}

\subsubsection{1}

\section{Three-Layer Formation}

First, we consider an isotropic formation with three horizontal layers, whose conductivity $\sigma$ of layers 1,2 and 3 are $1 \mathrm{~S} / \mathrm{m}, 0.01 \mathrm{~S} / \mathrm{m}$ and $1 \mathrm{~S} / \mathrm{m}$, respectively, as illustrated in Fig. 3.11. The LWD tools is moving inside a borehole filled with a lossy mud having $\sigma=2 \mathrm{~S} / \mathrm{m}$, and employ horizontal coil antennas. We truncate the radial domain using a PEC at $r_{N}=r_{N-1}+\alpha \delta_{N, \max }$, which means that the outermost radial layer thickness is $\alpha$ times the skin depth on the lowest conductive formation $(0.01 \mathrm{~S} / \mathrm{m})$. We assume the same truncation for the remaining axial regions. We do not employed the complex stretching in the PML layer because the frequency is low such that the spectrum of evanescent modes become dominant. In our simulations, we consider all modes that satisfy the criteria of axial attenuation $A_{d B} \geq-30 \mathrm{~dB}$ at $\Delta z=5 \mathrm{in}$. As the coil source do not present tilt in relation to its axial axis (i.e., $\theta_{T}=0$ ), from (3-258), only modal fields with azimuthal index $n=0$ will be excited.

The received voltages at antennas $\mathrm{RX}_{1}$ and $\mathrm{RX}_{2}$ due to a unit current excitation $\left(I_{T}=1 \mathrm{~A}\right.$ at $\left.2 \mathrm{MHz}\right)$ are depicted in Fig. 3.12 and Fig. 3.13 in respect to 5 values of $\alpha$ : from 0.25 to 0.75 , with steps of 0.125 . The ratio of the received voltages amplitude and phase as a function of the axial position $z_{T}$ is shown in Fig. 3.14 and 3.15, respectively. 
Fig. 3.16 shows the ratio of the $\mathrm{RX}_{2}$ and $\mathrm{RX}_{1}$ voltages as a function of the axial position $z_{T}$, for two excitation frequencies: $500 \mathrm{kHz}$ and $2 \mathrm{MHz}$, both for $I_{T}=1 \mathrm{~A}$. We truncated the radial domain using the same criteria described above, in which $r_{N}=r_{N-1}+0.75 \delta_{N, \max }$. Notice that $\delta_{N, \max }$ is a frequency dependent parameter: equal to 280.6113 -in for $500 \mathrm{kHz}$ and equal to 140.8920 in for $2 \mathrm{MHz}$. Very good agreement is observed versus the finite-difference time-domain (FDTD) results from $[5,6]$. The total CPU time required by our algorithm was 25 seconds and 6 seconds to simulate 300 observation points at $500 \mathrm{kHz}$ and $2 \mathrm{MHz}$, respectively.

In results shown above we have considered all modes that satisfy the criteria of axial attenuation $A_{d B}=-30 \mathrm{~dB}$ at $\Delta z=5 \mathrm{in}$. However this criteria is very conservative. In Fig. 3.17 we shown the convergence of the voltages for five values of $A_{d B}$ (from $-10 \mathrm{~dB}$ to $-30 \mathrm{~dB}$, with steps of $5 \mathrm{~dB}$ ). We employed the same radial truncation as used before: a PEC at $r_{N}=r_{N-1}+0.75 \delta_{N, \max }$. We can see that a criteria no more strong than $A_{d B}=-20 \mathrm{~dB}$ at $\Delta z=5 \mathrm{in}$ needs be used for practical proposals. Using this criteria, we need just employ 31 modal fields in the mode-matching approach for a coil source operating at $2 \mathrm{MHz}$, as shown in Table 3.1 .

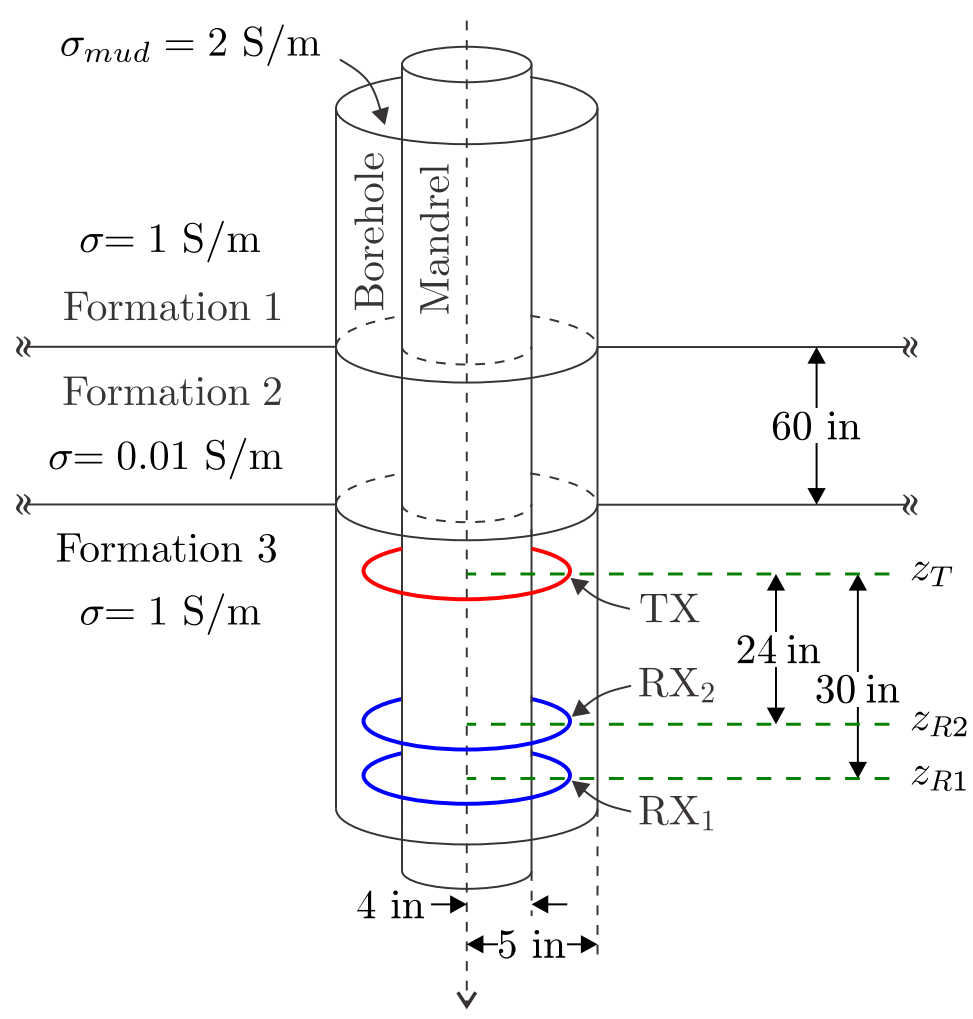

Figure 3.11: Geometry of a triaxial well-logging tool within a stratified geophysical formation. 

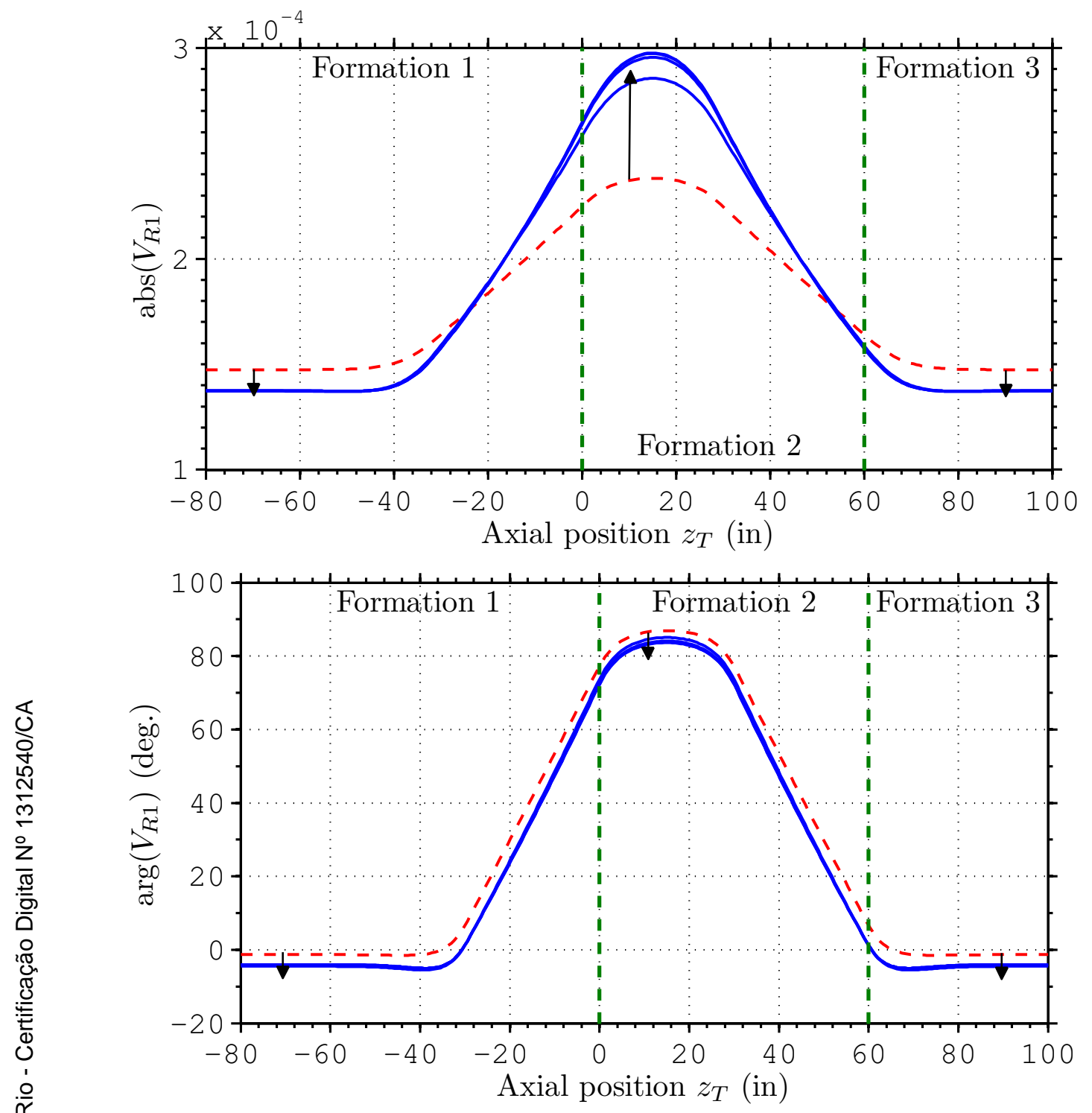

Figure 3.12: Voltage amplitude and phase received by antenna $\mathrm{RX}_{1}$. The response for $r_{N}=r_{N-1}+\alpha \delta_{N, \max }, \alpha=0.25$ (dashed-line) to $0.75 \mathrm{in}$, with steps of $0.125 \mathrm{in}$. Vertical arrows indicate the convergence sense.

Table 3.1: Number of modes for $A_{d B}$ at 5 in

\begin{tabular}{lccccccc}
\hline & \multicolumn{3}{c}{$500 \mathrm{kHz}$} & & \multicolumn{3}{c}{$2 \mathrm{MHz}$} \\
\cline { 2 - 4 } \cline { 6 - 8 }$A_{d B}(\mathrm{~dB})$ & Region 1 & Region 2 & Region 3 & & Region 1 & Region 2 & Region 3 \\
\hline-10 & 31 & 31 & 31 & & 15 & 15 & 15 \\
-15 & 47 & 47 & 47 & & 23 & 23 & 23 \\
-20 & 61 & 61 & 61 & & 31 & 31 & 31 \\
-25 & 77 & 77 & 77 & & 39 & 39 & 39 \\
-30 & 93 & 93 & 93 & & 47 & 47 & 47 \\
\hline
\end{tabular}



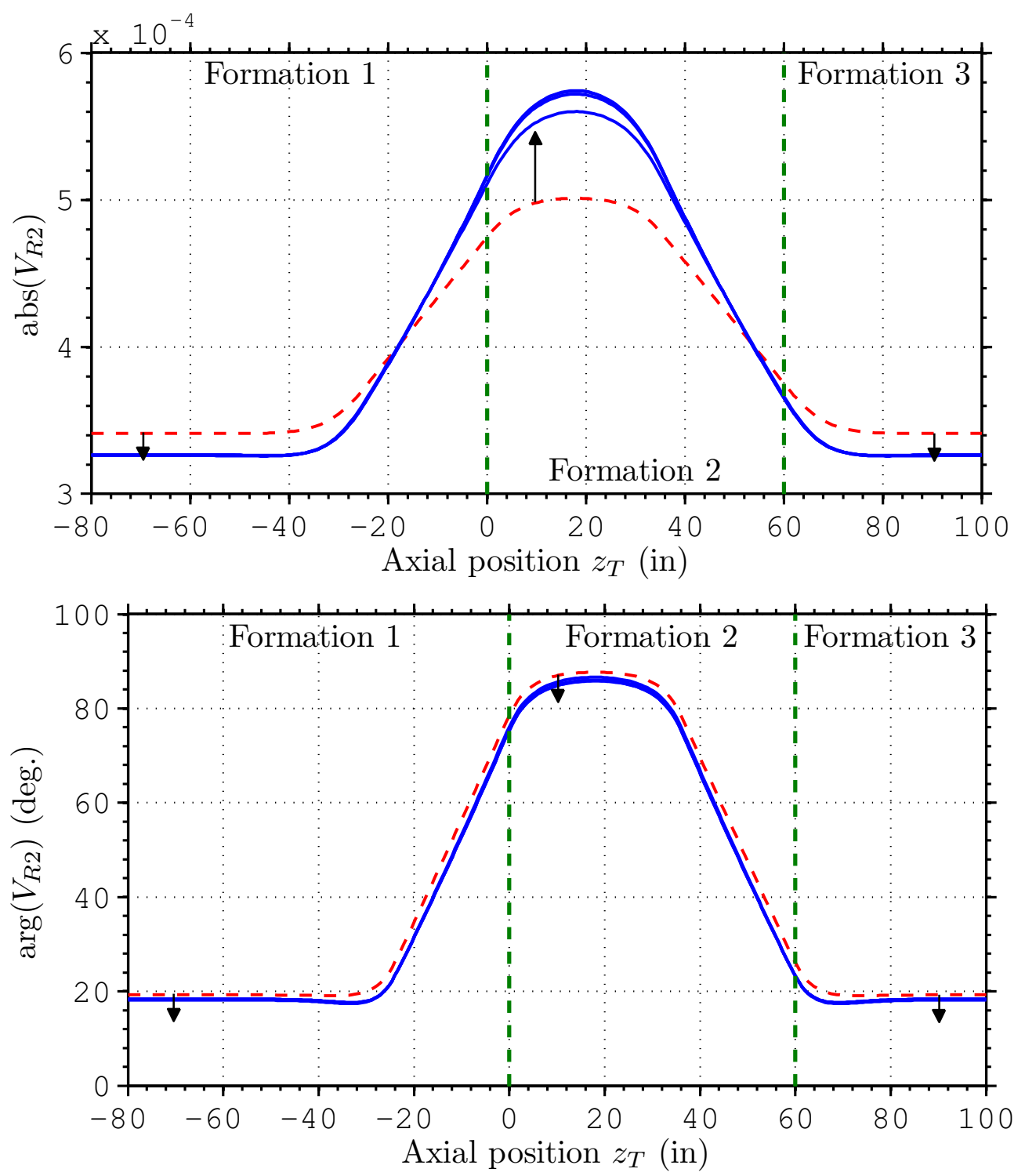

Figure 3.13: Voltage amplitude and phase received by antenna $\mathrm{RX}_{2}$. The responses for $r_{N}=r_{N-1}+\alpha \delta_{N, \max }, \alpha=0.25$ (dashed-line) to 0.75 , with steps of 0.125 are shown. Vertical arrows indicate the convergence sense. 


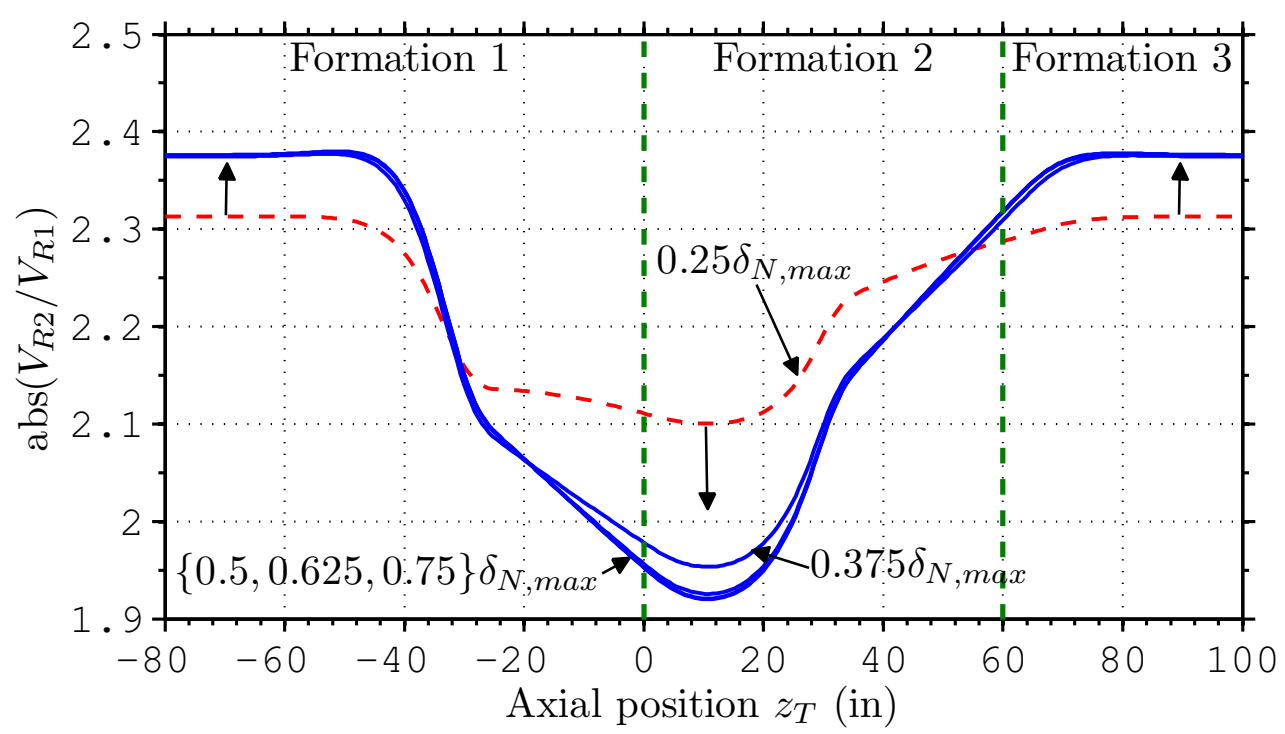

Figure 3.14: Relative amplitude voltage received by two antennas moving with one transmitter antenna across a bed layer $(z=0$ to $z=60$ in). The responses for $r_{N}=r_{N-1}+\alpha \delta_{N, \max }, \alpha=0.25$ (dashed-line) to 0.75 , with steps of 0.125 are shown. Vertical arrows indicate the convergence sense.

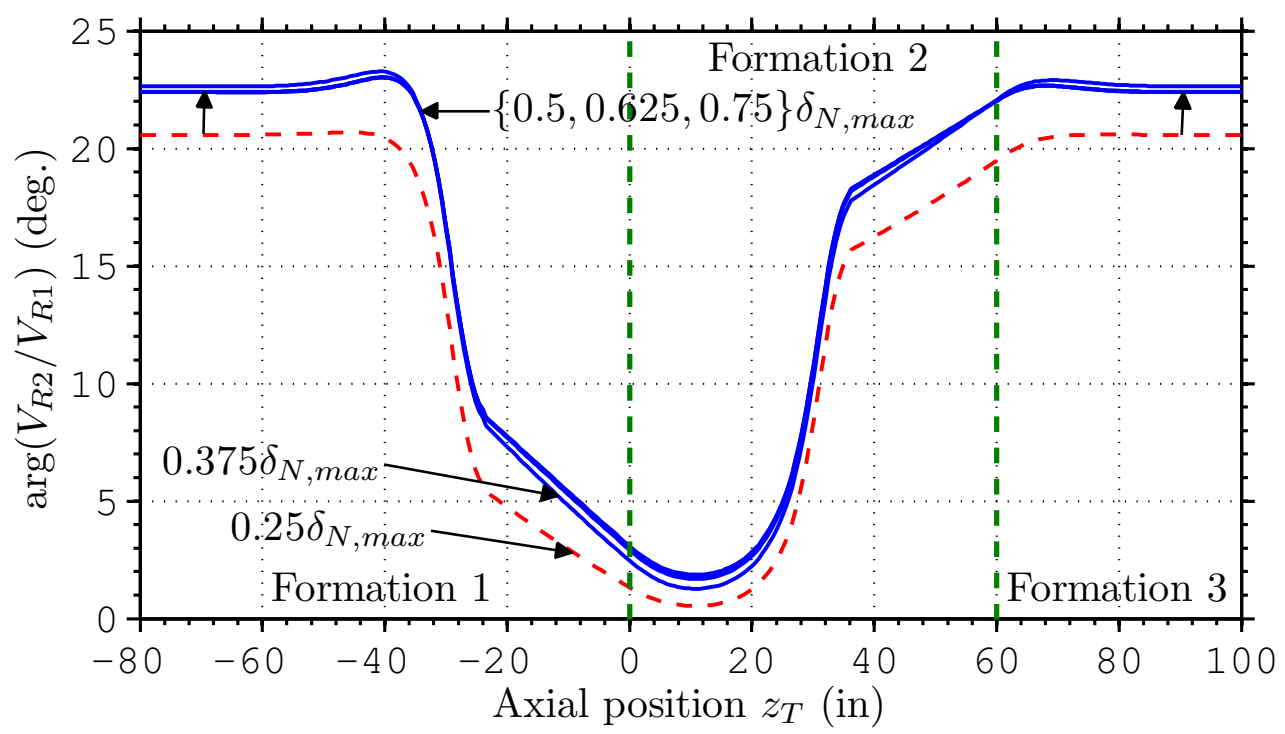

Figure 3.15: Relative phase voltage received by two antennas moving with one transmitter antenna across a bed layer ( $z=0$ to $z=60 \mathrm{in}$ ). The responses for $r_{N}=r_{N-1}+\alpha \delta_{N, \max }, \alpha=0.25$ (dashed-line) to 0.75 , with steps of 0.125 are shown. Vertical arrows indicate the convergence sense. 


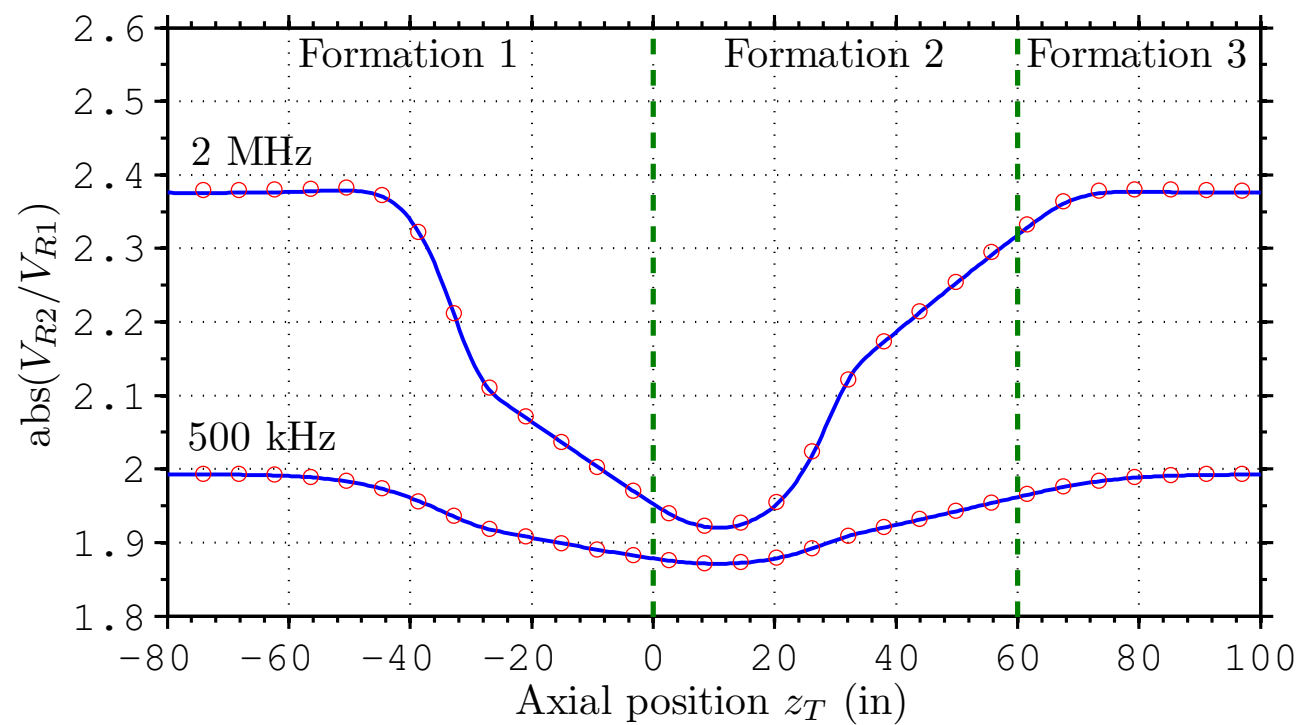

Figure 3.16: Voltage ratio for a triaxial LWD logging tool operating at $500 \mathrm{kHz}$ and $2 \mathrm{MHz}$. The results from the present method are shown by solid lines. The small circles are FDTD results from $[5,6]$. 


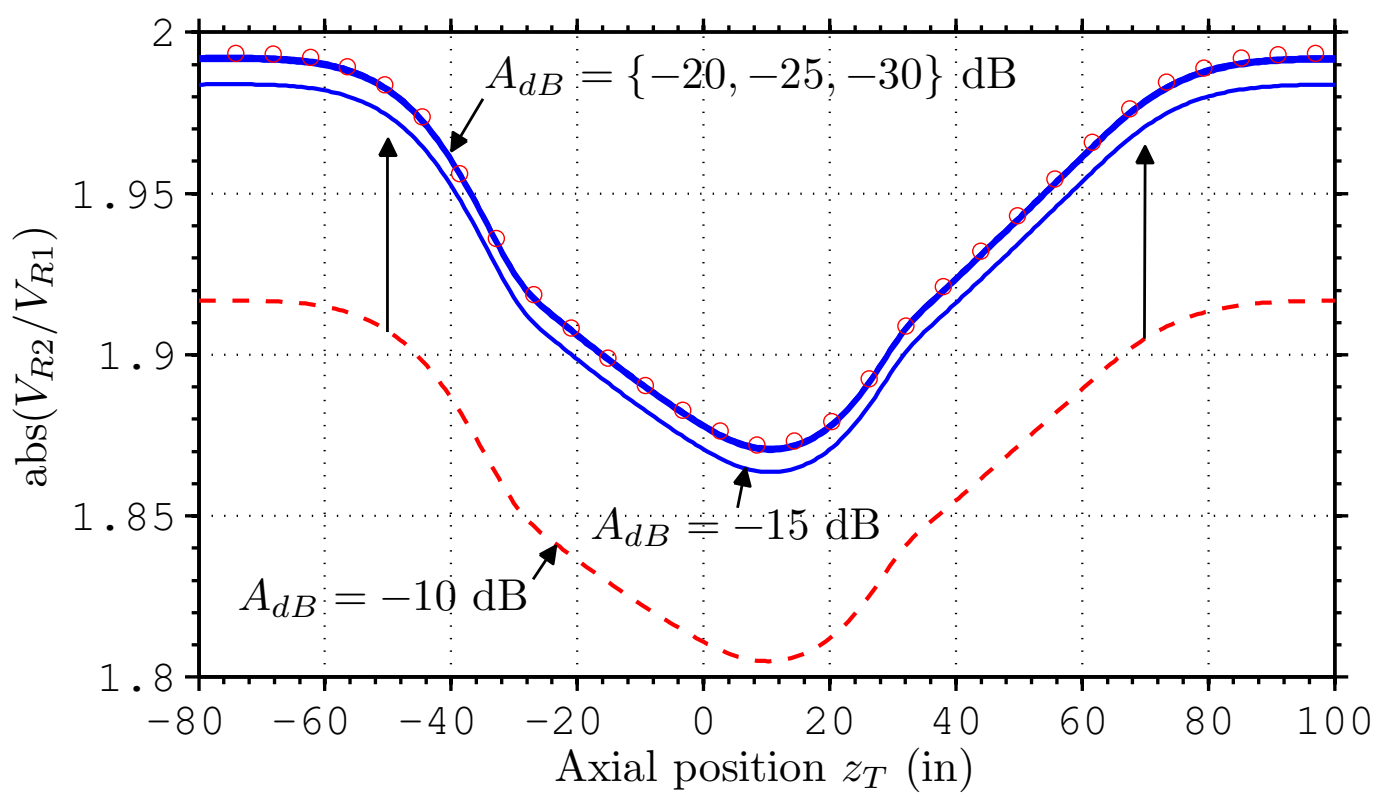

(a) $500 \mathrm{kHz}$

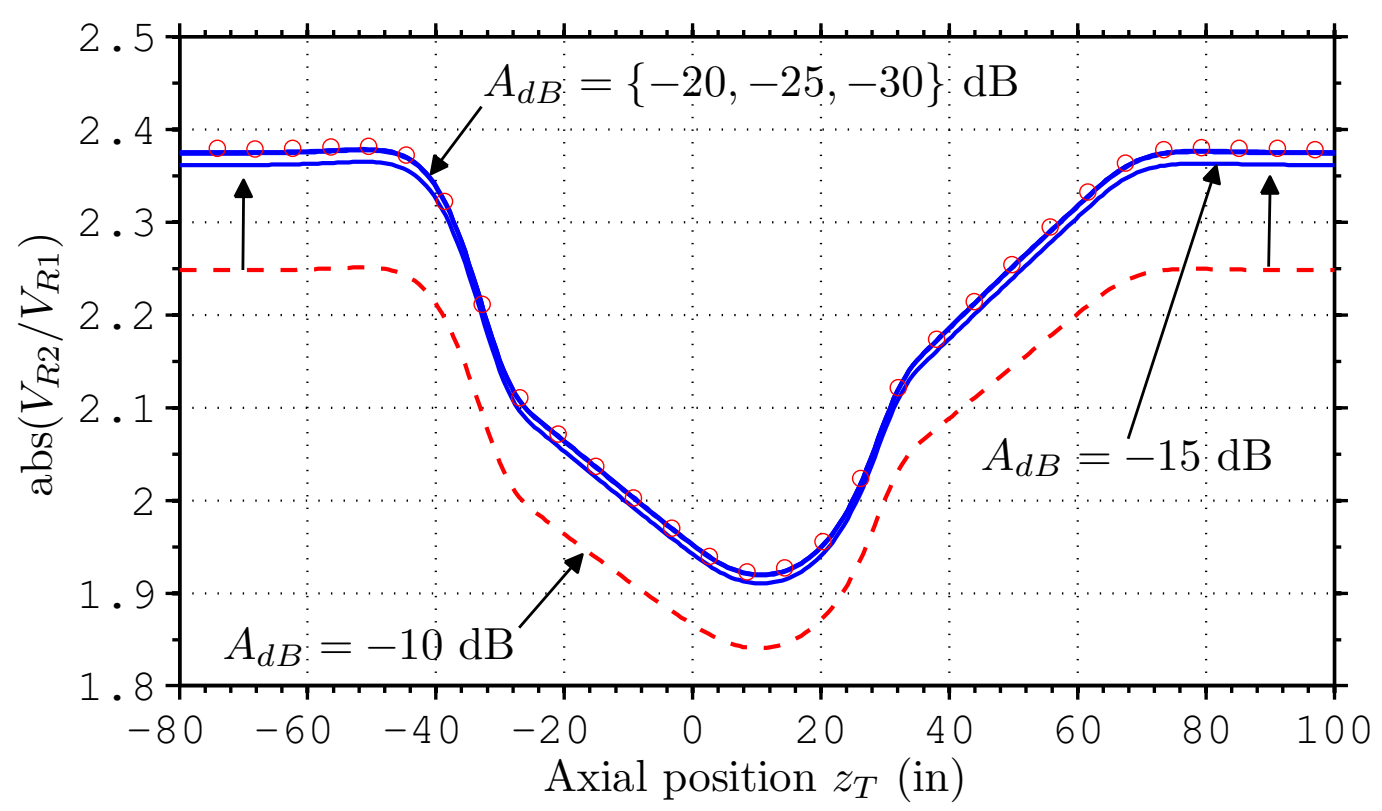

(b) $2 \mathrm{MHz}$

Figure 3.17: Convergence of the voltage amplitude ratio for $A_{d B}$ at $\Delta z=5$ in. The response for five values of $A_{d B}$ (from $-10 \mathrm{~dB}$ to $-30 \mathrm{~dB}$, with steps of $5 \mathrm{~dB})$ are presented. Vertical arrows indicate the convergence sense. 


\subsubsection{2}

\section{Borehole With Invasion Zone}

As a second example, we consider a LWD tool operating at $2 \mathrm{MHz}$, again using horizontal coil antennas. The borehole is filled with salt water having $\sigma=10 \mathrm{~S} / \mathrm{m}$ and the tool is now traversing a 60 -in bed layer with conductivity equal to $5 \times 10^{-4} \mathrm{~S} / \mathrm{m}$. The formations bellow and above the bed layer have high-contrast conductivities: $5 \mathrm{~S} / \mathrm{m}$ and $1 \mathrm{~S} / \mathrm{m}$, respectively. Only fields with azimuthal harmonic index $n=0$ will be excited, and we have considered all modes that satisfy the axial attenuation criteria of $-50 \mathrm{~dB}$ at 5 in, resulting in 41 modal fields. Fig. 3.18 shows the voltage ratio and the phase difference measured by the triaxial LWD tool as a function of the axial position $z_{T}$. Also, we show results for a 10-in-deep invasion zone with conductivity equal to $5 \mathrm{~S} / \mathrm{m}$ at intermediary formation, as depicted in Fig. 3.19. Good agreement is observed versus the results from the finite volumes method used in [7]. The total CPU time required by our algorithm was 16 seconds to simulate 300 observation points for the non-invaded problem. About the same CPU time has been taken by the invaded problem.

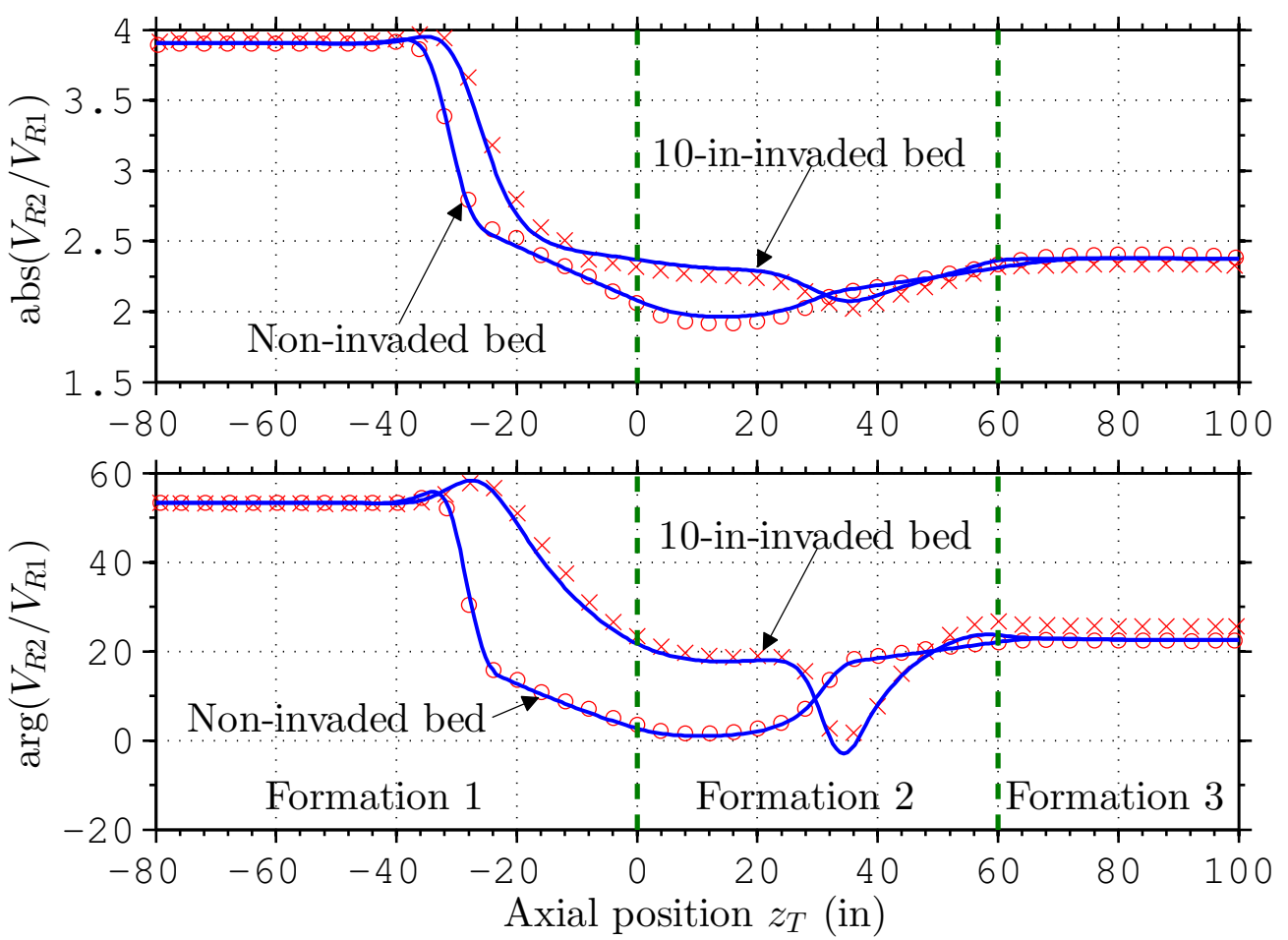

Figure 3.18: Voltage ratio and phase difference for a triaxial LWD logging tool traversing a bed layer with and without invasion. The results from the present algorithm are indicated by solid lines. The small circles and crosses are FV results from [7]. 


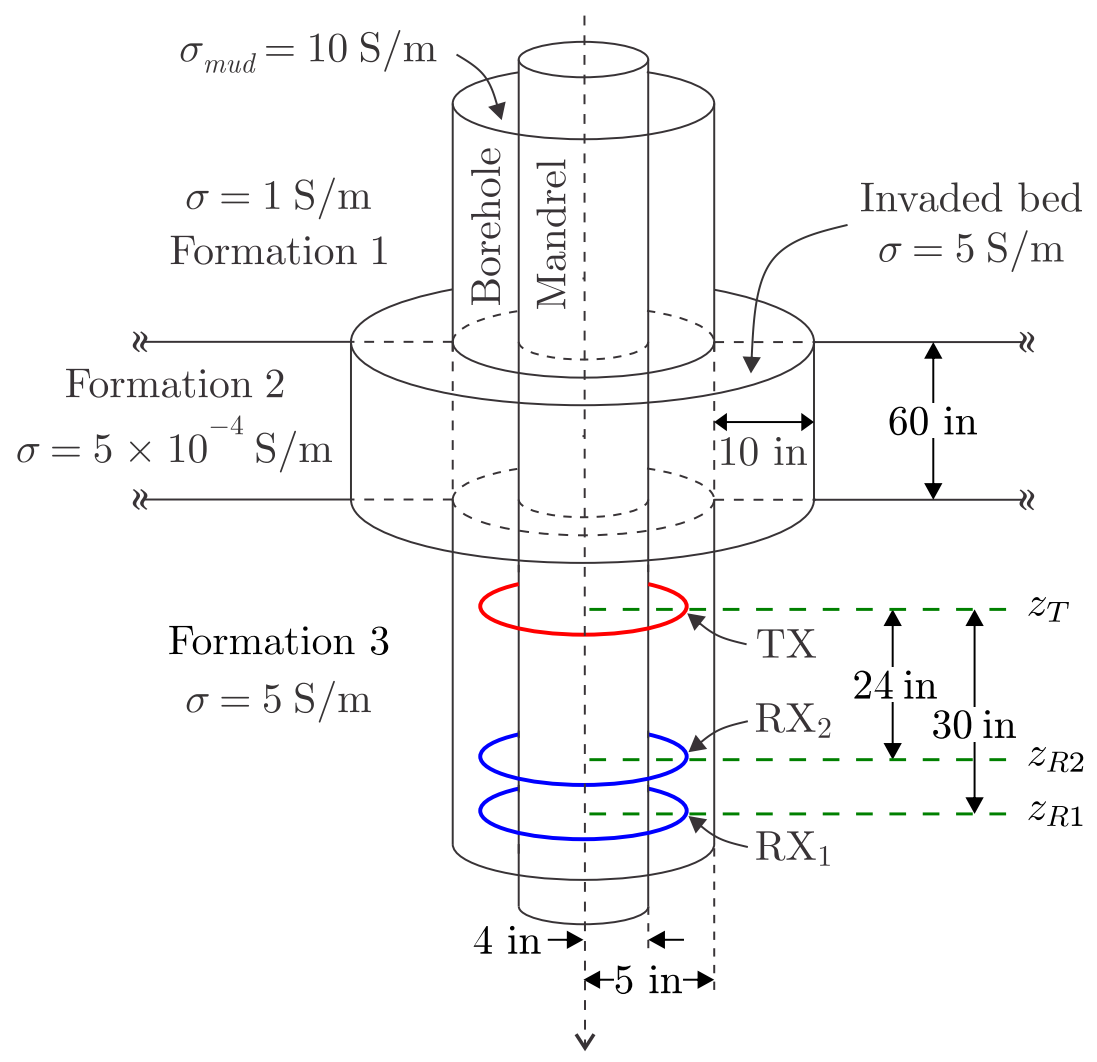

Figure 3.19: Geometry of a triaxial well-logging tool within a stratified geophysical formation. A 10-in-invasion zone is present in formation 2.

\subsection{2}

\section{Tilted-Coil Antennas LWD Tools}

\subsubsection{1}

\section{Isotropic Formations}

As a first problem involving TCAs, we consider the case of a triaxial TCA LWD tool in a vertical-well borehole filled with oil-based mud having $\sigma=0.0005 \mathrm{~S} / \mathrm{m}$ traversing an isotropic Earth formation with two horizontal layers, as illustrated in Fig. 3.20. Surrounding the borehole, the conductivity of top formation $(z<0)$ is $5 \mathrm{~S} / \mathrm{m}$. The bottom formation $(z>0)$ is characterized by $1 \mathrm{~S} / \mathrm{m}$.

We truncate the radial domain at $r_{N}=60 \mathrm{in}$, which means that the outermost radial layer thickness are about 4 times the skin depth on the less conductivity formation $(1 \mathrm{~S} / \mathrm{m})$. We do not employed the complex stretching in the PML layer, as suggested in [17], because the frequency is low, and the formations losses are very hight. The back-PML layer is assumed as a PEC.

We have considered all modes attenuated up to $60 \mathrm{~dB}$ at 5 in for all simulations shown above, which results in 49 modes at each region. Also, we have considered azimuthal index up to $|n|=4$. Fig. 3.21 to Fig. 3.28 plot 


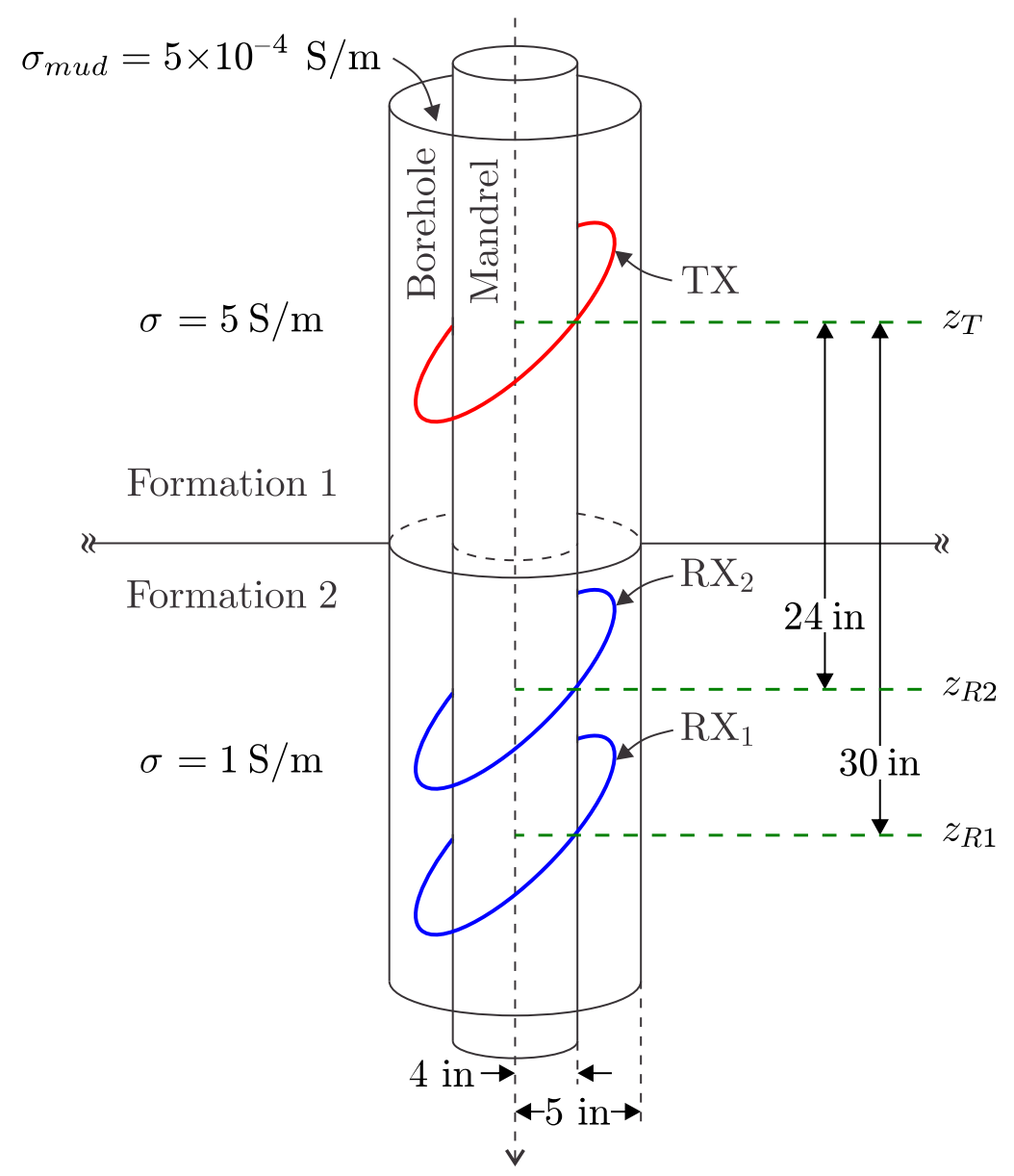

Figure 3.20: Geometry of a triaxial TCA well-logging tool within a stratified geophysical formation.

the voltage due to a unit current excitation $\left(I_{T}=1 \mathrm{~A}\right.$ at $\left.2 \mathrm{MHz}\right)$ at the receiver antennas $\mathrm{RX}_{1}$ and $\mathrm{RX}_{2}$ as a function of the axial position $z_{T}$ of the tool (abscissa in the plots), considering a transmitter TCA with fixed tilt angle of $45^{\circ}$, and four different receiver TCA tilt angles: $\theta_{R}=10^{\circ}, 20^{\circ}, 30^{\circ}$ and $40^{\circ}$. We compare the FDTD (circles) and R-NMM (dots) results in a two-layer isotropic formation obtained (from graphical reading) in [6, pp. 106-107], with the results of our formulations (solid lines). Very good agreement is observed versus the results from [6]. The CPU time required by our algorithm was less than 15 seconds to simulate the contribution of each azimuthal harmonic for 300 observation points.

We can see a small difference between the FDTD results compared with those from the mode-matching approaches (both the radial and axial). These differences are more pronounced when the antennas are crossing the junction at $z=0$, which leads us to believe that the deviations are related to the fact that the antennas geometry are not conformal with the finite-difference grid employed in [6]. 


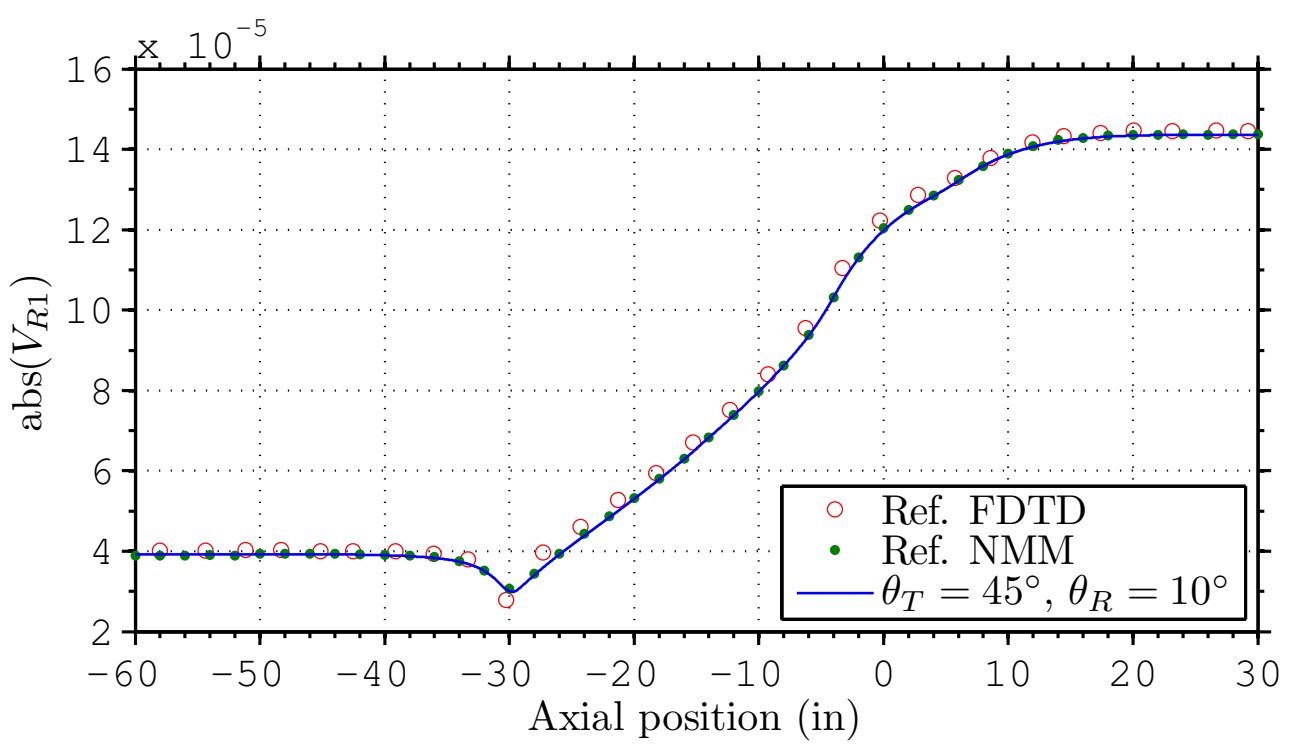

(a) Voltage amplitude

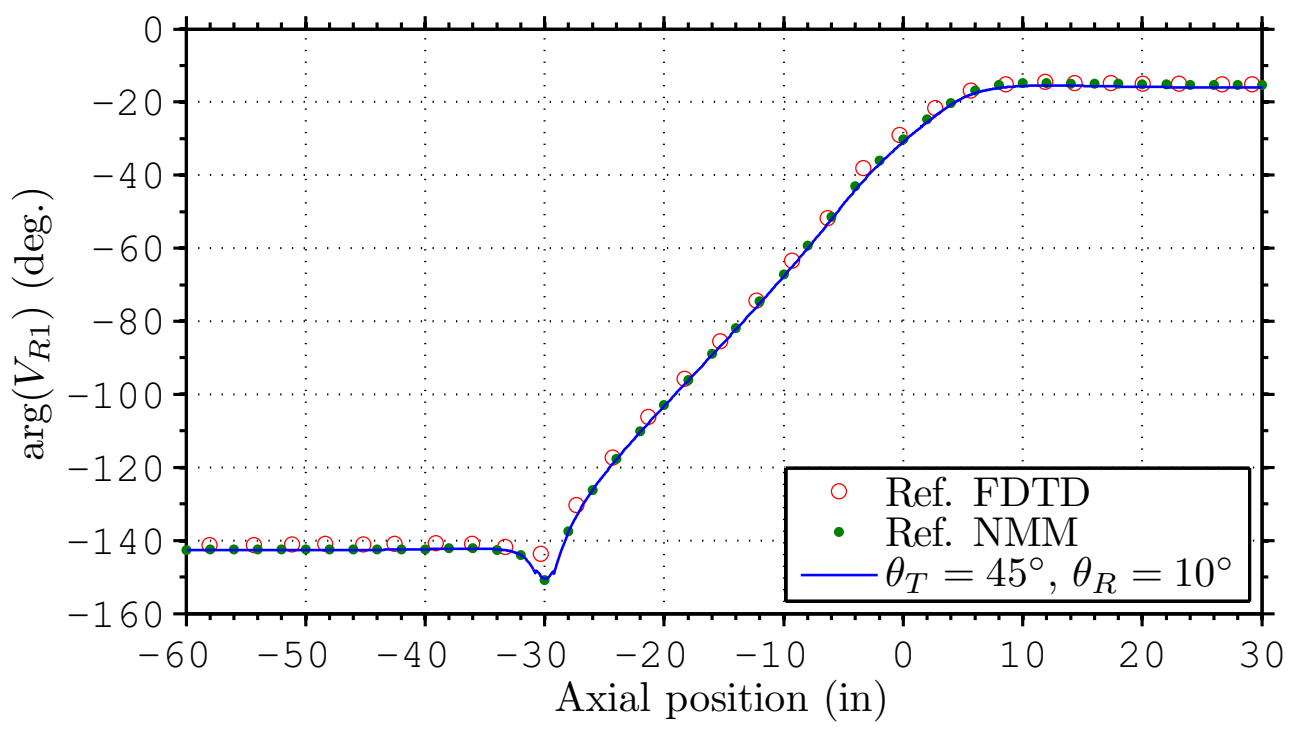

(b) Voltage phase

Figure 3.21: Voltage at TCA receiver $\mathrm{RX}_{1}$ with $\theta_{R}=10^{\circ}$ due to a unit current excitation transmitting TCA with $\theta_{T}=45^{\circ}$. The results from the present algorithm are indicated by solid lines. The small circles and dots are FDTD and R-NMM results, respectively, from [6, pp. 106-107]. 


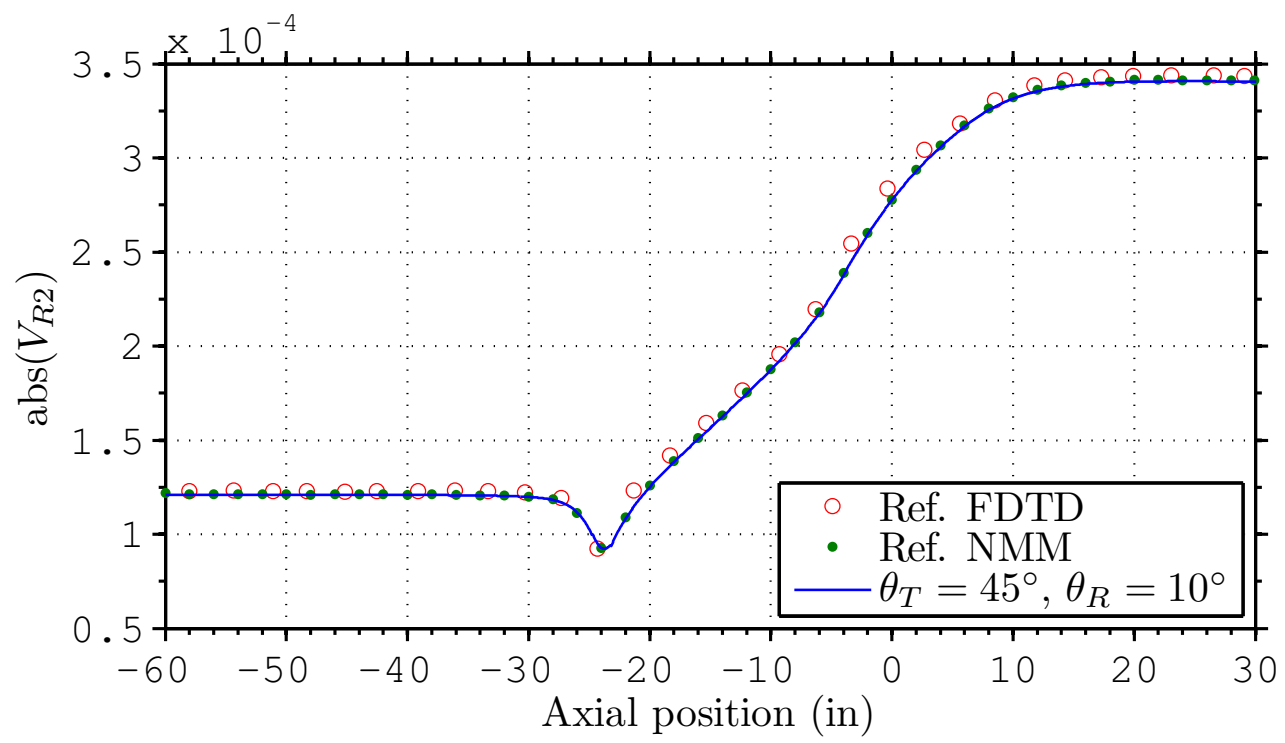

(a) Voltage amplitude

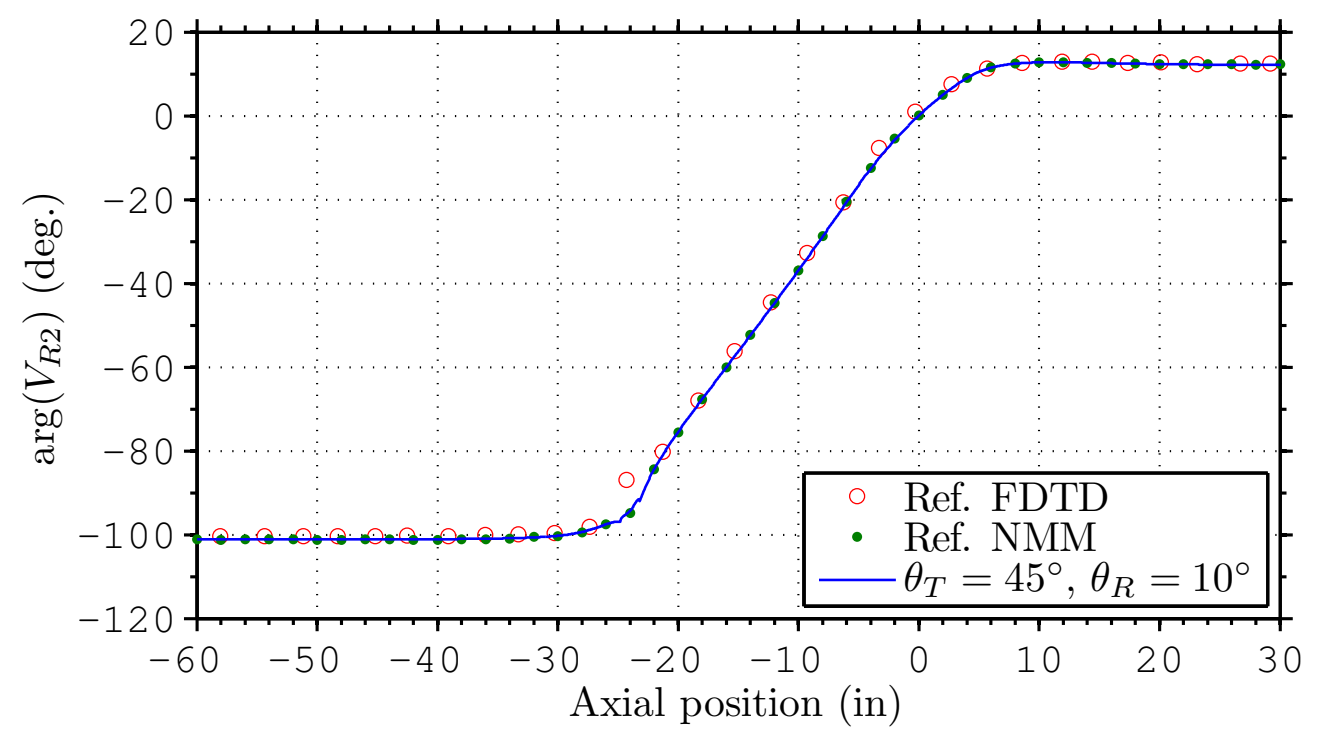

(b) Voltage phase

Figure 3.22: Voltage at TCA receiver $\mathrm{RX}_{2}$ with $\theta_{R}=10^{\circ}$ due to a unit current excitation transmitting TCA with $\theta_{T}=45^{\circ}$. The results from the present algorithm are indicated by solid lines. The small circles and dots are FDTD and R-NMM results, respectively, from [6, pp. 106-107]. 


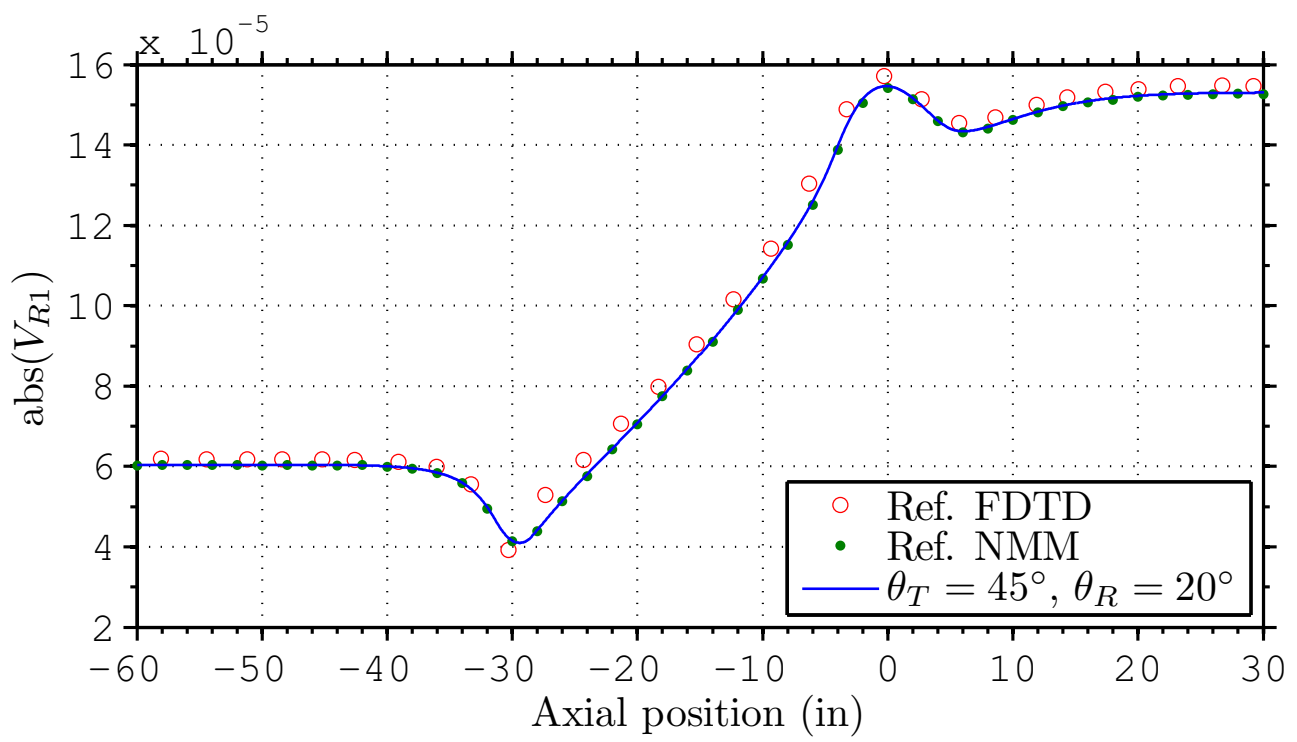

(a) Voltage amplitude

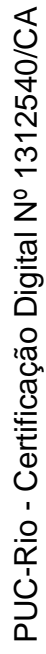

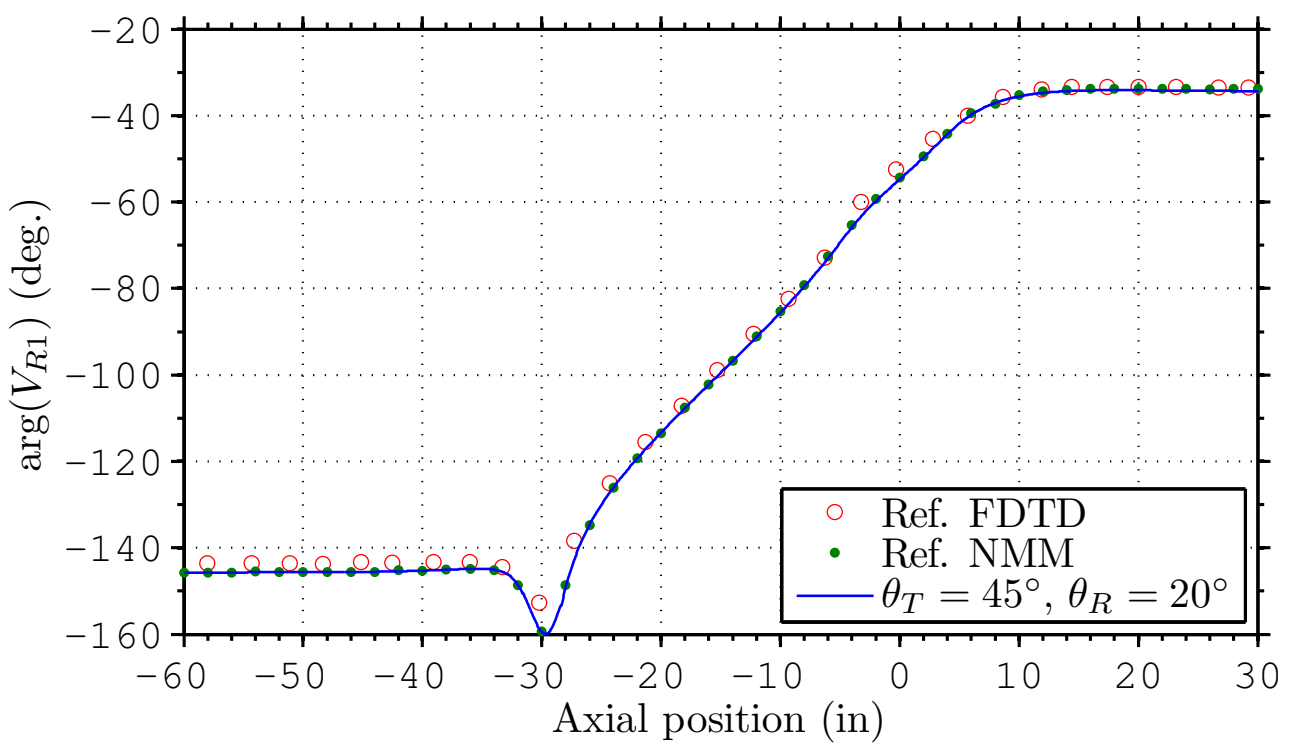

(b) Voltage phase

Figure 3.23: Voltage at TCA receiver $\mathrm{RX}_{1}$ with $\theta_{R}=20^{\circ}$ due to a unit current excitation transmitting TCA with $\theta_{T}=45^{\circ}$. The results from the present algorithm are indicated by solid lines. The small circles and dots are FDTD and R-NMM results, respectively, from [6, pp. 106-107]. 


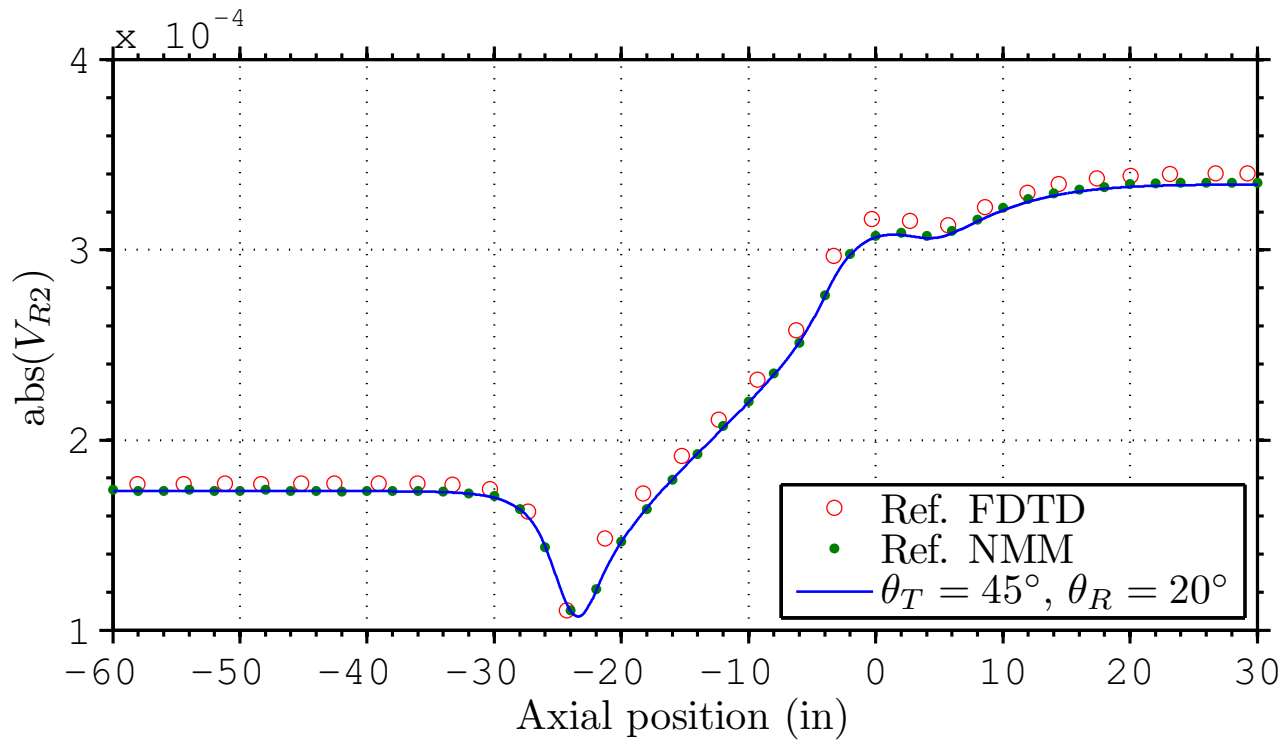

(a) Voltage amplitude

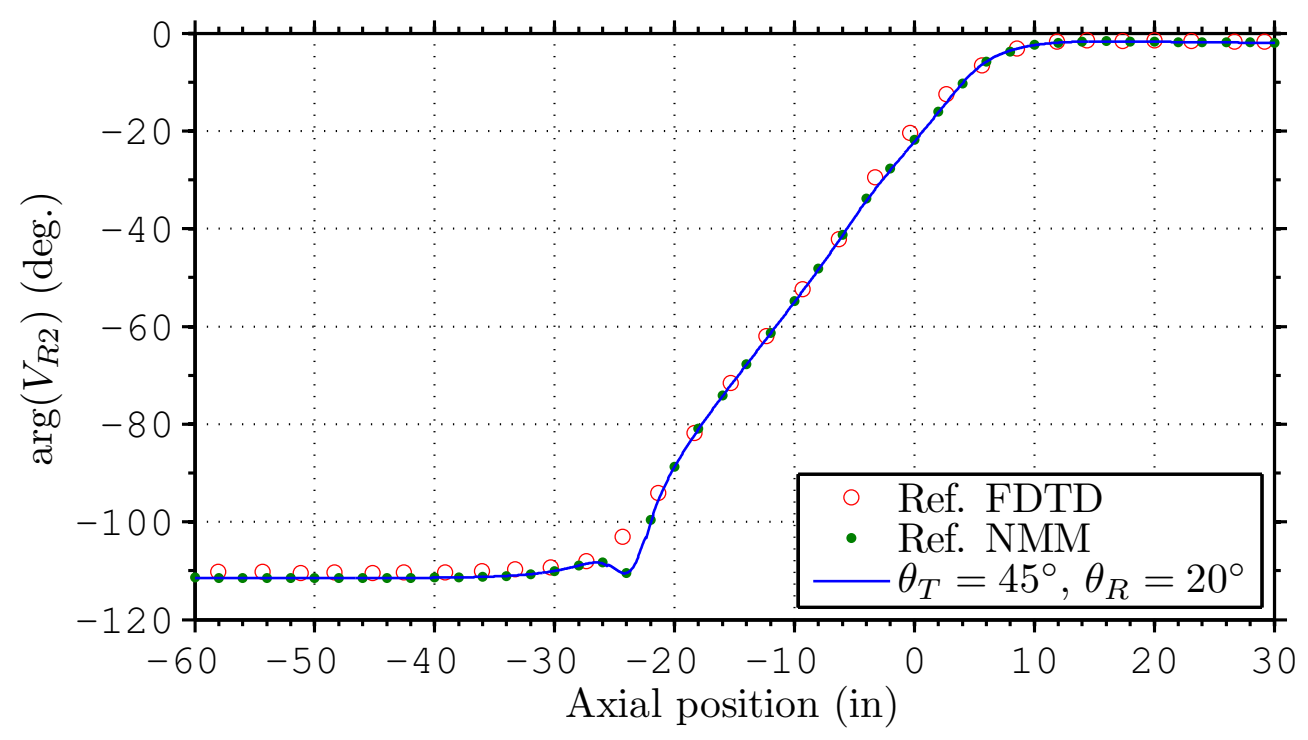

(b) Voltage phase

Figure 3.24: Voltage at TCA receiver $\mathrm{RX}_{2}$ with $\theta_{R}=20^{\circ}$ due to a unit current excitation transmitting TCA with $\theta_{T}=45^{\circ}$. The results from the present algorithm are indicated by solid lines. The small circles and dots are FDTD and R-NMM results, respectively, from [6, pp. 106-107]. 


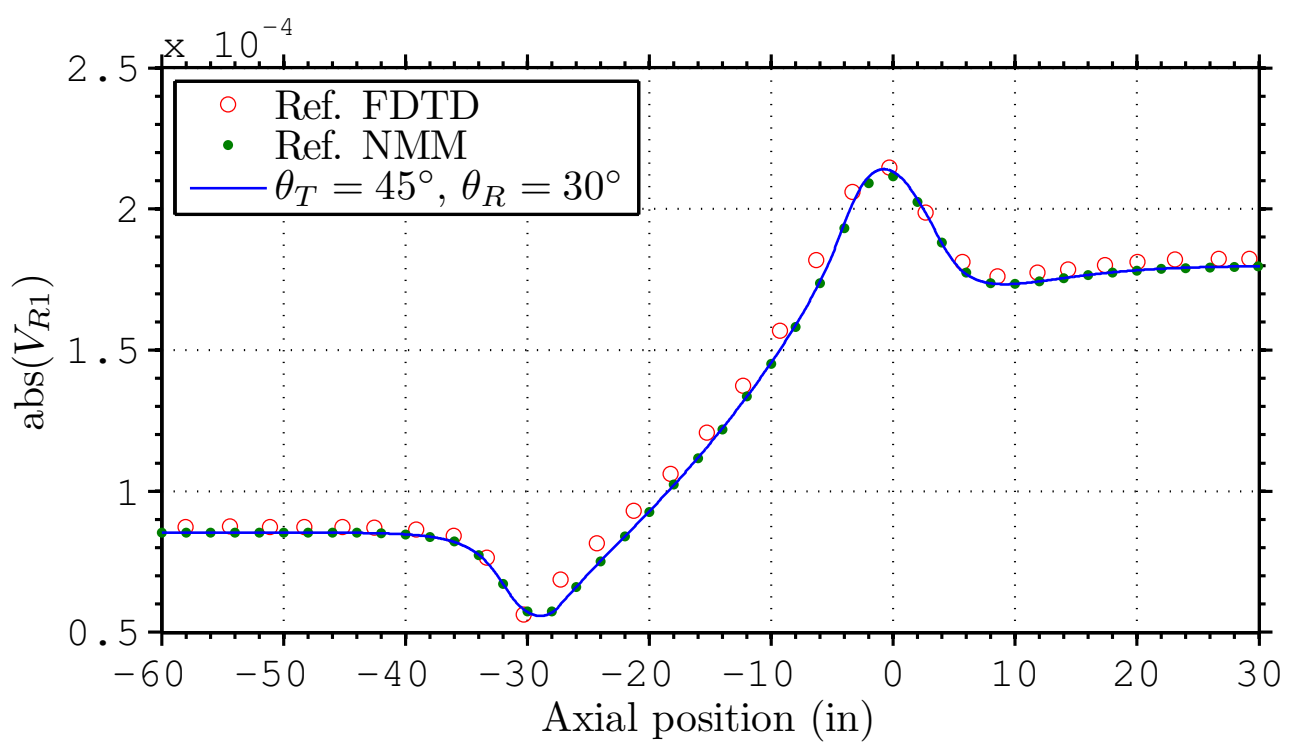

(a) Voltage amplitude

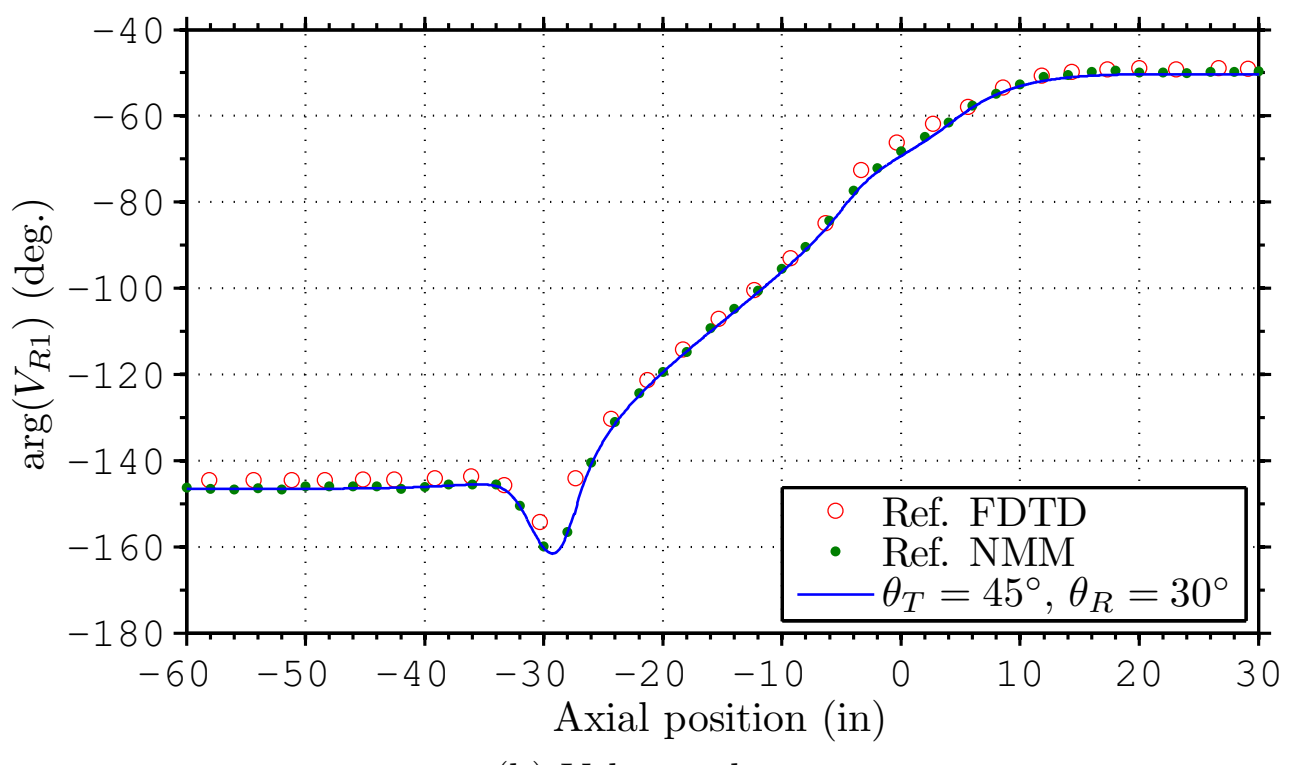

(b) Voltage phase

Figure 3.25: Voltage at TCA receiver $\mathrm{RX}_{1}$ with $\theta_{R}=30^{\circ}$ due to a unit current excitation transmitting TCA with $\theta_{T}=45^{\circ}$. The results from the present algorithm are indicated by solid lines. The small circles and dots are FDTD and R-NMM results, respectively, from [6, pp. 106-107]. 


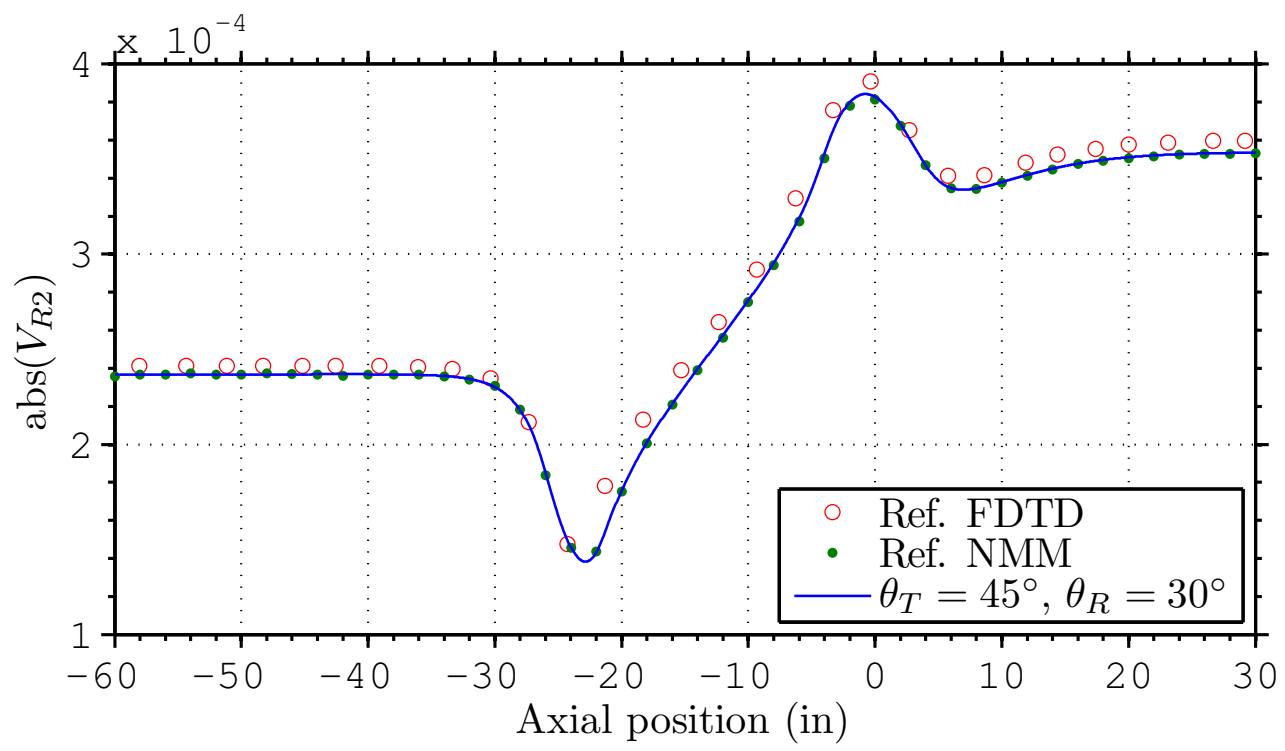

(a) Voltage amplitude

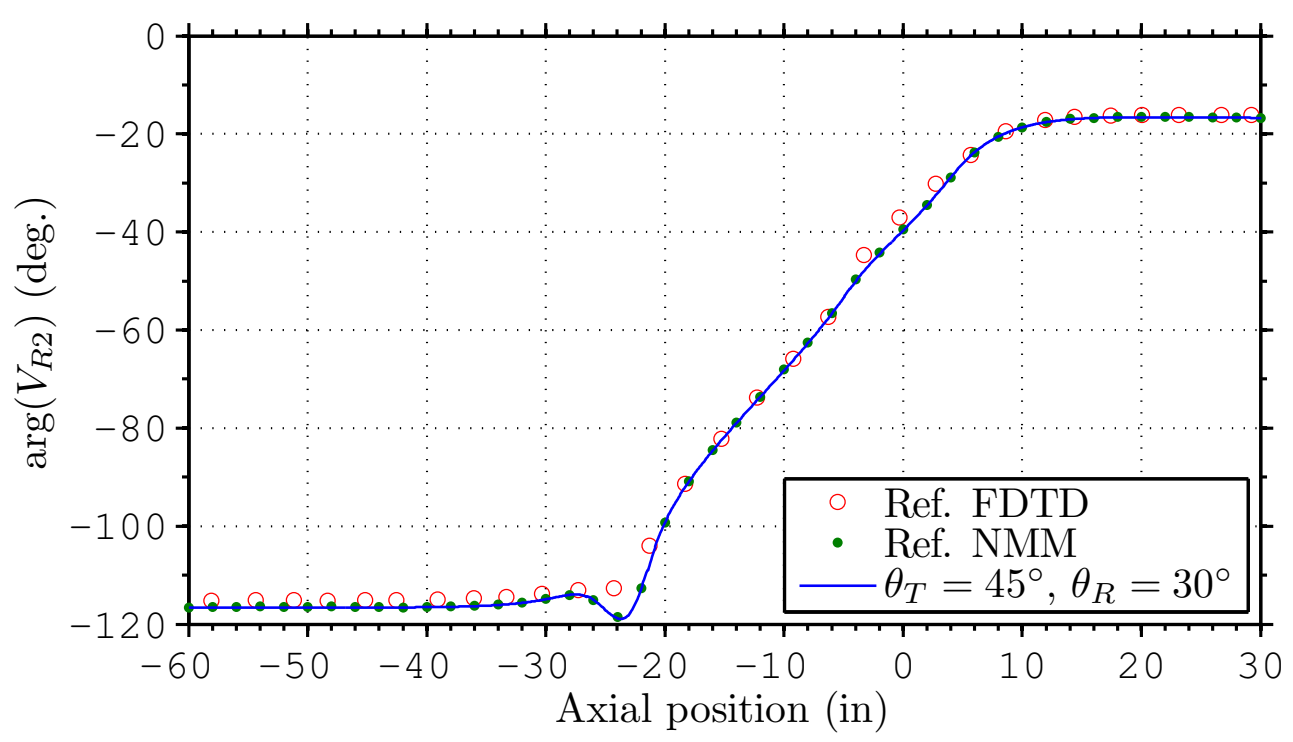

(b) Voltage phase

Figure 3.26: Voltage at TCA receiver $\mathrm{RX}_{2}$ with $\theta_{R}=30^{\circ}$ due to a unit current excitation transmitting TCA with $\theta_{T}=45^{\circ}$. The results from the present algorithm are indicated by solid lines. The small circles and dots are FDTD and R-NMM results, respectively, from [6, pp. 106-107]. 


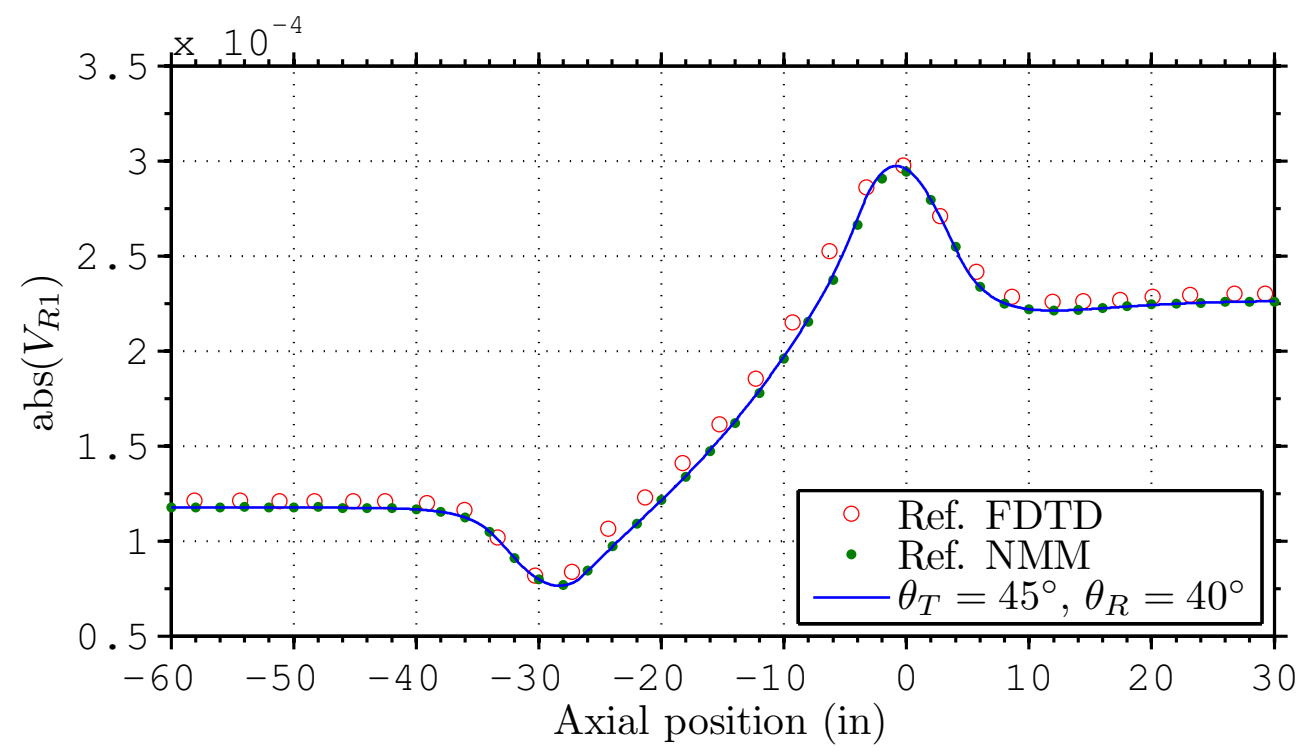

(a) Voltage amplitude

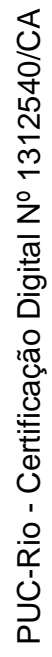

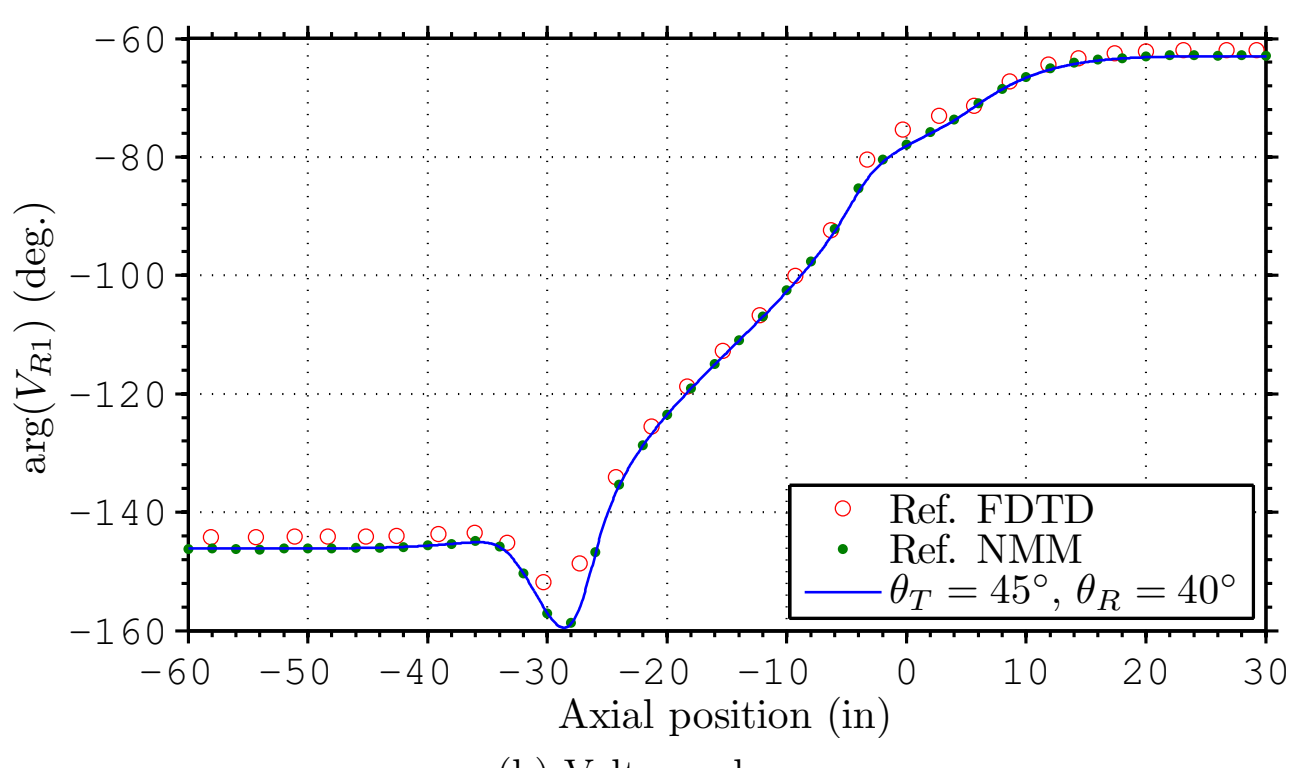

(b) Voltage phase

Figure 3.27: Voltage at TCA receiver $\mathrm{RX}_{1}$ with $\theta_{R}=40^{\circ}$ due to a unit current excitation transmitting TCA with $\theta_{T}=45^{\circ}$. The results from the present algorithm are indicated by solid lines. The small circles and dots are FDTD and R-NMM results, respectively, from [6, pp. 106-107]. 


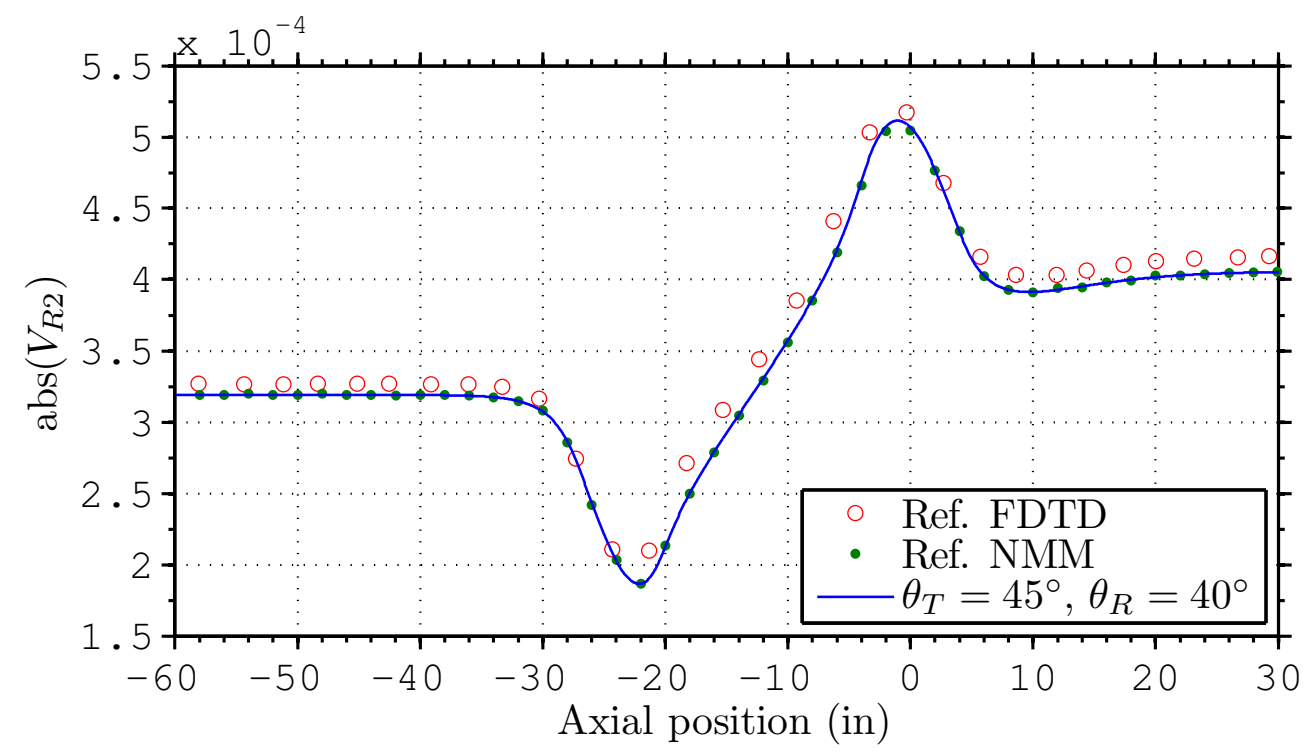

(a) Voltage amplitude

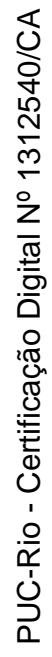

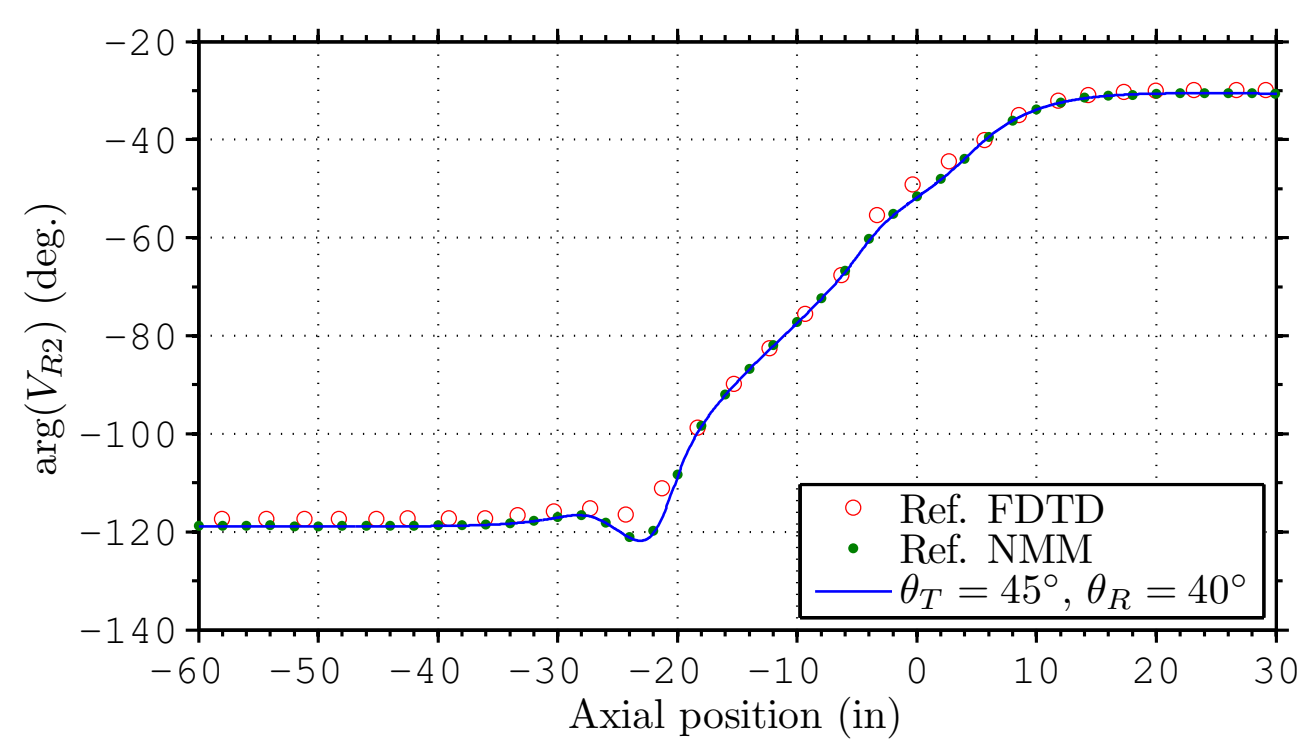

(b) Voltage phase

Figure 3.28: Voltage at TCA receiver $\mathrm{RX}_{2}$ with $\theta_{R}=40^{\circ}$ due to a unit current excitation transmitting TCA with $\theta_{T}=45^{\circ}$. The results from the present algorithm are indicated by solid lines. The small circles and dots are FDTD and R-NMM results, respectively, from [6, pp. 106-107]. 
As said before, we have employed the fields contribution of the azimuthal index $n=0, \pm 1, \pm 2, \pm 3, \pm 4$. However, we have verified that the main contribution comes from fields associated with $n=0$ and $n= \pm 1$.

Fig. 3.29 to 3.32 show the absolute values of the received voltages for each orthogonal azimuthal index. We clearly see that the main contribution comes from $n=0$ as $\theta_{R}$ is small, but for larger $\theta_{R}$, the contribution of $n= \pm 1$ becomes dominant. For practical purposes, we do not need to include high order azimuthal index such as $|n|>1$ in order to proper analyze TCA antennas in logging well environments. Also, the criteria used above to include a modal solution in the mode-matching is very conservative $\left(A_{d B}=-60 \mathrm{~dB}\right.$ at $\Delta z=5$ in).

In Fig. 3.33 and Fig. 3.34 we show the convergence behavior of the received fields in relation to the axial convergence criteria. We select the voltage received by antenna $\mathrm{RX}_{2}$ using $\theta_{R}=40^{\circ}$, and the number of modes used in each simulations is depicted in Table 3.2. Notice that using fields with axial attenuation lower than $-20 \mathrm{~dB}$ at $5 \mathrm{in}$, the received voltages are virtually indistinguishable. By using all fields with axial attenuation up to $-20 \mathrm{~dB}$ at 5 in and the $n=0,1$ azimuthal harmonics, the total CPU time required by our algorithm was 6 seconds to simulate 300 observation points. However, $A_{d B}$ higher than $-20 \mathrm{~dB}$ is not enough to correctly capture the fields behavior at the vicinity of the interface between formations 1 and 2, as depicted in Fig. 3.34.

From the above, we conclude that only a few number of modes excited by the source have significant fields after propagate the distance between the source and a receiving antenna. However, the presence of the interface between the axial formations scatters the incident fields, acting as a new equivalent source at the junction. Thus, we need to include a number of modal fields in order to accurately account the fields when the antennas (both TX as $\mathrm{RX}_{1}$ and $\mathrm{RX}_{2}$ ) are crossing or near the junction.

The results shown above confirm that the analytical approach introduced in Section 3.5.6 can efficiently model TCA antennas crossing different axial regions. In this way, we have verified that the axial mode-matching can be employed in combination with excitation sources with non-zero span along the axial direction. As far as we know, until now only radial mode-matching formulations are reported in the literature as able to model this kind of problem. 


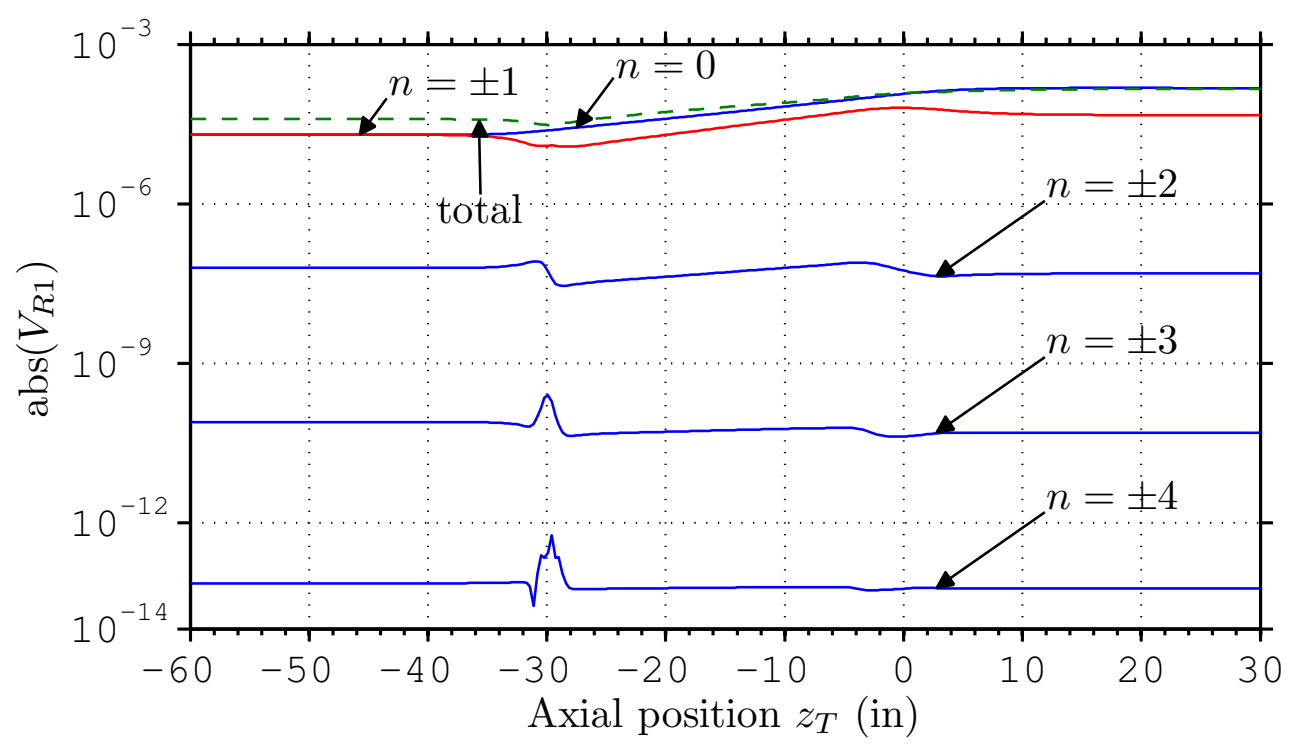

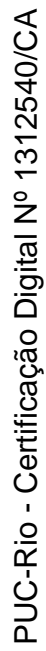

(a) Voltage amplitude $V_{R 1}$

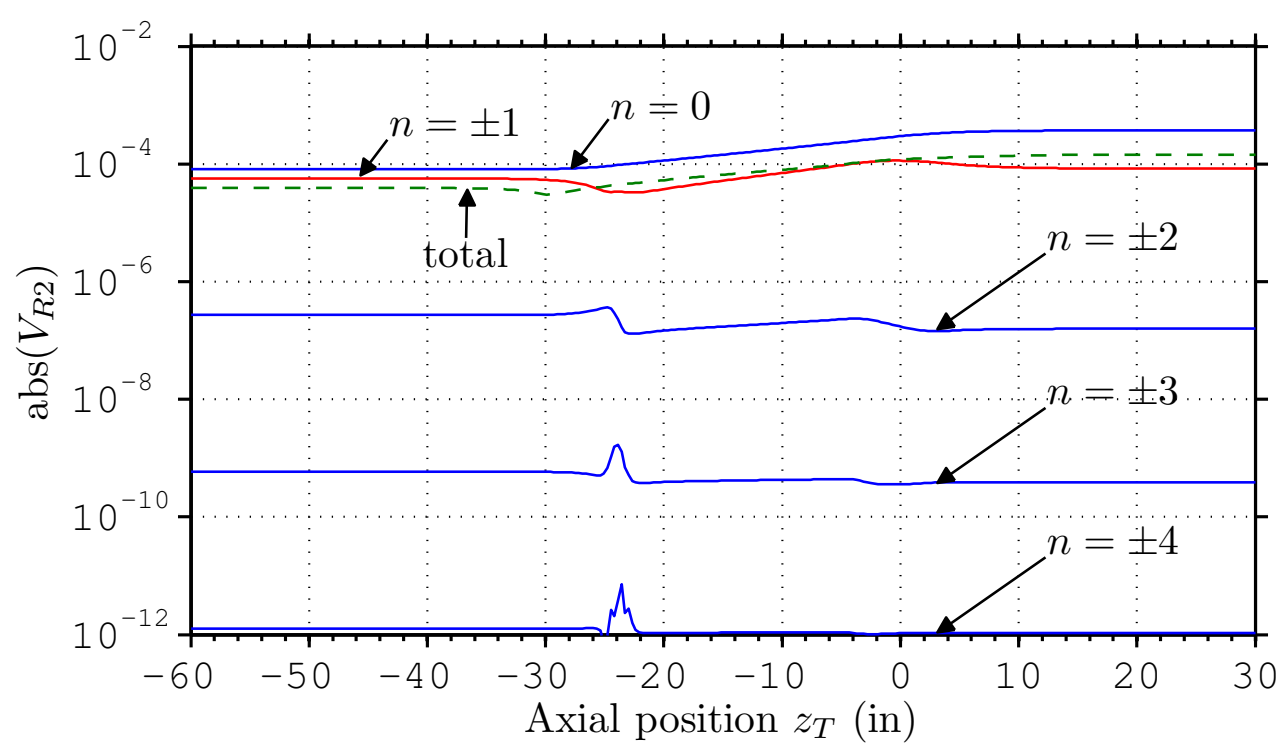

(b) Voltage amplitude $V_{R 2}$

Figure 3.29: Amplitude of the received voltages $V_{R 1}$ and $V_{R 2}$ for $\theta_{R}=10^{\circ}$ in terms of its azimuthal contributions. The total voltages are shown in dashed lines. 


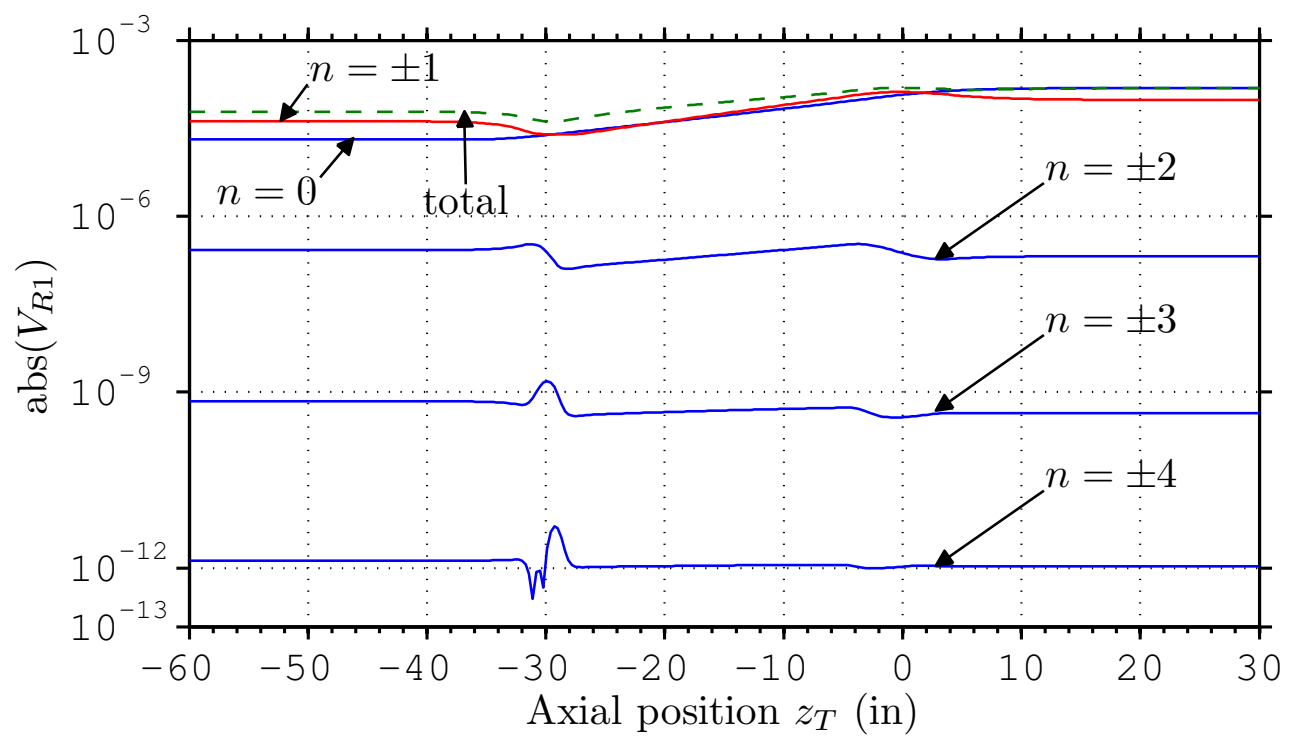

(a) Voltage amplitude $V_{R 1}$

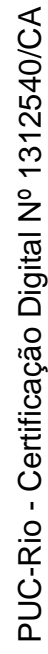

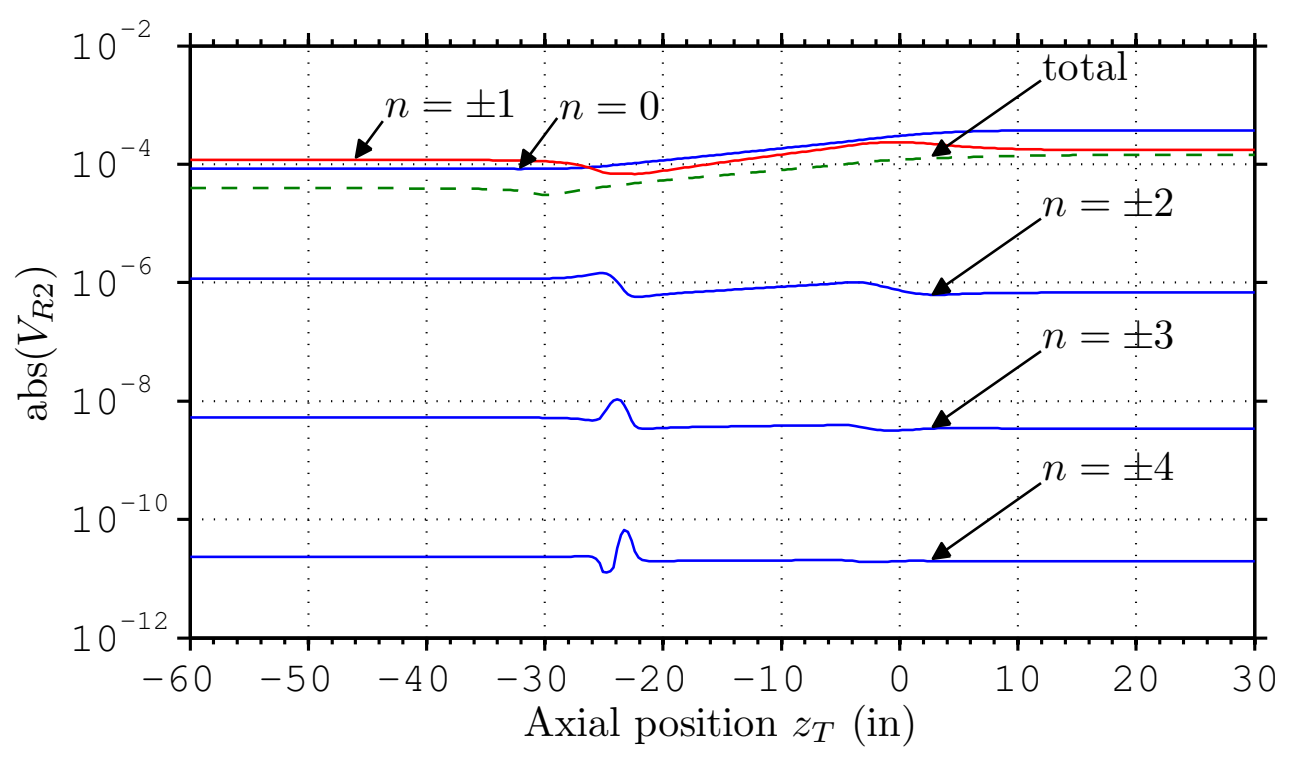

(b) Voltage amplitude $V_{R 2}$

Figure 3.30: Amplitude of the received voltages $V_{R 1}$ and $V_{R 2}$ for $\theta_{R}=20^{\circ}$ in terms of its azimuthal contributions. The total voltages are shown in dashed lines. 


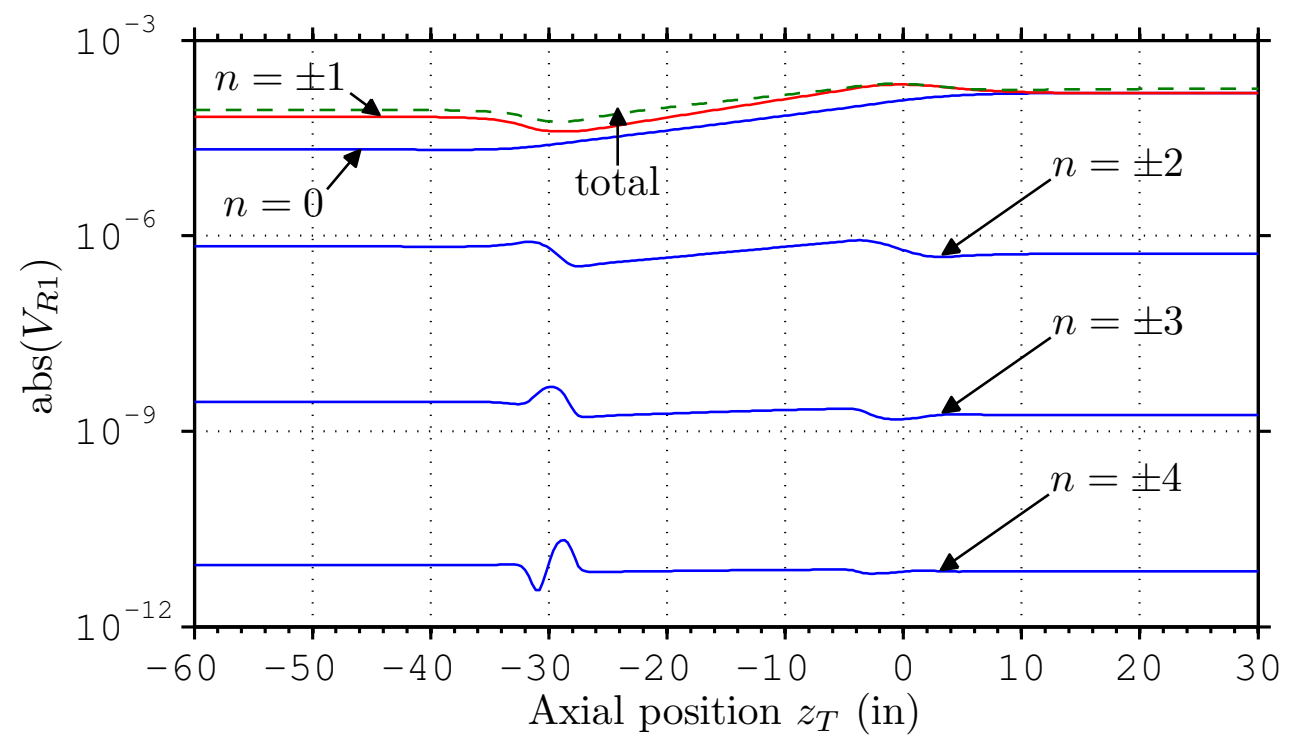

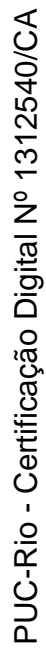

(a) Voltage amplitude $V_{R 1}$

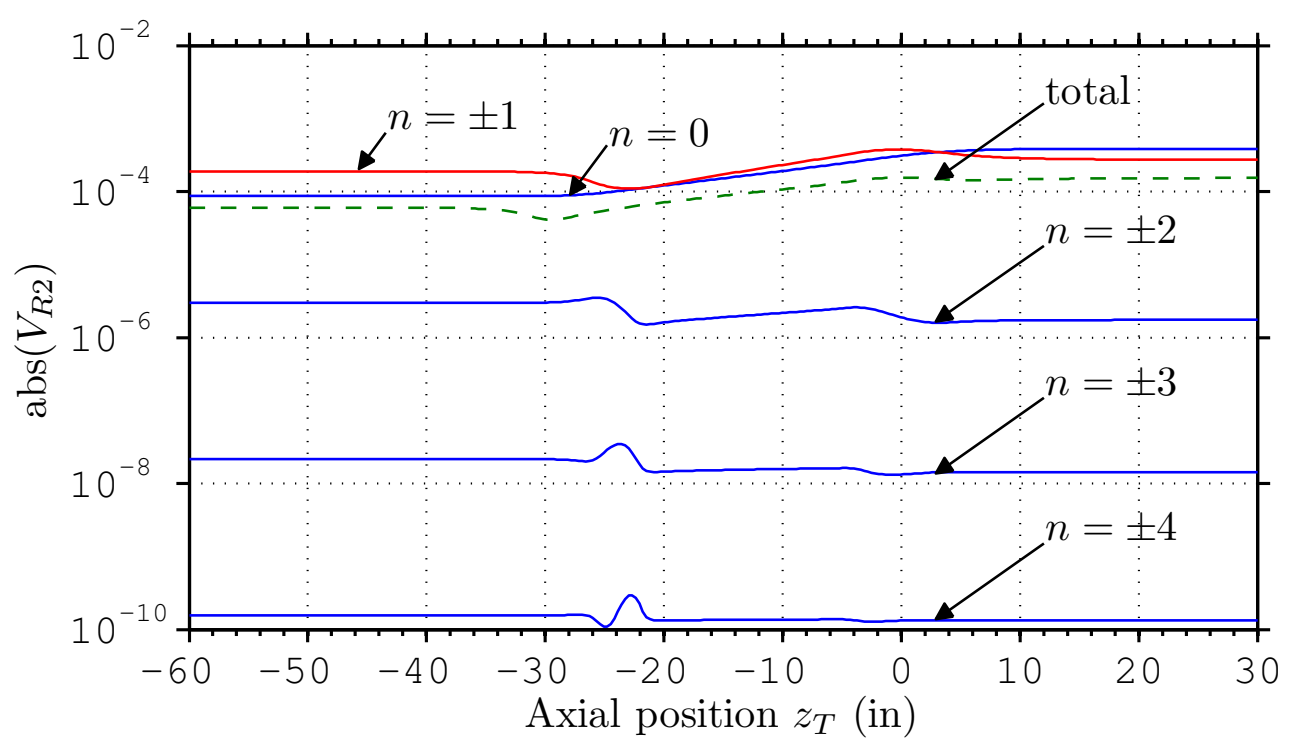

(b) Voltage amplitude $V_{R 2}$

Figure 3.31: Amplitude of the received voltages $V_{R 1}$ and $V_{R 2}$ for $\theta_{R}=30^{\circ}$ in terms of its azimuthal contributions. The total voltages are shown in dashed lines. 


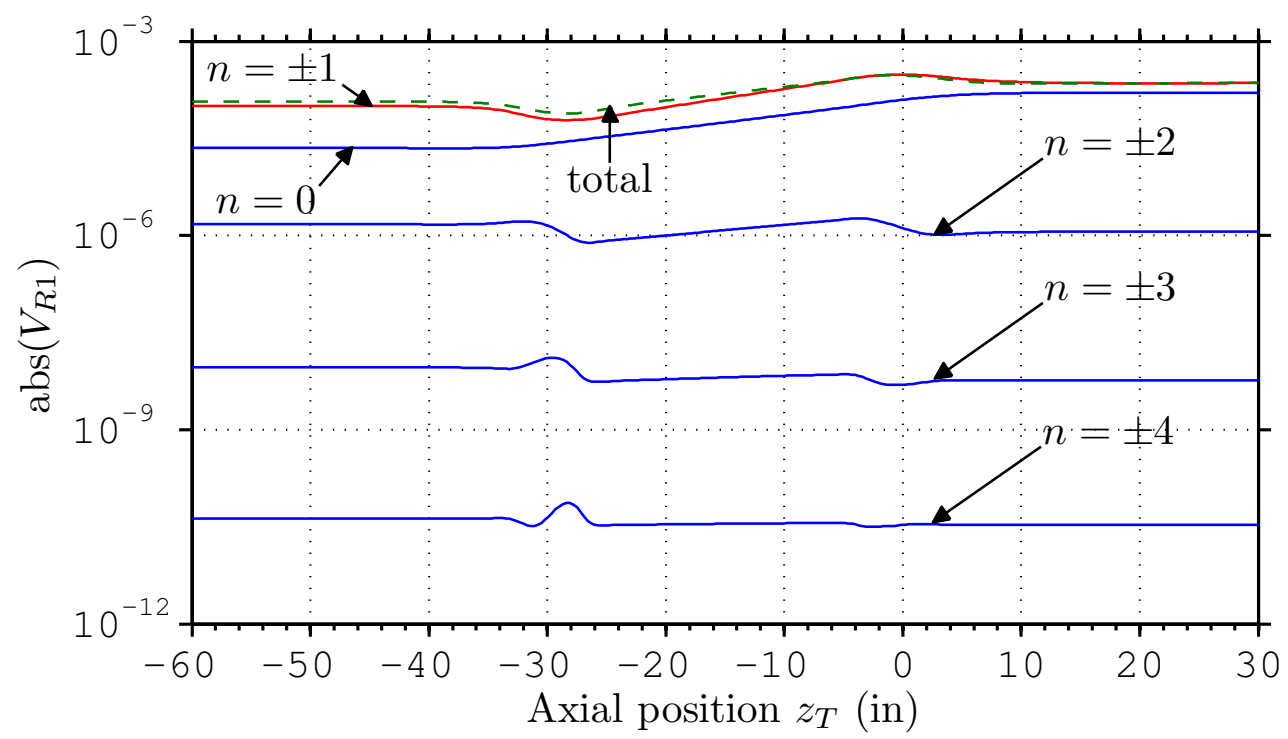

(a) Voltage amplitude $V_{R 1}$

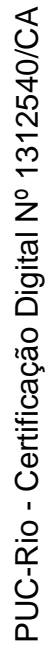

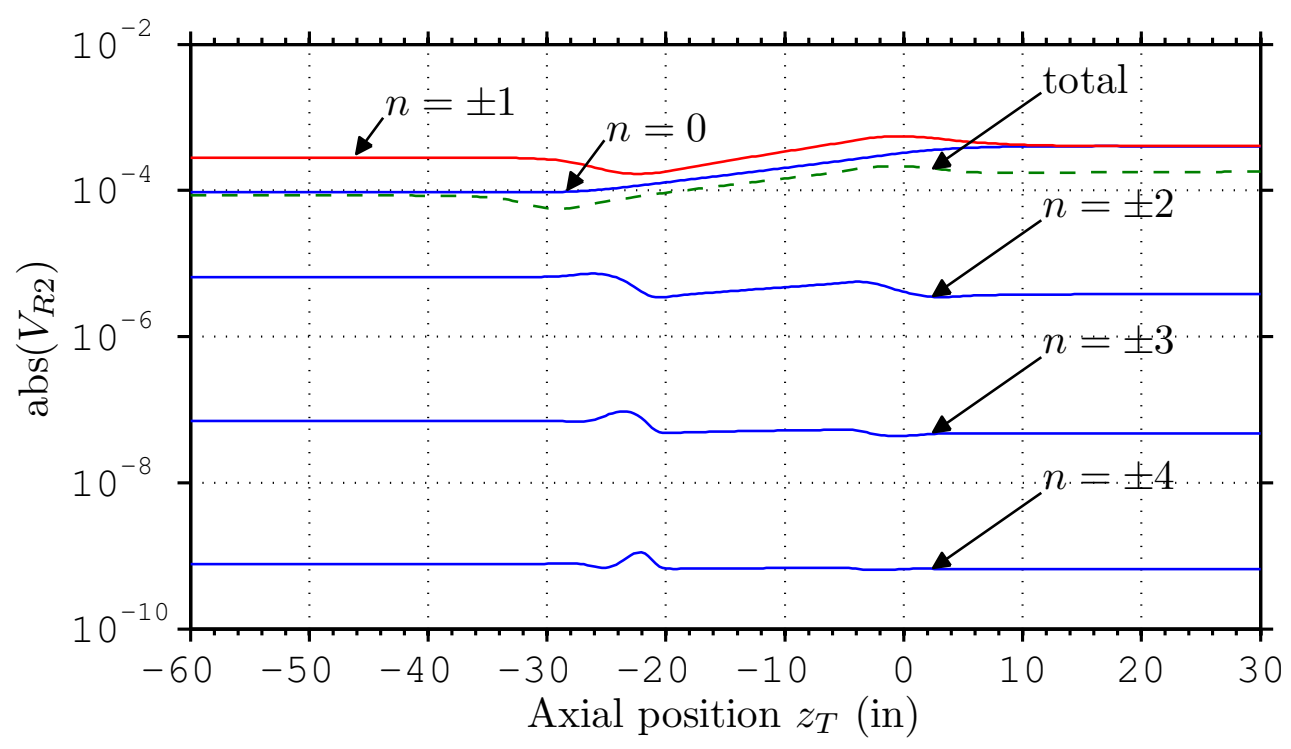

(b) Voltage amplitude $V_{R 2}$

Figure 3.32: Amplitude of the received voltages $V_{R 1}$ and $V_{R 2}$ for $\theta_{R}=40^{\circ}$ in terms of its azimuthal contributions. The total voltages are shown in dashed lines. 


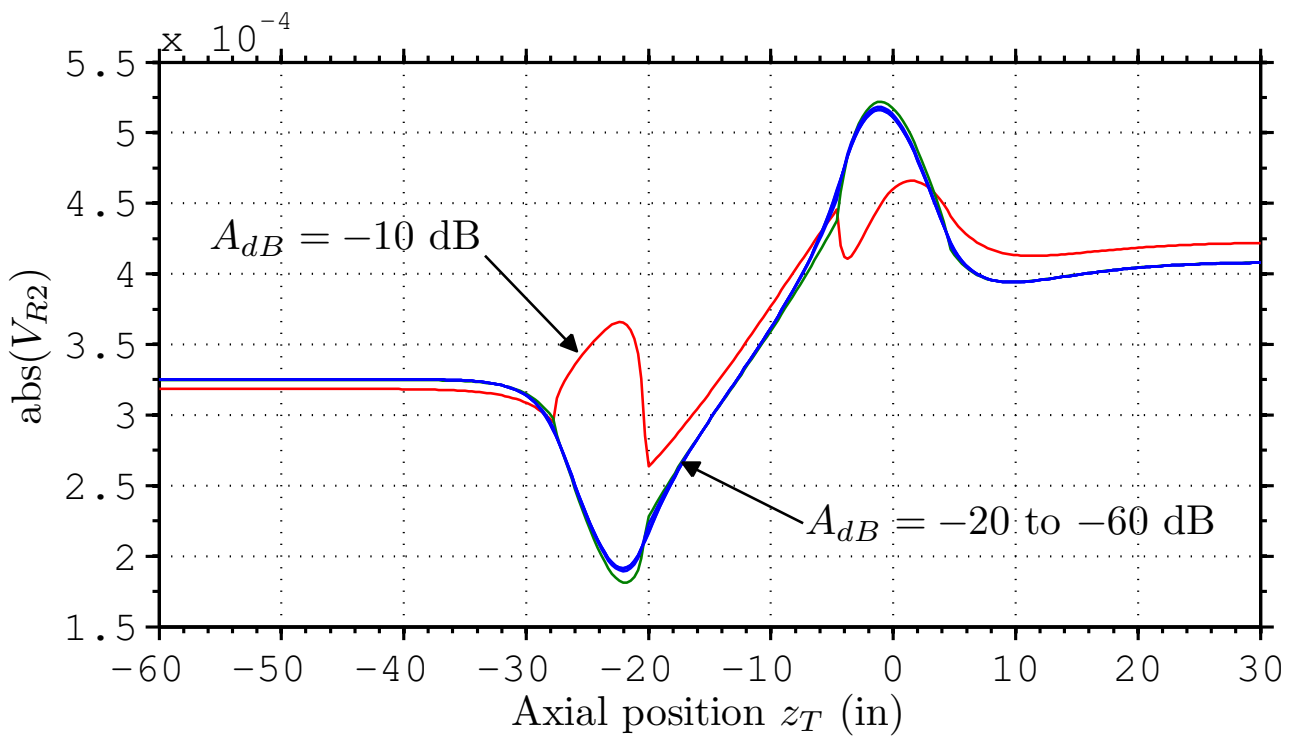

(a) Voltage amplitude

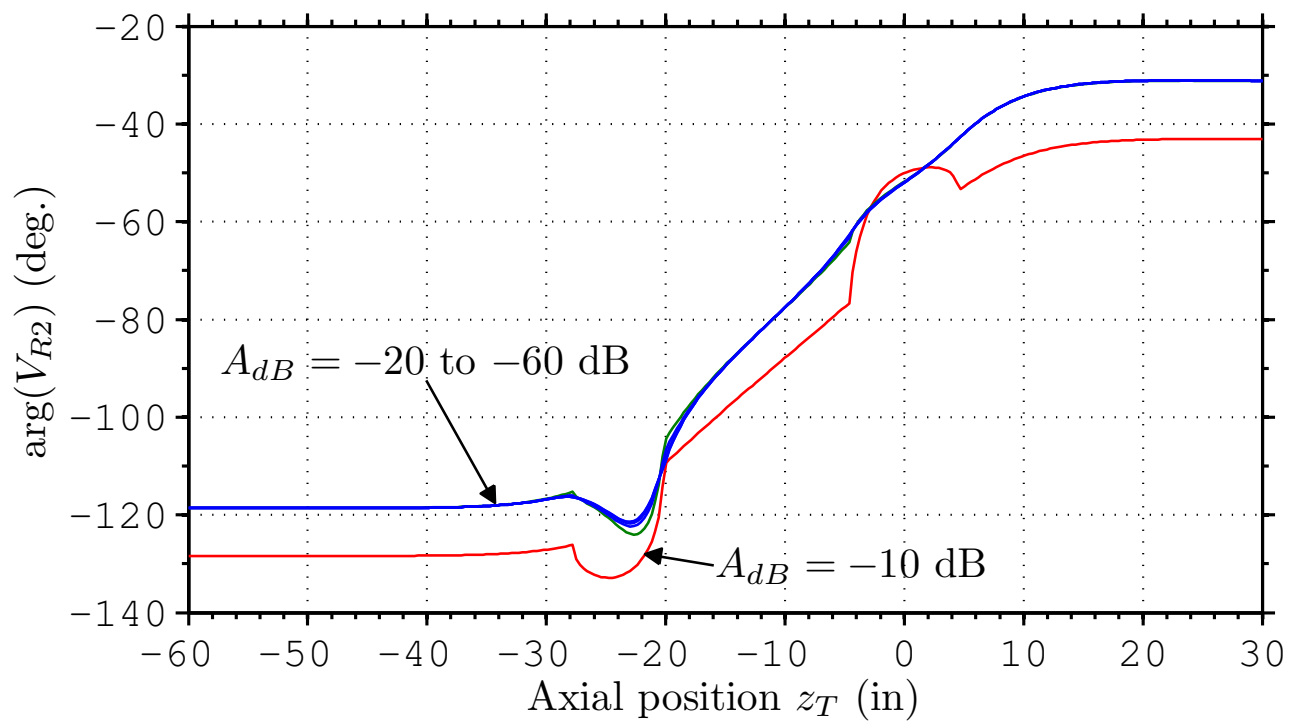

(b) Voltage phase

Figure 3.33: Convergence of the voltage amplitude and phase received by antenna $\operatorname{RX}_{2}\left(\theta_{R}=40^{\circ}\right)$ for $A_{d B}$ at $\Delta z=5 \mathrm{in}$. The response from six values of $A_{d B}$ (from $-10 \mathrm{~dB}$ to $-60 \mathrm{~dB}$, with steps of $10 \mathrm{~dB}$ ) are presented. 


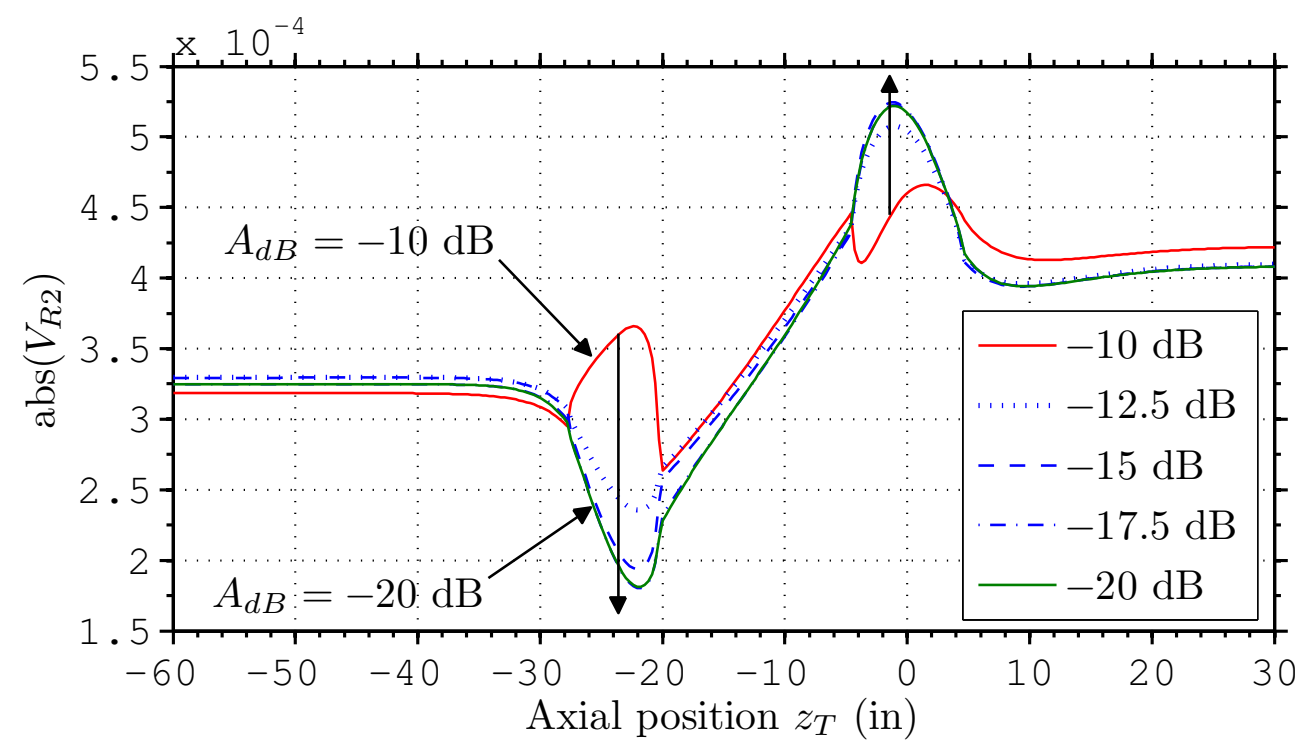

(a) Voltage amplitude

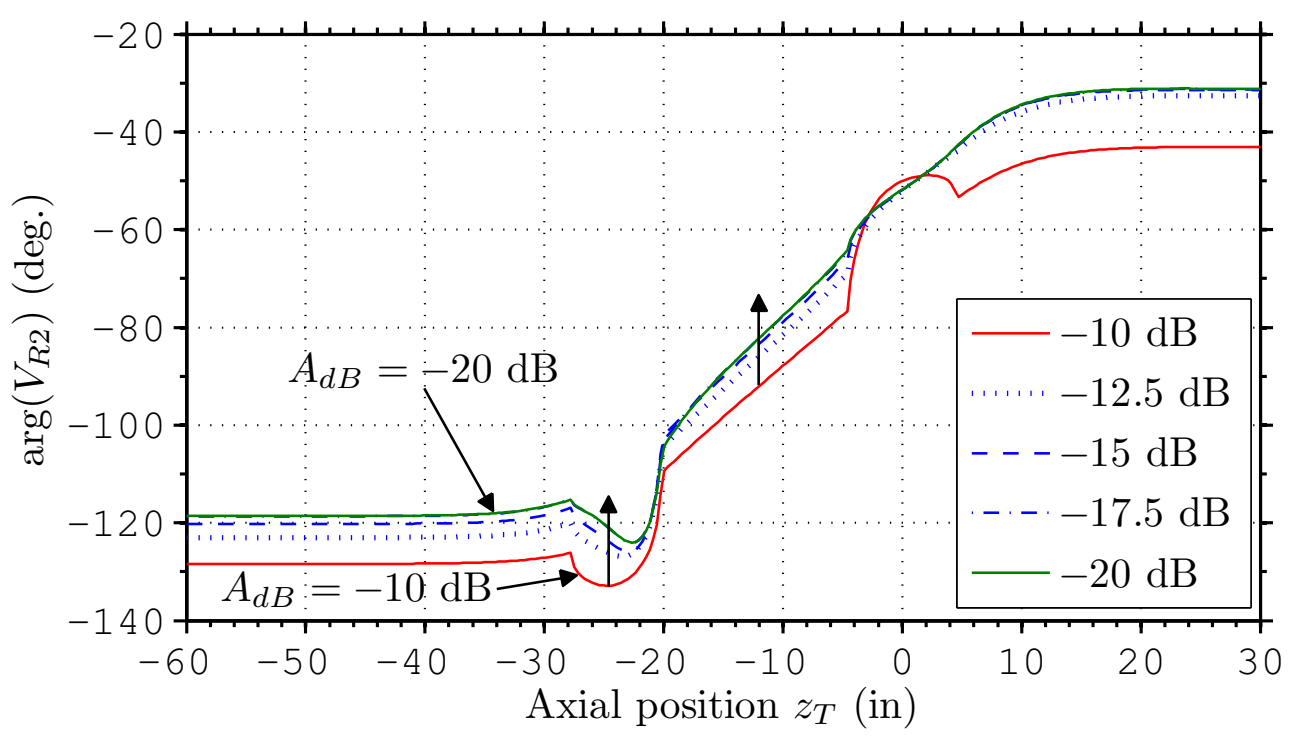

(b) Voltage phase

Figure 3.34: Convergence of the voltage amplitude and phase received by antenna $\operatorname{RX}_{2}\left(\theta_{R}=40^{\circ}\right)$ for $A_{d B}$ at $\Delta z=5 \mathrm{in}$. The response for five values of $A_{d B}$ (from $-10 \mathrm{~dB}$ to $-20 \mathrm{~dB}$, with steps of $2.5 \mathrm{~dB}$ ) are presented. Vertical arrows indicate the convergence sense. 
Table 3.2: Number of modes for $A_{d B}$ at 5 in

\begin{tabular}{lccccc}
\hline & \multicolumn{2}{c}{$n=0$} & & \multicolumn{2}{c}{$n= \pm 1$} \\
\cline { 2 - 3 } \cline { 5 - 6 }$A_{d B}(\mathrm{~dB})$ & Region 1 & Region 2 & & Region 1 & Region 2 \\
\hline-10 & 7 & 8 & & 7 & 8 \\
-12.5 & 10 & 11 & & 10 & 10 \\
-15 & 12 & 13 & & 12 & 12 \\
-17.5 & 15 & 15 & & 14 & 15 \\
-20 & 17 & 17 & & 16 & 17 \\
-30 & 25 & 25 & & 25 & 25 \\
-40 & 33 & 33 & & 33 & 33 \\
-50 & 41 & 41 & & 41 & 41 \\
-60 & 49 & 49 & & 49 & 49 \\
\hline
\end{tabular}

\subsubsection{2}

\section{Anisotropic Formations}

We now consider a formation similar to the latter problem, but with anisotropic formations. Surrounding the borehole, the horizontal and vertical components of the conductivity of the formation $1(z<0)$ are $5 \mathrm{~S} / \mathrm{m}$ and $1 \mathrm{~S} / \mathrm{m}$. Formation $2(z>0)$ is characterized by $1 \mathrm{~S} / \mathrm{m}$ and $5 \mathrm{~S} / \mathrm{m}$, respectively, as shown in Fig. 3.35.

Fig. 3.36 to Fig. 3.43 plot the voltage due to a unit current excitation $\left(I_{T}=1 \mathrm{~A}\right.$ at $\left.2 \mathrm{MHz}\right)$ at the receiver antennas $\mathrm{RX}_{1}$ and $\mathrm{RX}_{2}$ as a function of the axial position of the tool, considering a transmitter TCA with fixed tilt angle of $45^{\circ}$, and four different receiver TCA tilt angles: $\theta_{R}=15^{\circ}, 25^{\circ}, 35^{\circ}$ and $45^{\circ}$. The results obtained shown good agreement with those from $[6,8]$. The CPU time required by our algorithm is less than 19 seconds to simulate the contribution of each azimuthal harmonic for 300 observation points.

As noted before, the orthogonality of the azimuthal harmonics allow us to analyze the contribution of each $n$ to the total received voltage in (3-274). In Fig. 3.44 we shown the voltage amplitude (in decibels) at TCA antenna $\mathrm{RX}_{2}$ considered in the last example, as in Fig. 3.43(a), but now for different tilt angles $\theta_{R}$. Only the first 4 harmonics, $n=0,1,2$ and 3 , are depicted because higher order ones show negligible amplitudes. In the case $\theta_{R}=0$, the receiver TCA becomes an ordinary coil antenna and, as predicted before, only transversal electric modes associated with $n=0$ are present. It is observed that the amplitudes of the harmonics increase with the antenna tilt, which is expected from the behavior of the Bessel function with the complex-valued argument $\rho_{R} \tan \theta_{R} k_{z, n p}$. As expected, the relevant contribution to the received voltages comes from the azimuthal harmonics $n=0,1$. For small $\theta_{R}$, the main contribution comes from $n=0$, but for larger $\theta_{R}$, the contribution of $n=1$ 
becomes dominant.

We believe that the above observations based on the analytical form of the fields can provide some guidance for the inverse problem associated to LWD tools, i.e., where the measured voltages can be used to estimate the soil formation conductivity.

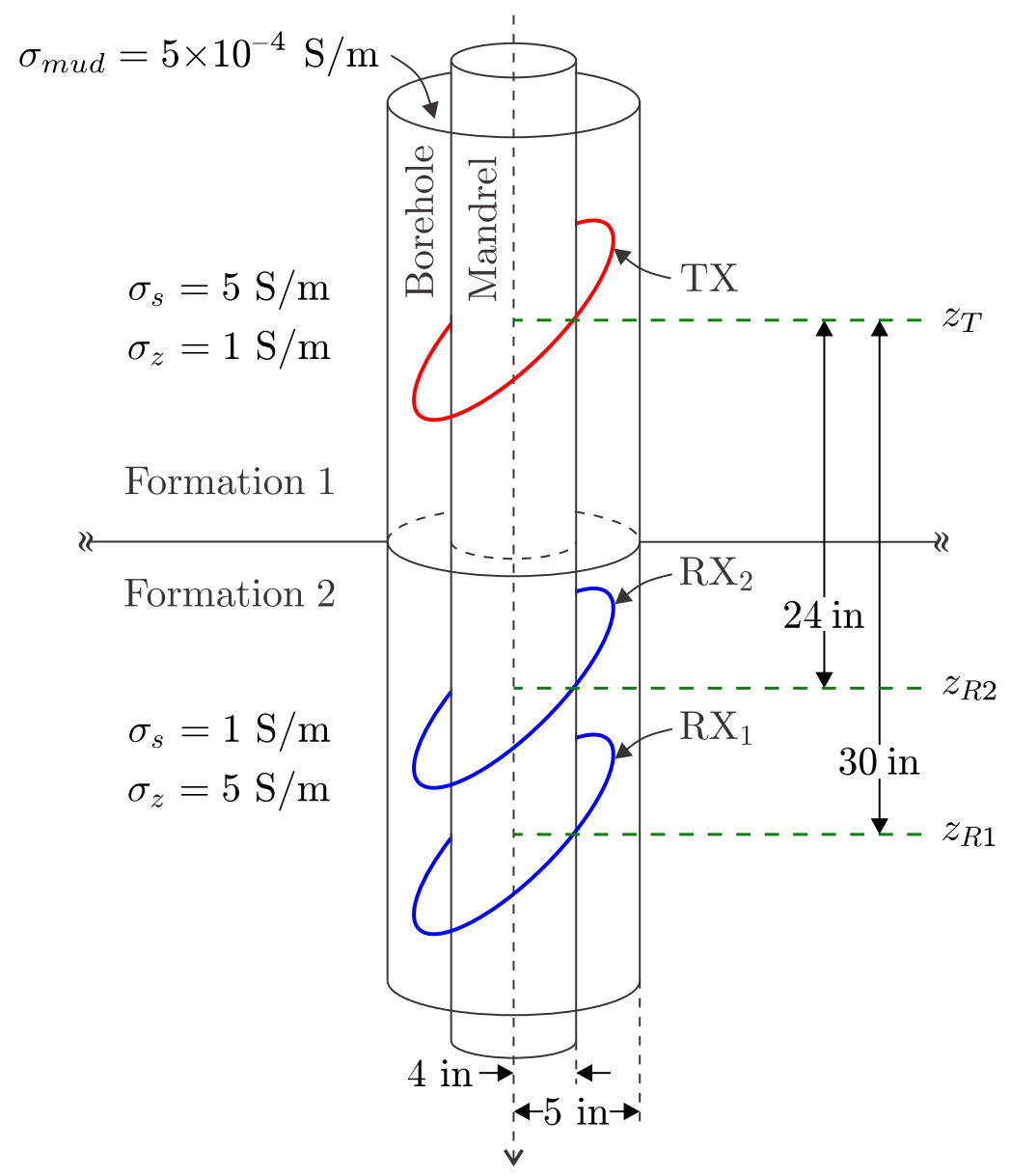

Figure 3.35: Geometry of a triaxial TCA well-logging tool within an anisotropic stratified geophysical formation. 


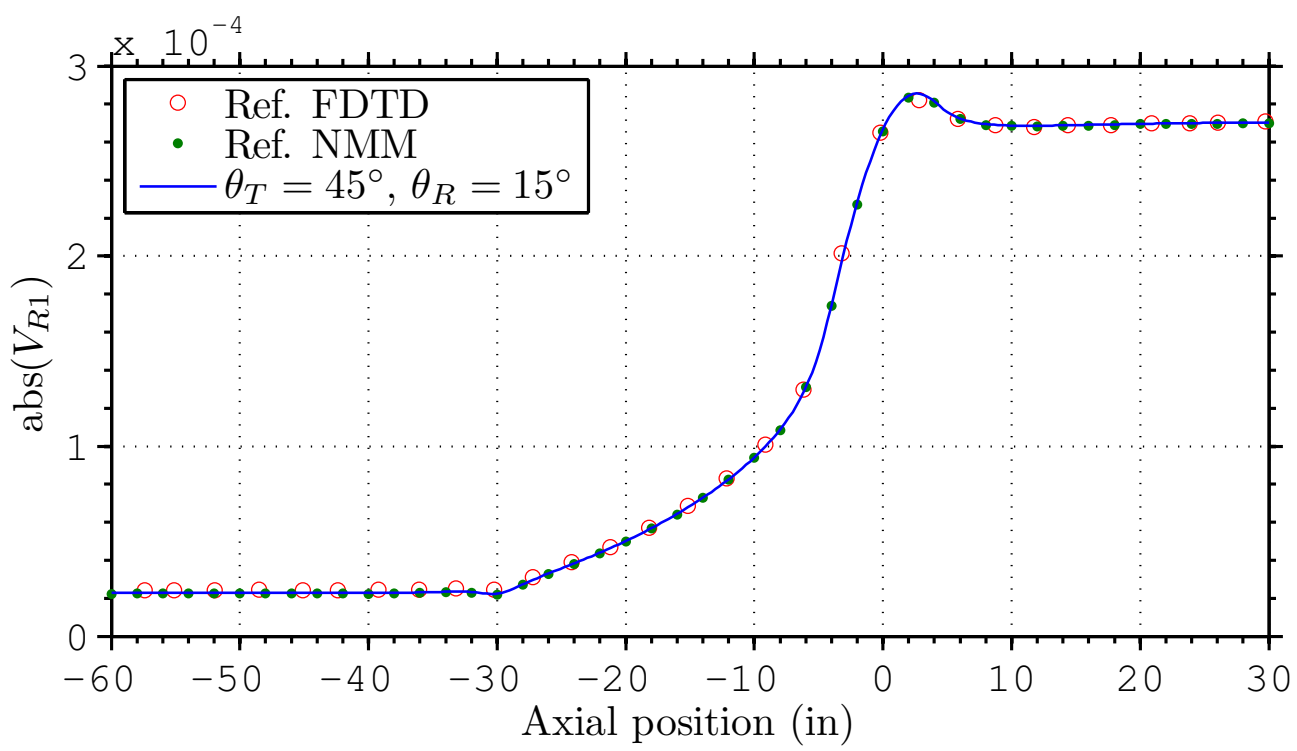

(a) Voltage amplitude

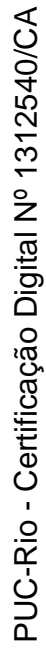

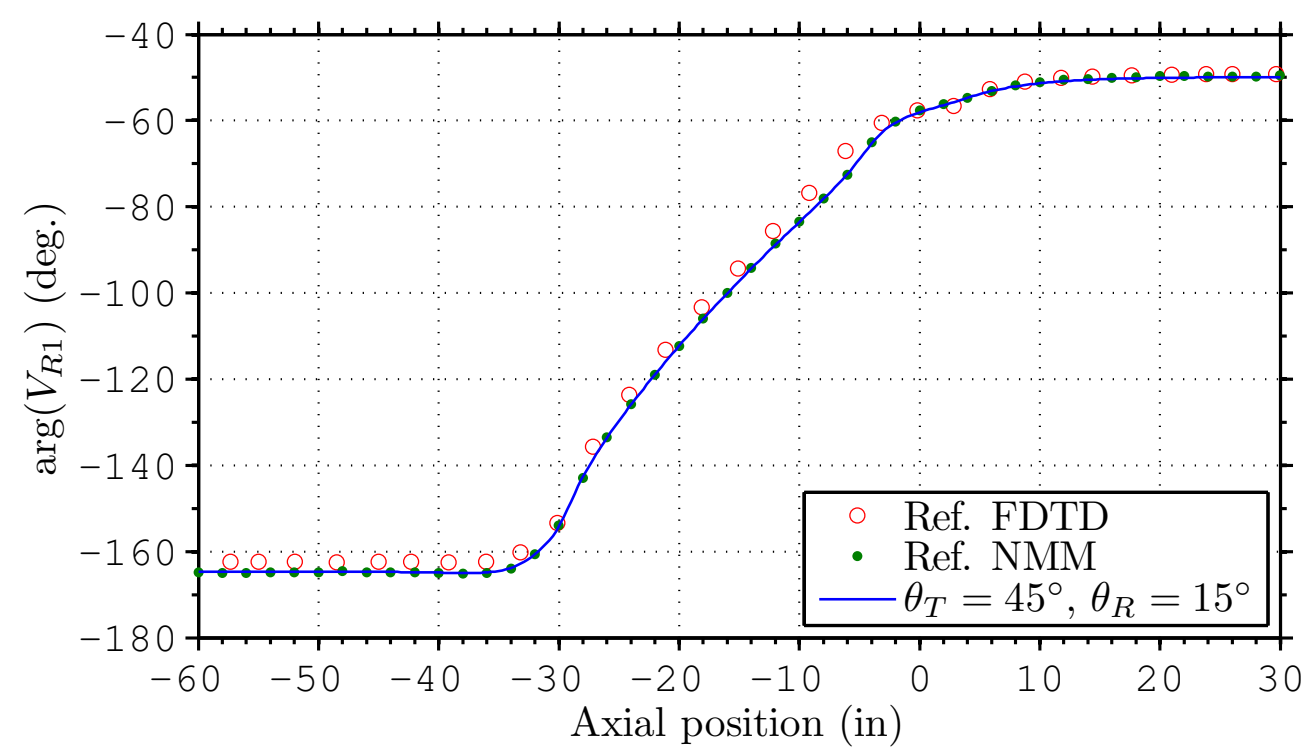

(b) Voltage phase

Figure 3.36: Voltage at TCA receiver $\mathrm{RX}_{1}$ with $\theta_{R}=15^{\circ}$ due to a unit current excitation transmitting TCA with $\theta_{T}=45^{\circ}$. The results from the present algorithm are indicated by solid lines. The small circles and dots are FDTD and R-NMM results, respectively, from [6, pp. 108-109], [8]. 


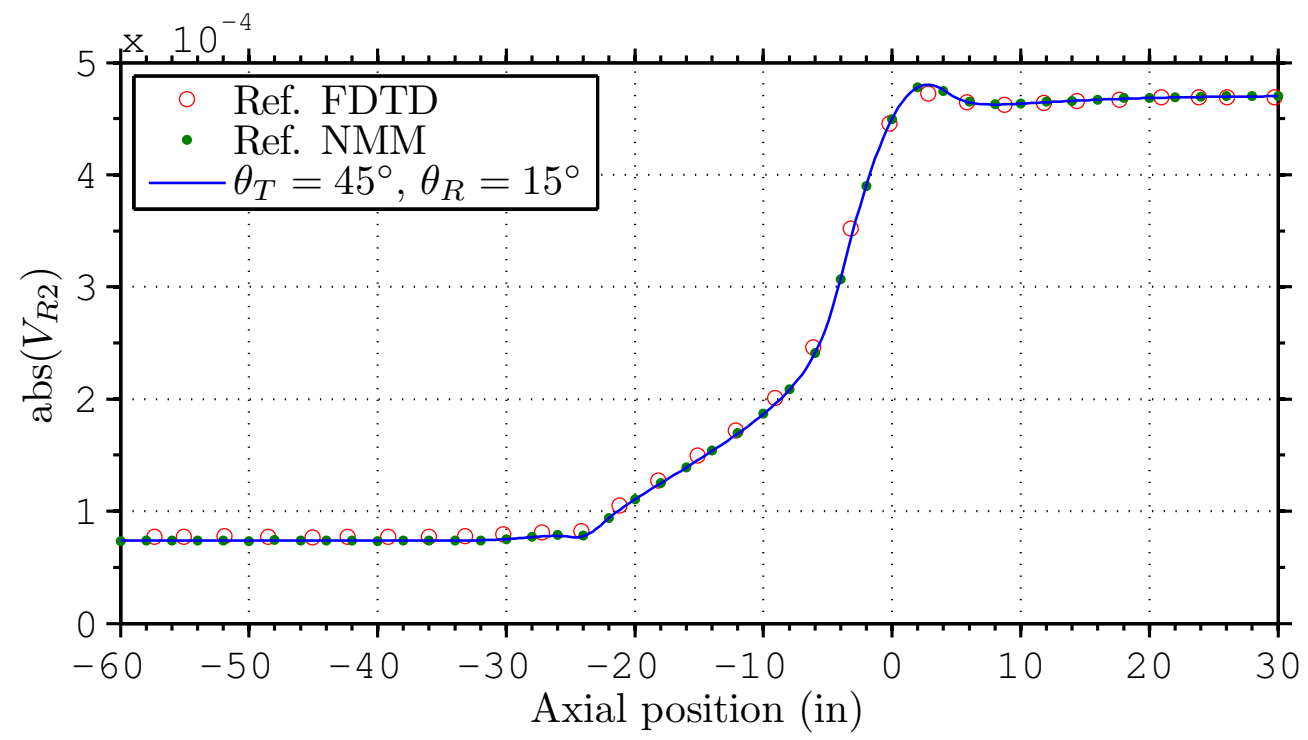

(a) Voltage amplitude

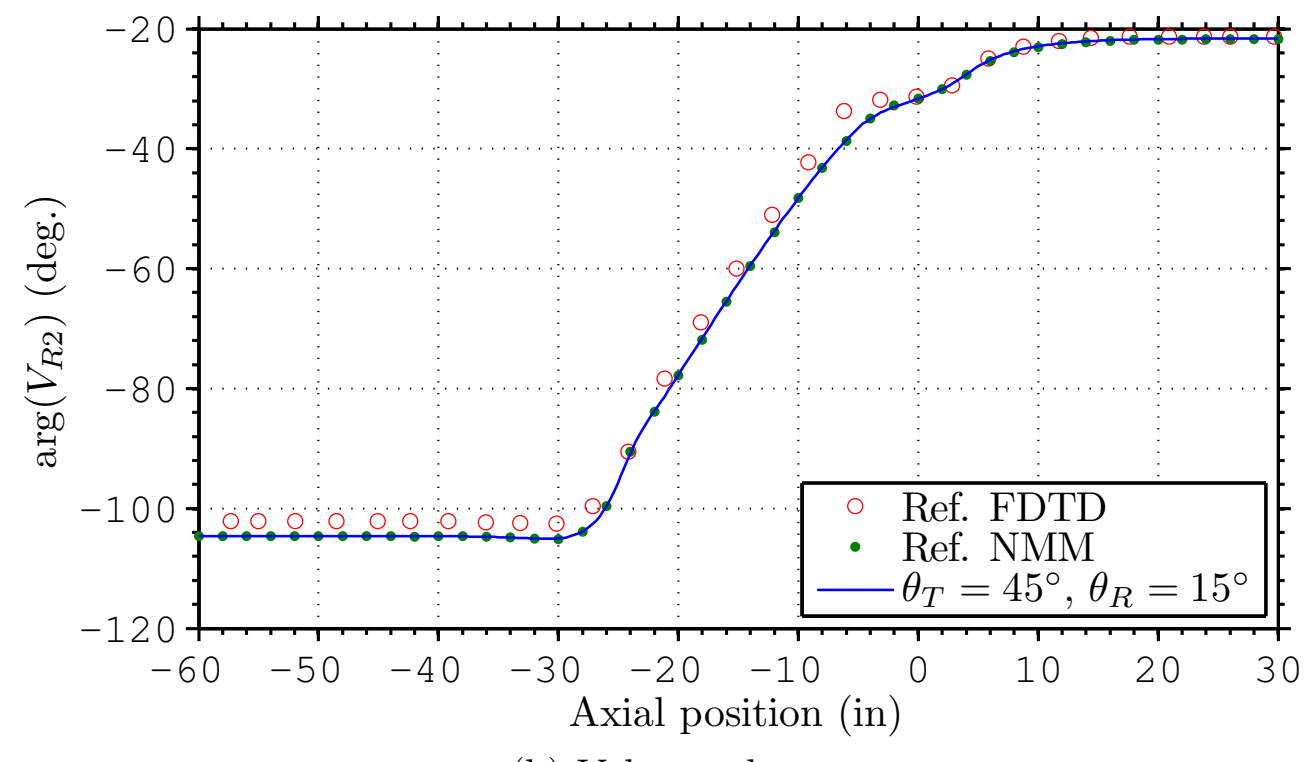

(b) Voltage phase

Figure 3.37: Voltage at TCA receiver $\mathrm{RX}_{2}$ with $\theta_{R}=15^{\circ}$ due to a unit current excitation transmitting TCA with $\theta_{T}=45^{\circ}$. The results from the present algorithm are indicated by solid lines. The small circles and dots are FDTD and R-NMM results, respectively, from [6, pp. 108-109], [8]. 


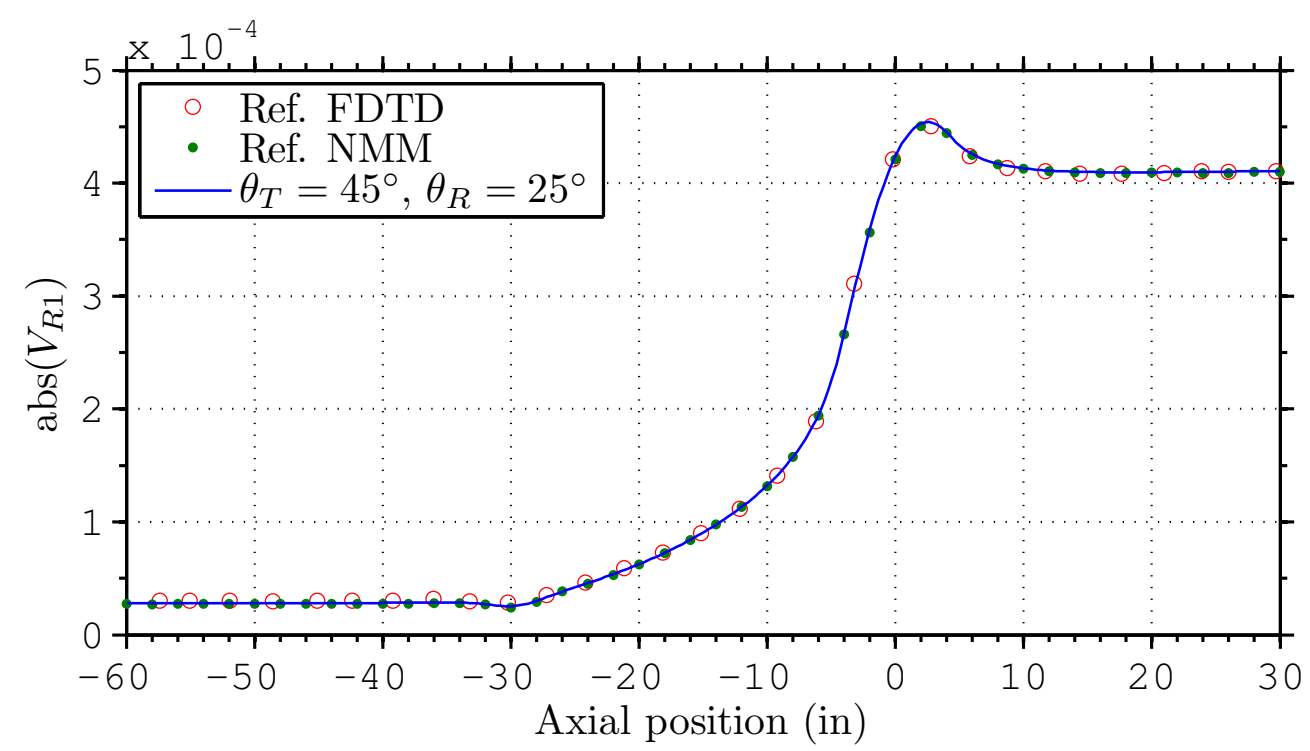

(a) Voltage amplitude

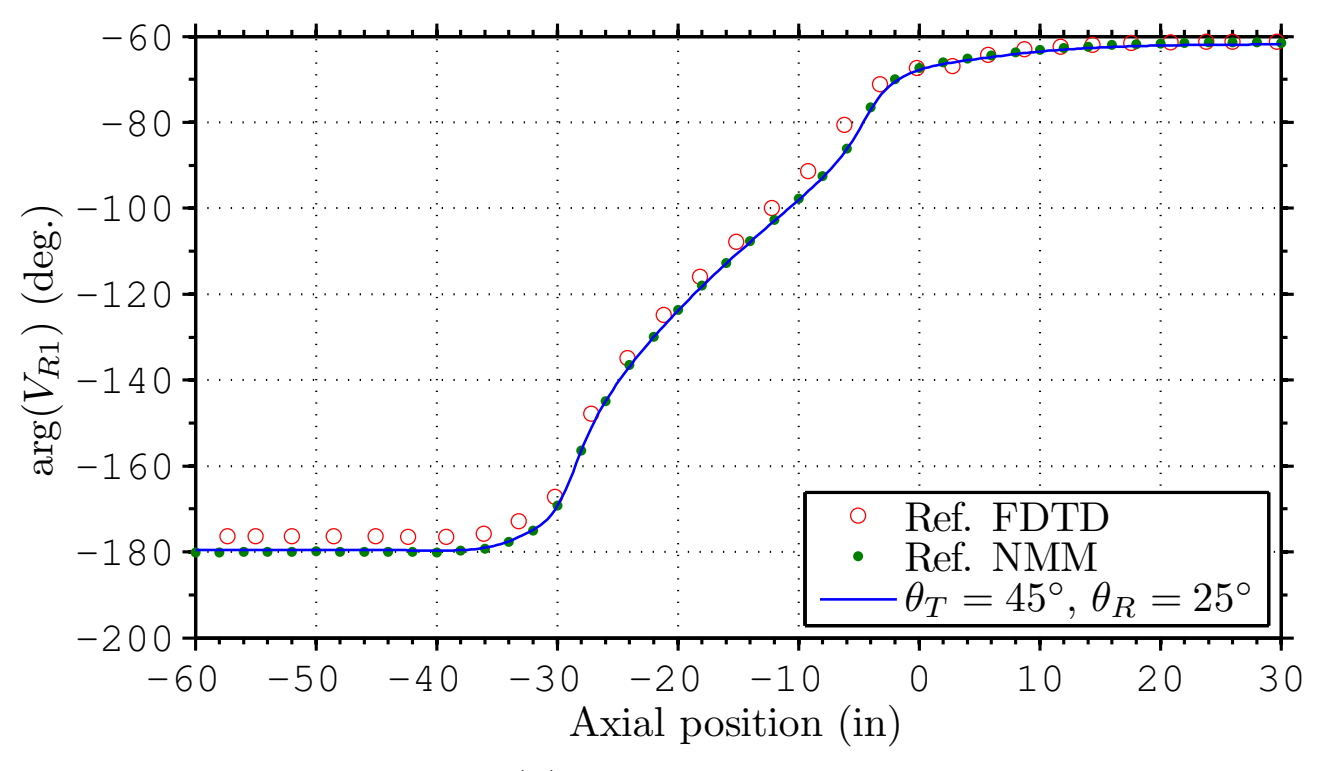

(b) Voltage phase

Figure 3.38: Voltage at TCA receiver $\mathrm{RX}_{1}$ with $\theta_{R}=25^{\circ}$ due to a unit current excitation transmitting TCA with $\theta_{T}=45^{\circ}$. The results from the present algorithm are indicated by solid lines. The small circles and dots are FDTD and R-NMM results, respectively, from [6, pp. 108-109], [8]. 


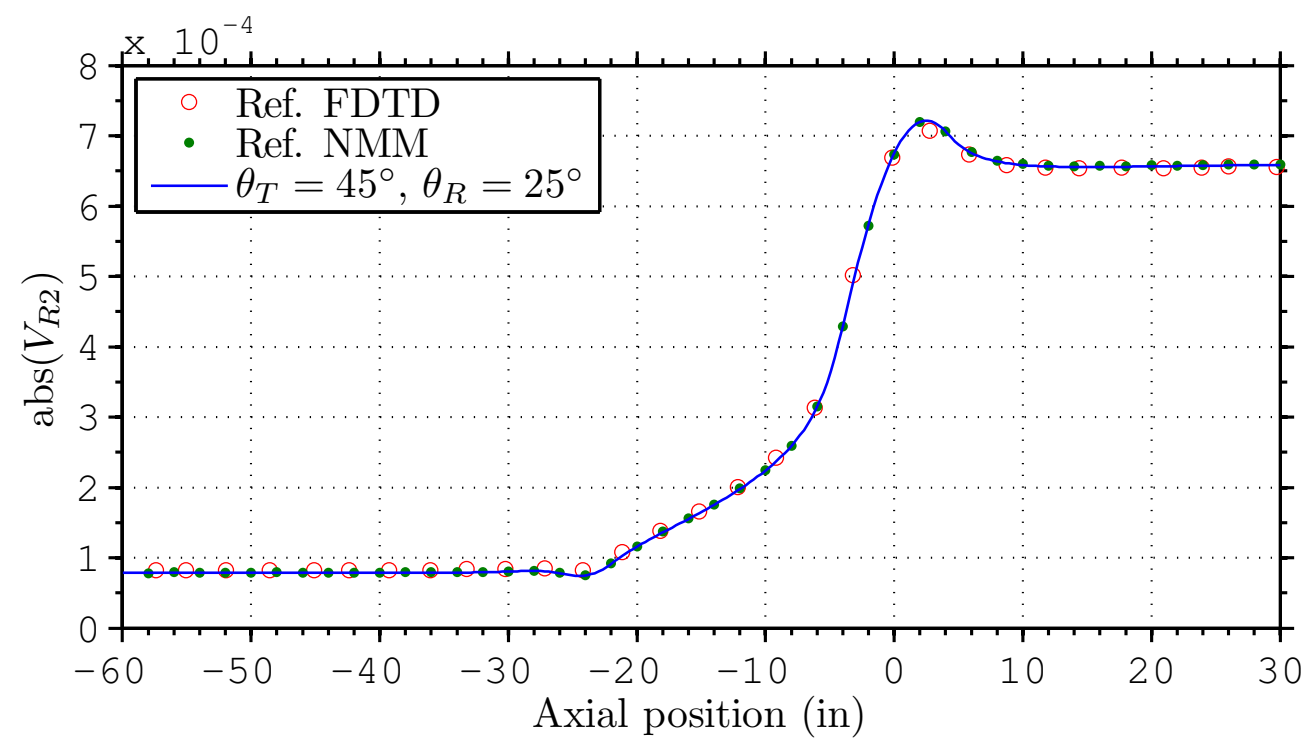

(a) Voltage amplitude

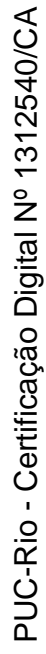

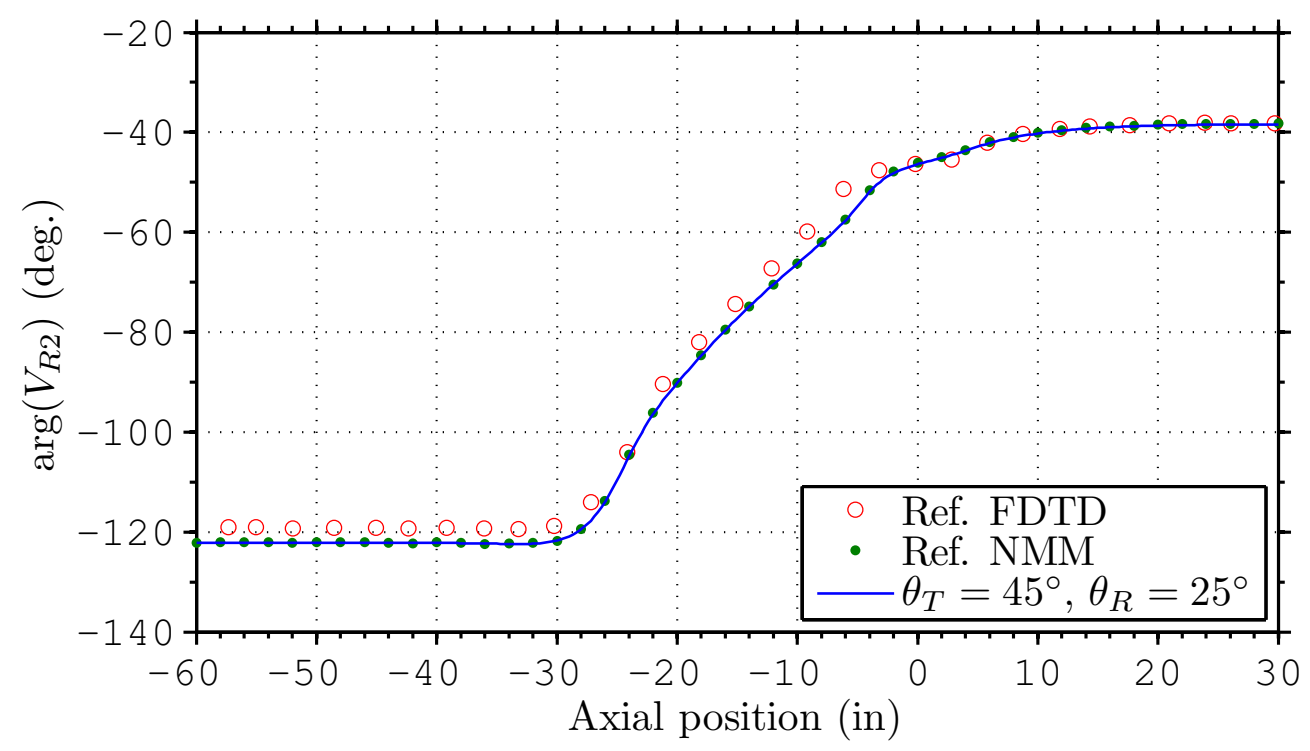

(b) Voltage phase

Figure 3.39: Voltage at TCA receiver $\mathrm{RX}_{2}$ with $\theta_{R}=25^{\circ}$ due to a unit current excitation transmitting TCA with $\theta_{T}=45^{\circ}$. The results from the present algorithm are indicated by solid lines. The small circles and dots are FDTD and R-NMM results, respectively, from [6, pp. 108-109], [8]. 


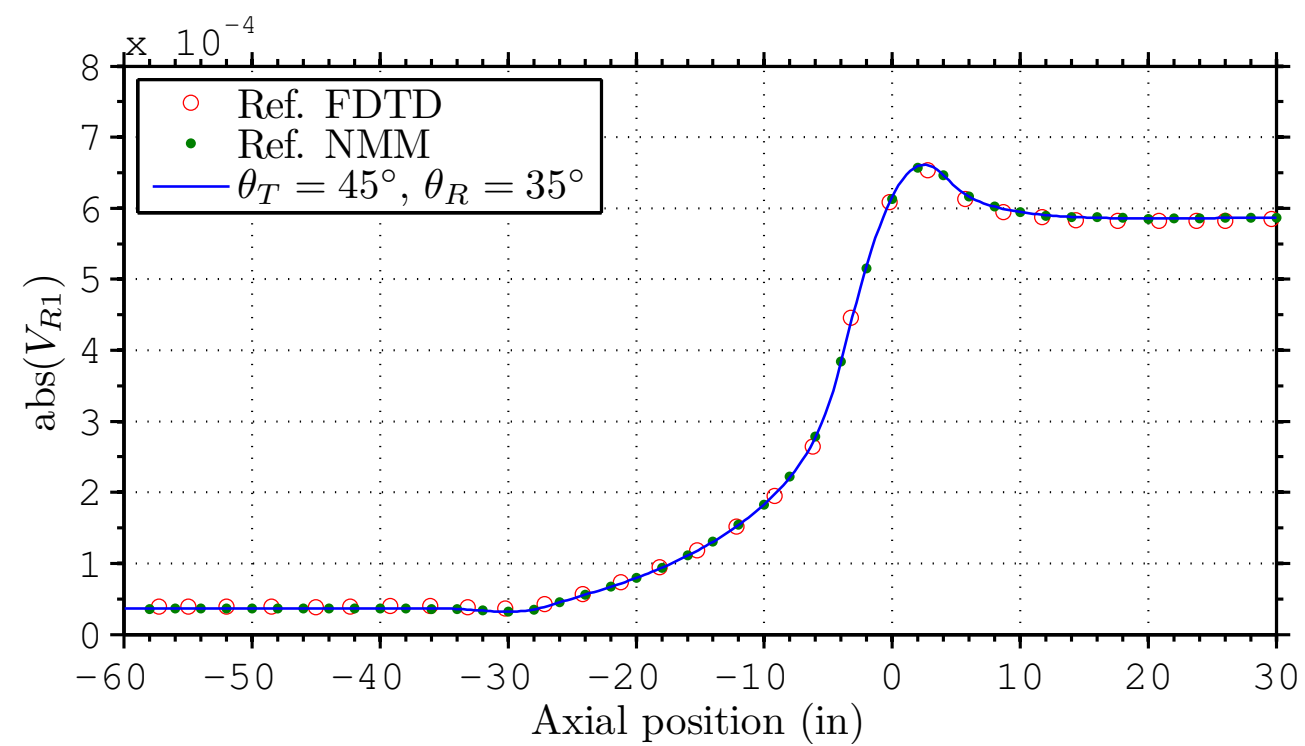

(a) Voltage amplitude

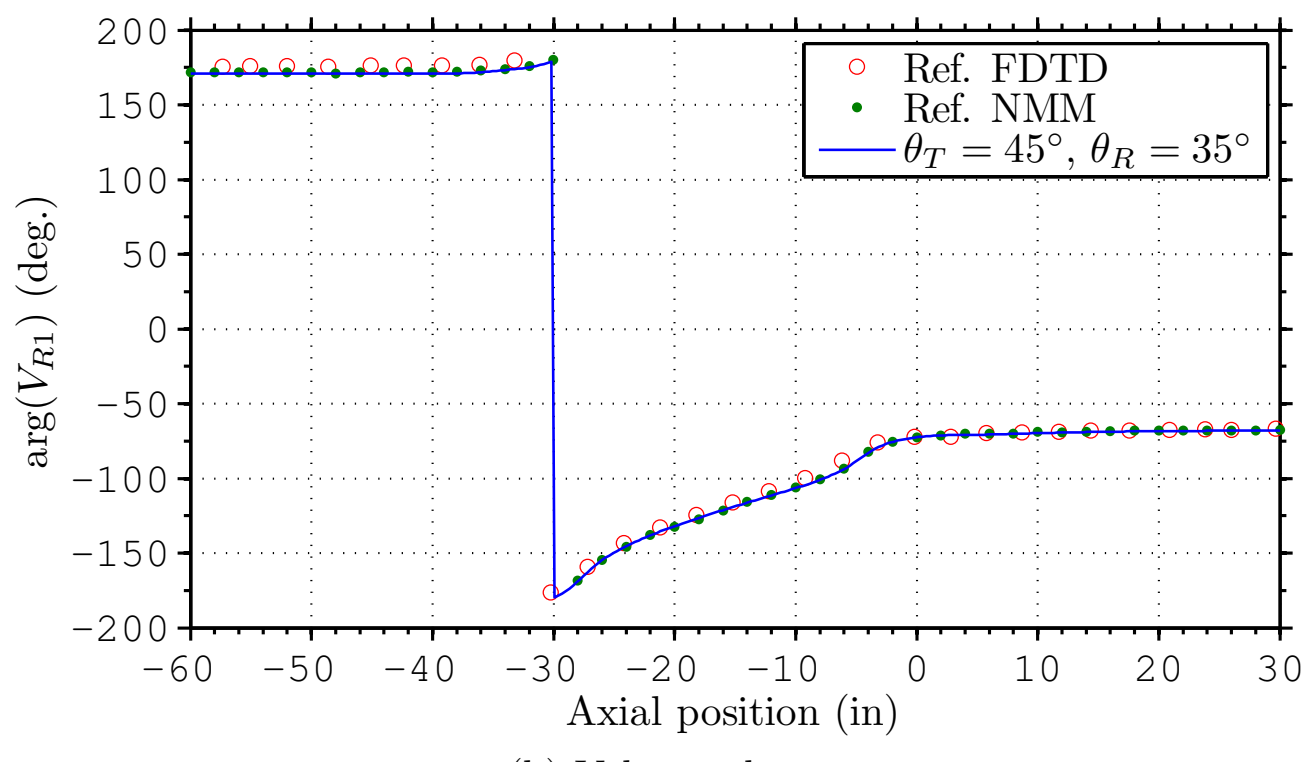

(b) Voltage phase

Figure 3.40: Voltage at TCA receiver $\mathrm{RX}_{1}$ with $\theta_{R}=35^{\circ}$ due to a unit current excitation transmitting TCA with $\theta_{T}=45^{\circ}$. The results from the present algorithm are indicated by solid lines. The small circles and dots are FDTD and R-NMM results, respectively, from [6, pp. 108-109], [8]. 


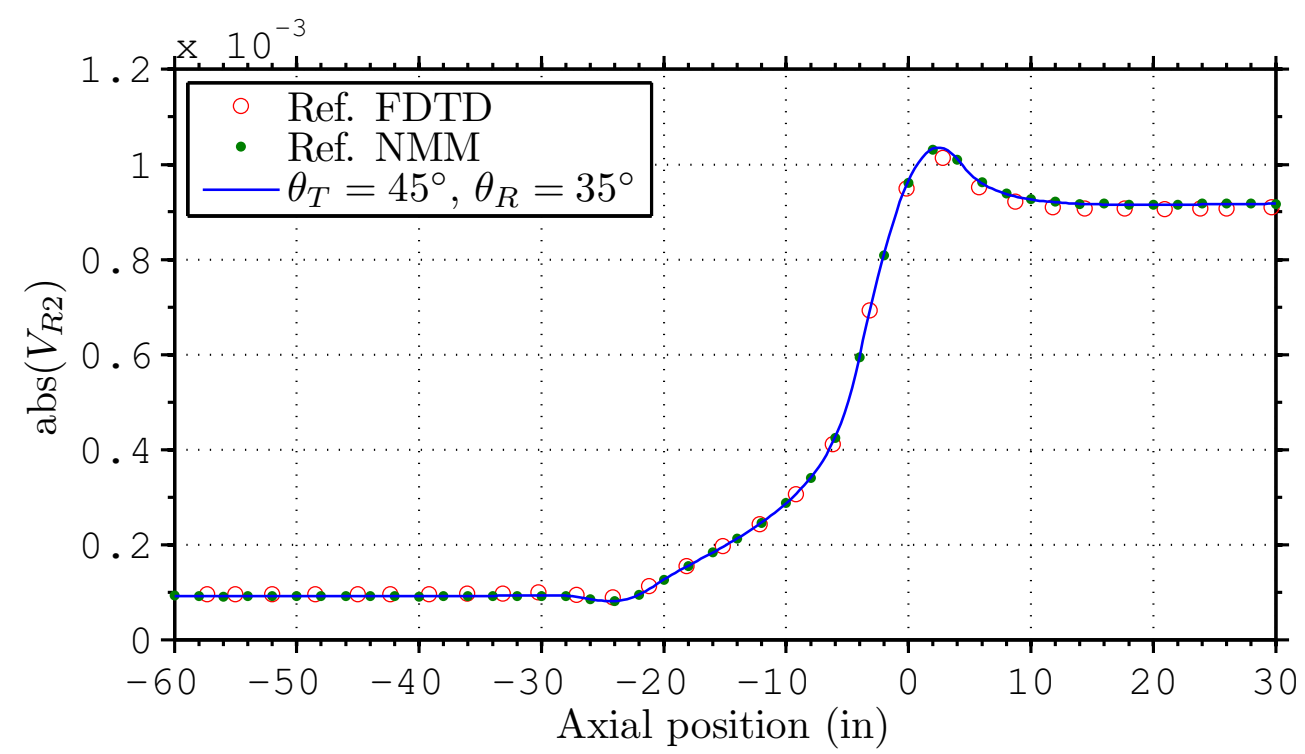

(a) Voltage amplitude

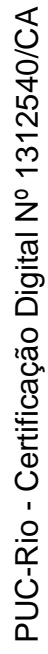

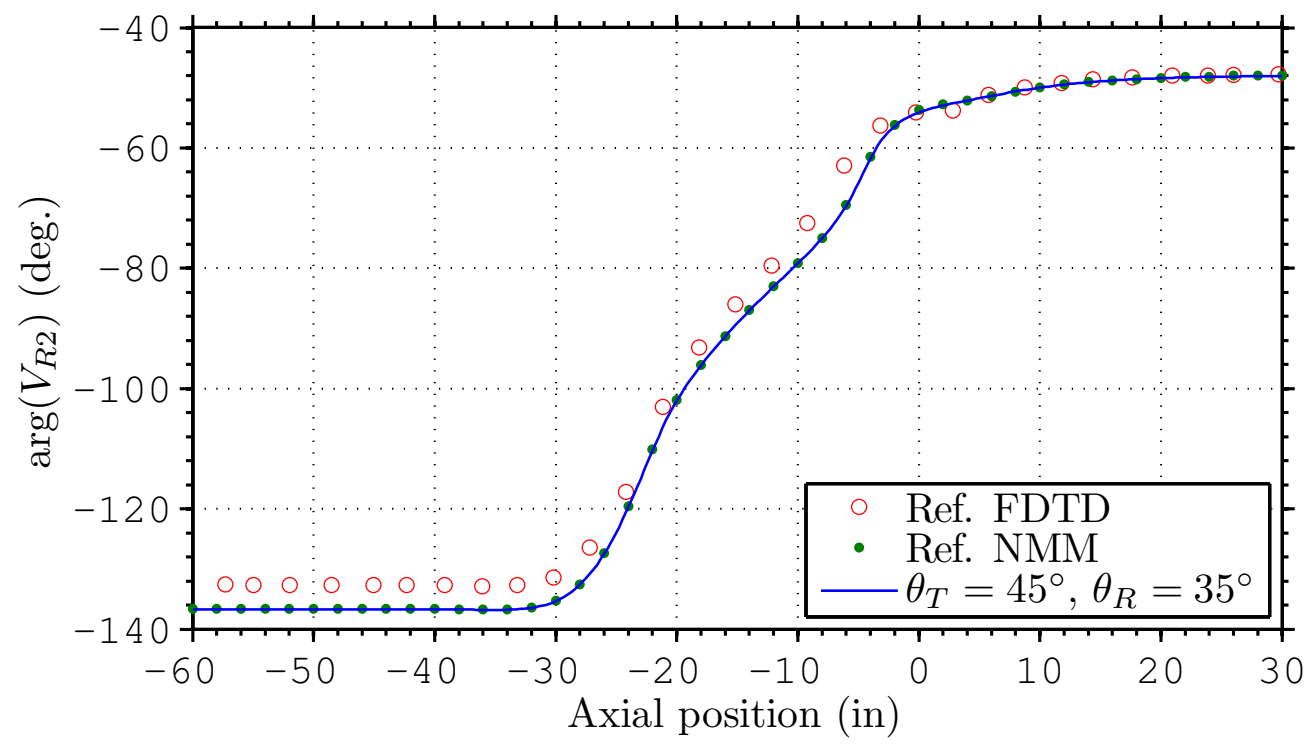

(b) Voltage phase

Figure 3.41: Voltage at TCA receiver $\mathrm{RX}_{2}$ with $\theta_{R}=35^{\circ}$ due to a unit current excitation transmitting TCA with $\theta_{T}=45^{\circ}$. The results from the present algorithm are indicated by solid lines. The small circles and dots are FDTD and R-NMM results, respectively, from [6, pp. 108-109], [8]. 


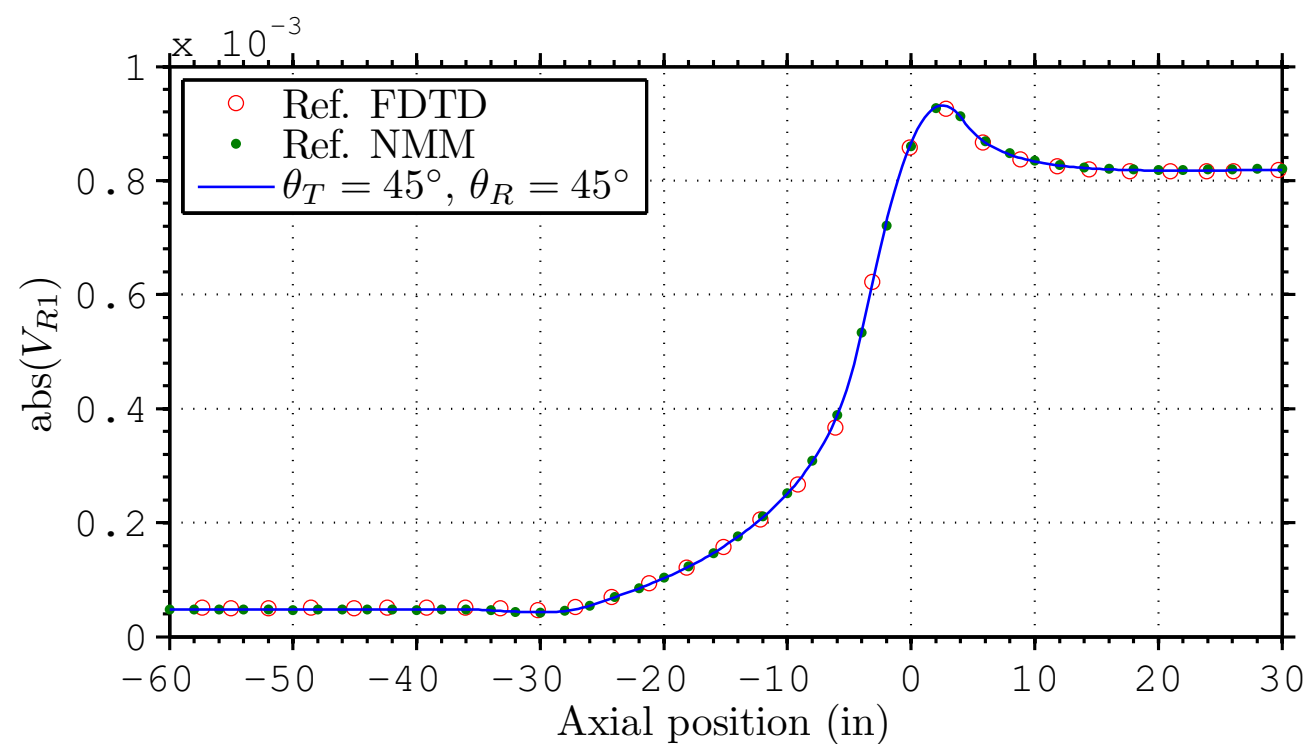

(a) Voltage amplitude

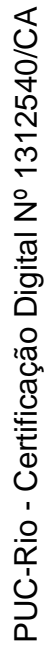

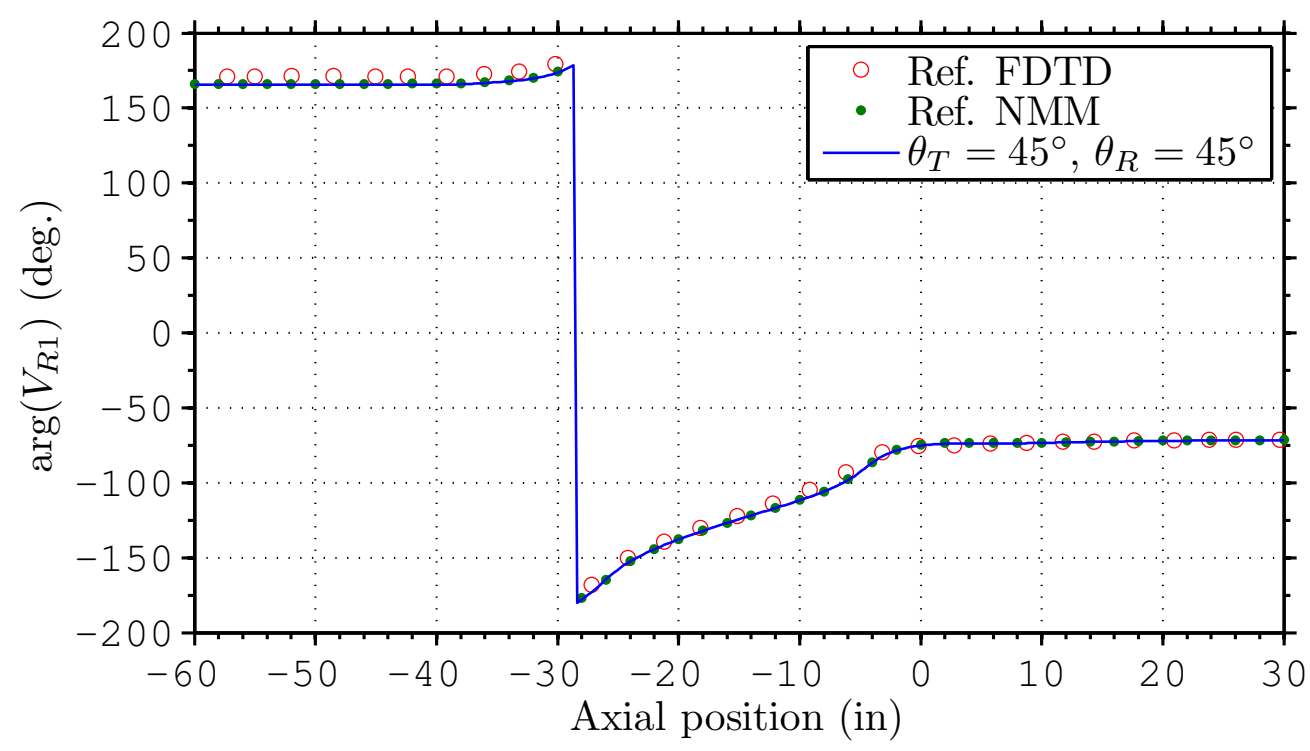

(b) Voltage phase

Figure 3.42: Voltage at TCA receiver $\mathrm{RX}_{1}$ with $\theta_{R}=45^{\circ}$ due to a unit current excitation transmitting TCA with $\theta_{T}=45^{\circ}$. The results from the present algorithm are indicated by solid lines. The small circles and dots are FDTD and R-NMM results, respectively, from [6, pp. 108-109], [8]. 


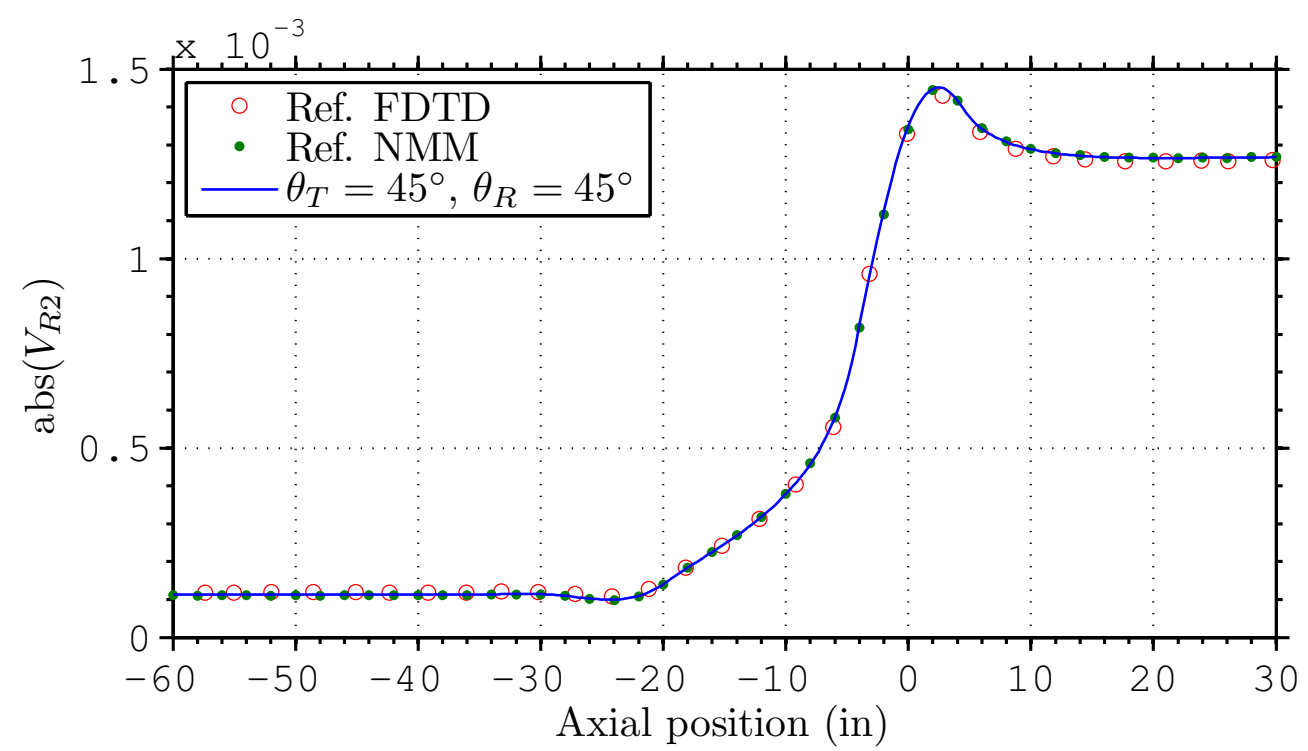

(a) Voltage amplitude

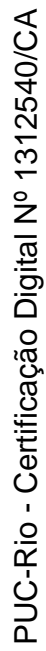

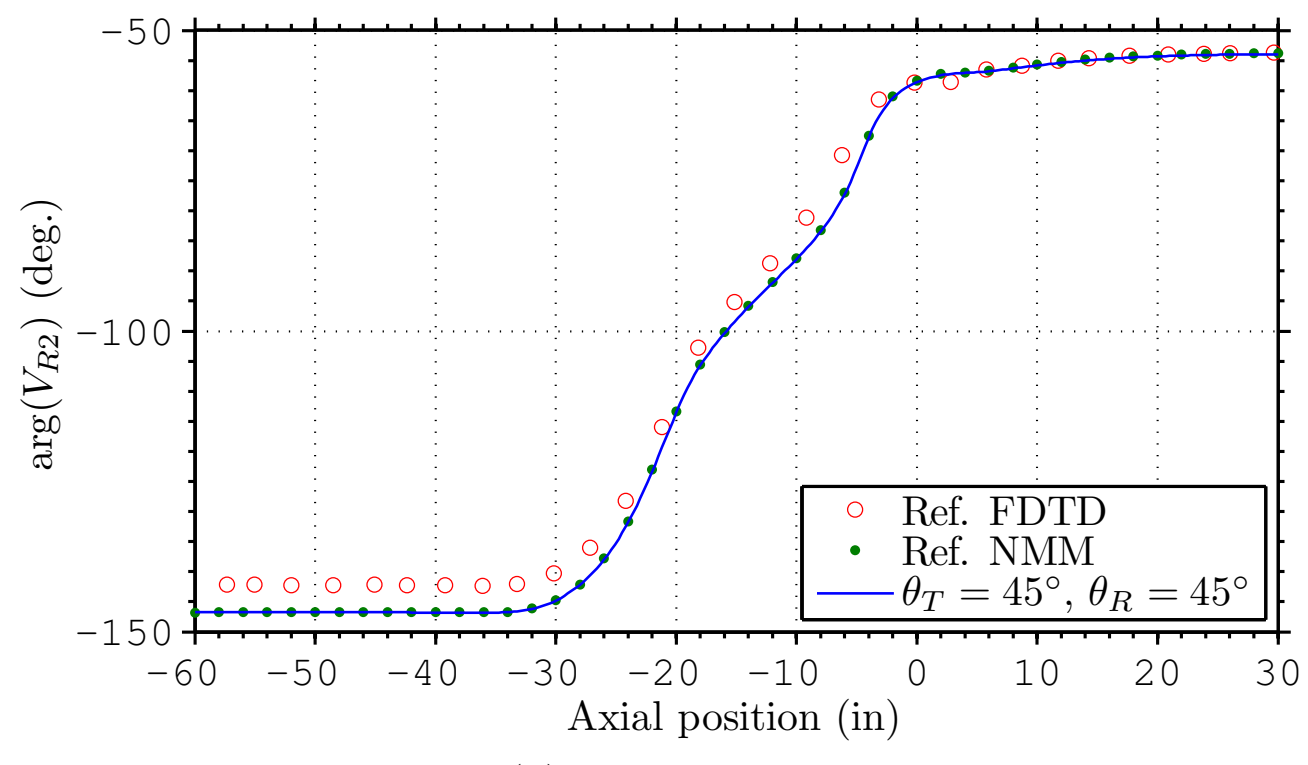

(b) Voltage phase

Figure 3.43: Voltage at TCA receiver $\mathrm{RX}_{2}$ with $\theta_{R}=45^{\circ}$ due to a unit current excitation transmitting TCA with $\theta_{T}=45^{\circ}$. The results from the present algorithm are indicated by solid lines. The small circles and dots are FDTD and R-NMM results, respectively, from [6, pp. 108-109], [8]. 

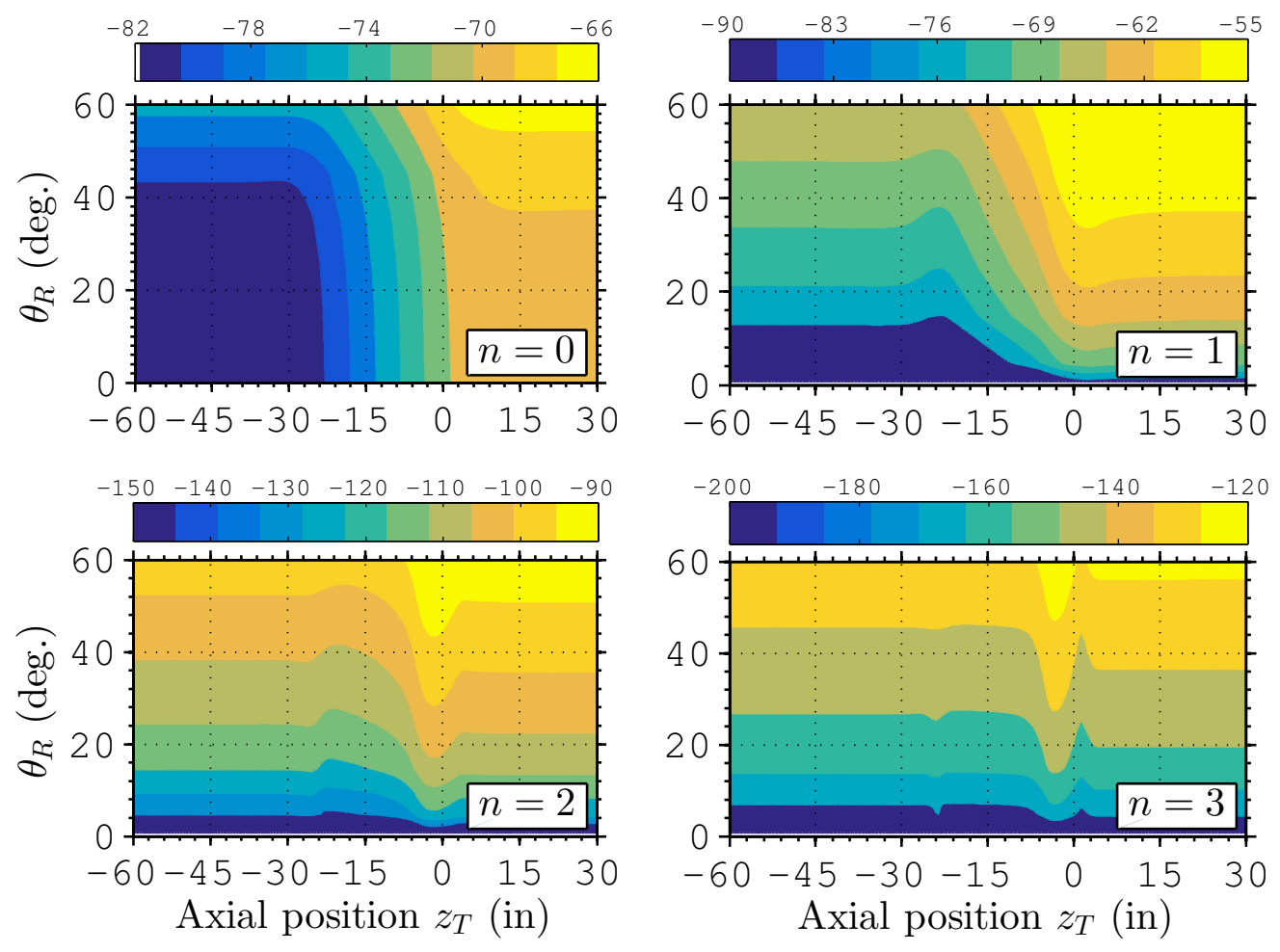

Figure 3.44: Voltage amplitude in decibel, $20 \log _{10}\left(\operatorname{abs}\left(V_{R 2}\right)\right)$, at TCA receiver $\mathrm{RX}_{2}$ due a transmitter TCA with tilt angle of $45^{\circ}$. The contribution of the azimuthal harmonics $n=0,1,2$ and 3 are depicted. 


\section{8}

\section{Comparison of the Proposed Method Versus the R-NMM}

For many years, LWD problems where efficiently solved using a 1D-FE discretization along each horizontal layer of a cylindrical stratified medium $[10,24,26,31]$, and then, in the vertical direction, a mode-matching procedure is used to match the fields between each horizontal layer. As said before, this approach is denoted as axial-NMM (A-NMM).

A typical LWD tool moves with the downhole drilling so that, at each new tool position along the well, it is necessary to recalculate several matrices incorporating the reflections from the horizontal layers above and below the source and the receiving antennas (these recursive calculations are presented in Appendix B). This is a drawback of the A-NMM-based, as pointed out in [46]. In $[6,8,27,28,46]$, an alternative NMM approach was proposed to circumvent this issue in LWD applications. As both transmitting and receiving LWD antennas are placed around a metallic mandrel inside the borehole and typically have the same radius, they are located in the same radial layer regardless of the the axial position of the tool in the logging well. Therefore, it becomes more efficient to first perform a mode expansion of the fields along vertical direction and then a mode-matching along each radial layer. According to previous statements, this approach is denoted as radial-NMM (R-NMM).

Recently, an improved R-NMM technique was proposed in [9]. In former R-NMM formulations, the vertical dependence of electromagnetic fields where expanded by using sinusoidal [27] and quadratic B-splines [8] basis functions to represent vertical eigenmodes. The accuracy of second order B-splines versus others not curl-conforming basis where proved in [6, pp. 88-90]. Motivated by this, the authors of [9] have investigated the use of cubic B-splines local basis functions because its high order nature as well as its ability to handle with multiple knots. The novelties introduced in [9] have allowed a R-NMM approach that can improve the numerical efficiency of the traditional NMM algorithms reported so far. In this section, numerical simulations for LWD sensors in typical geophysical environments are presented to compare some aspects of the improved R-NMM versus the proposed method. The R-NMM results and simulation details were obtained by personal communication with the authors of [9].

We consider a triaxial well-logging tool consisting of one transmitter and two receivers coil antennas in a vertical-well borehole traversing different Earth formations. In all cases, the axial positions of the transmitter TX, and receivers $\mathrm{RX}_{2}$ and $\mathrm{RX} \mathrm{X}_{1}$ are $z_{T}, z_{T}+24$ in and $z_{T}+30$ in, respectively, where 1 in $=2.54 \times 10^{-2} \mathrm{~m}$. The antennas are 4.5 -in-radius coils and are wrapped 


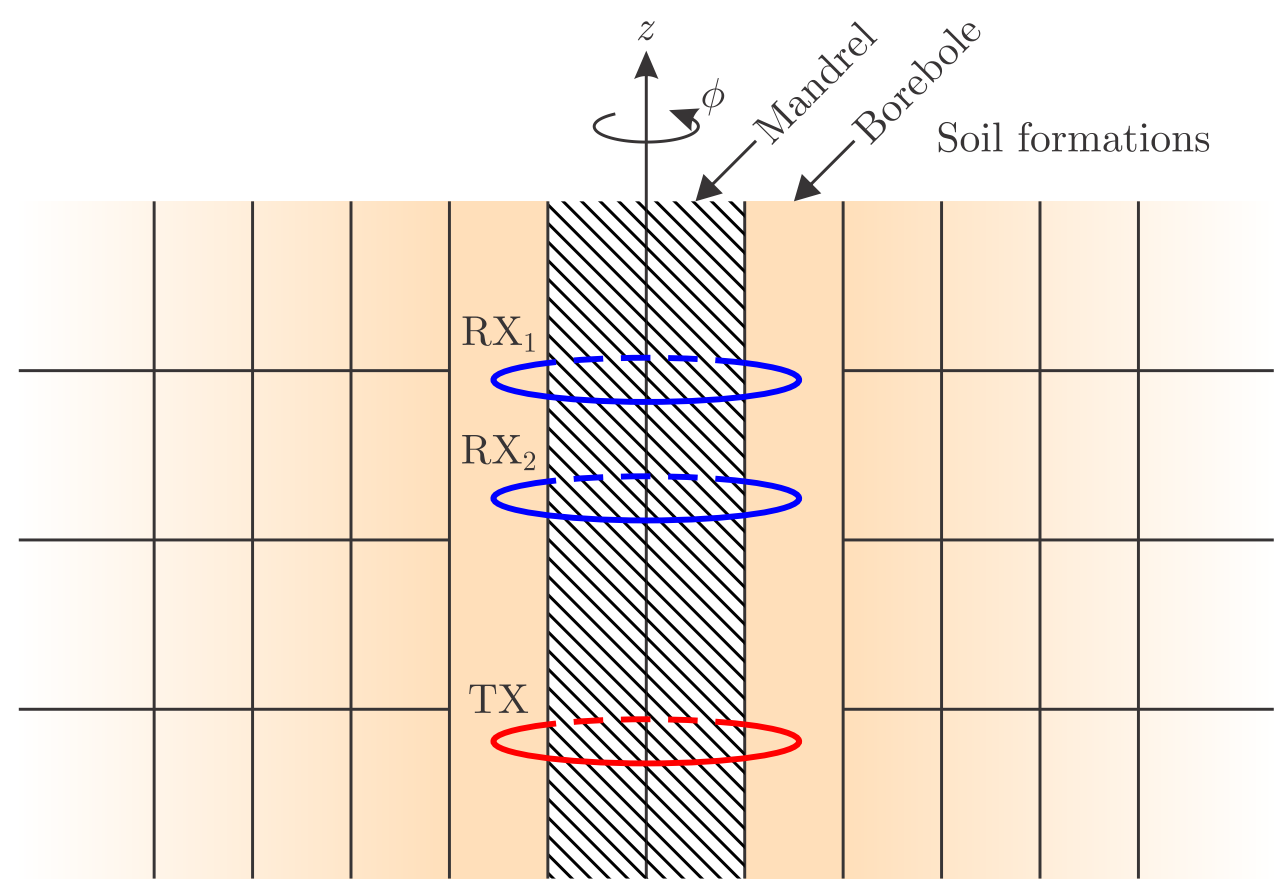

Figure 3.45: Geometry of a triaxial LWD tool (antennas TX, $\mathrm{RX}_{1}$ and $\mathrm{RX}_{2}$ ) within a stratified geophysical formation.

around a 4-in-radius metallic mandrel and inside a 5-in-radius borehole, as depicted in Fig. 3.45. We assume a perfect electric conductor (PEC) media on the metallic mandrel. A perfectly matched layer (PML) was used to truncate the axial and radial domain for the R-NMM [9] and the proposed formulations, respectively, where the back-PML media is also assumed as a PEC.

We consider in all cases that the borehole is filled with an oil-based mud having conductivity $(\sigma)$ equal to $0.0005 \mathrm{~S} / \mathrm{m}$, and to explore the ability of the basis functions proposed in [9] to correctly represent fields along the stratifications, we consider a high contrast of $\sigma$ between borehole and the soil formations. In addition, only non-magnetic media are considered, and the real part of the complex permittivity is assumed equal to $8.85 \times 10^{-12} \mathrm{~F} / \mathrm{m}$, i.e., the vacuum permittivity. These assumptions are typical for modeling lowfrequency LWD tools.

\subsection{1}

\section{Case 1: Radial Invasion Zone}

We first consider the particular 2D configuration described in Fig. 3.46, where the soil formation (with $\sigma=5 \mathrm{~S} / \mathrm{m}$ ) is assumed axially invariant, but the low porosity of the media surrounding the borehole creates an invasion zone with thickness $d$. For geophysical prospection applications, the output parameters of interest are not directly the voltages at $\mathrm{RX}_{2}$ and $\mathrm{RX}_{1}$, but instead the amplitude ratio (AR) and phase difference $(\mathrm{PD})$ between these 


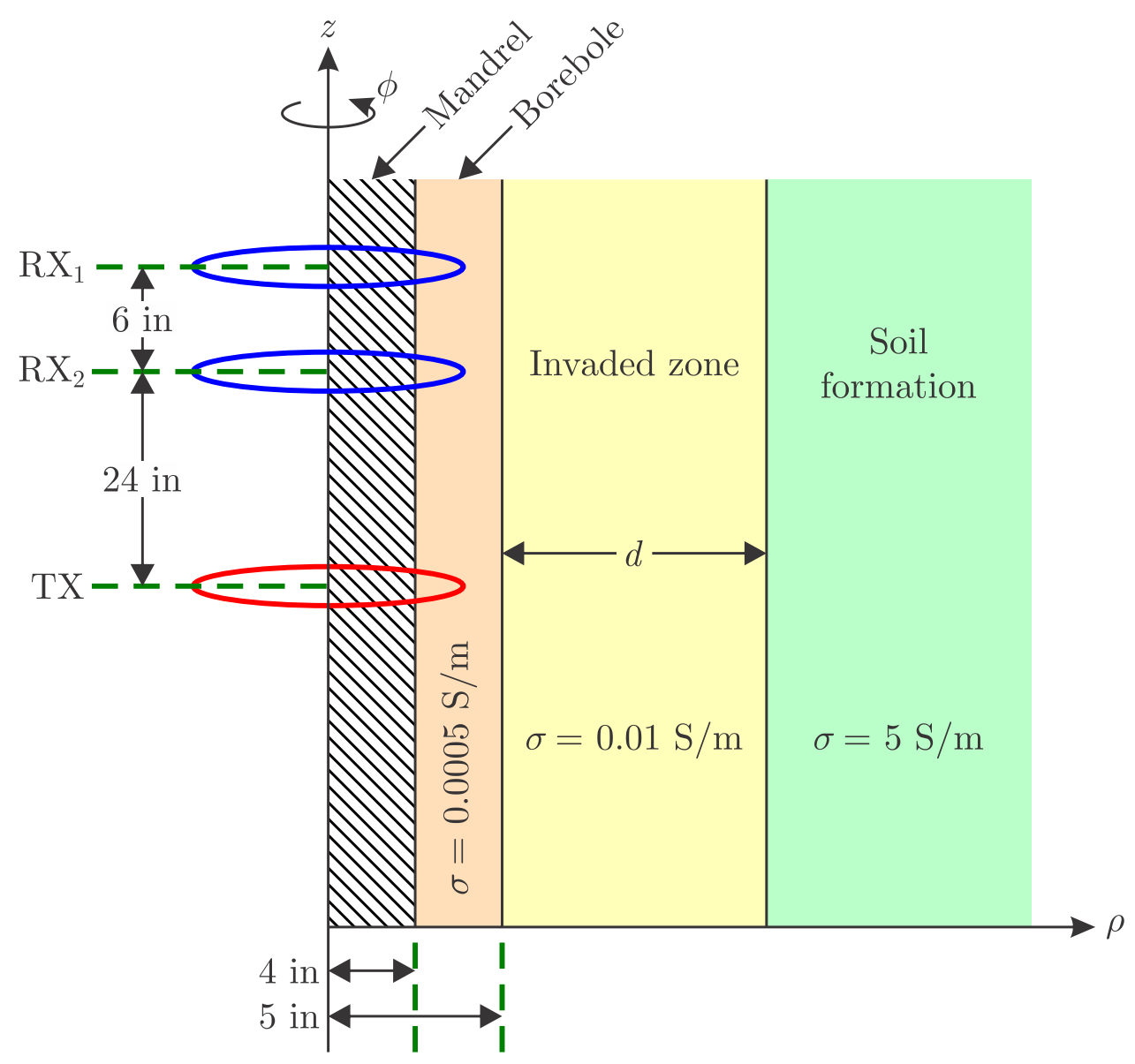

Figure 3.46: Geometry of a triaxial well-logging tool within the geophysical formation for the case 1 .

voltages. Fig. 3.47 shows $\mathrm{AR}$ and $\mathrm{PD}$ results as a function of the invasion thickness $d$, due to an excitation antenna TX operating at $2 \mathrm{MHz}$. The RNMM results are in good agreement with those from the presented method, with a relative error no larger than $0.5 \%$ over the range of investigation. The $\mathrm{R}-$ NMM's CPU time required for each configuration of $d$ was about 517 seconds to account 180 modes using a double-precision Fortran code running on an ordinary laptop. For comparison, our algorithm required about 31 seconds using 74 modes.

\subsection{2}

\section{Case 2: Vertical Bed Layer}

As a second case, we consider now the particular 3D configuration described in Fig. 3.48, where the LWD tool is traversing a three-layer formation with conductivities $5 \mathrm{~S} / \mathrm{m}, 0.01 \mathrm{~S} / \mathrm{m}$ and $5 \mathrm{~S} / \mathrm{m}$. Notice that the conductivity profile can vary by a factor of $10^{4}$ times between the borehole and formations 1 and 3. Fig. 3.49 shows AR and PD results for several thicknesses $h$ in formation 2 as a function of the axial position of the transmitting $z_{T}$; that is again 


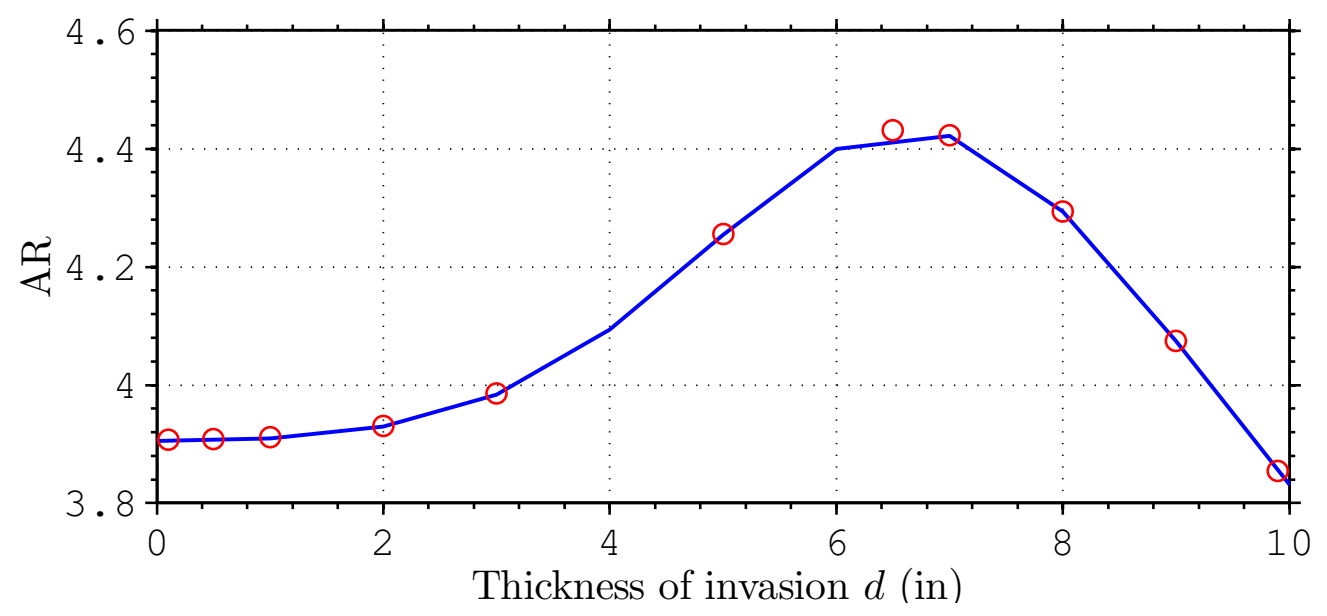

(a) Voltage amplitude ratio

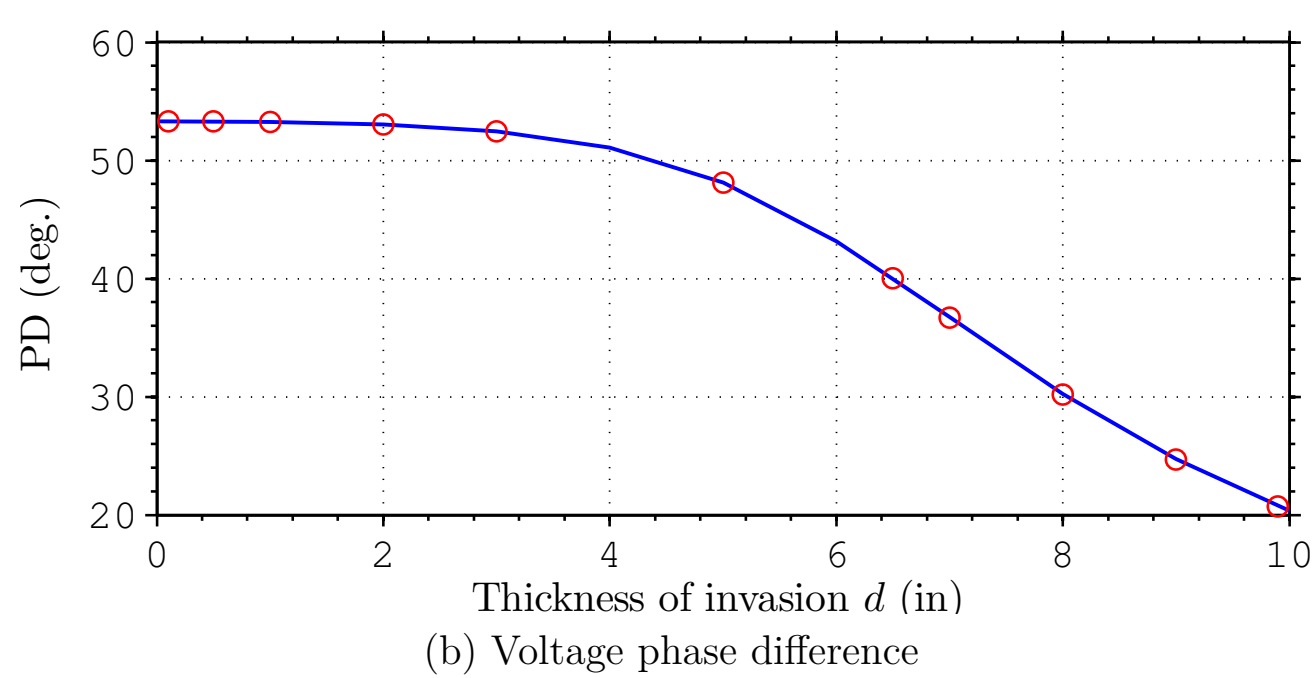

Figure 3.47: Voltage amplitude ratio and phase difference for the triaxial LWD tool depicted in case 1 (see Fig. 3.46) as a function of the invasion thickness $d$. The results from our (A-NMM-based) algorithm and from the R-NMM [9] are indicated by solid lines and small circles, respectively.

operating at $2 \mathrm{MHz}$. The R-NMM and A-NMM results are in agreement, with a relative error no larger than $1 \%$ over the range of investigation. The RNMM's CPU time required for each configuration of $h$ was about 28 minutes to account 230 modes. The A-NMM algorithm, on other hand, required no more than 49 seconds using 73 modes.

\subsection{3}

\section{Case 3: Complex Soil Formation}

Finally, we now consider a more complex soil formation composed by four vertical layers and an intricate invasion zone, whose 3D configuration is depicted in Fig. 3.50. Fig. 3.51 shows AR and PD results for a triaxial LWD tool traversing the bed layers as a function of the axial position of the transmitting 


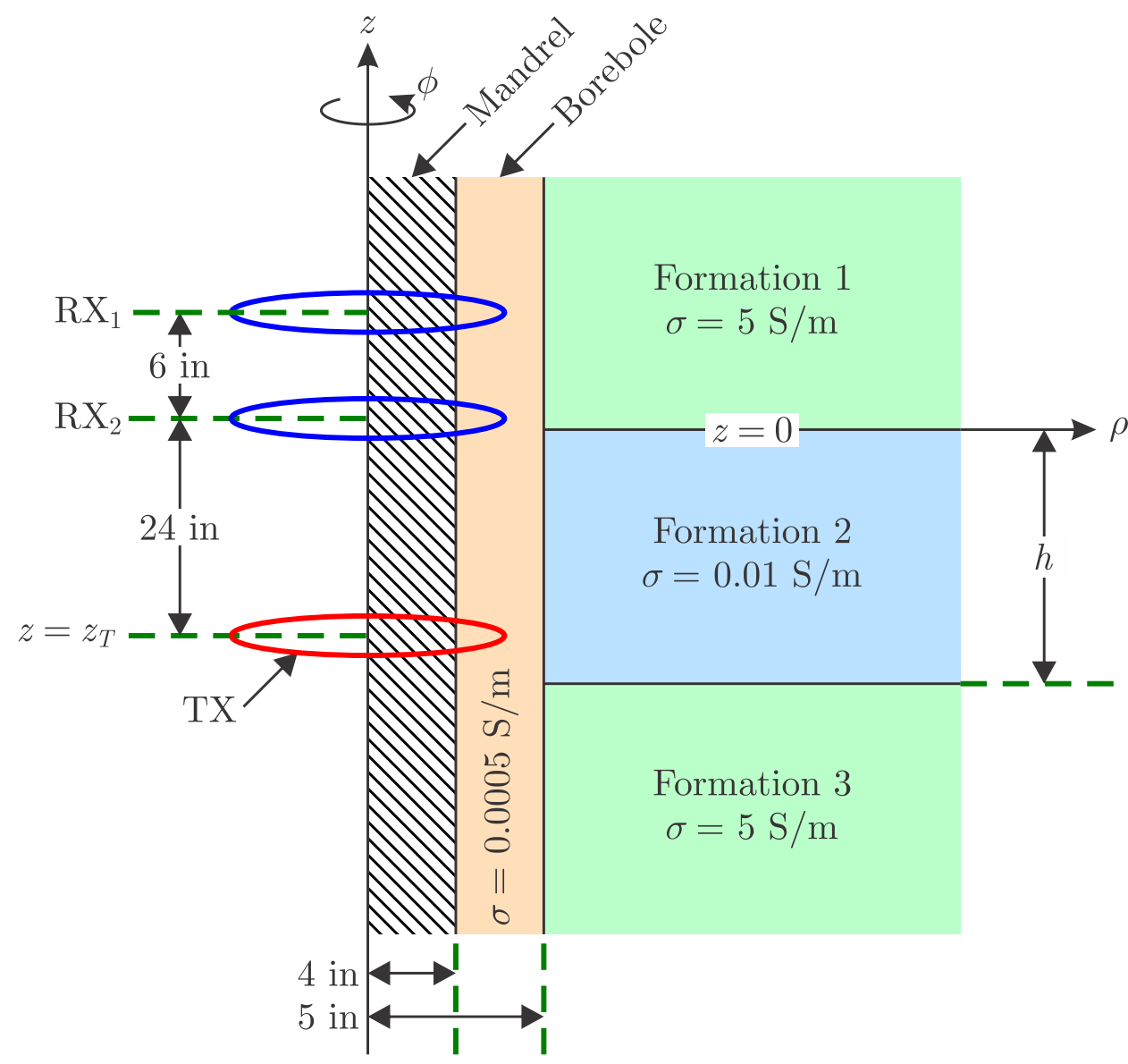

Figure 3.48: Geometry of a triaxial well-logging tool within the geophysical formation for the case 2 .

antenna (viz. $z_{T}$ ) for three typical operating frequencies: $100 \mathrm{kHz}, 500 \mathrm{kHz}$ and $2 \mathrm{MHz}$. The R-NMM and A-NMM results are in very good conformity, with a relative error no larger than $0.3 \%$. The R-NMM's CPU time required for each frequency simulations was about 17 minutes to account 212 modes. The A-NMM algorithm required no more than 114 seconds using 60 modes.

From results in Fig. 3.51 become clear that the resolution of the LWD tool operating at $2 \mathrm{MHz}$ is improved compared to the others analyzed low frequency configurations.

\subsection{4}

\section{Comparison Summary}

In this section, we showed numerical results for LWD tools responses with a restricted computational effort via NMM-based formulations. Typically, an ordinary R-NMM simulation required about $1 / 4$ of the CPU time of an FDTD simulation [8]. In [9], it was verified that the multiplicity of knots in cubic Bsplines basis functions can reduces the domain discretization in about $30 \%$ compared to the ordinary R-NMM for typical LWD applications. In addition, 


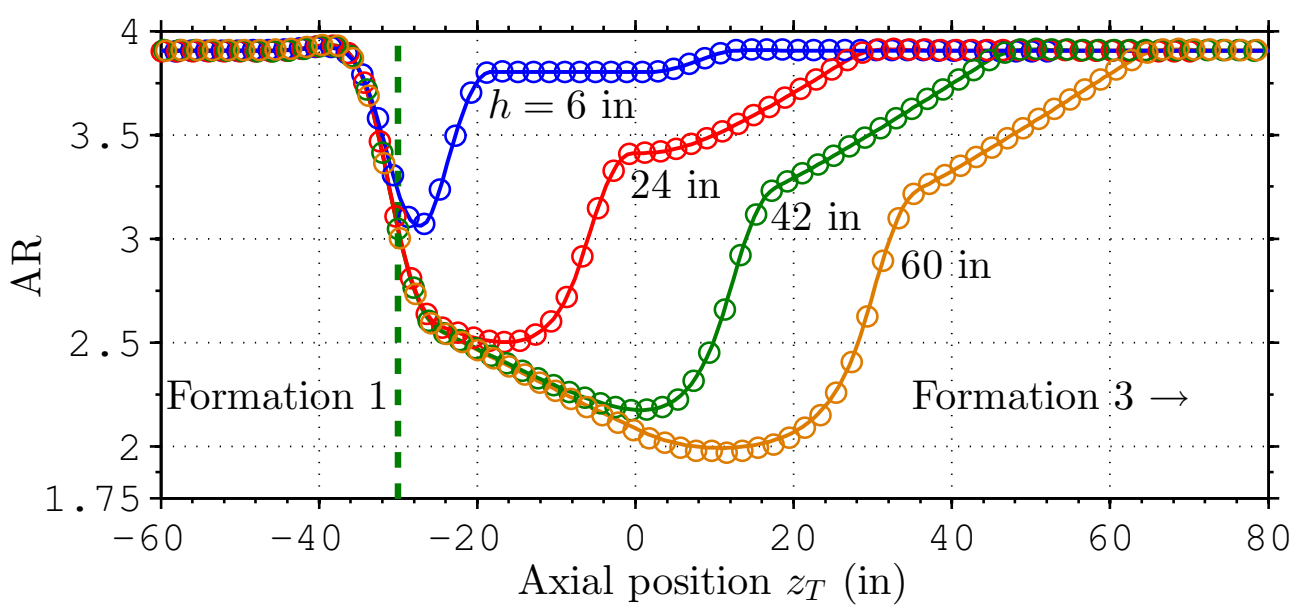

(a) Voltage amplitude ratio

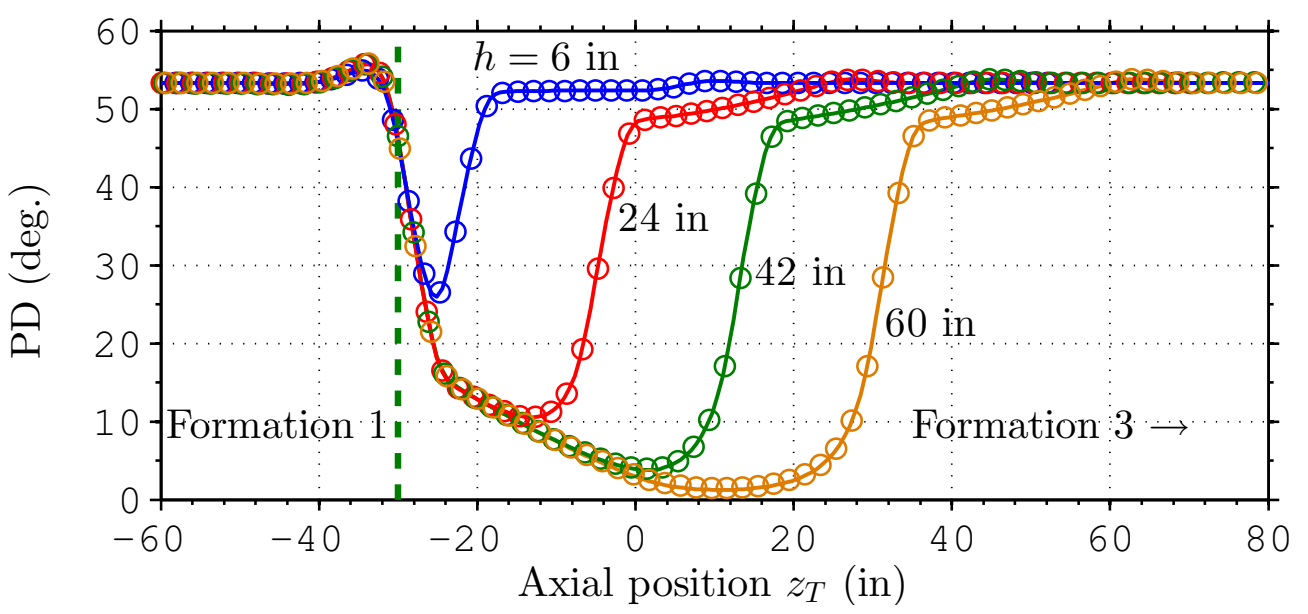

(b) Voltage phase difference

Figure 3.49: Voltage amplitude ratio and phase difference for a triaxial LWD tool traversing the bed layers depicted in case 2 (see Fig. 3.48) for several bed layer thickness $h$. The results from our (A-NMM-based) algorithm and from the R-NMM [9] are indicated by solid lines and small circles, respectively.

the A-NMM-based formulation described in the chapter required just a fraction of that simulation time.

It is important to observe that the problem scenarios we have consider here present high conductivity contrasts such the problem was modeled as a layered cylindrical structure. For others scenarios in which the soil formation profile changes smoothly in vertical direction, the fields can be more naturally represented by using the R-NMM associated with cubic B-splines basis functions. 


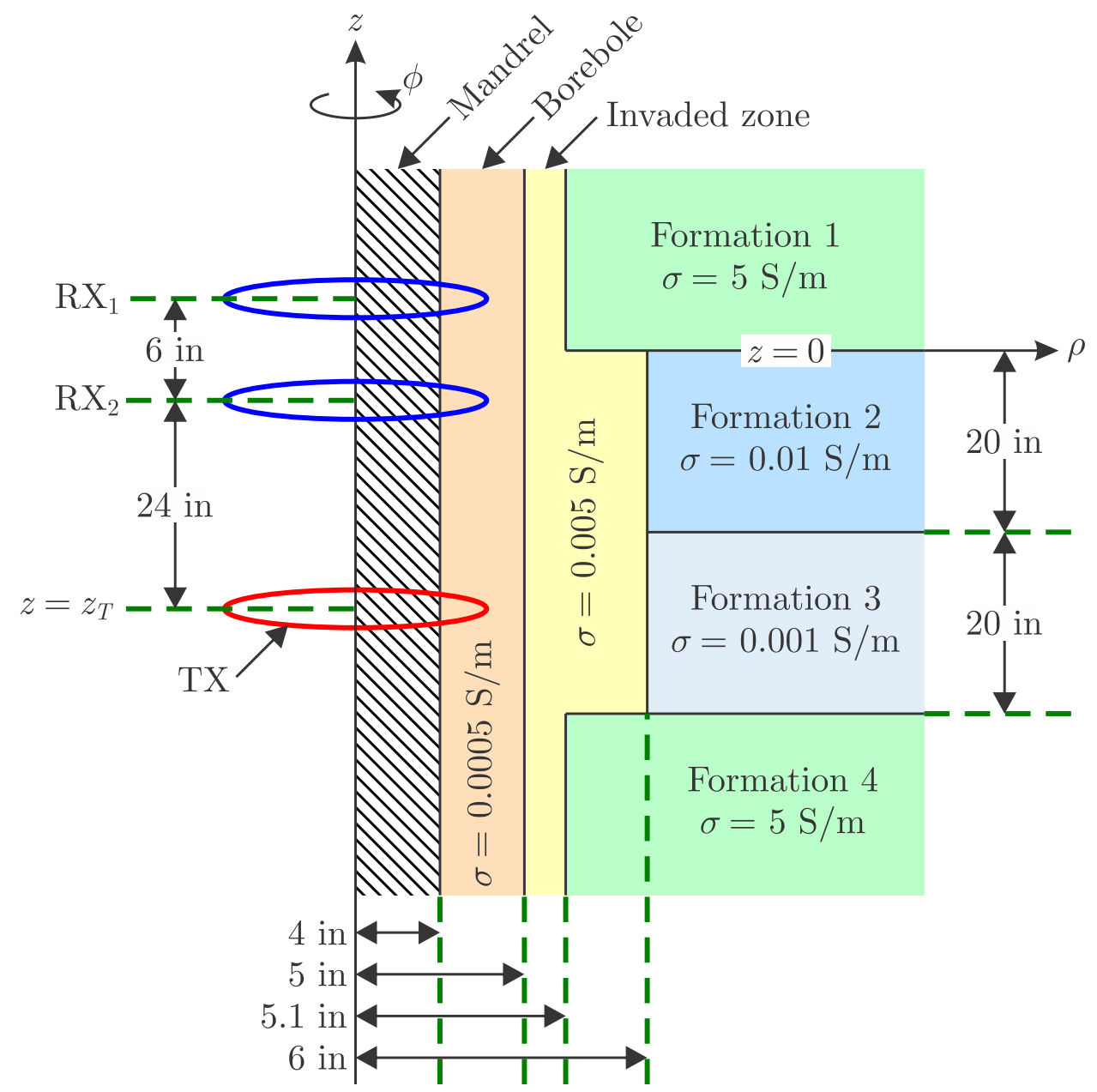

Figure 3.50: Geometry of a triaxial well-logging tool within the geophysical formation for the case 3 . 


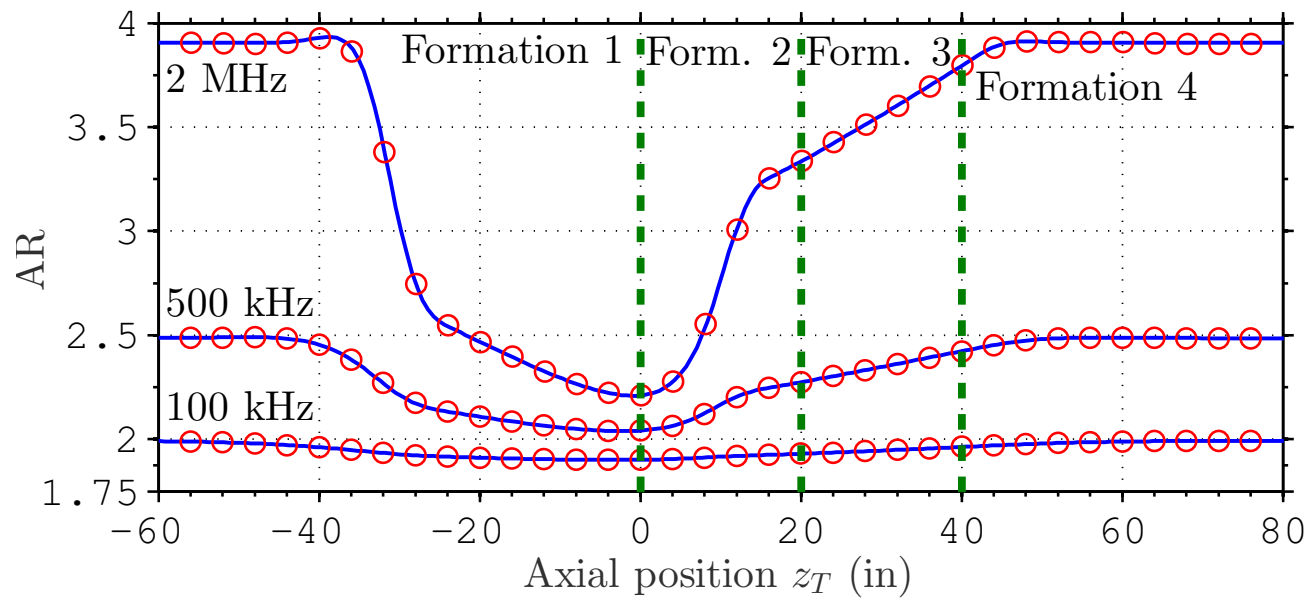

(a) Voltage amplitude ratio

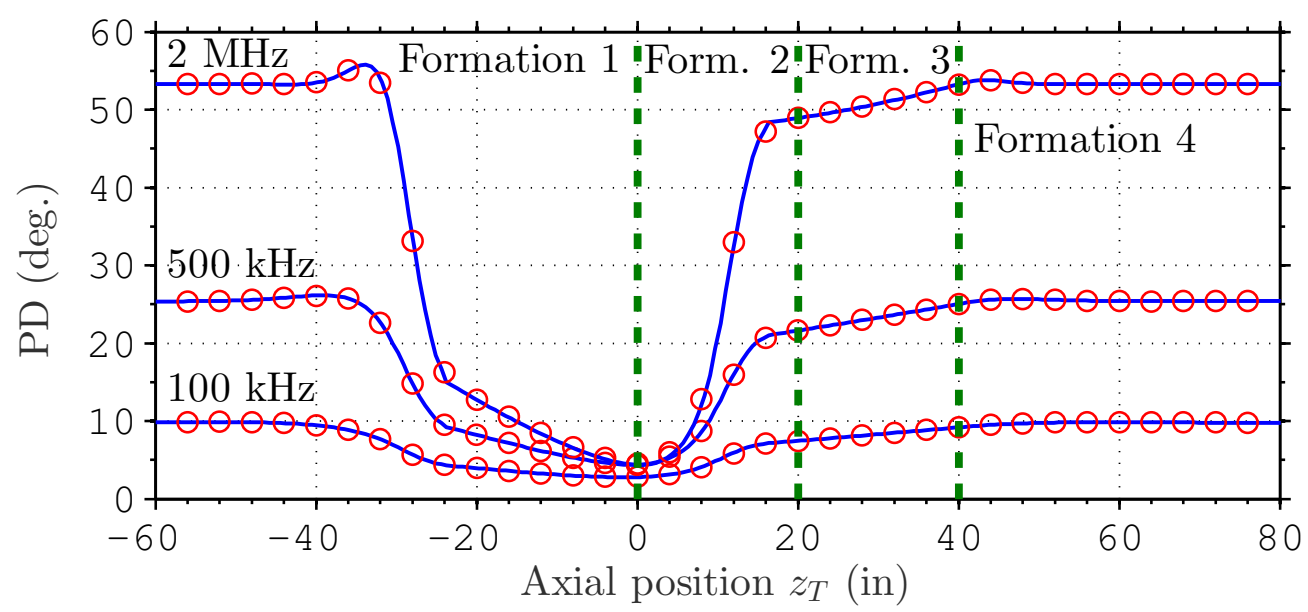

(b) Voltage phase difference

Figure 3.51: Voltage amplitude ratio and phase difference for a triaxial LWD tool traversing the bed layers depicted in case 3 (see Fig. 3.50). The results from our (A-NMM-based) algorithm and from the R-NMM [9] are indicated by solid lines and small circles, respectively. 


\section{9 Modeling of LWD Sensors Inside Mandrel Grooves}

As previously mentioned in Chapter 2, to grantee the drill bit lubrication, we need to wash out the drilling cuttings. To maximize the flow of the mud and cuttings back to the surface, some LWD tools employ wire loop antennas placed inside grooves around the metallic mandrel. The numerical simulation of this kind of structure are not commonly encountered in the literature due the some complications in its modeling. Both R-NMM and A-NMM approach can not be easily generalized to modeling sources inside such indentations due some enforcements of the NMM. In practice, however, we can use a tool calibration as a reference to proper approximate the real-world LWD tool response, as described in [34]. To be more clear, in geophysical prospecting using LWD tools the parameters of interest are the amplitude ratio (AR) and phase difference $(\mathrm{PD})$ between the voltages at two antennas $[6,34]$ :

$$
A R=20 \log _{10}\left(V_{R 2} / V_{R 1}\right)
$$

$$
P D=\arg \left(V_{R 2}\right)-\arg \left(v_{R 1}\right) .
$$

The individual value of the antenna voltages are not of (direct) interest, but instead its values compared to a reference; i.e., the voltage $V_{R 2}$ in relation to $V_{R 1}$. Experimental analysis of resistivity logging-sensors have showed that we can consider numerical simulations of LWD tools with antennas placed around the mandrel (as in the examples showed in Section 3.7, where coil antennas are placed in the middle between the mandrel and the borehole border) to generate $A R$ and $P D$ data and then we can perform measurements of a real LWD tool (using mandrel-embedded antennas) to calibrate the simulated tool [34]. This approach is very practical, however, we believe realistic simulations for LWD tools can render important information to the design of novel resistivity logging-sensors more accurate than the conventional technology. For this reason, in this section we explore the influence of LWD sensors housed inside grooves around the mandrel.

\subsection{1}

\section{Horizontal-Coil Antennas}

According to the mode-matching formulation presented in Section 3.4.1, the inclusion of indentations on the mandrel can be easily simulated just assuming that the innermost radial-layer (the PEC mandrel) now presents two distinct radius, as illustrated in Fig. 3.52. By assuming the grooves present the radial-width $d$ and axial-height $h$, we can model one groove by as the ordinary 


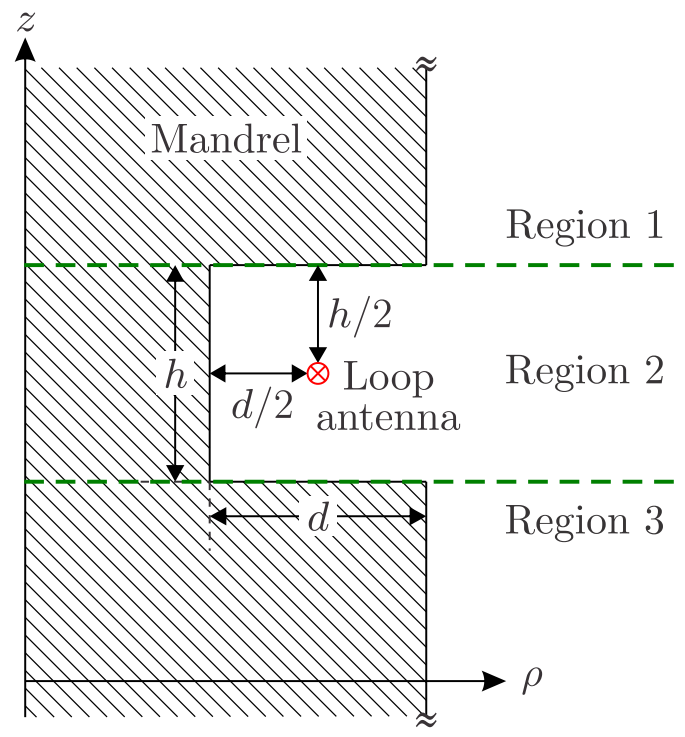

Figure 3.52: Side view of a coil antenna (depicted by $\otimes$ ) placed within a groove on the metallic mandrel.

junction between an intermediate waveguide (region 2, in which innermost radius is located at $\rho=r_{0}-d$ ) with the two adjacent waveguides (in regions 1 and 3 , whose innermost radial domain start at $\rho=r_{0}$ ).

We consider the case of a 5 -in borehole filled with an oil-based mud having $\sigma=5 \times 10^{-4}$ and now the triaxial logging tool consist of one transmitter and two receivers horizontal-coil antennas inside a formation with conductivity $1 \mathrm{~S} / \mathrm{m}$. The transmitter (TX) operates at $2 \mathrm{MHz}$, and will induces voltages at receivers $\mathrm{RX}_{1}$ and $\mathrm{RX}_{2}$ placed 30-in and 24-in axially away from the source. The antennas are wrapped within grooves with $d=h=1$ in on a 4 -in metallic mandrel, as illustrated in Fig. 3.52 and Fig. 3.53(a).

Fig. 3.54 and Fig. 3.55 show the voltage amplitude ratio (AR) and phase difference $(\mathrm{PD})$ received by the triaxial LWD tool for several pertinent values for axial attenuation $\left(A_{d B}\right.$ at $\left.0.5 \mathrm{in}\right)$ and the radial truncation in function of the thickness of outermost radial layer $(2,3,4$ and 5 times the skin depth of soil formation). We can verify a convergence of both $A R$ and $P D$ as the radial domain is as large as $4 \delta_{N}$.

Now using the truncating the radial space at 60 in (about 4 times the skin depth of the formation) and using all fields with axial attenuation less than $A_{d B}=30 \mathrm{~dB}$ at $0.5 \mathrm{in}$, we explored the LWD tool response for a parametric variation of the groove widths and heights. Fig. 3.56 shows results for $A R$ and $P D$ for 1 -in $\leq d \leq 2$-in and 1 -in $\leq h \leq 30$-in.

For small groove heights, in the range of $h<6$-in, $A R$ ans $P D$ vary subtly for the analyzed widths $d$ : AR changes about $1 \mathrm{~dB}$ and are mainly influenced by values of $h$, and the PD variation is almost negligible; less than 


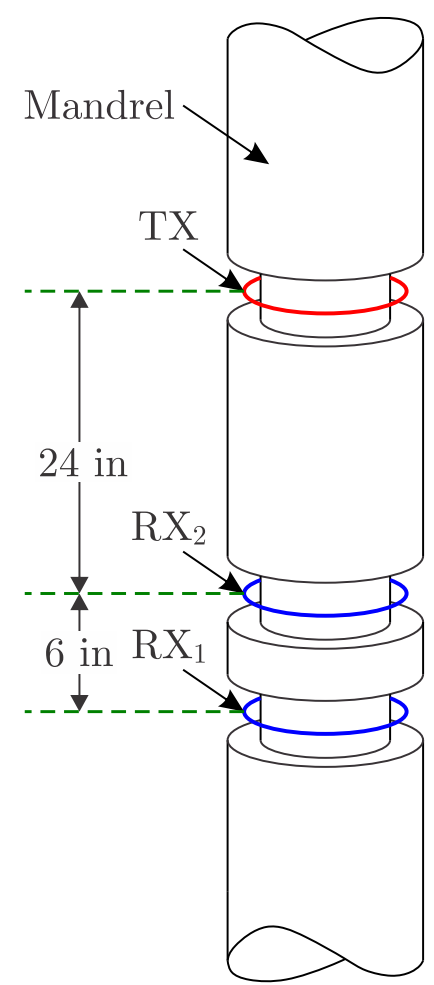

(a) $h<6$ in

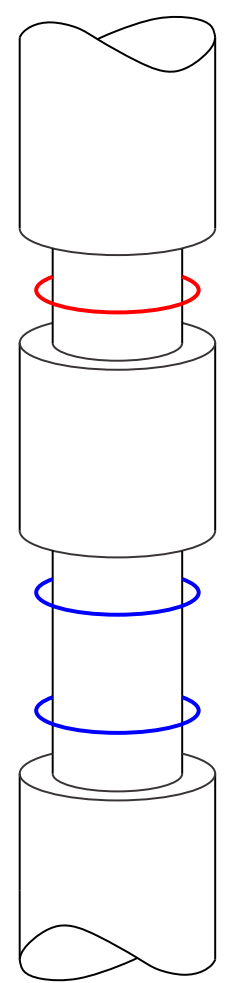

(b) $6 \leq h<24$ in

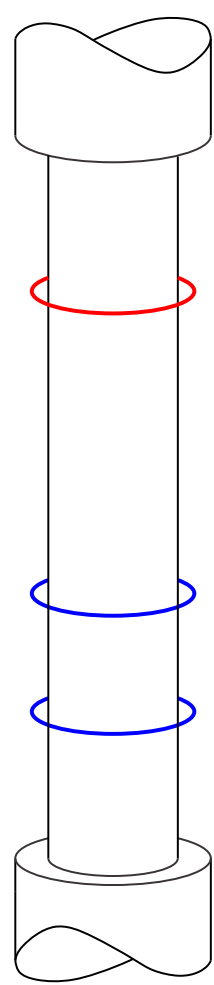

(c) $h \geq 24$ in

Figure 3.53: LWD tool for different groove heights.

one degree.

Despite the small variations in $A R$ and $P D$ described above, recall that these are relative parameters, since we well know that the induced voltages goes to zero as $d \rightarrow 0$; this is due the azimuthal currents along the antennas becoming close to the PEC layer of mandrel.

However, we verify that when the groove heights are in the range 6in $\leq h<24$-in (see Fig. 3.53(b)), a significantly variation as observed in $A R$ results, and in this case the groove width is a relevant parameter.

In the last case, when the groove heights is bigger than 24-in (see Fig. 3.53(c)), the responses of $A R$ and $P D$ again recover its groove-width independence. As far as $h$ increases, the tool response resembles that expected for an infinitely-long mandrel without indentation. 


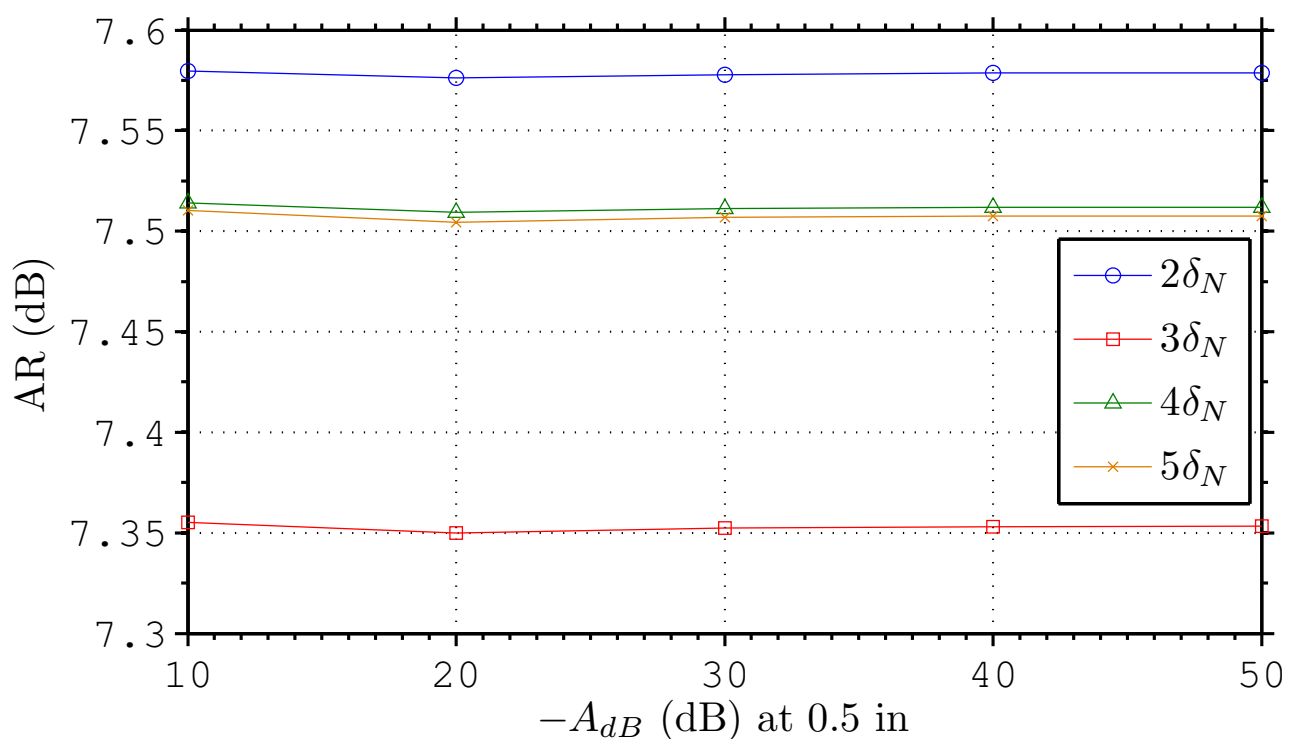

Figure 3.54: Convergence of the voltage amplitude ratio received by a LWD tool whose antennas are placed inside grooves $(d=h=1$ in) on the conductor mandrel for $A_{d B}$ at $\Delta z=0.5 \mathrm{in}$. The radial domain is truncated at $r_{N}=r_{N-1}+\alpha \delta_{N}, \alpha=2,3,4$ and 5 .

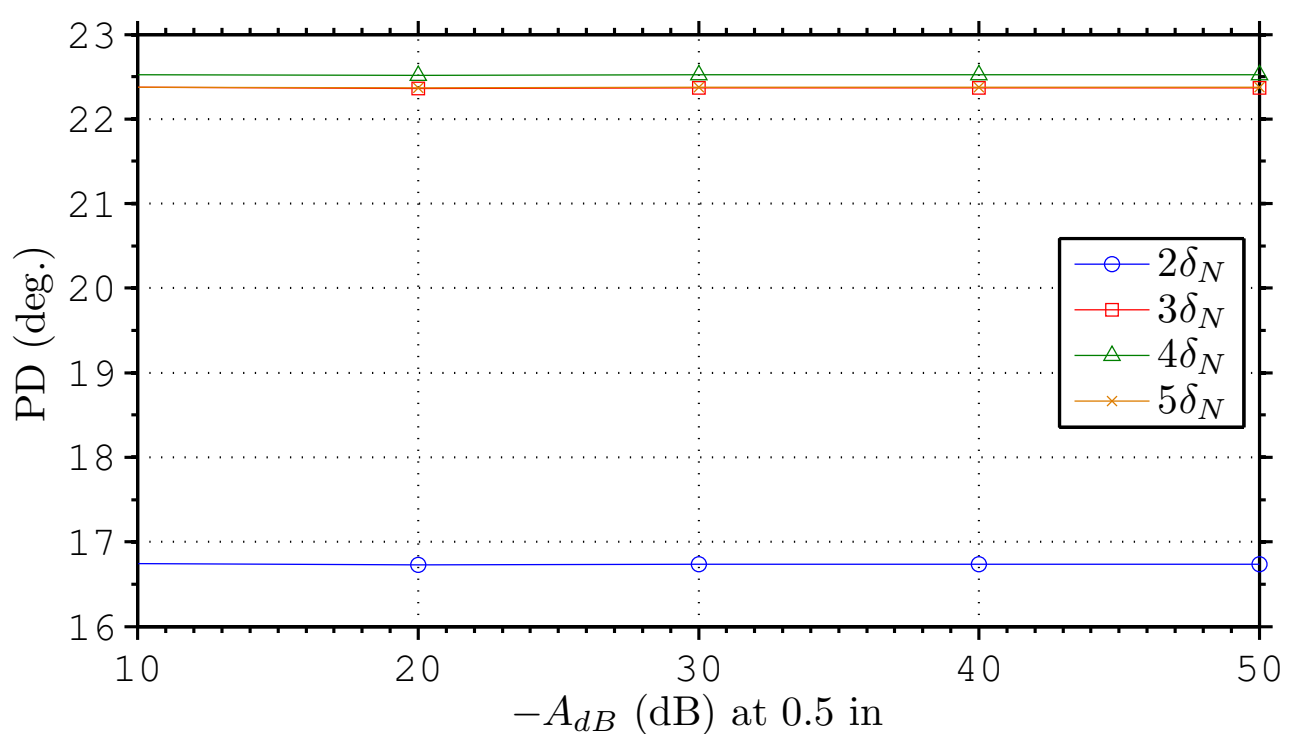

Figure 3.55: Convergence of the voltage phase difference received by a LWD tool whose antennas are placed inside grooves $(d=h=1$ in) on the conductor mandrel for $A_{d B}$ at $\Delta z=0.5 \mathrm{in}$. The radial domain is truncated at $r_{N}=r_{N-1}+\alpha \delta_{N}, \alpha=2,3,4$ and 5 . 

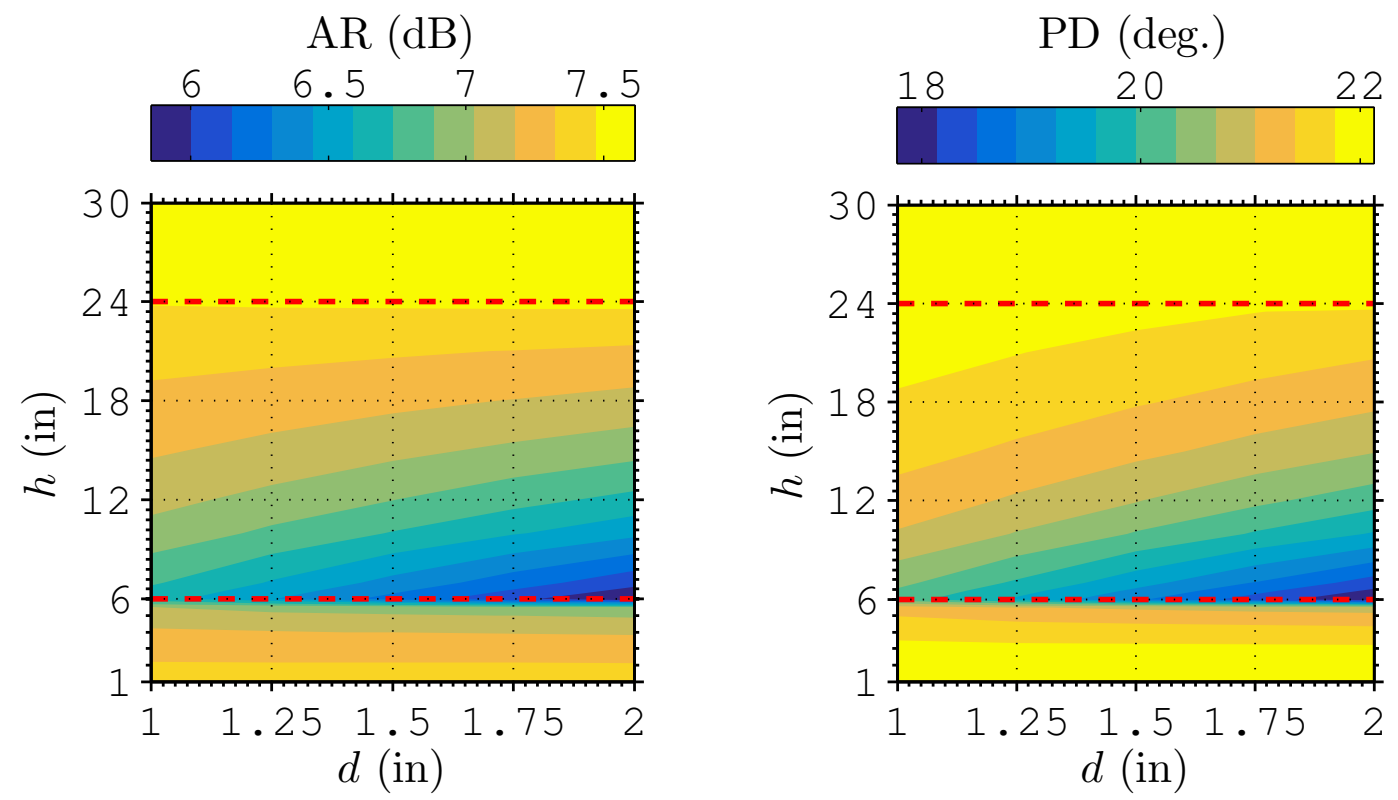

Figure 3.56: Voltage amplitude ratio and phase difference received by a LWD tool whose antennas are placed inside grooves on the conductor mandrel. Horizontal dashed lines indicate two relevant groove height: $h=6$ in and 24 in.

\subsection{2}

\section{Tilted-Coil Antennas}

To further explore some aspects of realistic LWD logging-sensors, we now will analyze TCA within mandrel indentations. Instead of install the TCAs around the mandrel, we consider the antennas are wounded inside grooves on the mandrel pipe, as illustrated in Fig. 3.57. The mandrel presents a radius of $4 \mathrm{in}$, and the indentations are 2-in-depth in radial direction. The TCA antennas are placed at $\rho=3$ in. The axial position of the antenna TX is on middle of a 8-in-groove, as shown in Fig. 3.57. The receivers TCA antennas $\mathrm{RX}_{1}$ and $\mathrm{RX}_{2}$ are placed inside a 14-in-groove, such these receivers are 30in and 24-in far from the transmitter, respectively. We consider a borehole filled with oil-based mud having the isotropic conductivity $\sigma=5 \times 10^{-4} \mathrm{~S} / \mathrm{m}$. The surrounding soil formation is an anisotropic media, and its horizontal and vertical component of the conductivity are $5 \mathrm{~S} / \mathrm{m}$ and $1 \mathrm{~S} / \mathrm{m}$, respectively. Also, we consider that all antennas are azimuthally aligned, i.e., $\phi_{T}=\phi_{R 1}=\phi_{R 2}$. Assuming a unit current excitation at $2 \mathrm{MHz}$, the tilt angle of each TCA antenna will be investigated in the following. We truncate the radial domain at $60 \mathrm{in}$, and have include all modes whose axial attenuation is less than $30 \mathrm{~dB}$ at 1 in; a very conservative criteria. Again, only azimuthal indices $n=0,1$ are included in our simulations.

Fig. 3.58 shows results for the voltage at TCA receiver $\mathrm{RX}_{1}$ for various 
values of $\theta_{T}$ and $\theta_{R 1}$, in which the tilt angles vary from 0 to $45^{\circ}$. Similarly, Fig. 3.59 presents the voltage at $\mathrm{RX}_{2}$ for several tilt angles associated to this receiver antenna. We can see that as the tilt angle of TCAs increases, the received voltage becomes higher as well.
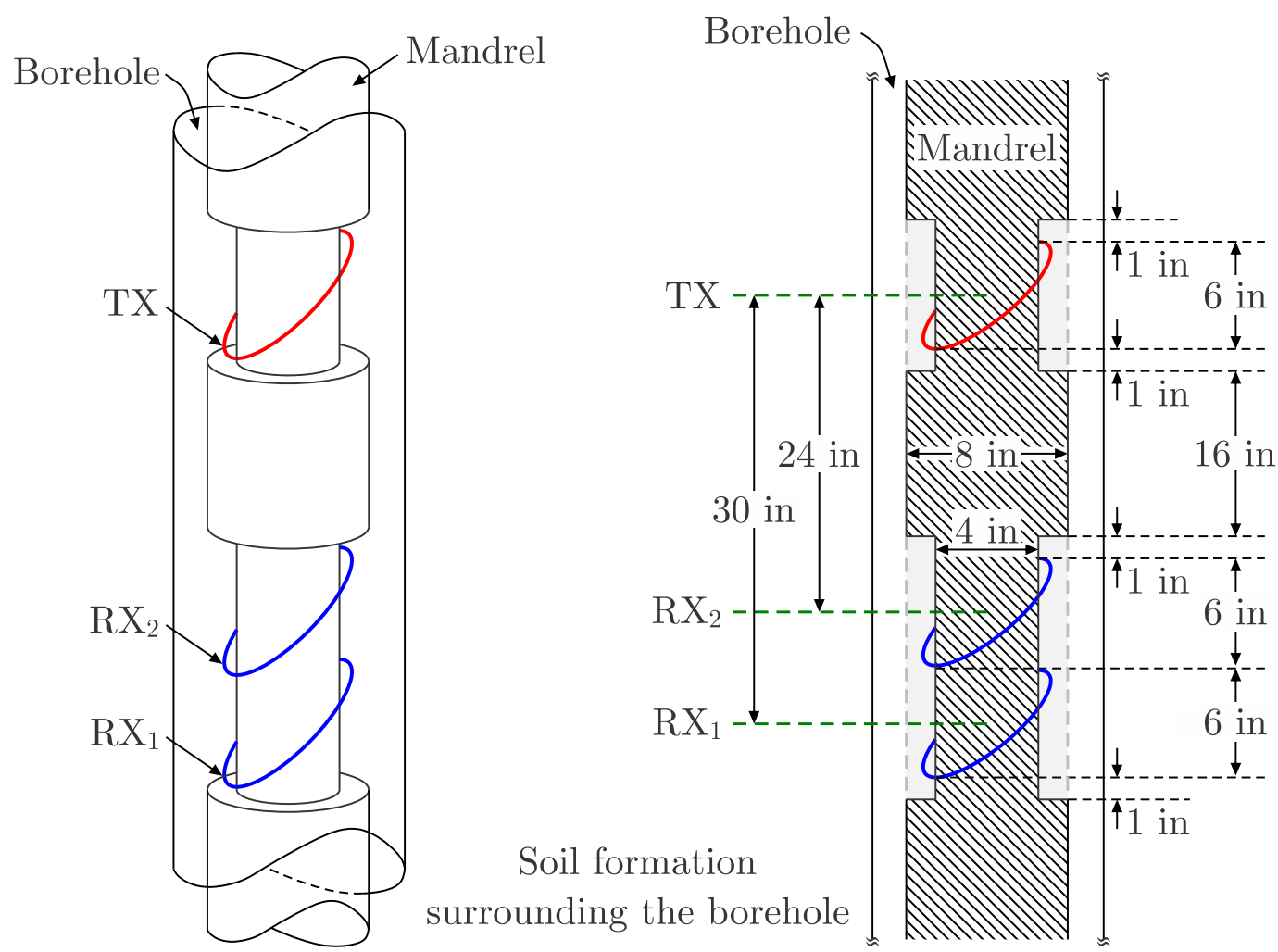

Figure 3.57: Model employed to simulate the mandrel indentations. The details of the antennas positions and the dimensions of grooves on mandrel are shown on the right, in which the TCAs have $\rho_{T}=\rho_{R 1}=\rho_{R 2}=3 \mathrm{in}$.
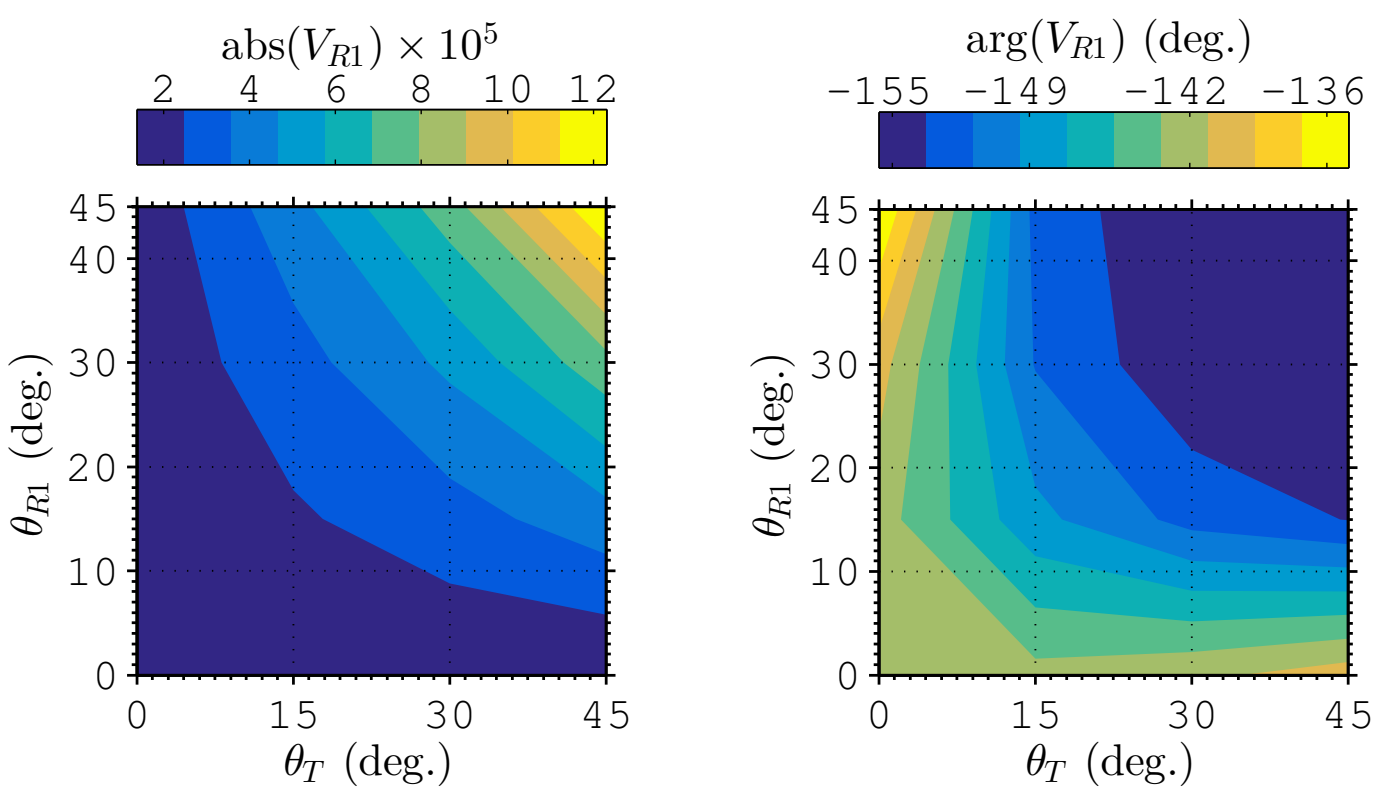

Figure 3.58: Voltage at TCA receiver $\mathrm{RX}_{1}$ for various values of $\theta_{T}$ and $\theta_{R 1}$. 

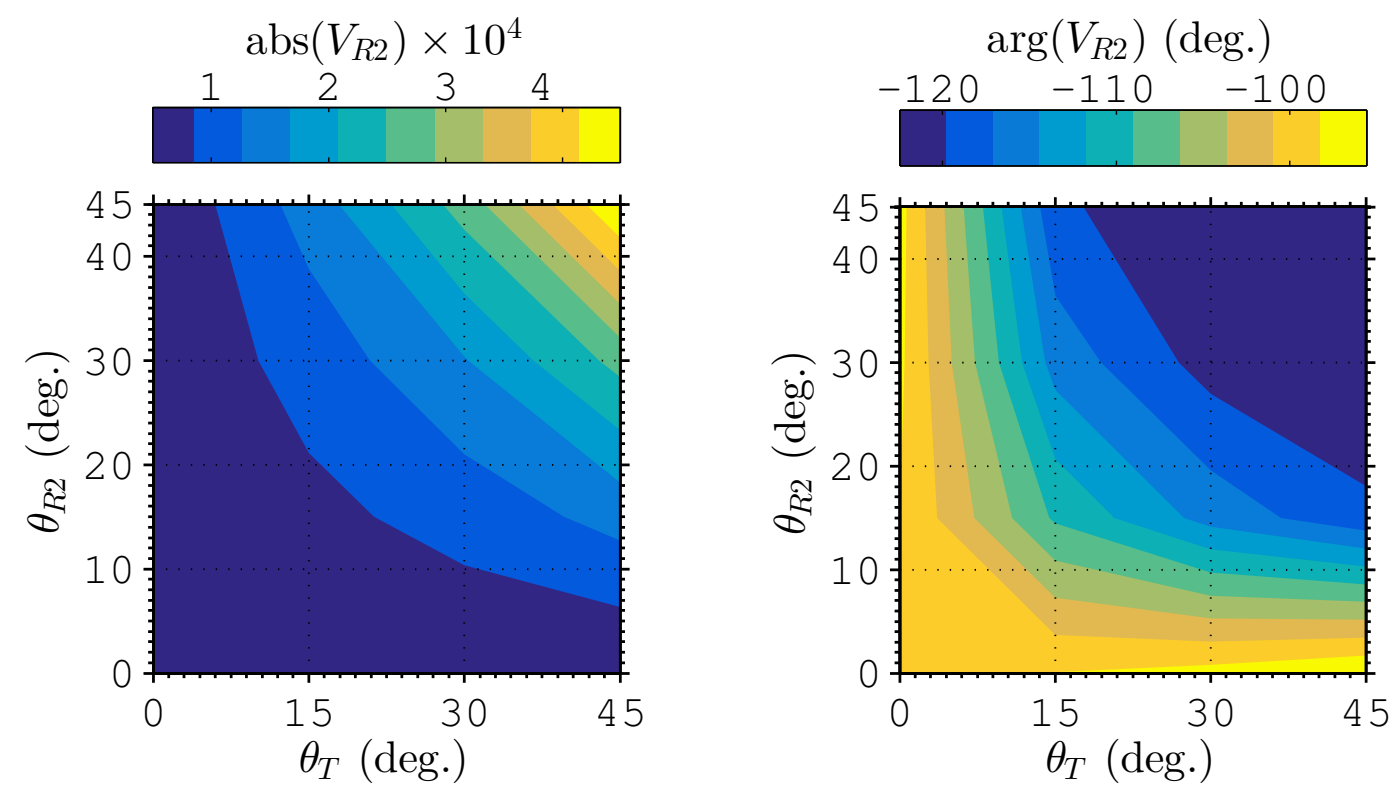

Figure 3.59: Voltage at TCA receiver $\mathrm{RX}_{2}$ for various values of $\theta_{T}$ and $\theta_{R 2}$.

Proper combination of the results showed above in Fig. 3.58 and Fig. 3.59 allow us to describe AR and PD responses for a desired LWD tool under investigation. Considering fixed transmitter TCA tilt angles $\theta_{T}=0,15^{\circ}, 30^{\circ}$ and $45^{\circ}$, we can derive the results shown in Fig. 3.60 to Fig. 3.63.

For $\theta_{T}=0$, only fields associated to azimuthal order $n=0$ are excited, such $\mathrm{AR}$ and $\mathrm{PD}$ due tilts in receivers observed in Fig. 3.60 cover the range of $8.4 \mathrm{~dB}$ to $9.8 \mathrm{~dB}$ and $37^{\circ}$ to $46^{\circ}$, respectively. As said before, for $\theta_{T}>0$, the $n=0$ and $n=1$ harmonics are the main contribution to received voltage. In Fig. 3.61 we can clearly see the effects of fields with azimuthal index $n=1$ when $\theta_{T}=15^{\circ}$ : now the variations of $\mathrm{AR}$ and $\mathrm{PD}$ are over $1.5 \mathrm{~dB}$ to $17 \mathrm{~dB}$ and $23^{\circ}$ to $55^{\circ}$. This range of variation increase with $\theta_{T}$, as illustrated in Fig. 3.62 (for $\theta_{T}=30^{\circ}$ ) and in Fig. 3.63 (for $\theta_{T}=45^{\circ}$ ). Notably, in these last two cases we can observe negative values for AR; i.e., for some combinations of $\theta_{R 1}$ and $\theta_{R 2}$ the voltage received at $\mathrm{RX}_{1}$ is larger than that in $\mathrm{RX}_{2}$ (which is closer to TX). 

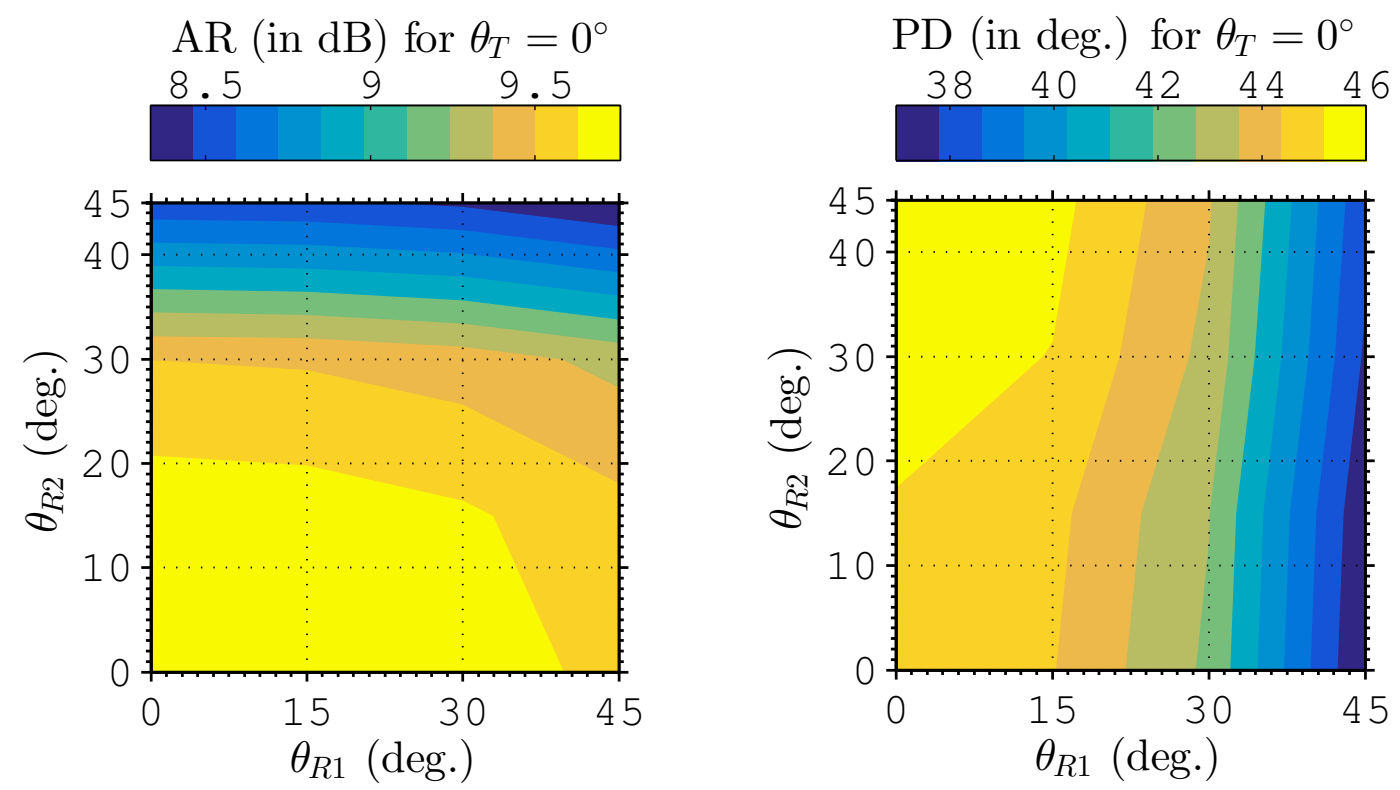

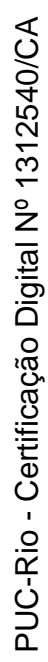

Figure 3.60: Amplitude ratio and phase difference response for a transmitter TCA with $\theta_{T}=0$ in respect to the tilt angle of receivers.
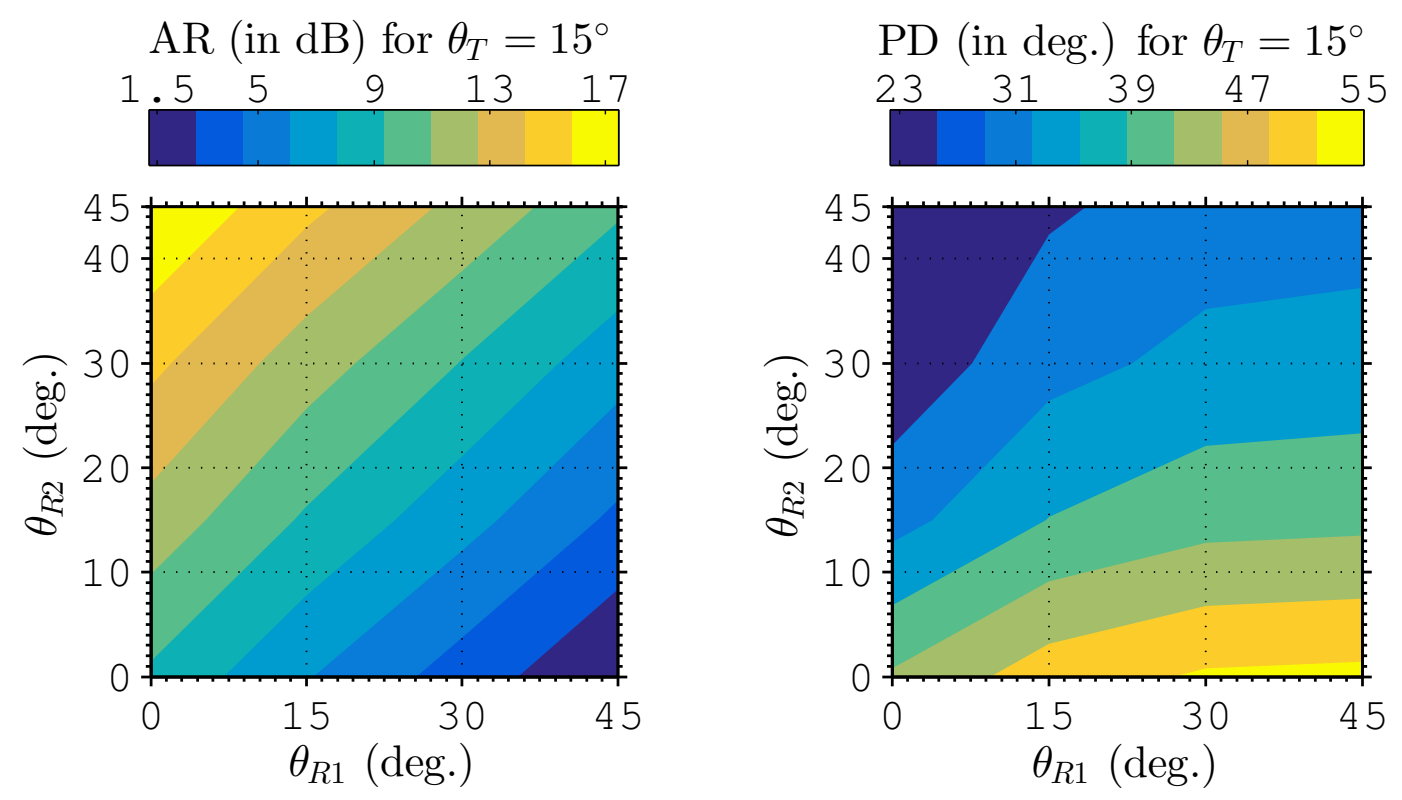

Figure 3.61: Amplitude ratio and phase difference response for a transmitter TCA with $\theta_{T}=15^{\circ}$ in respect to the tilt angle of receivers. 

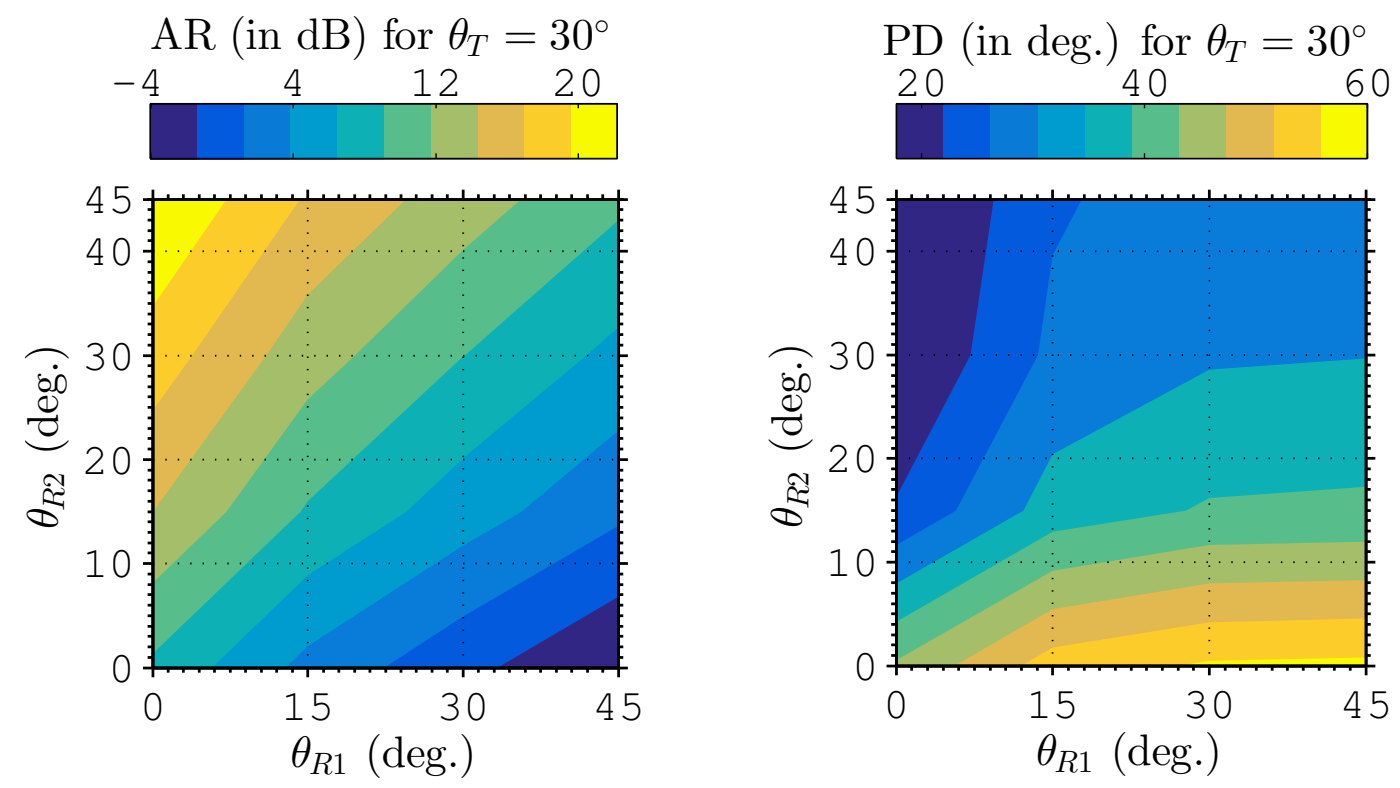

Figure 3.62: Amplitude ratio and phase difference response for a transmitter

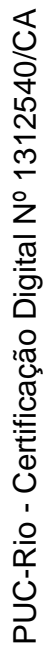
TCA with $\theta_{T}=30^{\circ}$ in respect to the tilt angle of receivers.

$\mathrm{AR}$ (in $\mathrm{dB}$ ) for $\theta_{T}=45^{\circ}$
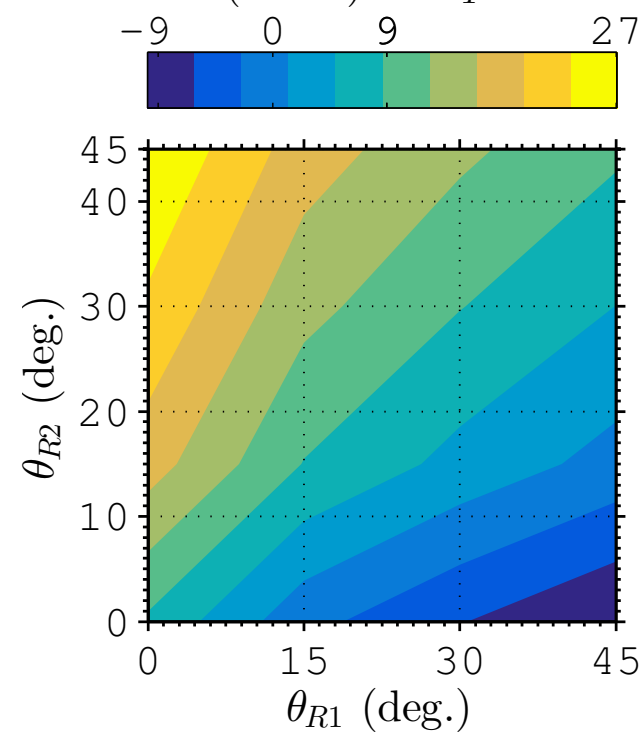

$\mathrm{PD}$ (in deg.) for $\theta_{T}=45^{\circ}$

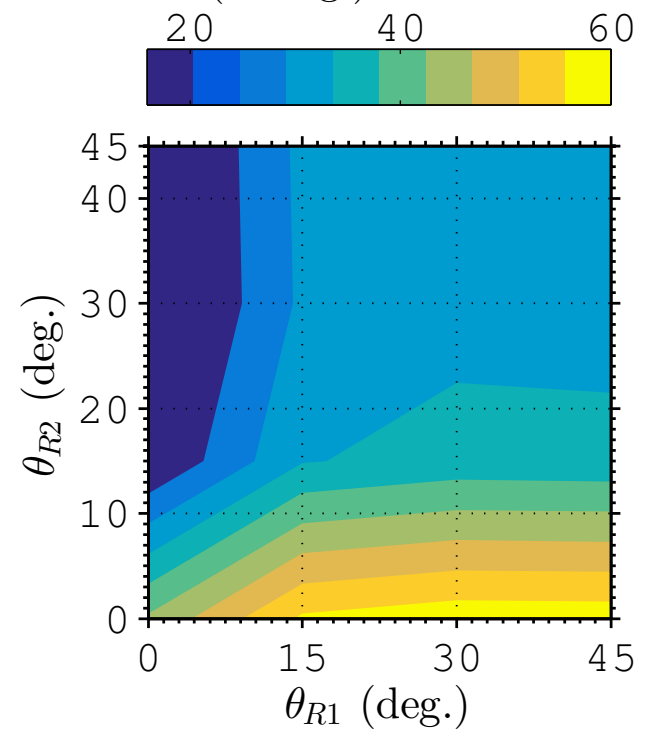

Figure 3.63: Amplitude ratio and phase difference response for a transmitter TCA with $\theta_{T}=45^{\circ}$ in respect to the tilt angle of receivers. 


\subsection{0}

\section{Some Investigations on Wireless Telemetry for Oilfield Applications}

Real-time LWD tools acquire information about the soil surrounding the drill bit. The data are then sent to the surface equipment in order to be processed. In several situations where wired telemetry is impossible, the data is sent by mud-pulse telemetry at bit rate of few bits per second. In the following we will explore the wireless telemetry as an alternative communications channel between the downhole sensors and the oilfield surface infrastructure.

\subsection{1}

\section{Low-Frequency Wireless Telemetry in Deep Oil Fields}

Transmission of logging signals along an oil well can be achieved using electrical cables or optical fibers connecting the Earth's surface to the downhole instrumentation. However, the prohibitive cost of the cabling, and the possibility of the cable tangling, breaking, and/or poor electrical contact renders this technique less than ideal for deep oil wells [121]. Wireless telemetry systems can overcome these challenges, but to date it has been restricted to very low frequencies and is strongly affected by losses on the annulus region.

Wireless telemetry systems and methods designed for application to wells are described in $[121,122]$ for cased boreholes having a tubing string. This system is mounted inside the casing pipe, in the region called the annulus, as illustrated in Fig. 3.64. This region is bounded by the center tubing of the production column (internal pipe) and the conductor casing (external pipe). According to [121], this system requires a substantially non-conductive fluid in the annulus (such crude oil or air) to acts as a wireless telemetry communication channel. The high conductivity of typical oil-based mud or problems with brine leakage into the casing result in severe attenuation for deep wells even at low frequencies. The remarks in $[121,122]$ are reasonable when the well is modeled as a coaxial cavity. However, a more careful analysis, considering the finite conductivity of the (metallic) pipes reveals that electromagnetic fields can be concentrated outside the casing, enabling the propagation over long axial distances with relatively low attenuation. In this section, a rigorous analysis is performed to quantify this statement.

Fig. 3.64 illustrates a well for petroleum production using wireless telemetry. In this system, transmitter and receiver antennas are mounted inside the annulus region. In LWD problems, the electromagnetic excitation is usually a current loop $[8,48,123]$, which primarily excites $\mathrm{TE}^{z}$ modes. We performed preliminary simulations that showed a high attenuation of $\mathrm{TE}^{z}$ modes in a cased borehole. In contrast, the attenuation of $\mathrm{TM}^{z}$ modes is substantially 


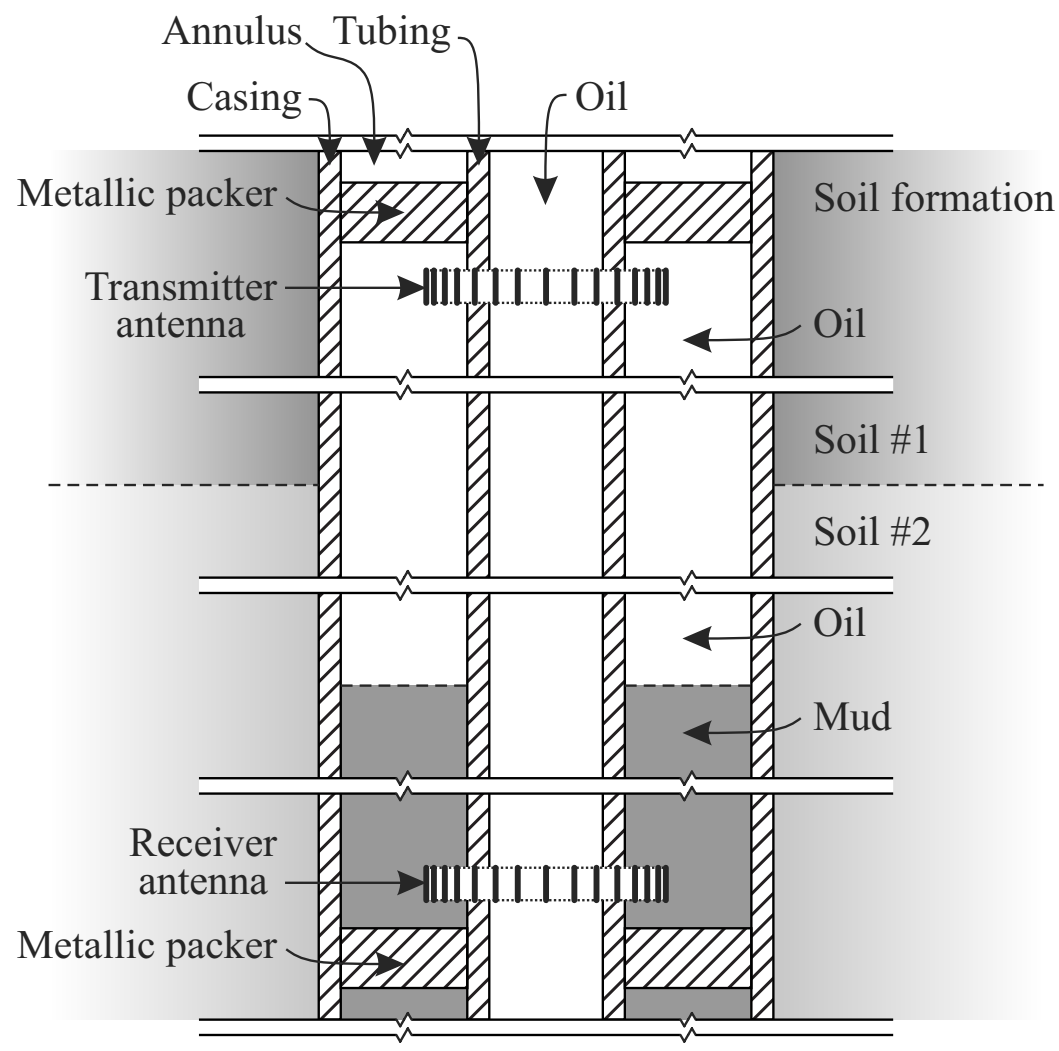

Figure 3.64: Sectional view of an oil well using wireless telemetry.

lower. In order to excite only $\mathrm{TM}^{z}$ fields, it is assumed that the transmitter antenna is a toroidal coil, mounted around the inner tubing of the well. The receiving antenna is identical to the transmitting one. For the problem at hand, the wavelength is very long compared to the size of the borehole and we can simplify the transmitter antenna model using the equivalence principle [55, p. 106], and modeling it as a magnetic source as described in Section 3.5.3. A radially stratified waveguide is used to model the oil well cross-section, where each layer is described as an isotropic and homogeneous media.

We consider an oil well described by the parameters listed in Table 3.3. All simulations consider antennas placed at $\rho=3.81 \mathrm{~cm}$, operating at $1 \mathrm{~Hz}$. We truncate the radial domain at $r_{N}=100 \mathrm{~m}$ and the PML extends over $50 \mathrm{~m}$ using $s_{\rho}=1+i 0.01$. We used $10 \mathrm{TM}_{0 p}^{z}$ modes to obtain convergence. In Fig. 3.65, we shown results of the attenuation along a 500-m-long oil well for several mud conductivities. The attenuation is determined by examining the quantity $20 \log _{10}\left(V_{R 1} / V_{R 2}\right)$, where $V_{R 1,2}$ are the received voltages by a fixed antenna at $z_{R 1}=z_{T}$ and by a moving antenna at $z_{R 2}=z_{R}$, in the range $z_{R}-z_{T}=0$ to $500 \mathrm{~m}$.

For a low-loss mud $\left(\sigma=10^{-2} \mathrm{~S} / \mathrm{m}\right)$ the attenuation is mainly determined by the guided TEM-like mode. However, for high-loss mud, we observe an interesting phenomena that can be illustrated by examining the case $\sigma=$ 
Table 3.3: Dimensions and parameters of the cased oil well.

\begin{tabular}{lccccc}
\hline & Inner diameter $(\mathrm{cm})$ & Thickness $(\mathrm{cm})$ & $\sigma(\mathrm{S} / \mathrm{m})$ & $\mu_{r}$ & $\epsilon_{r}$ \\
\hline Internal pipe & 7.3025 & 0.635 & $4.5 \times 10^{6}$ & 500 & 1 \\
External pipe & 13.97 & 0.9525 & $4.5 \times 10^{6}$ & 500 & 1 \\
Oil & & & 2.985 & 1 & 14 \\
Mud & & $10^{-2}$ to 10 & 1 & 14 \\
Soil formation & & $3.5 \times 10^{-2}$ & 1 & 1 \\
\hline
\end{tabular}

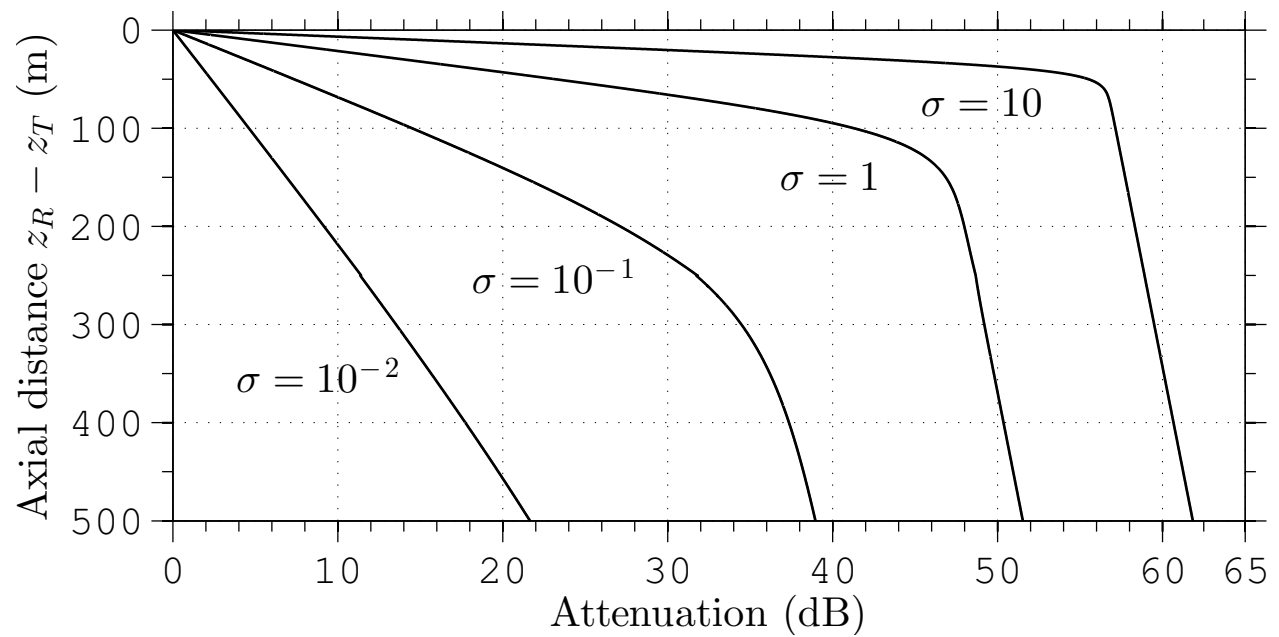

Figure 3.65: Influence of the oil-based mud conductivities ( $\sigma$, given in $\mathrm{S} / \mathrm{m}$ ) in the attenuation of the received signal along the axial distance from the source.

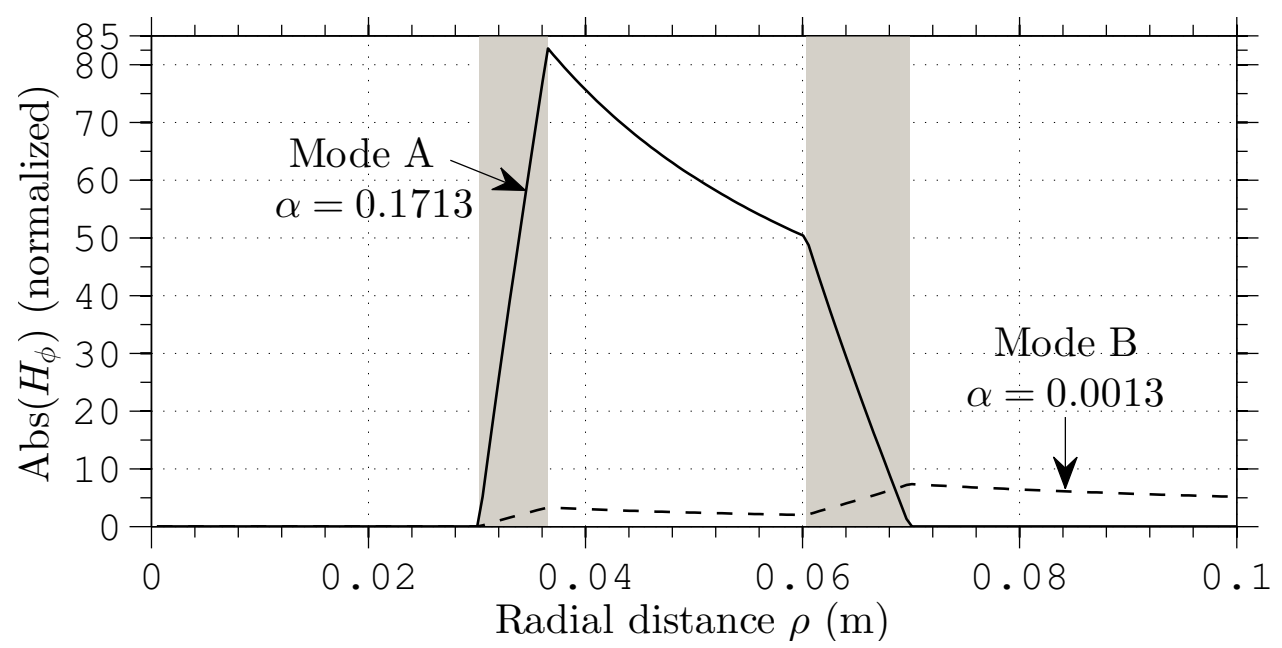

Figure 3.66: Transversal magnetic field $H_{\phi}$ of the modes $A$ and $B$. The axial attenuation constant $\alpha=\Im m\left(k_{z}\right)$ of each mode is presented. Metallic pipe layers are shaded.

$10 \mathrm{~S} / \mathrm{m}$. For modes with less attenuation, identified as $A$ and $B$ in Fig. 3.66, we note that the TEM-like mode $A$ has a larger amplitude compared with the mode $B$ at the source radial position $\rho=3.81 \mathrm{~cm}$. Thus, near the source $\left(z_{R} \approx z_{T}\right)$, the magnetic field $H_{\phi}$ is mainly determined by the mode $A$. 
However, the field of the mode $A$ is dominant only along the first $50 \mathrm{~m}$ (see Fig. 3.65 for $\sigma=10 \mathrm{~S} / \mathrm{m}$ ), after this, the mode $B$, which has smaller attenuation constant ( $\alpha=0.0013$, as shown in Fig. 3.66), becomes dominant. By analyzing the other curves in Fig. 3.65, for $\sigma=10^{-1}$ and $\sigma=1 \mathrm{~S} / \mathrm{m}$, we can observe a similar behavior: near the source, a TEM-like mode is dominant, but sufficiently far away from the source the mode $B$ becomes the main contributor for the field. Therefore, for long distances, the fields become confined near to the external casing (according to mode $B$ profile), enabling the wireless telemetry despite the presence of high lossy medium in the annular region. We used ten $\mathrm{TM}_{0 p}^{z}$ modes to ensure convergence of the received fields. A simplified model based on the two principal modes can be used for deep wells, i.e. large $\left|z_{R}-z_{T}\right|$, with high-loss mud. For low-loss mud and small $\left|z_{R}-z_{T}\right|$, one TEMlike mode may suffice (see the curve for $\sigma=10^{-2} \mathrm{~S} / \mathrm{m}$ in Fig. 3.65 and the dot-dashed line in Fig. 3.67).

As a second example, we consider a oil well using high-loss mud filling the annulus region $(\sigma=10 \mathrm{~S} / \mathrm{m})$, and a layer boundary between soil formations \#1 and \#2 (see Fig. 3.64) at $250 \mathrm{~m}$ away from the source. We show the results of the axial attenuation along a 500-m-long oil well in Fig. 3.67. It is important to note the attenuation is overestimated when we consider the first mode only (similar to mode $A$ in Fig. 3.66).

In this section, we have present a computationally efficient method to analyze wireless telemetry in ultra-deep oil wells when high-loss drilling fluids (mud) are present in the oil well annulus region. For wells with these characteristics, we observed a gradual evolution on the radial profile of the field propagating along the well. For long distances, the field becomes mostly

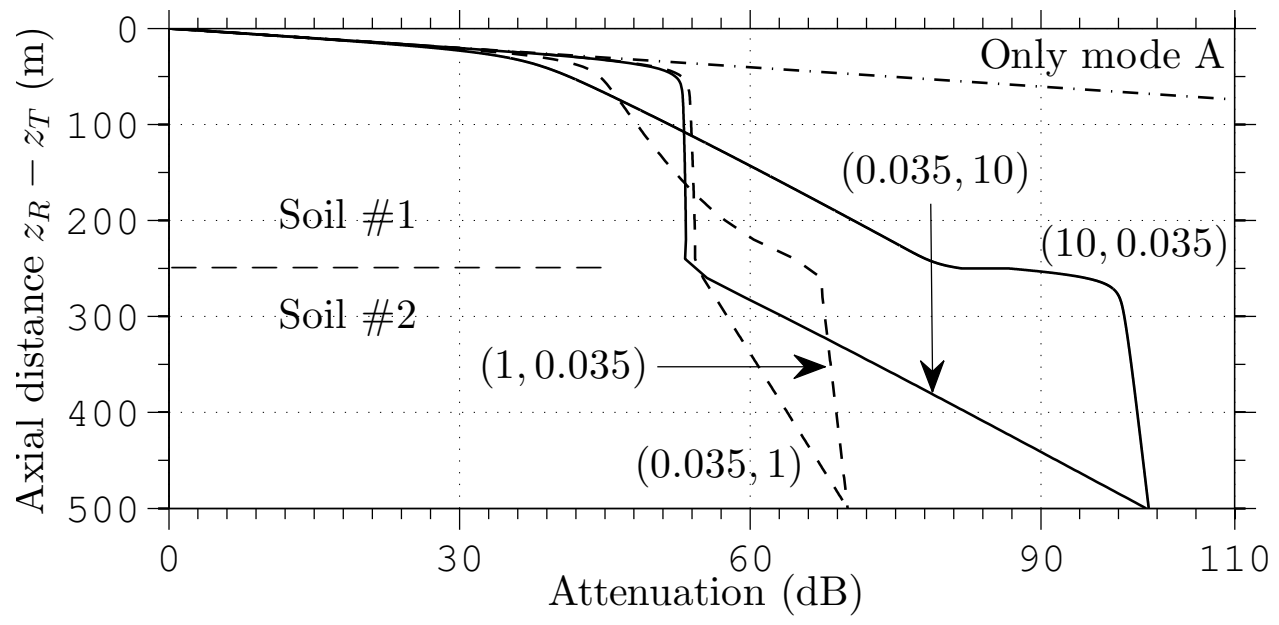

Figure 3.67: Influence of the soil formations conductivities $\left(\sigma_{\# 1}, \sigma_{\# 2}\right)$ in the attenuation of the received signal along the axial distance from the source. The annulus has high losses $(\sigma=10 \mathrm{~S} / \mathrm{m})$. 
confined around the external well casing, which enables wireless telemetry links from the Earth's surface to the downhole antenna despite the presence of a high loss medium in the annular region.

\subsection{2}

\section{High-Frequency Wireless Communications in Tunnels}

The electromagnetic propagation along circular tunnels and boreholes has important applications to wireless communication at LWD at lowfrequency geophysics problems [10] as well as in high-frequency $[11,12]$ communications. In some applications where the borehole diameter is small compared to the wavelength, at the interest region of geophysics (near the source), the fields are dominated by the continuum mode (or its equivalent PML modes as described in Section 3.3.2). In contrast, case the borehole diameter is large compared to the wavelength $[11,12]$, the contribution of the continuum mode is negligible, and far from the source at high-frequency only a few guided modes need to be considered.

In order to explore the technique introduced in this chapter for the modeling of high-frequency electromagnetic propagation in the Earth's formations, we initially consider the problem shown in [10], where a circular crosssection borehole with a center conducting pipe is excited by an electric loop antenna (mounted around the pipe) that operates at $25 \mathrm{MHz}$. At this frequency, the influence of the conducting currents can be more significant compared to that of the low-frequency LWD tools we have analyzed before in this chapter. In some low-conductive environments, the electromagnetic modeling requires the proper selection of the real part for the permittivity; this is because the (possible) negligible imaginary part proportional to $\sigma / \omega$ (in isotropic media).

We consider one transmitting (at $z_{T}$ ) and two receiving antennas (at $z_{R 1}$ and at $z_{R 2}$ ) that are moving through the discontinuity between the regions 1 and 2. The axial position of the antennas are given by $z_{T}=z-95.25 \mathrm{~cm}$, $z_{R 1}=z_{T}+63.5 \mathrm{~cm}$ and $z_{R 2}=z_{T}+127 \mathrm{~cm}$ (for more details, see $[10,109]$ ). The antennas are identical 3.81-cm-radius circular electric loops. The waveguide of the region 1 is characterized by: $r_{0}=1.27 \mathrm{~cm}, r_{1}=10.16 \mathrm{~cm}, \epsilon_{1}=80 \epsilon_{0}+i / \omega$, $\epsilon_{2}=\epsilon_{0}+i 0.05 / \omega$. The region 2 is characterized by the same parameters, except for the soil formation: $\epsilon_{2}=20 \epsilon_{0}+i 0.2 / \omega$. Notice the media is isotropic and nonmagnetic, but unlike to other sensors presented in Section 3.7 and Section 3.9, here the values for the relative permittivity constants $\epsilon_{r}$ are greater than 1 .

In Fig. 3.68, we show the results of the relative voltage amplitudes and phases received by the two antennas moving (together the transmitter) across the axial discontinuity at $z=0$. The results obtained using the presented 


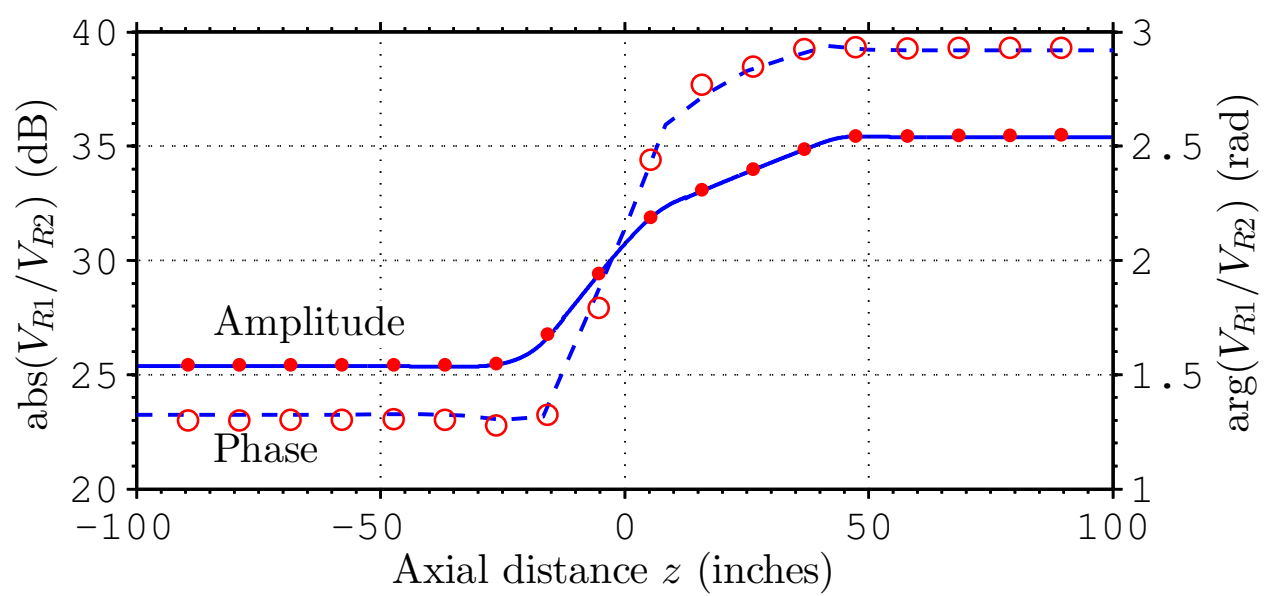

Figure 3.68: Relative voltage received by two antennas moving with one transmitter antenna across a bed boundary. The results of our approach are depicted by the solid (for amplitude) and dashed (for phase) lines. The small $\operatorname{dots}(\bullet$ for amplitude and o for phase) are the results from [10] (from graphical reading).

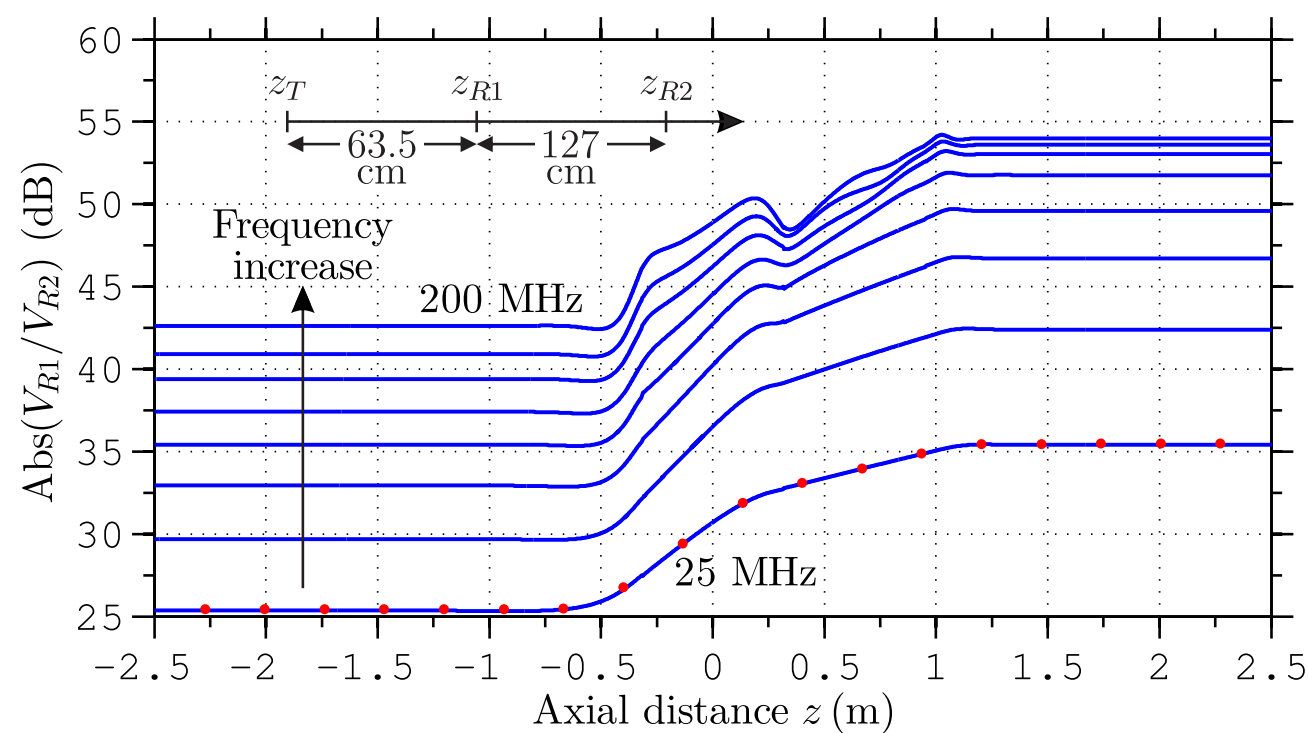

Figure 3.69: Relative voltage received by two antennas moving with one transmitter antenna across a bed boundary. The results of our approach are depicted by the solid lines. The small dots are the results from [10].

formulation are in agreement with those shown in [10]. We have considered all the eigenvalues that meet $A_{d B}=-30 \mathrm{~dB}$ at $\Delta z=5 \mathrm{~cm}$.

Additionally, in order to verify the rise of guided modes, we perform the series of simulations on the same structure show in [10] by varying the operating frequency up to $200 \mathrm{MHz}$, with steps of $25 \mathrm{MHz}$, as shown in Fig. 3.69. Note that with the frequency increase the received voltages become perturbed near the junction. To analyze this effect, in Fig. 3.70, we show the normalized electric fields $E_{\phi}$ for the two principal modes of the region 


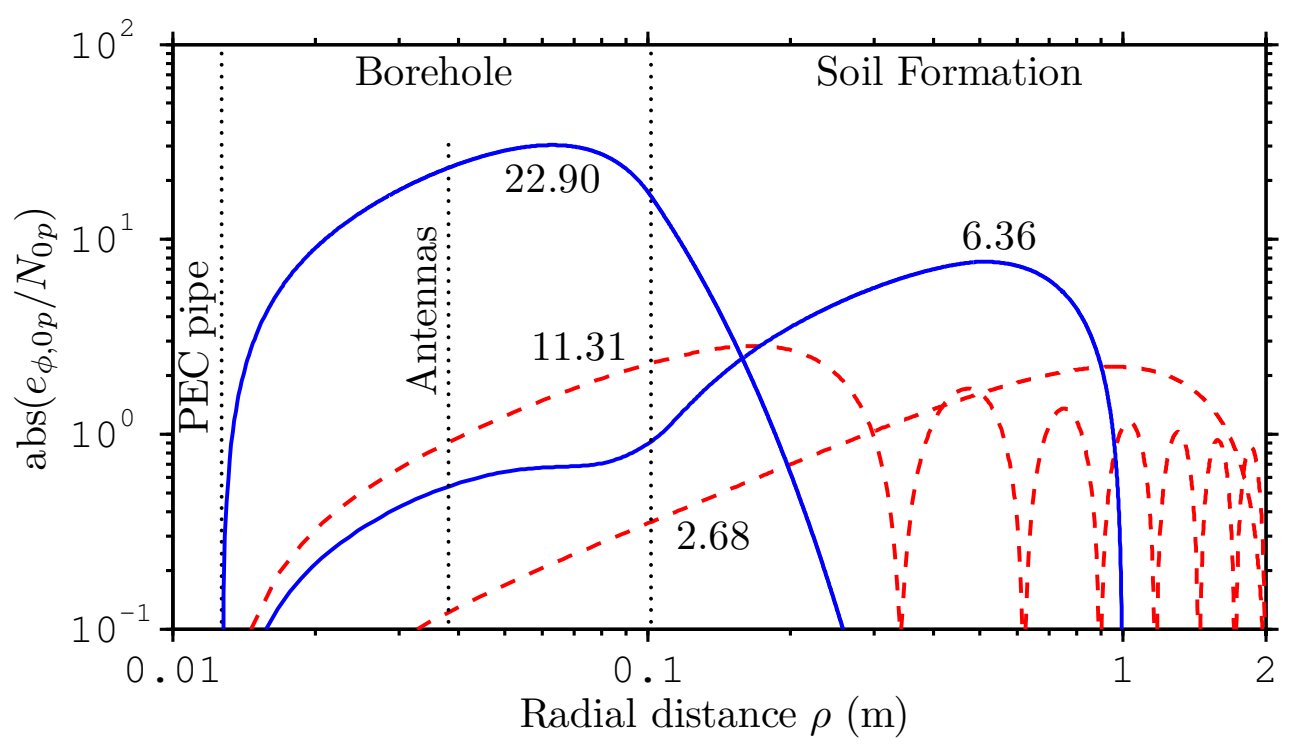

Figure 3.70: Normalized modal $E_{\phi}$ fields for $25 \mathrm{MHz}$ (dashed line) and for $200 \mathrm{MHz}$ (solid line). The value of $\Im m\left(k_{z, 0 p}\right)$ is shown for each field.

1, for both $25 \mathrm{MHz}$ and $200 \mathrm{MHz}$. The axial attenuation constants are also shown. Note that we have used $r_{N}=2 \mathrm{~m}$ for $25 \mathrm{MHz}$, and $r_{N}=1 \mathrm{~m}$ for $200 \mathrm{MHz}$. At high-frequency, we can verify that the guided mode is dominant only near the axial discontinuity, after this, the mode with small attenuation constant overcomes the initial amplitude differences and becomes dominant. These modes have high attenuation compared to the PML modes outside the borehole, and thereby, these modes are only relevant near the discontinuity at $z=0$.

In contrast to the problems in [10], we consider now the example shown in [108], where a system operates at $1 \mathrm{GHz}$, in a 2-m-radius tunnel $\left(r_{0}=0, r_{1}=2 \mathrm{~m}, r_{N} \rightarrow \infty\right)$, with $\epsilon_{1\{s, z\}}=\epsilon_{0}$ and $\epsilon_{2\{s, z\}}=(10-j) \epsilon_{0}$. For this problem, we have calculated the hybrid modes eigenvalues with azimuthal dependence $n=1$ for the unbounded waveguide. This problem was solved employing a PML to bound the radial space. We consider two cases: PML 1 using $\tilde{r}_{N}=3+i 0.03 \mathrm{~m}$, and PML 2 using $\tilde{r}_{N}=3+i 0.3 \mathrm{~m}$. For both cases, the PML extends over $2.5 \mathrm{~m}<\rho<3 \mathrm{~m}$. The eigenvalues for these cases are shown in Fig. 3.71, and are in agreement with the guided modes of the unbounded waveguide. The axial electric field of the mode identified by $A$ in Fig. 3.71 is shown in Fig. 3.72. Note the continuity of the field across the interface in $\rho=2 \mathrm{~m}$, and also note the significantly field damping inside the PML for $\rho>2.5 \mathrm{~m}$.

We consider now an example shown in [11], where a tunnel with circular cross-section is excited by an electric loop antenna. This system operates at $1 \mathrm{GHz}$, in a 2-m-radius tunnel $\left(r_{0}=0, r_{1}=2 \mathrm{~m}, r_{N} \rightarrow \infty\right)$, with $\epsilon_{1\{s, z\}}=\epsilon_{0}$, 


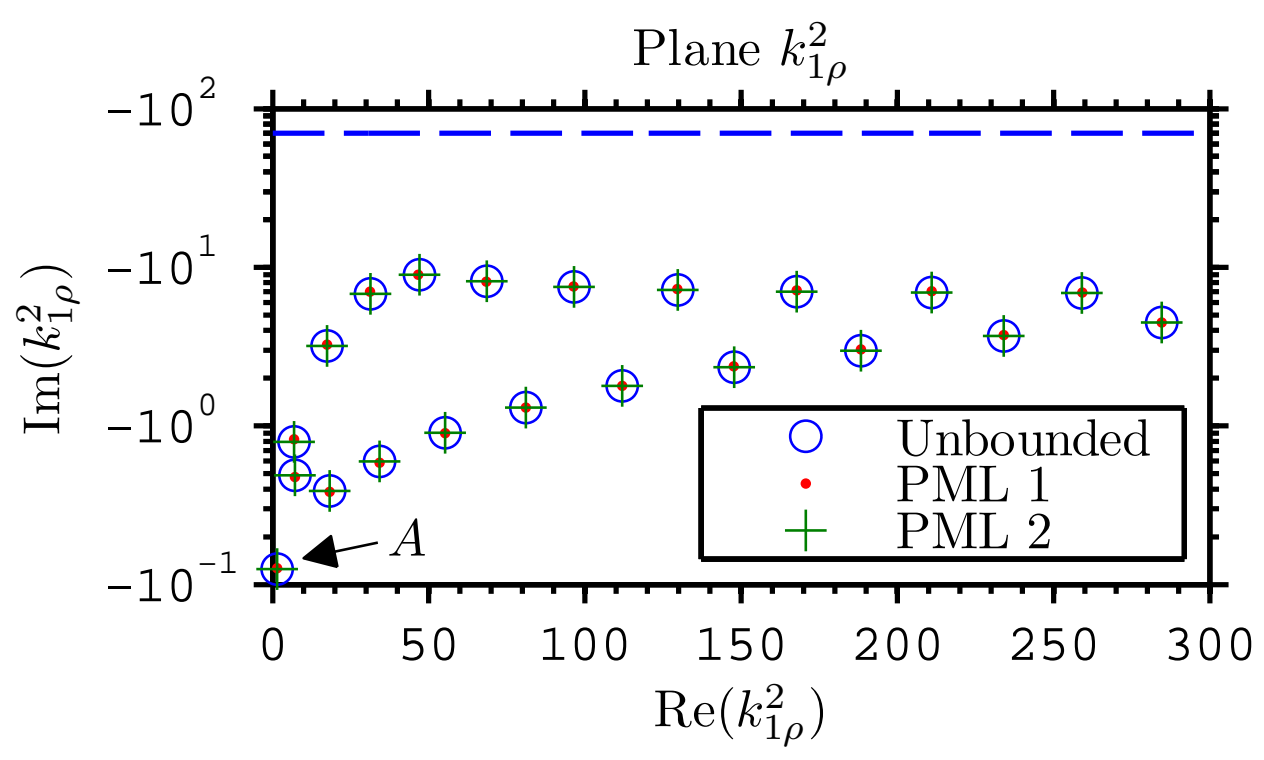

Figure 3.71: Eigenvalues $k_{1 \rho}^{2}$ for $1 \mathrm{GHz}$ hybrid $\mathrm{HE}_{1 p}$ and $\mathrm{EH}_{1 p}$ modes for a 2-m-radius tunnel, with $\epsilon_{1\{s, z\}}=\epsilon_{0}$ and $\epsilon_{2\{s, z\}}=(10+i) \epsilon_{0}$. There is branchcut associated to the unbounded problem, and it is depicted by the horizontal dashed-line.

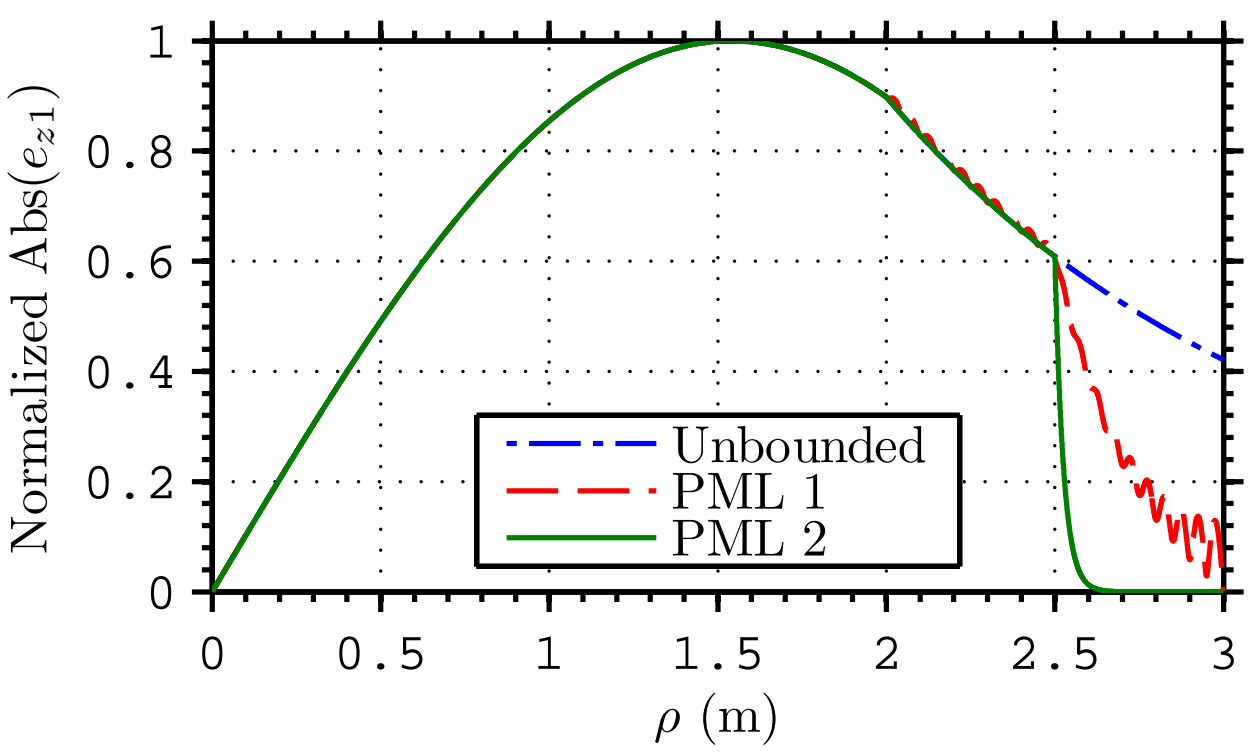

Figure 3.72: Axial electric field of the mode $A\left(k_{1 \rho}=-1.1968+i 0.0523\right)$ for the unbounded $\left(r_{N} \rightarrow \infty\right)$ waveguide, and for the bounded PML 1 $\left(\tilde{r}_{N}=3+i 0.03 \mathrm{~m}\right)$ and PML $2\left(\tilde{r}_{N}=3+i 0.3 \mathrm{~m}\right)$.

$\epsilon_{2 r\{s, z\}}=12$ and $\sigma_{2\{s, z\}}=0.02 \mathrm{~S} / \mathrm{m}$. The electric loop has the electric moment $j_{T}=I_{T} \rho_{T}$, with $\rho_{T}=0.1 \mathrm{~m}$, and due the electric current $I_{T}$. According the formalism shown in Section 3.5.4, this source is a particular case of a TCA with $\theta_{T}=0$.

It is important to observer that the complete solution of the fields of an unbounded waveguide must into account both the discrete (guided) plus the continuum modes [103], [15, 333-334]. For the presented formulation, the 
solution is approximated by a set of discrete modes (guided plus PML modes). In [11], only the guided modes are included in the solution because in the highfrequency, for fields far from the source, the continuum modes is negligible. In the same way as the formulation in [11], we considered $16 \mathrm{TE}^{z}$ guided modes to ensure the convergence.

In Fig. 3.73, we shown the results of the axial variation of the field $E_{\phi}$. The results obtained using the presented formulation show good agreement with those in [11]. As expected, the solutions including or not a PML show negligible differences. For this reason, our results shown in Fig. 3.73 employed only the guided modes of the unbounded waveguide $\left(s_{\rho}=1\right)$.

Next, we consider an example shown in [12], where a tunnel with circular cross-section is excited by a $\hat{x}$-oriented elementary electric current source with moment of $2 \mathrm{~A} \cdot \mathrm{m}$ at origin. This system operates at $1 \mathrm{GHz}$, in a $2 \mathrm{~m}$ radius tunnel, with $\epsilon_{1}=\epsilon_{0}, \epsilon_{2}=12 \epsilon_{0}+i 0.02 / \omega$. Note that the fields in a radially stratified waveguide in general are hybrid modes, but, from Section 3.5.1, we can show that this source excites only hybrid modes with azimuthal dependence $n= \pm 1$. In Fig. 3.74, we shown the results of the axial variation of the field $E_{x}$ at $\rho=0.5 \mathrm{~m}$ and $\phi=90^{\circ}$.

The results obtained by using the presented formulation show good agreement with those in [12], where only the guided modes are included in far-field solution because the contribution of the continuum mode is negligible at high-frequency. Our solution showed negligible differences with and without the PML $\left(r_{P M L}=2.5 \mathrm{~m}, r_{N}=3 \mathrm{~m}, \alpha_{P M L}=0.2\right)$. We consider 13 hybrid modes to ensure the convergence of the fields; the same number of modes used in $[12]$.

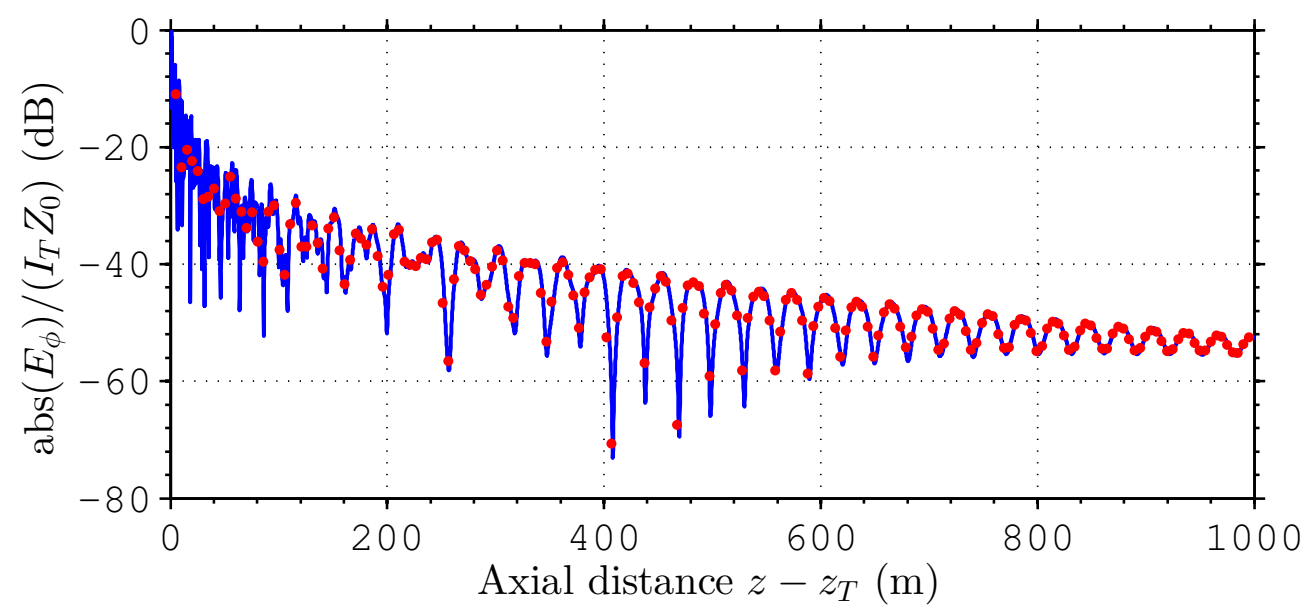

Figure 3.73: Comparison of the electric field along the axial distance. The results of our approach are depicted by the solid line. The small dots are the results from [11]. The free-space impedance is $Z_{0}$. 


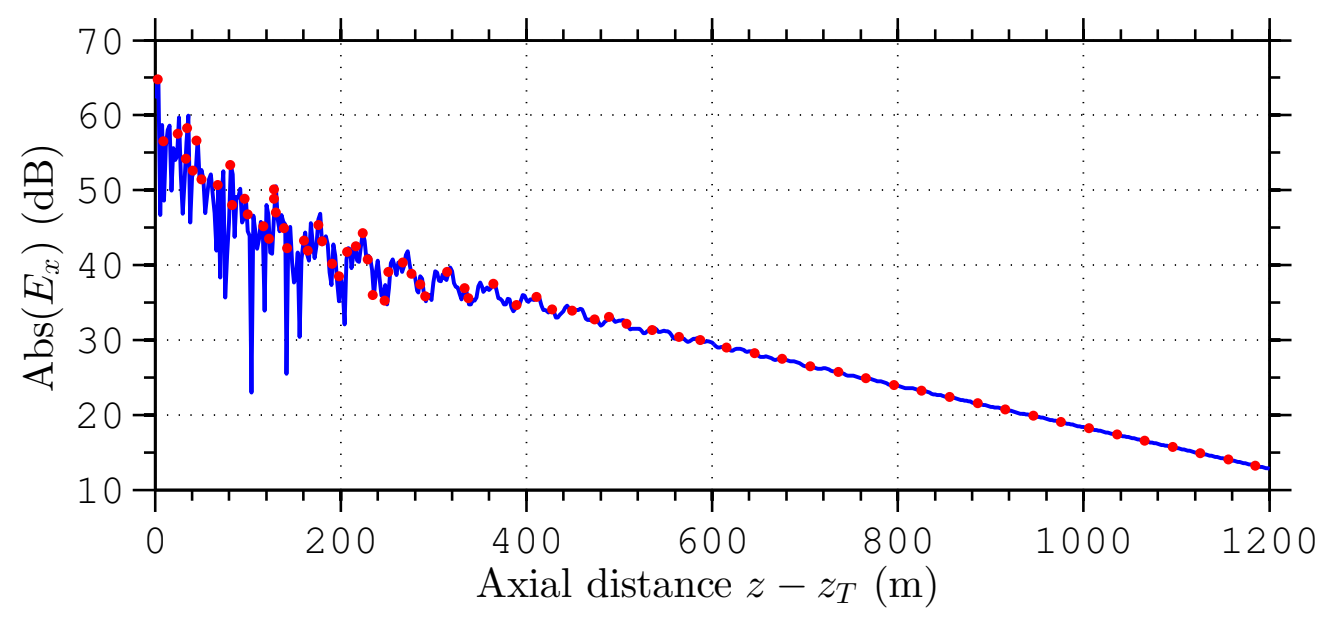

Figure 3.74: Comparison of the electric field along the axial distance. The results of our approach are depicted by the solid line. The small dots are the results from [12].

\subsection{1}

\section{Preliminary Conclusion}

We have introduced a new pseudo-analytical formulation to model tiltedcoil antenna LWD tools in anisotropic formations. The combination of wellknown closed-form solutions of Maxwell's equations in cylindrical coordinates with an efficient PML allow us to express the fields in radially-layered Earth formations as a sum of discrete modes. The TCA source is expanded in terms of modal fields, and then the radiated fields along vertical formations are found using an analytical axial mode-matching. We showed numerical results to validate the method and illustrate its ability to analyze directional well-logging tools. We also presented numerical simulations of TCA housed inside grooves into a metallic mandrel, revealing some features that can be incorporated into the design of novel LWD sensors. 


\section{4}

\section{Analysis of Well-Logging Tools in Directional Wells}

\section{1 \\ Introduction}

The introduction of tilted-coil antennas (TCAs) have provided the azimuthal sensitivity to logging-while-drilling (LWD) tools. This technology enables the anticipation of lithological variations in the soil formation surrounding the oil well so that the proactive geosteering allows the exploration of deviated and horizontal wells by means of real-time adjustments in the drilling direction to steer the well towards a target region.

Compared to convention (i.e., strictly vertical) drilling, directional drilling yield several advantages for oil and gas exploration as oilfield productivity can be increased by deploying multiple wells drilled from a single platform. Additional benefits of directional drilling include: (1) drilling of a new well to intersect and recover an uncontrolled well, (2) sidetrack an old well to prospect new reservoirs, (3) horizontal wells placement, among others.

Electromagnetic simulations of complex directional wells can be done by brute-force techniques such as finite-differences (FDs) [124,125], finite-elements (FEs) [20,126], finite-volumes (FVs) [45] or method of moments (MoM) [127]; however, their computational cost can become prohibitive for large problems. As the borehole orientation is not conformal with typical discretization grids employed in those brute-force algorithms, a very fine space discretization is necessary for the sake of accurately capture the well curvature effects. In order to avoid large errors introduced in these staircase approximation, the transformation optics (TO) [51, Sec. 7.8], [128-130] approach can be used to maps the space of complex-shaped boreholes into others more feasible to be handled in usual algorithms. As a consequence of the TO mapping, however, the equivalent problem will be comprised by highly non-homogeneous media.

As an alternative, pseudo-analytical methods based on the Fourier-Bessel transform [14] or numerical mode-matching (NMM) $[8,15]$ can be used to model geophysical formations comprised of both radial and axial stratifications, as shown in Chapter 3. However, the modeling of more complex geophysical formations as those of directional wells remains a challenging task to such 
pseudo-analytical methods.

In this chapter, we describe a new pseudo-analytical technique to analyze the electromagnetic propagation along directional wells, where axial bending is present. First, we further simplify the complex three-dimensional (3D) oriented well (shown in Fig. 4.1) to be modeled as sections of toroidal structures (shown in Fig. 4.2). Generally speaking, now we need to express the field solutions for Maxwell's equations in toroidal coordinates.

The Helmholtz equation in toroidal coordinates is not separable as the product of three functions, each one with an exclusive dependence with one spacial coordinate [13, p. 112-115], such the exact eigenmode solutions involve complicated hypergeometric functions [131]. A more feasible approach to decouple and solve the fields in toroidal structure was presented in [51] using a perturbation solution in terms of the torus curvature, viz., $R^{-1}$, such the zeroth-order solution recover the well-known shape of the eigenfields in cylindrical coordinates. This perturbation method was used to model several toroidal cavities and waveguides solving Maxwell's equations by coupled mode theory (CMT) $[132,133]$ or as a direct solution in the perturbation theory for

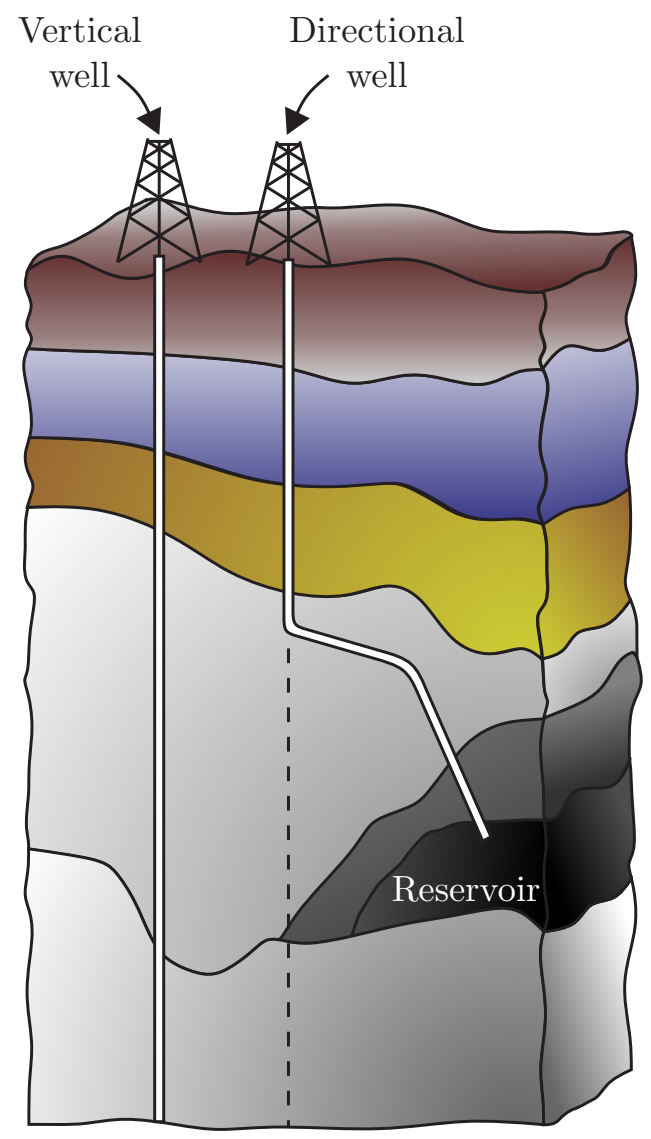

Figure 4.1: Geometry of vertical and directional wells. 


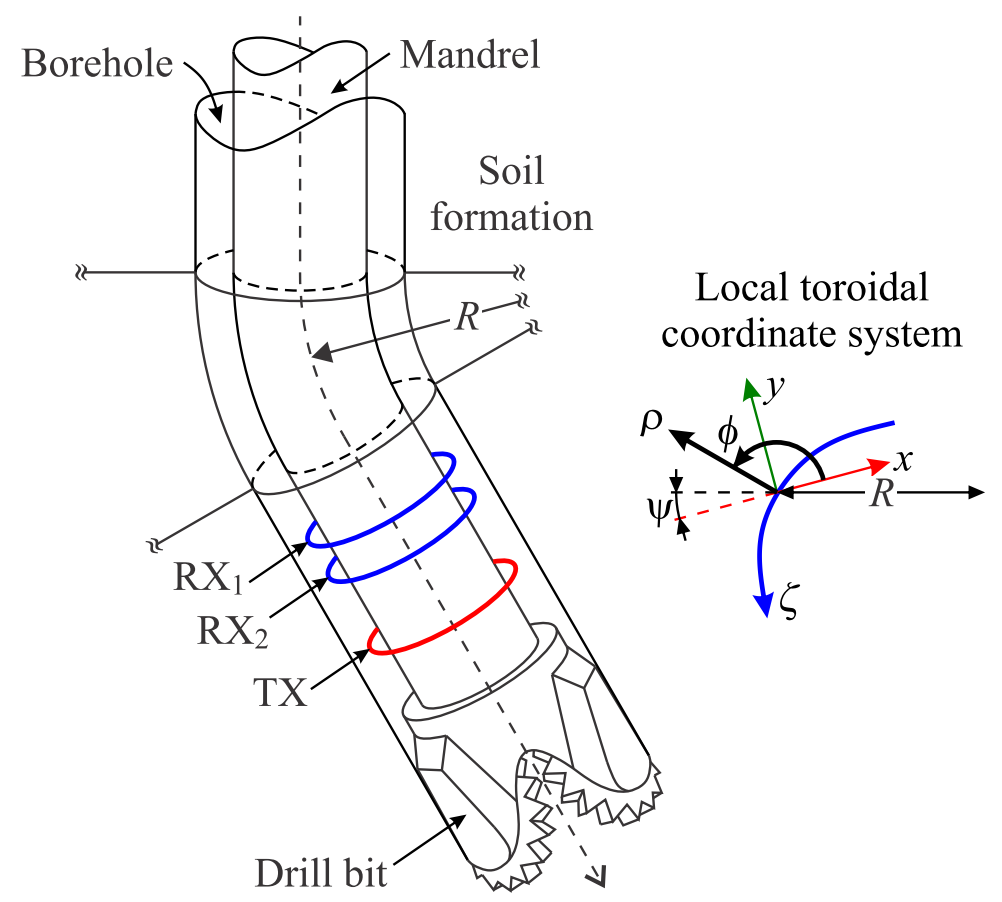

Figure 4.2: Geometry of a well-logging sensor within a directional well. An auxiliary coordinate system is presented.

differential equations (PTDE) ${ }^{1}[51$, Ch. 6], [135-142].

In CMT, the solutions are often expressed in terms of first-order (coupled) differential equations. In this problem, the coupling coefficients are expressed as surface integrals; analytical solutions are rare and sometimes a numerical integration is required. In a final step, the coupled system of differential equations must be numerically solved using an iterative Runge-Kutta method.

In PTDE, we can in general enforce a zeroth-order solution in the shape of a well-know differential equation; such as the scalar Helmholtz equation in cylindrical coordinates. In the next step, we seek recursively high-order perturbation corrections for the original problem: for each perturbation order, we need to solve a new non-homogeneous second-order linear differential equation, that depends on all the lower-order solutions. This approach does not rely on numerical solutions for coupling integrals or iterative methods, but the complications to solve new (and not tabulated) differential equations restrict its application to the modeling of simple hollow cavities excited by the main modal fields.

In this chapter, we extend the range of applicability classical PTDE methods by considering the modeling of finite-curvature sectors of radiallystratified toroidal waveguides. Each toroidal waveguide sector considered here has a constant torus curvature radius and are filled with radially-stratified

\footnotetext{
${ }^{1}$ Also known as Rayleigh-Schrödinger Perturbation Theory [134, Ch. 15].
} 
and isotropic media. New formulas are derived to describe the finite-curvature problem, such Maxwell's equations are expressed as a series of the torus curvature $\left(R^{-1}\right)$ (see Fig. 4.2 for a more clear illustration), which naturally recover the previously presented solution derived in Chapter 3. Next, using a generalization of the axial mode-matching we can proper satisfy the boundary conditions between each waveguide junction. The excitations are represented by a set of modal amplitudes, which are then combined with the new straightto-bend (or bend-to-bend) generalized scattering matrices (GSMs), similarly to the procedure used in Chapter 3.

The remaining of this chapter is organized as follows. Section 4.2 presents a detailed description of the electromagnetic fields into our particular toroidal coordinate system. We employ a local toroidal coordinate system that preserves the polar cross-section along the bend direction, and presents concentric radial surfaces which suitably match with our desired boundary conditions. In contrast to straight cylindrical guides, the axial fields are no longer decoupled in toroidal structures. We have employed new bi-complex fields to decouple the original problem. Also, we present a new alternative way to derive decoupled wave equations in toroidal coordinates without bi-complex numbers. We present the first-order perturbation corrections for the electromagnetic fields in toroidal structures. The field solutions are expressed in terms of a series of the torus curvature, whose zeroth-order solution reduces exactly to the wellknown fields in cylindrical coordinates. Section 4.3 and Section 4.4 present formulas to match the boundary conditions of a radially-stratified and axiallylayered toroidal waveguide, respectively. The modeling of LWD sensors as coil transmitters and receivers antennas is considered in Section 4.5. An analytical study predicts that the axial finite-curvature of directional boreholes only support azimuthally non-symmetric hybrid modal fields (with all fieldcomponents present). In Section 4.6, preliminary results demonstrate the ability of our method to accurately modeling LWD tools in deviated drilling conditions. Finally, Section 4.7 provides preliminary concluding remarks.

\section{2}

\section{Electromagnetic Fields in Toroidal Coordinates}

Consider a circular-cross-section structure aligned with the axial direction $z$, as shown in Fig. 4.3. The electromagnetic fields for problems having boundaries coincident with cylindrical coordinates $(\rho, \phi, z)$ are well-know [55, Ch. 6], [15, Ch. 3], and the method of separation of variables can be used to find the field solutions. Note that the axis $z$ in this structure presents an infinite radius of curvature. Now consider that the axial axis was perturbed, and 

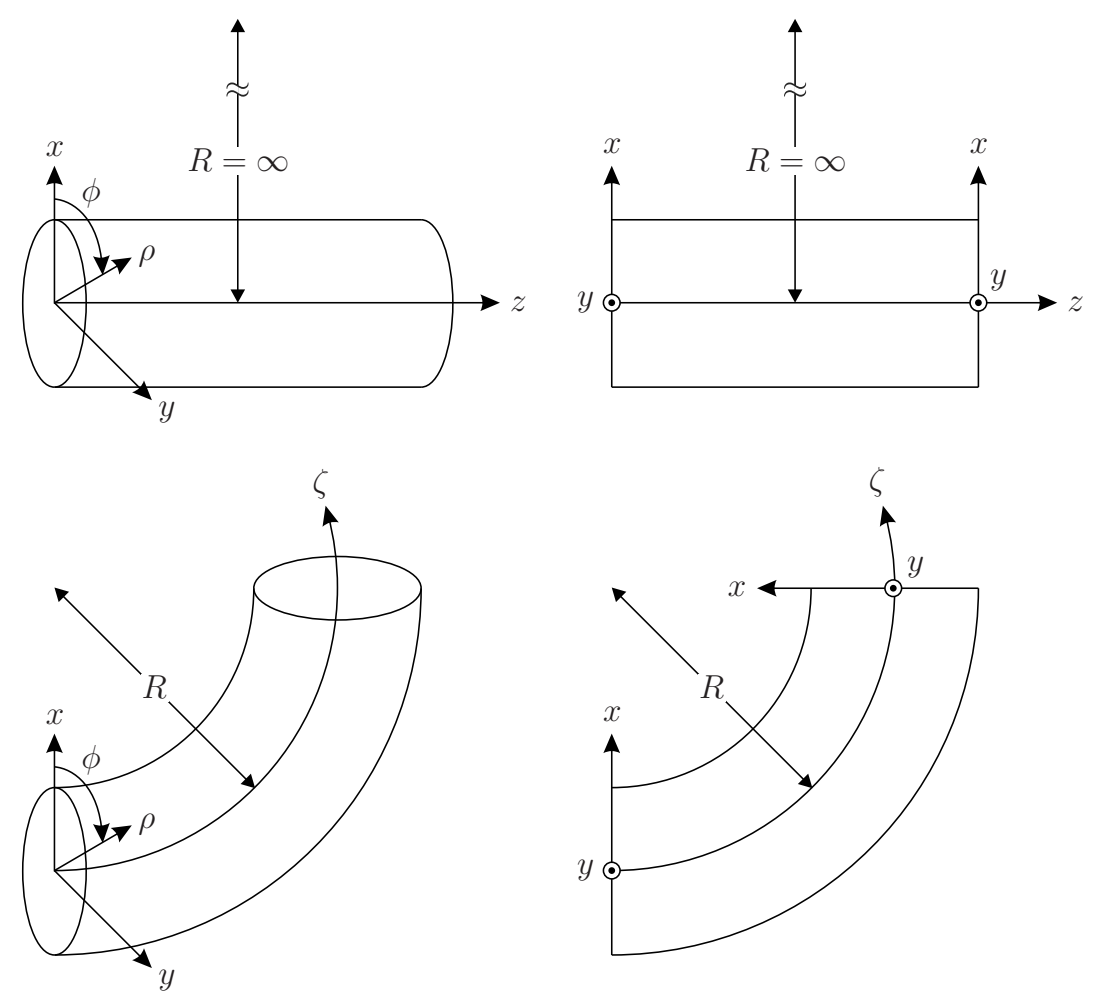

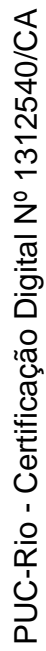

Figure 4.3: Cylindrical $(\rho, \phi, z)$ and toroidal $(\rho, \phi, \zeta)$ coordinate systems.

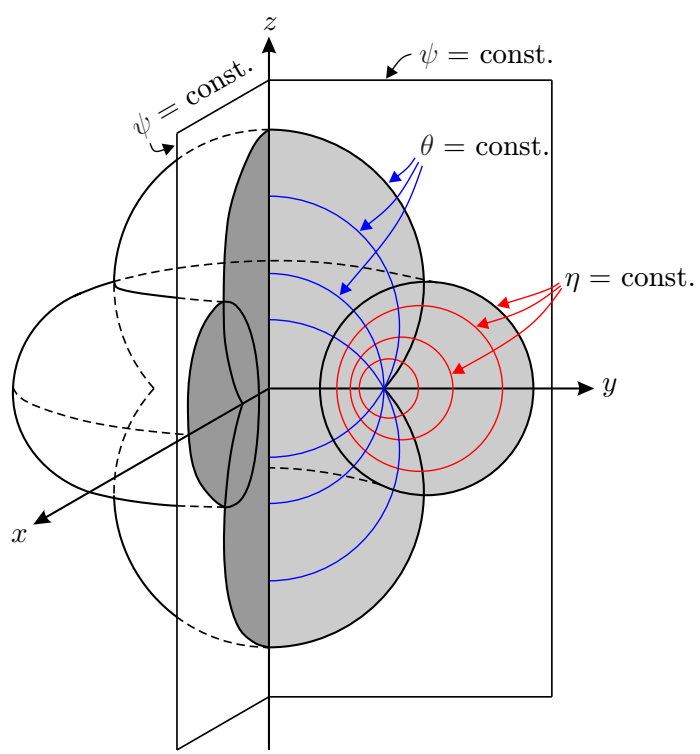

Figure 4.4: Toroidal coordinates $(\eta, \theta, \psi)[13$, p. 113, Fig. 4.04]. The coordinate surfaces are toroids $(\eta=$ const.), spherical bowls $(\theta=$ const.) and half-planes $(\psi=$ const. $)$.

presents a constant radius of curvature $R$.

The choice of a coordinate system conformal with the positions of our boundary conditions is quite appropriate to simplify the problem at hand. At first glance, a standard toroidal coordinate system $(\eta, \theta, \psi)$ [13, pp. 112-115] appear a good choice. Our interest is to model curved waveguides with several 
radial stratifications, such that the transversal cross-section fits nicely with the polar coordinates. However, the surfaces of constant $\eta$ are not concentric as we can see in Fig. 4.4. Furthermore, we have another severe complication: the Helmholtz differential equation is not separable in toroidal coordinates.

Our efforts are to represent the fields into the bend structure in a way that they resemble the straight waveguide modes. Thus, to analyze this structure, we employ our local toroidal coordinates $(\rho, \phi, \zeta)$ defined in details in Appendix $\mathrm{C}$, which employ a polar cross-section coordinates along the bend direction $\zeta$ as shown in Fig. 4.3. This coordinate system presents concentric radial surfaces which match neatly with the boundaries of our problem, as shown in Fig. C.1. The metric coefficients are given by

$$
h_{\rho}=1, \quad h_{\phi}=\rho, \quad h_{\zeta}=h=1-R^{-1} \rho \cos \phi .
$$

We assume that the center of curvature points to $\phi=0$. This direction could be placed at a general angle $\phi=\psi$, such that the axial metric coefficient turns into $h_{\zeta}=1-R^{-1} \rho \cos (\phi+\psi)$.

Assuming time harmonic fields in the form $\exp (-i \omega t)$, the Maxwell's curls equations in an isotropic and source-free medium in a curvilinear coordinate system are given by [51, p. 21], [143, p. 50]

$$
\begin{aligned}
& \frac{\partial\left(h_{3} E_{3}\right)}{\partial u_{2}}-\frac{\partial\left(h_{2} E_{2}\right)}{\partial u_{3}}=i \omega \mu h_{2} h_{3} H_{1} \\
& \frac{\partial\left(h_{1} E_{1}\right)}{\partial u_{3}}-\frac{\partial\left(h_{3} E_{3}\right)}{\partial u_{1}}=i \omega \mu h_{3} h_{1} H_{2} \\
& \frac{\partial\left(h_{2} E_{2}\right)}{\partial u_{1}}-\frac{\partial\left(h_{1} E_{1}\right)}{\partial u_{2}}=i \omega \mu h_{1} h_{2} H_{3}
\end{aligned}
$$

$$
\begin{aligned}
& \frac{\partial\left(h_{3} H_{3}\right)}{\partial u_{2}}-\frac{\partial\left(h_{2} H_{2}\right)}{\partial u_{3}}=-i \omega \epsilon h_{2} h_{3} E_{1}, \\
& \frac{\partial\left(h_{1} H_{1}\right)}{\partial u_{3}}-\frac{\partial\left(h_{3} H_{3}\right)}{\partial u_{1}}=-i \omega \epsilon h_{3} h_{1} E_{2}, \\
& \frac{\partial\left(h_{2} H_{2}\right)}{\partial u_{1}}-\frac{\partial\left(h_{1} H_{1}\right)}{\partial u_{2}}=-i \omega \epsilon h_{1} h_{2} E_{3} .
\end{aligned}
$$

By using the metric coefficients in (4-1), defining the new axial fields

$$
E=h E_{3}, \quad H=h H_{3},
$$

and $Z=(\mu / \epsilon)^{1 / 2}$ and $Y=(\epsilon / \mu)^{1 / 2}$, for the toroidal coordinate system 
$\left(u_{1}, u_{2}, u_{3}\right)=(\rho, \phi, \zeta)$, we can derive

$$
\begin{aligned}
\frac{1}{\rho h}\left(\frac{\partial E}{\partial \phi}-\rho \frac{\partial E_{\phi}}{\partial \zeta}\right) & =i k Z H_{\rho} \\
\frac{1}{h}\left(\frac{\partial E_{\rho}}{\partial \zeta}-\frac{\partial E}{\partial \rho}\right) & =i k Z H_{\phi} \\
\frac{h}{\rho}\left(\frac{\partial\left(\rho E_{\phi}\right)}{\partial \rho}-\frac{\partial E_{\rho}}{\partial \phi}\right) & =i k Z H \\
\frac{1}{\rho h}\left(\frac{\partial H}{\partial \phi}-\rho \frac{\partial H_{\phi}}{\partial \zeta}\right) & =-i k Y E_{\rho} \\
\frac{1}{h}\left(\frac{\partial H_{\rho}}{\partial \zeta}-\frac{\partial H}{\partial \rho}\right) & =-i k Y E_{\phi} \\
\frac{h}{\rho}\left(\frac{\partial\left(\rho H_{\phi}\right)}{\partial \rho}-\frac{\partial H_{\rho}}{\partial \phi}\right) & =-i k Y E .
\end{aligned}
$$

Noting that $h=h_{\zeta}$ is independent of $\zeta$, we can seek field solutions with uniform propagation in the form $\exp \left(i k_{\zeta} \zeta\right)=\exp (\gamma \zeta)$, where $\partial / \partial \zeta$ can be replaced by $\gamma$.

\subsection{1}

\section{Decoupling Axial Fields using Bi-Complex Systems}

In order to solve the Maxwell's equations (4-4a) to (4-4f) we need first decouple the individual field components. To proceed, we will use a procedure introduced in $[51,144]$, in which new transversal fields are defined by

$$
E_{t}=E_{\rho}+j E_{\phi}, \quad H_{t}=H_{\rho}+j H_{\phi},
$$

where a new complex variable $j$ was introduced, such as $j^{2}=-1$, but $i j=j i \neq-1$. We can also define the differential operators $D$ and $D^{*}$ as

$$
D, D^{*} \equiv \frac{\partial}{\partial \rho} \pm j \frac{1}{\rho} \frac{\partial}{\partial \phi}
$$

so that the pairs of equations (4-4a) and (4-4b), and (4-4d) and (4-4e) can be combined and written in the short forms

$$
\begin{gathered}
D(E)-\gamma E_{t}=j i k h Z H_{t}, \text { and } \\
D(H)-\gamma H_{t}=-j i k h Y E_{t} .
\end{gathered}
$$

By isolating $H_{t}$ in (4-7b) and replacing in (4-7a), and performing a similar procedure in (4-7b), after some manipulations we can write (4-7) as 


$$
\begin{gathered}
k_{t}^{2} E_{t}=\gamma D(E)-j i k h Z D(H), \text { and } \\
k_{t}^{2} H_{t}=\gamma D(H)+j i k h Y D(E),
\end{gathered}
$$

where we introduced the parameter

$$
k_{t}^{2}=k^{2} h^{2}+\gamma^{2}
$$

It is important to observer that $k_{t}$ is not the transversal propagation constant, since $h$ is function of $\rho$ and $\phi$.

To further analysis, we use the following properties of the operator $D^{*}$ in cylindrical coordinates:

$$
-\Re e_{j}\left[j D^{*}\left(\rho E_{t}\right)\right]=\frac{\partial}{\partial \rho}\left(\rho E_{\phi}\right)-\frac{\partial E_{\rho}}{\partial \phi},
$$

and also a similar relation to $H_{t}$ :

$$
-\Re e_{j}\left[j D^{*}\left(\rho H_{t}\right)\right]=\frac{\partial}{\partial \rho}\left(\rho H_{\phi}\right)-\frac{\partial H_{\rho}}{\partial \phi} .
$$

In the above system of bi-complex ${ }^{2}$ equations, the operator $\Re e_{j}$ take the real part of the quantities in the complex variable $j$. For simplifying the notation, the subscript $j$ in $\Re e_{j}$ will be omitted in what follows, and $\Re e$ and $\Im m$ shall be understood as operators that only act over $j$.

By noting that $(4-10) \times h \rho^{-1}$ is equal to $(4-4 \mathrm{c})$, we can write

$$
-\frac{h}{\rho} \Re e\left[j D^{*}\left(\rho E_{t}\right)\right]=i k Z H
$$

and as $(4-11) \times h \rho^{-1}$ is equal to (4-4f), we can verify that

$$
-\frac{h}{\rho} \Re e\left[j D^{*}\left(\rho H_{t}\right)\right]=-i k Y E .
$$

The results in (4-12) and (4-13) can be used into the equations in (4-8) to get a new pair of differential equations using only the axial fields $E$ and $H$. Applying the operator $-h \rho^{-1} \Re e\left(j D^{*}\right)$ over $(4-8) \times \rho k_{t}^{-2}$, and using the results of the last two equations above, we can find

$$
\begin{aligned}
i k \rho Z H & =-h \Re e\left\{D^{*}\left\{\rho k_{t}^{-2}[j \gamma D(E)+i k h Z D(H)]\right\}\right\}, \text { and } \\
-i k \rho Y E & =-h \Re e\left\{D^{*}\left\{\rho k_{t}^{-2}[j \gamma D(H)-i k h Y D(E)]\right\}\right\} .
\end{aligned}
$$

The relationships in (4-14) and (4-15) form a pair of coupled equations for $E$ and $H$, and before trying to solve it, seems convenient to perform some pertinent observations.

${ }^{2}$ Note that there are two complex variables $(i$ and $j$ ) in the pair of coupled equation (4-10) and (4-11). 
We can verify that $[51$, p. 61$]$

$$
D^{*}[\rho D(f)]=\rho \nabla_{t}^{2} f
$$

where $f$ is any differentiable function and $\nabla_{t}^{2}$ is the transversal Laplacian operator

$$
\nabla_{t}^{2}=\frac{1}{\rho} \frac{\partial}{\partial \rho}\left(\rho \frac{\partial}{\partial \rho}\right)+\frac{1}{\rho^{2}} \frac{\partial^{2}}{\partial \phi^{2}}
$$

Note that both the cylindrical and the toroidal coordinate systems used here share the same transversal coordinates $\rho$ and $\phi$. Then, the $\nabla_{t}^{2}$ is equal to the well-know transversal Laplacian in cylindrical coordinates shown in [55, p. 449], [15, p. 162]. However, the Laplacian operator in toroidal coordinates assumes the form $\nabla^{2}=\nabla_{t}^{2}+1 / h_{\zeta}^{2} \partial^{2} / \partial \zeta^{2}$.

\subsubsection{1}

\section{The Curvature Vanishing Special Case}

For the particular case when $R \rightarrow \infty$, the toroidal structure recover the cylindrical form. For this case, the axial metric coefficient becomes $h \rightarrow 1$, and hence $k_{t}$ indeed becomes the radial propagation constant. Moreover, the axial coordinate $\zeta \rightarrow z$. By using (4-16) in both (4-14) and (4-15), we can clearly see that the first term in the right-hand side (RHS) of these equations become purely imaginary in term of $j$. As we are interested just in the real (in $j$ ) parcels of the RHS, the first term in the RHS of (4-14) and (4-15) become zero. Now, we can verify that the equations (4-14) and (4-15) become decoupled, simplifying to

$$
\begin{aligned}
& \left(\nabla_{t}^{2}+k_{\rho}^{2}\right) H_{z}=0, \text { and } \\
& \left(\nabla_{t}^{2}+k_{\rho}^{2}\right) E_{z}=0,
\end{aligned}
$$

that are the well-know Helmholtz equations for cylindrical structures [54, p. 165]. Note that $k_{\rho}=\left.k_{t}\right|_{R \rightarrow \infty}$ is the radial propagation constant.

\subsubsection{2}

\section{Solving Coupled Differential Equations}

In last section, we have obtained the coupled differential equations (4-7a) and (4-7b). Also, we have expressed these equations in an equivalent form, given by (4-14) and (4-15) . To decouple these equations, we follow the process shown in [51, Ch. 2] and [144]. To take the decouple process we need to combine these equations in such a way that the axial $(E$ and $H)$ and transversal $\left(E_{t}\right.$ and $H_{t}$ ) fields come together in a form involving a single combinations. In the first step, we combine (4-7a) and (4-7b) using an unspecified multiplier $N$. 
Note that, at this point, there is no restrictions to $N$, but we now that this parameter may be complex in term of both $i$ and/or $j$. Hence, (4-7a) multiplied by $N$ and added to (4-7b) results in

$$
D(E+N H)-\gamma\left(E_{t}+N H_{t}\right)=-j i k h\left(N Y E_{t}-Z H_{t}\right) .
$$

Note that we need to choose $N$ so that the combination of $E_{t}$ and $N H_{t}$ in left and in the right sides of the above equation is the same. As in the right-hand side of the equation there is a negative sign along with $H_{t}$, we can easily verify that $N$ must include a complex variable ( $i$ or $j$ ). Also, note that we have both $i$ and $j$ multiplying the combination of $E_{t}$ and $H_{t}$ in the right-hand side of (4-20). Accordingly, if we rewrite (4-20) as

$$
D(E+N H)-\gamma\left(E_{t}+N H_{t}\right)=j k h\left(-i N Y E_{t}+i Z H_{t}\right),
$$

we can see that $N=i Z$ allow us to combine the fields in a single form. Furthermore, note that $N=Z / i$ also allow us to combine (4-7a) and (4-7b). Then, we can verify that

$$
N= \pm N_{0}= \pm i Z
$$

satisfies $(4-20)^{3}$.

Now, if we define a new field $F$, which is a linear combination of $E$ and $H$ in the form

$$
F_{ \pm}=E \pm N_{0} H
$$

and by analogy defining $F_{t, \pm}$ as

$$
F_{t, \pm}=E_{t} \pm N_{0} H_{t}
$$

we can rewrite (4-20) as

$$
D\left(F_{ \pm}\right)-\gamma F_{t, \pm}= \pm j k h F_{t, \pm}
$$

To proceed, we need to eliminate the transversal fields in (4-25). The combination of the equations (4-14) and (4-15) as $-(4-15) \times Z \pm N_{0} \times(4-14) \times Y$ leads to

$$
\begin{aligned}
& i k \rho\left(E \pm N_{0} H\right)= \\
& \quad-h \Re e\left\{D^{*}\left\{\rho k_{t}^{-2}\left[j \gamma D\left(-Z H \pm N_{0} Y E\right)+i k h D\left(E \pm N_{0} H\right)\right]\right\}\right\} .
\end{aligned}
$$

Using the previous definitions for $F_{ \pm}$and noting that $-Z H \pm N_{0} Y E= \pm i F_{ \pm}$,

${ }^{3}$ It is important to be noted that $N_{0}=i Z$ is just one of the possible ways of combining fields in (4-20). This equation is also satisfied by $N_{0}=j Z$. But, as we expect fields that are real in terms of $j$, we must select the form $N_{0}=i Z$. 
we can find

$$
\begin{aligned}
i k \rho\left(F_{ \pm}\right) & =-h \Re e\left\{D^{*}\left\{\rho k_{t}^{-2}\left[ \pm i j \gamma D\left(F_{ \pm}\right)+i k h D\left(F_{ \pm}\right)\right]\right\}\right\} \\
& =-i h \Re e\left\{D^{*}\left[\rho k_{t}^{-2}( \pm j \gamma+k h) D\left(F_{ \pm}\right)\right]\right\}
\end{aligned}
$$

Multiplying $i$ in both sides of the above equation, and noting that

$$
\frac{k h \pm j \gamma}{k_{t}^{2}}=\frac{k h \pm j \gamma}{k^{2} h^{2}+\gamma^{2}}=\frac{k h \pm j \gamma}{(k h \pm j \gamma)(k h \mp j \gamma)}=\frac{1}{k h \mp j \gamma}
$$

we can find

$$
-k \rho F_{ \pm}=h \Re e\left\{D^{*}\left[\frac{\rho}{k h \mp j \gamma} D\left(F_{ \pm}\right)\right]\right\}
$$

which is one pair $\left(F_{-}\right.$and $\left.F_{+}\right)$of uncoupled equations. After we found the solution for $F_{ \pm}$, the axial fields $E$ and $H$ can be easily recovered by using

$$
\begin{gathered}
E=\frac{F_{+}+F_{-}}{2}, \text { and } \\
H=\frac{F_{+}-F_{-}}{2 N_{0}} .
\end{gathered}
$$

Remembering that $E=h_{\zeta} E_{\zeta}$ and $H=h_{\zeta} H_{\zeta}$, at this point is more practical to work with the axial fields $E_{\zeta}$ and $H_{\zeta}$ instead of using $E$ and $H$. We can do this by using the new field $G$, defined by

$$
G_{ \pm}=E_{\zeta} \pm N_{0} H_{\zeta}=\frac{F_{ \pm}}{h} .
$$

Replacing (4-33) in (4-30) $\times h^{-1}$ we can write

$$
-k \rho G_{ \pm}=\Re e\left\{D^{*}\left[\frac{\rho}{k h \mp j \gamma} D\left(h G_{ \pm}\right)\right]\right\} .
$$

To continue toward the solution, consider $f_{1}$ and $f_{2}$, both functions of $\rho$ and $\phi$. We can show that

$$
D\left(f_{1} f_{2}\right)=f_{1} D f_{2}+f_{2} D f_{1}
$$

Taking the conjugate with respect to $j$ in (4-35) we can derive a similar equation for $D^{*}$.

Using the result in (4-35) into (4-34), we can write

$$
-k \rho G_{ \pm}=\Re e\left\{D^{*}\left[\frac{h}{k h \mp j \gamma} \rho D\left(G_{ \pm}\right)+\frac{\rho}{k h \mp j \gamma} G_{ \pm} D(h)\right]\right\} .
$$

By using the conjugate version of (4-35) in terms of $j$, the above equation 
takes the shape of

$$
\begin{aligned}
-k \rho G_{ \pm} \stackrel{\Re e}{=} \frac{h}{k h \mp j \gamma} D^{*}\left(\rho D G_{ \pm}\right)+\rho D\left(G_{ \pm}\right) D^{*}\left(\frac{h}{k h \mp j \gamma}\right) \\
+\frac{\rho}{k h \mp j \gamma} D^{*}\left[G_{ \pm} D(h)\right]+G_{ \pm} D(h) D^{*}\left(\frac{\rho}{k h \mp j \gamma}\right)
\end{aligned}
$$

where the symbol $\stackrel{\Re e}{=}$ shall be understood as the real part in respect to $j$ from the expression on the right-hand side of equality; i.e., LHS $\stackrel{\Re e}{=}$ RHS is equivalent to LHS $=\Re e_{j}(\mathrm{RHS})$.

Using

$$
\begin{aligned}
D(h) & =\left(\frac{\partial}{\partial \rho}+j \frac{1}{\rho} \frac{\partial}{\partial \phi}\right)\left(1-R^{-1} \rho \cos \phi\right) \\
& =R^{-1}(-\cos \phi+j \sin \phi),
\end{aligned}
$$

we can write

$$
\begin{aligned}
-k \rho G_{ \pm} \stackrel{\Re e}{=} \frac{h}{k h \mp j \gamma} D^{*}\left(\rho D G^{ \pm}\right)+\rho D\left(G_{ \pm}\right) D^{*}\left(\frac{h}{k h \mp j \gamma}\right) \\
+R^{-1}\left\{\frac{\rho}{k h \mp j \gamma} D^{*}\left[G_{ \pm}(-\cos \phi+j \sin \phi)\right]\right. \\
\left.+G_{ \pm}(-\cos \phi+j \sin \phi) D^{*}\left(\frac{\rho}{k h \mp j \gamma}\right)\right\} .
\end{aligned}
$$

From (4-16), we found $D^{*}\left[\rho D\left(G_{ \pm}\right)\right]=\rho \nabla G_{ \pm}$, and by using

$$
\begin{gathered}
D^{*}\left(\frac{h}{k h \mp j \gamma}\right)= \pm R^{-1} \frac{j \gamma(\cos \phi+j \sin \phi)}{(k h \mp j \gamma)^{2}}, \\
D^{*}\left(\frac{\rho}{k h \mp j \gamma}\right)=\frac{k h \mp j \gamma+R^{-1} k \rho(\cos \phi+j \sin \phi)}{(k h \mp j \gamma)^{2}},
\end{gathered}
$$

$D^{*}\left[G_{ \pm}(-\cos \phi+j \sin \phi)\right]=-\frac{j}{\rho}(\sin \phi+j \cos \phi) G_{ \pm}+(-\cos \phi+j \sin \phi) D^{*} G_{ \pm}$,

we can derive

$$
\begin{aligned}
-k \rho G_{ \pm} & \stackrel{\Re e}{=} \frac{h}{k h \mp j \gamma} \rho \nabla_{t}^{2} G_{ \pm} \pm R^{-1} \rho D\left(G_{ \pm}\right) \frac{j \gamma(\cos \phi+j \sin \phi)}{(k h \mp j \gamma)^{2}} \\
+ & R^{-1} \frac{\rho}{k h \mp j \gamma}\left[-\frac{j}{\rho}(\sin \phi+j \cos \phi)+(-\cos \phi+j \sin \phi) D^{*}\left(G_{ \pm}\right)\right] \\
+ & R^{-1} \frac{k h \mp j \gamma+R^{-1} k \rho(\cos \phi+j \sin \phi) \rho}{(k h \mp j \gamma)^{2}}(-\cos \phi+j \sin \phi) G_{ \pm} .
\end{aligned}
$$


In order to find the real part of the right side of the equality in the above equation, the following identities are helpful [116, p. 16]:

$$
\begin{aligned}
\Re e_{j}\left(\frac{a+j b}{c \mp j d}\right) & =\frac{a c \mp b d}{c^{2}+d^{2}}, \text { and } \\
\Re e_{j}\left[\frac{a+j b}{(c \mp j d)^{2}}\right] & =\frac{a\left(c^{2}-d^{2}\right) \mp 2 b c d}{\left(c^{2}+d^{2}\right)^{2}}
\end{aligned}
$$

where the parameters $a, b, c$ and $d$ are real in terms of $j$. By using the identities (4-44) and (4-45) in (4-43), after a series of laborious but straightforward simplifications, we can express (4-43) as ${ }^{4}$

$$
\begin{aligned}
& \left(\nabla_{t}^{2}+\frac{k_{t}^{2}}{h^{2}}\right) G_{ \pm}=\frac{R^{-1}}{k_{t}^{2}}\left[R^{-1}\left(k^{2}-\gamma^{2} / h^{2}\right) G_{ \pm}\right. \\
+ & \left.h\left(k^{2}+3 \gamma^{2} / h^{2}\right)\left(\cos \phi \frac{\partial G_{ \pm}}{\partial \rho}-\frac{\sin \phi}{\rho} \frac{\partial G_{ \pm}}{\partial \phi}\right) \pm 2 \gamma k\left(\sin \phi \frac{\partial G_{ \pm}}{\partial \rho}+\frac{\cos \phi}{\rho} \frac{\partial G_{ \pm}}{\partial \phi}\right)\right] .
\end{aligned}
$$

The wave equation for $G_{ \pm}$given in (4-46) seems to have no exact solution. To proceed with the solution, it is important to note that on the LHS of (4-46) the term $k_{t}^{2} / h^{2}$ is proportional to $\gamma^{2} / h^{2}$. On the RHS of (4-46) we can see that the first term is of the order of $R^{-2}$, and the remainder as $R^{-1}$, apart from the variation of $h$ and $G_{ \pm}$itself with $R^{-1}$. As observed before, for $R \rightarrow \infty$ the field $G_{ \pm}$has analytical solution in terms of cylindrical functions. In this way, a perturbation solution can be sought as a power series in $R^{-1}$, assuming

$$
\begin{gathered}
G_{ \pm}=\left(G_{0, \pm}+R^{-1} G_{1, \pm}+R^{-2} G_{2, \pm}+\cdots\right) \exp (\gamma \zeta) \\
\gamma^{2}=\gamma_{0}^{2}\left(1+R^{-1} \alpha_{1}+R^{-2} \alpha_{2}+\cdots\right)
\end{gathered}
$$

where the terms with subscript 0 refer to the solutions of an axially straight structure. To be precise, $G_{0}$ and $\gamma_{0}$ are the well-know fields and propagation constants that satisfies the Maxwell equations in cylindrical coordinates.

Before replacing the series (4-47) into the differential equation (4-46), we verify that is more appropriate first multiply the both sides of (4-46) by $k_{t}^{2} h^{2}$,

\footnotetext{
${ }^{4}$ The wave equation in (4-46) was derived for a bent structure with radius of curvature $R$. In this equation the direction of the center of curvature is at $\phi=0$. Case the curvature is not aligned with the local $x$-axis (see Fig. 4.3), but instead it is rotated at an angle $\psi$, the axial metric coefficient becomes $h_{\zeta}=1-R^{-1} \rho \cos (\phi+\psi)$ [145]. In this case, we need to replace the argument of the trigonometric functions in (4-46) such that $\phi \rightarrow \phi+\psi$.
} 
resulting in

$$
\begin{aligned}
& \left(h^{2} k_{t}^{2} \nabla_{t}^{2}+k_{t}^{4}\right) G_{ \pm}=R^{-2}\left(k^{2} h^{2}-\gamma^{2}\right) G_{ \pm} \\
& +R^{-1} h\left(k^{2} h^{2}+3 \gamma^{2}\right)\left[\cos \phi \frac{\partial G_{ \pm}}{\partial \rho}-\frac{\sin \phi}{\rho} \frac{\partial G_{ \pm}}{\partial \phi}\right] \\
& \quad \pm 2 R^{-1} h^{2} \gamma k\left[\sin \phi \frac{\partial G_{ \pm}}{\partial \rho}+\frac{\cos \phi}{\rho} \frac{\partial G_{ \pm}}{\partial \phi}\right]
\end{aligned}
$$

Note that the axial dependence $\exp (\gamma \zeta)$ was omitted.

As said before, we assume that the zeroth-order solution $G_{0, \pm}$ is wellknow, and at this moment we are looking for the first-order solution $G_{1, \pm}$. Considering only the terms of order up to $R^{-1}$, it is apparent that the RHS of (4-49) can be simplified. The term proportional to $R^{-2}$ can be overlooked. Also, the other two remaining term are proportional to $R^{-1}$, so we just need to use the series up $R^{0}$ for $\gamma, h$ and $G_{ \pm}$:

$$
\begin{aligned}
&\left(h^{2} k_{t}^{2} \nabla_{t}^{2}+k_{t}^{4}\right) G_{ \pm}=R^{-1}\left(k^{2}+3 \gamma_{0}^{2}\right)\left[\cos \phi \frac{\partial G_{0, \pm}}{\partial \rho}-\frac{\sin \phi}{\rho} \frac{\partial G_{0, \pm}}{\partial \phi}\right] \\
& \pm 2 R^{-1} \gamma_{0} k\left[\sin \phi \frac{\partial G_{0, \pm}}{\partial \rho}+\frac{\cos \phi}{\rho} \frac{\partial G_{0, \pm}}{\partial \phi}\right]
\end{aligned}
$$

The left-hand side (LHS) of the above equation contains terms with orders beyond $R^{-1}$, but we can replace

$$
\begin{aligned}
h^{2} k_{t}^{2} & =\left(1-2 R^{-1} \rho \cos \phi\right)\left[k_{0, t}^{2}+R^{-1}\left(\gamma_{0}^{2} \alpha_{1}-2 k^{2} \rho \cos \phi\right)\right]+O\left(R^{-2}\right) \\
& =k_{0, t}^{2}+R^{-1}\left(\gamma_{0}^{2} \alpha_{1}-2 \rho \cos \phi\left(k^{2}+k_{0, t}^{2}\right)\right)+O\left(R^{-2}\right)
\end{aligned}
$$

and

$$
\begin{aligned}
k_{t}^{4} & =\left[k^{2}\left(1-2 R^{-1} \rho \cos \phi\right)+\gamma_{0}^{2}\left(1+\alpha_{1} R^{-1}\right)+O\left(R^{-2}\right)\right]^{2} \\
& =k_{0, t}^{4}+2 k_{0, t}^{2} R^{-1}\left(\gamma_{0}^{2} \alpha_{1}-2 k^{2} \rho \cos \phi\right)+O\left(R^{-2}\right),
\end{aligned}
$$

into the LHS of (4-50) and retaining only the terms of order up to $R^{-1}$ :

$$
\begin{aligned}
& \left(h^{2} k_{t}^{2} \nabla_{t}^{2}+k_{t}^{4}\right) G_{ \pm}=\left\{\left\{k_{\rho}^{2}+R^{-1}\left[\gamma_{0}^{2} \alpha_{1}-2 \rho \cos \phi\left(k^{2}+k_{\rho}^{2}\right)\right]\right\} \nabla_{t}^{2}\right. \\
& \left.+k_{\rho}^{4}+2 k_{\rho}^{2} R^{-1}\left(\gamma_{0}^{2} \alpha_{1}-2 k^{2} \rho \cos \phi\right)\right\} G_{0, \pm}+R^{-1}\left(k_{\rho}^{2} \nabla_{t}^{2}+k_{\rho}^{4}\right) G_{1, \pm}
\end{aligned}
$$


or, in a more suitable form:

$$
\begin{gathered}
\left(h^{2} k_{t}^{2} \nabla_{t}^{2}+k_{t}^{4}\right) G_{ \pm}=\left\{k_{\rho}^{2}+R^{-1}\left[\gamma_{0}^{2} \alpha_{1}-2 \rho \cos \phi\left(k^{2}+k_{\rho}^{2}\right)\right]\right\}\left(\nabla_{t}^{2}+k_{\rho}^{2}\right) G_{0, \pm} \\
\quad+k_{\rho}^{2} R^{-1}\left[\gamma_{0}^{2} \alpha_{1}+2 \rho \cos \phi\left(k_{\rho}^{2}-k^{2}\right)\right] G_{0, \pm}+k_{\rho}^{2} R^{-1}\left(\nabla_{t}^{2}+k_{\rho}^{2}\right) G_{1, \pm} .
\end{gathered}
$$

With a view to simplifying the notation in the equations above, we have define the parcel of $k_{t}$ of order $R^{0}$, i.e., is the radial propagations constant of the straight structure $\left(R^{-1}=0\right)$, such as

$$
k_{\rho}^{2}=k_{0, t}^{2}=k^{2}+\gamma_{0}^{2} .
$$

Replacing the above simplifications back in (4-50) we can write

$$
\begin{gathered}
\left\{k_{\rho}^{2}+R^{-1}\left[\gamma_{0}^{2} \alpha_{1}-2 \rho \cos \phi\left(k^{2}+k_{\rho}^{2}\right)\right]\right\}\left(\nabla_{t}^{2}+k_{\rho}^{2}\right) G_{0, \pm} \\
+k_{\rho}^{2} R^{-1} \gamma_{0}^{2}\left(\alpha_{1}+2 \rho \cos \phi\right) G_{0, \pm}+k_{\rho}^{2} R^{-1}\left(\nabla_{t}^{2}+k_{\rho}^{2}\right) G_{1, \pm}= \\
R^{-1}\left(k^{2}+3 \gamma_{0}^{2}\right)\left[\cos \phi \frac{\partial G_{0, \pm}}{\partial \rho}-\frac{\sin \phi}{\rho} \frac{\partial G_{0, \pm}}{\partial \phi}\right] \\
\pm 2 R^{-1} \gamma_{0} k\left[\sin \phi \frac{\partial G_{0, \pm}}{\partial \rho}+\frac{\cos \phi}{\rho} \frac{\partial G_{0, \pm}}{\partial \phi}\right]
\end{gathered}
$$

Taking into account that the linear combination of (4-19) and (4-18) can be written in the form $\left(\nabla_{t}^{2}+k_{\rho}^{2}\right) G_{0, \pm}=0$, the first term in (4-56) should be zero. Eliminating the first term and multiplying the resulting equation by $k_{\rho}^{-2} R$, we obtain a differential equation independent of $R$ given by

$$
\begin{aligned}
\left(\nabla_{t}^{2}+k_{\rho}^{2}\right) G_{1, \pm} & =-\gamma_{0}^{2}\left(\alpha_{1}+2 \rho \cos \phi\right) G_{0, \pm} \\
+k_{\rho}^{-2}\left\{\left(k^{2}+3 \gamma_{0}^{2}\right)\right. & {\left[\cos \phi \frac{\partial G_{0, \pm}}{\partial \rho}-\frac{\sin \phi}{\rho} \frac{\partial G_{0, \pm}}{\partial \phi}\right] } \\
& \left. \pm 2 \gamma_{0} k\left[\sin \phi \frac{\partial G_{0, \pm}}{\partial \rho}+\frac{\cos \phi}{\rho} \frac{\partial G_{0, \pm}}{\partial \phi}\right]\right\}
\end{aligned}
$$

\subsubsection{3}

\section{Verification of the First Order Differential Equation}

In [51] the effects of the curvature in a circular waveguide was analyzed. The coupling between the degenerated modes $\mathrm{TE}_{01}^{z}$ and $\mathrm{TM}_{11}^{z}$ can be formulated from their zeroth-order axial fields

$$
\begin{aligned}
& H_{z}=J_{0}\left(k_{\rho} \rho\right) \exp \left(\gamma_{0} \zeta\right), \text { and } \\
& E_{z}=J_{1}\left(k_{\rho} \rho\right) \sin \phi \exp \left(\gamma_{0} \zeta\right),
\end{aligned}
$$


where $k_{\rho}$ is the first root of $J_{1}\left(k_{\rho} r_{N}\right)=0$ for a hollow circular waveguide truncated at $\rho=r_{N}$ with an PEC wall. Using the above axial fields, the zeroth-order solution can be defined by

$$
G_{0, \pm}=J_{1}\left(k_{\rho} \rho\right) \sin \phi \pm M J_{0}\left(k_{\rho} \rho\right)
$$

where $M$ is an arbitrary constant. It should be observed that the zeroth-order field in (4-58) and (4-59) does not share the same azimuthal dependence such we can anticipate that $M \neq i Z$.

Plugging $G_{0, \pm}$ into (4-57), and using

$$
\begin{gathered}
\frac{\partial G_{0, \pm}}{\partial \rho}=\left(k_{\rho} J_{0}\left(k_{\rho} \rho\right)-\frac{J_{1}\left(k_{\rho} \rho\right)}{\rho}\right) \sin \phi \mp M k_{\rho} J_{1}\left(k_{\rho} \rho\right), \text { and } \\
\frac{\partial G_{0, \pm}}{\partial \phi}=J_{1}\left(k_{\rho} \rho\right) \cos \phi,
\end{gathered}
$$

we can derive

$$
\begin{aligned}
& \left(\nabla_{t}^{2}+k_{\rho}^{2}\right) G_{1, \pm}=-\gamma_{0}^{2}\left(\alpha_{1}+2 \rho \cos \phi\right)\left(J_{1} \sin \phi \pm M J_{0}\right) \\
& +k_{\rho}^{-2}\left\{\left(k^{2}+3 \gamma_{0}^{2}\right)\left\{\cos \phi\left[\left(k_{\rho} J_{0}-\frac{J_{1}}{\rho}\right) \sin \phi \mp M k_{\rho} J_{1}\right]-\frac{\sin \phi}{\rho} J_{1} \cos \phi\right\}\right. \\
& \left.\quad \pm 2 \gamma_{0} k\left\{\sin \phi\left[\left(k_{\rho} J_{0}-\frac{J_{1}}{\rho}\right) \sin \phi \mp M k_{\rho} J_{1}\right]+\frac{\cos \phi}{\rho} J_{1} \cos \phi\right\}\right\},
\end{aligned}
$$

were we have dropped the argument of the Bessel functions. The above equation can be rewrite as

$$
\begin{aligned}
& \left(\nabla_{t}^{2}+k_{\rho}^{2}\right) G_{1, \pm}=-\gamma_{0}^{2}\left(\alpha_{1}+2 \rho \cos \phi\right)\left(J_{1} \sin \phi \pm M J_{0}\right) \\
& +k_{\rho}^{-2}\left\{\left(k^{2}+3 \gamma_{0}^{2}\right)\left[-2 \frac{J_{1}}{\rho} \sin \phi \cos \phi+k_{\rho} J_{0} \sin \phi \cos \phi \mp M k_{\rho} J_{1} \cos \phi\right]\right. \\
& \left.\quad \pm 2 \gamma_{0} k\left[\frac{J_{1}}{\rho}\left(\cos ^{2} \phi-\sin ^{2} \phi\right)+k_{\rho} J_{0} \sin ^{2} \phi \mp M k_{\rho} J_{1} \sin \phi\right]\right\} .
\end{aligned}
$$

By using the trigonometric identities

$$
\begin{gathered}
\sin \phi \cos \phi=\frac{1}{2} \sin 2 \phi, \text { and } \\
\cos ^{2} \phi-\sin ^{2} \phi=\cos 2 \phi,
\end{gathered}
$$


we can show that (4-64) becomes

$$
\begin{aligned}
&\left(\nabla_{t}^{2}+k_{\rho}^{2}\right) G_{1, \pm}=-\gamma_{0}^{2}\left(\alpha_{1}+2 \rho \cos \phi\right)\left(J_{1} \sin \phi \pm M J_{0}\right) \\
&+k_{\rho}^{-2}\left\{\left(k^{2}+3 \gamma_{0}^{2}\right)\left[k_{\rho} J_{0} \sin \phi \cos \phi-J_{1}\left( \pm M k_{\rho} \cos \phi+\frac{\sin 2 \phi}{\rho}\right)\right]\right. \\
&\left.\quad \pm 2 \gamma_{0} k\left[k_{\rho} J_{0} \sin ^{2} \phi-J_{1}\left( \pm M k_{\rho} \sin \phi-\frac{\cos 2 \phi}{\rho}\right)\right]\right\}
\end{aligned}
$$

The result shown above is identical to one obtained in [51, p. 63, eq. 6.24] .

\subsection{2}

\section{Alternative Decoupling Approach}

Here we will derive an alternative approach to decouple the fields in the toroidal coordinates. Instead the employment of the bi-complex parameters $i$ and $j$, we can combine the axial fields in (4-4c) and (4-4f) in the following matrix form

$$
i \omega \frac{\rho}{h}\left[\begin{array}{c}
\mu H \\
-\epsilon E
\end{array}\right]=\frac{\partial}{\partial \rho}\left\{\rho\left[\begin{array}{c}
E_{\phi} \\
h_{\phi}
\end{array}\right]\right\}-\frac{\partial}{\partial \phi}\left[\begin{array}{c}
E_{\rho} \\
H_{\rho}
\end{array}\right] .
$$

Substituting (4-194) and (4-195) in the equation above, we obtain

$$
\begin{aligned}
i \omega \frac{\rho}{h}\left[\begin{array}{c}
\mu H \\
-\epsilon E
\end{array}\right]=\frac{\partial}{\partial \rho}\left\{\frac{1}{k_{t}^{2}}\left[\begin{array}{cc}
\gamma \frac{\partial}{\partial \phi} & -h i \omega \mu \rho \frac{\partial}{\partial \rho} \\
h i \omega \epsilon \rho \frac{\partial}{\partial \rho} & \gamma \frac{\partial}{\partial \phi}
\end{array}\right]\left[\begin{array}{l}
E \\
H
\end{array}\right]\right\} \\
-\frac{1}{\rho} \frac{\partial}{\partial \phi}\left\{\frac{1}{k_{t}^{2}}\left[\begin{array}{cc}
\gamma \rho \frac{\partial}{\partial \rho} & h i \omega \mu \frac{\partial}{\partial \phi} \\
-h i \omega \epsilon \frac{\partial}{\partial \phi} & \gamma \rho \frac{\partial}{\partial \rho}
\end{array}\right]\left[\begin{array}{l}
E \\
H
\end{array}\right]\right\} .
\end{aligned}
$$

Using the product rule for the derivatives, the equation above becomes

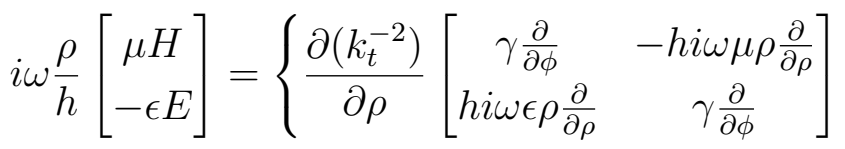

$$
\begin{aligned}
& +k_{t}^{-2}\left[\begin{array}{cc}
\gamma \frac{\partial}{\partial \rho} \frac{\partial}{\partial \phi} & -i \omega \mu \frac{\partial}{\partial \rho}\left(h \rho \frac{\partial}{\partial \rho}\right) \\
i \omega \epsilon \frac{\partial}{\partial \rho}\left(h \rho \frac{\partial}{\partial \rho}\right) & \gamma \frac{\partial}{\partial \rho} \frac{\partial}{\partial \phi}
\end{array}\right]-\frac{1}{\rho} \frac{\partial\left(k_{t}^{-2}\right)}{\partial \phi}\left[\begin{array}{cc}
\gamma \rho \frac{\partial}{\partial \rho} & h i \omega \mu \frac{\partial}{\partial \phi} \\
-h i \omega \epsilon \frac{\partial}{\partial \phi} & \gamma \rho \frac{\partial}{\partial \rho}
\end{array}\right] \\
& \left.-\frac{k_{t}^{-2}}{\rho}\left[\begin{array}{cc}
\gamma \rho \frac{\partial}{\partial \phi} \frac{\partial}{\partial \rho} & i \omega \mu \frac{\partial}{\partial \phi}\left(h \frac{\partial}{\partial \phi}\right) \\
-i \omega \epsilon \frac{\partial}{\partial \phi}\left(h \frac{\partial}{\partial \phi}\right) & \gamma \rho \frac{\partial}{\partial \phi} \frac{\partial}{\partial \rho}
\end{array}\right]\right\}\left[\begin{array}{l}
E \\
H
\end{array}\right] \text {. }
\end{aligned}
$$

Using

$$
\begin{gathered}
\frac{\partial\left(k_{t}^{-2}\right)}{\partial \rho}=2 R^{-1} k^{2} h \frac{\cos \phi}{k_{t}^{4}}, \text { and } \\
\frac{\partial\left(k_{t}^{-2}\right)}{\partial \phi}=-2 R^{-1} k^{2} h \frac{\rho \sin \phi}{k_{t}^{4}}
\end{gathered}
$$


we can derive

$$
\begin{aligned}
& i \omega \frac{\rho}{h}\left[\begin{array}{c}
\mu H \\
-\epsilon E
\end{array}\right]=\left\{2 k_{t}^{-4} R^{-1} k^{2} h \cos \phi\left[\begin{array}{cc}
\gamma \frac{\partial}{\partial \phi} & -h i \omega \mu \rho \frac{\partial}{\partial \rho} \\
h i \omega \epsilon \rho \frac{\partial}{\partial \rho} & \gamma \frac{\partial}{\partial \phi}
\end{array}\right]\right. \\
& +2 k_{t}^{-4} R^{-1} k^{2} h \sin \phi\left[\begin{array}{cc}
\gamma \rho \frac{\partial}{\partial \rho} & h i \omega \mu \frac{\partial}{\partial \phi} \\
-h i \omega \epsilon \frac{\partial}{\partial \phi} & \gamma \rho \frac{\partial}{\partial \rho}
\end{array}\right] \\
& \left.+k_{t}^{-2}\left[\begin{array}{cc}
0 & -i \omega \mu\left[\frac{\partial}{\partial \rho}\left(h \rho \frac{\partial}{\partial \rho}\right)+\frac{1}{\rho} \frac{\partial}{\partial \phi}\left(h \frac{\partial}{\partial \phi}\right)\right] \\
i \omega \epsilon\left[\frac{\partial}{\partial \rho}\left(h \rho \frac{\partial}{\partial \rho}\right)+\frac{1}{\rho} \frac{\partial}{\partial \phi}\left(h \frac{\partial}{\partial \phi}\right)\right] & 0
\end{array}\right]\right\}\left[\begin{array}{l}
E \\
H
\end{array}\right] \text {. }
\end{aligned}
$$

To simplify the above equation, we can start with

$$
\begin{aligned}
\frac{\partial}{\partial \rho}\left(h \rho \frac{\partial}{\partial \rho}\right)+\frac{1}{\rho} \frac{\partial}{\partial \phi}\left(h \frac{\partial}{\partial \phi}\right)=h \frac{\partial}{\partial \rho}\left(\rho \frac{\partial}{\partial \rho}\right) & +\frac{h}{\rho} \frac{\partial^{2}}{\partial \phi^{2}} \\
& +\frac{\partial h}{\partial \rho}\left(\rho \frac{\partial}{\partial \rho}\right)+\frac{1}{\rho} \frac{\partial h}{\partial \phi} \frac{\partial}{\partial \phi}
\end{aligned}
$$

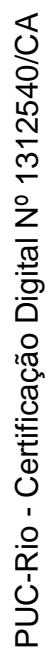

Noting that the first two terms are equal to $\rho h \nabla_{t}^{2}$, and using

$$
\frac{\partial h}{\partial \rho}=-R^{-1} \cos \phi, \quad \frac{\partial h}{\partial \phi}=R^{-1} \rho \sin \phi,
$$

we obtain

$$
\frac{\partial}{\partial \rho}\left(h \rho \frac{\partial}{\partial \rho}\right)+\frac{1}{\rho} \frac{\partial}{\partial \phi}\left(h \frac{\partial}{\partial \phi}\right)=\rho h \nabla_{t}^{2}-R^{-1} \rho \cos \phi \frac{\partial}{\partial \rho}+R^{-1} \sin \phi \frac{\partial}{\partial \phi} .
$$

Substituting the above in (4-73) allow us to write

$$
\begin{aligned}
& i \omega \frac{\rho}{h}\left[\begin{array}{c}
\mu H \\
-\epsilon E
\end{array}\right]=\left\{2 k_{t}^{-4} R^{-1} k^{2} h \cos \phi\left[\begin{array}{cc}
\gamma \frac{\partial}{\partial \phi} & -h i \omega \mu \rho \frac{\partial}{\partial \rho} \\
h i \omega \epsilon \rho \frac{\partial}{\partial \rho} & \gamma \frac{\partial}{\partial \phi}
\end{array}\right]\right. \\
& +2 k_{t}^{-4} R^{-1} k^{2} h \sin \phi\left[\begin{array}{cc}
\gamma \rho \frac{\partial}{\partial \rho} & h i \omega \mu \frac{\partial}{\partial \phi} \\
-h i \omega \epsilon \frac{\partial}{\partial \phi} & \gamma \rho \frac{\partial}{\partial \rho}
\end{array}\right]+k_{t}^{-2} \rho h\left[\begin{array}{cc}
0 & -i \omega \mu \nabla_{t}^{2} \\
i \omega \epsilon \nabla_{t}^{2} & 0
\end{array}\right] \\
& \left.+R^{-1} k_{t}^{-2}\left[\begin{array}{cc}
0 & -i \omega \mu\left(-\rho \cos \phi \frac{\partial}{\partial \rho}+\sin \phi \frac{\partial}{\partial \phi}\right) \\
i \omega \epsilon\left(-\rho \cos \phi \frac{\partial}{\partial \rho}+\sin \phi \frac{\partial}{\partial \phi}\right) & 0
\end{array}\right]\right\}\left[\begin{array}{c}
E \\
H
\end{array}\right] .
\end{aligned}
$$

Multiplying the first line of the equation above by $k_{t}^{2}(i \omega \mu \rho h)^{-1}$ and 
multiplying the second line by $k_{t}^{2}(-i \omega \epsilon \rho h)^{-1}$, we can obtain

$$
\begin{aligned}
&\left(\nabla_{t}^{2}+\frac{k_{t}^{2}}{h^{2}}\right)\left[\begin{array}{c}
H \\
E
\end{array}\right]=\left\{2 k_{t}^{-2} R^{-1} k^{2} \cos \phi\left[\begin{array}{cc}
\frac{\gamma}{i \omega \mu} \frac{1}{\rho} \frac{\partial}{\partial \phi} & -h \frac{\partial}{\partial \rho} \\
-h \frac{\partial}{\partial \rho} & -\frac{\gamma}{i \omega \epsilon} \frac{1}{\rho} \frac{\partial}{\partial \phi}
\end{array}\right]\right. \\
&+2 k_{t}^{-2} R^{-1} k^{2} \sin \phi\left[\begin{array}{cc}
\frac{\gamma}{i \omega \mu} \frac{\partial}{\partial \rho} & h \frac{1}{\rho} \frac{\partial}{\partial \phi} \\
h \frac{1}{\rho} \frac{\partial}{\partial \phi} & -\frac{\gamma}{i \omega \epsilon} \frac{\partial}{\partial \rho}
\end{array}\right] \\
&\left.+R^{-1} h^{-1}\left[\begin{array}{cc}
0 & \left(\cos \phi \frac{\partial}{\partial \rho}-\frac{1}{\rho} \sin \phi \frac{\partial}{\partial \phi}\right) \\
\left(\cos \phi \frac{\partial}{\partial \rho}-\frac{1}{\rho} \sin \phi \frac{\partial}{\partial \phi}\right) & 0
\end{array}\right]\right\}\left[\begin{array}{l}
E \\
H
\end{array}\right] .
\end{aligned}
$$

Defining

$$
\begin{aligned}
L_{D} & =2 R^{-1} \frac{k^{2} h}{k_{t}^{2}}\left(-\cos \phi \frac{\partial}{\partial \rho}+\frac{1}{\rho} \sin \phi \frac{\partial}{\partial \phi}\right)+R^{-1} h^{-1}\left(\cos \phi \frac{\partial}{\partial \rho}-\frac{1}{\rho} \sin \phi \frac{\partial}{\partial \phi}\right) \\
& =R^{-1} h^{-1}\left(\frac{2 k^{2} h^{2}}{k_{t}^{2}}-1\right)\left(-\cos \phi \frac{\partial}{\partial \rho}+\frac{\sin \phi}{\rho} \frac{\partial}{\partial \phi}\right) \\
& =R^{-1} \frac{k^{2} h^{2}-\gamma^{2}}{h k_{t}^{2}}\left(-\cos \phi \frac{\partial}{\partial \rho}+\frac{\sin \phi}{\rho} \frac{\partial}{\partial \phi}\right), \text { and } \\
L_{A} & =-2 R^{-1} \frac{k^{2}}{k_{t}^{2}} \frac{\gamma}{i \omega}\left(\frac{\cos \phi}{\rho} \frac{\partial}{\partial \phi}+\sin \phi \frac{\partial}{\partial \rho}\right)
\end{aligned}
$$

we can rewrite (4-78) in a compact form:

$$
\begin{aligned}
\left(\nabla_{t}^{2}+\frac{k_{t}^{2}}{h^{2}}\right)\left[\begin{array}{l}
H \\
E
\end{array}\right] & =\left[\begin{array}{cc}
-\mu^{-1} L_{A} & L_{D} \\
L_{D} & \epsilon^{-1} L_{A}
\end{array}\right]\left[\begin{array}{l}
E \\
H
\end{array}\right] \\
& =\left[\begin{array}{cc}
L_{D} & -\mu^{-1} L_{A} \\
\epsilon^{-1} L_{A} & L_{D}
\end{array}\right]\left[\begin{array}{c}
H \\
E
\end{array}\right],
\end{aligned}
$$

or

$$
\left(\nabla_{t}^{2}+\frac{k_{t}^{2}}{h^{2}}\right)\left[\begin{array}{l}
E \\
H
\end{array}\right]=\left[\begin{array}{cc}
L_{D} & \epsilon^{-1} L_{A} \\
-\mu^{-1} L_{A} & L_{D}
\end{array}\right]\left[\begin{array}{l}
E \\
H
\end{array}\right] .
$$

An equation more symmetric than the above can be derived if we define

$$
\begin{aligned}
L_{C} & =\frac{1}{\sqrt{\mu \epsilon}} L_{A} \\
& =2 R^{-1} \frac{k}{k_{t}^{2}} i \gamma\left(\frac{\cos \phi}{\rho} \frac{\partial}{\partial \phi}+\sin \phi \frac{\partial}{\partial \rho}\right),
\end{aligned}
$$

such as (4-85) becomes

$$
\left(\nabla_{t}^{2}+\frac{k_{t}^{2}}{h^{2}}\right)\left[\begin{array}{l}
E \\
H
\end{array}\right]=\left[\begin{array}{cc}
L_{D} & \sqrt{\frac{\mu}{\epsilon}} L_{C} \\
-\sqrt{\frac{\epsilon}{\mu}} L_{C} & L_{D}
\end{array}\right]\left[\begin{array}{l}
E \\
H
\end{array}\right]
$$


Multiplying the second line of the above equation by $Z=(\mu / \epsilon)^{1 / 2}$, we obtain ${ }^{5}$

$$
\left(\nabla_{t}^{2}+\frac{k_{t}^{2}}{h^{2}}\right)\left[\begin{array}{c}
E \\
Z H
\end{array}\right]=\left[\begin{array}{cc}
L_{D} & L_{C} \\
-L_{C} & L_{D}
\end{array}\right]\left[\begin{array}{c}
E \\
Z H
\end{array}\right] .
$$

Introducing the differential operators

$$
\begin{gathered}
L_{d}=\left(\nabla_{t}^{2}+\frac{k_{t}^{2}}{h^{2}}\right)-L_{D}, \\
L_{a}=-L_{C},
\end{gathered}
$$

we can write

$$
\left[\begin{array}{cc}
L_{d} & L_{a} \\
-L_{a} & L_{d}
\end{array}\right]\left[\begin{array}{c}
E \\
Z H
\end{array}\right]=\left[\begin{array}{l}
0 \\
0
\end{array}\right]
$$

We will call as $\overline{\bar{M}}$ the matrix operator show above, and the vector of the axial fields as $\bar{F}$ such as

$$
\overline{\bar{M}} \bar{F}=\overline{0}
$$

Then, we can decompose this matrix into the form

$$
\overline{\bar{M}}=\overline{\bar{P}} \overline{\bar{D}} \overline{\bar{P}}^{-1}
$$

where $\overline{\bar{P}}$ is a matrix composed of the eigenvectors of $\overline{\bar{M}}$, and $\overline{\bar{D}}$ is the diagonal matrix constructed from the corresponding eigenvalues. Namely

$$
\begin{array}{cc}
\overline{\bar{D}}=\left[\begin{array}{cc}
L_{d}-i L_{a} & 0 \\
0 & L_{d}+i L_{a}
\end{array}\right], \\
\overline{\bar{P}}=\frac{1}{2}\left[\begin{array}{cc}
1 & 1 \\
-i & i
\end{array}\right], & \overline{\bar{P}}^{-1}=\left[\begin{array}{cc}
1 & i \\
1 & -i
\end{array}\right] .
\end{array}
$$

By substituting the decomposed form of $\overline{\bar{M}}$ into (4-93), we can obtain

$$
\overline{\bar{P}} \overline{\bar{D}} \overline{\bar{P}}^{-1} \bar{F}=\overline{0}
$$

which we can reduce to

$$
\overline{\bar{D}} \overline{\bar{P}}^{-1} \bar{F}=\overline{0} \text {. }
$$

Now, we can look for the vector given by $\overline{\bar{P}}^{-1} \bar{F}$, namely

$$
\overline{\bar{P}}^{-1} \bar{F}=\left[\begin{array}{l}
E+i Z H \\
E-i Z H
\end{array}\right]
$$

which reduces our equation to the form

${ }^{5}$ A matrix equation similar to (4-89) can be found in [138, eq. 2] or in [140, eq. 6], but with some change in the notation. The approach shown in this section, unlike the method presented in $[138,140]$, do not employ any bi-complex field to decouple and solve the axial fields $E$ and $H$. 


$$
\left[\begin{array}{cc}
L_{d}-i L_{a} & 0 \\
0 & L_{d}+i L_{a}
\end{array}\right]\left[\begin{array}{l}
E+i Z H \\
E-i Z H
\end{array}\right]=\left[\begin{array}{l}
0 \\
0
\end{array}\right],
$$

or, alternatively, we can write

$$
\left(L_{d} \mp i L_{a}\right)(E \pm i Z H)=0 .
$$

At this point we can use the field $F_{ \pm}$as defined in (4-23), and additionally, substituting $L_{d}$ and $L_{a}$ by theirs forms in (4-90) and (4-91) allow us to obtain a decoupled differential equation given by

$$
\left(\nabla_{t}^{2}+\frac{k_{t}^{2}}{h^{2}}\right) F_{ \pm}=L_{ \pm} F_{ \pm}
$$

where

$$
L_{ \pm}=L_{D} \mp i L_{C}
$$

The axial fields $E$ and $H$ can be separated from $F_{ \pm}$by using simple relations shown in (4-31) and (4-32).

It is important to be observed that in order to obtain the decoupled equation shown above there is no bi-complex variables. Here, we have employed a matrix differential operator to combine the axial fields in (4-89), and after a diagonalization process, we have obtained a decoupled wave equation. In Section 4.2.1, instead, the new complex variable $j$ was used to help us in the decoupling process.

Returning to the decoupled differential equation (4-102), it is more expedite to work with the fields $G_{ \pm}$defined in (4-33) in place of the hypothetic fields $F_{ \pm}$. From (4-33), we have $F_{ \pm}=h G_{ \pm}$that allow us to rewrite (4-102) as

$$
\begin{aligned}
h\left(\nabla_{t}^{2}+\frac{k_{t}^{2}}{h^{2}}\right) G_{ \pm}+G_{ \pm} \nabla_{t}^{2} h+ & 2\left(\frac{\partial G_{ \pm}}{\partial \rho} \frac{\partial h}{\partial \rho}+\frac{1}{\rho^{2}} \frac{\partial G_{ \pm}}{\partial \phi} \frac{\partial h}{\partial \phi}\right)= \\
& h\left(L_{D} \mp i L_{C}\right) G_{ \pm}+G_{ \pm}\left(L_{D} \mp i L_{C}\right) h .
\end{aligned}
$$

We can verify that $h$ is harmonic in $(\rho, \phi)$, i.e., satisfies the twodimensional Laplace equation $\nabla_{t}^{2} h=0$ [145], such that we can derive

$$
\begin{aligned}
& h\left(\nabla_{t}^{2}+\frac{k_{t}^{2}}{h^{2}}\right) G_{ \pm}-2 R^{-1}\left(\cos \phi \frac{\partial}{\partial \rho}+\right.\left.\frac{\sin \phi}{\rho} \frac{\partial}{\partial \rho}\right) G_{ \pm}= \\
& h\left(L_{D} \mp i L_{C}\right) G_{ \pm}+G_{ \pm}\left(L_{D} \mp i L_{C}\right) h .
\end{aligned}
$$

Using (4-81) and (4-87) we can easily shown that $L_{C} h$ vanish, such the last term in RHS of the above equation reduces to

$$
G_{ \pm}\left(L_{D} \mp i L_{C}\right) h=R^{-2} \frac{k^{2} h^{2}-\gamma^{2}}{h k_{t}^{2}} G_{ \pm} .
$$


Then, after we dividing both sides of (4-105) by $h$, with help of (4-81) and (4-87), we can finally obtain

$$
\begin{aligned}
&\left(\nabla_{t}^{2}+\frac{k_{t}^{2}}{h^{2}}\right) G_{ \pm}=R^{-1} \frac{2}{h}\left(\cos \phi \frac{\partial}{\partial \rho}+\frac{\sin \phi}{\rho} \frac{\partial}{\partial \rho}\right) \\
&+R^{-1} \frac{k^{2} h^{2}-\gamma^{2}}{h k_{t}^{2}}\left(-\cos \phi \frac{\partial G_{ \pm}}{\partial \rho}+\frac{\sin \phi}{\rho} \frac{\partial G_{ \pm}}{\partial \phi}\right) \\
& \pm 2 R^{-1} \frac{k}{k_{t}^{2}} \gamma\left(\frac{\cos \phi}{\rho} \frac{\partial G_{ \pm}}{\partial \phi}+\sin \phi \frac{\partial G_{ \pm}}{\partial \rho}\right)+R^{-2} \frac{k^{2} h^{2}-\gamma^{2}}{h^{2} k_{t}^{2}} G_{ \pm},
\end{aligned}
$$

or, in a more compact shape, we can write

$$
\begin{aligned}
& \left(\nabla_{t}^{2}+\frac{k_{t}^{2}}{h^{2}}\right) G_{ \pm}=\frac{R^{-1}}{k_{t}^{2}}\left[R^{-1}\left(k^{2}-\gamma^{2} / h^{2}\right) G_{ \pm}\right. \\
+ & \left.h\left(k^{2}+3 \gamma^{2} / h^{2}\right)\left(\cos \phi \frac{\partial G_{ \pm}}{\partial \rho}-\frac{\sin \phi}{\rho} \frac{\partial G_{ \pm}}{\partial \phi}\right) \pm 2 \gamma k\left(\sin \phi \frac{\partial G_{ \pm}}{\partial \rho}+\frac{\cos \phi}{\rho} \frac{\partial G_{ \pm}}{\partial \phi}\right)\right] .
\end{aligned}
$$

The above differential equation is exactly equal to (4-46) derived in Section 4.2.1. The approach proposed here allow us to decouple the differential equations for the axial fields (by means of the auxiliary fields $G_{ \pm}$) merely by carrying out a matrix digitalization, instead of employing a bi-complex variable as in $[51,144]$.

\subsection{3}

\section{Solution for Non-Degenerate Modal Fields}

The elementary wave function for the zeroth-order vanishing curvature ( $R^{-1}=0$, i.e., for a cylindrical waveguide) is given by

$$
G_{0, \pm}(\rho, \phi)=E_{z} \pm M H_{z}
$$

where $E_{z}$ and $H_{z}$ are the axial fields in cylindrical coordinates, and $M$ is an arbitrary constant to be determined. Note that the axial dependence in form $\exp \left(\gamma_{0} z\right)$ are assumed and omitted in the above.

As said before, the above defined constant $M$ combines the axial electric and magnetic fields and can be determined by the boundary conditions. However, the zeroth-order solution (in radially-stratified cylindrically waveguides) only support non-degenerated and orthogonal modes and we can anticipate that $M=N_{0}=i Z$. This is possible because each modal fields is characterized by a single eigenvalue $k_{z}$. In contrast, this is not true for example in an homogeneous circular waveguide where the modes $\mathrm{TE}_{0 p}^{z}$ and $\mathrm{TM}_{1 p}^{z}, p=1,2,3, \ldots$, are degenerated and we need to work with their linear combination into $G_{0, \pm}(\rho, \phi)$, 
as analyzed in Section 4.2.1.3 and in [51, p. 62]. Then, our elementary wave function for non-degenerated modes can be written as

$$
\begin{aligned}
G_{0, \pm}(\rho, \phi) & =\left\{H_{n}^{(1)}\left(k_{\rho} \rho\right) a^{e}+J_{n}\left(k_{\rho} \rho\right) b^{e} \pm N_{0}\left[H_{n}^{(1)}\left(k_{\rho} \rho\right) a^{h}+J_{n}\left(k_{\rho} \rho\right) b^{h}\right]\right\} e^{i n \phi} \\
& =B_{n, \pm}^{e h}\left(k_{\rho} \rho\right) \exp (\text { in } \phi), \quad n=0,1,-1,2,-2, \ldots
\end{aligned}
$$

As an aid to simplifying the notation, we have introduced $B_{n, \pm}^{e h}$ as the linear combination of unperturbed fields, and we will assume that these fields are already known from Chapter 3. In addition, we will drop temporarily the subscript \pm by using just $B_{n}^{e h}$, restoring it later as needed.

By replacing (4-111) in (4-57), and by using the differential operator

$$
\begin{aligned}
L & =\frac{\partial^{2}}{\partial \rho^{2}}+\frac{1}{\rho} \frac{\partial}{\partial \rho}+\frac{1}{\rho^{2}} \frac{\partial^{2}}{\partial \phi^{2}}+k_{\rho}^{2} \\
& =\nabla_{t}^{2}+k_{\rho}^{2},
\end{aligned}
$$

we can write

$$
\begin{aligned}
L G_{1, \pm} & =-\gamma_{0}^{2}\left(\alpha_{1}+2 \rho \cos \phi\right) B_{n}^{e h}\left(k_{\rho} \rho\right) e^{i n \phi} \\
+ & k_{\rho}^{-2}\left\{\left(k^{2}+3 \gamma_{0}^{2}\right)\left[\cos \phi k_{\rho} B_{n}^{\prime e h}\left(k_{\rho} \rho\right) e^{i n \phi}-\frac{\sin \phi}{\rho} i n B_{n}^{e h}\left(k_{\rho} \rho\right) e^{i n \phi}\right]\right. \\
& \left.\quad \pm 2 \gamma_{0} k\left[\sin \phi k_{\rho} B_{n}^{\prime e h}\left(k_{\rho} \rho\right) e^{i n \phi}+\frac{\cos \phi}{\rho} i n B_{n}^{e h}\left(k_{\rho} \rho\right) e^{i n \phi}\right]\right\}
\end{aligned}
$$

where the superscript prime means the derivative with respect to the argument of the cylindrical functions.

From the trigonometric identities

$$
\begin{aligned}
e^{i n \phi} \sin \phi & =\frac{i}{2}\left[e^{i(n-1) \phi}-e^{i(n+1) \phi}\right], \\
e^{i n \phi} \cos \phi & =\frac{1}{2}\left[e^{i(n-1) \phi}+e^{i(n+1) \phi}\right],
\end{aligned}
$$


we can write

$$
\begin{gathered}
L G_{1, \pm}=e^{i n \phi}\left[-\gamma_{0}^{2} \alpha_{1} B_{n}^{e h}\left(k_{\rho} \rho\right)\right] \\
+e^{i(n-1) \phi}\left[-\gamma_{0}^{2} \rho B_{n}^{e h}\left(k_{\rho} \rho\right)+\frac{k_{\rho}^{-1}}{2}\left(k^{2}+3 \gamma_{0}^{2}\right) B_{n}^{\prime e h}\left(k_{\rho} \rho\right)+\frac{n k_{\rho}^{-2}}{2 \rho}\left(k^{2}+3 \gamma_{0}^{2}\right) B_{n}^{e h}\left(k_{\rho} \rho\right)\right. \\
\left. \pm i k_{\rho}^{-1} \gamma_{0} k B_{n}^{\prime e h}\left(k_{\rho} \rho\right) \pm \frac{i n k_{\rho}^{-2} \gamma_{0} k}{\rho} B_{n}^{e h}\left(k_{\rho} \rho\right)\right] \\
+e^{i(n+1) \phi}\left[-\gamma_{0}^{2} \rho B_{n}^{e h}\left(k_{\rho} \rho\right)+\frac{k_{\rho}^{-1}}{2}\left(k^{2}+3 \gamma_{0}^{2}\right) B_{n}^{\prime e h}\left(k_{\rho} \rho\right)-\frac{n k_{\rho}^{-2}}{2 \rho}\left(k^{2}+3 \gamma_{0}^{2}\right) B_{n}^{e h}\left(k_{\rho} \rho\right)\right. \\
\left.\mp i k_{\rho}^{-1} \gamma_{0} k B_{n}^{\prime e h}\left(k_{\rho} \rho\right) \pm \frac{i n k_{\rho}^{-2} \gamma_{0} k}{\rho} B_{n}^{e h}\left(k_{\rho} \rho\right)\right] . \quad(4-117)
\end{gathered}
$$

Using the following properties of the cylindrical functions [55, p. 463]

$$
B_{n}^{\prime}\left(k_{\rho} \rho\right)= \pm B_{n \mp 1}\left(k_{\rho} \rho\right) \mp \frac{n}{k_{\rho} \rho} B_{n}\left(k_{\rho} \rho\right),
$$

and after some simplifications, we can finally obtain

$$
\begin{aligned}
& L G_{1, \pm}=e^{i n \phi}\left[-\gamma_{0}^{2} \alpha_{1} B_{n}^{e h}\left(k_{\rho} \rho\right)\right] \\
& \quad+e^{i(n-1) \phi}\left[-\gamma_{0}^{2} \rho B_{n}^{e h}\left(k_{\rho} \rho\right)+k_{\rho}^{-1}\left(\frac{k^{2}+3 \gamma_{0}^{2}}{2} \pm i \gamma_{0} k\right) B_{n-1}^{e h}\left(k_{\rho} \rho\right)\right] \\
& +e^{i(n+1) \phi}\left[-\gamma_{0}^{2} \rho B_{n}^{e h}\left(k_{\rho} \rho\right)+k_{\rho}^{-1}\left(-\frac{k^{2}+3 \gamma_{0}^{2}}{2} \pm i \gamma_{0} k\right) B_{n+1}^{e h}\left(k_{\rho} \rho\right)\right]
\end{aligned}
$$

\subsubsection{1}

\section{Verification of the Perturbation Solution in a Curved Coaxial Waveguide}

The effects of the curvature in a coaxial waveguide was analyzed in [137]. Here, we will use our equations to solve the particular problem presented in [137], i.e., the first-order correction fields for the fundamental $\mathrm{TEM}^{z}$ mode. The direct application of (4-119) presents some problems: the fundamental $\mathrm{TEM}^{z}$ mode of the unperturbed waveguide does not present axial fields, viz., $E_{z}=H_{z}=0$. To overcome this limitation, we consider instead the excitation from the mode $\mathrm{TM}_{00}$ to $z$. This is an asymmetric mode $(n=0)$ in the limit case when $k_{\rho} \rightarrow 0$. We can verify that this mode presents 3 null-field components, namely, $H_{z}=E_{\phi}=H_{\rho}=0$. Furthermore, the non-null remaining fields are given by

$$
\begin{gathered}
E_{0, \zeta}=H_{0}\left(k_{\rho} \rho\right) a_{0}^{e}+J_{0}\left(k_{\rho} \rho\right) b_{0}^{e}, \\
E_{0, \rho}=\frac{i k_{z}}{k_{\rho}}\left[H_{0}^{\prime}\left(k_{\rho} \rho\right) a_{0}^{e}+J_{0}^{\prime}\left(k_{\rho} \rho\right) b_{0}^{e}\right], \\
H_{0, \phi}=\frac{i \omega \epsilon}{k_{\rho}}\left[H_{0}^{\prime}\left(k_{\rho} \rho\right) a_{0}^{e}+J_{0}^{\prime}\left(k_{\rho} \rho\right) b_{0}^{e}\right],
\end{gathered}
$$


where $a_{0}^{e}$ and $b_{0}^{e}$ are constant parameters to be determined by the boundary conditions. Using the small argument approximations for the cylindrical function, we can write [55, p. 462]

$$
\begin{gathered}
H_{0}\left(k_{\rho} \rho\right) \approx \frac{2 i}{\pi} \log \left(k_{\rho} \rho\right), \\
H_{0}^{\prime}\left(k_{\rho} \rho\right)=-H_{1}\left(k_{\rho} \rho\right) \approx \frac{2 i}{\pi k_{\rho} \rho}, \\
J_{0}\left(k_{\rho} \rho\right) \approx 1, \\
J_{0}^{\prime}\left(k_{\rho} \rho\right)=-J_{1}\left(k_{\rho} \rho\right) \approx-\frac{k_{\rho} \rho}{2} .
\end{gathered}
$$

In the coaxial waveguide at hand, there is a PEC wall at $\rho=r_{0}$, such we need to satisfy the condition

$$
H_{0, \phi}\left(\rho=r_{0}\right)=\frac{I_{0}}{2 \pi r_{0}},
$$

which allow us to find $a_{0}^{e}$ :

$$
a_{0}^{e}=-\frac{I_{0} k_{\rho}^{2}}{4 \omega \epsilon} .
$$

By combining the above results, and noting that $k_{z} \rightarrow k$ as $k_{\rho} \rightarrow 0$, the $\mathrm{TM}_{00}^{z}$ fields becomes

$$
\begin{gathered}
E_{0, \zeta}=0, \\
E_{0, \rho}=Z \frac{I_{0}}{2 \pi \rho}, \text { and } \\
H_{0, \phi}=\frac{I_{0}}{2 \pi \rho},
\end{gathered}
$$

that correspond to the same field components for the $\mathrm{TEM}^{z}$ mode in a coaxial waveguide employed in [137]. The above derivation allow us to find $a_{0}^{e}$ using (4-128). Note that $b_{0}^{e}$ becomes zero as $k_{\rho} \rightarrow 0$. Also, like any $\mathrm{TM}^{z}$ mode, we have $a_{n}^{h}=b_{n}^{h}=0$ for all $n$. This allow us to write

$$
\begin{gathered}
B_{0, \pm}^{e h} \rightarrow 0, \\
B_{-1, \pm}^{e h}=H_{-1}\left(k_{\rho} \rho\right) a_{0}^{e} \rightarrow-\frac{I_{0}}{2 \pi \rho} \frac{i k_{\rho}}{\omega \epsilon}, \\
B_{1, \pm}^{e h}=H_{1}\left(k_{\rho} \rho\right) a_{0}^{e} \rightarrow \frac{I_{0}}{2 \pi \rho} \frac{i k_{\rho}}{\omega \epsilon} .
\end{gathered}
$$

The above equations can be used in (4-119), resulting in

$$
\begin{aligned}
L G_{1, \pm}=e^{-i \phi}\left[k_{\rho}^{-1}\left(\frac{k^{2}+3 \gamma_{0}^{2}}{2} \pm i \gamma_{0} k\right)\left(-\frac{I_{0}}{2 \pi \rho} \frac{i k_{\rho}}{\omega \epsilon}\right)\right] \\
+e^{i \phi}\left[k_{\rho}^{-1}\left(-\frac{k^{2}+3 \gamma_{0}^{2}}{2} \pm i \gamma_{0} k\right)\left(\frac{I_{0}}{2 \pi \rho} \frac{i k_{\rho}}{\omega \epsilon}\right)\right] .
\end{aligned}
$$


Note that $i \gamma_{0}=k$ and $\gamma_{0}^{2}=-k^{2}$, and from the boundary conditions, their is no first-order correction on the propagation constant: $\alpha_{1}=0$. Also, we can simplify the above equation to a more compact form:

$$
L G_{1, \pm}=\frac{I_{0}}{2 \pi \rho} \frac{i}{\omega \epsilon}\left[e^{-i \phi}\left(k^{2} \mp k^{2}\right)+e^{i \phi}\left(k^{2} \pm k^{2}\right)\right] .
$$

The first-order axial field corrections can be recovered from $G_{1, \pm}$ by using $E_{1, \zeta}=\left(G_{1,+}+G_{1,-}\right) / 2$ and $H_{1, \zeta}=\left(G_{1,+}-G_{1,-}\right) /(2 i Z)$, so we can derive

$$
\begin{gathered}
L E_{1, \zeta}=Z \frac{i k I_{0}}{\pi \rho} \cos (\phi), \text { and } \\
L H_{1, \zeta}=-\frac{i k I_{0}}{\pi \rho} \sin (\phi) .
\end{gathered}
$$

The above results are identical to ones shown in [137, eq. 8 and eq. 11].

\subsection{4}

\section{Solving the First-Order Correction Field}

In order to solve $G_{1, \pm}$ in (4-119) it is convenient make use an approach similar to one used in [139], in which the orthogonality of the azimuthal harmonic functions allows to establish the ansatz for the particular solution of $G_{1, \pm}$ :

$$
G_{1, \pm}^{p}=R_{1}^{n}(\rho) e^{i n \phi}+R_{1}^{n-1}(\rho) e^{i(n-1) \phi}+R_{1}^{n+1}(\rho) e^{i(n+1) \phi} .
$$

Thus, we admit that the homogeneous solution of $G_{1, \pm}$ satisfies

$$
L G_{1, \pm}^{h}=L_{n} G_{1, \pm}^{n} e^{i n \phi}+L_{n-1} G_{1, \pm}^{n-1} e^{i(n-1) \phi}+L_{n+1} G_{1, \pm}^{n+1} e^{i(n+1) \phi},
$$

where the Bessel differential operator of order integer order $m=n, n \pm 1$ is given by

$$
L_{m}=\frac{d^{2}}{d \rho^{2}}+\frac{1}{\rho} \frac{d}{d \rho}+\left(k_{\rho}^{2}-\frac{m^{2}}{\rho^{2}}\right) .
$$

By comparing (4-139) and (4-119), we can derive the following equations for $R_{1}^{n}(\rho), R_{1}^{n-1}(\rho)$ and $R_{1}^{n+1}(\rho)$ :

$$
\begin{gathered}
L_{n} R_{1}^{n}=-\gamma_{0}^{2} \alpha_{1} B_{n}^{e h}, \\
L_{n-1} R_{1}^{n-1}=-\gamma_{0}^{2} \rho B_{n}^{e h}\left(k_{\rho} \rho\right)+k_{\rho}^{-1}\left(\frac{k^{2}+3 \gamma_{0}^{2}}{2} \pm i \gamma_{0} k\right) B_{n-1}^{e h}\left(k_{\rho} \rho\right), \\
L_{n+1} R_{1}^{n+1}=-\gamma_{0}^{2} \rho B_{n}^{e h}\left(k_{\rho} \rho\right)+k_{\rho}^{-1}\left(-\frac{k^{2}+3 \gamma_{0}^{2}}{2} \pm i \gamma_{0} k\right) B_{n+1}^{e h}\left(k_{\rho} \rho\right) .
\end{gathered}
$$

To aid in solving (4-142) we can pursue solutions of the operator $L_{m}$ over 
$\rho^{q} B_{p}\left(k_{\rho} \rho\right):$

$$
L_{m}\left[\rho^{q} B_{p}\left(k_{\rho} \rho\right)\right]=\left(\frac{d^{2}}{d \rho^{2}}+\frac{1}{\rho} \frac{d}{d \rho}\right)\left[\rho^{q} B_{p}\left(k_{\rho} \rho\right)\right]+\left(k_{\rho}^{2}-\frac{m^{2}}{\rho^{2}}\right)\left[\rho^{q} B_{p}\left(k_{\rho} \rho\right)\right] .
$$

By omitting the argument of the cylindrical functions, we can write

$$
\begin{array}{r}
L_{m}\left[\rho^{q} B_{p}\left(k_{\rho} \rho\right)\right]=\frac{d^{2} \rho^{q}}{d \rho^{2}} B_{p}+2 \frac{d \rho^{q}}{d \rho} \frac{d B_{p}}{d \rho}+\frac{d^{2} B_{p}}{d \rho^{2}} \rho^{q}+\frac{1}{\rho}\left\{\rho^{q} k_{\rho} B_{p}^{\prime}+q \rho^{q-1} B_{p}\right\} \\
+k_{\rho}^{2} \rho^{q} B_{p}-m^{2} \rho^{q-2} B_{p}, \quad(4-1
\end{array}
$$

which we can reduce to

$$
\begin{aligned}
L_{m}\left[\rho^{q} B_{p}\left(k_{\rho} \rho\right)\right]=q(q & -1) \rho^{q-2} B_{p}+2 q \rho^{q-1} k_{\rho} B_{p}^{\prime}+\rho^{q} \frac{k_{\rho}^{2}}{4}\left(B_{p-2}-2 B_{p}+B_{p+2}\right) \\
& +\rho^{q-1} k_{\rho} B_{p}^{\prime}+q \rho^{q-2} B_{p}+k_{\rho}^{2} \rho^{q} B_{p}-m^{2} \rho^{q-2} B_{p} . \quad(4-145)
\end{aligned}
$$

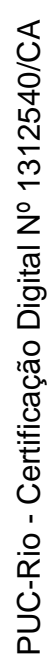

Expanding the RHS of the above equation, we have found

$$
\begin{array}{r}
L_{m}\left[\rho^{q} B_{p}\left(k_{\rho} \rho\right)\right]=q^{2} \rho^{q-2} B_{p}-q \rho^{q-2} B_{p}+2 q \rho^{q-1} k_{\rho} B_{p}^{\prime}+\rho^{q} \frac{k_{\rho}^{2}}{4}\left(B_{p-2}-2 B_{p}+B_{p+2}\right) \\
+\rho^{q-1} k_{\rho} B_{p}^{\prime}+q \rho^{q-2} B_{p}+k_{\rho}^{2} \rho^{q} B_{p}-m^{2} \rho^{q-2} B_{p} . \quad(4-146)
\end{array}
$$

After a simple simplification, we can obtain

$$
\begin{aligned}
L_{m}\left[\rho^{q} B_{p}\left(k_{\rho} \rho\right)\right]= & \left(q^{2}-m^{2}\right) \rho^{q-2} B_{p}+2 q \rho^{q-1} k_{\rho} B_{p}^{\prime} \\
& +\rho^{q} \frac{k_{\rho}^{2}}{4}\left(B_{p-2}-2 B_{p}+B_{p+2}\right)+\rho^{q-1} k_{\rho} B_{p}^{\prime}+k_{\rho}^{2} \rho^{q} B_{p} .
\end{aligned}
$$

Employing the identities $B_{p \pm 1}=2(p \pm 1) /\left(k_{\rho} \rho\right) B_{p \pm 1}-B_{p}[55$, p. 463], we can derive

$$
\rho^{q} \frac{k_{\rho}^{2}}{4}\left(B_{p-2}+B_{p+2}\right)=-\frac{1}{2} \rho^{q} k_{\rho}^{2} B_{p}+p^{2} \rho^{q-2} B_{p}-\rho^{q-1} k_{\rho} B_{p}^{\prime},
$$

that allows to rewrite $L_{m}\left[\rho^{q} B_{p}\left(k_{\rho} \rho\right)\right]$ as

$$
\begin{aligned}
L_{m}\left[\rho^{q} B_{p}\left(k_{\rho} \rho\right)\right]=\left(q^{2}-\right. & \left.m^{2}\right) \rho^{q-2} B_{p}+2 q \rho^{q-1} k_{\rho} B_{p}^{\prime}-\frac{1}{2} \rho^{q} k_{\rho}^{2} B_{p}+p^{2} \rho^{q-2} B_{p} \\
& -\rho^{q-1} k_{\rho} B_{p}^{\prime}-\frac{1}{2} \rho^{q} k_{\rho}^{2} B_{p}+\rho^{q-1} k_{\rho} B_{p}^{\prime}+\rho^{q} k_{\rho}^{2} B_{p}
\end{aligned}
$$

After some simplifications, the last equation reduces to

$$
L_{m}\left[\rho^{q} B_{p}\left(k_{\rho} \rho\right)\right]=\left(p^{2}+q^{2}-m^{2}\right) \rho^{q-2} B_{p}+2 q \rho^{q-1} k_{\rho} B_{p}^{\prime} .
$$


We can derive another pair of useful equation using $B_{p}^{\prime}= \pm B_{p \mp 1} \mp p /\left(k_{\rho} \rho\right) B_{p}$, resulting in

$$
\begin{aligned}
L_{m}\left[\rho^{q} B_{p}\left(k_{\rho} \rho\right)\right] & =\left(p^{2}+q^{2}-m^{2}\right) \rho^{q-2} B_{p}\left(k_{\rho} \rho\right)+2 q k_{\rho} \rho^{q-1} B_{p}^{\prime}\left(k_{\rho} \rho\right) \\
& =\left[(p-q)^{2}-m^{2}\right] \rho^{q-2} B_{p}\left(k_{\rho} \rho\right)+2 q k_{\rho} \rho^{q-1} B_{p-1}\left(k_{\rho} \rho\right) \\
& =\left[(p+q)^{2}-m^{2}\right] \rho^{q-2} B_{p}\left(k_{\rho} \rho\right)-2 q k_{\rho} \rho^{q-1} B_{p+1}\left(k_{\rho} \rho\right),
\end{aligned}
$$

which is a generalization of the methods used in [51, p. 63] and $[139]^{6}$ for a linear combination of cylindrical functions $B_{p}$ of integer order $p$.

We can find useful results using (4-152) with $p=n+1, q=1$ and $m=n$, i.e.,

$$
L_{n}\left[\rho B_{n+1}\left(k_{\rho} \rho\right)\right]=2 k_{\rho} B_{n}\left(k_{\rho} \rho\right) .
$$

Alternatively, by using (4-153) with $p=n-1, q=1$ and $m=n$, we verify that

$$
L_{n}\left[\rho B_{n-1}\left(k_{\rho} \rho\right)\right]=-2 k_{\rho} B_{n}\left(k_{\rho} \rho\right)
$$

also must be satisfied. By subtracting (4-155) from (4-154), after some manipulation we can write

$$
\begin{aligned}
L_{n}\left\{\frac{\rho}{4 k_{\rho}}\left[B_{n+1}\left(k_{\rho} \rho\right)-B_{n-1}\left(k_{\rho} \rho\right)\right]\right\} & =B_{n}\left(k_{\rho} \rho\right), \\
L_{n}\left[-\frac{\rho}{2 k_{\rho}} B_{n}^{\prime}\left(k_{\rho} \rho\right)\right] & =B_{n}\left(k_{\rho} \rho\right),
\end{aligned}
$$

that allow us to figure out $R_{1}^{n}$ in (4-142a) except for a constant factor. The above equation also can be used to solve $L_{n \pm 1}[f(\rho)]=B_{n \pm 1}^{e h}\left(k_{\rho} \rho\right)$, and we can derive the proper function $f(\rho)$ that satisfies the last terms in (4-142b) and (4-142c).

At first glance, it may sound strange that the Bessel differential equation of order $n$ with an inhomogeneous term $B_{n}\left(k_{\rho} \rho\right)$ presents three particular solutions: $\rho B_{n+1}\left(k_{\rho} \rho\right) /\left(2 k_{\rho}\right),-\rho B_{n-1}\left(k_{\rho} \rho\right) /\left(2 k_{\rho}\right)$ and $2 \rho B_{n}^{\prime}\left(k_{\rho} \rho\right) /\left(2 k_{\rho}\right)$. But note that the Bessel operator of order $n$ applied to $\rho B_{n}^{\prime}\left(k_{\rho} \rho\right)$ leads to

$$
L_{n}\left[B_{n}^{\prime}\left(k_{\rho} \rho\right)\right]= \pm L_{n}\left[\rho B_{n \mp 1}\left(k_{\rho} \rho\right)\right] \mp \frac{n}{k_{\rho}} L_{n}\left[B_{n}\left(k_{\rho} \rho\right)\right],
$$

in which the last term clearly satisfies the homogeneous Bessel differential equation, and becomes zero.

In order to find $R_{1}^{n-1}$, except for a constant factor, we need to find $p, q$ and $m$ which satisfy

$$
L_{n-1}\left[\rho^{q} B_{p}\left(k_{\rho} \rho\right)\right]=\rho B_{n}\left(k_{\rho} \rho\right), \text { and }
$$

${ }^{6}$ An equation similar to (4-151) shown in [139, eq. 45] is incorrect. The last term in the said equation should instead be equal to the last term shown in (4-151). 


$$
L_{n-1}\left[\rho^{q} B_{p}\left(k_{\rho} \rho\right)\right]=B_{n-1}\left(k_{\rho} \rho\right) .
$$

By choosing proper combinations of $m, p$ and $q$ in (4-152) we can find:

$$
\begin{gathered}
\left.\begin{array}{c}
m=n-1 \\
p=n+1 \\
q=2
\end{array}\right\}: L_{n-1}\left[\rho^{2} B_{n+1}\left(k_{\rho} \rho\right)\right]=4 k_{\rho} \rho B_{n}\left(k_{\rho} \rho\right), \\
\left.\begin{array}{c}
m=n-1 \\
p=n \\
q=1
\end{array}\right\}: L_{n-1}\left[\rho B_{n}\left(k_{\rho} \rho\right)\right]=2 k_{\rho} B_{n-1}\left(k_{\rho} \rho\right),
\end{gathered}
$$

that allow us to solve $R_{1}^{n-1}$ in (4-142b).

Proceeding in a similar manner, we can solve $R_{1}^{n+1}$ choosing the following values for $m, p$ and $q$ in (4-153):

$$
\begin{gathered}
\left.\begin{array}{c}
m=n+1 \\
\begin{array}{c}
p= \\
q=2
\end{array}
\end{array}\right\}: L_{n+1}\left[\rho^{2} B_{n-1}\left(k_{\rho} \rho\right)\right]=-4 k_{\rho} \rho B_{n}\left(k_{\rho} \rho\right), \\
\left.\begin{array}{c}
m=n+1 \\
p=n \\
q=1
\end{array}\right\}: L_{n+1}\left[\rho B_{n}\left(k_{\rho} \rho\right)\right]=-2 k_{\rho} B_{n+1}\left(k_{\rho} \rho\right) .
\end{gathered}
$$

In short, we can satisfy (4-142) using

$$
\begin{gathered}
L_{n}\left[-\frac{\rho}{2 k_{\rho}} B_{n}^{\prime}\right]=B_{n}, \\
L_{n-1}\left(\frac{\rho^{2}}{4 k_{\rho}} B_{n+1}\right)=\rho B_{n}, \\
L_{n-1}\left(\frac{\rho}{2 k_{\rho}} B_{n}\right)=B_{n-1}, \\
L_{n+1}\left(-\frac{\rho^{2}}{4 k_{\rho}} B_{n-1}\right)=\rho B_{n}, \\
L_{n+1}\left(-\frac{\rho}{2 k_{\rho}} B_{n}\right)=B_{n+1},
\end{gathered}
$$

where the argument of cylindrical functions is omitted for the sake of brevity.

With the results in (4-165) we can solve the particular solution of (4-119) for each azimuthal dependent term; namely, for terms associated with $\exp (i m \phi), m=n, n \pm 1$.

The homogeneous solution of (4-119) must satisfies (4-140), and based 
on the linear independence of the terms $\exp (i m \phi)$, we can write

$$
\begin{gathered}
L_{n} G_{1, \pm}^{n}=0, \\
L_{n-1} G_{1, \pm}^{n-1}=0, \\
L_{n+1} G_{1, \pm}^{n+1}=0,
\end{gathered}
$$

whose solutions for $G_{1, \pm}^{m}$ can be written as combinations of cylindrical function of order $m$, e.g.:

$$
\begin{gathered}
G_{1, \pm}^{n}=A_{1, \pm}^{n} H_{n}\left(k_{\rho} \rho\right)+B_{1, \pm}^{n} J_{n}\left(k_{\rho} \rho\right), \\
G_{1, \pm}^{n-1}=A_{1, \pm}^{n-1} H_{n-1}\left(k_{\rho} \rho\right)+B_{1, \pm}^{n-1} J_{n-1}\left(k_{\rho} \rho\right), \\
G_{1, \pm}^{n+1}=A_{1, \pm}^{n+1} H_{n+1}\left(k_{\rho} \rho\right)+B_{1, \pm}^{n+1} J_{n+1}\left(k_{\rho} \rho\right),
\end{gathered}
$$

where $H_{m}$ and $J_{m}$ are the first kind Hankel and Bessel functions of integer order $m$. The constants $A_{1, \pm}^{m}$ and $B_{1, \pm}^{m}$ will be determined on the application of the boundary conditions. Note that instead of $H_{m}$ and $J_{m}$ we could have chosen any pair of linear independent cylindrical functions.

Accordingly, we get the general solution for $G_{1, \pm}$ as

$$
\begin{aligned}
& G_{1, \pm}=e^{i n \phi}\left[\frac{\gamma_{0}^{2} \alpha_{1} \rho}{2 k_{\rho}} B_{n, \pm}^{\prime e h}+A_{1, \pm}^{n} H_{n, \pm}+B_{1, \pm}^{n} J_{n, \pm}\right] \\
& +e^{i(n-1) \phi}\left[-\frac{\gamma_{0}^{2} \rho^{2}}{4 k_{\rho}} B_{n+1, \pm}^{e h}+\frac{\rho}{2 k_{\rho}^{2}}\left(\frac{k^{2}+3 \gamma_{0}^{2}}{2} \pm i \gamma_{0} k\right) B_{n, \pm}^{e h}\right. \\
& \left.+A_{1, \pm}^{n-1} H_{n-1, \pm}+B_{1, \pm}^{n-1} J_{n-1, \pm}\right] \\
& +e^{i(n+1) \phi}\left[\frac{\gamma_{0}^{2} \rho^{2}}{4 k_{\rho}} B_{n-1, \pm}^{e h}+\frac{\rho}{2 k_{\rho}^{2}}\left(\frac{k^{2}+3 \gamma_{0}^{2}}{2} \mp i \gamma_{0} k\right) B_{n, \pm}^{e h}\right. \\
& \left.+A_{1, \pm}^{n+1} H_{n+1, \pm}+B_{1, \pm}^{n+1} J_{n+1, \pm}\right] \text {. }
\end{aligned}
$$

As said before, the arbitrary multiplying constants $A_{1, \pm}^{m}$ and $B_{1, \pm}^{m}, m=$ $n, n \mp 1$, arise as solutions of the homogeneous equation of (4-119), and will be determined later by applying the boundary conditions. Note that we have restored the subscript \pm in $B_{n, \pm}^{e h}$, but the argument of cylindrical functions remains omitted to shorten the notation.

Taking into account the terms of order $R^{-1}$, due to the linear independence of the exponentials $\exp (i n \phi), \exp [i(n-1) \phi]$ and $\exp [i(n+1) \phi]$, the boundary conditions split up into three equations, one to each exponential 
term ${ }^{7}$.

\subsection{5}

\section{First-Order Correction to Axial Fields}

The perturbation solution for $G_{ \pm}$up to the order $R^{-1}$ is such as

$$
G_{ \pm}=E_{\zeta} \pm N_{0} H_{\zeta}=\left(G_{0, \pm}+R^{-1} G_{1, \pm}\right) e^{\gamma \zeta}
$$

The axial electric field can be recovered from $G_{ \pm}$by using

$$
E_{\zeta}=\frac{G_{+}+G_{-}}{2}
$$

and similarly, we can establish the following equation for the axial magnetic field

$$
H_{\zeta}=\frac{G_{+}-G_{-}}{2 N_{0}}
$$

To recover the axial fields, noting the sign of \pm (or $\mp$ ) that came with the term $i \gamma_{0} k$ in (4-168), the following relationships are relevant. Let us now consider

$$
\begin{gathered}
B_{ \pm}=(a \pm b)\left(B^{e} \pm N_{0} B^{h}\right), \\
\frac{B_{+}+B_{-}}{2}=a B^{e}+b N_{0} B^{h}, \text { and } \\
\frac{B_{+}-B_{-}}{2 N_{0}}=\frac{b B^{e}+a N_{0} B^{h}}{N_{0}}=\frac{b B^{e}}{N_{0}}+a B^{h} .
\end{gathered}
$$

then:

Using the above relationships, we have found

$$
\begin{aligned}
E_{\zeta}=\left[B_{n}^{e}\right. & \left.+R^{-1}\left(\frac{\gamma_{0}^{2} \alpha_{1} \rho}{2 k_{\rho}} B_{n}^{\prime e}+\frac{A_{1,+}^{n}+A_{1,-}^{n}}{2} H_{n}+\frac{B_{1,+}^{n}+B_{1,-}^{n}}{2} J_{n}\right)\right] e^{i n \phi} e^{\gamma \zeta} \\
& +R^{-1}\left[-\frac{\gamma_{0}^{2} \rho^{2}}{4 k_{\rho}} B_{n+1}^{e}+\frac{\rho}{2 k_{\rho}^{2}}\left(\frac{k^{2}+3 \gamma_{0}^{2}}{2} B_{n}^{e}+i \gamma_{0} k N_{0} B_{n}^{h}\right)\right. \\
& \left.+\frac{A_{1,+}^{n-1}+A_{1,-}^{n-1}}{2} H_{n-1}+\frac{B_{1,+}^{n-1}+B_{1,-}^{n-1}}{2} J_{n-1}\right] e^{i(n-1) \phi} e^{\gamma \zeta} \\
& +R^{-1}\left[\frac{\gamma_{0}^{2} \rho^{2}}{4 k_{\rho}} B_{n-1}^{e}+\frac{\rho}{2 k_{\rho}^{2}}\left(\frac{k^{2}+3 \gamma_{0}^{2}}{2} B_{n}^{e}-i \gamma_{0} k N_{0} B_{n}^{h}\right)\right. \\
+ & \left.\frac{A_{1,+}^{n+1}+A_{1,-}^{n+1}}{2} H_{n+1}+\frac{B_{1,+}^{n+1}+B_{1,-}^{n+1}}{2} J_{n+1}\right] e^{i(n+1) \phi} e^{\gamma \zeta}, \text { and }(4-175)
\end{aligned}
$$

\footnotetext{
${ }^{7}$ It is important to observe that in (4-168), the terms $A_{1, \pm}^{n} H_{n, \pm} \exp (i n \phi)$ and $B_{1, \pm}^{n} J_{n, \pm} \exp (i n \phi)$ are linearly dependent of the zeroth-order solution $G_{0, \pm}$ defined in (4-111). This can be a clue that both $A_{1, \pm}^{n}$ and $B_{1, \pm}^{n}$ are zero.
} 


$$
\begin{aligned}
H_{\zeta}=\left[B_{n}^{h}+\right. & \left.R^{-1}\left(\frac{\gamma_{0}^{2} \alpha_{1} \rho}{2 k_{\rho}} B_{n}^{\prime h}+\frac{A_{1,+}^{n}-A_{1,-}^{n}}{2 N_{0}} H_{n}+\frac{B_{1,+}^{n}-B_{1,-}^{n}}{2 N_{0}} J_{n}\right)\right] e^{i n \phi} e^{\gamma \zeta} \\
& +R^{-1}\left[-\frac{\gamma_{0}^{2} \rho^{2}}{4 k_{\rho}} B_{n+1}^{h}+\frac{\rho}{2 k_{\rho}^{2}}\left(\frac{k^{2}+3 \gamma_{0}^{2}}{2} B_{n}^{h}+\frac{i \gamma_{0} k}{N_{0}} B_{n}^{e}\right)\right. \\
& \left.+\frac{A_{1,+}^{n-1}-A_{1,-}^{n-1}}{2 N_{0}} H_{n-1}+\frac{B_{1,+}^{n-1}-B_{1,-}^{n-1}}{2 N_{0}} J_{n-1}\right] e^{i(n-1) \phi} e^{\gamma \zeta} \\
& +R^{-1}\left[\frac{\gamma_{0}^{2} \rho^{2}}{4 k_{\rho}} B_{n-1}^{h}+\frac{\rho}{2 k_{\rho}^{2}}\left(\frac{k^{2}+3 \gamma_{0}^{2}}{2} B_{n}^{h}-\frac{i \gamma_{0} k}{N_{0}} B_{n}^{e}\right)\right. \\
& \left.\left.+\frac{A_{1,+}^{n+1}-A_{1,-}^{n+1}}{2 N_{0}} H_{n+1}+\frac{B_{1,+}^{n+1}-B_{1,-}^{n+1}}{2 N_{0}} J_{n+1}\right] e^{i(n+1) \phi} e^{\gamma \zeta}\right\} . \quad \text { (4-176) }
\end{aligned}
$$

The axial field can be written in a compact form, highlighting the azimuthal dependence and the order in terms of $R^{-1}$ in the shape of

$$
\begin{array}{r}
{\left[\begin{array}{c}
E_{\zeta} \\
H_{\zeta}
\end{array}\right]=\left\{\left[\begin{array}{l}
E_{0, \zeta}^{n} \\
H_{0, \zeta}^{n}
\end{array}\right]+R^{-1}\left[\begin{array}{c}
E_{1, \zeta}^{n} \\
H_{1, \zeta}^{n}
\end{array}\right]\right\} e^{i n \phi} e^{\gamma \zeta}+R^{-1}\left[\begin{array}{l}
E_{1, \zeta}^{n-1} \\
H_{1, \zeta}^{n-1}
\end{array}\right] e^{i(n-1) \phi-i \psi} e^{\gamma \zeta}} \\
+R^{-1}\left[\begin{array}{c}
E_{1, \zeta}^{n+1} \\
H_{1, \zeta}^{n+1}
\end{array}\right] e^{i(n+1) \phi+i \psi} e^{\gamma \zeta},
\end{array}
$$

where the zeroth-order curvature vanishing axial fields are given by $E_{0, \zeta}^{n}=B_{n}^{e}$ and $H_{0, \zeta}^{n}=B_{n}^{h}$.

The portion of the axial fields proportional to $R^{-1} \exp [i n \phi]$ are obtained comparing (4-177) with (4-175) and (4-176), so that:

$$
\left[\begin{array}{l}
E_{1, \zeta}^{n} \\
H_{1, \zeta}^{n}
\end{array}\right]=\alpha_{1} \frac{\gamma_{0}^{2} \rho}{2 k_{\rho}}\left[\begin{array}{l}
B_{n}^{\prime e} \\
B_{n}^{\prime h}
\end{array}\right]+H_{n}\left[\begin{array}{l}
a_{1, n}^{e} \\
a_{1, n}^{h}
\end{array}\right]+J_{n}\left[\begin{array}{l}
b_{1, n}^{e} \\
b_{1, n}^{h}
\end{array}\right],
$$

where

$$
\begin{gathered}
{\left[\begin{array}{l}
a_{1, m}^{e} \\
b_{1, m}^{e}
\end{array}\right]=\frac{1}{2}\left[\begin{array}{l}
A_{1,+}^{m}+A_{1,-}^{m} \\
B_{1,+}^{m}+B_{1,-}^{m}
\end{array}\right],} \\
{\left[\begin{array}{l}
a_{1, m}^{h} \\
b_{1, m}^{h}
\end{array}\right]=\frac{1}{2 N_{0}}\left[\begin{array}{l}
A_{1,+}^{m}-A_{1,-}^{m} \\
B_{1,+}^{m}-B_{1,-}^{m}
\end{array}\right] .}
\end{gathered}
$$

Note that the four unknowns in $A_{1, \pm}$ and $B_{1, \pm}$ where transformed to other four unknowns in $a_{1, m}^{e, h}$ and $b_{1, m}^{e, h}$. These constants will be determined later by applying the boundary conditions.

The portion of the axial fields proportional to $R^{-1} \exp [i(n \pm 1) \phi]$ are derived comparing (4-177) with (4-175) and (4-176), so that:

$$
\begin{aligned}
& E_{1, \zeta}^{n \pm 1}=R_{\zeta, n \pm 1}^{e}+a_{1, n \pm 1}^{e} H_{n \pm 1}+b_{1, n \pm 1}^{e} J_{n \pm 1}, \\
& H_{1, \zeta}^{n \pm 1}=R_{\zeta, n \pm 1}^{h}+a_{1, n \pm 1}^{h} H_{n \pm 1}+b_{1, n \pm 1}^{h} J_{n \pm 1},
\end{aligned}
$$

where 


$$
\begin{gathered}
R_{\zeta, n \pm 1}^{e}=R_{\zeta, n \pm 1}^{e}\left(k_{\rho} \rho\right)= \pm \frac{\gamma_{0}^{2} \rho^{2}}{4 k_{\rho}} B_{n \mp 1}^{e}+\frac{\rho}{2 k_{\rho}^{2}}\left(\frac{k^{2}+3 \gamma_{0}^{2}}{2} B_{n}^{e} \mp i \gamma_{0} k N_{0} B_{n}^{h}\right), \\
R_{\zeta, n \pm 1}^{h}=R_{\zeta, n \pm 1}^{h}\left(k_{\rho} \rho\right)= \pm \frac{\gamma_{0}^{2} \rho^{2}}{4 k_{\rho}} B_{n \mp 1}^{h}+\frac{\rho}{2 k_{\rho}^{2}}\left(\frac{k^{2}+3 \gamma_{0}^{2}}{2} B_{n}^{h} \mp \frac{i \gamma_{0} k}{N_{0}} B_{n}^{e}\right) .
\end{gathered}
$$

In a compact manner, for $m=n \pm 1$, we can write

$$
\left[\begin{array}{c}
E_{1, \zeta}^{m} \\
H_{1, \zeta}^{m}
\end{array}\right]=\left[\begin{array}{c}
R_{\zeta, m}^{e} \\
R_{\zeta, m}^{h}
\end{array}\right]+H_{m}\left[\begin{array}{l}
a_{1, m}^{e} \\
a_{1, m}^{h}
\end{array}\right]+J_{m}\left[\begin{array}{l}
b_{1, m}^{e} \\
b_{1, m}^{h}
\end{array}\right] .
$$

Recalling that

$$
B_{m}^{e, h}=H_{m}\left(k_{\rho} \rho\right) a_{0, n}^{e, h}+J_{m}\left(k_{\rho} \rho\right) b_{0, n}^{e, h}
$$

we can define

$$
\bar{R}_{\zeta, m}=\left[\begin{array}{c}
R_{\zeta, m}^{e} \\
R_{\zeta, m}^{h}
\end{array}\right]=\overline{\bar{H}}_{\zeta, m}^{p} \bar{a}_{0, n}+\overline{\bar{J}}_{\zeta, m}^{p} \bar{b}_{0, n}
$$

where

$$
\overline{\bar{B}}_{\zeta, n}^{p}=\alpha_{1} \frac{\gamma_{0}^{2} \rho}{2 k_{\rho}}\left[\begin{array}{cc}
B_{n}^{\prime}\left(k_{\rho} \rho\right) & 0 \\
0 & B_{n}^{\prime}\left(k_{\rho} \rho\right)
\end{array}\right] \text {, }
$$

$$
\begin{aligned}
\overline{\bar{B}}_{\zeta, n \pm 1}^{p}= \pm \frac{\gamma_{0}^{2} \rho^{2}}{4 k_{\rho}}\left[\begin{array}{cc}
B_{n \mp 1}\left(k_{\rho} \rho\right) & 0 \\
0 & B_{n \mp 1}\left(k_{\rho} \rho\right)
\end{array}\right] \\
+\frac{\rho}{2 k_{\rho}^{2}}\left[\begin{array}{cc}
\frac{k^{2}+3 \gamma_{0}^{2}}{2} B_{n}\left(k_{\rho} \rho\right) & \mp i \gamma_{0} k N_{0} B_{n}\left(k_{\rho} \rho\right) \\
\mp \frac{i \gamma_{0} k}{N_{0}} B_{n}\left(k_{\rho} \rho\right) & \frac{k^{2}+3 \gamma_{0}^{2}}{2} B_{n}\left(k_{\rho} \rho\right)
\end{array}\right]
\end{aligned}
$$

At this point, the first-order correction to propagation constant $\alpha_{1}$ is an unknown. Then, it is appropriated to redefine $\overline{\bar{B}}_{\zeta, n}^{p}$ such as

$$
\overline{\bar{B}}_{\zeta, n}^{\hat{p}}=\frac{1}{\alpha_{1}} \overline{\bar{B}}_{\zeta, n}^{p}=\frac{\gamma_{0}^{2} \rho}{2 k_{\rho}} B_{n}^{\prime}\left(k_{\rho} \rho\right) \overline{\bar{I}}
$$

Also, using $N_{0}=i(\mu / \epsilon)^{1 / 2}$, we can derive $\overline{\bar{B}}_{\zeta, n \pm 1}$ in a more compact shape:

$$
\overline{\bar{B}}_{\zeta, n \pm 1}^{p}= \pm \frac{\gamma_{0}^{2} \rho^{2}}{4 k_{\rho}} B_{n \mp 1}\left(k_{\rho} \rho\right) \overline{\bar{I}}+\frac{\rho}{2 k_{\rho}^{2}} B_{n}\left(k_{\rho} \rho\right)\left[\begin{array}{cc}
\frac{k^{2}+3 \gamma_{0}^{2}}{2} & \pm \gamma_{0} \omega \mu \\
\mp \gamma_{0} \omega \epsilon & \frac{k^{2}+3 \gamma_{0}^{2}}{2}
\end{array}\right] .
$$

Thus, for $m=n, n \pm 1$, we can write

$$
\begin{aligned}
{\left[\begin{array}{c}
E_{1, \zeta}^{m} \\
H_{1, \zeta}^{m}
\end{array}\right] } & =\bar{R}_{\zeta m}+\overline{\bar{H}}_{\zeta, m} \bar{a}_{1, m}+\overline{\bar{J}}_{\zeta, m} \bar{b}_{1, m} \\
& =\overline{\bar{H}}_{\zeta, m}^{p} \bar{a}_{0, n}+\overline{\bar{J}}_{\zeta, m}^{p} \bar{b}_{0, n}+\overline{\bar{H}}_{m} \bar{a}_{1, m}+\overline{\bar{J}}_{m} \bar{b}_{1, m}
\end{aligned}
$$

where $\overline{\bar{B}}_{\zeta, m}=B_{m}\left(k_{\rho} \rho\right) \overline{\bar{I}}$, with $B_{m}=H_{m}$ or $J_{m}$ being first kinds Hankel or Bessel function of order $m$. For $m=n$ we must use $\overline{\bar{B}}_{\zeta, n}^{p}=\alpha_{1} \overline{\bar{B}}_{\zeta, n}^{\hat{p}}$ to apply the 
boundary conditions for the fields associated to $\exp (i n \phi)$ because $\alpha_{1}$ is still an unknown quantity.

\subsection{6}

\section{First-Order Correction to Transversal Fields}

The proper combinations of (4-4a), (4-4b), (4-4d) and (4-4e) allow us to write the fields transversal to $\zeta$ from the axial fields $E$ and $H$ as

$$
\begin{gathered}
{\left[\begin{array}{c}
E_{\phi} \\
H_{\phi}
\end{array}\right]=\frac{1}{k_{t}^{2} \rho}\left[\begin{array}{cc}
\gamma \frac{\partial}{\partial \phi} & -h_{\zeta} i \omega \mu \rho \frac{\partial}{\partial \rho} \\
h_{\zeta} i \omega \epsilon \rho \frac{\partial}{\partial \rho} & \gamma \frac{\partial}{\partial \phi}
\end{array}\right]\left[\begin{array}{l}
E \\
H
\end{array}\right] \text {, and }} \\
{\left[\begin{array}{l}
E_{\rho} \\
H_{\rho}
\end{array}\right]=\frac{1}{k_{t}^{2} \rho}\left[\begin{array}{cc}
\gamma \rho \frac{\partial}{\partial \rho} & h_{\zeta} i \omega \mu \frac{\partial}{\partial \phi} \\
-h_{\zeta} i \omega \epsilon \frac{\partial}{\partial \phi} & \gamma \rho \frac{\partial}{\partial \rho}
\end{array}\right]\left[\begin{array}{l}
E \\
H
\end{array}\right] .}
\end{gathered}
$$

Recollecting that $E=h_{\zeta} E_{\zeta}$ and $H=h_{\zeta} H_{\zeta}$, we can use

$$
\begin{gathered}
\frac{\partial}{\partial \phi}\left(h_{\zeta} G_{\zeta}\right)=h_{\zeta} \frac{\partial G_{\zeta}}{\partial \phi}+R^{-1} \rho \sin (\phi+\psi) G_{\zeta}, \text { and } \\
\frac{\partial}{\partial \rho}\left(h_{\zeta} G_{\zeta}\right)=h_{\zeta} \frac{\partial G_{\zeta}}{\partial \rho}-R^{-1} \cos (\phi+\psi) G_{\zeta},
\end{gathered}
$$

to derive a direct relation between the axial and the transversal fields:

$$
\begin{aligned}
& {\left[\begin{array}{l}
E_{\phi} \\
H_{\phi}
\end{array}\right]=} \frac{h_{\zeta}^{2}}{k_{t}^{2} \rho}\left[\begin{array}{cc}
\frac{\gamma}{h_{\zeta}} \frac{\partial}{\partial \phi} & -i \omega \mu \rho \frac{\partial}{\partial \rho} \\
i \omega \epsilon \rho \frac{\partial}{\partial \rho} & \frac{\gamma}{h_{\zeta}} \frac{\partial}{\partial \phi}
\end{array}\right]\left[\begin{array}{l}
E_{\zeta} \\
H_{\zeta}
\end{array}\right] \\
&+R^{-1} \frac{h_{\zeta}}{k_{t}^{2}}\left[\begin{array}{cc}
\frac{\gamma}{h_{\zeta}} \sin (\phi+\psi) & i \omega \mu \cos (\phi+\psi) \\
-i \omega \epsilon \cos (\phi+\psi) & \frac{\gamma}{h_{\zeta}} \sin (\phi+\psi)
\end{array}\right]\left[\begin{array}{l}
E_{\zeta} \\
H_{\zeta}
\end{array}\right], \text { and } \\
& {\left[\begin{array}{c}
E_{\rho} \\
H_{\rho}
\end{array}\right]=\frac{h_{\zeta}^{2}}{k_{t}^{2} \rho}\left[\begin{array}{ccc}
\frac{\gamma}{h_{\zeta}} \rho \frac{\partial}{\partial \rho} & i \omega \mu \frac{\partial}{\partial \phi} \\
-i \omega \epsilon \frac{\partial}{\partial \phi} & \frac{\gamma}{h_{\zeta}} \rho \frac{\partial}{\partial \rho}
\end{array}\right]\left[\begin{array}{l}
E_{\zeta} \\
H_{\zeta}
\end{array}\right] . } \\
&+R^{-1} \frac{h_{\zeta}}{k_{t}^{2}}\left[\begin{array}{ll}
-\frac{\gamma}{h_{\zeta}} \cos (\phi+\psi) & i \omega \mu \sin (\phi+\psi) \\
-i \omega \epsilon \sin (\phi+\psi) & -\frac{\gamma}{h_{\zeta}} \cos (\phi+\psi)
\end{array}\right]\left[\begin{array}{l}
E_{\zeta} \\
H_{\zeta}
\end{array}\right] .
\end{aligned}
$$

At this point, we should truncate the transversal fields up to the order $R^{-1}$. For this purpose, the following approximations are useful:

$$
\begin{gathered}
\gamma=\gamma_{0} \sqrt{1+R^{-1} \alpha_{1}+O\left(R^{-2}\right)}=\gamma_{0}\left(1+R^{-1} \frac{\alpha_{1}}{2}\right)+O\left(R^{-2}\right), \\
\frac{h_{\zeta} \gamma}{k^{2} h_{\zeta}^{2}+\gamma^{2}}=\frac{\gamma_{0}}{k_{\rho}^{2}}-R^{-1} \frac{\gamma_{0}\left(-k^{2}+\gamma_{0}^{2}\right)}{2 k_{\rho}^{4}}\left[\alpha_{1}+2 \rho \cos (\phi+\psi)\right]+O\left(R^{-2}\right), \\
\frac{h_{\zeta}^{2}}{k^{2} h_{\zeta}^{2}+\gamma^{2}}=\frac{1}{k_{\rho}^{2}}-R^{-1} \frac{\gamma_{0}^{2}}{k_{\rho}^{4}}\left[\alpha_{1}+2 \rho \cos (\phi+\psi)\right]+O\left(R^{-2}\right),
\end{gathered}
$$




$$
\begin{aligned}
\frac{\gamma}{k^{2} h_{\zeta}^{2}+\gamma^{2}} & =\frac{\gamma_{0}}{k_{\rho}^{2}}+O\left(R^{-1}\right), \\
\frac{h_{\zeta}}{k^{2} h_{\zeta}^{2}+\gamma^{2}} & =\frac{1}{k_{\rho}^{2}}+O\left(R^{-1}\right)
\end{aligned}
$$

Considering the previous approximations, now we can write

$$
\begin{aligned}
& {\left[\begin{array}{c}
E_{\phi} \\
H_{\phi}
\end{array}\right]=\left\{\frac{1}{k_{\rho}^{2} \rho}\left[\begin{array}{cc}
\gamma_{0} \frac{\partial}{\partial \phi} & -i \omega \mu \rho \frac{\partial}{\partial \rho} \\
i \omega \epsilon \rho \frac{\partial}{\partial \rho} & \gamma_{0} \frac{\partial}{\partial \phi}
\end{array}\right]\right.} \\
& -R^{-1} \frac{\gamma_{0}^{2}\left[\alpha_{1}+2 \rho \cos (\phi+\psi)\right]}{k_{\rho}^{4} \rho}\left[\begin{array}{cc}
\frac{\gamma_{0}^{2}-k^{2}}{2 \gamma_{0}} \frac{\partial}{\partial \phi} & -i \omega \mu \rho \frac{\partial}{\partial \rho} \\
i \omega \epsilon \rho \frac{\partial}{\partial \rho} & \frac{\gamma_{0}^{2}-k^{2}}{2 \gamma_{0}} \frac{\partial}{\partial \phi}
\end{array}\right] \\
& \left.+R^{-1} \frac{1}{k_{\rho}^{2}}\left[\begin{array}{cc}
\gamma_{0} \sin (\phi+\psi) & i \omega \mu \cos (\phi+\psi) \\
-i \omega \epsilon \cos (\phi+\psi) & \gamma_{0} \sin (\phi+\psi)
\end{array}\right]\right\}\left[\begin{array}{c}
E_{z} \\
H_{z}
\end{array}\right] \\
& +R^{-1} \frac{1}{k_{\rho}^{2} \rho}\left[\begin{array}{cc}
\gamma_{0} \frac{\partial}{\partial \phi} & -i \omega \mu \rho \frac{\partial}{\partial \rho} \\
i \omega \epsilon \rho \frac{\partial}{\partial \rho} & \gamma_{0} \frac{\partial}{\partial \phi}
\end{array}\right]\left[\begin{array}{c}
E_{1, \zeta} \\
H_{1, \zeta}
\end{array}\right], \text { and }
\end{aligned}
$$

$$
\begin{aligned}
& {\left[\begin{array}{l}
E_{\rho} \\
H_{\rho}
\end{array}\right]=\left\{\frac{1}{k_{\rho}^{2} \rho}\left[\begin{array}{cc}
\gamma_{0} \rho \frac{\partial}{\partial \rho} & i \omega \mu \frac{\partial}{\partial \phi} \\
-i \omega \epsilon \frac{\partial}{\partial \phi} & \gamma_{0} \rho \frac{\partial}{\partial \rho}
\end{array}\right]\right.} \\
& -R^{-1} \frac{\gamma_{0}^{2}\left[\alpha_{1}+2 \rho \cos (\phi+\psi)\right]}{k_{\rho}^{4} \rho}\left[\begin{array}{cc}
\frac{\gamma_{0}^{2}-k^{2}}{2 \gamma_{0}} \rho \frac{\partial}{\partial \rho} & i \omega \mu \frac{\partial}{\partial \phi} \\
-i \omega \epsilon \frac{\partial}{\partial \phi} & \frac{\gamma_{0}^{2}-k^{2}}{2 \gamma_{0}} \rho \frac{\partial}{\partial \rho}
\end{array}\right] \\
& \left.+R^{-1} \frac{1}{k_{\rho}^{2}}\left[\begin{array}{cc}
-\gamma_{0} \cos (\phi+\psi) & i \omega \mu \sin (\phi+\psi) \\
-i \omega \epsilon \sin (\phi+\psi) & -\gamma_{0} \cos (\phi+\psi)
\end{array}\right]\right\}\left[\begin{array}{l}
E_{z} \\
H_{z}
\end{array}\right] \\
& +R^{-1} \frac{1}{k_{\rho}^{2} \rho}\left[\begin{array}{cc}
\gamma_{0} \rho \frac{\partial}{\partial \rho} & i \omega \mu \frac{\partial}{\partial \phi} \\
-i \omega \epsilon \frac{\partial}{\partial \phi} & \gamma_{0} \rho \frac{\partial}{\partial \rho}
\end{array}\right]\left[\begin{array}{c}
E_{1, \zeta} \\
H_{1, \zeta}
\end{array}\right],
\end{aligned}
$$

where the axial fields are written such as $G_{\zeta}=G_{z}+R^{-1} G_{1, \zeta}$.

Noting that the zeroth-order solutions are proportional to $\exp (i n \phi)$, we can use the relations

$$
\begin{aligned}
\cos (\phi+\psi) e^{i n \phi} & =\frac{1}{2}\left[e^{i(n-1) \phi-i \psi}+e^{i(n+1) \phi+i \psi}\right], \text { and } \\
\sin (\phi+\psi) e^{i n \phi} & =\frac{i}{2}\left[e^{i(n-1) \phi-i \psi}-e^{i(n+1) \phi+i \psi}\right],
\end{aligned}
$$

to rewrite the transversal field components in the form

$$
\begin{array}{r}
{\left[\begin{array}{l}
E_{\phi} \\
H_{\phi}
\end{array}\right]=\left\{\left[\begin{array}{l}
E_{0, \phi}^{n} \\
H_{0, \phi}^{n}
\end{array}\right]+R^{-1}\left[\begin{array}{l}
E_{1, \phi}^{n} \\
H_{1, \phi}^{n}
\end{array}\right]\right\} e^{i n \phi} e^{\gamma \zeta}+R^{-1}\left[\begin{array}{l}
E_{1, \phi}^{n-1} \\
H_{1, \phi}^{n-1}
\end{array}\right] e^{i(n-1) \phi-i \psi} e^{\gamma \zeta}} \\
+R^{-1}\left[\begin{array}{l}
E_{1, \phi}^{n+1} \\
H_{1, \phi}^{n+1}
\end{array}\right] e^{i(n+1) \phi+i \psi} e^{\gamma \zeta},
\end{array}
$$




$$
\begin{array}{r}
{\left[\begin{array}{l}
E_{\rho} \\
H_{\rho}
\end{array}\right]=\left\{\left[\begin{array}{l}
E_{0, \rho}^{n} \\
H_{0, \rho}^{n}
\end{array}\right]+R^{-1}\left[\begin{array}{l}
E_{1, \rho}^{n} \\
H_{1, \rho}^{n}
\end{array}\right]\right\} e^{i n \phi} e^{\gamma \zeta}+R^{-1}\left[\begin{array}{l}
E_{1, \rho}^{n-1} \\
H_{1, \rho}^{n-1}
\end{array}\right] e^{i(n-1) \phi-i \psi} e^{\gamma \zeta}} \\
+R^{-1}\left[\begin{array}{l}
E_{1, \rho}^{n+1} \\
H_{1, \rho}^{n+1}
\end{array}\right] e^{i(n+1) \phi+i \psi} e^{\gamma \zeta},
\end{array}
$$

where the zeroth-order curvature vanishing fields are given by $\left\{E_{0, t}^{n}, H_{0, t}^{n}\right\} \exp (i n \phi+\gamma \zeta), t=\rho, \phi$. The remaining terms are given by

$$
\begin{aligned}
& {\left[\begin{array}{l}
E_{1, \phi}^{n} \\
H_{1, \phi}^{n}
\end{array}\right]=\frac{1}{k_{\rho}^{2} \rho}\left[\begin{array}{cc}
\gamma_{0} i n & -i \omega \mu \rho \frac{\partial}{\partial \rho} \\
i \omega \epsilon \rho \frac{\partial}{\partial \rho} & \gamma_{0} i n
\end{array}\right]\left[\begin{array}{l}
E_{1, \zeta}^{n} \\
H_{1, \zeta}^{n}
\end{array}\right]} \\
& -\frac{\gamma_{0}^{2} \alpha_{1}}{k_{\rho}^{4} \rho}\left[\begin{array}{cc}
\frac{\gamma_{0}^{2}-k^{2}}{2 \gamma_{0}} i n & -i \omega \mu \rho \frac{\partial}{\partial \rho} \\
i \omega \epsilon \rho \frac{\partial}{\partial \rho} & \frac{\gamma_{0}^{2}-k^{2}}{2 \gamma_{0}} i n
\end{array}\right]\left[\begin{array}{c}
E_{0, \zeta}^{n} \\
H_{0, \zeta}^{n}
\end{array}\right], \\
& {\left[\begin{array}{l}
E_{1, \phi}^{n \pm 1} \\
H_{1, \phi}^{n \pm 1}
\end{array}\right]=-\frac{\gamma_{0}^{2}}{k_{\rho}^{4}}\left[\begin{array}{cc}
\frac{\gamma_{0}^{2}-k^{2}}{2 \gamma_{0}} i n & -i \omega \mu \rho \frac{\partial}{\partial \rho} \\
i \omega \epsilon \rho \frac{\partial}{\partial \rho} & \frac{\gamma_{0}^{2}-k^{2}}{2 \gamma_{0}} i n
\end{array}\right]\left[\begin{array}{l}
E_{0, \zeta}^{n} \\
H_{0, \zeta}^{n}
\end{array}\right]} \\
& +\frac{i}{2 k_{\rho}^{2}}\left[\begin{array}{cc}
\mp \gamma_{0} & \omega \mu \\
-\omega \epsilon & \mp \gamma_{0}
\end{array}\right]\left[\begin{array}{l}
E_{0, \zeta}^{n} \\
H_{0, \zeta}^{n}
\end{array}\right]+\frac{1}{k_{\rho}^{2} \rho}\left[\begin{array}{cc}
\gamma_{0} i(n \pm 1) & -i \omega \mu \rho \frac{\partial}{\partial \rho} \\
i \omega \epsilon \rho \frac{\partial}{\partial \rho} & \gamma_{0} i(n \pm 1)
\end{array}\right]\left[\begin{array}{l}
E_{1, \zeta}^{n \pm 1} \\
H_{1, \zeta}^{n \pm 1}
\end{array}\right], \\
& {\left[\begin{array}{c}
E_{1, \rho}^{n} \\
H_{1, \rho}^{n}
\end{array}\right]=\frac{1}{k_{\rho}^{2} \rho}\left[\begin{array}{cc}
\gamma_{0} \rho \frac{\partial}{\partial \rho} & i \omega \mu(i n) \\
-i \omega \epsilon(i n) & \gamma_{0} \rho \frac{\partial}{\partial \rho}
\end{array}\right]\left[\begin{array}{l}
E_{1, \zeta}^{n} \\
H_{1, \zeta}^{n}
\end{array}\right]} \\
& -\frac{\gamma_{0}^{2} \alpha_{1}}{k_{\rho}^{4} \rho}\left[\begin{array}{cc}
\frac{\gamma_{0}^{2}-k^{2}}{2 \gamma_{0}} \rho \frac{\partial}{\partial \rho} & i \omega \mu(i n) \\
-i \omega \epsilon(i n) & \frac{\gamma_{0}^{2}-k^{2}}{2 \gamma_{0}} \rho \frac{\partial}{\partial \rho}
\end{array}\right]\left[\begin{array}{c}
E_{0, \zeta}^{n} \\
H_{0, \zeta}^{n}
\end{array}\right] \text {, } \\
& {\left[\begin{array}{l}
E_{1, \rho}^{n \pm 1} \\
H_{1, \rho}^{n \pm 1}
\end{array}\right]=-\frac{\gamma_{0}^{2}}{k_{\rho}^{4}}\left[\begin{array}{cc}
\frac{\gamma_{0}^{2}-k^{2}}{2 \gamma_{0}} \rho \frac{\partial}{\partial \rho} & i \omega \mu(i n) \\
-i \omega \epsilon(i n) & \frac{\gamma_{0}^{2}-k^{2}}{2 \gamma_{0}} \rho \frac{\partial}{\partial \rho}
\end{array}\right]\left[\begin{array}{l}
E_{0, \zeta}^{n} \\
H_{0, \zeta}^{n}
\end{array}\right]} \\
& +\frac{1}{2 k_{\rho}^{2}}\left[\begin{array}{cc}
-\gamma_{0} & \mp i \omega \mu i \\
\pm i \omega \epsilon i & -\gamma_{0}
\end{array}\right]\left[\begin{array}{l}
E_{0, \zeta}^{n} \\
H_{0, \zeta}^{n}
\end{array}\right]+\frac{1}{k_{\rho}^{2} \rho}\left[\begin{array}{cc}
\gamma_{0} \rho \frac{\partial}{\partial \rho} & i \omega \mu i(n \pm 1) \\
-i \omega \epsilon i(n \pm 1) & \gamma_{0} \rho \frac{\partial}{\partial \rho}
\end{array}\right]\left[\begin{array}{l}
E_{1, \zeta}^{n \pm 1} \\
H_{1, \zeta}^{n \pm 1}
\end{array}\right] \text {. }
\end{aligned}
$$

We must simplify the equations (4-211) to (4-214) in order to detach the parameters $\alpha_{1}, a_{1, m}^{e, h}$ and $b_{1, m}^{e, h}$, for $m=n, n \pm 1$, in order to assist the application of the appropriated boundary conditions. For this purpose, in the following sections each of these equations will be simplified. 


\subsubsection{1}

\section{Simplification of the Equation (4-211)}

Defining

$$
\begin{gathered}
\overline{\bar{D}}_{\phi, m}=\frac{1}{k_{\rho}^{2} \rho}\left[\begin{array}{cc}
\gamma_{0} i m & -i \omega \mu \rho \frac{\partial}{\partial \rho} \\
i \omega \epsilon \rho \frac{\partial}{\partial \rho} & \gamma_{0} i m
\end{array}\right], \\
\overline{\bar{B}}_{\phi, n}^{a}=-\frac{\gamma_{0}^{2}}{k_{\rho}^{4} \rho}\left[\begin{array}{cc}
\frac{\gamma_{0}^{2}-k^{2}}{2 \gamma_{0}} i n B_{n}\left(k_{\rho} \rho\right) & -i \omega \mu k_{\rho} \rho B_{n}^{\prime}\left(k_{\rho} \rho\right) \\
i \omega \epsilon k_{\rho} \rho B_{n}^{\prime}\left(k_{\rho} \rho\right) & \frac{\gamma_{0}^{2}-k^{2}}{2 \gamma_{0}} i n B_{n}\left(k_{\rho} \rho\right)
\end{array}\right],
\end{gathered}
$$

we can rewrite (4-211) as

$$
\begin{aligned}
{\left[\begin{array}{c}
E_{1, \phi}^{n} \\
H_{1, \phi}^{n}
\end{array}\right]=\overline{\bar{D}}_{\phi, n}\left[\alpha_{1} \overline{\bar{H}}_{\zeta, n}^{\hat{p}} \bar{a}_{0, n}+\alpha_{1} \overline{\bar{J}}_{\zeta, n}^{\hat{p}} \bar{b}_{0, n}\right.} & \left.+\overline{\bar{H}}_{n} \bar{a}_{1, n}+\overline{\bar{J}}_{n} \bar{b}_{1, n}\right] \\
& +\alpha_{1} \overline{\bar{H}}_{\phi, n}^{a} \bar{a}_{0, n}+\alpha_{1} \overline{\bar{J}}_{\phi, n}^{a} \bar{b}_{0, n} .
\end{aligned}
$$

Introducing the new matrices $\overline{\bar{B}}_{\phi, n}^{\hat{p}}$ and $\overline{\bar{B}}_{\phi, m}$ such as

$$
\begin{gathered}
\overline{\bar{B}}_{\phi, n}^{\hat{p}}=\overline{\bar{D}}_{\phi, n} \overline{\bar{B}}_{\zeta, n}^{\hat{p}}+\overline{\bar{B}}_{\phi, n}^{a} \\
=\frac{\gamma_{0}^{2}}{2 k_{\rho}^{4} \rho}\left[\begin{array}{cc}
i n\left(\gamma_{0} k_{\rho} \rho B_{n}^{\prime}-\frac{\gamma_{0}^{2}-k^{2}}{\gamma_{0}} B_{n}\right) & i \omega \mu\left[\left(k_{\rho}^{2} \rho^{2}-n^{2}\right) B_{n}+2 k_{\rho} \rho B_{n}^{\prime}\right] \\
-i \omega \epsilon\left[\left(k_{\rho}^{2} \rho^{2}-n^{2}\right) B_{n}+2 k_{\rho} \rho B_{n}^{\prime}\right] & i n\left(\gamma_{0} k_{\rho} \rho B_{n}^{\prime}-\frac{\gamma_{0}^{2}-k^{2}}{\gamma_{0}} B_{n}\right)
\end{array}\right], \\
\overline{\bar{B}}_{\phi, m}=\overline{\bar{D}}_{\phi, m} \overline{\bar{B}}_{m} \\
=\frac{1}{k_{\rho}^{2} \rho}\left[\begin{array}{cc}
\gamma_{0} i m B_{m} & -i \omega \mu k_{\rho} \rho B_{m}^{\prime} \\
i \omega \epsilon k_{\rho} \rho B_{m}^{\prime} & \gamma_{0} i m B_{m}
\end{array}\right],
\end{gathered}
$$

where $B_{m}=H_{m}$ or $J_{m}$, we can obtain

$$
\begin{aligned}
{\left[\begin{array}{c}
E_{1, \phi}^{n} \\
H_{1, \phi}^{n}
\end{array}\right] } & =\alpha_{1} \overline{\bar{H}}_{\phi, n}^{\hat{p}} \bar{a}_{0, n}+\alpha_{1} \overline{\bar{J}}_{\phi, n}^{\hat{p}} \bar{b}_{0, n}+\overline{\bar{H}}_{\phi, n} \bar{a}_{1, n}+\overline{\bar{J}}_{\phi, n} \bar{b}_{1, n} \\
& =\alpha_{1} \bar{R}_{\phi, n}+\overline{\bar{H}}_{\phi, n} \bar{a}_{1, n}+\overline{\bar{J}}_{\phi, n} \bar{b}_{1, n} .
\end{aligned}
$$

\subsubsection{2}

\section{Simplification of the Equation (4-213)}

Defining

$$
\begin{gathered}
\overline{\bar{D}}_{\rho, m}=\frac{1}{k_{\rho}^{2} \rho}\left[\begin{array}{cc}
\gamma_{0} \rho \frac{\partial}{\partial \rho} & -m \omega \mu \\
m \omega \epsilon & \gamma_{0} \rho \frac{\partial}{\partial \rho}
\end{array}\right] \\
\overline{\bar{B}}_{\rho, n}^{a}=-\frac{\gamma_{0}^{2}}{k_{\rho}^{4} \rho}\left[\begin{array}{cc}
\frac{\gamma_{0}^{2}-k^{2}}{2 \gamma_{0}} k_{\rho} \rho B_{n}^{\prime}\left(k_{\rho} \rho\right) & -n \omega \mu B_{n}\left(k_{\rho} \rho\right) \\
n \omega \epsilon B_{n}\left(k_{\rho} \rho\right) & \frac{\gamma_{0}^{2}-k^{2}}{2 \gamma_{0}} k_{\rho} \rho B_{n}^{\prime}\left(k_{\rho} \rho\right)
\end{array}\right],
\end{gathered}
$$




$$
\begin{aligned}
{\left[\begin{array}{c}
E_{1, \rho}^{n} \\
H_{1, \rho}^{n}
\end{array}\right]=\frac{1}{k_{\rho}^{2} \rho}\left[\begin{array}{cc}
\gamma_{0} \rho \frac{\partial}{\partial \rho} & i \omega \mu(i n) \\
-i \omega \epsilon(i n) & \gamma_{0} \rho \frac{\partial}{\partial \rho}
\end{array}\right]\left[\begin{array}{l}
E_{1, \zeta}^{n} \\
H_{1, \zeta}^{n}
\end{array}\right] } \\
-\frac{\gamma_{0}^{2} \alpha_{1}}{k_{\rho}^{4} \rho}\left[\begin{array}{cc}
\frac{\gamma_{0}^{2}-k^{2}}{2 \gamma_{0}} \rho \frac{\partial}{\partial \rho} & i \omega \mu(i n) \\
-i \omega \epsilon(i n) & \frac{\gamma_{0}^{2}-k^{2}}{2 \gamma_{0}} \rho \frac{\partial}{\partial \rho}
\end{array}\right]\left[\begin{array}{l}
E_{0, \zeta}^{n} \\
H_{0, \zeta}^{n}
\end{array}\right],
\end{aligned}
$$

we can rewrite (4-213) as

$$
\begin{aligned}
{\left[\begin{array}{l}
E_{1, \rho}^{n} \\
H_{1, \rho}^{n}
\end{array}\right]=\overline{\bar{D}}_{\rho, n}\left[\alpha_{1} \overline{\bar{H}}_{\zeta, n}^{\hat{p}} \bar{a}_{0, n}+\alpha_{1} \overline{\bar{J}}_{\zeta, n}^{\hat{p}} \bar{b}_{0, n}+\right.} & \left.\overline{\bar{H}}_{n} \bar{a}_{1, n}+\overline{\bar{J}}_{n} \bar{b}_{1, n}\right] \\
& +\alpha_{1} \overline{\bar{H}}_{\rho, n}^{a} \bar{a}_{0, n}+\alpha_{1} \overline{\bar{J}}_{\rho, n}^{a} \bar{b}_{0, n} .
\end{aligned}
$$

Introducing the new matrices $\overline{\bar{B}}_{\rho, n}^{\hat{p}}$ and $\overline{\bar{B}}_{\rho, m}$ such as

$$
\begin{gathered}
\overline{\bar{B}}_{\rho, n}^{\hat{p}}=\overline{\bar{D}}_{\rho, n} \overline{\bar{B}}_{\zeta, n}^{\hat{p}}+\overline{\bar{B}}_{\rho, n}^{a} \\
=\frac{\gamma_{0}^{2}}{2 k_{\rho}^{4} \rho}\left[\begin{array}{cc}
-\gamma_{0}\left(k_{\rho}^{2} \rho^{2}-n^{2}\right) B_{n}-\frac{\gamma_{0}^{2}-k^{2}}{\gamma_{0}} k_{\rho} \rho B_{n}^{\prime} & -n \omega \mu\left(k_{\rho} \rho B_{n}^{\prime}-2 B_{n}\right) \\
n \omega \epsilon\left(k_{\rho} \rho B_{n}^{\prime}-2 B_{n}\right) & -\gamma_{0}\left(k_{\rho}^{2} \rho^{2}-n^{2}\right) B_{n}-\frac{\gamma_{0}^{2}-k^{2}}{\gamma_{0}} k_{\rho} \rho B_{n}^{\prime}
\end{array}\right], \\
\quad(4-226) \\
\overline{\bar{B}}_{\rho, m}= \\
=\frac{\overline{\bar{D}}_{\rho, m} \overline{\bar{B}}_{m}}{k_{\rho}^{2} \rho}\left[\begin{array}{cc}
\gamma_{0} k_{\rho} \rho B_{m}^{\prime} & -n \omega \mu B_{m} \\
n \omega \epsilon B_{m} & \gamma_{0} k_{\rho} \rho B_{m}^{\prime}
\end{array}\right],
\end{gathered}
$$

where $B_{m}=H_{m}$ or $J_{m}$, we can obtain

$$
\begin{aligned}
{\left[\begin{array}{c}
E_{1, \rho}^{n} \\
H_{1, \rho}^{n}
\end{array}\right] } & =\alpha_{1} \overline{\bar{H}}_{\rho, n}^{\hat{p}} \bar{a}_{0, n}+\alpha_{1} \overline{\bar{J}}_{\rho, n}^{\hat{p}} \bar{b}_{0, n}+\overline{\bar{H}}_{\rho, n} \bar{a}_{1, n}+\overline{\bar{J}}_{\rho, n} \bar{b}_{1, n} \\
& =\alpha_{1} \bar{R}_{\rho, n}+\overline{\bar{H}}_{\rho, n} \bar{a}_{1, n}+\overline{\bar{J}}_{\rho, n} \bar{b}_{1, n} .
\end{aligned}
$$

\subsubsection{3}

\section{Simplification of the Equation (4-212)}

Defining $\overline{\bar{B}}_{\phi, n}^{b}{ }^{8}$ and $\overline{\bar{B}}_{\phi, n}^{c}$ as

$$
\overline{\bar{B}}_{\phi, n}^{b}=-\frac{\gamma_{0}^{2}}{k_{\rho}^{4}}\left[\begin{array}{cc}
\frac{\gamma_{0}^{2}-k^{2}}{2 \gamma_{0}} i n B_{n}\left(k_{\rho} \rho\right) & -i \omega \mu k_{\rho} \rho B_{n}^{\prime}\left(k_{\rho} \rho\right) \\
i \omega \epsilon k_{\rho} \rho B_{n}^{\prime}\left(k_{\rho} \rho\right) & \frac{\gamma_{0}^{2}-k^{2}}{2 \gamma_{0}} i n B_{n}\left(k_{\rho} \rho\right)
\end{array}\right],
$$

${ }^{8}$ Please note that $\overline{\bar{B}}_{\phi, n}^{a}=\rho^{-1} \overline{\bar{B}}_{\phi, n}^{b} ;$ cf. (4-216). 


$$
\overline{\bar{B}}_{\phi, n}^{c}=\frac{B_{n}}{2 k_{\rho}^{2}}\left[\begin{array}{cc}
\mp i \gamma_{0} & i \omega \mu \\
-i \omega \epsilon & \mp i \gamma_{0}
\end{array}\right],
$$

and using $\overline{\bar{D}}_{\phi, m}$ in (4-215), we can rewrite (4-212) as

$$
\begin{aligned}
& {\left[\begin{array}{c}
E_{1, \phi}^{n \pm 1} \\
H_{1, \phi}^{n \pm 1}
\end{array}\right]=\overline{\bar{H}}_{\phi, n}^{b} \bar{a}_{0, n}+\overline{\bar{J}}_{\phi, n}^{b} \bar{b}_{0, n}+\overline{\bar{H}}_{\phi, n}^{c} \bar{a}_{0, n}+\overline{\bar{J}}_{\phi, n}^{c} \bar{b}_{0, n}} \\
& \quad+\overline{\bar{D}}_{\phi, n \pm 1}\left[\overline{\bar{H}}_{\zeta, n \pm 1}^{p} \bar{a}_{0, n}+\overline{\bar{J}}_{\zeta, n \pm 1}^{p} \bar{b}_{0, n}+\overline{\bar{H}}_{n \pm 1} \bar{a}_{1, n \pm 1}+\overline{\bar{J}}_{n \pm 1} \bar{b}_{1, n \pm 1}\right] .
\end{aligned}
$$

Introducing the new matrix $\overline{\bar{B}}_{\phi, n \pm 1}^{p}$ as

$$
\overline{\bar{B}}_{\phi, n \pm 1}^{p}=\overline{\bar{B}}_{\phi, n}^{b}+\overline{\bar{B}}_{\phi, n}^{c}+\overline{\bar{D}}_{\phi, n \pm 1} \overline{\bar{B}}_{\zeta, n \pm 1}^{p},
$$

we can obtain

$$
\begin{aligned}
{\left[\begin{array}{c}
E_{1, \phi}^{n \pm 1} \\
H_{1, \phi}^{n \pm 1}
\end{array}\right] } & =\overline{\bar{H}}_{\phi, n \pm 1}^{p} \bar{a}_{0, n}+\overline{\bar{J}}_{\phi, n \pm 1}^{p} \bar{b}_{0, n}+\overline{\bar{H}}_{\phi, n \pm 1} \bar{a}_{1, n \pm 1}+\overline{\bar{J}}_{\phi, n \pm 1} \bar{b}_{1, n \pm 1} \\
& =\bar{R}_{\phi, n \pm 1}+\overline{\bar{H}}_{\phi, n \pm 1} \bar{a}_{1, n \pm 1}+\overline{\bar{J}}_{\phi, n \pm 1} \bar{b}_{1, n \pm 1},
\end{aligned}
$$

where

$$
\begin{aligned}
\overline{\bar{B}}_{\phi, n \pm 1} & =\overline{\bar{D}}_{\phi, n \pm 1} B_{n \pm 1}\left(k_{\rho} \rho\right) \\
& =\frac{1}{k_{\rho}^{2} \rho}\left[\begin{array}{cc}
\gamma_{0} i(n \pm 1) B_{n \pm 1}\left(k_{\rho} \rho\right) & -i \omega \mu k_{\rho} \rho B_{n \pm 1}^{\prime}\left(k_{\rho} \rho\right) \\
i \omega \epsilon k_{\rho} \rho B_{n \pm 1}^{\prime}\left(k_{\rho} \rho\right) & \gamma_{0} i(n \pm 1) B_{n \pm 1}\left(k_{\rho} \rho\right)
\end{array}\right]
\end{aligned}
$$

After a series of cumbersome manipulations, we found a simplified version for $\overline{\bar{B}}_{\phi, n \pm 1}^{p}$ given by

$$
\overline{\bar{B}}_{\phi, n \pm 1}^{p}=\frac{1}{4 k_{\rho}^{3}}\left[\begin{array}{cc}
i \gamma_{0} f_{11, \pm} & -i \omega \mu f_{12, \pm} \\
i \omega \epsilon f_{12, \pm} & i \gamma_{0} f_{11, \pm}
\end{array}\right]
$$

where

$$
\begin{gathered}
f_{11, \pm}=\left[2 k^{2} \pm \gamma_{0}^{2}(n \pm 1)\right] \rho B_{n \mp 1}\left(k_{\rho} \rho\right)+k_{\rho}(n \pm 1) B_{n}\left(k_{\rho} \rho\right), \text { and } \\
f_{12, \pm}=\left(\gamma_{0}^{2} n \pm k^{2}\right) \rho B_{n \mp 1}\left(k_{\rho} \rho\right)-k_{\rho}\left[\gamma_{0}^{2} \rho^{2} \pm(n \pm 1)\right) B_{n}\left(k_{\rho} \rho\right) .
\end{gathered}
$$

Note that in the above equations $B_{m}=H_{m}$ or $J_{m}$.

\subsubsection{4}

Simplification of the Equation (4-214)

Defining $\overline{\bar{B}}_{\rho, n}^{b}{ }^{9}$ and $\overline{\bar{B}}_{\rho, n}^{c}$ as

${ }^{9}$ Please note that $\overline{\bar{B}}_{\rho, n}^{a}=\rho^{-1} \overline{\bar{B}}_{\rho, n}^{b} ;$ cf. (4-223). 


$$
\begin{gathered}
\overline{\bar{B}}_{\rho, n}^{b}=-\frac{\gamma_{0}^{2}}{k_{\rho}^{4}}\left[\begin{array}{cc}
\frac{\gamma_{0}^{2}-k^{2}}{2 \gamma_{0}} k_{\rho} \rho B_{n}^{\prime}\left(k_{\rho} \rho\right) & -n \omega \mu B_{n}\left(k_{\rho} \rho\right) \\
n \omega \epsilon B_{n}\left(k_{\rho} \rho\right) & \frac{\gamma_{0}^{2}-k^{2}}{2 \gamma_{0}} k_{\rho} \rho B_{n}^{\prime}\left(k_{\rho} \rho\right)
\end{array}\right], \\
\overline{\bar{B}}_{\rho, n}^{c}=\frac{B_{n}}{2 k_{\rho}^{2}}\left[\begin{array}{cc}
-\gamma_{0} & \pm \omega \mu \\
\mp \omega \epsilon & -\gamma_{0}
\end{array}\right],
\end{gathered}
$$

and using $\overline{\bar{D}}_{\rho, m}$ in (4-222), we can rewrite (4-214) as

$$
\begin{aligned}
& {\left[\begin{array}{l}
E_{1, \rho}^{n \pm 1} \\
H_{1, \rho}^{n \pm 1}
\end{array}\right]} \\
& \quad=\overline{\bar{H}}_{\rho, n}^{b} \bar{a}_{0, n}+\overline{\bar{J}}_{\rho, n}^{b} \bar{b}_{0, n}+\overline{\bar{H}}_{\rho, n}^{c} \bar{a}_{0, n}+\overline{\bar{J}}_{\rho, n}^{c} \bar{b}_{0, n} \\
& \quad+\overline{\bar{D}}_{\rho, n \pm 1}\left[\overline{\bar{H}}_{\zeta, n \pm 1}^{p} \bar{a}_{0, n}+\overline{\bar{J}}_{\zeta, n \pm 1}^{p} \bar{b}_{0, n}+\overline{\bar{H}}_{n \pm 1} \bar{a}_{1, n \pm 1}+\overline{\bar{J}}_{n \pm 1} \bar{b}_{1, n \pm 1}\right] .
\end{aligned}
$$

Introducing the new matrix $\overline{\bar{B}}_{\rho, n \pm 1}^{p}$ as

$$
\overline{\bar{B}}_{\rho, n \pm 1}^{p}=\overline{\bar{B}}_{\rho, n}^{b}+\overline{\bar{B}}_{\rho, n}^{c}+\overline{\bar{D}}_{\rho, n \pm 1} \overline{\bar{B}}_{\zeta, n \pm 1}^{p},
$$

we can obtain

$$
\begin{aligned}
{\left[\begin{array}{c}
E_{1, \rho}^{n \pm 1} \\
H_{1, \rho}^{n \pm 1}
\end{array}\right] } & =\overline{\bar{H}}_{\rho, n \pm 1}^{p} \bar{a}_{0, n}+\overline{\bar{J}}_{\rho, n \pm 1}^{p} \bar{b}_{0, n}+\overline{\bar{H}}_{\rho, n \pm 1} \bar{a}_{1, n \pm 1}+\overline{\bar{J}}_{\rho, n \pm 1} \bar{b}_{1, n \pm 1} \\
& =\bar{R}_{\rho, n \pm 1}+\overline{\bar{H}}_{\rho, n \pm 1} \bar{a}_{1, n \pm 1}+\overline{\bar{J}}_{\rho, n \pm 1} \bar{b}_{1, n \pm 1}
\end{aligned}
$$

where

$$
\begin{aligned}
\overline{\bar{B}}_{\rho, n \pm 1} & =\overline{\bar{D}}_{\rho, n \pm 1} B_{n \pm 1}\left(k_{\rho} \rho\right) \\
& =\frac{1}{k_{\rho}^{2} \rho}\left[\begin{array}{cc}
\gamma_{0} k_{\rho} \rho B_{n \pm 1}^{\prime}\left(k_{\rho} \rho\right) & -(n \pm 1) \omega \mu B_{n \pm 1}\left(k_{\rho} \rho\right) \\
(n \pm 1) \omega \epsilon B_{n \pm 1}\left(k_{\rho} \rho\right) & \gamma_{0} k_{\rho} \rho B_{n \pm 1}^{\prime}\left(k_{\rho} \rho\right)
\end{array}\right] .
\end{aligned}
$$

After a series of cumbersome manipulations, we found a simplified version for $\overline{\bar{B}}_{\rho, n \pm 1}^{p}$ given by

where

$$
\overline{\bar{B}}_{\rho, n \pm 1}^{p}=\frac{1}{4 k_{\rho}^{3}}\left[\begin{array}{cc}
\gamma_{0} g_{11, \pm} & -\omega \mu g_{12, \pm} \\
\omega \epsilon g_{12, \pm} & \gamma_{0} g_{11, \pm}
\end{array}\right]
$$

$$
\begin{gathered}
g_{11, \pm}=\left[\gamma_{0}^{2}(n \pm 2) \pm 3 k^{2}\right] \rho B_{n \mp 1}\left(k_{\rho} \rho\right) \mp k_{\rho}\left(n \mp 1 \pm \gamma_{0}^{2} \rho^{2}\right) B_{n}\left(k_{\rho} \rho\right), \text { and } \\
g_{12, \pm}= \pm(n \mp 1)\left[\gamma_{0}^{2} \rho B_{n \mp 1}\left(k_{\rho} \rho\right) \pm k_{\rho} B_{n}\left(k_{\rho} \rho\right)\right] .
\end{gathered}
$$

\section{3}

\section{Fields Along Radial Stratifications}

In the following formulation, we consider that the bend structure shown in Fig. 4.3 is comprised by $N$ radial layers. Each layer extends over $r_{j-1}<\rho<$ $r_{j}, 0<\phi<2 \pi$, and is characterized by the constitutive parameters $\epsilon_{j}$ and $\mu_{j}$, 
$j=1,2, \cdots, N$. Note we assume each layer is a homogeneous and isotropic medium.

To find the proper values of $\alpha_{1}$ and $\bar{a}_{1, m}, \bar{b}_{1, m}$, for $m=n, n \pm 1$, for each layer, we must enforce the continuity of the fields (transversal to $\rho$ ) at the junctions between each layer.

For brevity, we can write all fields in (4-177), (4-209) and (4-210) in a compact manner using

$$
\begin{aligned}
\bar{G}_{\alpha}=\left(\bar{G}_{0, \alpha}^{n}+R^{-1} \bar{G}_{1, \alpha}^{n}\right) e^{i n \phi} e^{\gamma \zeta} & \\
& +R^{-1} \bar{G}_{1, \alpha}^{n-1} e^{i(n-1) \phi-i \psi} e^{\gamma \zeta}+R^{-1} \bar{G}_{1, \alpha}^{n+1} e^{i(n+1) \phi+i \psi} e^{\gamma \zeta},
\end{aligned}
$$

where $\bar{G}_{\alpha}=\left[E_{\alpha}, H_{\alpha}\right]^{t}$, and the polarization direction is $\alpha=\rho, \phi$ or $\zeta$.

Taking into account the azimuthal orthogonality in terms of $m \phi, m=$ $n, n \pm 1$, the boundary conditions splits up into three independent equations. Thus, we must satisfy the continuity of fields in terms of $\exp (i n \phi), \exp [i(n-$ 1) $\phi]$ and $\exp [i(n+1) \phi]$ apart.

\subsection{1}

\section{Continuity for Fields in Terms of $\exp (\operatorname{in} \phi)$}

The enforcement of boundary conditions for the fields $\bar{G}_{\zeta}$ and $\bar{G}_{\phi}$ in terms of $\exp ($ in $\phi)$ allow us to determine $\alpha_{1}, \bar{a}_{1, n}$ and $\bar{b}_{1, n}$. From (4-252), is clear that we must enforce the continuity of only $\bar{G}_{1, \zeta}^{n}$ and $\bar{G}_{1, \phi}^{n}$ because the zeroth-order non-perturbed fields $\bar{G}_{0, \zeta}^{n}$ and $\bar{G}_{0, \phi}^{n}$ already fulfill the boundary conditions. The enforcement of all boundary conditions for the continuity of the fields in a $N$-layers stratified cylindrical structure provide us a set of $d$ independent equations, which can be written as the homogeneous linear system:

$$
\left[\begin{array}{ll}
\bar{R}_{1, n} & \overline{\bar{M}}_{1, n}
\end{array}\right]\left[\begin{array}{c}
\alpha_{1} \\
\bar{A}_{1, n}
\end{array}\right]=\overline{0}
$$

where $\overline{\bar{M}}_{1, n}$ is a $d \times d$ square matrix, $\bar{R}_{1, n}$ and $\bar{A}_{1, n}$ are $d \times 1$ column vectors, and $\overline{0}$ is a $d \times 1$ mull column vector. The dimension $d$ is the number of boundary conditions.

The value of $d$ can be easily determined: for each radial layer we need to include 4 continuity conditions for the fields $E_{\zeta}, H_{\zeta}, E_{\phi}$ and $H_{\phi}$. Furthermore, case our stratified structured presents a hard wall (PEC or PMC) at $r_{0}$; i.e., $r_{0} \neq 0$; we need to include two additional conditions: $E_{\zeta}=E_{\phi}=0$ for a PEC, 
Table 4.1: Number of boundary conditions $d$

\begin{tabular}{c|cc} 
& $r_{0}=0$ & $r_{0} \neq 0$ \\
\hline$r_{N}=\infty$ & $4 N-4$ & $4 N-2$ \\
$r_{N} \neq \infty$ & $4 N-2$ & $4 N$
\end{tabular}

or $H_{\zeta}=H_{\phi}=0$ for a $\mathrm{PMC}^{10}$. The same idea should be used to include a hard wall at a finite $r_{N}$. Table 4.1 shows the number of boundary conditions in a $N$-layers stratified structure.

Turning back to (4-253), we can see that our problem has $(d+1)$ unknown and just $d$ equations. This system of equation is under-determined, but also is homogeneous and always has at least one solution: the trivial solution where all the unknowns $\alpha_{1}$ and $\bar{A}_{1, n}$ are zero.

If we assume for a moment that (4-253) has more solutions than the trivial, one can write

$$
\overline{\bar{M}}_{1, n} \bar{A}_{1, n}=-\alpha_{1} \bar{R}_{1, n}
$$

where we supposed $\alpha_{1} \neq 0$.

It is interesting to note that $\operatorname{det}\left(\overline{\bar{M}}_{1, n}\right)=0$ for any mode that satisfy the zeroth-order characteristic equation (3-91). This means that $\overline{\bar{M}}_{1, n}$ is not invertible, and only the trivial solution $\bar{A}_{1, n}=\overline{0}$ and $\alpha_{1}=0$ satisfies both (4-253) and (4-254). Then, we verify that there is no first-order correction for the propagation constant. Moreover, as we have anticipated, there is no perturbation correction for fields in terms of $\exp (i n \phi)$.

\subsection{2}

\section{Continuity for Fields in Terms of $\exp (i(n \pm 1) \phi)$}

To enforce the continuity of $\bar{G}_{1, \zeta}^{m}$ and $\bar{G}_{1, \phi}^{m}$, for $m=n \pm 1$, in a $N$-layers stratified cylindrical structure we need to simultaneously satisfy a set of $d$ linear equations, which can be written as

$$
\overline{\bar{M}}_{1, m} \bar{A}_{1, m}=\bar{R}_{1, m},
$$

where $\overline{\bar{M}}_{1, m}$ is a $d \times d$ square matrix given by

\footnotetext{
${ }^{10}$ It should be observed that we need to apply the boundary condition on the azimuthal component of the fields instead of use the derivative in $\rho$ of the axial ones, as usual in straight structures. From the azimuthal fields in a bend structure (see (4-4e) or (4-4b)) we clearly see that $\partial\left(h_{\zeta} H_{\zeta}\right) / \partial \rho=0$ (for PEC) or $\partial\left(h_{\zeta} E_{\zeta}\right) / \partial \rho=0$ (for PMC). Thus, depicting $G_{\zeta}$ as $E_{\zeta}$ or $H_{\zeta}$, we must satisfy
}

$$
h_{\zeta} \frac{\partial G_{\zeta}}{\partial \rho}+G_{\zeta} \frac{h_{\zeta}}{\partial \rho}=0 \quad \text { instead of } \quad \frac{\partial G_{\zeta}}{\partial \rho}=0 .
$$




$$
\overline{\bar{M}}_{1, m}=\left[\begin{array}{ccccc}
\overline{\bar{C}}_{1,0} & & & & \\
\overline{\bar{D}}_{1,1} & -\overline{\bar{D}}_{2,1} & & & \\
& \overline{\bar{D}}_{2,2} & -\overline{\bar{D}}_{3,2} & & \\
& & \ddots & \ddots & \\
& & & \overline{\bar{D}}_{N-1, N-1} & -\overline{\bar{D}}_{N, N-1} \\
& & & & \overline{\bar{C}}_{N, N}
\end{array}\right],
$$

and $\bar{A}_{1, m}$ and $\bar{R}_{1 . m}$ are $d \times 1$ column vectors given by

$$
\bar{A}_{1, m}=\left[\begin{array}{c}
\bar{a}_{m, 1} \\
\bar{b}_{m, 1} \\
\bar{a}_{m, 2} \\
\bar{b}_{m, 2} \\
\cdots \\
\bar{a}_{m, N} \\
\bar{b}_{m, N}
\end{array}\right], \quad \bar{R}_{1, m}=\left[\begin{array}{c}
\bar{E}_{1,0} \\
\bar{F}_{1,2} \\
\bar{F}_{2,3} \\
\vdots \\
\bar{F}_{N-1, N} \\
\bar{E}_{N, N}
\end{array}\right] .
$$

Notice that just the non-zero elements of $\overline{\bar{M}}_{1, m}$ are shown in (4-256), and we also have omitted the first index in vectors $\bar{a}_{m, j}=\bar{a}_{1, m, j}$ and $\bar{b}_{m, j}=\bar{b}_{1, m, j}$ to simplify the notation. The dimension $d$ is the number of boundary conditions given by Table 4.1 .

The sub-matrix $\overline{\bar{C}}_{1,0}$ and sub-vector $\bar{E}_{1,0}$ enforce the boundary conditions of a hard PEC wall placed at $\rho=r_{0}$. Similarly, $\overline{\bar{C}}_{N, N} \bar{E}_{N, N}$ enforce the boundary conditions of a hard PEC wall placed at $\rho=r_{N}$. The sub-matrices $\overline{\bar{D}}_{i, j}$ and $\overline{\bar{D}}_{i+1, j}$ in conjunction of the sub-vectors $\bar{F}_{i, i+1}$ enforce continuity of the fields between layer $j$ and $j+1$ at $\rho=r_{j}$. These matrices and vectors are given by

$$
\begin{aligned}
& \overline{\bar{C}}_{i, j}=\left[\begin{array}{cccc}
\left.\overline{\bar{H}}_{\zeta, m}\left(k_{i \rho} r_{j}\right)\right|_{11} & 0 & \left.\overline{\bar{J}}_{\zeta, m}\left(k_{i \rho} r_{j}\right)\right|_{11} & 0 \\
\left.\overline{\bar{H}}_{\phi, m}\left(k_{i \rho} r_{j}\right)\right|_{11} & \left.\overline{\bar{H}}_{\phi, m}\left(k_{i \rho} r_{j}\right)\right|_{12} & \left.\overline{\bar{J}}_{\phi, m}\left(k_{i \rho} r_{j}\right)\right|_{11} & \left.\overline{\bar{J}}_{\phi, m}\left(k_{i \rho} r_{j}\right)\right|_{12}
\end{array}\right], \\
& \overline{\bar{D}}_{i, j}=\left[\begin{array}{cc}
\overline{\bar{H}}_{\zeta, m}\left(k_{i \rho} r_{j}\right) & \overline{\bar{J}}_{\zeta, m}\left(k_{i \rho} r_{j}\right) \\
\overline{\bar{H}}_{\phi, m}\left(k_{i \rho} r_{j}\right) & \overline{\bar{J}}_{\phi, m}\left(k_{i \rho} r_{j}\right)
\end{array}\right], \\
& \bar{E}_{i, j}=\left[\begin{array}{l}
-\left.\bar{R}_{\zeta, m}\left(k_{i \rho} r_{j}\right)\right|_{1} \\
-\left.\bar{R}_{\phi, m}\left(k_{i \rho} r_{j}\right)\right|_{1}
\end{array}\right] \text {, and } \\
& \bar{F}_{i, i+1}=\left[\begin{array}{l}
-\bar{R}_{\zeta, m}\left(k_{i \rho} r_{i}\right)+\bar{R}_{\zeta, m}\left(k_{i+1, \rho} r_{i}\right) \\
-\bar{R}_{\phi, m}\left(k_{i \rho} r_{i}\right)+\bar{R}_{\phi, m}\left(k_{i+1, \rho} r_{i}\right)
\end{array}\right] .
\end{aligned}
$$

The matrices $\overline{\bar{B}}_{\zeta, m}$ and $\overline{\bar{B}}_{\phi, m}(\overline{\bar{B}}=\overline{\bar{H}}$ or $\overline{\bar{J}})$ have already been defined in previous sections for a single layer structure. These results can be easily generalized to take into account the additional stratifications. In layer $j$, we must employ 


$$
\begin{gathered}
\overline{\bar{B}}_{\zeta, m}\left(k_{j \rho} \rho\right)=\left[\begin{array}{cc}
B_{m}\left(k_{j \rho} \rho\right) & 0 \\
0 & B_{m}\left(k_{j \rho} \rho\right)
\end{array}\right] \text {, and } \\
\overline{\bar{B}}_{\phi, m}\left(k_{j \rho} \rho\right)=\frac{1}{k_{j \rho}^{2} \rho}\left[\begin{array}{cc}
-m k_{z} B_{m}\left(k_{j \rho} \rho\right) & -i \omega \mu_{j} k_{j \rho} \rho B_{m}^{\prime}\left(k_{j \rho} \rho\right) \\
i \omega \epsilon_{j} k_{j \rho} \rho B_{m}^{\prime}\left(k_{j \rho} \rho\right) & -m k_{z} B_{m}\left(k_{j \rho} \rho\right)
\end{array}\right] .
\end{gathered}
$$

The above formulation considers a hard wall placed at $\rho=r_{0}$ and another at $\rho=r_{N}$. Case $r_{0}=0$; i.e., there is no boundary condition to be matched at $\rho=r_{0}$; we need then to ensure finite fields in the first layer by enforcing $\bar{a}_{m, 1}=\overline{0}$ and we should not employ $\overline{\bar{C}}_{1,0}$ and $\bar{E}_{1,0}$. Besides that, the fist two columns of $\overline{\bar{D}}_{1,1}$ are no longer necessary.

Similarly, if we consider an unbounded waveguide; i.e., $r_{N} \rightarrow \infty$; there is no backward wave at last radial layer and $\bar{b}_{m, N}=\overline{0}$. In this case, we should not employ $\overline{\bar{C}}_{N, N}$ and $\overline{\bar{E}}_{N, N}$, and the last two columns of $\overline{\bar{D}}_{N, N-1}$ are no longer necessary.

The simple inversion of matrix $\overline{\bar{M}}_{1, m}$ in (4-255) allow us to find the unknowns $\bar{A}_{1, m}$, namely,

$$
\bar{A}_{1, m}=\bar{M}_{1, m}^{-1} \bar{R}_{1, m}
$$

\subsection{3}

\section{Mirror Modes and Fields Symmetry}

In Section 3.3.5 we have showed that exist symmetric relations for the fields in a cylindrically-stratified waveguide (zeroth-order fields in our toroidal structure). Here we will generalize these results for perturbed fields in toroidal coordinates.

Decomposing the field components in terms of the axial and transversal in relation to $\zeta$ as

$$
\mathbf{E}=\mathbf{E}_{t}+\hat{\zeta} E_{\zeta}, \quad \mathbf{H}=\mathbf{H}_{t}+\hat{\zeta} H_{\zeta}
$$

we can write the Maxwell's curls equations in (4-2) as

$$
\begin{aligned}
& \frac{i k_{\zeta}}{h_{\zeta}} \hat{\zeta} \times \mathbf{E}_{t}+\frac{1}{h_{\zeta}} \nabla_{t} \times\left(\hat{\zeta} h_{\zeta} E_{\zeta}\right)=i \omega \mu \mathbf{H}_{t}, \\
& \frac{i k_{\zeta}}{h_{\zeta}} \hat{\zeta} \times \mathbf{H}_{t}+\frac{1}{h_{\zeta}} \nabla_{t} \times\left(\hat{\zeta} h_{\zeta} H_{\zeta}\right)=-i \omega \epsilon \mathbf{E}_{t}, \\
& \nabla_{t} \times \mathbf{E}_{t}=i \omega \mu \hat{\zeta} H_{\zeta} \\
& \nabla_{t} \times \mathbf{H}_{t}=-i \omega \epsilon \hat{\zeta} E_{\zeta}
\end{aligned}
$$

where

$$
\nabla=\nabla_{t}+\hat{\zeta} \frac{i k_{\zeta}}{h_{\zeta}}
$$

and the two-dimensional (transversal) nabla operator is given by 


$$
\nabla_{t}=\hat{\rho} \frac{\partial}{\partial \rho}+\hat{\phi} \frac{1}{\rho} \frac{\partial}{\partial \phi}
$$

By the vector multiplication of $\hat{\zeta}$ on both sides of the equations in (4-266) and using

$$
\begin{gathered}
\hat{\zeta} \times\left(\nabla_{t} \times \hat{\zeta}\left(h_{\zeta} G_{\zeta}\right)\right)=\nabla_{t}\left(h_{\zeta} G_{\zeta}\right), \text { and } \\
\hat{\zeta} \times\left(\hat{\zeta} \times \mathbf{G}_{t}\right)=-\mathbf{G}_{t},
\end{gathered}
$$

and after a few manipulations, we can express the transversal fields as a combinations of the axial ones using ${ }^{11}$

$$
\begin{aligned}
& \mathbf{E}_{t}=\frac{h_{\zeta}}{k_{t}^{2}}\left[\frac{i k_{\zeta}}{h_{\zeta}} \nabla_{t}\left(h_{\zeta} E_{\zeta}\right)+i \omega \mu \nabla_{t} \times\left(\hat{\zeta} h_{\zeta} H_{\zeta}\right)\right], \\
& \mathbf{H}_{t}=\frac{h_{\zeta}}{k_{t}^{2}}\left[\frac{i k_{\zeta}}{h_{\zeta}} \nabla_{t}\left(h_{\zeta} H_{\zeta}\right)-i \omega \epsilon \nabla_{t} \times\left(\hat{\zeta} h_{\zeta} E_{\zeta}\right)\right] .
\end{aligned}
$$

If the fields associated with $\left(k_{\zeta}, \mathbf{E}_{t}, E_{\zeta}, \mathbf{H}_{t}, H_{\zeta}\right)$ are a solution for (4-266) and (4-267), the mirror mode $\left(-k_{\zeta}, \mathbf{E}_{t},-E_{\zeta},-\mathbf{H}_{t}, H_{\zeta}\right)$ is also a solution for Maxwell's equations. In this manner, we can write the $(n p)$ th modal fields as

$$
\begin{gathered}
\mathbf{E}_{n p}^{ \pm}(\rho, \phi)=\left[\mathbf{E}_{t, n p}^{+}(\rho, \phi) \pm \hat{\zeta} E_{\zeta, n p}^{+}(\rho, \phi)\right] e^{ \pm i k_{\zeta, n p} \zeta}, \text { and } \\
\mathbf{H}_{n p}^{ \pm}(\rho, \phi)=\left[ \pm \mathbf{H}_{t, n p}^{+}(\rho, \phi)+\hat{\zeta} H_{\zeta, n p}^{+}(\rho, \phi)\right] e^{ \pm i k_{\zeta, n p} \zeta},
\end{gathered}
$$

for fields propagating to $\pm \zeta$. Note that the fields are symmetric in relation to $\zeta$-axis such as

$$
\begin{array}{ll}
\mathbf{E}_{t, n p}^{-}(\rho, \phi)=\mathbf{E}_{t, n p}^{+}(\rho, \phi) & \mathbf{H}_{t, n p}^{-}(\rho, \phi)=-\mathbf{H}_{t, n p}^{+}(\rho, \phi), \\
\mathbf{E}_{\zeta, n p}^{-}(\rho, \phi)=-\mathbf{E}_{\zeta, n p}^{+}(\rho, \phi) & \mathbf{H}_{\zeta, n p}^{-}(\rho, \phi)=\mathbf{H}_{\zeta, n p}^{+}(\rho, \phi),
\end{array}
$$

For simplify our notation, we will assumed that the fields $G_{\alpha, n p}^{+}=G_{\alpha, n p}$, $G=\{E, H\}, \alpha=\{t, \zeta\}$, were derived for the forward axial wavenumber $k_{\zeta, n p}$ such as $\Im m\left(k_{\zeta, n p}\right) \geq 0$. In practical terms, the superscript + will no longer be employed here.

From the results in Section 4.3.1, we can verify that there is no first-order perturbation correction for the axial wavenumber in our toroidal coordinate system, i.e., $\alpha_{1}=0$ and consequently $k_{\zeta, n p}=k_{z, n p}$. Thus, we can continue employing the all fields and symmetry relations for the zeroth-order solution derived in (3-128), (3-129) and (3-130). In other words, for $n>0$, the $(n p)$ th

\footnotetext{
${ }^{11}$ Note that the derivative of the unit vectors in toroidal coordinates are given in (C-14)(C-16), such $\partial \hat{\rho} / \partial \zeta \neq 0$ and $\partial \hat{\phi} / \partial \zeta \neq 0$, but $\partial \hat{\zeta} / \partial \rho=\partial \hat{\zeta} / \partial \phi=0$. Also, observe that the fields in (4-272) and (4-272) can be arranged in the matrix forms shown in (4-194) and (4-195).
} 
zeroth-order modal fields satisfy:

$$
\begin{array}{ll}
E_{0, \rho,-n p}^{n}=-(-1)^{n} E_{0, \rho, n p}^{n} & H_{0, \rho,-n p}^{n}=(-1)^{n} H_{0, \rho, n p}^{n} \\
E_{0, \phi,-n p}^{n}=(-1)^{n} E_{0, \phi, n p}^{n} & H_{0, \phi,-n p}^{n}=-(-1)^{n} H_{0, \phi, n p}^{n} \\
E_{0, \zeta,-n p}^{n}=-(-1)^{n} E_{0, \zeta, n p}^{n} & H_{0, \zeta,-n p}^{n}=(-1)^{n} H_{0, \zeta, n p}^{n} .
\end{array}
$$

We could further simplify our analysis using a series of symmetry relations derived from the first-order fields in (4-192), (4-234), (4-245) and (4-255), as follows.

For $n=0$, the $(0 p)$ th first-order modal field satisfies:

$$
\begin{array}{ll}
E_{1, \rho, 0 p}^{n-1}=E_{1, \rho, 0 p}^{n+1} & H_{1, \rho, 0 p}^{n-1}=-H_{1, \rho, 0 p}^{n+1} \\
E_{1, \phi, 0 p}^{n-1}=-E_{1, \phi, 0 p}^{n+1} & H_{1, \phi, 0 p}^{n-1}=H_{1, \phi, 0 p}^{n+1} \\
E_{1, \zeta, 0 p}^{n-1}=E_{1, \zeta, 0 p}^{n+1} & H_{1, \zeta, 0 p}^{n-1}=-H_{1, \zeta, 0 p}^{n+1} .
\end{array}
$$

For $n>0$, the $(n p)$ th first-order modal field satisfies:

$$
\begin{array}{ll}
E_{1, \rho,-n p}^{n \pm 1}=-(-1)^{n} E_{1, \rho, n p}^{n \mp 1} & H_{1, \rho,-n p}^{n \pm 1}=(-1)^{n} H_{1, \rho, n p}^{n \mp 1} \\
E_{1, \phi,-n p}^{n \pm 1}=(-1)^{n} E_{1, \phi, n p}^{n \mp 1} & H_{1, \phi,-n p}^{n \pm 1}=-(-1)^{n} H_{1, \phi, n p}^{n \mp 1} \\
E_{1, \zeta,-n p}^{n \pm 1}=-(-1)^{n} E_{1, \zeta, n p}^{n \mp 1} & H_{1, \zeta,-n p}^{n \pm 1}=(-1)^{n} H_{1, \zeta, n p}^{n \mp 1} .
\end{array}
$$

\section{4}

\section{Fields Along Axial Stratifications}

\subsection{1}

\section{Axial Mode-Matching}

The fields transversal to $\zeta$ in the axial region $j$ can be written as

$$
\begin{aligned}
\mathbf{E}_{j t}= & \sum_{n=-\infty}^{\infty} \sum_{p=1}^{\infty}\left(a_{j, n p}^{+} e^{i k_{z, n p} \zeta}+a_{j, n p}^{-} e^{-i k_{z, n p} \zeta}\right)\left\{\mathbf{E}_{j t}^{n}\left(k_{\rho, n p} \rho\right) e^{i n \phi}\right. \\
& \left.+R_{j}^{-1} \mathbf{E}_{j t}^{n-1}\left(k_{\rho, n p} \rho\right) e^{i(n-1) \phi-i \psi_{j}}+R_{j}^{-1} \mathbf{E}_{j t}^{n+1}\left(k_{\rho, n p} \rho\right) e^{i(n+1) \phi+i \psi_{j}}\right\}, \\
\mathbf{H}_{j t}= & \sum_{n^{\prime}=-\infty}^{\infty} \sum_{p^{\prime}=1}^{\infty}\left(a_{j, n p}^{+} e^{i k_{z, n p} \zeta}-a_{j, n p}^{-} e^{-i k_{z, n p} \zeta}\right)\left\{\mathbf{H}_{j t}^{n^{\prime}}\left(k_{\rho, n^{\prime} p^{\prime}} \rho\right) e^{i n^{\prime} \phi}\right. \\
& \left.+R_{j}^{-1} \mathbf{H}_{j t}^{n^{\prime}-1}\left(k_{\rho, n^{\prime} p^{\prime}} \rho\right) e^{i\left(n^{\prime}-1\right) \phi-i \psi_{j}}+R_{j}^{-1} \mathbf{H}_{j t}^{n^{\prime}+1}\left(k_{\rho, n^{\prime} p^{\prime}} \rho\right) e^{i\left(n^{\prime}+1\right) \phi+i \psi_{j}}\right\} .
\end{aligned}
$$

Suppose a junction between the region 1 and region 2 at $\zeta=\zeta_{1}$, as depicted in Fig. 4.5. Then, we must match the transversal fields such that 


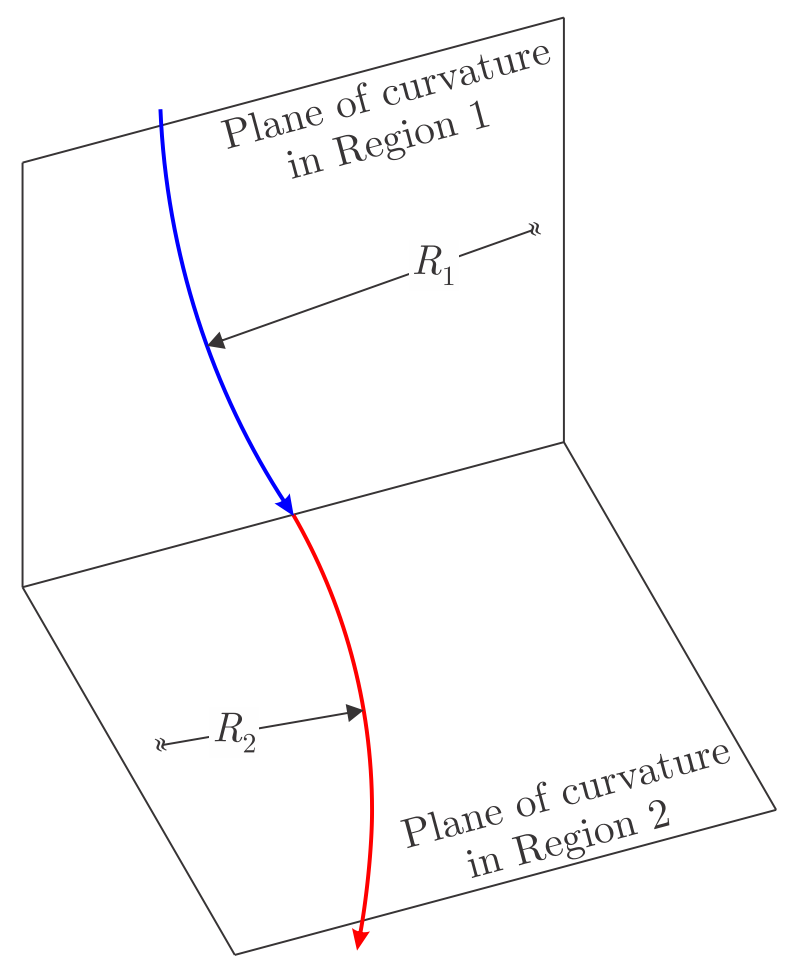

(a) Planes of curvature

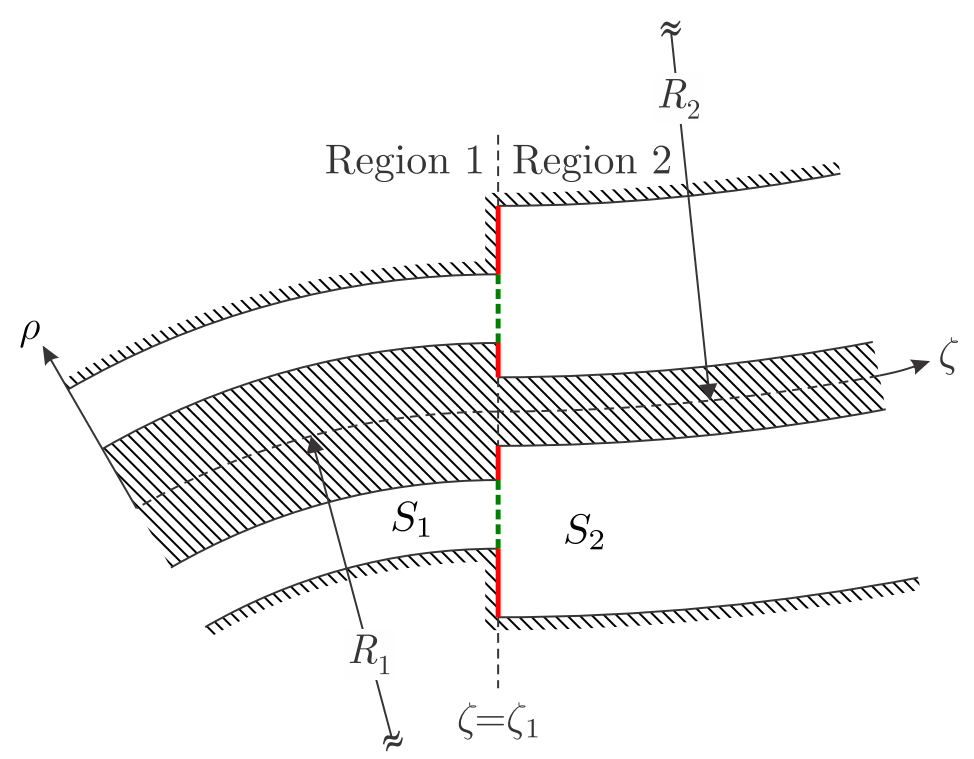

(b) Transversal view at the junction

Figure 4.5: Junction between two semi-infinitely-long waveguides.

$$
\begin{aligned}
& \left.\begin{array}{rl}
\mathbf{E}_{1 t} & =\mathbf{E}_{2 t} \\
\mathbf{H}_{1 t} & =\mathbf{H}_{2 t}
\end{array}\right\} \text { inside } S_{1}, \\
& \mathbf{E}_{1 t}=0 \text { inside } S_{2}-S_{1},
\end{aligned}
$$

where the waveguide cross-sections in regions 1 and 2 are depicted by $S_{1}$ and $S_{2}$, respectively. To shorting the notation, consider to write the fields as 


$$
\begin{gathered}
\mathbf{E}_{j t}=\sum_{m}^{\infty}\left(A_{j, m}^{+}+A_{j, m}^{-}\right) \mathbf{E}_{j t, m}, \\
\mathbf{H}_{j t}=\sum_{m^{\prime}}^{\infty}\left(A_{j, m^{\prime}}^{+}-A_{j, m^{\prime}}^{-}\right) \mathbf{H}_{j t, m^{\prime}},
\end{gathered}
$$

where the field amplitudes at $\zeta=\zeta_{1}$ are $A_{j, m}^{ \pm}=a_{j, m}^{ \pm} \exp \left( \pm i k_{z, m} \zeta_{1}\right)$, and the $m$ th transversal field $\mathbf{G}_{j t, m}, \mathbf{G}=\mathbf{E}$ or $\mathbf{H}$, can be write as a function of the radius of curvature in region $j$ (viz., $R_{j}$ ) as

$$
\mathbf{G}_{j t, m}=\mathbf{G}_{j t, m}^{(0)}(\rho, \phi)+R_{j}^{-1} \mathbf{G}_{j t, m}^{(1)}(\rho, \phi)+O\left(R_{j}^{-2}\right) .
$$

Considering $M$ modes in the region 1 , and $N$ modes in the region 2 , from the continuity of the transversal electric fields at $\zeta=\zeta_{1}$ we can obtain

$$
\sum_{m=1}^{M}\left(A_{1, m}^{+}+A_{1, m}^{-}\right) \mathbf{E}_{1 t, m}=\sum_{n=1}^{N}\left(A_{2, n}^{+}+A_{2, n}^{-}\right) \mathbf{E}_{2 t, n} .
$$

Multiplying both sides of the above equation by $\times \mathbf{H}_{2 t, n^{\prime}}\left(n^{\prime}=1,2,3, \cdots, N\right)$ and integrand over the surface $S_{2}$ (in the direction $\zeta>0$ ), we can obtain a set of $N$ equations:

$$
\begin{aligned}
& \sum_{m=1}^{M}\left(A_{1, m}^{+}+A_{1, m}^{-}\right) \int_{S_{2}}\left(\mathbf{E}_{1 t, m} \times \mathbf{H}_{2 t, n^{\prime}}\right) \cdot \hat{\zeta} d S= \\
& \quad \sum_{n=1}^{N}\left(A_{2, n}^{+}+A_{2, n}^{-}\right) \int_{S_{2}}\left(\mathbf{E}_{2 t, n} \times \mathbf{H}_{2 t, n^{\prime}}\right) \cdot \hat{\zeta} d S, \text { for } n^{\prime}=1,2, \ldots, N .
\end{aligned}
$$

We can reduce the integral in the LHS of the above equation to be over $S_{1}$ instead $S_{2}$ because $\mathbf{E}_{1 t}=0$ inside $S_{2}-S_{1}$. The reaction of the $m$ th field in region $i$ to the $n$th mode in region $j$ can be defined as

$$
\begin{aligned}
\left\langle\mathbf{E}_{i t, m}, \mathbf{H}_{j t, n}\right\rangle_{\zeta} & =\int_{S_{i}}\left(\mathbf{E}_{i t, m} \times \mathbf{H}_{j t, n}\right) \cdot \hat{\zeta} d S \\
& =\int_{0}^{r_{i}} \int_{0}^{2 \pi}\left(E_{i \rho, m} H_{j \phi, n}-E_{i \phi, m} H_{j \rho, n}\right) \rho d \phi d \rho \\
& =X_{i m, j n} .
\end{aligned}
$$

The self-reaction $X_{j n, j n}$ can be defined as $Q_{j n}$. In this way, the set of equations in (4-293) can be written in a matrix form as

$$
\overline{\bar{X}}_{12}\left(\bar{A}_{1}^{+}+\bar{A}_{1}^{-}\right)=\overline{\bar{Q}}_{2}\left(\bar{A}_{2}^{+}+\bar{A}_{2}^{-}\right)
$$

where the elements of the matrices $\overline{\bar{X}}_{12}$ and $\overline{\bar{Q}}_{2}$ are given by

$$
\left.\overline{\bar{X}}_{12}\right|_{n, m},=X_{1 m, 2 n}
$$




$$
\left.\overline{\bar{Q}}_{2}\right|_{n, m},=X_{2 m, 2 n}
$$

and the modal amplitudes at the junction $A_{j, m}^{ \pm}$were proper combined into the vectors $\bar{A}_{j}^{ \pm}$, for $j=1$ or 2 .

Following the above steps for the continuity of the magnetic field at $\zeta=\zeta_{1}$, we can derive

$$
\overline{\bar{X}}_{12}^{t}\left(\bar{A}_{2}^{+}-\bar{A}_{2}^{-}\right)=\overline{\bar{Q}}_{1}^{t}\left(\bar{A}_{1}^{+}-\bar{A}_{1}^{-}\right) .
$$

Combining the (4-297) and (4-300) we can relate the forward and backward modal amplitudes at the junction $\left(\zeta=\zeta_{1}\right)$ as

$$
\left[\begin{array}{c}
\bar{A}_{1}^{-} \\
\bar{A}_{2}^{+}
\end{array}\right]=\left[\begin{array}{cc}
\overline{\bar{R}}_{12}^{(\zeta)} & \overline{\bar{T}}_{21}^{(\zeta)} \\
\overline{\bar{T}}_{12}^{(\zeta)} & \overline{\bar{R}}_{21}^{(\zeta)}
\end{array}\right]\left[\begin{array}{c}
\bar{A}_{1}^{+} \\
\bar{A}_{2}^{-}
\end{array}\right],
$$

where scattering sub-matrices are given by

$$
\begin{aligned}
\overline{\bar{R}}_{12}^{(\zeta)} & =\left(\overline{\bar{Q}}_{1}^{t}+\overline{\bar{X}}_{12}^{t} \overline{\bar{Q}}_{2}^{-1} \overline{\bar{X}}_{12}\right)^{-1}\left(\overline{\bar{Q}}_{1}^{t}-\overline{\bar{X}}_{12}^{t} \overline{\bar{Q}}_{2}^{-1} \overline{\bar{X}}_{12}\right), \\
\overline{\bar{T}}_{21}^{(\zeta)} & =2\left(\overline{\bar{Q}}_{1}^{t}+\overline{\bar{X}}_{12}^{t} \overline{\bar{Q}}_{2}^{-1} \overline{\bar{X}}_{12}\right)^{-1} \overline{\bar{X}}_{12}^{t}, \\
\overline{\bar{T}}_{12}^{(\zeta)} & =2\left(\overline{\bar{Q}}_{2}+\overline{\bar{X}}_{12}\left(\overline{\bar{Q}}_{1}^{t}\right)^{-1} \overline{\bar{X}}_{12}^{t}\right)^{-1} \overline{\bar{X}}_{12}, \text { and } \\
\overline{\bar{R}}_{21}^{(\zeta)} & =-\left(\overline{\bar{Q}}_{2}+\overline{\bar{X}}_{12}\left(\overline{\bar{Q}}_{1}^{t}\right)^{-1} \overline{\bar{X}}_{12}^{t}\right)^{-1}\left(\overline{\bar{Q}}_{2}-\overline{\bar{X}}_{12}\left(\overline{\bar{Q}}_{1}^{t}\right)^{-1} \overline{\bar{X}}_{12}^{t}\right) .
\end{aligned}
$$

The above matrices and amplitude vectors have the following dimensions (rows, columns):

$$
\begin{aligned}
\operatorname{dim}\left(\bar{A}_{1}^{ \pm}\right) & =(M, 1), \\
\operatorname{dim}\left(\bar{A}_{2}^{ \pm}\right) & =(N, 1), \\
\operatorname{dim}\left(\overline{\bar{X}}_{12}\right) & =(N, M), \\
\operatorname{dim}\left(\overline{\bar{Q}}_{1}\right) & =(M, M), \\
\operatorname{dim}\left(\overline{\bar{Q}}_{2}\right) & =(N, N),
\end{aligned}
$$

where the total number of modes in regions 1 and 2 are given by

$$
\begin{aligned}
& M=\sum_{n=-N_{1 \phi}}^{N_{1 \phi}} N_{1 \rho}(n), \\
& N=\sum_{n=-N_{2 \phi}}^{N_{2 \phi}} N_{2 \rho}(n),
\end{aligned}
$$

where $N_{j \phi}$ is associated to each azimuthal dependence in $\phi$ considered in the modal field expansion. Furthermore, for each $N_{j \phi}$ we have a set of $N_{j \rho}$ modal fields.

The modal amplitudes can be written as 


$$
\bar{A}_{j}^{ \pm}=\left[\begin{array}{c}
\bar{A}_{j,-N_{\phi}}^{ \pm} \\
\vdots \\
\bar{A}_{j,-1}^{ \pm} \\
\bar{A}_{j, 0}^{ \pm} \\
\bar{A}_{j,+1}^{ \pm} \\
\vdots \\
\bar{A}_{j, N_{\phi}}^{ \pm}
\end{array}\right]
$$

where

$$
\bar{A}_{j, n}^{ \pm}=\left[\begin{array}{c}
A_{j, n 1}^{ \pm} \\
A_{j, n 2}^{ \pm} \\
\vdots \\
A_{j, n p}^{ \pm} \\
\vdots
\end{array}\right] .
$$

The reaction matrix will then assume the form

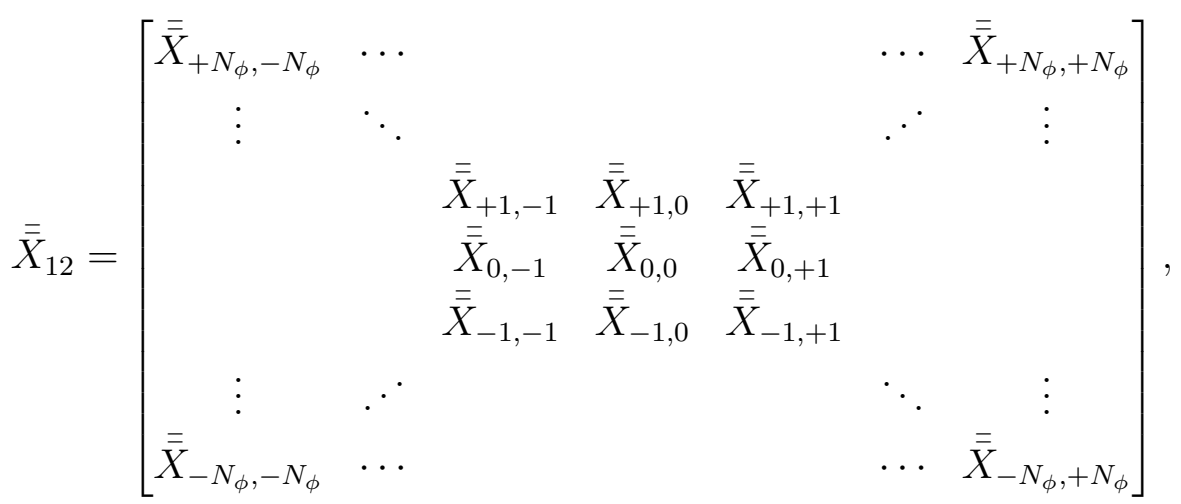

or

$$
\left.\overline{\bar{X}}_{12}\right|_{n^{\prime}, n}=\overline{\bar{X}}_{n^{\prime}, n}
$$

Considering the perturbed fields up to the first-order in relation to the curvature radius $R_{j}$, we can write

$$
X_{1(n p), 2\left(n^{\prime} p^{\prime}\right)} \approx X_{1(n p), 2\left(n^{\prime} p^{\prime}\right)}^{(0,0)}+R_{2}^{-1} X_{1(n p), 2\left(n^{\prime} p^{\prime}\right)}^{(0,1)}+R_{1}^{-1} X_{1(n p), 2\left(n^{\prime} p^{\prime}\right)}^{(1,0)} .
$$

We can substantially simplify the reactions in the above equation taking into account the azimuthal harmonic dependence of the fields. Note that the zeroth-order curvature vanishing fields are proportionals to $\exp ($ in $\phi)$ whereas the first-order fields are proportional to $\exp [i(n \pm 1) \phi]$. Accordingly, we can easy ascertain that 


$$
\begin{gathered}
X_{1(n p), 2\left(n^{\prime} p^{\prime}\right)}^{(0,0)}=2 \pi \delta_{n,-n^{\prime}} \int_{\rho_{1}}\left(\mathbf{E}_{1 t, n p}^{n} \times \mathbf{H}_{2 t, n^{\prime} p^{\prime}}^{n}\right) \cdot \hat{\zeta} \rho d \rho, \\
X_{1(n p), 2\left(n^{\prime} p^{\prime}\right)}^{(0,1)}=2 \pi e^{-i \psi_{2}} \delta_{n,-\left(n^{\prime}-1\right)} \int_{\rho_{1}}\left(\mathbf{E}_{1 t, n p}^{n} \times \mathbf{H}_{2 t, n^{\prime} p^{\prime}}^{n-1}\right) \cdot \hat{\zeta} \rho d \rho \\
+2 \pi e^{i \psi_{2}} \delta_{n,-\left(n^{\prime}+1\right)} \int_{\rho_{1}}\left(\mathbf{E}_{1 t, n p}^{n} \times \mathbf{H}_{2 t, n^{\prime} p^{\prime}}^{n+1}\right) \cdot \hat{\zeta} \rho d \rho, \\
X_{1(n p), 2\left(n^{\prime} p^{\prime}\right)}^{(1,0)}=2 \pi e^{-i \psi_{1}} \delta_{n-1,-n^{\prime}} \int_{\rho_{1}}\left(\mathbf{E}_{1 t, n p}^{n-1} \times \mathbf{H}_{2 t, n^{\prime} p^{\prime}}^{n}\right) \cdot \hat{\zeta} \rho d \rho \\
+2 \pi e^{i \psi_{1}} \delta_{n+1,-n^{\prime}} \int_{\rho_{1}}\left(\mathbf{E}_{1 t, n p}^{n+1} \times \mathbf{H}_{2 t, n^{\prime} p^{\prime}}^{n}\right) \cdot \hat{\zeta} \rho d \rho,
\end{gathered}
$$

and we can verify that $\overline{\bar{X}}_{12}$ is a tridiagonal block matrix because

$$
\overline{\bar{X}}_{n^{\prime}, n}=\overline{\overline{0}} \text { for }\left|n+n^{\prime}\right|>1 \text {. }
$$

We can further derive the following specializations:

$$
\left.\overline{\bar{X}}_{n^{\prime}, n}\right|_{p^{\prime}, p}=2 \pi I_{1(n p), 2\left(n^{\prime} p^{\prime}\right)}^{(n, n)} \text {, for } n=-n^{\prime},
$$

$\left.\overline{\bar{X}}_{n^{\prime}, n}\right|_{p^{\prime}, p}=2 \pi\left[R_{2}^{-1} e^{-i \psi_{2}} I_{1(n p), 2\left(n^{\prime} p^{\prime}\right)}^{(n, n-1)}+R_{1}^{-1} e^{-i \psi_{1}} I_{1(n p), 2\left(n^{\prime} p^{\prime}\right)}^{(n-1, n)}\right]$, for $n=-\left(n^{\prime}-1\right)$,

$$
\left.\overline{\bar{X}}_{n^{\prime}, n}\right|_{p^{\prime}, p}=2 \pi\left[R_{2}^{-1} e^{i \psi_{2}} I_{1(n p), 2\left(n^{\prime} p^{\prime}\right)}^{(n, n+1)}+R_{1}^{-1} e^{i \psi_{1}} I_{1(n p), 2\left(n^{\prime} p^{\prime}\right)}^{(n+1, n)}\right] \text {, for } n=-\left(n^{\prime}+1\right) \text {. }
$$

where we have introduced integrals along the radial space in region 1 of five kinds, given by

$$
\begin{gathered}
I_{1(n p), 2\left(n^{\prime} p^{\prime}\right)}^{(n, n)}=\int_{\rho_{1}}\left(E_{1 \rho, n p}^{n} H_{2 \phi, n^{\prime} p^{\prime}}^{n}-E_{1 \phi, n p}^{n} H_{2 \rho, n^{\prime} p^{\prime}}^{n}\right) \rho d \rho, \\
I_{1(n p), 2\left(n^{\prime} p^{\prime}\right)}^{(n, n-1)}=\int_{\rho_{1}}\left(E_{1 \rho, n p}^{n} H_{2 \phi, n^{\prime} p^{\prime}}^{n-1}-E_{1 \phi, n p}^{n} H_{2 \rho, n^{\prime} p^{\prime}}^{n-1}\right) \rho d \rho, \\
I_{1(n p), 2\left(n^{\prime} p^{\prime}\right)}^{(n-1, n)}=\int_{\rho_{1}}\left(E_{1 \rho, n p}^{n-1} H_{2 \phi, n^{\prime} p^{\prime}}^{n}-E_{1 \phi, n p}^{n-1} H_{2 \rho, n^{\prime} p^{\prime}}^{n}\right) \rho d \rho, \\
I_{1(n p), 2\left(n^{\prime} p^{\prime}\right)}^{(n, n+1)}=\int_{\rho_{1}}\left(E_{1 \rho, n p}^{n} H_{2 \phi, n^{\prime} p^{\prime}}^{n+1}-E_{1 \phi, n p}^{n} H_{2 \rho, n^{\prime} p^{\prime}}^{n+1}\right) \rho d \rho, \text { and } \\
I_{1(n p), 2\left(n^{\prime} p^{\prime}\right)}^{(n+1, n)}=\int_{\rho_{1}}\left(E_{1 \rho, n p}^{n+1} H_{2 \phi, n^{\prime} p^{\prime}}^{n}-E_{1 \phi, n p}^{n+1} H_{2 \rho, n^{\prime} p^{\prime}}^{n}\right) \rho d \rho .
\end{gathered}
$$

It is clear that (4-324) is precisely the reaction integral between the zerothorder fields previously derived in Section 3.4.1.1. 


\section{5}

\section{Mode Excitation from Sources Inside a Toroidal Waveguide}

Consider a generic source inside a toroidal waveguide, located between two planes at $\zeta=\zeta_{T}^{ \pm}=\zeta_{T} \pm \delta$ as shown in Fig. 4.6. This source generates the fields $\mathbf{E}^{+}$and $\mathbf{H}^{+}$traveling in the positive $\zeta$-direction, and the fields $\mathbf{E}^{-}$and $\mathbf{H}^{-}$traveling to the opposite direction. The electromagnetic fields generated by this source can be expressed in terms of the waveguide modes as

$$
\begin{gathered}
\mathbf{E}^{ \pm}=\sum_{m=1}^{M} A_{m}^{ \pm} \mathbf{E}_{m}^{ \pm}(\rho, \phi) e^{ \pm i k_{\zeta, m}\left(\zeta-\zeta_{T}\right)}, \text { and } \\
\mathbf{H}^{ \pm}=\sum_{m=1}^{M} A_{m}^{ \pm} \mathbf{H}_{m}^{ \pm}(\rho, \phi) e^{ \pm i k_{\zeta, m}\left(\zeta-\zeta_{T}\right)},
\end{gathered}
$$

for $\zeta \gtrless \zeta_{T}^{ \pm}$. Here we used the single index $m$ to represent any possible mode of the toroidal waveguide.

We can determine the unknown amplitudes $A_{m}^{ \pm}$by using the Lorentz reciprocity theorem

$$
\int_{S_{0}^{ \pm}}\left(\mathbf{E}_{1} \times \mathbf{H}_{2}-\mathbf{E}_{2} \times \mathbf{H}_{1}\right) \cdot d \mathbf{s}=\int_{V}\left(\mathbf{E}_{2} \cdot \mathbf{J}_{1}-\mathbf{E}_{1} \cdot \mathbf{J}_{2}+\mathbf{H}_{1} \cdot \mathbf{M}_{2}-\mathbf{H}_{2} \cdot \mathbf{M}_{1}\right) d v
$$

where the surface integral is over the cross-section at $\zeta=\zeta_{T}^{ \pm}$.

By selecting $\mathbf{E}_{1}$ and $\mathbf{H}_{1}$ as the fields radiated be the set of sources $\mathbf{J}_{1}=\mathbf{J}$ and $\mathbf{M}_{1}=\mathbf{M}$, and assuming that there is no more sources $\left(\mathbf{J}_{2}=\mathbf{M}_{2}=\mathbf{0}\right)$, let

$$
\mathbf{E}_{1}=\mathbf{E}^{ \pm}, \text {for } \zeta \gtrless \zeta_{T}^{ \pm},
$$

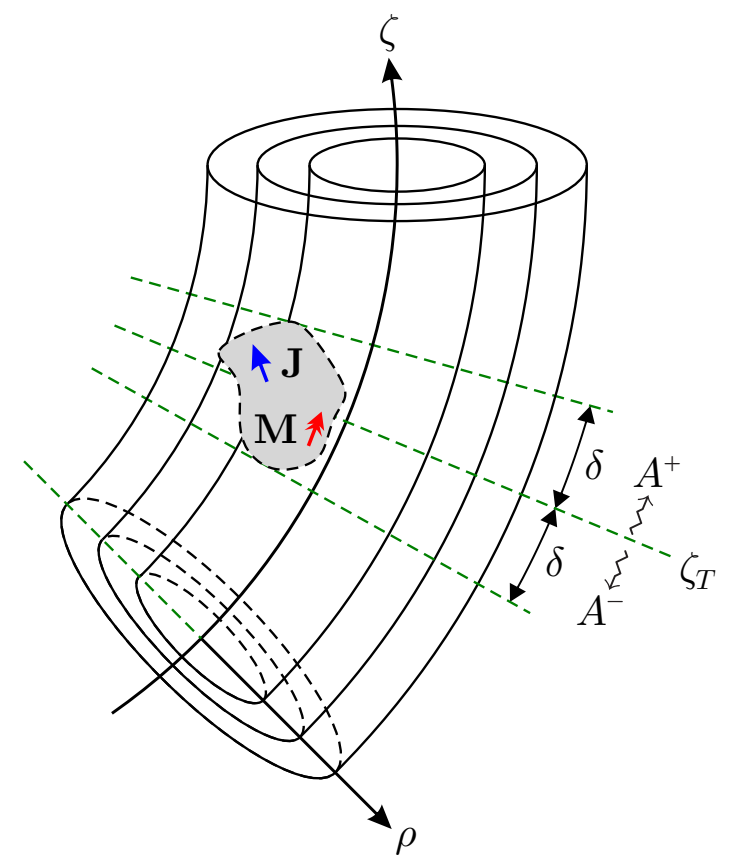

Figure 4.6: Sources inside a radially-stratified and axially-toroidal waveguide. 


$$
\mathbf{H}_{1}=\mathbf{H}^{ \pm}, \text {for } \zeta \gtrless \zeta_{T}^{ \pm},
$$

and let $\mathbf{E}_{2}$ and $\mathbf{H}_{2}$ the $m^{\prime}$ th waveguide mode traveling to negative $\zeta$-direction:

$$
\begin{gathered}
\mathbf{E}_{2}=\mathbf{E}_{m^{\prime}}^{-}, \\
\mathbf{H}_{2}=\mathbf{H}_{m^{\prime}}^{-},
\end{gathered}
$$

for $m^{\prime}=1,2, \ldots, M$. Now, we can write (4-334) as a set of $M$ equations, i.e.,

$$
\begin{aligned}
\int_{S_{0}^{+}}\left[\left(\sum_{m} A_{m}^{+} \mathbf{E}_{m}^{+}\right) \times \mathbf{H}_{m^{\prime}}^{-}-\mathbf{E}_{m^{\prime}}^{-} \times\left(\sum_{m} A_{m}^{+} \mathbf{H}_{m}^{+}\right)\right] \cdot d \mathbf{s} \\
+\int_{S_{0}^{-}}\left[\left(\sum_{m} A_{m}^{-} \mathbf{E}_{m}^{-}\right) \times \mathbf{H}_{m^{\prime}}^{-}-\mathbf{E}_{m^{\prime}}^{-} \times\left(\sum_{m} A_{m}^{-} \mathbf{H}_{m}^{-}\right)\right] \cdot d \mathbf{s} \\
=\int_{V}\left(\mathbf{E}_{m^{\prime}}^{-} \cdot \mathbf{J}-\mathbf{H}_{m^{\prime}}^{-} \cdot \mathbf{M}\right) d v, \text { for } m^{\prime}=1,2, \ldots, M
\end{aligned}
$$

Noting that the vector normal to surface $S_{0}^{+}(\hat{n}=\hat{\zeta})$ is in opposite direction to the vector normal to surface $S_{0}^{-}(\hat{n}=-\hat{\zeta})$ and using the relations in shown in Section 4.3.3 (decomposing the field components in terms of the axial and transversal components in relation to $\zeta$ ), we can simplify the last equation to obtain

$$
\begin{aligned}
&-\int_{S_{0}} {\left[\left(\sum_{m} A_{m}^{+} \mathbf{E}_{t m}\right) \times \mathbf{H}_{t m^{\prime}}+\mathbf{E}_{t m^{\prime}} \times\left(\sum_{m} A_{m}^{+} \mathbf{H}_{t m}\right)\right] \cdot \hat{\zeta} d s } \\
&- \int_{S_{0}}\left[-\left(\sum_{m} A_{m}^{-} \mathbf{E}_{t m}\right) \times \mathbf{H}_{t m^{\prime}}+\mathbf{E}_{t m^{\prime}} \times\left(\sum_{m} A_{m}^{-} \mathbf{H}_{t m}\right)\right] \cdot \hat{\zeta} d s \\
&= \int_{V}\left[\left(\mathbf{E}_{t m^{\prime}}-\hat{\zeta} E_{\zeta m^{\prime}}\right) \cdot \mathbf{J}-\left(-\mathbf{H}_{t m^{\prime}}+\hat{\zeta} H_{\zeta m^{\prime}}\right) \cdot \mathbf{M}\right] e^{-i k_{\zeta m^{\prime}} \zeta} d v \\
& \text { for } m^{\prime}=1,2, \ldots, M .
\end{aligned}
$$

Introducing the matrix $\overline{\bar{Q}}$, which combines the reaction integrals $Q_{m, m^{\prime}}$ (over the cross-section $S_{0}$ ) of the modal fields such as

$$
\left.\overline{\bar{Q}}\right|_{m^{\prime}, m}=Q_{m, m^{\prime}}=\int_{S_{0}}\left(\mathbf{E}_{t m} \times \mathbf{H}_{t m^{\prime}}\right) \cdot \hat{\zeta} \rho d \rho d \phi,
$$

and defining the column vectors $\bar{F}^{+}$and $\bar{A}^{ \pm}$such as

$$
\begin{gathered}
\left.\bar{F}^{+}\right|_{m^{\prime}}=F_{m^{\prime}}^{+}, \\
\left.\bar{A}^{ \pm}\right|_{m}=A_{m}^{ \pm},
\end{gathered}
$$




$$
F_{m^{\prime}}^{+}=\int_{V}\left[\left(\mathbf{E}_{t m^{\prime}}-\hat{\zeta} E_{\zeta m^{\prime}}\right) \cdot \mathbf{J}-\left(-\mathbf{H}_{t m^{\prime}}+\hat{\zeta} H_{\zeta m^{\prime}}\right) \cdot \mathbf{M}\right] e^{-i k_{\zeta m^{\prime}}\left(\zeta-\zeta_{T}\right)} d v
$$

we can rewrite (4-340) as the matrix equation

$$
-\left(\overline{\bar{Q}}+\overline{\bar{Q}}^{t}\right) \bar{A}^{+}+\left(\overline{\bar{Q}}-\overline{\bar{Q}}^{t}\right) \bar{A}^{-}=\bar{F}^{+} .
$$

By repeating the above procedure but now selecting

$$
\begin{gathered}
\mathbf{E}_{2}=\mathbf{E}_{m^{\prime}}^{+}, \\
\mathbf{H}_{2}=\mathbf{H}_{m^{\prime}}^{+},
\end{gathered}
$$

instead of (4-337) and (4-338), we find a set of $M$ equations:

$$
\begin{array}{r}
\int_{S_{0}}\left[\left(\sum_{m} A_{m}^{+} \mathbf{E}_{t m}\right) \times \mathbf{H}_{t m^{\prime}}-\mathbf{E}_{t m^{\prime}} \times\left(\sum_{m} A_{m}^{+} \mathbf{H}_{t m}\right)\right] \cdot \hat{\zeta} d s \\
-\int_{S_{0}}\left[\left(\sum_{m} A_{m}^{-} \mathbf{E}_{t m}\right) \times \mathbf{H}_{t m^{\prime}}+\mathbf{E}_{t m^{\prime}} \times\left(\sum_{m} A_{m}^{-} \mathbf{H}_{t m}\right)\right] \cdot \hat{\zeta} d s \\
=\int_{V}\left[\left(\mathbf{E}_{t m^{\prime}}+\hat{\zeta} E_{\zeta m^{\prime}}\right) \cdot \mathbf{J}-\left(\mathbf{H}_{t m^{\prime}}+\hat{\zeta} H_{\zeta m^{\prime}}\right) \cdot \mathbf{M}\right] e^{i k_{\zeta m^{\prime}} \zeta} d v \\
\text { for } m^{\prime}=1,2, \ldots, M .
\end{array}
$$

Now, we can rewrite (4-348) as a matrix equation:

$$
\left(\overline{\bar{Q}}-\overline{\bar{Q}}^{t}\right) \bar{A}^{+}-\left(\overline{\bar{Q}}+\overline{\bar{Q}}^{t}\right) \bar{A}^{-}=\bar{F}^{-},
$$

where the column vectors $\bar{F}^{-}$is such as

$$
\left.\bar{F}^{-}\right|_{m^{\prime}}=F_{m^{\prime}}^{-},
$$

where $F_{m^{\prime}}^{-}$can be expressed similarly to (4-344):

$$
\begin{aligned}
F_{m^{\prime}}^{ \pm}=\int_{V}\left[\left(\mathbf{E}_{t m^{\prime}} \mp \hat{\zeta} E_{\zeta m^{\prime}}\right) \cdot \mathbf{J}-\left(\mp \mathbf{H}_{t m^{\prime}}\right.\right. & \left.\left.+\hat{\zeta} H_{\zeta m^{\prime}}\right) \cdot \mathbf{M}\right] \\
& \times e^{\mp i k_{\zeta m^{\prime}}\left(\zeta-\zeta_{T}\right)} h_{\zeta} \rho d \rho d \phi d \zeta .
\end{aligned}
$$

To save our notation, we can introduce two new matrices:

$$
\begin{gathered}
\overline{\bar{D}}=\overline{\bar{Q}}+\overline{\bar{Q}}^{t}, \text { and } \\
\overline{\bar{C}}=\overline{\bar{Q}}-\overline{\bar{Q}}^{t} .
\end{gathered}
$$

We must take care to avoid the inversion the antisymmetric matrix $\overline{\bar{C}}$ when solving $\bar{A}^{ \pm}$from the coupled equations (4-345) and (4-349). In this way, we can derive 


$$
\begin{gathered}
\bar{A}^{+}=(\overline{\bar{C}} \overline{\bar{D}}-1 \overline{\bar{C}}-\overline{\bar{D}})^{-1}\left(\bar{F}^{+}+\overline{\bar{C}} \overline{\bar{D}}-1 \bar{F}^{-}\right), \text {and } \\
\bar{A}^{-}=\left(\overline{\bar{C}} \overline{\bar{D}}^{-1} \overline{\bar{C}}-\overline{\bar{D}}\right)^{-1}\left(\bar{F}^{-}+\overline{\bar{C}} \overline{\bar{D}}^{-1} \bar{F}^{+}\right) .
\end{gathered}
$$

If we introduce the normalization matrix

$$
\overline{\bar{N}}=\overline{\bar{C}} \overline{\bar{D}}^{-1} \overline{\bar{C}}-\overline{\bar{D}}
$$

and the a column vector combining the source amplitudes such as

$$
\bar{S}^{ \pm}=\bar{F}^{ \pm}+\overline{\bar{C}} \overline{\bar{D}}^{-1} \bar{F}^{\mp}
$$

the forward and backward amplitudes of the fields can be written as

$$
\bar{A}^{ \pm}=\overline{\bar{N}}^{-1} \bar{S}^{ \pm}
$$

It should be observed that the modal fields in a toroidal coordinate system are no longer orthogonal over the cross-section $S_{0}$, and the condition (3-201) can not be used here.

\subsection{1}

\section{The Dirac Delta in Toroidal Coordinates}

In our toroidal coordinate system we can write the Dirac delta distribution as

$$
\begin{aligned}
\delta\left(\mathbf{r}-\mathbf{r}^{\prime}\right) & =\frac{1}{h_{\rho} h_{\phi} h_{\zeta}} \delta\left(\rho-\rho^{\prime}\right) \delta\left(\phi-\phi^{\prime}\right) \delta\left(\zeta-\zeta^{\prime}\right) \\
& =\frac{1}{\rho\left[1-R^{-1} \rho \cos (\phi+\psi)\right]} \delta\left(\rho-\rho^{\prime}\right) \delta\left(\phi-\phi^{\prime}\right) \delta\left(\zeta-\zeta^{\prime}\right) .
\end{aligned}
$$

We can express the above as a series of the curvature by means of the Maclaurin series for $h_{\zeta}^{-1}$ using [116, p. 15]

$$
\frac{1}{1-R^{-1} \rho \cos (\phi+\psi)}=1+R^{-1} \rho \cos (\phi+\psi)+R^{-2} \rho^{2} \cos ^{2}(\phi+\psi)+O\left(R^{-3}\right)
$$

Furthermore, by employing the Fourier series expansion of the azimuthal variation $[15$, Appendix C]

$$
\delta\left(\phi-\phi^{\prime}\right)=\frac{1}{2 \pi} \sum_{n=-\infty}^{\infty} e^{i n\left(\phi-\phi^{\prime}\right)},
$$


we can derive a first-order approximation to $\delta\left(\mathbf{r}-\mathbf{r}^{\prime}\right)$ given by

$$
\begin{aligned}
\delta\left(\mathbf{r}-\mathbf{r}^{\prime}\right) \approx \frac{\delta\left(\rho-\rho^{\prime}\right)}{2 \pi \rho} \delta\left(\zeta-\zeta^{\prime}\right) \sum_{n=-\infty}^{\infty}\left[e^{i n \phi}\right. & +\frac{\rho}{2 R} e^{i(n-1) \phi-i \psi} \\
& \left.+\frac{\rho}{2 R} e^{i(n+1) \phi+i \psi}\right] e^{-i n \phi^{\prime}}
\end{aligned}
$$

It should be observed that the above, in addition to a proper polarization, can be used to describe a generic source. Hence a given source distribution can be expanded in terms of $\exp (i n \phi)$ and $\exp [i(n \pm 1) \phi]$ harmonics that will excite fields with these azimuthal dependences.

\subsection{2}

\section{Coil Antenna Sources}

Consider a circular coil antenna, placed on the plane $\zeta=\zeta_{T}$ and covering $0 \leq \phi \leq 2 \pi$ using a constant radius $\rho=\rho_{T}$ in our toroidal coordinate system, as shown in Fig. 4.7. The normal to its plane is $\hat{n}_{T}=\hat{\zeta}$, and the coordinates of the winding satisfy the equation $\hat{n}_{T} \cdot \boldsymbol{r}_{T}$ and is given by

$$
\boldsymbol{r}_{T}=\hat{\rho} \rho_{T}
$$

The electrical current density of the antenna can be written in the form

$$
\mathbf{J}=\frac{1}{2 \pi} \frac{\delta\left(\rho-\rho_{T}\right)}{\rho} \frac{\delta\left(\zeta-\zeta_{T}\right)}{h_{\zeta}} \hat{\phi}
$$

The current density in (4-365) must be divergenceless vector, such we can easily verify that $\boldsymbol{\nabla} \cdot \mathbf{J}=0$, as shown below:

$$
\begin{aligned}
\boldsymbol{\nabla} \cdot \mathbf{J} & =\frac{1}{h_{\rho} h_{\phi} h_{\zeta}}\left[\frac{\partial}{\partial \rho}\left(J_{\rho} h_{\phi} h_{\zeta}\right)+\frac{\partial}{\partial \phi}\left(J_{\phi} h_{\rho} h_{\zeta}\right)+\frac{\partial}{\partial \zeta} J_{\zeta} h_{\rho} h_{\phi}\right] \\
& =\frac{1}{\rho h_{\zeta}} \frac{\partial}{\partial \phi}\left(J_{\phi} h_{\zeta}\right)=0 .
\end{aligned}
$$

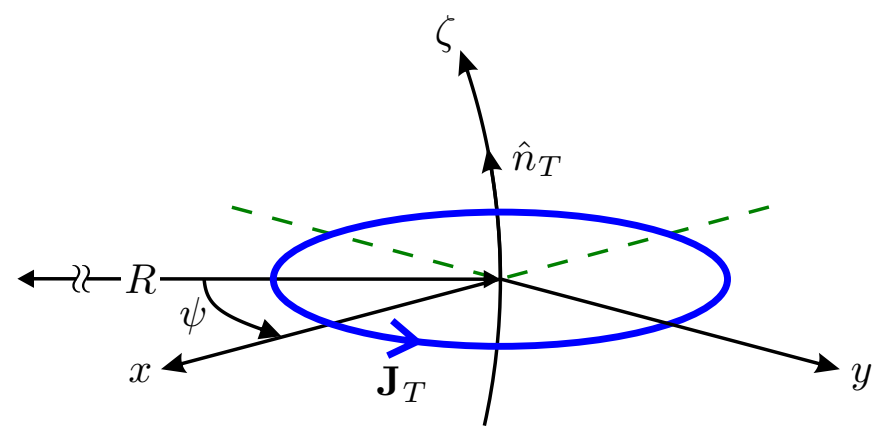

Figure 4.7: Transmitting coil antenna with current density $\mathbf{J}_{T}$ placed at a plane of constant $\zeta$. 
The current $I_{T}$ in the coil is given by the surface integral of $\mathbf{J}$ over its infinitesimal cross-section, i.e.,

$$
\begin{aligned}
I_{T} & =\int_{\zeta} \int_{\rho} \mathbf{J} \cdot \hat{\phi} h_{\rho} h_{\zeta} d \rho d \zeta \\
& =\frac{1}{2 \pi \rho_{T}} .
\end{aligned}
$$

Note that the magnitude of $\mathbf{J}$ varies with $\phi$, but the total current flowing along the coil is constant and equal to $I_{T}$. Taking that in consideration, we can redefine the current distribution in (4-365) such as

$$
\mathbf{J}=I_{T} \boldsymbol{\delta}_{T}
$$

where

$$
\boldsymbol{\delta}_{T}=\delta\left(\rho-\rho_{T}\right) \frac{\delta\left(\zeta-\zeta_{T}\right)}{h_{\zeta}} \hat{\phi}
$$

By substituting (4-370) into (4-351) we can easily show that

$$
F_{m^{\prime}}^{ \pm}=I_{T} \rho_{T} \int_{-\pi}^{\pi} E_{\phi, m^{\prime}}\left(\rho_{T}, \phi\right) d \phi .
$$

Using the fields of the toroidal waveguide, the above equation becomes

$$
\begin{aligned}
F_{n^{\prime} p^{\prime}}^{ \pm} & =I_{T} \rho_{T} \int_{-\pi}^{\pi}\left[E_{0, \phi, n^{\prime} p^{\prime}}^{n}\left(\rho_{T}\right) e^{i n^{\prime} \phi}\right. \\
& \left.+R^{-1} E_{1, \phi, n^{\prime} p^{\prime}}^{n-1}\left(\rho_{T}\right) e^{i\left(n^{\prime}-1\right) \phi-i \psi}+R^{-1} E_{1, \phi, n^{\prime} p^{\prime}}^{n+1}\left(\rho_{T}\right) e^{i\left(n^{\prime}+1\right) \phi+i \psi}\right] d \phi,
\end{aligned}
$$

where the single index $m^{\prime}$ was replaced by $n^{\prime} p^{\prime}$. These indices come from the zeroth-order fields related to $B_{n^{\prime}}\left(k_{\rho, n^{\prime} p^{\prime}} \rho\right) \exp \left(i n^{\prime} \phi\right)$. By solving the integral over $\phi$ in (4-373), we can derive

$$
\begin{aligned}
F_{n^{\prime} p^{\prime}}^{ \pm}= & I_{T} 2 \pi \rho_{T} \delta_{n^{\prime}, 0} E_{0, \phi, n^{\prime} p^{\prime}}^{n}\left(\rho_{T}\right) \\
& +I_{T} 2 \pi \rho_{T} R^{-1}\left[\delta_{n^{\prime}, 1} e^{-i \psi} E_{1, \phi, n^{\prime} p^{\prime}}^{n-1}\left(\rho_{T}\right)+\delta_{n^{\prime},-1} e^{i \psi} E_{1, \phi, n^{\prime} p^{\prime}}^{n+1}\left(\rho_{T}\right)\right],
\end{aligned}
$$

where $\delta_{i, j}$ the Kronecker delta. From (4-284), we can see that $E_{1, \phi, 1 p^{\prime}}^{n-1}\left(\rho_{T}\right)=$ $-E_{1, \phi,-1 p^{\prime}}^{n+1}\left(\rho_{T}\right)$, which allow us to find the symmetry relation

$$
e^{-i \psi} F_{-1 p^{\prime}}^{ \pm}=-e^{i \psi} F_{1 p^{\prime}}^{ \pm}
$$

In addition, from (4-374) it is clear that the fields radiated by the source defined in (4-370) are symmetric in relation to plane $\zeta=\zeta_{T}$, i.e.,

$$
F_{n^{\prime} p^{\prime}}^{-}=F_{n^{\prime} p^{\prime}}^{+}
$$


Clearly, (4-374) shows that a coil antenna will excite both the zeroth-order and the first-order perturbed fields. Our solution must then include the zeroth-order-axial-symmetric fields derived from $\exp ($ in $\phi)$ in which $n=0$. Additionally, the first-order fields derived from the zeroth-order ones with azimuthal index $n=1$ and with the form $\exp (i(n-1) \phi)$ must also be included. Finally, the first-order fields derived from the zeroth-order ones with azimuthal index $n=-1$ and with the form $\exp (i(n+1) \phi)$ must also be added with the previous fields.

Based on the above considerations, our solution require the zeroth-order fields with azimuthal index $n=0$, and $n= \pm 1$. Then, it is appropriated to write $\bar{A}^{ \pm}, \bar{F}^{ \pm}$and $\overline{\bar{Q}}$ by using sub-vectors and sub-matrices associated with these azimuthal indices:

$$
\begin{aligned}
& \bar{A}^{ \pm}=\left[\begin{array}{c}
\bar{A}_{-1}^{ \pm} \\
\bar{A}_{0}^{ \pm} \\
\bar{A}_{+1}^{ \pm}
\end{array}\right], \\
& \bar{F}_{+1}^{ \pm}=\left[\begin{array}{c}
\bar{F}_{0}^{ \pm} \\
\bar{F}_{-1}^{ \pm}
\end{array}\right], \\
& \overline{\bar{Q}}=\left[\begin{array}{ccc}
\overline{\bar{Q}}_{+1,-1} & \overline{\bar{Q}}_{+1,0} & \overline{\bar{Q}}_{+1,+1} \\
\overline{\bar{Q}}_{0,-1} & \overline{\bar{Q}}_{0,0} & \overline{\bar{Q}}_{0,+1} \\
\overline{\bar{Q}}_{-1,-1} & \overline{\bar{Q}}_{-1,0} & \overline{\bar{Q}}_{-1,+1}
\end{array}\right], \text { or }\left.\quad \overline{\bar{Q}}\right|_{n^{\prime}, n}=\overline{\bar{Q}}_{n^{\prime}, n} .
\end{aligned}
$$

From the zeroth-order solution, we know that the modal amplitude of the field associated with $\exp (i n \phi)$ comes from the excitation linked to $\exp (-i n \phi)$. This fact motivate us to assembly (4-377) and (4-379) to be consistent with the definition in (4-378), and also allow $\overline{\bar{Q}}$ to reduces to a diagonal matrix for the curvature vanishing scenario $\left(R^{-1}=0\right)$. In this spacial case our fields become decoupled in terms of each azimuthal index $( \pm n)$. Furthermore, these zeroth-order fields are orthogonal one to each other, and the condition shown in (3-201) enable to express $A_{n p}^{ \pm}$directly linked to $F_{-n p}^{ \pm}$(or its corresponding term in the source amplitude vector $\bar{S}$ from (4-357): $S_{-n p}^{ \pm}$). The sub-vectors $\bar{A}_{n}^{ \pm}$and $\bar{F}_{n^{\prime}}^{ \pm}$are assembled as follows:

$$
\bar{A}_{n}^{ \pm}=\left[\begin{array}{c}
A_{n, 1}^{ \pm} \\
A_{n, 2}^{ \pm} \\
\vdots \\
A_{n, p}^{ \pm} \\
\vdots
\end{array}\right], \text { and }
$$




$$
\bar{F}_{n^{\prime}}^{ \pm}=\left[\begin{array}{c}
F_{n^{\prime}, 1}^{ \pm} \\
F_{n^{\prime}, 2}^{ \pm} \\
\vdots \\
F_{n^{\prime}, p^{\prime}}^{ \pm} \\
\vdots
\end{array}\right]
$$

Notice that under conditions (4-375) and (4-376), specifically for coil source with the current (4-365), we have $e^{-i \psi} \bar{F}_{-n^{\prime}}^{ \pm}=(-1)^{n^{\prime}} e^{i \psi} \bar{F}_{n^{\prime}}^{ \pm}$and $\bar{F}_{n^{\prime}}^{+}=$ $\bar{F}_{n^{\prime}}^{-}$. Consequently, we get the following relations for the modal excitation amplitudes:

$$
e^{-i \psi} A_{n p}^{ \pm}=(-1)^{n} e^{i \psi} A_{-n p}^{ \pm}, \quad \text { and } \quad A_{n p}^{+}=A_{n p}^{-}
$$

which allow us to reduces the numerical effort in the above vector calculations.

The sub-matrices $\overline{\bar{Q}}_{n^{\prime}, n}$ are assembled by the following manner:

$$
\left.\overline{\bar{Q}}_{n^{\prime}, n}\right|_{p^{\prime}, p}=Q_{n p, n^{\prime} p^{\prime}}=\int_{S_{0}}\left(\mathbf{E}_{t, n p} \times \mathbf{H}_{t, n^{\prime} p^{\prime}}\right) \cdot \hat{\zeta} \rho d \rho d \phi .
$$

From the above equation, it is easy to show that the sub-matrices $\overline{\bar{Q}}_{0,0}$ and $\overline{\bar{Q}}_{ \pm 1, \mp 1}$ in (4-379) will diagonal matrices, whose its entries are exactly equal to the reaction integrals in cylindrical coordinates coupling two zeroth-order fields. The entries of sub-matrices $\overline{\bar{Q}}_{ \pm 1, \pm 1}$, however, couples two first-order fields. The resulting amplitudes will be proportional to $R^{-2}$ and this highorder term will be neglected. Finally, the same inspection allow us to verify that entries in the remaining sub-matrices in (4-379) present amplitudes of order $O\left(R^{-1}\right)$ that need to be included in our solution.

\subsection{3}

\section{Received Voltage}

By using the same procedure employed in Section 3.5.5, but now for receivers sensors inside toroidal structures, the voltage induced at a coil antenna can be written as

$$
V_{R}=-\int_{V}\left(\mathbf{E}^{+}+\mathbf{E}^{-}\right) \cdot \boldsymbol{\delta}_{R} d v
$$

where $\boldsymbol{\delta}_{R}$ is defined similarly to (4-371), but using the subscript substitution $T \rightarrow R$. We can now decouple the forward and the backward contributions for the received voltage as

$$
V_{R}=\sum_{n=-\infty}^{\infty} \sum_{p=1}^{\infty}\left(V_{R, n p}^{+}+V_{R, n p}^{-}\right)
$$


We can express the forward and backward voltages as a function of the modal field amplitudes at the receiver position, $A_{R, n p}^{ \pm}$, such as

$$
V_{R, n p}^{ \pm}=-A_{r, n p}^{ \pm} \int_{V} \mathbf{E}_{n p}^{ \pm}(\rho, \phi, \zeta) \cdot \hat{\phi} \delta\left(\rho-\rho_{T}\right) \frac{\delta\left(\zeta-\zeta_{R}\right)}{h_{\zeta}} \rho h_{\zeta} d \rho d \phi d \zeta
$$

for a receiving coil antenna with radius of $\rho_{R}$ and axially placed at $\zeta=\zeta_{R}$. By substituting (4-209) into the above, after a little algebra we obtain

$$
\begin{aligned}
V_{R, n p}^{ \pm} & =-A_{R, n p}^{ \pm} \rho_{R} \int_{-\pi}^{\pi}\left[E_{0, \phi, n p}^{n}\left(\rho_{R}\right) e^{i n \phi}\right. \\
& \left.+R^{-1} E_{1, \phi, n p}^{n-1}\left(\rho_{R}\right) e^{i(n-1) \phi-i \psi}+R^{-1} E_{1, \phi, n p}^{n+1}\left(\rho_{R}\right) e^{i(n+1) \phi+i \psi}\right] d \phi .
\end{aligned}
$$

Then, solving the above integrals yields

$$
\begin{aligned}
& V_{R, n p}^{ \pm}=-A_{R, n p}^{ \pm} 2 \pi \rho_{R} \\
& \quad \times\left[\delta_{0, n} E_{0, \phi, n p}^{n}\left(\rho_{R}\right)+R^{-1} e^{-i \psi} \delta_{n, 1} E_{1, \phi, n p}^{n-1}\left(\rho_{R}\right)+R^{-1} e^{i \psi} \delta_{n,-1} E_{1, \phi, n p}^{n+1}\left(\rho_{R}\right)\right],
\end{aligned}
$$

where $\delta_{i, j}$ is the Kronecker delta. Notice that the axial positions of transmitting and receiving antennas are embedded into $A_{R, n p}^{ \pm}$. The procedure required to transfer the modal source amplitudes at $\zeta=\zeta_{T}$ to a desired observation point at $\zeta=\zeta_{R}$ can be obtained by means of the formulas presented in Appendix B with little changes: the orthogonality condition in (B-2) is no longer true in toroidal structures, and now we need to incorporate all the azimuthal harmonics into the transfer matrices; similarly to that used in (4-377)-(4-379).

A special case of practical interest is when both transmitting and receiving antennas are placed inside an infinitely-long axial waveguide. In this case, for $\zeta_{R}>\zeta_{T}$ there is no backward propagating waves inducing voltages at receiver and thereby we must calculate the received voltage as $V_{R}=\sum_{n, p} V_{R, n p}^{+}$. Otherwise, when $\zeta_{R}<\zeta_{T}$, the absence of forward waves lead us to conclude that the received voltage is now given by $V_{R}=\sum_{n, p} V_{R, n p}^{-}$. Consequently, due the symmetries in (4-382) and (4-284) we can rewrite $V_{R}^{ \pm}$as

$$
V_{R}^{ \pm}=-2 \pi \rho_{R} \sum_{p=1}^{\infty}\left[A_{R, 0 p}^{ \pm} E_{0, \phi, n p}^{n}\left(\rho_{R}\right)+2 R^{-1} A_{R,+1 p}^{ \pm} E_{1, \phi,+1 p}^{n-1}\left(\rho_{R}\right)\right] .
$$

We can identify the first term of the above as the voltage induced at a coil antenna inside an ordinary cylindrical waveguide, i.e., a weighted sum of the azimuthal component of the radiated electric field as predicted by the formulas in Section 3.5.5 for $\theta_{R, T}=0$. In addition, the second term is a first-order perturbation correction due to a finite curvature $R^{-1}$. 


\subsection{4}

\section{First-Order Approximated Solution for the Excitation Amplitudes}

The voltage expressed in (4-389) is proportional to the amplitudes $A_{R}^{ \pm}$, that are axial translations of the excitation amplitudes $A_{T}^{ \pm}$embedded into vector (4-358); namely, $A_{R, n p}^{ \pm}=A_{T, n p}^{ \pm} e^{i k_{\zeta, n p}\left(\zeta_{R}-\zeta_{T}\right)}$. These amplitudes, however, could present terms with orders higher than $O\left(R^{-1}\right)$. This motivate us to further simplify the excitation amplitudes aiming to obtain a physical picture of the most important perturbation correction due the finite-curvature $R^{-1}$.

As stated before, all the off-diagonal entries of matrix $\overline{\bar{Q}}$ are proportional to $R^{-1}$. For the propose of our analysis, we can decompose this diagonallydominant matrix as

$$
\overline{\bar{Q}}=\overline{\bar{Q}}_{D}+R^{-1} \overline{\bar{Q}}_{C}
$$

where $\overline{\bar{Q}}_{D}$ is a diagonal matrix associated with the zeroth-order fields and $\overline{\bar{Q}}_{C}$ has only zeros in its diagonal elements. We can use the above to approximate (4-358) considering terms up to $R^{-1}$. After some tedious but straightforward manipulations, we can find

$$
\begin{aligned}
& \bar{A}^{ \pm}=-\frac{1}{2} \overline{\bar{Q}}_{D}^{-1} \bar{F}^{0, \pm}-\frac{R^{-1}}{2} \overline{\bar{Q}}_{D}^{-1} \bar{F}^{1, \pm} \\
& +\frac{R^{-1}}{4} \overline{\bar{Q}}_{D}^{-1}\left(\overline{\bar{Q}}_{C}+\overline{\bar{Q}}_{C}^{t}\right) \overline{\bar{Q}}_{D}^{-1} \bar{F}^{0, \pm}-\frac{R^{-1}}{4} \overline{\bar{Q}}_{D}^{-1}\left(\overline{\bar{Q}}_{C}-\overline{\bar{Q}}_{C}^{t}\right) \overline{\bar{Q}}_{D}^{-1} \bar{F}^{0, \mp}+O\left(R^{-2}\right),
\end{aligned}
$$

where we have decomposed the excitation vector as $\bar{F}^{ \pm}=\bar{F}^{0, \pm}+R^{-1} \bar{F}^{1, \pm}$. We can identify a zeroth-order normalization matrix as $\overline{\bar{N}}^{0}=-2 \overline{\bar{Q}}_{D}$, so that $\bar{A}^{0, \pm}=\left(\overline{\bar{N}}^{0}\right)^{-1} \bar{F}^{0, \pm}$ are the same source amplitudes derived for the zeroth-order and curvature vanishing problem. Consequently, we can obtain

$$
\bar{A}^{ \pm} \approx \bar{A}^{0, \pm}+R^{-1}\left(\overline{\bar{N}}^{0}\right)^{-1}\left[\bar{F}^{1, \pm}+\left(\overline{\bar{Q}}_{C}+\overline{\bar{Q}}_{C}^{t}\right) \bar{A}^{0, \pm}-\left(\overline{\bar{Q}}_{C}-\overline{\bar{Q}}_{C}^{t}\right) \bar{A}^{0, \mp}\right] .
$$

It is interesting to be observed that the source amplitudes in the toroidal structures are linked directly to the zeroth-order terms $\bar{A}^{0, \pm}$, as depicted in the first term of RHS of the above equation. In addition, the first-order correction terms are all normalized by the above-defined matrix $\overline{\bar{N}}^{0}$ (which is a diagonal matrix and its entries are equal to those expected in the nonperturbed problem). The term $\bar{F}^{1, \pm}$ is a consequence of the new shape of fields in toroidal structures. The remaining two terms revels a coupling of the zeroth-order amplitudes $\bar{A}^{0, \pm}$ due the curvature. The third term couples forward/backward (zeroth-order) waves with the forward/backward solution amplitudes. The last term, instead, couples $\bar{A}^{0, \mp}$ into $\bar{A}^{ \pm}$. This means that the 
curvature causes a coupling between the axial forward and backward zerothorder fields.

\section{6}

\section{Numerical Results and Validation}

\subsection{1}

\section{Electromagnetic Field Characteristics in a Radially-Stratified Waveguide}

To illustrate the application of the solution derived in this chapter, we now will present simulation results for the electromagnetic field patterns and propagation characteristics in radially-stratified and axially-toroidal media. We consider a typical scenario of geophysical exploration, in which a 4-inradius metallic mandrel is inside a 5-in-radius borehole filled with oil-based mud having conductivity equal to $5 \times 10^{-4} \mathrm{~S} / \mathrm{m}$. Outside borehole, the soil formation has conductivity equal to $1 \mathrm{~S} / \mathrm{m}$. We also assume that source excitation operates at $2 \mathrm{MHz}$, so that the mandrel layer is assumed as a PEC. The radial domain is truncated at $\tilde{r}_{N}=60+i 20 \mathrm{in}$, with a PML over $\rho>40 \mathrm{in}$.

The axial propagation constants for the first modes (sorted in ascending order according to $\left.\Im m\left(k_{\zeta}\right)\right)$ with azimuthal dependence $n=0, \pm 1, \pm 2, \pm 3$ (with respect to the zeroth-order solution) are shown in Fig. 4.8. As previously mentioned in Section 4.3.1, there is no first-order correction (in respect to the torus curvature) for the perturbed propagation constant, so that $k_{\zeta}=k_{z}$.

We can better understand the finite-curvature effects in toroidal waveg-
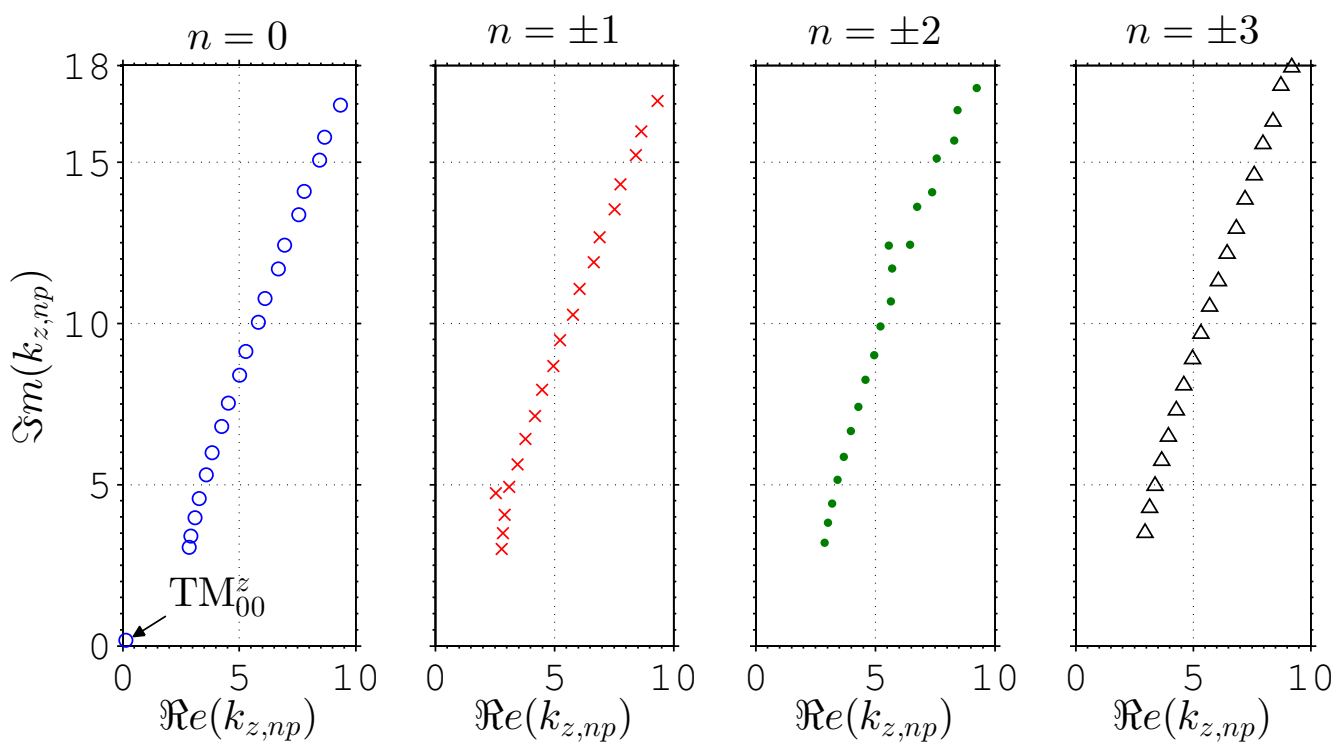

Figure 4.8: Axial propagation constants $k_{z, n p}$ for a cylindrical waveguide with $r_{0}=4$-in, $r_{1}=5$-in and $\tilde{r}_{N}=60$-in $+i 20$-in, where layer 1 has $\sigma_{1}=5 \times 10^{-4} \mathrm{~S} / \mathrm{m}$ and layer 2 has $\sigma_{2}=1 \mathrm{~S} / \mathrm{m}$. 
uides by analyzing the fields distribution shown in Table 4.2 to Table 4.11 . We show the axial field patterns over the waveguide cross-section for the first five modes with $n=0$ and the first five modes with $n=+1$. Notice these fields are not yet normalized in respect to (4-358), and only relative comparisons are allowed for fields coming from a common eigenvalue $k_{\zeta, n p}$. The modes for $n=0$ will be designed as modes $\# 1, \# 2, \# 3$, \#4 and \#5, while modes for $n=+1$ will be $\# 6$, \#7, \#8, \#9 and \#10. High-order modes will not be shown here due its negligible contribution to the total fields. Also notice that at $\rho=40$ in, fields enter into the PML and are then absorbed in the radial direction. The PML layer inner boundary is depicted by dashed-circles.

From Fig. 4.8 and Table 4.2 (see mode $\# 1$ for $n=0$ with $R \rightarrow \infty$ ), we can clear recognize the presence of the fundamental transversal magnetic (in relation to $z$-axis) $\mathrm{TM}_{00}^{z}$ mode. We can shown this mode is a degeneration of the transversal electromagnetic mode $\left(\mathrm{TEM}^{z}\right)$ that occur in hollow coaxial waveguides $[119$, Ch. 2$]$. In contrast to $\mathrm{TEM}^{z}$ modes, mode $\# 1$ presents a $E_{z}$ field component. Also for mode \#1, we can see the emergence of a $H_{\zeta}$ field component as the radius of curvature decreases. The main pattern of $E_{\zeta}$ does not change significantly for the analyzed curvature scenarios due the small value of the radial wavenumber associated to the zeroth-order solution.

The curvature-vanishing version of mode $\# 2$ is recognized as a $\mathrm{TM}_{01}^{z}$ field, as depicted in Table 4.3. Again, we can see the emergence of a $H_{\zeta}$ field component as the radius of curvature decreases, and now the pattern of $E_{\zeta}$ changes significantly when compared to corresponding zeroth-oder azimuthally-symmetric fields.

By analyzing Table 4.4, we can recognize mode $\# 3$ as a $\mathrm{TE}_{01}^{z}$ field when $R \rightarrow \infty$. Now, we can see the emergence of a $E_{\zeta}$ field component as the radius of curvature decreases, and also the pattern distribution of $H_{\zeta}$ over the cross-section now becomes more concentrated in the direction of the center of curvature; that points to positive $x$-axis.

Similarly, modes \#4 and \#5 (shown in Table 4.5, and Table 4.6,) are recognized as $\mathrm{TM}_{02}^{z}$ and $\mathrm{TE}_{02}^{z}$ as $R \rightarrow \infty$, respectively. As in the case of mode \#2 (or \#3), we can now see the emergence of a $H_{\zeta}$ (or $E_{\zeta}$ ) field component as the radius of curvature decreases, and also an associated pattern disturbance of $E_{\zeta}$ (or $H_{\zeta}$ ) over the cross-section concentrating the fields into the direction of the center of curvature.

Modes \#6 to \#10 are associated with the hybrid mode with $n=+1$ in case of $R \rightarrow \infty$. In Table 4.7 to Table 4.11 we can verify several modifications in the original axial field patterns (for $R \rightarrow \infty$ ) compared to results for finite $R$. Notice that the zeroth-order solutions are associated with an azimuthal 
harmonic in shape of $e^{i n \phi}$. The combinations of $n=+1$ and $n=-1$ harmonics can give rise to azimuthal cosine-dependent fields. We only show the $n=+1$ harmonic, but we can recover the corresponding $n=-1$ complementary fields using relations shown in (4-281) and (4-284). 
Table 4.2: Axial field patterns for the mode \#1 (for $n=0$ ) in a toroidal waveguide with $\tilde{r}_{N}=60$-in $+i 20$-in as a function of the radius of curvature $R$. The PML layer is place at $\rho>40$-in and its inner boundary is indicated by the dashed-circles. The center of curvature points to positive $x$-axis.

\begin{tabular}{lll}
\hline$R($ in $)$ & $\operatorname{abs}\left(E_{\zeta}\right)$ & $\operatorname{abs}\left(H_{\zeta}\right)$ \\
\hline
\end{tabular}

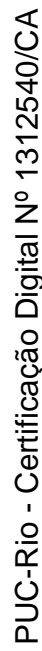

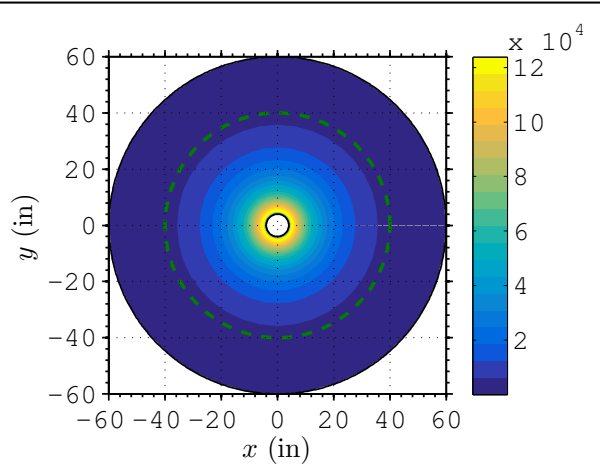

\section{Null field}
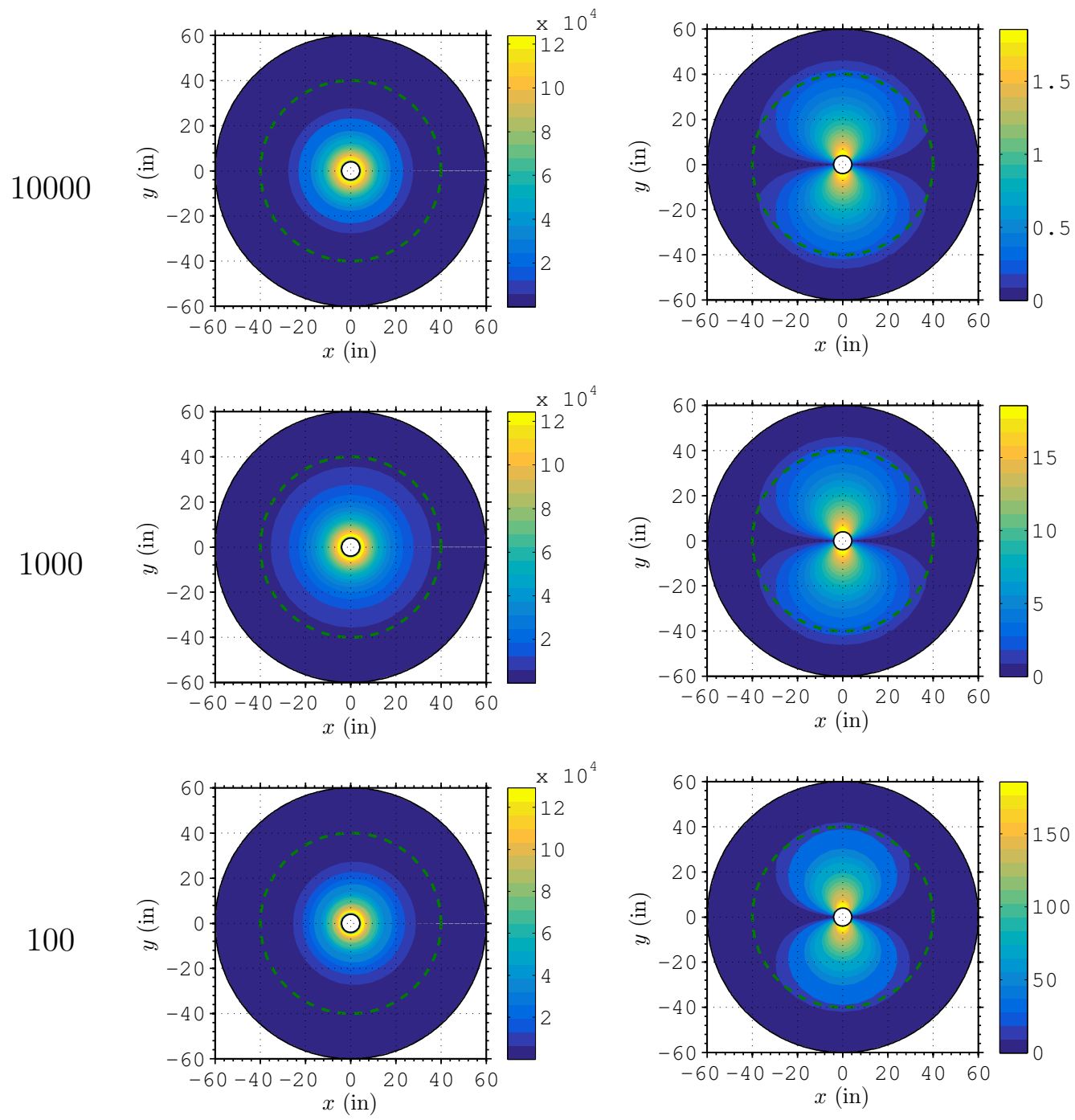
Table 4.3: Axial field patterns for the mode $\# 2$ (for $n=0$ ) in a toroidal waveguide with $\tilde{r}_{N}=60$-in $+i 20$-in as a function of the radius of curvature $R$. The PML layer is place at $\rho>40$-in and its inner boundary is indicated by the dashed-circles. The center of curvature points to positive $x$-axis.

\begin{tabular}{lll}
\hline$R$ (in) & $\operatorname{abs}\left(E_{\zeta}\right)$ & $\operatorname{abs}\left(H_{\zeta}\right)$ \\
\hline
\end{tabular}

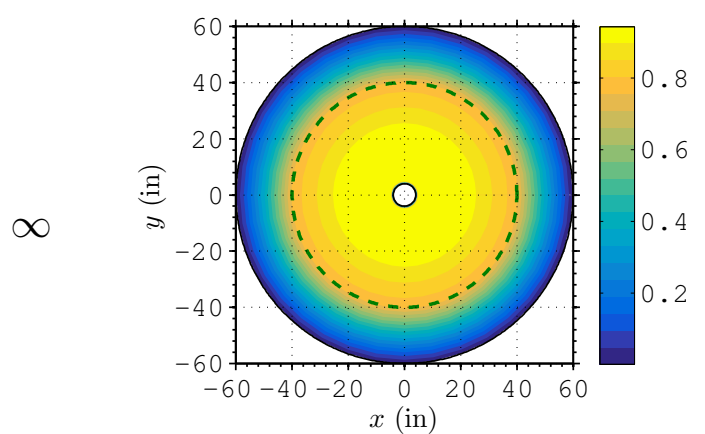

\section{Null field}
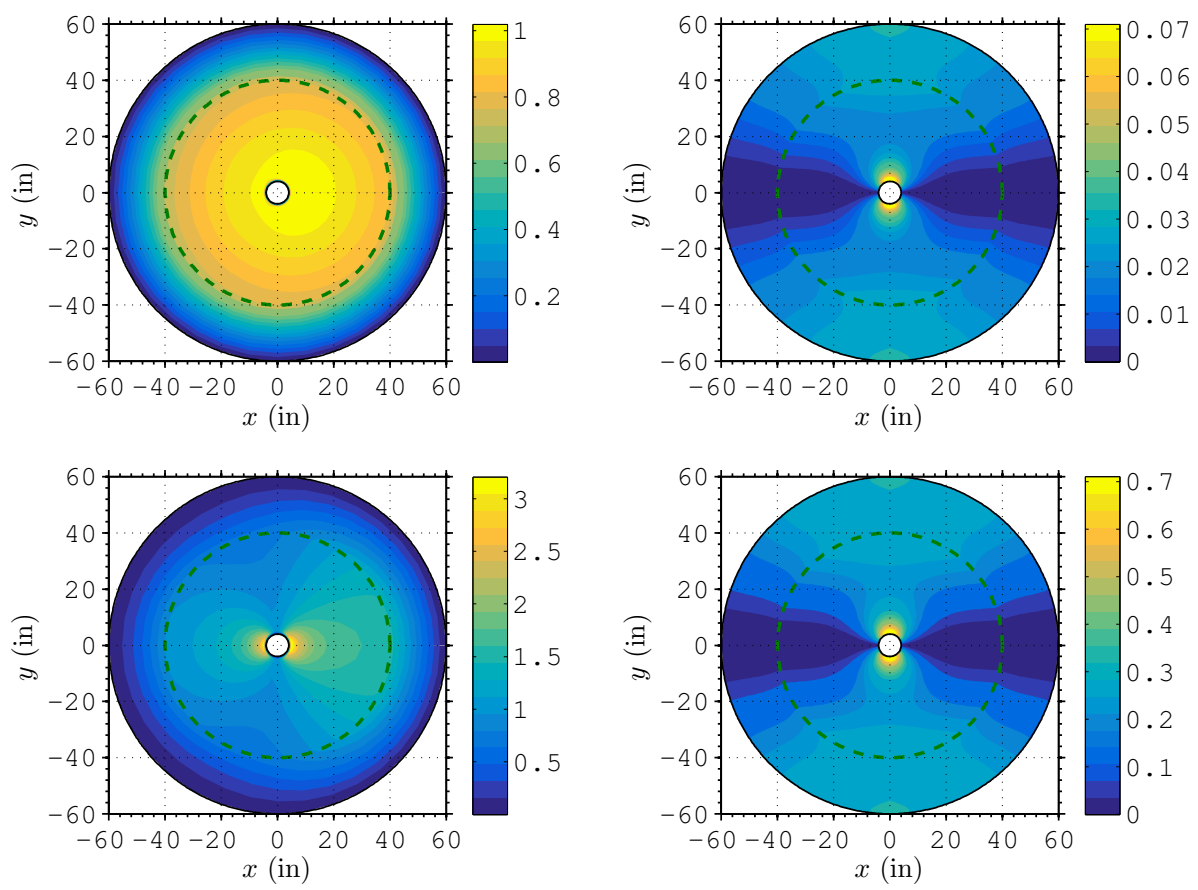

100
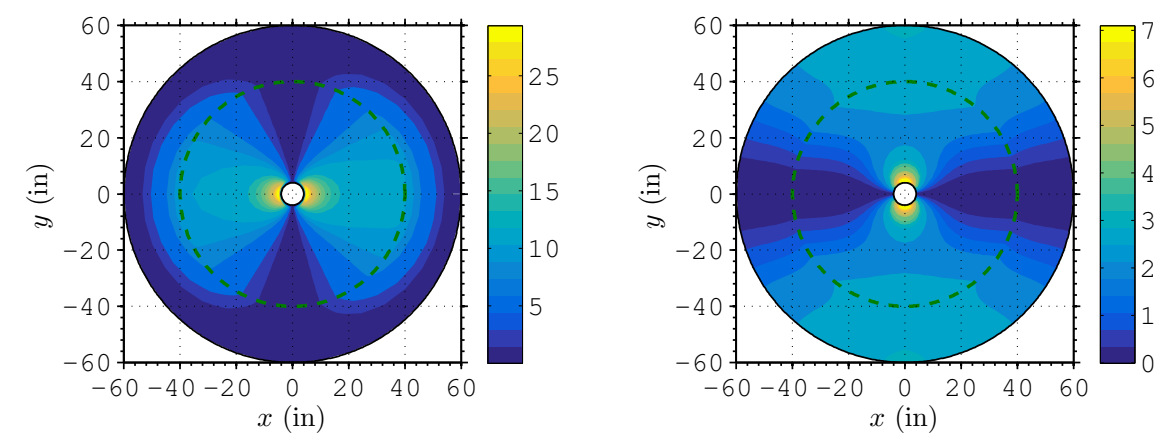
Table 4.4: Axial field patterns for the mode $\# 3$ (for $n=0$ ) in a toroidal waveguide with $\tilde{r}_{N}=60$-in $+i 20$-in as a function of the radius of curvature $R$. The PML layer is place at $\rho>40$-in and its inner boundary is indicated by the dashed-circles. The center of curvature points to positive $x$-axis.

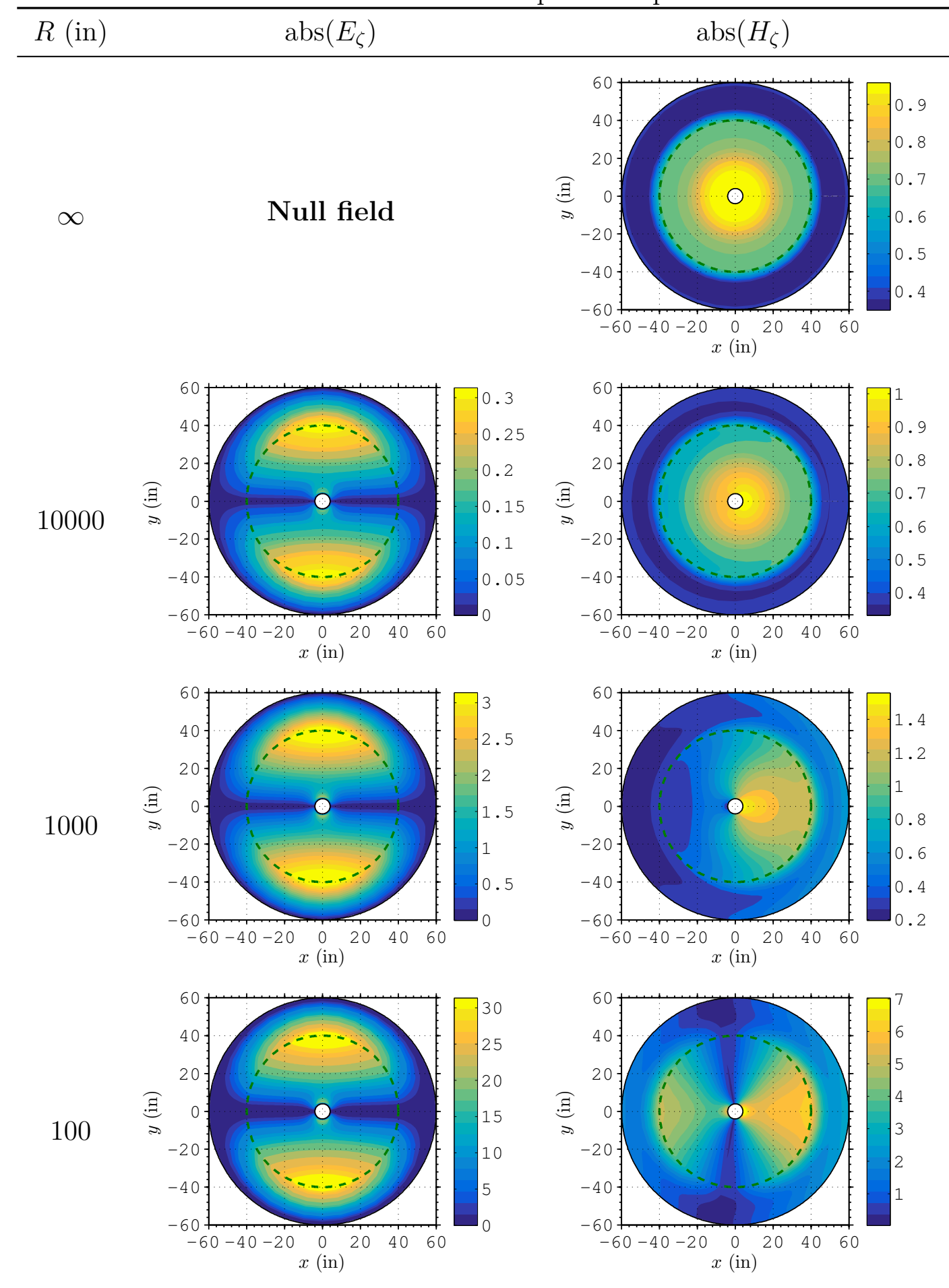


Table 4.5: Axial field patterns for the mode $\# 4$ (for $n=0$ ) in a toroidal waveguide with $\tilde{r}_{N}=60$-in $+i 20$-in as a function of the radius of curvature $R$. The PML layer is place at $\rho>40$-in and its inner boundary is indicated by the dashed-circles. The center of curvature points to positive $x$-axis.

\begin{tabular}{lll}
\hline$R$ (in) & $\operatorname{abs}\left(E_{\zeta}\right)$ & $\operatorname{abs}\left(H_{\zeta}\right)$ \\
\hline
\end{tabular}

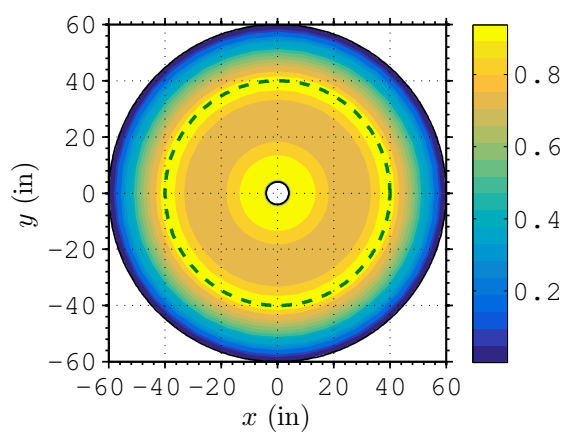

\section{Null field}
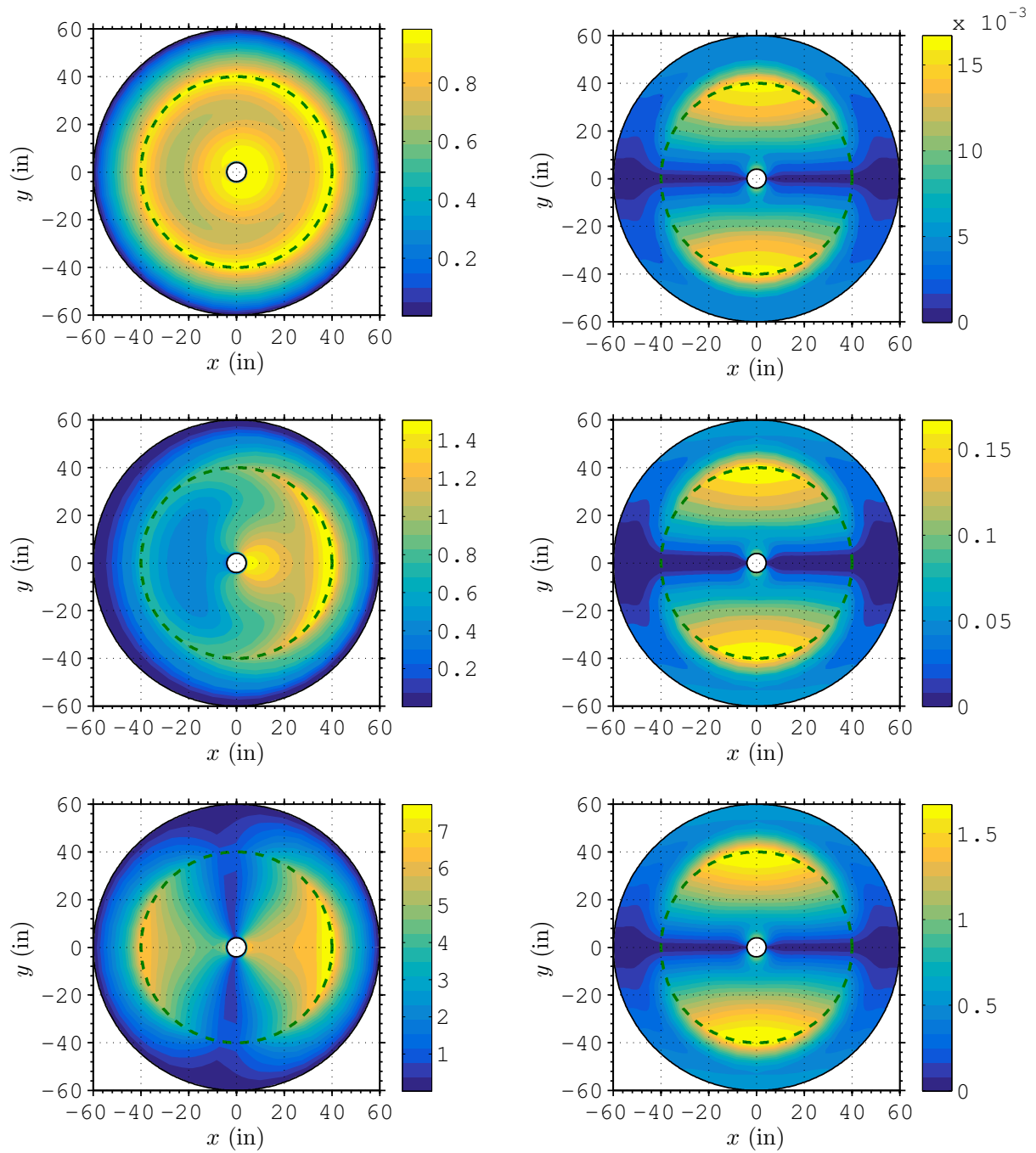
Table 4.6: Axial field patterns for the mode \#5 (for $n=0$ ) in a toroidal waveguide with $\tilde{r}_{N}=60$-in $+i 20$-in as a function of the radius of curvature $R$. The PML layer is place at $\rho>40$-in and its inner boundary is indicated by the dashed-circles. The center of curvature points to positive $x$-axis.

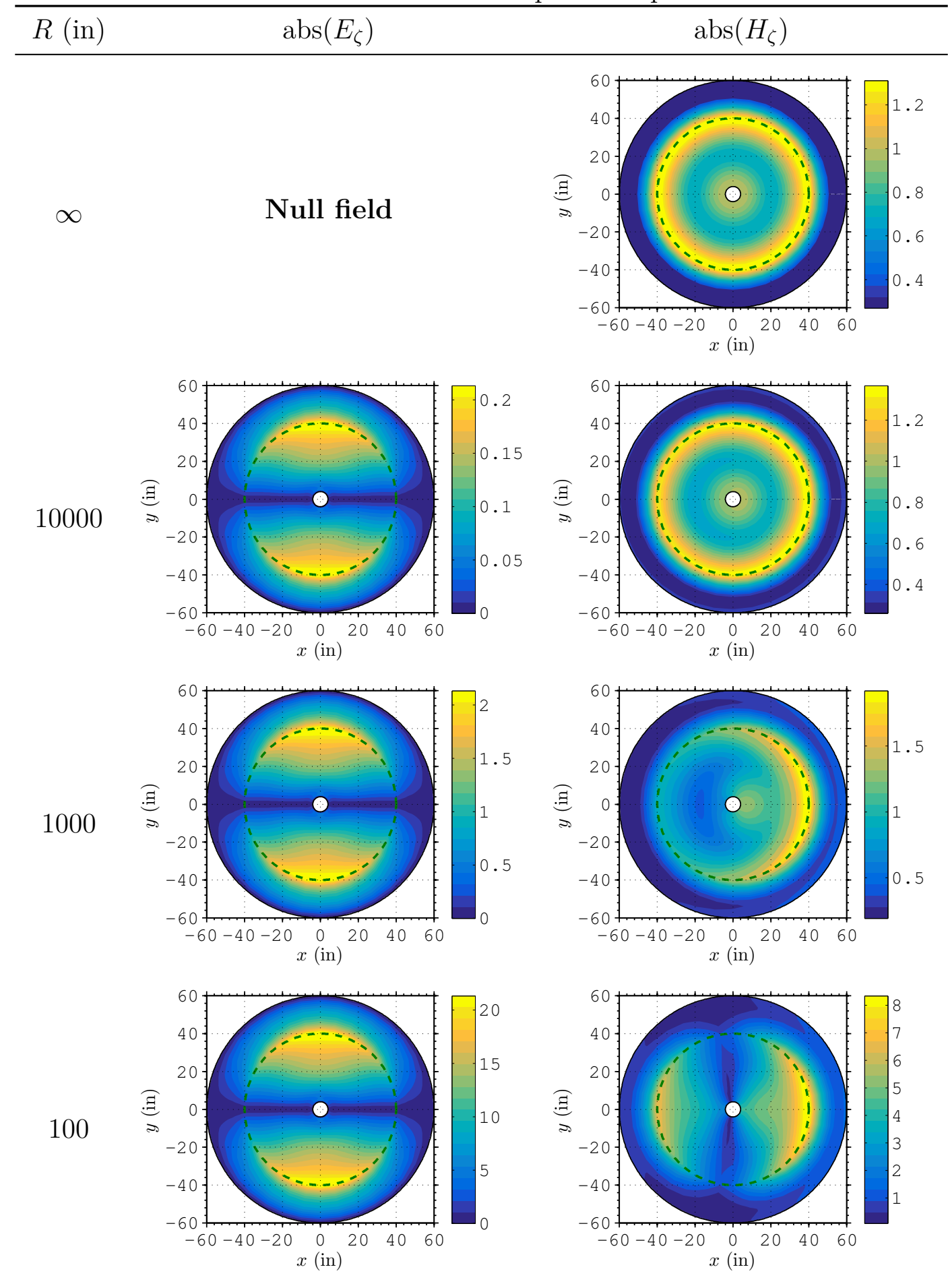


Table 4.7: Axial field patterns for the mode $\# 6$ (for $n=+1$ ) in a toroidal waveguide with $\tilde{r}_{N}=60$-in $+i 20$-in as a function of the radius of curvature $R$. The PML layer is place at $\rho>40$-in and its inner boundary is indicated by the dashed-circles. The center of curvature points to positive $x$-axis.

\begin{tabular}{lll}
\hline$R$ (in) & $\operatorname{abs}\left(E_{\zeta}\right)$ & $\operatorname{abs}\left(H_{\zeta}\right)$ \\
\hline
\end{tabular}

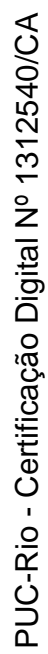
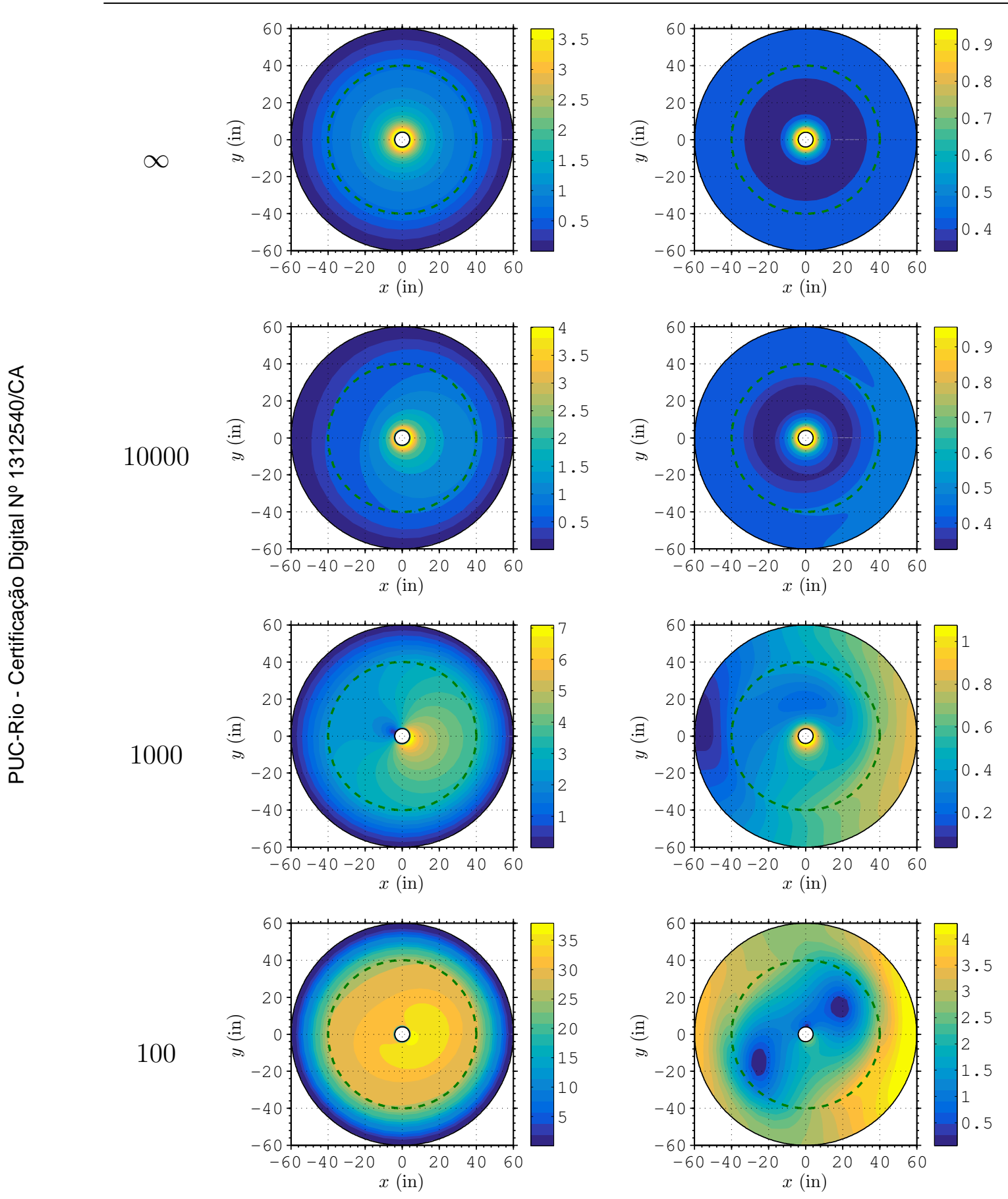
Table 4.8: Axial field patterns for the mode \#7 (for $n=+1$ ) in a toroidal waveguide with $\tilde{r}_{N}=60$-in $+i 20$-in as a function of the radius of curvature $R$. The PML layer is place at $\rho>40$-in and its inner boundary is indicated by the dashed-circles. The center of curvature points to positive $x$-axis.

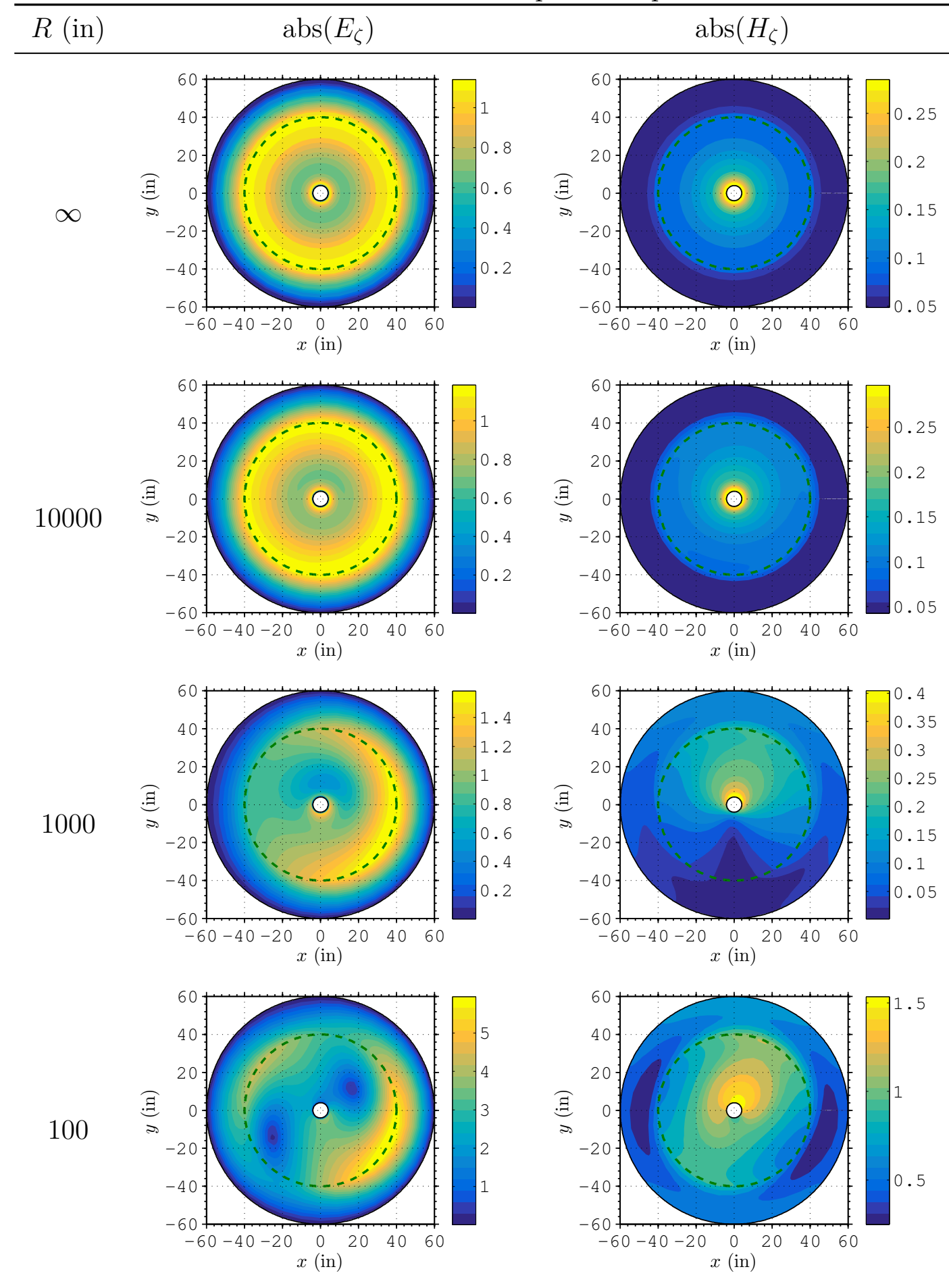


Table 4.9: Axial field patterns for the mode \#8 (for $n=+1$ ) in a toroidal waveguide with $\tilde{r}_{N}=60$-in $+i 20$-in as a function of the radius of curvature $R$. The PML layer is place at $\rho>40$-in and its inner boundary is indicated by the dashed-circles. The center of curvature points to positive $x$-axis.
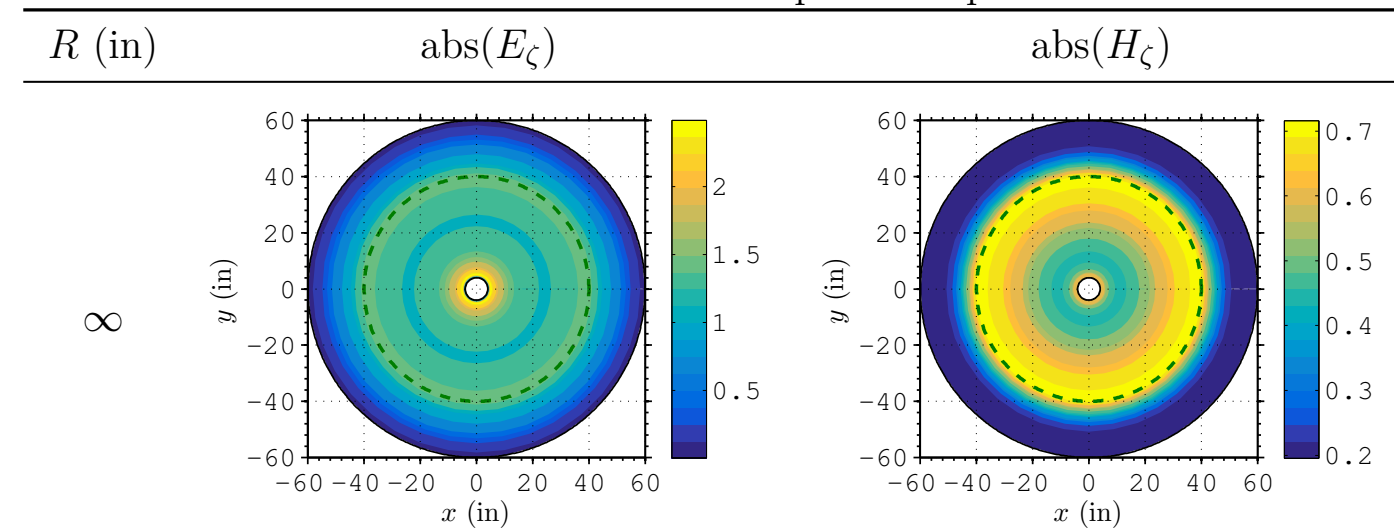

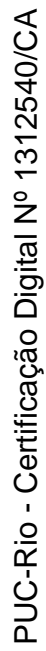
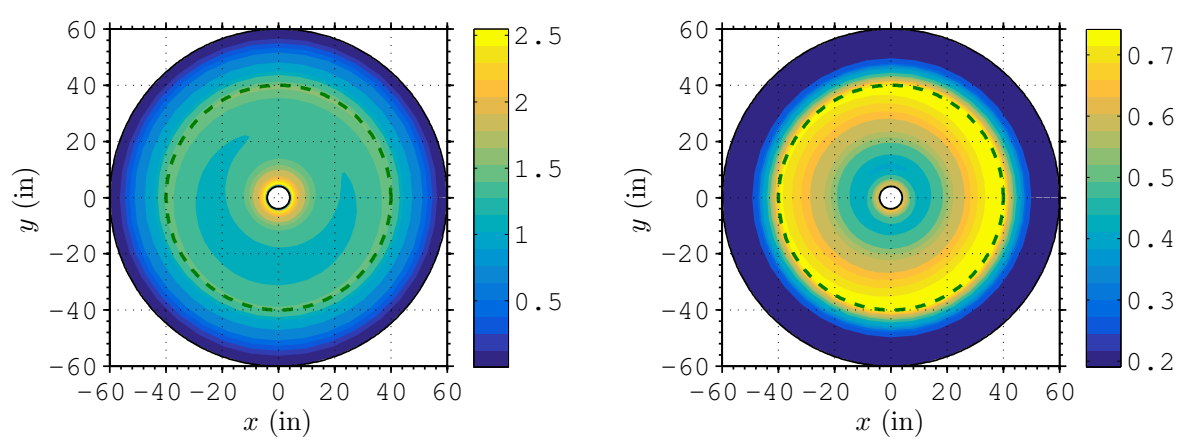

1000
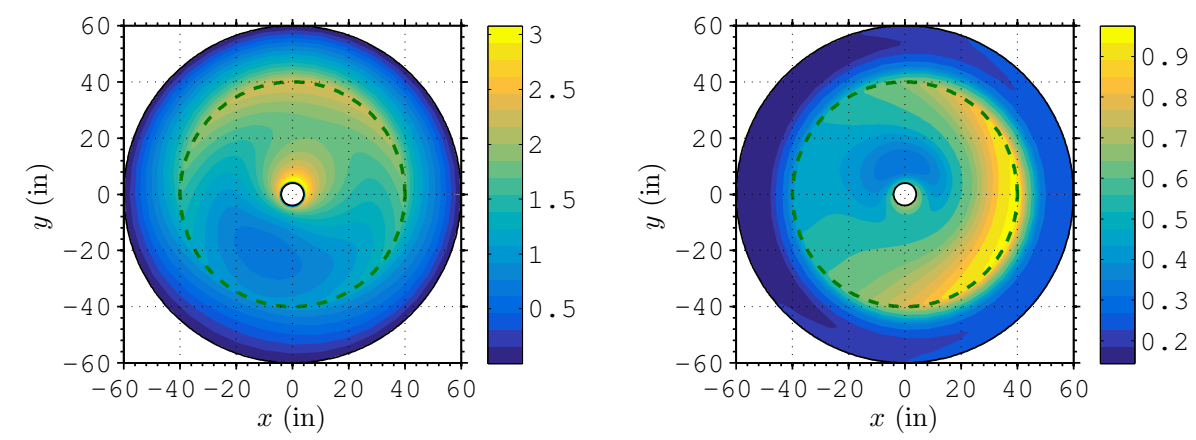

100
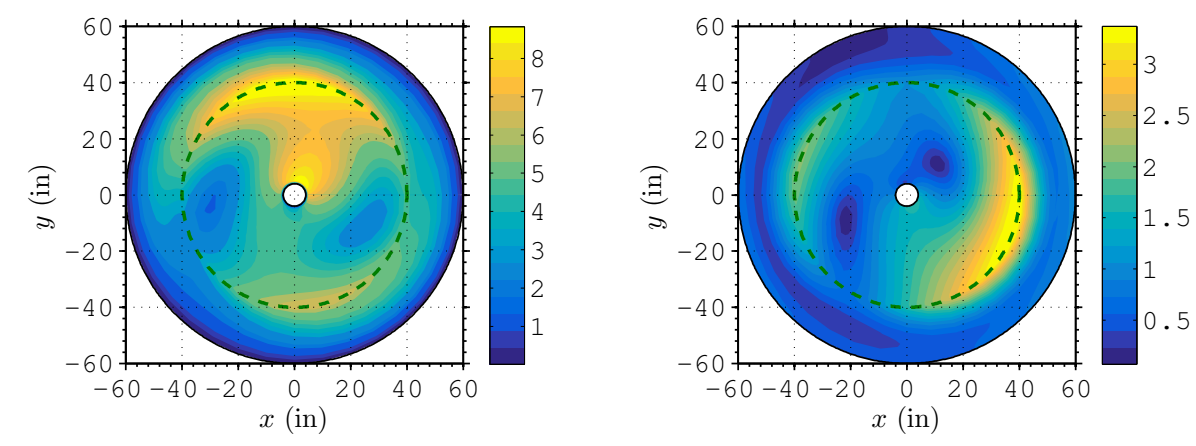
Table 4.10: Axial field patterns for the mode \#9 (for $n=+1$ ) in a toroidal waveguide with $\tilde{r}_{N}=60$-in $+i 20$-in as a function of the radius of curvature $R$. The PML layer is place at $\rho>40$-in and its inner boundary is indicated by the dashed-circles. The center of curvature points to positive $x$-axis.

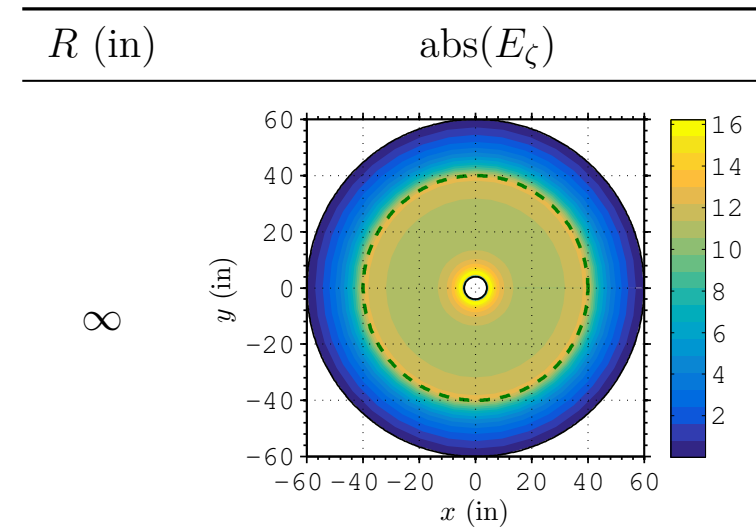
$\operatorname{abs}\left(H_{\zeta}\right)$

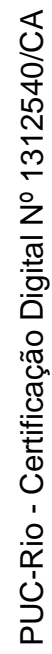
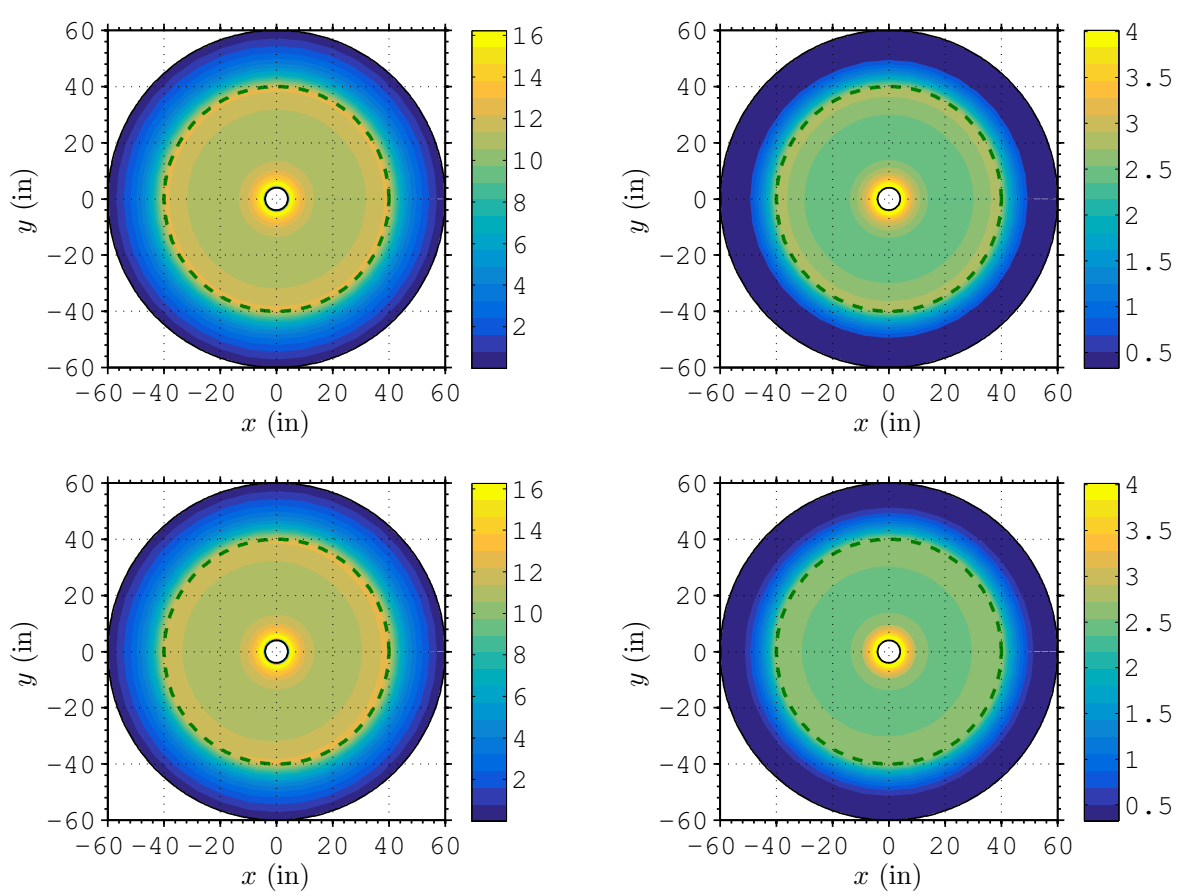

10000
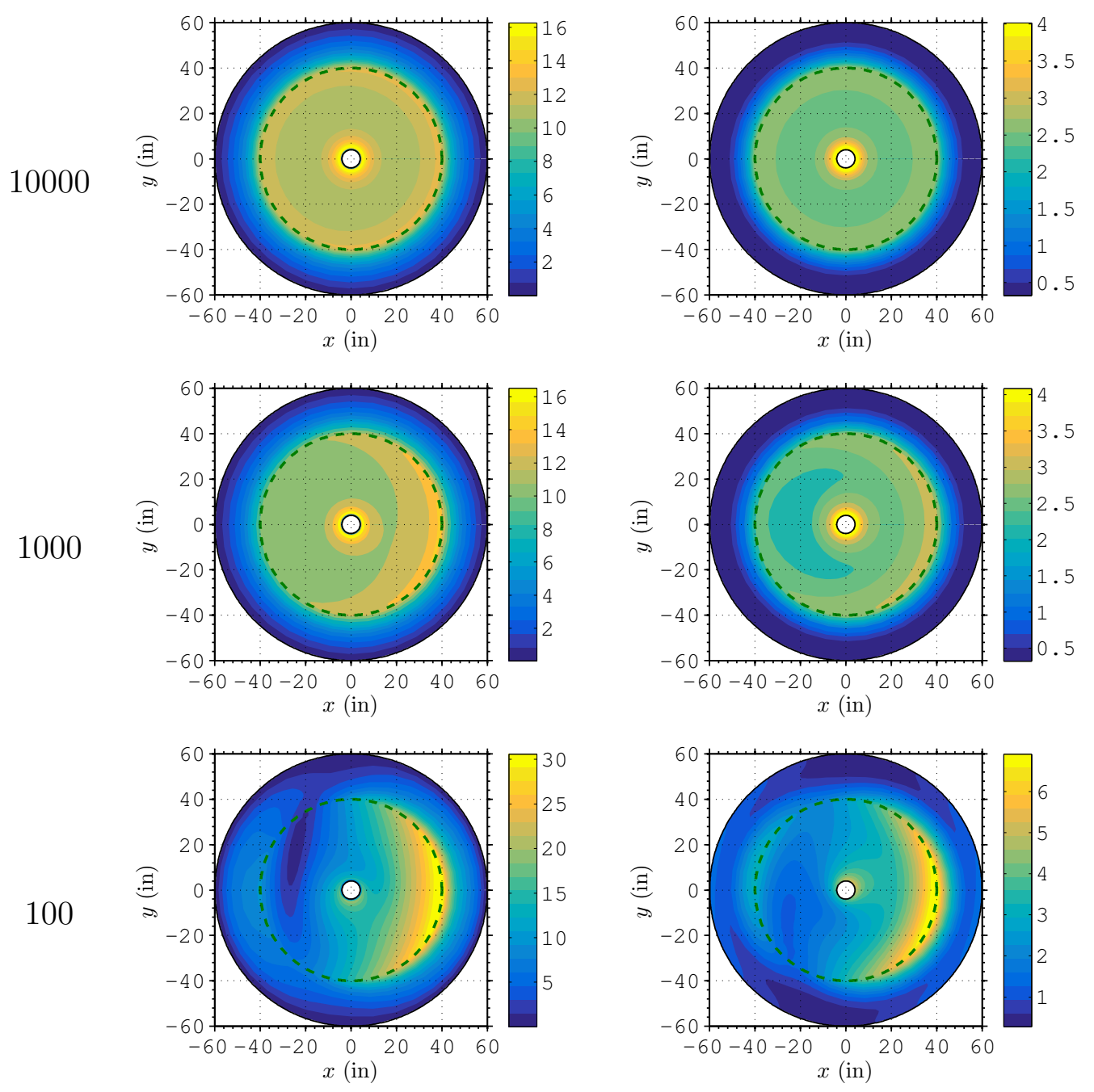
Table 4.11: Axial field patterns for the mode $\# 10$ (for $n=+1$ ) in a toroidal waveguide with $\tilde{r}_{N}=60$-in $+i 20$-in as a function of the radius of curvature $R$. The PML layer is place at $\rho>40$-in and its inner boundary is indicated by the dashed-circles. The center of curvature points to positive $x$-axis.

\begin{tabular}{lll}
\hline$R$ (in) & $\operatorname{abs}\left(E_{\zeta}\right)$ & $\operatorname{abs}\left(H_{\zeta}\right)$ \\
\hline
\end{tabular}
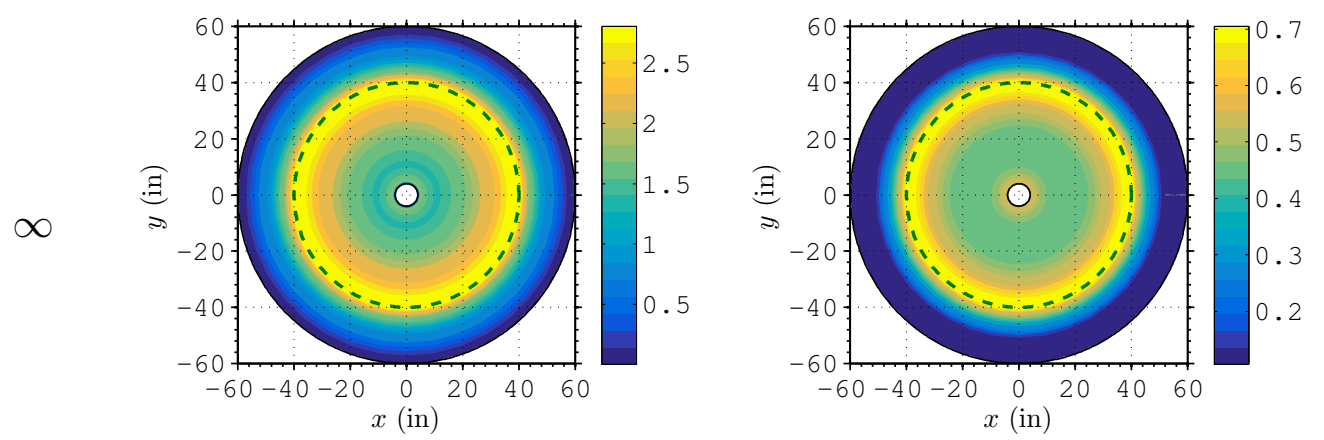

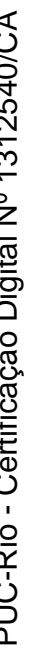
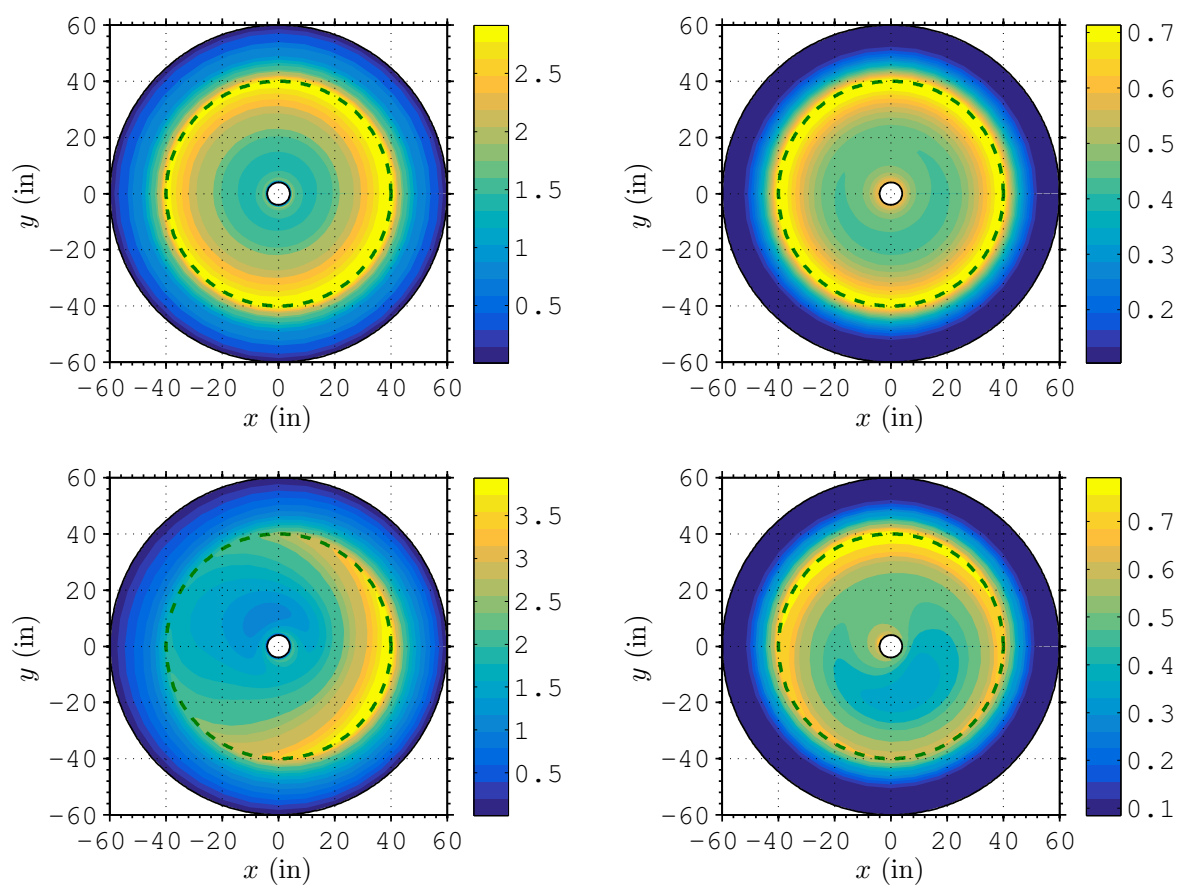

100
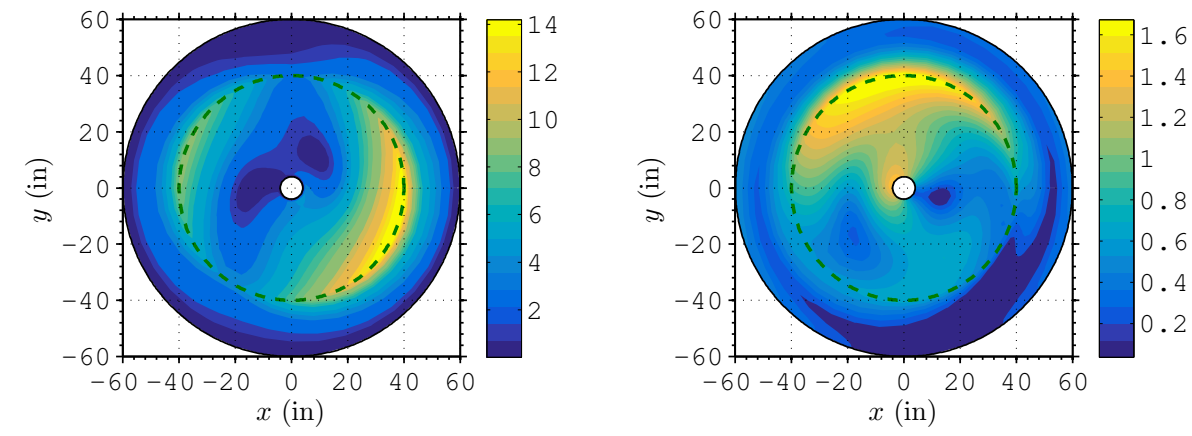
In LWD applications, the azimuthal electric field (around the mandrel) is of great interest because we need this field to account the source excitation and to calculate the induced voltages, as described above in Section 4.5.2. Proper combinations of axial fields shown in Table 4.2 to Table 4.11 (according to (4-194) and (4-209)) allow us to derive the corresponding $E_{\phi}$ components depicted below in Table 4.12 to Table 4.16 (associated with $n=0$ ) and Table 4.17 to Table 4.21 (associated with $n=+1$ ). In all plots shown into these tables, we again shown a dashed-circle at $\rho=40$ in indicating the PML inner boundary. Also, we show a zoom near the mandrel, and another dashed-circle at $\rho=5$ in depicts the interface between the inner borehole and the surrounding soil formation. We can now see in a clear fashion the real implications of the curvature in the fields distribution around the mandrel, where normally coil antennas are wrapped.

Modes \#1, \#2 and \#4 (associated with zeroth-order $\mathrm{TM}^{z}$ fields) are not excited in ordinary cylindrical structures $(R \rightarrow \infty)$, but for finite curvatures there is a non-null $E_{\phi}$ component (of course, proportional to $R^{-1}$ ) that now may possibly be excited.

A new inspection in (4-374) revels that the field components $E_{1, \phi, \mp 1 p^{\prime}}^{n \pm 1}(\rho)$ will contribute to the amplitude excitation of the perturbed versions of $\mathrm{TM}_{0 p^{\prime}}^{z}$ modes. But, in view of the symmetry relations presented in (4-284), the corresponding contribution of each term in (4-374) are in opposite phase: annulling the excitation. Although the significantly fields pattern variations, modes \#1, \#2, \#4 and all other that come from (zeroth-order) transversal magnetic fields will not be excited, and do not change the LWD sensor response.

In the case of the excitation of any of the remaining modes coming from the (zeroth-order) $\mathrm{TE}_{0 p}^{z}$ (e.g., modes \#3 and \#5) or from the hybrid fields (e.g., modes \#6 to \#10), we need to incorporate the said fields to correctly capture the curvature effects in our analysis. 
Table 4.12: Azimuthal electric field patterns for the mode $\# 1$ (for $n=0$ ) in a toroidal waveguide with $\tilde{r}_{N}=60$-in $+i 20$-in as a function of the radius of curvature $R$. The borehole-to-formation and the PML inner boundaries are indicated by the dashed-circles. The center of curvature points to positive $x$ axis.

\begin{tabular}{ll}
\hline$R($ in $)$ & $\operatorname{abs}\left(E_{\phi}\right)$ \\
\hline
\end{tabular}

$\infty$

Null field

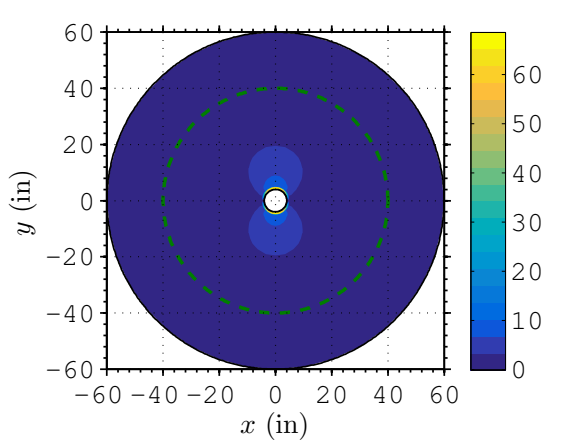

1000

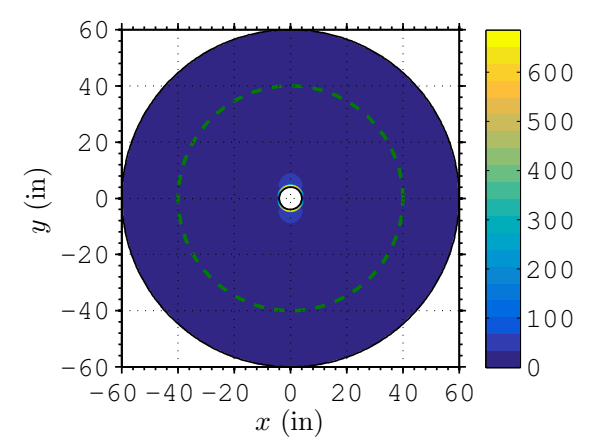

100
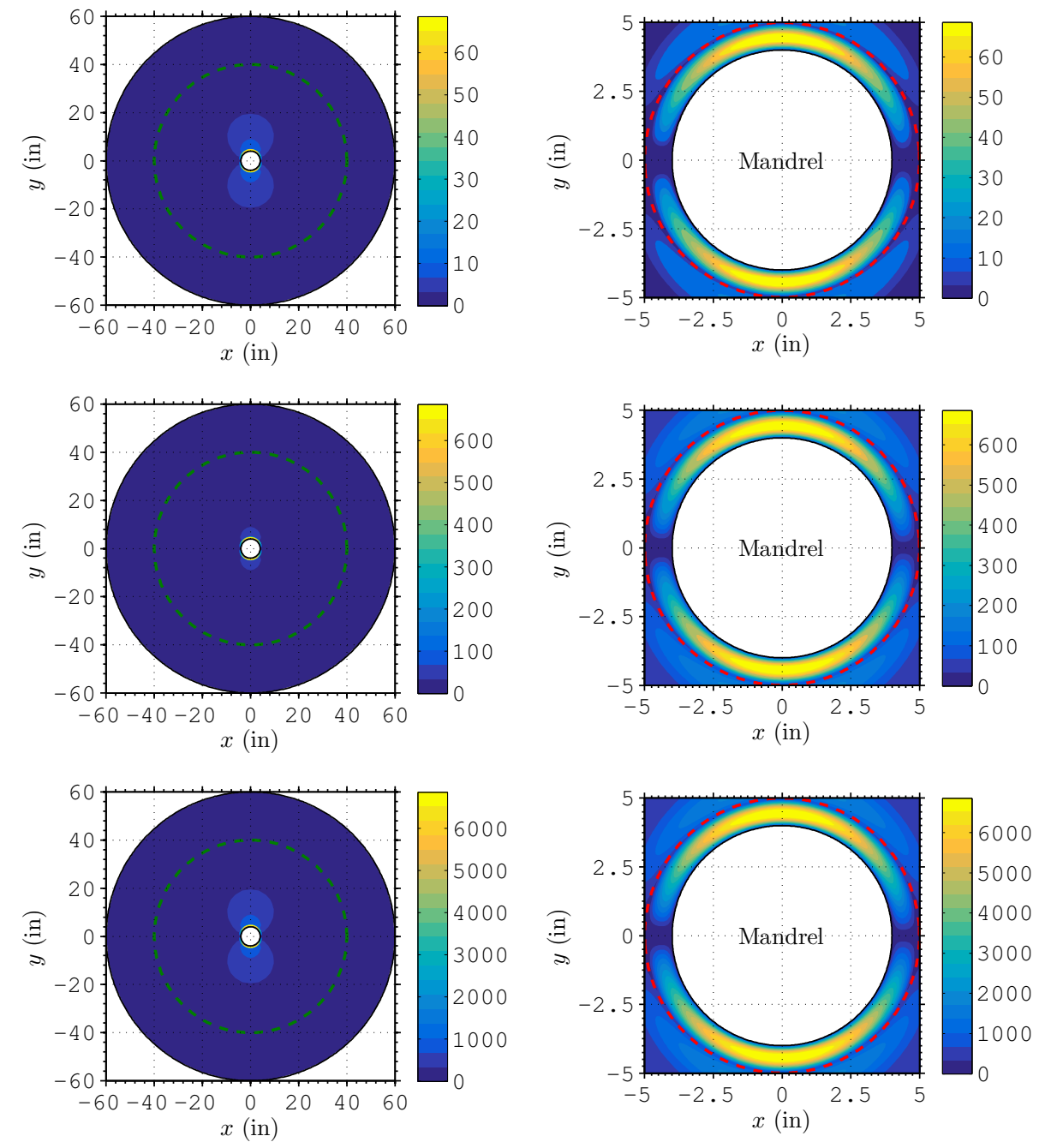
Table 4.13: Azimuthal electric field patterns for the mode \#2 (for $n=0$ ) in a toroidal waveguide with $\tilde{r}_{N}=60$-in $+i 20$-in as a function of the radius of curvature $R$. The borehole-to-formation and the PML inner boundaries are indicated by the dashed-circles. The center of curvature points to positive $x$ axis.

\begin{tabular}{ll}
\hline$R($ in $)$ & $\operatorname{abs}\left(E_{\phi}\right)$ \\
\hline
\end{tabular}

$\infty$

Null field

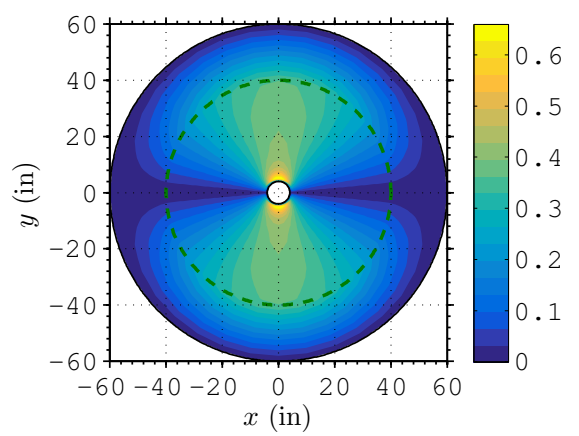

1000

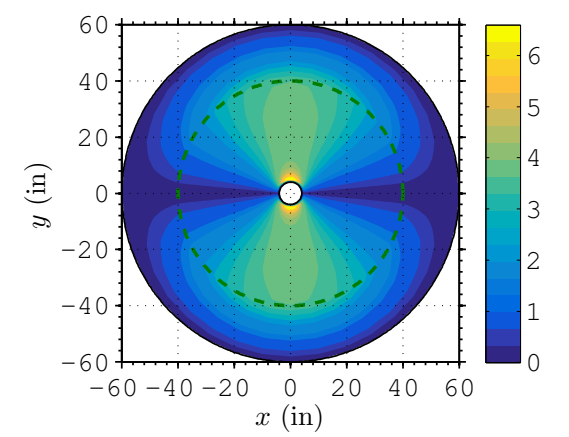

100

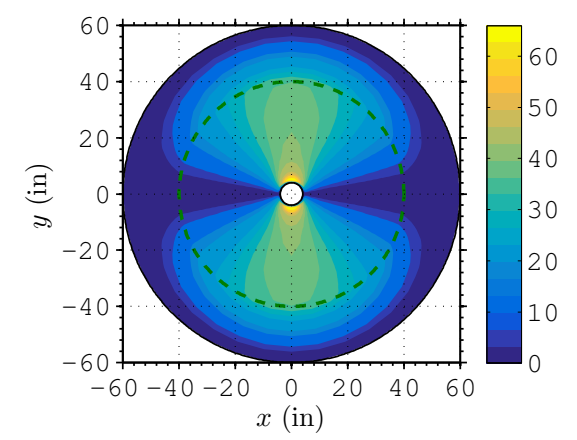

\section{Null field}
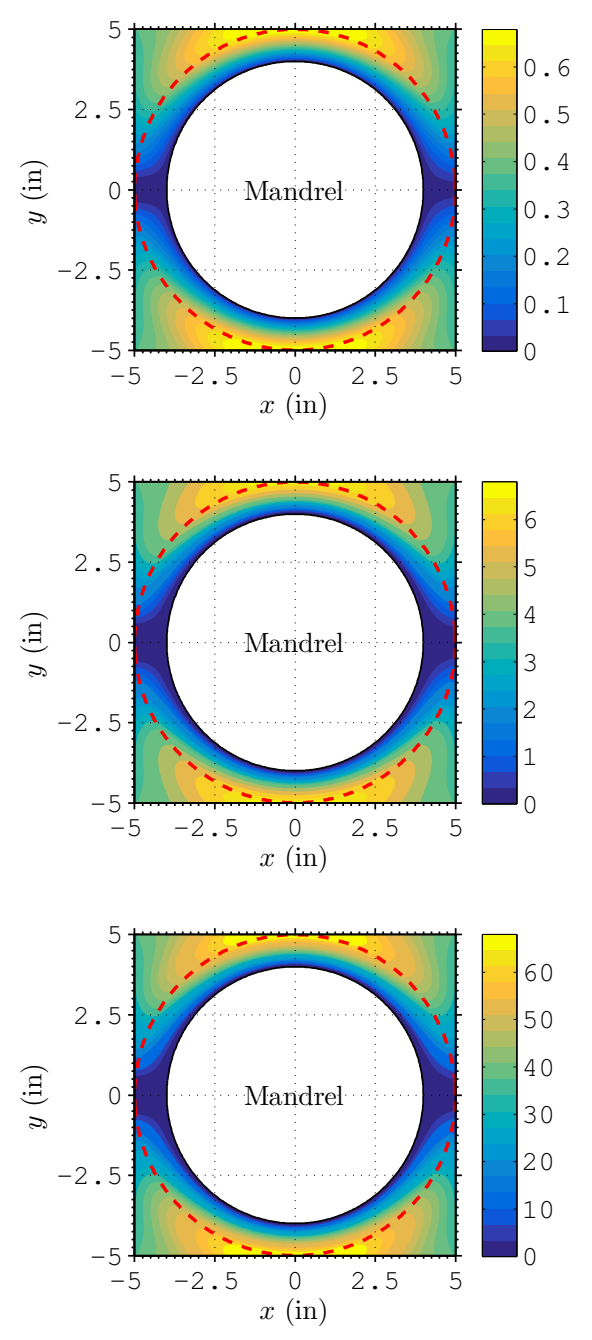
Table 4.14: Azimuthal electric field patterns for the mode \#3 (for $n=0$ ) in a toroidal waveguide with $\tilde{r}_{N}=60$-in $+i 20$-in as a function of the radius of curvature $R$. The borehole-to-formation and the PML inner boundaries are indicated by the dashed-circles. The center of curvature points to positive $x$ axis.

\begin{tabular}{cc}
\hline$R($ in $)$ & $\operatorname{abs}\left(E_{\phi}\right)$ \\
\hline
\end{tabular}
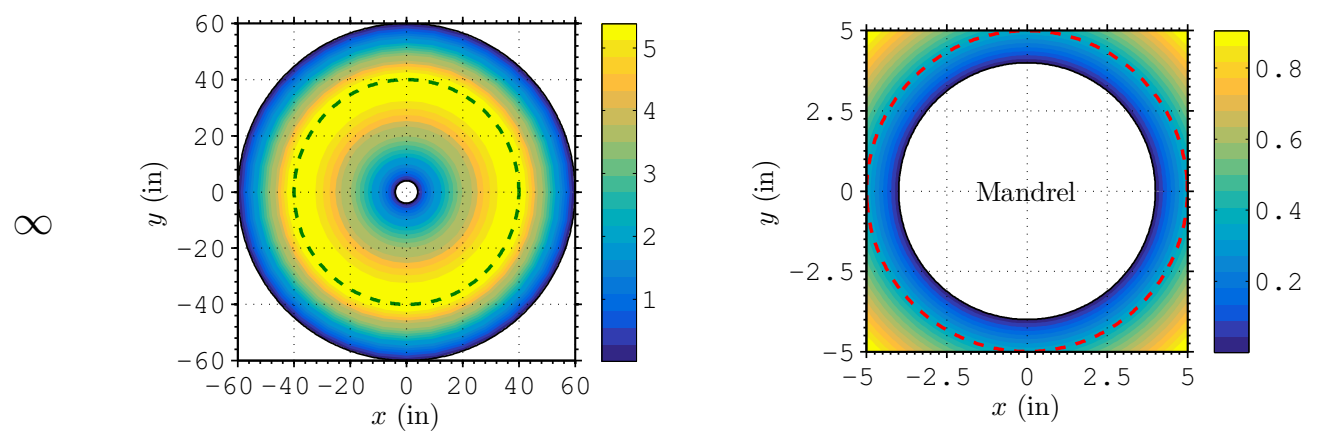

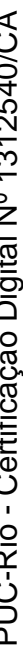
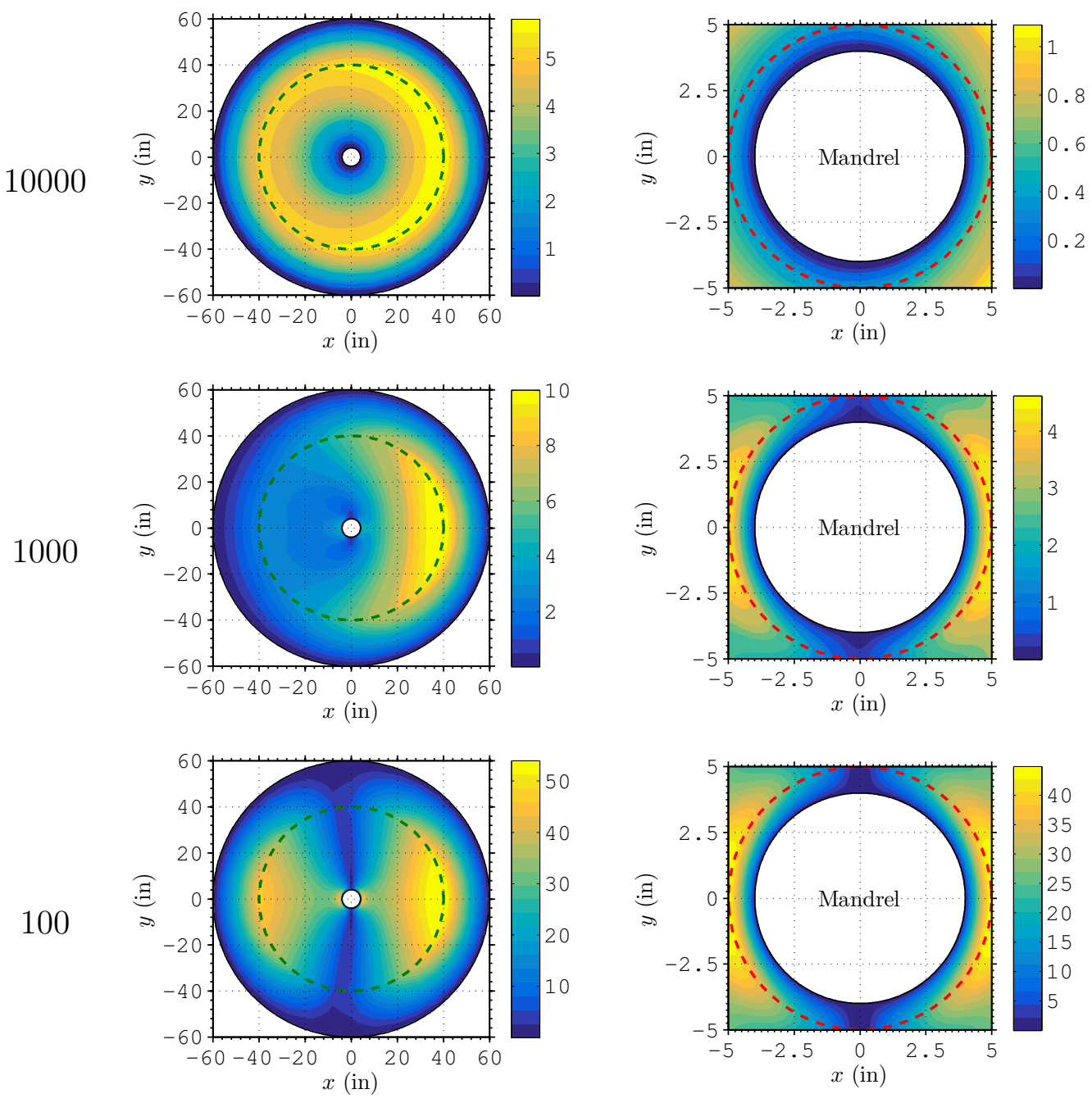
Table 4.15: Azimuthal electric field patterns for the mode \#4 (for $n=0$ ) in a toroidal waveguide with $\tilde{r}_{N}=60$-in $+i 20$-in as a function of the radius of curvature $R$. The borehole-to-formation and the PML inner boundaries are indicated by the dashed-circles. The center of curvature points to positive $x$ axis.

\begin{tabular}{lll}
\hline$R($ in $)$ & $\operatorname{abs}\left(E_{\phi}\right)$ & $\operatorname{abs}\left(E_{\phi}\right)$ near the mandrel \\
\hline
\end{tabular}

$\infty$

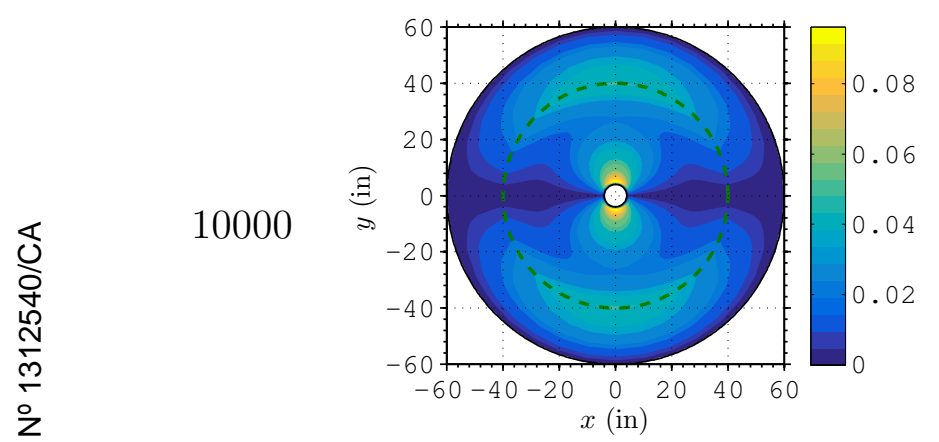

1000

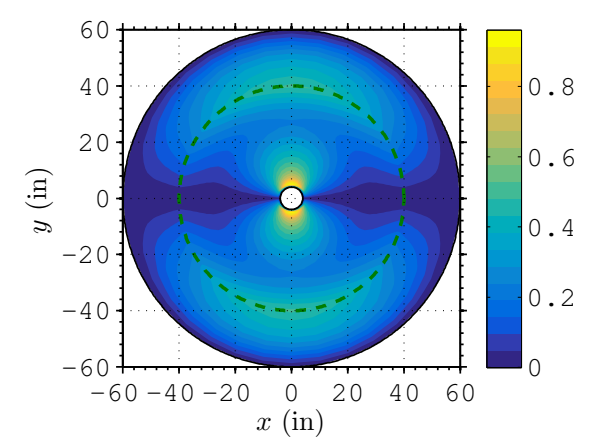

100

\section{Null field}
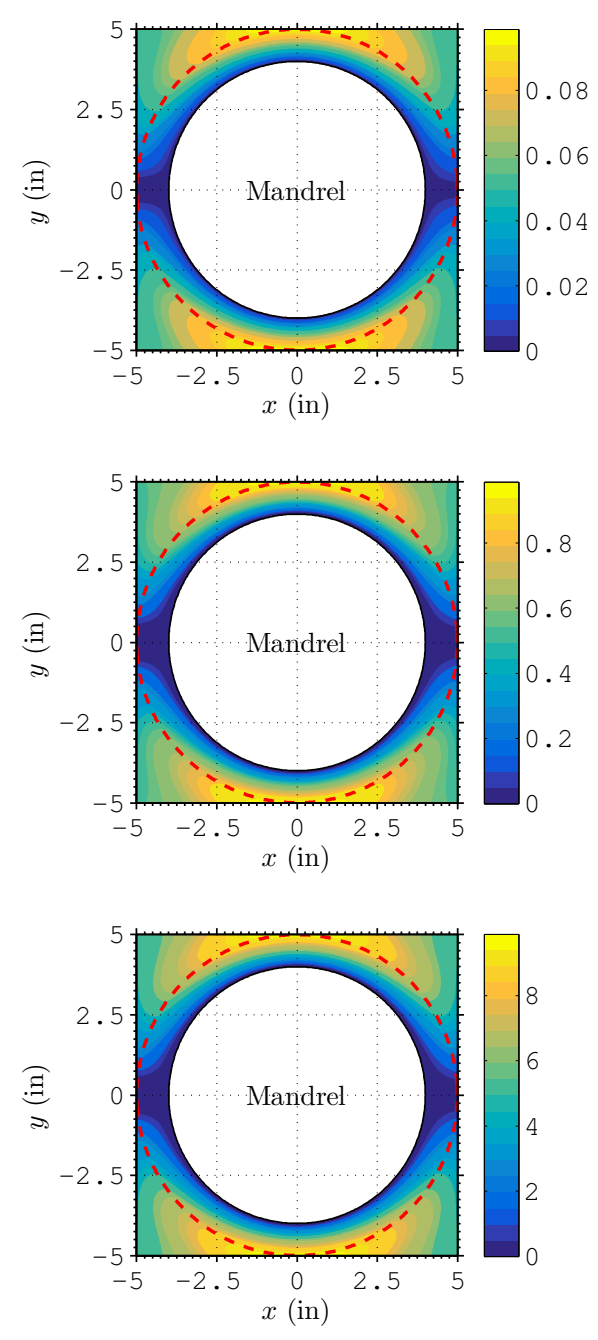
Table 4.16: Azimuthal electric field patterns for the mode \#5 (for $n=0$ ) in a toroidal waveguide with $\tilde{r}_{N}=60$-in $+i 20$-in as a function of the radius of curvature $R$. The borehole-to-formation and the PML inner boundaries are indicated by the dashed-circles. The center of curvature points to positive $x$ axis.

\begin{tabular}{cc}
\hline$R($ in $)$ & $\operatorname{abs}\left(E_{\phi}\right)$ \\
\hline
\end{tabular}
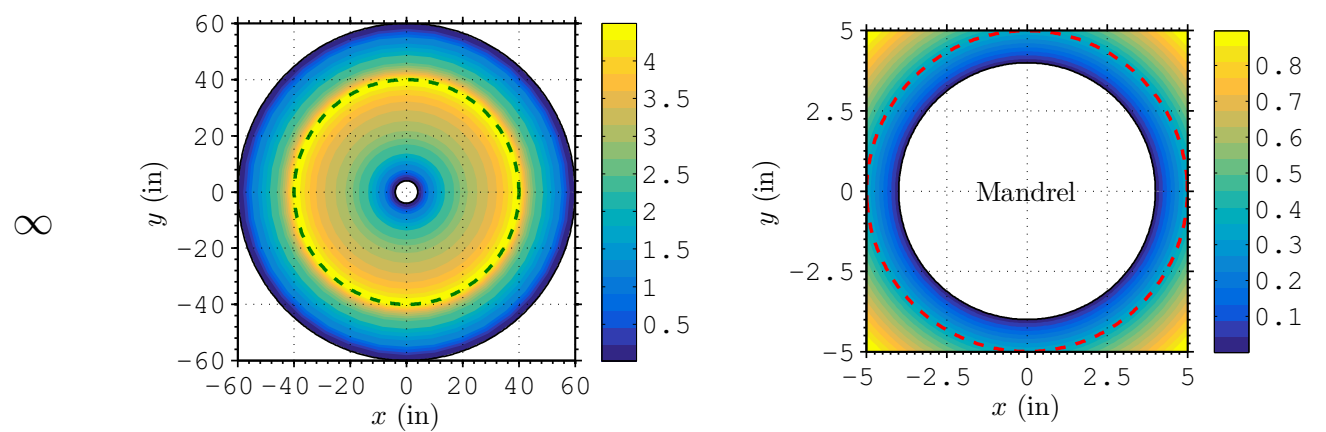

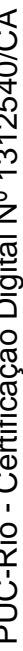
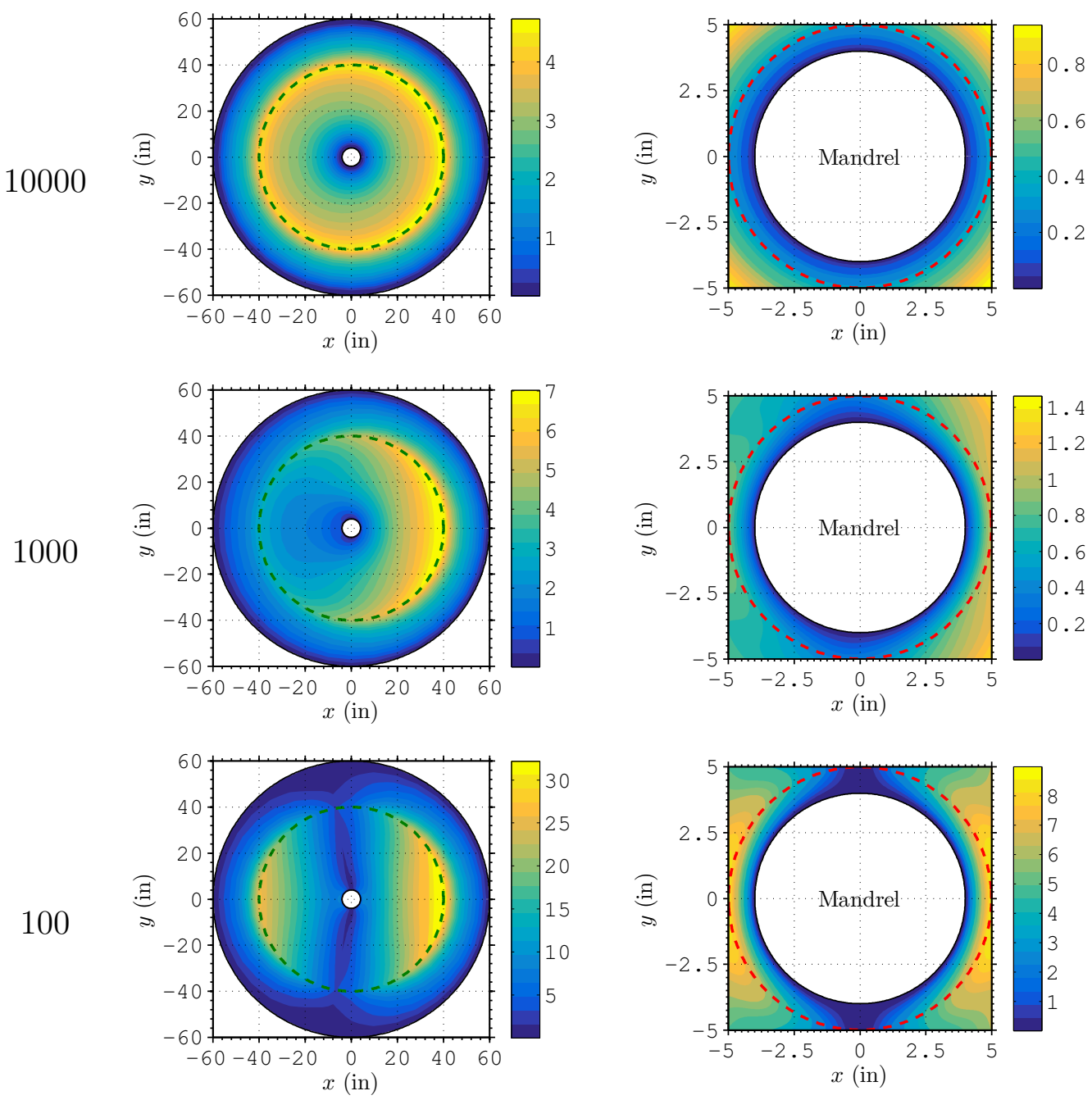
Table 4.17: Azimuthal electric field patterns for the mode $\# 6$ (for $n=+1$ ) in a toroidal waveguide with $\tilde{r}_{N}=60$-in $+i 20$-in as a function of the radius of curvature $R$. The borehole-to-formation and the PML inner boundaries are indicated by the dashed-circles. The center of curvature points to positive $x$ axis.

\begin{tabular}{lll}
\hline$R($ in $)$ & $\operatorname{abs}\left(E_{\phi}\right)$ & $\operatorname{abs}\left(E_{\phi}\right)$ near the mandrel \\
\hline
\end{tabular}
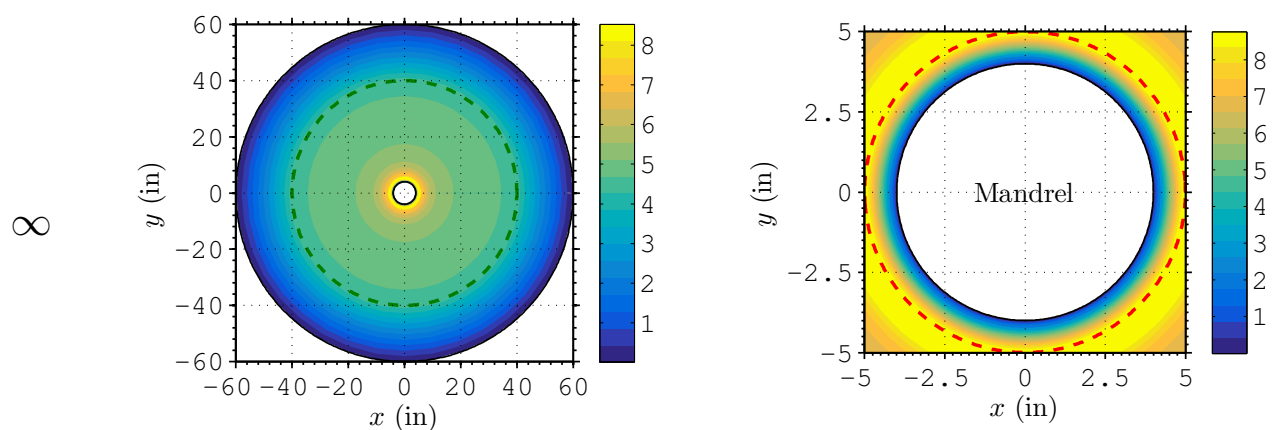

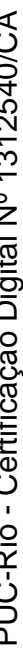
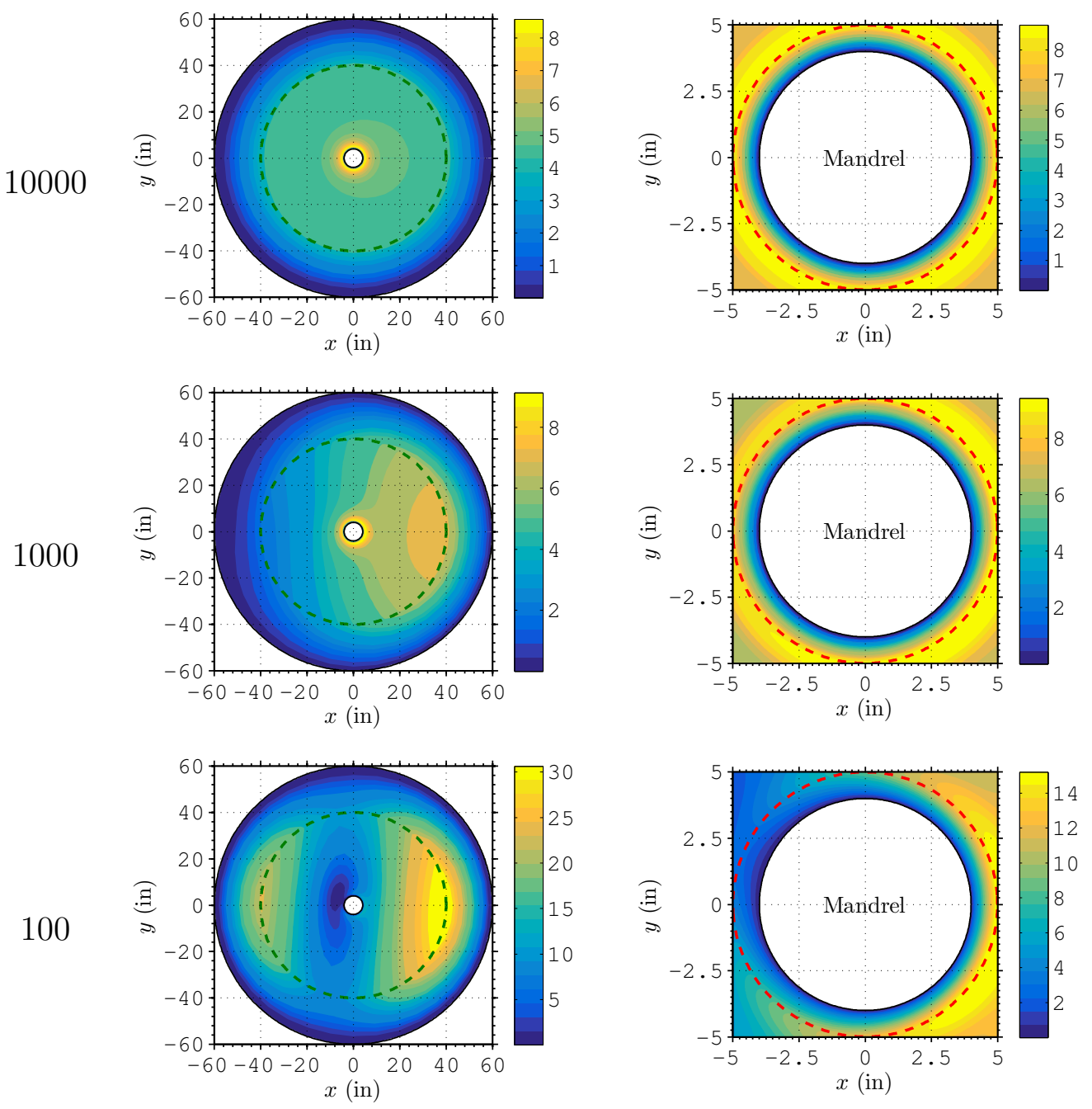
Table 4.18: Azimuthal electric field patterns for the mode $\# 7$ (for $n=+1$ ) in a toroidal waveguide with $\tilde{r}_{N}=60$-in $+i 20$-in as a function of the radius of curvature $R$. The borehole-to-formation and the PML inner boundaries are indicated by the dashed-circles. The center of curvature points to positive $x$ axis.

\begin{tabular}{cc}
\hline$R($ in $)$ & $\operatorname{abs}\left(E_{\phi}\right)$ \\
\hline
\end{tabular}
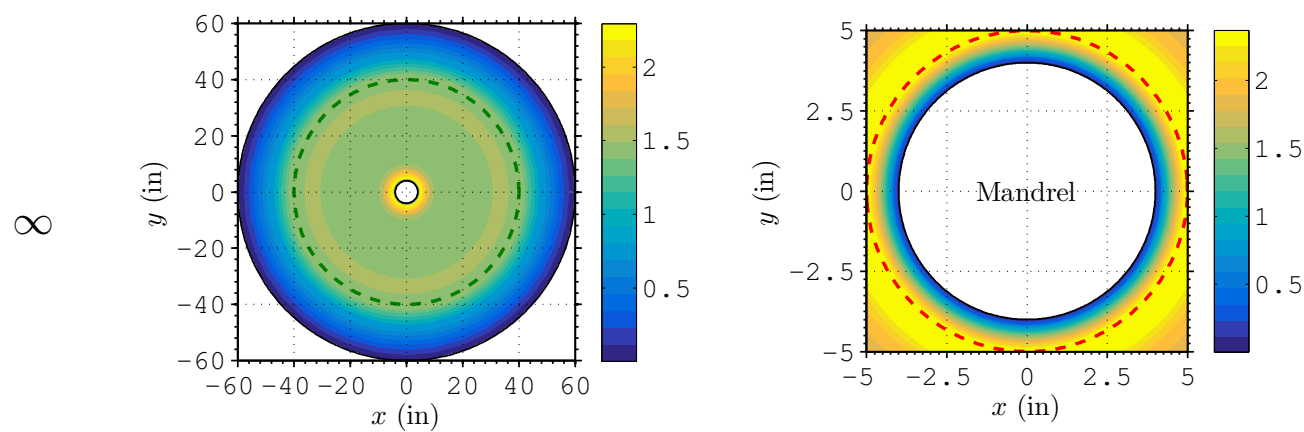

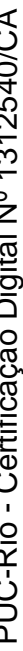
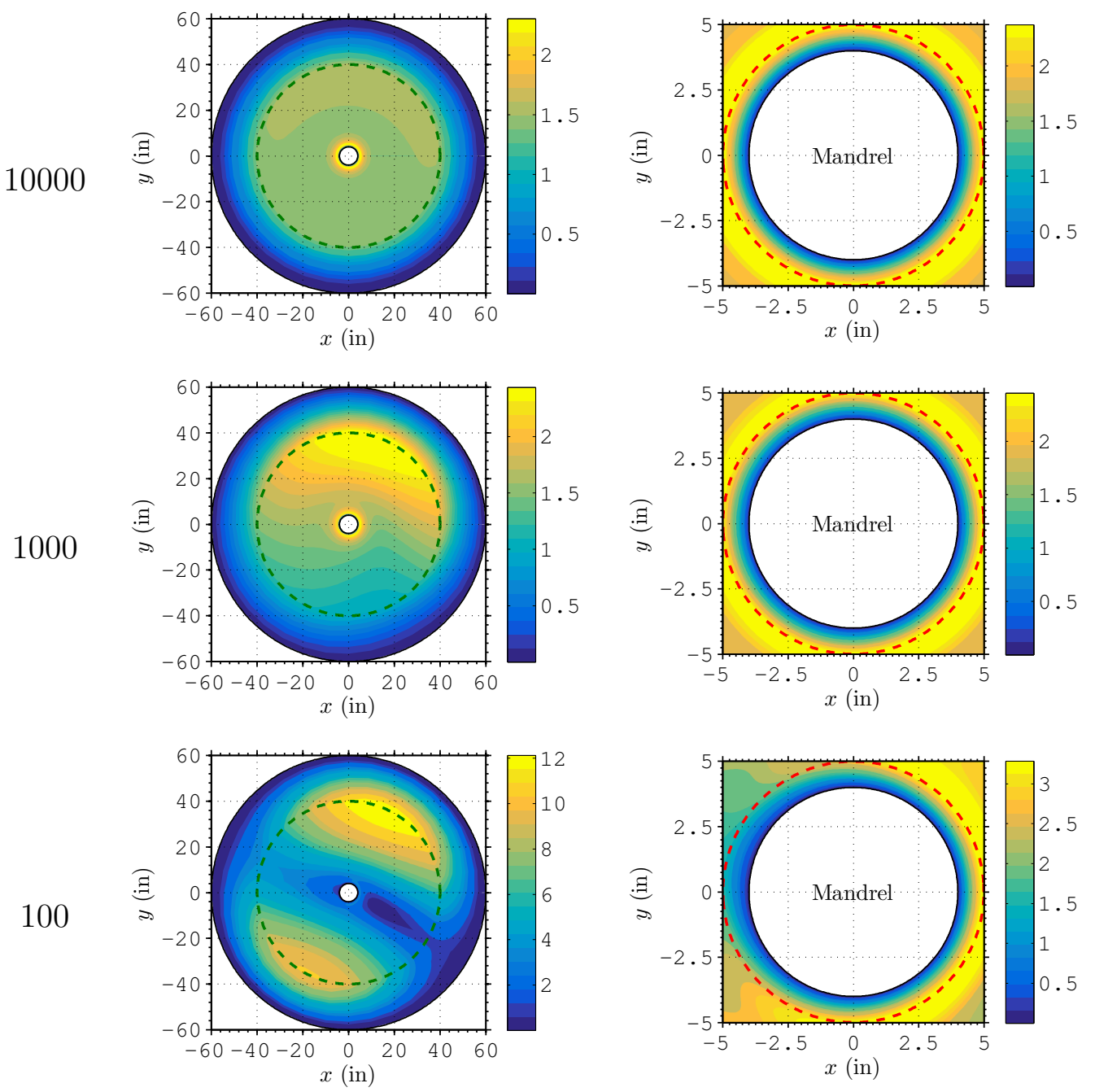
Table 4.19: Azimuthal electric field patterns for the mode $\# 8$ (for $n=+1$ ) in a toroidal waveguide with $\tilde{r}_{N}=60$-in $+i 20$-in as a function of the radius of curvature $R$. The borehole-to-formation and the PML inner boundaries are indicated by the dashed-circles. The center of curvature points to positive $x$ axis.

\begin{tabular}{cc}
\hline$R($ in $)$ & $\operatorname{abs}\left(E_{\phi}\right)$ \\
\hline
\end{tabular}
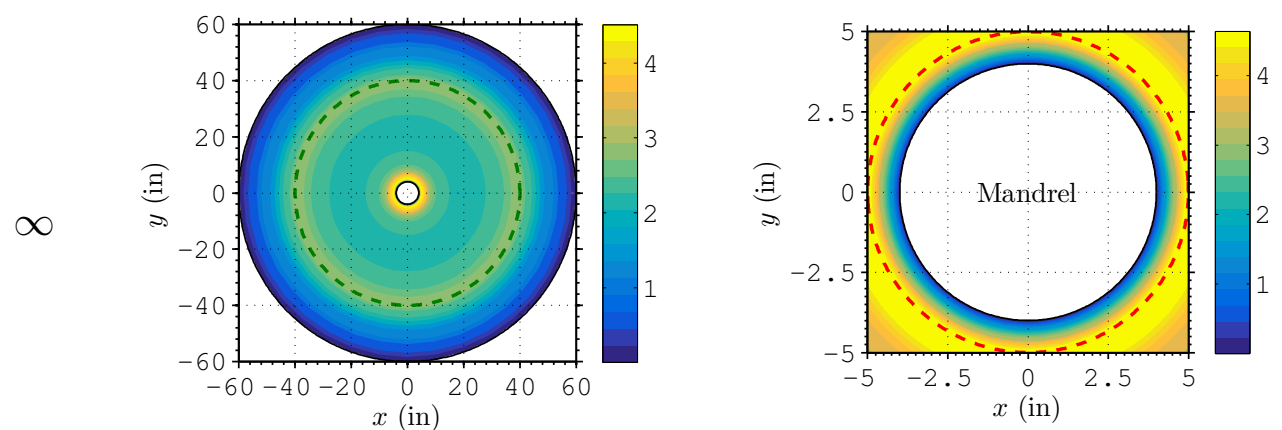

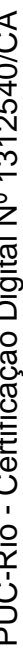
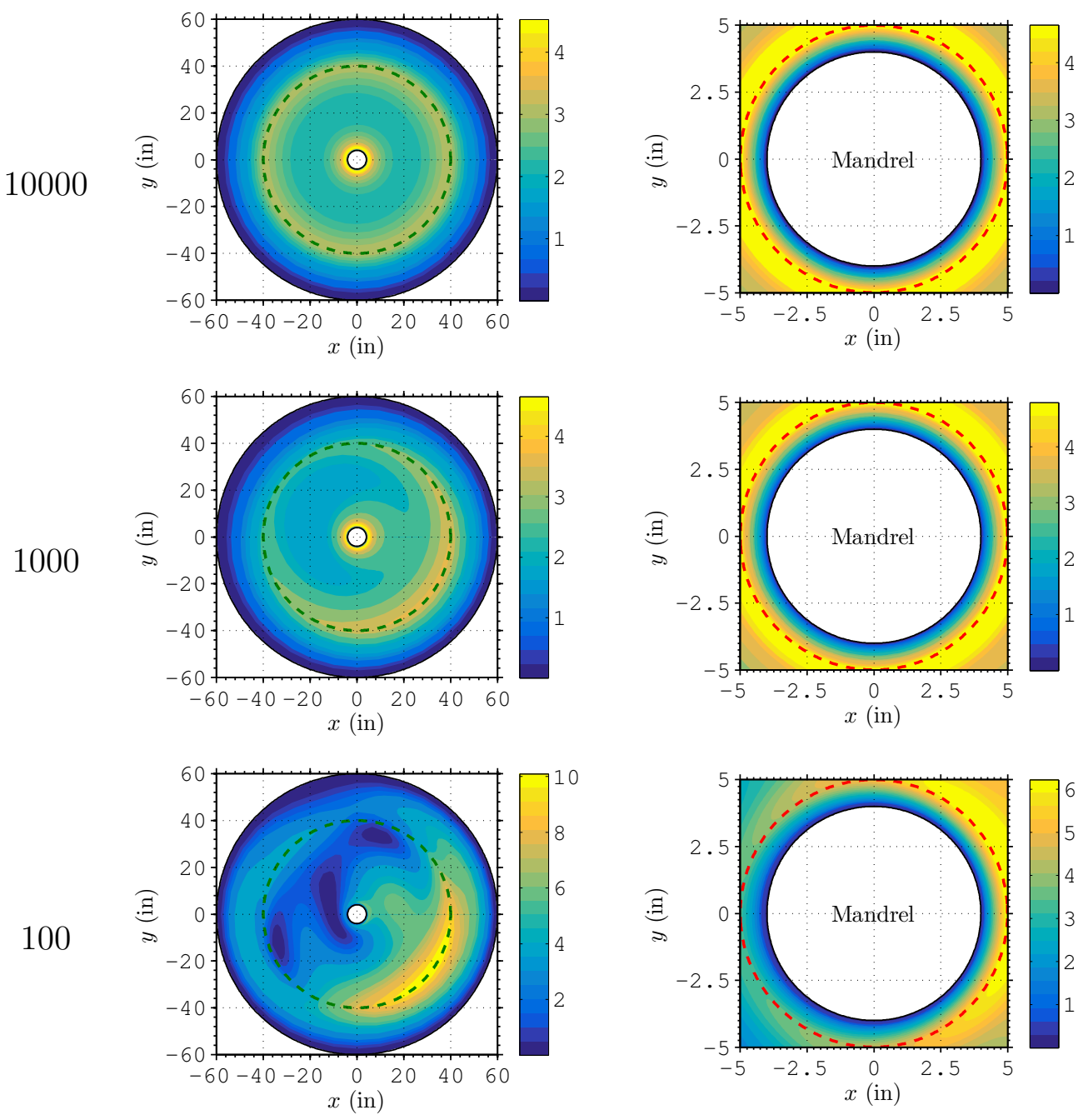
Table 4.20: Azimuthal electric field patterns for the mode $\# 9$ (for $n=+1$ ) in a toroidal waveguide with $\tilde{r}_{N}=60$-in $+i 20$-in as a function of the radius of curvature $R$. The borehole-to-formation and the PML inner boundaries are indicated by the dashed-circles. The center of curvature points to positive $x$ axis.

\begin{tabular}{lll}
\hline$R($ in $)$ & $\operatorname{abs}\left(E_{\phi}\right)$ & $\operatorname{abs}\left(E_{\phi}\right)$ near the mandrel \\
\hline
\end{tabular}
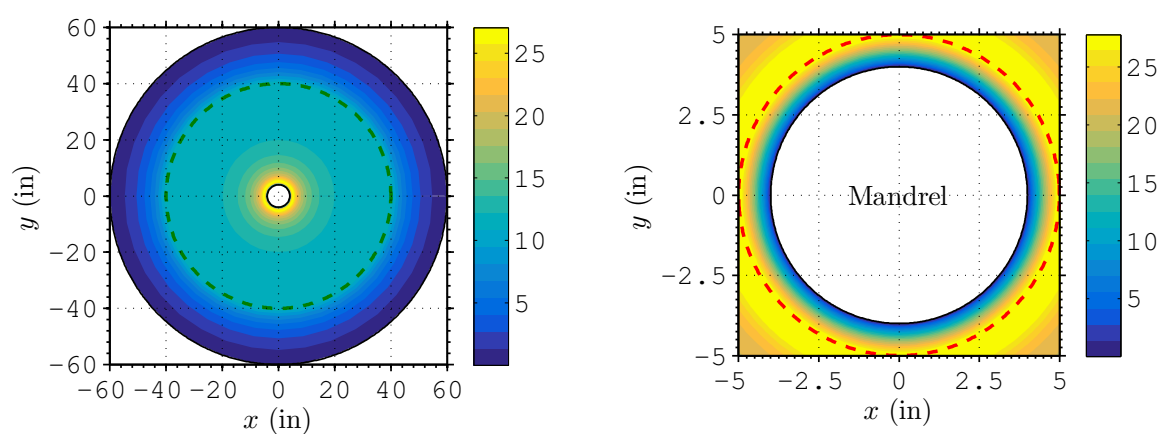

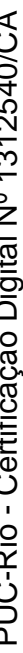
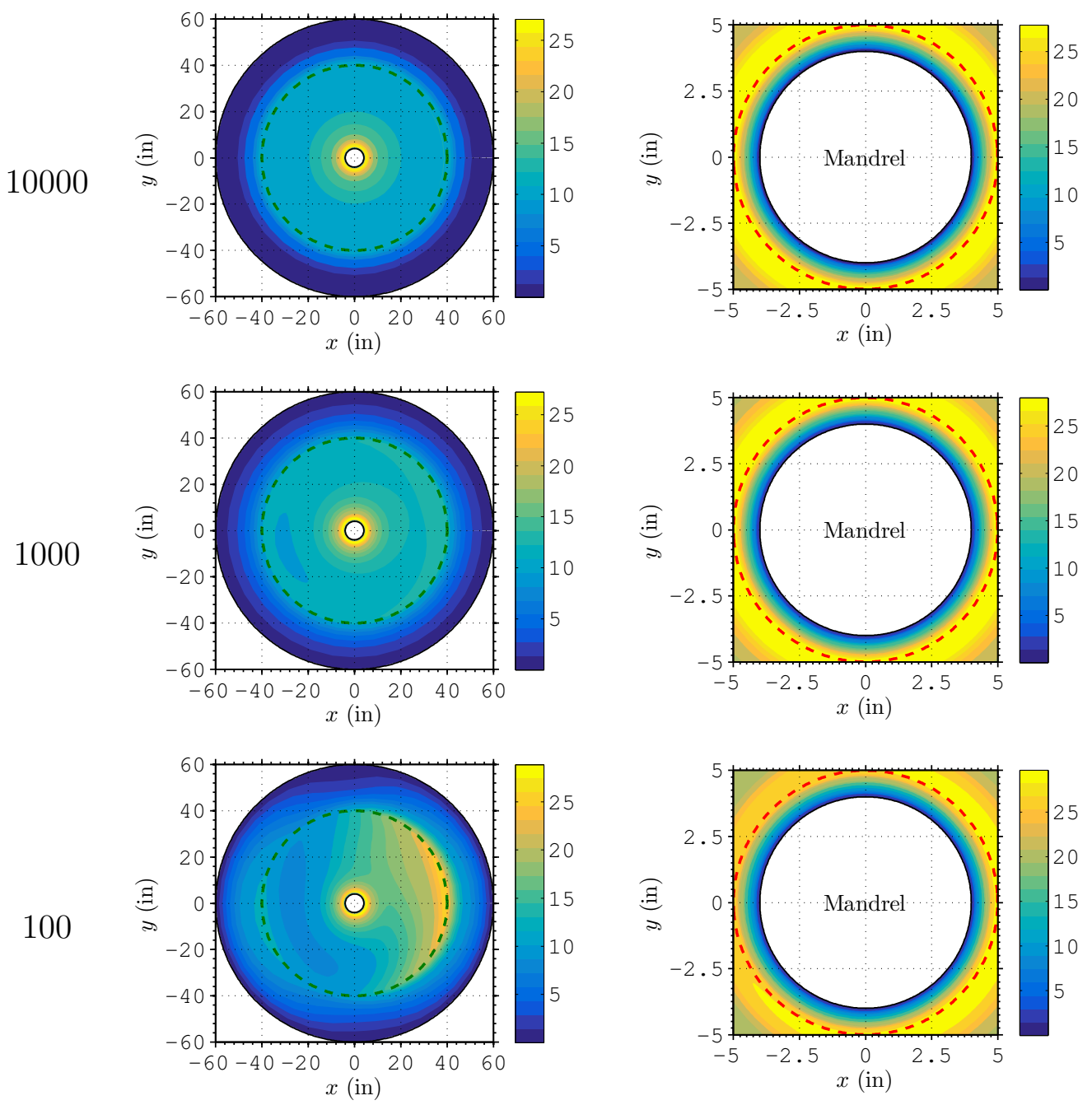
Table 4.21: Azimuthal electric field patterns for the mode \#10 (for $n=+1)$ in a toroidal waveguide with $\tilde{r}_{N}=60$-in $+i 20$-in as a function of the radius of curvature $R$. The borehole-to-formation and the PML inner boundaries are indicated by the dashed-circles. The center of curvature points to positive $x$ axis.

\begin{tabular}{ccc}
\hline$R($ in $)$ & $\operatorname{abs}\left(E_{\phi}\right)$ & $\operatorname{abs}\left(E_{\phi}\right)$ near the mandrel \\
\hline
\end{tabular}
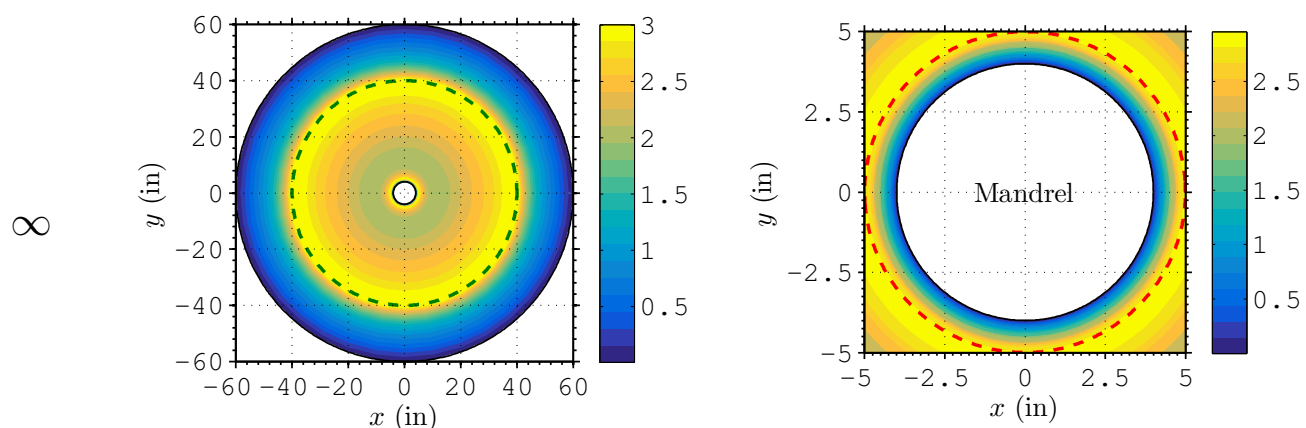

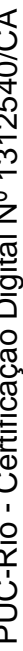
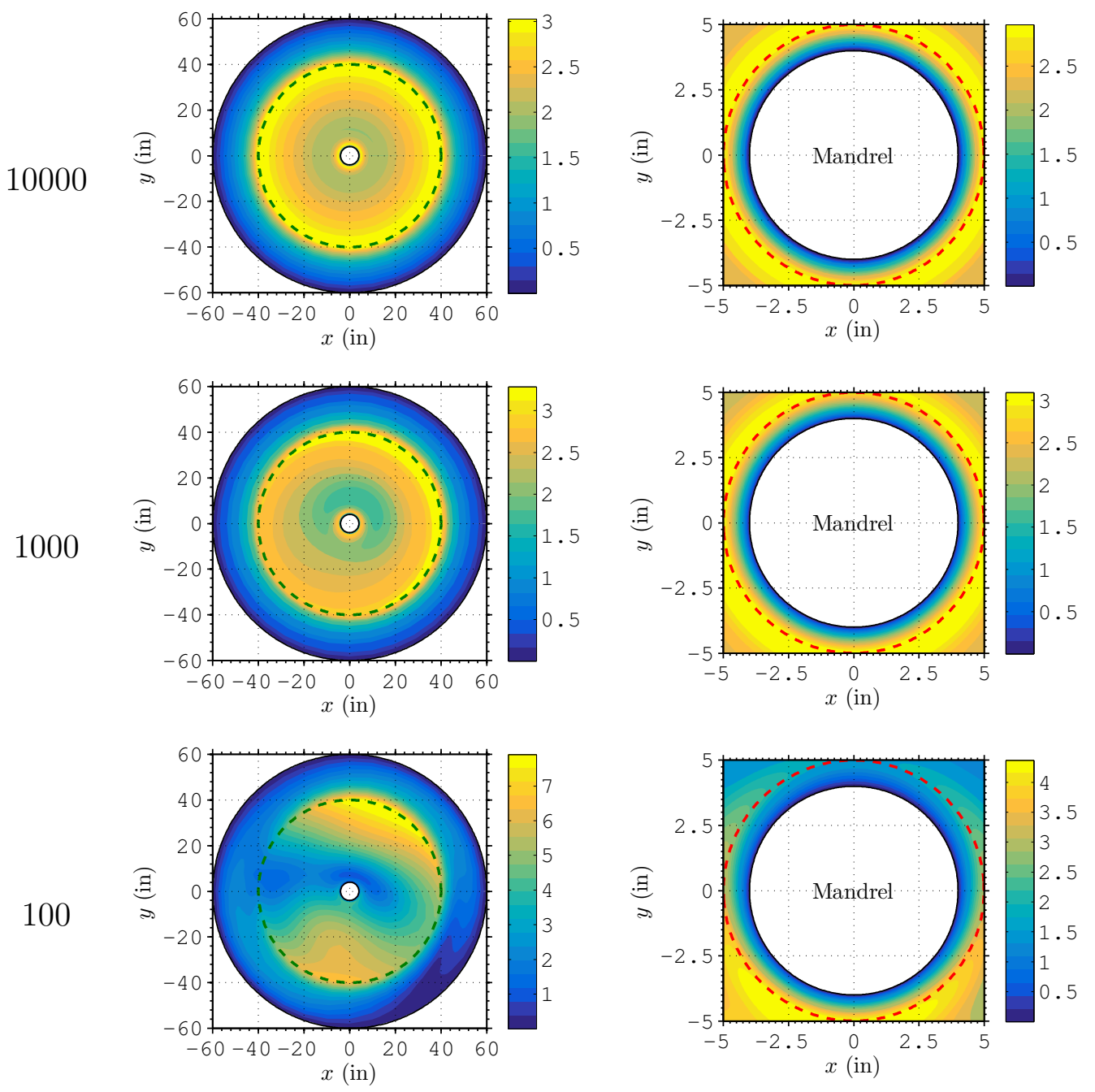
As an illustrative example, consider now a transmitting 4.5-in-radius coil antenna operating at $2 \mathrm{MHz}$ wrapped around the mandrel of the structure defined above. In Fig. 4.9 and Fig. 4.10 we show results for the induced voltage (amplitude and phase, respectively) due a unit current excitation $\left(I_{T}=1 \mathrm{~A}\right)$ at a 4.5-in-radius receiver antenna located 10-in to 100-in away from the source. We consider 40 modes with azimuthal index $n=0$ and others 40 for $n= \pm 1$; corresponding to an axial attenuation of about $A_{d B}=-50 \mathrm{~dB}$ at 5 in. Configurations with radius of curvature greater than 1000 in does not disturb significantly the solution if compared to response of a straight borehole. Solutions for $R=500$ in and $R=250$ in, however, change dramatically the

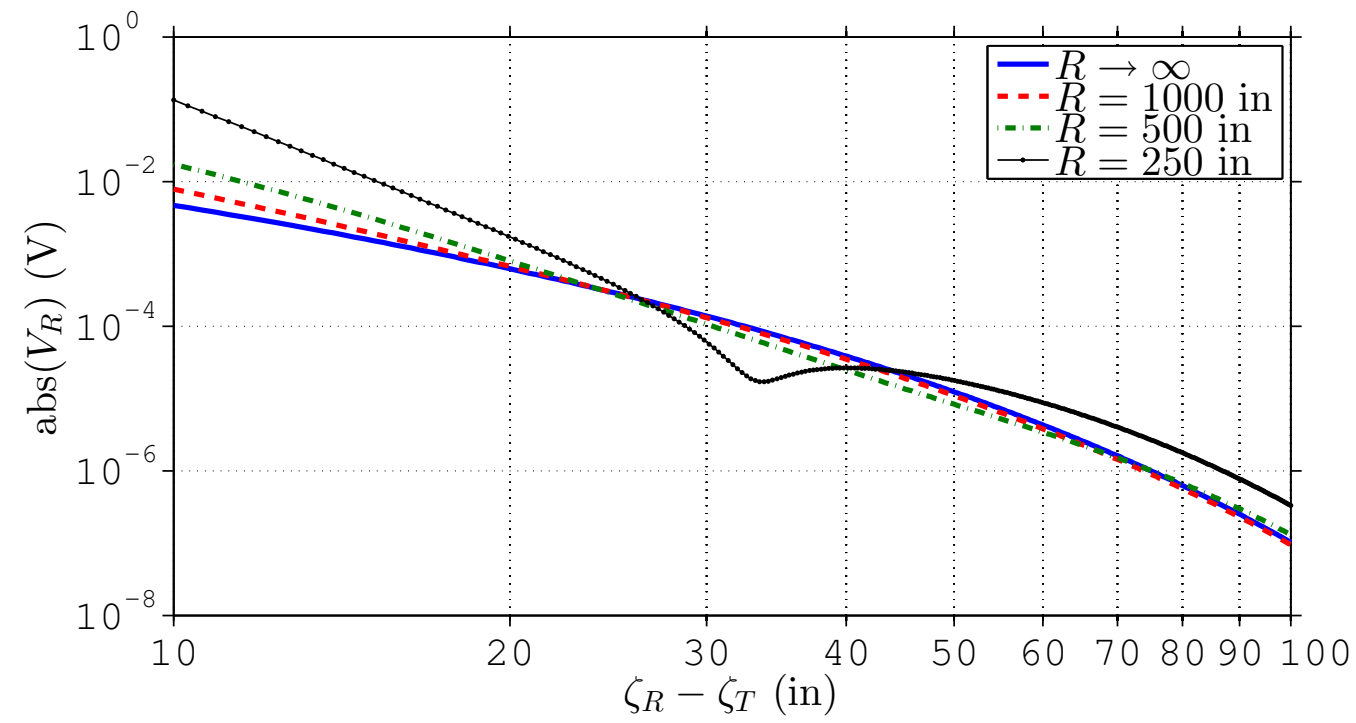

Figure 4.9: Voltage amplitude received by a coil antenna located $\zeta_{R}-\zeta_{T}$ away from the source and inside a bent borehole with curvature $R^{-1}$.

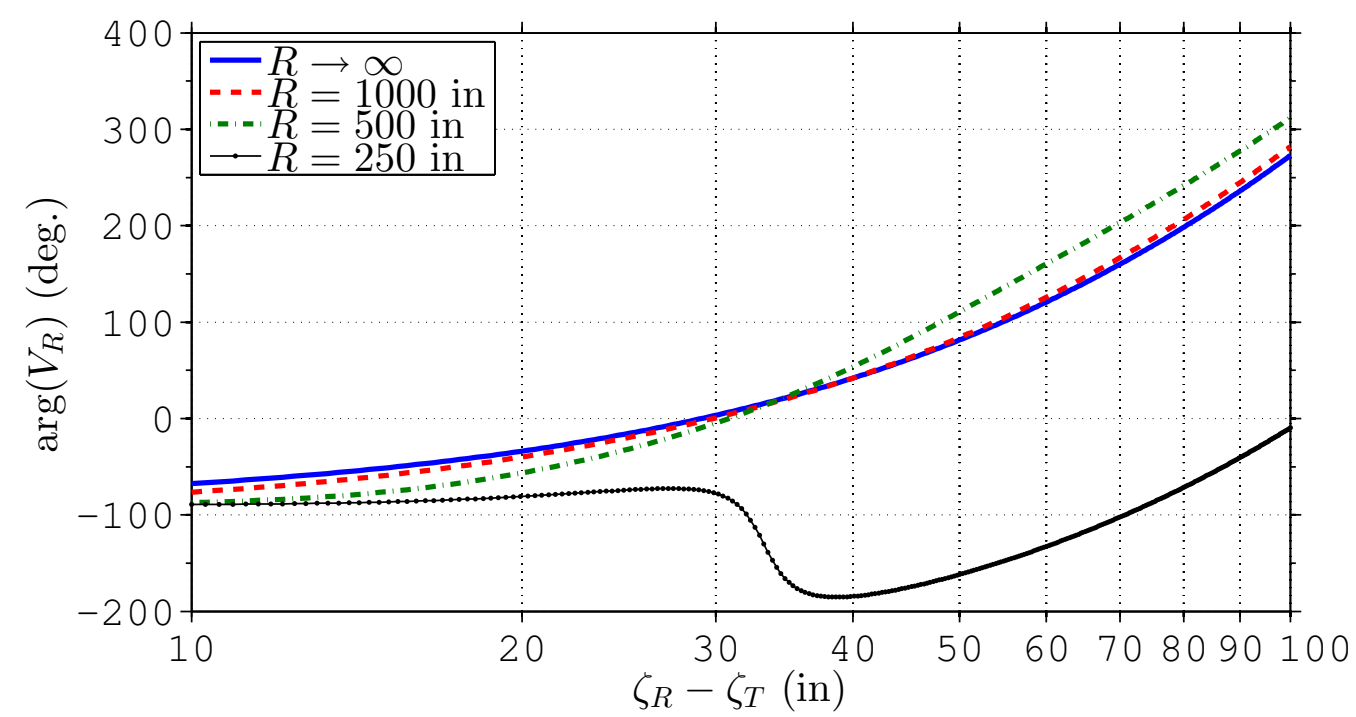

Figure 4.10: Voltage phase received by a coil antenna located $\zeta_{R}-\zeta_{T}$ away from the source and inside a bent borehole with curvature $R^{-1}$. 
LWD response when compared to that of an ordinary vertical well. This may be an indication that the formulation using only perturbation corrections up to $O\left(R^{-1}\right)$ are no longer sufficient to model directional wells with relatively small radius of curvature. In next section we will compare our solution with another method in order to verify the efficacy and the correctness of the formulation presented in this chapter.

\subsection{2}

\section{LWD Tools Inside a Directional-Well Borehole}

In this section we present simulation results of a triaxial logging tool consisting of one transmitter and two receivers inside a directional-well borehole of constant curvature. Each antenna consist of a 5.5-in-radius coil wrapped around a 4-in-radius metallic mandrel inside a 7-in-radius borehole, where 1 in $=2.54 \times 10^{-2} \mathrm{~m}$. The borehole is filled with oil-based mud having conductivity equal to $5 \times 10^{-4} \mathrm{~S} / \mathrm{m}$ and the soil formation has conductivity equal to $1 \mathrm{~S} / \mathrm{m}$. The receivers $\mathrm{RX}_{2}$ and $\mathrm{RX}_{1}$ are placed axially (along the $\zeta$-axis) at 24-in and 30-in away from the TX antenna, respectively. Fig. 4.11 and Fig. 4.12 show the voltages (e.m.f.) evaluated at $\mathrm{RX}_{1}$ and $\mathrm{RX}_{2}$ for a $\mathrm{LWD}$ tool operating in the frequency range of $500 \mathrm{kHz}$ to $2 \mathrm{MHz}$, for three curvature configurations: $R \rightarrow \infty, R=400$ in and $R=200 \mathrm{in}$. Good agreement is observed versus the finite-difference time-domain (FDTD) results from CST [146]. The radial domain was truncated at 10-in in order to reduce the mesh size required by the FDTD model. For the sources considered here, only azimuthally independent $\mathrm{TE}^{z}$ modes would be produced in vertical borehole. In contrast, the finitecurvature case couples the axial fields and excites azimuthally non-symmetric hybrid modal fields. We have employed perturbed corrections up to order $R^{-1}$, and 10 modes with azimuthal indices 0 and \pm 1 .

In our simulations, we first find the curvature-vanishing solutions, and its fields are then used to determine the corresponding correction terms as a function of $R$. In this way, in one simulation we can simultaneously find the voltages for $R \rightarrow \infty, 400$ in and 200 in. Our numerical results were obtained using a double-precision Fortran code running on a PC with a 2.93-GHz Intel Xeon W3540 processor. The CPU time required by our algorithm to simulate the LWD tool response for one frequency was no longer than 52.81 seconds. The total CPU time required in the simulation of the 11 frequencies shown in Fig. 4.11 and Fig. 4.12 was 8.94 minutes, requiring 9.10 MB of memory.

The CST's FDTD results were obtained using a dedicated Workstation with a 2.10-GHz Intel Xeon E5-2620 v2 twelve-core processor. The CPU time required by the FDTD solution to simulate the LWD tool response in a bent 


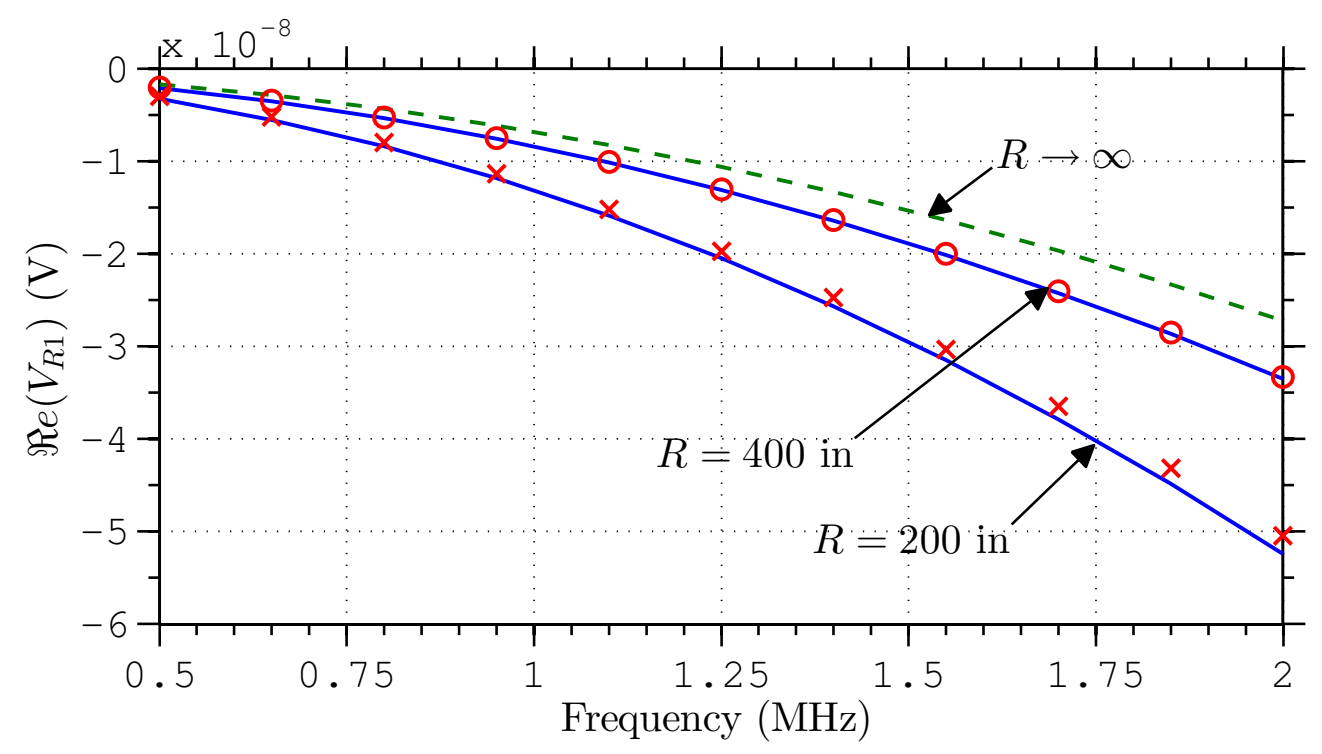

Figure 4.11: Voltages at $\mathrm{RX}_{1}$. Zeroth-order results are shown in dashed-line. The combination of the zeroth- and first-order perturbation corrections are indicated by solid lines. The small circles (for $R=400$ in) and crosses (for $R=200$ in) are FDTD results.

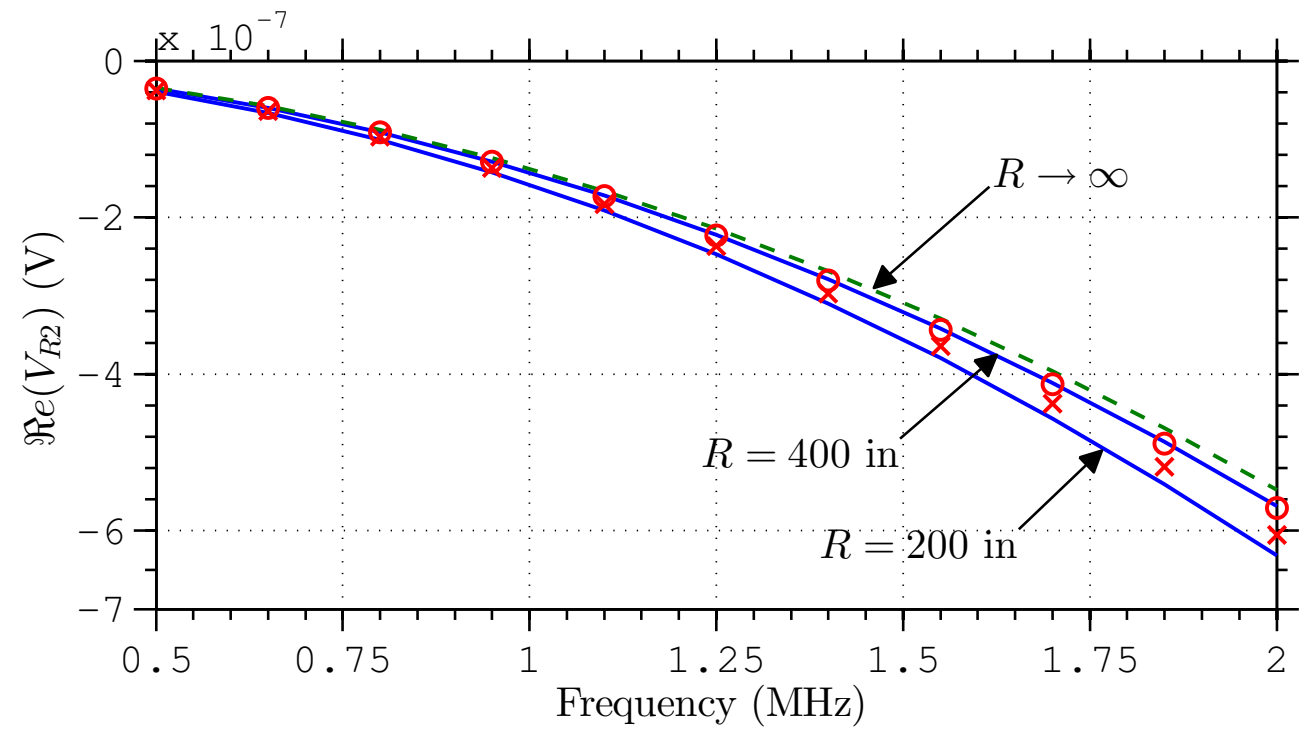

Figure 4.12: Voltages at $\mathrm{RX}_{2}$. Zeroth-order results are shown in dashed-line. The combination of the zeroth- and first-order perturbation corrections are indicated by solid lines. The small circles (for $R=400$ in) and crosses (for $R=200 \mathrm{in}$ ) are FDTD results.

borehole with $R=400$ in was 5.18 hours, requiring 2.96 GB of memory in a simulation with 5,675,184 mesh cells. In the FDTD solution, at each new bent configuration we need to re-simulate the entire structure, such for the case in which $R=200$ in, the CPU time, memory and number of mesh cells required by the FDTD were 16.40 hours, 6.04 GB and 10,772,190 cells, respectively.

It is important to mentioned some limitations on the FDTD model 
we have employed. Among them, we can highlight the finite size of space simulation. Fig. 4.13 depicts the schematic used in the CST modeling in the case $R=200$ in, where we have used a domain with $\Delta X=30$ in, $\Delta Y=120$ in and $\Delta Z=20 \mathrm{in}$. The same model was employed in the case $R=400 \mathrm{in}$, but with $\Delta X=25$ in.

To prevent very long simulations time, the FDTD model do not employed PMLs (in any direction) due the large number of mesh cells required to simulate the absorbing layers. This motivate us to simulate a radially-bounded structure, using a radial domain truncated by a PEC layer at $\rho=10$ in (in our local toroidal coordinate system). The axial space also have employed an ordinary PEC boundary condition to truncate its domain, but at a distance $\Delta Y / 2$ away from the transmitting antennas, as shown in Fig. 4.13. This model looks more like a cavity, and some undesirable reflections can arise from the axial domain

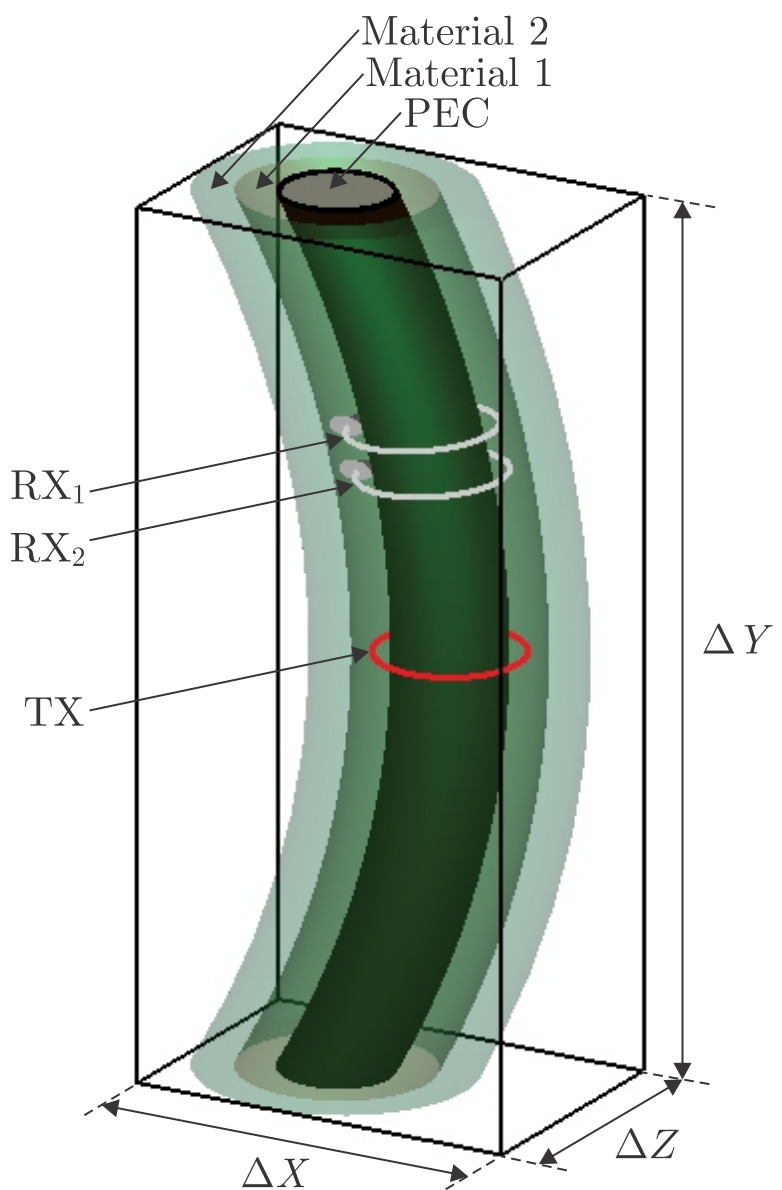

Global Cartesian coordinate system

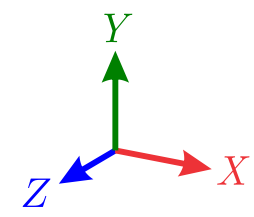

Figure 4.13: Schematic used in the CST modeling in the case $R=200 \mathrm{in}$. The spacial domain is limited by a parallelepiped with dimension $\Delta X \times \Delta Y \times \Delta Z$ on the global Cartesian coordinate system $(X, Y, Z)$ using a PEC boundary condition. To improve the visualization of small details, the aspect ratio of coordinates $X / Y$ and $Z / Y$ is 2 by 1 . The center of curvature points to the negative $X$-axis. 
truncation used in CST (in the $Y$-direction).

Another limitation in the FDTD model is the discretization grid: CST provide only Cartesian grid, which are not conformal with the radiallystratified toroidal structures at hand. As a consequence, severe staircase errors can occur if a large number of mesh cells was not employed to correctly capture the curvature effects. In order to try to minimize these errors, the transmitting antenna is a thin $1 / 20$-in-diameter wire placed aligned with the plane $Z-X$. The receiving antennas, on the other hand, will not be conformal like the source but sometimes we can accurately compute the induced voltages.

We performed several simulations using FDTD until we found a $\Delta Y$ that is large enough to not disturb the solution at the receiving antennas region. In addition, we have performed simulations varying the grid configuration until convergence be observed.

The CST's results showed in Fig. 4.11 for the case $R=200$ in will be identified herein as CST sim. 13, and as noted in the said figure, some differences can be observed between the FDTD solution versus the proposed technique. One possible justification to that deviations is that the first-order correction we have presented are not enough to precisely capture the field variations in toroidal coordinates, such high order corrections could be needed. But, we have explored discretization more finer than that in CST sim. 13. In Fig. 4.14 we shown CST's results for the voltage received in antenna $\mathrm{RX}_{2}$ for a progressive mesh refinement in simulations 12, 13, and 14. The details of those simulations are summarized in Table 4.22. We can clear see that the FDTD results in CST sim. 14 appear a little bit closer to our solution. Most important: when we look for the imaginary part of the phasor voltage in Fig. 4.14(b), we note relative good agreement with our results. Notice that the imaginary part of the voltage is about 10 times greater than the real part depicted in Fig. 4.14(a).

In Fig. 4.15(a) and Fig. 4.15(b) we show the schematics we have used in the CST modeling. As said before, in order to avoid very long simulations, the axial domain was truncated using homogeneous Dirichlet boundary condition for the electric field. In other words we have used a PEC at the top and at

Table 4.22: Computational cost in FDTD simulations.

\begin{tabular}{cccc}
\hline CST sim. \# & Mesh cells & CPU time & Memory (GB) \\
\hline 12 & 7082235 & $9 \mathrm{~h}, 37 \mathrm{~min}, 35 \mathrm{~s}$ & 4.038932 \\
13 & 10772190 & $16 \mathrm{~h}, 24 \mathrm{~min}, 13 \mathrm{~s}$ & 6.035560 \\
14 & 22796246 & $59 \mathrm{~h}, 33 \mathrm{~min}, 2 \mathrm{~s}$ & 12.500736 \\
18 & 56135750 & $138 \mathrm{~h}, 43 \mathrm{~min}, 40 \mathrm{~s}$ & 19.228004 \\
\hline
\end{tabular}




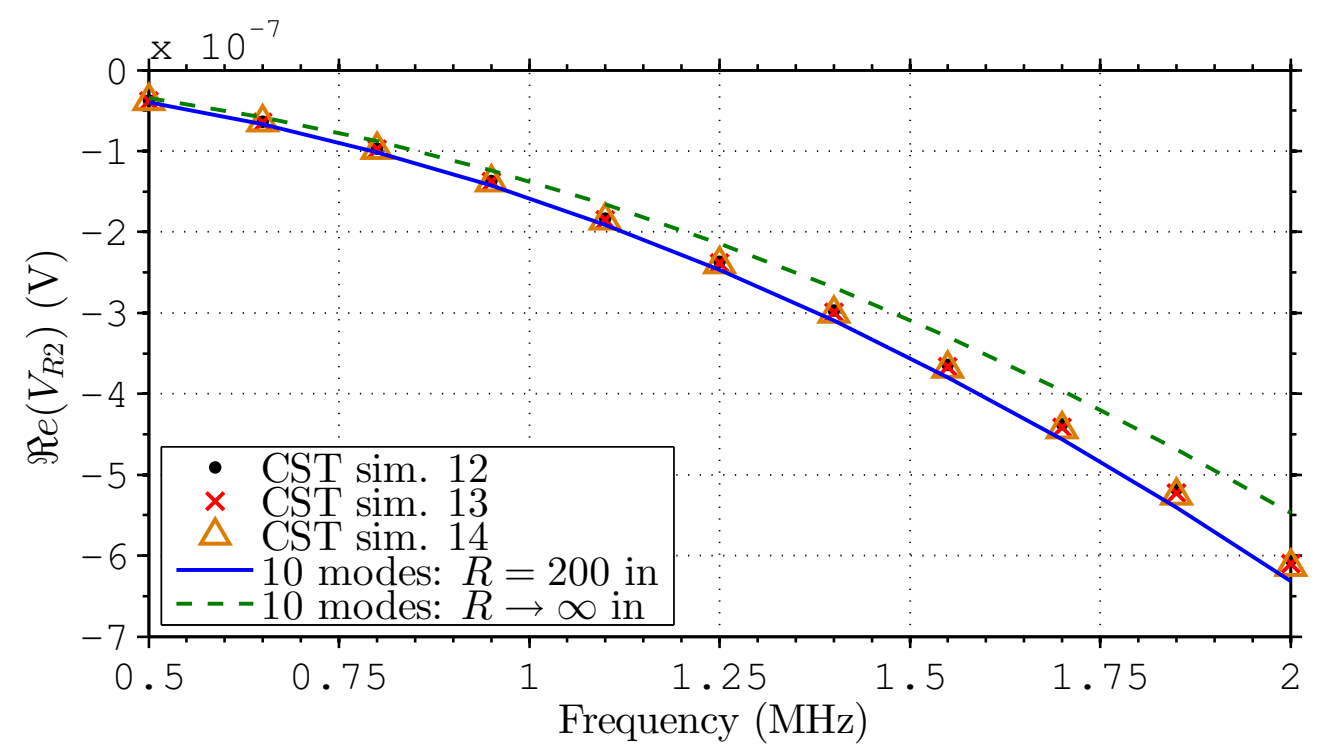

(a) Real part

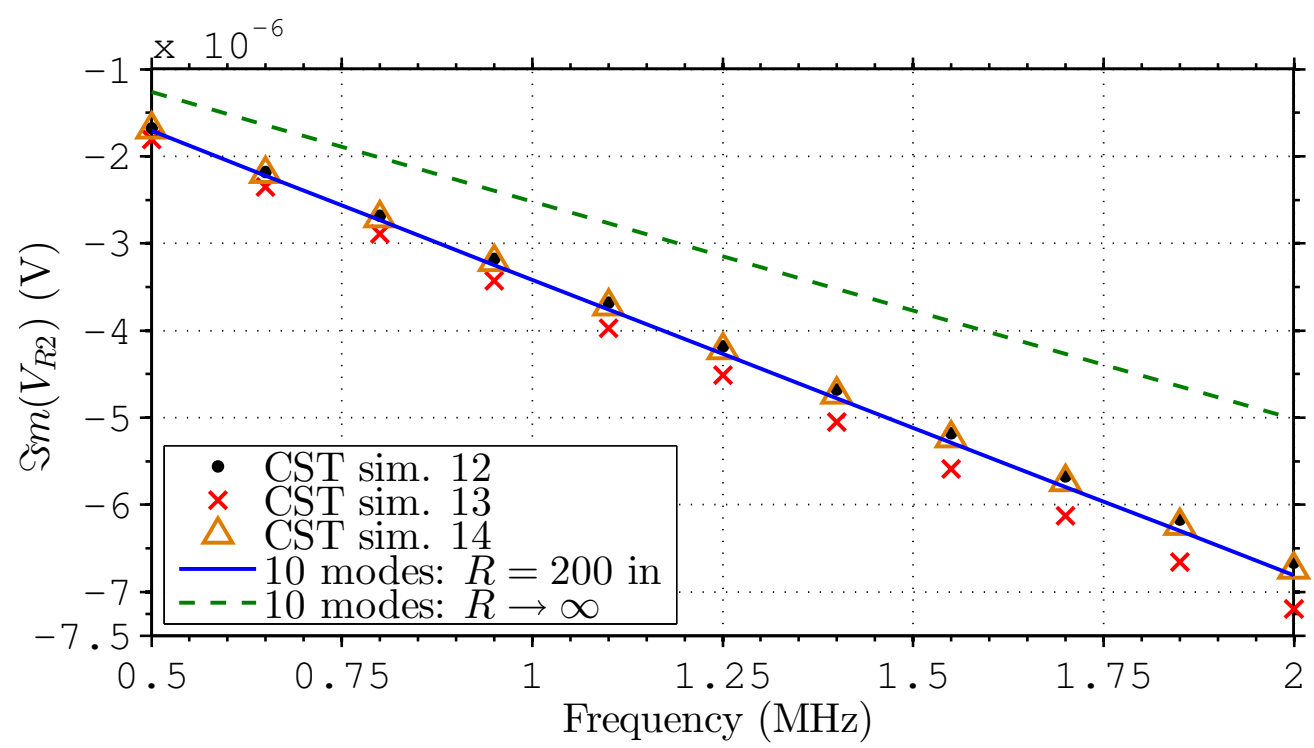

(b) Imaginary part

Figure 4.14: Voltage at $\mathrm{RX}_{2}$ for $R=200$ in. Zeroth-order results are shown in dashed-lines. The combination of the zeroth- and 1st-order perturbation corrections are indicated by solid lines. The small dots, crosses, and triangles are FDTD results for simulations 12, 13, and 14, respectively.

the bottom of the domain, such the $\zeta$-space has about 121 in for the case $R=400 \mathrm{in}$. For the case in which the radius of curvature is $200 \mathrm{in}$, the increment in the axial domain was only one-inch compared to the case in which $R=400 \mathrm{in}$. Such we believed that an undesirable reflection from the upper boundary can disturb the received voltages. In order to investigate this effect, we consider a longer axial domain, as showed in Fig. 4.15(c), where now the $\zeta$-space extends over $200 \mathrm{in.}$

The FDTD solutions we have obtained for the structure depicted in 


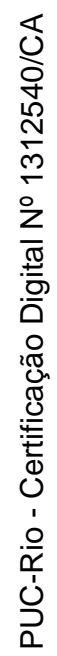
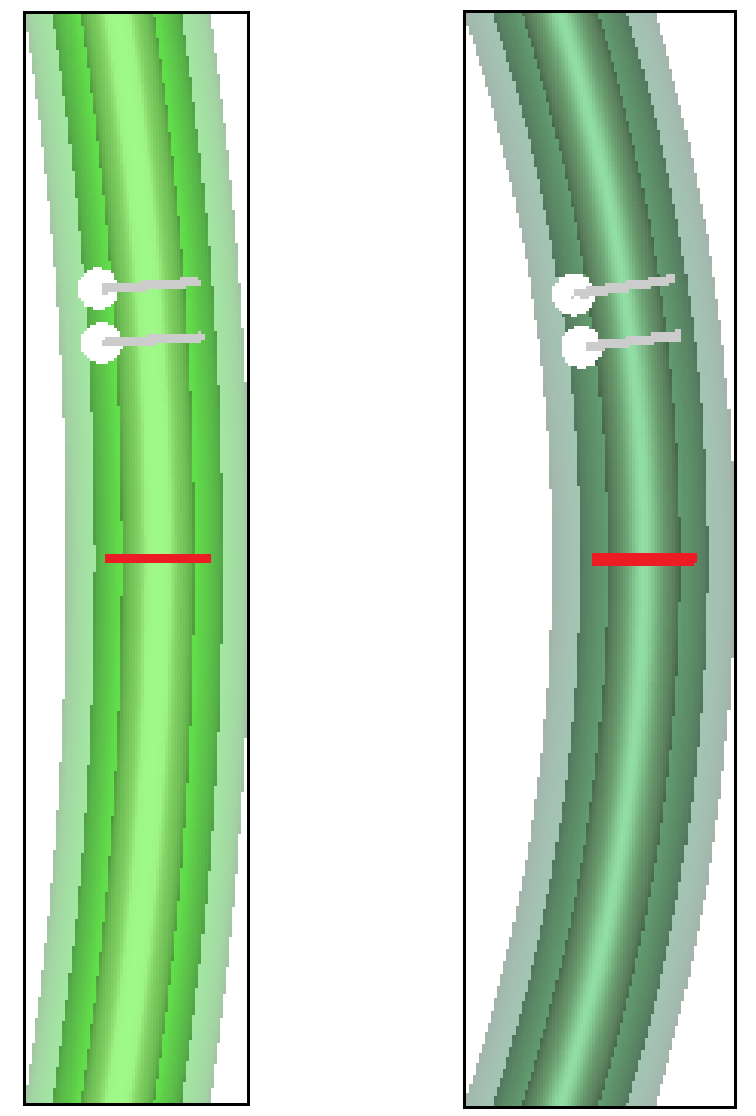

(a) $R=400 \mathrm{in}$, $\Delta \zeta \approx 121 \mathrm{in}$
(b) $R=200$ in,
$\Delta \zeta \approx 122$ in

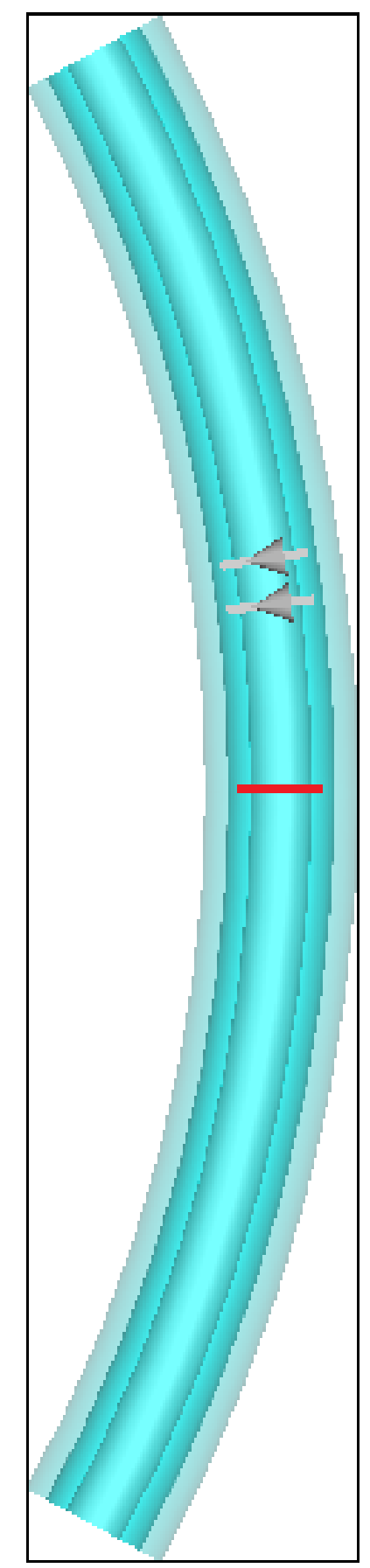
(c) $R=200 \mathrm{in}$, $\Delta \zeta=R$

Figure 4.15: Schematics used in the CST modeling. The aspect ratio of horizontal $(X)$ and vertical $(Y)$ coordinates is 1 by 1 . In all cases, the center of curvature points to the left side of the page.

Fig. 4.15(c) will be identified herein by the label CST sim. 18, and are presented in Fig. 4.16. In this figure, we can clear see that the CST's results for the voltage at $\mathrm{RX}_{2}$ appear more close to those we have derived with the 


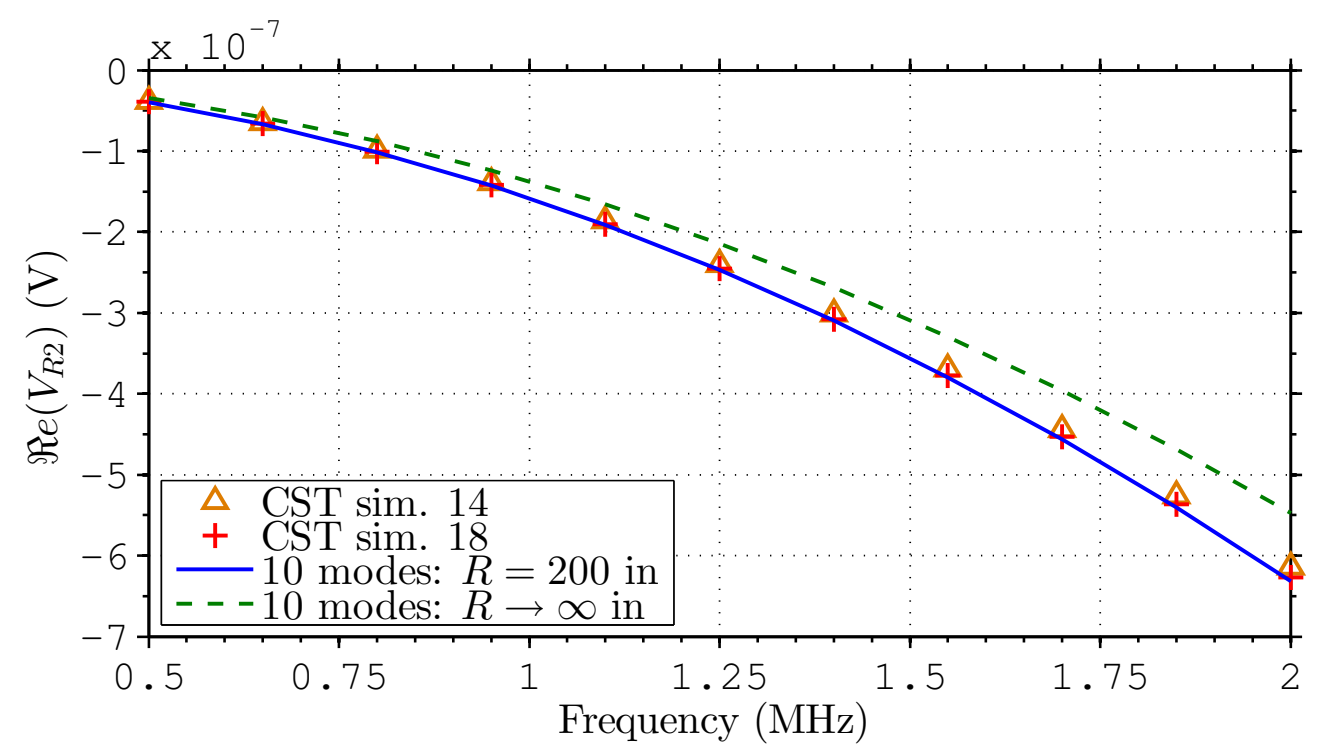

Figure 4.16: Voltages at $\mathrm{RX}_{2}$ for $R=200$ in.

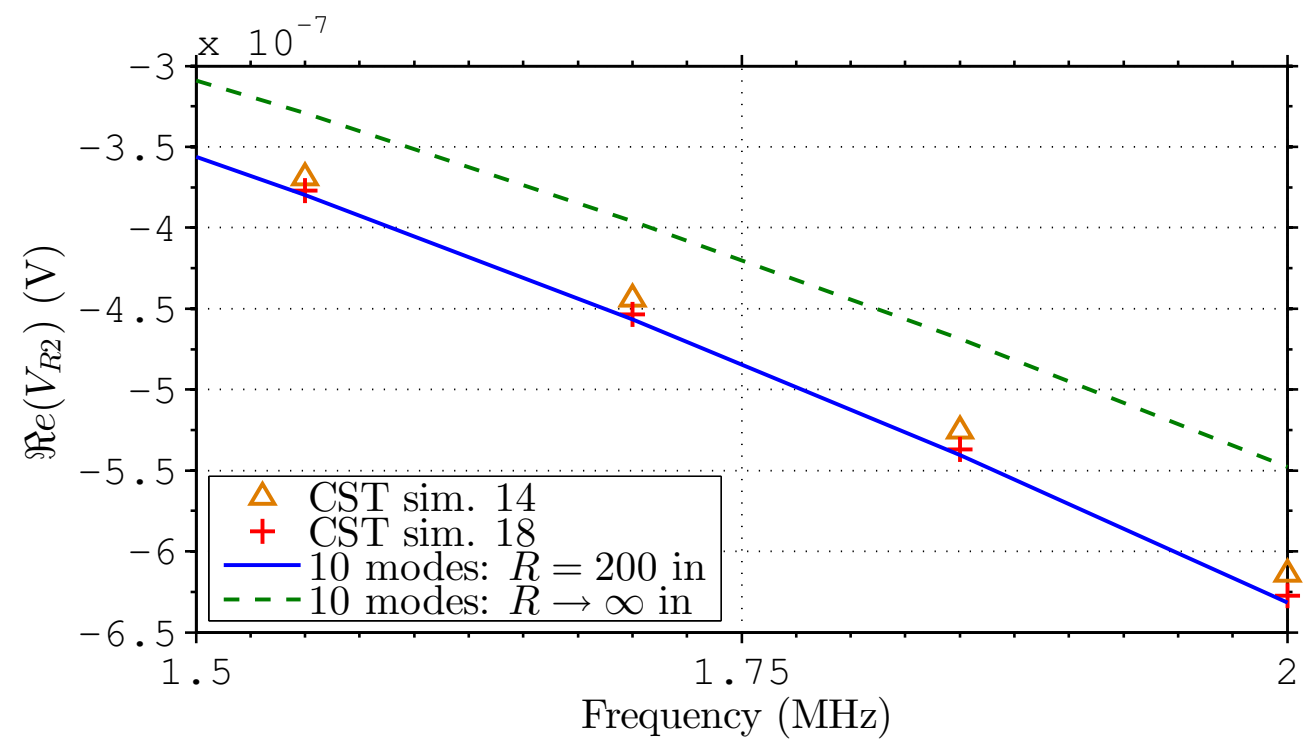

Figure 4.17: Voltages at $\mathrm{RX}_{2}$ for $R=200 \mathrm{in}$; Zoom near $1.75 \mathrm{MHz}$.

proposed algorithm. Now, looking at the high frequency range of operation, we have verified that the proposed solution has a relative error of about $1 \%$ as depicted in Fig. 4.17. To achieve that precision in the FDTD, however, we have required a relatively high numerical cost. In Table 4.22 we show the computational effort required for several simulations we have mentioned above. The simulation 18, for example, that have used an enlarged axial domain, required more than 56 million of meshcells and almost 6 days of simulation in a dedicated workstation. In contrast, as said before, the algorithm we have proposed take about 9 minutes into an ordinary laptop, using about $9 \mathrm{MB}$ of memory. 
In typical LWD applications, the curvature of the well is very small compared to the borehole radius: according to Table 2.1 in Chapter 2, drilling technologies available today allow deviated wells with radius of curvature no less than about $20 \mathrm{ft}$ (or $240 \mathrm{in}$ ). We have verified that corrections solutions up to the first-order are enough to correctly capture the effect of the finitecurvature in such conditions.

\section{7}

\section{Preliminary Conclusion}

We have introduced a new formulation to model the response of welllogging tools in directional wells. The combination of closed-form solutions of Maxwell's equations in cylindrical coordinates with a perturbation technique allow us to express the fields inside an axially-toroidal and radially-layered Earth formation. Our field solutions are presented as a series in terms of the radius of curvature of the bend structure. The zeroth-order perturbation correction is exactly equal to the solution in cylindrical coordinates. The firstorder perturbation solution shows that an important effect of the curvature is the excitation of new azimuthal-dependent fields other than the usual in cylindrical coordinates. As a consequence, the modal fields are no longer orthogonal. Numerical results showed that the presented method can accurately model the electromagnetic propagation inside curved boreholes. The computational cost of the novel technique we have introduced here is very low: compared to FDTD, the perturbation approach is several orders of magnitude more efficient in both terms of CPU time and memory requirements. 


\section{5}

\section{Analysis of Well-Logging Tools in Dipping-Bed Boundaries: A Mathematical Description}

\section{1 \\ Introduction}

Logging-while-drilling (LWD) resistivity tools often acquired logging data along deviated or horizontal holes. In this case, the drilling environment is no longer conformal with the cylindrical coordinate system. An illustration of a LWD tool in a three-layer formation with an dipping bed is shown in Fig. 5.1(a). This three-dimensional (3D) geometry can be handled by means of numerical techniques as 3D finite-difference time-domain (FDTD) $[17,19,68]$ or 3D finite-volumes (FV) [45].

As far as we know, pseudo-analytical formulations are not applicable to simulate 3D Earth formations similar to those of the dipping bed problem. However, a simplified version of the original problem was solved in $[6,8,16]$ by means of the Fourier-Bessel transform. This pseudo-analytical solution do not incorporates the borehole and neither the mandrel, so that the geophysical formations model becomes an ordinary axially-layered media, as depicted in Fig. 5.1(b). Additionally, the coil sensors were approximated by magnetic dipoles moving along an axis deviated from the axial one by the angle $\theta_{d}$.

To explore the mode-matching technique, we propose a novel approach to analyze dipping-bed boundaries including the mandrel, the borehole and additional invasion zones. Our idea is to take advantage of the analytical solution derived in Chapter 3 and Chapter 4 to solve the radial stratifications in combination with a new mode-matching along oblique surfaces.

\section{2}

\section{Mode-Matching along Oblique Surfaces}

Consider the junctions between the waveguides of regions 1 and 2 along a planar surface as shown in Fig. 5.2. Note that this surface is oblique in relation to the longitudinal axis at each considered region. The waveguide in region 1 is tilted by an angle $\theta_{1}$ and azimuthally rotated by an angle of $\phi_{1}$ in relation to the coordinates of the oblique junction surface. Similarly, regions 2 is axially 


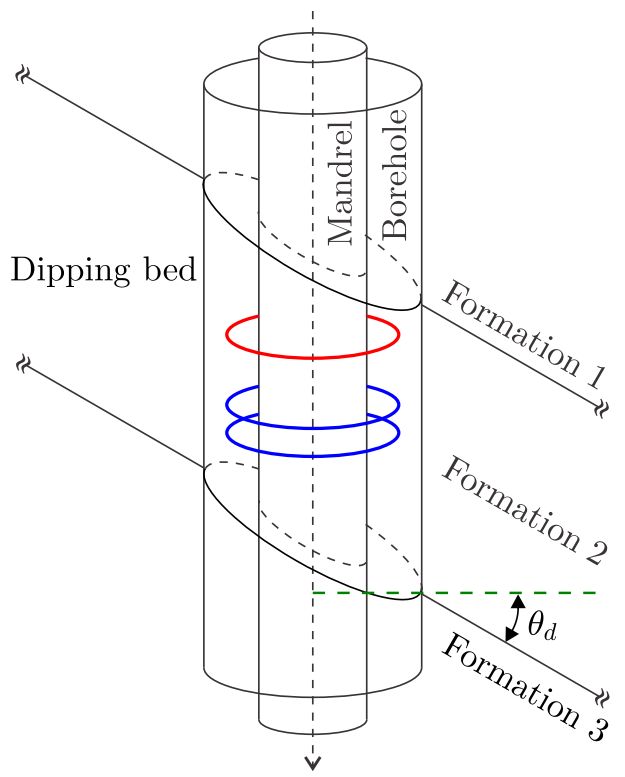

Drill direction

(a) Dipping bed formation

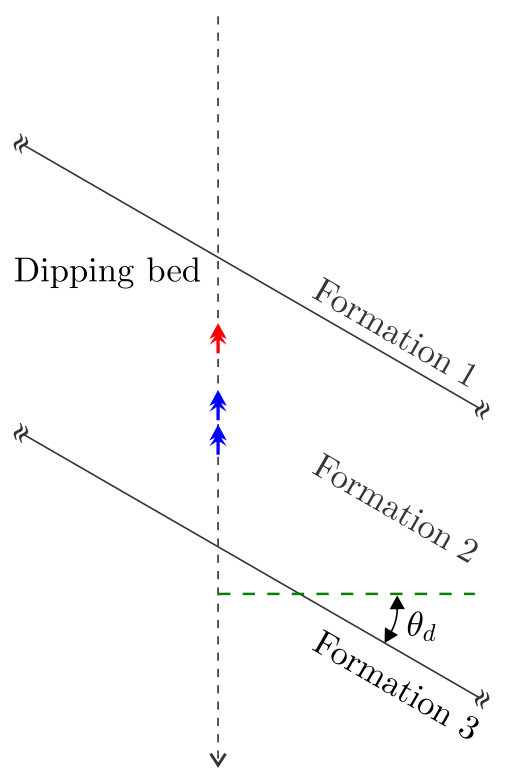

Drill direction

(b) Approximated model

Figure 5.1: Illustration of a LWD tool in a dipping bed. (a) Geometry of the triaxial coil LWD tool. (b) A simplified model of the tool using magnetic dipole sources in the absence of mandrel and borehole.

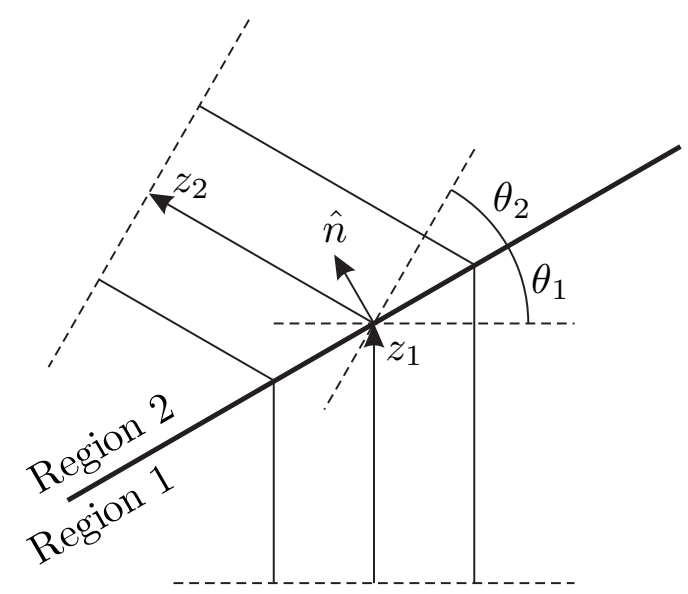

(a) Oblique junction of type 1

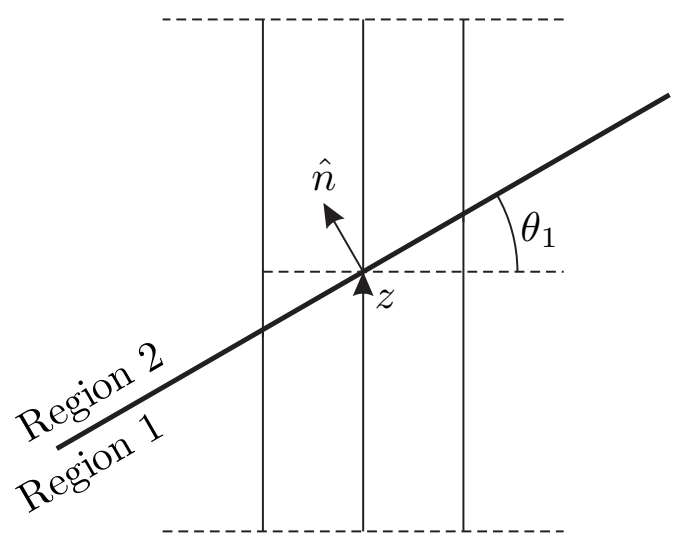

(b) Oblique junction of type 2

Figure 5.2: Waveguide junctions at oblique surfaces.

and azimuthally rotated in relation to the junction surface by the angles $\theta_{2}$ and $\phi_{2}$, respectively. For a moment, assume that $\theta_{1}=-\theta_{2}$ and $\phi_{2}=\phi_{1}$ (see Fig. 5.2(b)).

The coordinates of tilt plane $S_{i}$ are defined by $f_{i}(x, y, z)=0$, where

$$
\begin{aligned}
f_{i}(x, y, z) & =x \sin \theta_{i} \cos \phi_{i}+y \sin \theta_{i} \sin \phi_{i}+\left(z-z_{1}\right) \cos \theta_{i} \\
& =\rho \sin \theta_{i} \cos \left(\phi-\phi_{i}\right)+\left(z-z_{1}\right) \cos \theta_{i},
\end{aligned}
$$


and the normal vector to $S_{i}$ is given by $\nabla f_{i}$, namely:

$$
\begin{aligned}
\hat{n} & =\hat{x} \sin \theta_{i} \cos \phi_{i}+\hat{y} \sin \theta_{i} \sin \phi_{i}+\hat{z} \cos \theta_{i} \\
& =\hat{\rho} \sin \theta_{i} \cos \left(\phi-\phi_{i}\right)-\hat{\phi} \sin \theta_{i} \sin \left(\phi-\phi_{i}\right)+\hat{z} \cos \theta_{i} .
\end{aligned}
$$

The electric and magnetic fields at the region $j$ can be written as

$$
\begin{aligned}
\mathbf{E}_{j} & =\sum_{p}^{\infty} a_{j, p}^{+} e^{i k_{j z, p} z} \mathbf{E}_{j, p}^{+}+a_{j, n}^{-} e^{-i k_{j z, p} z} \mathbf{E}_{j, p}^{-}, \\
\mathbf{H}_{j} & =\sum_{p}^{\infty} a_{j, p}^{+} e^{i k_{j z, p} z} \mathbf{H}_{j, p}^{+}+a_{j, p}^{-} e^{-i k_{j z, p} z} \mathbf{H}_{j, p}^{-},
\end{aligned}
$$

where the $p$ th modal field is given by

$$
\begin{gathered}
\mathbf{E}_{j, p}^{ \pm}=\hat{\rho} E_{j \rho, p}+\hat{\phi} E_{j \phi, p} \pm \hat{z} E_{j z, p}, \\
\mathbf{H}_{j, p}^{ \pm}= \pm \hat{\rho} H_{j \rho, p} \pm \hat{\phi} H_{j \phi, p}+\hat{z} H_{j z, p} .
\end{gathered}
$$

Denoting the cross-sections in regions 1 and 2 over the oblique plane as $S_{1}$ and $S_{2}$, at junction we must match the fields transversal to $\hat{n}$ such that

$$
\begin{aligned}
& \left.\begin{array}{l}
\hat{n} \times \mathbf{E}_{1}=\hat{n} \times \mathbf{E}_{2} \\
\hat{n} \times \mathbf{H}_{1}=\hat{n} \times \mathbf{H}_{2}
\end{array}\right\} \text { inside } S_{1}, \\
& \hat{n} \times \mathbf{E}_{1}=0 \text { inside } S_{2}-S_{1},
\end{aligned}
$$

where we have assumed that $S_{1} \in S_{2}$.

Considering $M$ mode in the region 1 and $N$ modes in the region 2, from the continuity of the transversal electric fields at the junction we can obtain

$$
\begin{gathered}
\sum_{m=1}^{M} a_{1, m}^{+} e^{i k_{1 z, m} \zeta(\rho, \phi)} \hat{n} \times \mathbf{E}_{1, m}^{+}+a_{1, m}^{-} e^{-i k_{1 z, m} \zeta(\rho, \phi)} \hat{n} \times \mathbf{E}_{1, m}^{-}= \\
\sum_{n=1}^{N} a_{2, n}^{+} e^{i k_{2 z, n} \zeta(\rho, \phi)} \hat{n} \times \mathbf{H}_{2, n}^{+}+a_{2, n}^{-} e^{-i k_{2 z, n} \zeta(\rho, \phi)} \hat{n} \times \mathbf{H}_{2, n}^{-},
\end{gathered}
$$

where $\zeta(\rho, \phi)=z_{1}-\rho \tan \theta_{i} \cos \left(\phi-\phi_{i}\right)$. Taking the dot product of the above vector equation with the term $\exp \left(i k_{2 z, n^{\prime}} \zeta(\rho, \phi)\right) \mathbf{H}_{2, n^{\prime}}^{+}\left(\right.$for $\left.n^{\prime}=1,2, \ldots, N\right)$, and integrating the result over the cross-section $S_{2}$ (in the direction for 
$\zeta(\rho, \phi)>0)$, we can obtain a set of $N$ equations:

$$
\begin{gathered}
\sum_{m=1}^{M} \iint_{S_{1}}\left[a_{1, m}^{+} e^{i\left(k_{1 z, m}+k_{2 z, n^{\prime}}\right) \zeta(\rho, \phi)} \mathbf{E}_{1, m}^{+} \times \mathbf{H}_{2, n^{\prime}}^{+}\right. \\
\left.+a_{1, m}^{-} e^{-i\left(k_{1 z, m}-k_{2 z, n^{\prime}}\right) \zeta(\rho, \phi)} \mathbf{E}_{1, m}^{-} \times \mathbf{H}_{2, n^{\prime}}^{+}\right] \cdot \hat{n} \rho d \rho d \phi= \\
\sum_{n=1}^{N} \iint_{S_{2}}\left[a_{2, n}^{+} e^{i\left(k_{2 z, n}+k_{2 z, n^{\prime}}\right) \zeta(\rho, \phi)} \mathbf{E}_{2, n}^{+} \times \mathbf{H}_{2, n^{\prime}}^{+}\right. \\
\left.+a_{2, n}^{-} e^{-i\left(k_{2 z, n}-k_{2 z, n^{\prime}}\right) \zeta(\rho, \phi)} \mathbf{E}_{2, n}^{-} \times \mathbf{H}_{2, n^{\prime}}^{+}\right] \cdot \hat{n} \rho d \rho d \phi
\end{gathered}
$$

where we have employed the scalar triple product property $(\hat{n} \times \mathbf{E}) \cdot \mathbf{H}=$ $(\mathbf{E} \times \mathbf{H}) \cdot \hat{n}$. Note that we can also reduce the integral in the left-hand side of (5-12) to be over $S_{1}$ instead $S_{2}$ because $\mathbf{E}_{1}=0$ inside $S_{2}-S_{1}$. The above set of equations can be written in a matrix form as

$$
\overline{\bar{X}}_{1,2}^{+} \bar{a}_{1}^{+}+\overline{\bar{X}}_{1,2}^{-} \bar{a}_{1}^{-}=\overline{\bar{X}}_{2,2}^{+} \bar{a}_{2}^{+}+\overline{\bar{X}}_{2,2}^{-} \bar{a}_{2}^{-},
$$

where the reaction integrals

$$
X_{i(m), j(n)}^{ \pm}=\iint_{S_{i}} e^{i\left( \pm k_{i z, m}+k_{j z, n}\right) \zeta(\rho, \phi)} \mathbf{E}_{i, m}^{ \pm} \times \mathbf{H}_{j, n}^{+} \cdot \hat{n} \rho d \rho d \phi
$$

were written as the matrix

$$
\left.\overline{\bar{X}}_{i, j}^{ \pm}\right|_{n, m}=X_{i(m), j(n)}^{ \pm}
$$

and the modal amplitudes were depicted as the column vector

$$
\left.\bar{a}_{j}^{ \pm}\right|_{m}=a_{j, m}^{ \pm} .
$$

From the continuity of the transversal magnetic fields at the junction we must enforce

$$
\begin{gathered}
\sum_{m=1}^{M} a_{1, m}^{+} e^{i k_{1 z, m} \zeta(\rho, \phi)} \hat{n} \times \mathbf{H}_{1, m}^{+}+a_{1, m}^{-} e^{-i k_{1 z, m} \zeta(\rho, \phi)} \hat{n} \times \mathbf{H}_{1, m}^{-}= \\
\sum_{n=1}^{N} a_{2, n}^{+} e^{i k_{2 z, n} \zeta(\rho, \phi)} \hat{n} \times \mathbf{H}_{2, n}^{+}+a_{2, n}^{-} e^{-i k_{2 z, n} \zeta(\rho, \phi)} \hat{n} \times \mathbf{H}_{2, n}^{-}
\end{gathered}
$$

Now, taking the dot product of the above vector equation with the term $\exp \left(i k_{1 z, m^{\prime}} \zeta(\rho, \phi)\right) \mathbf{E}_{1, m^{\prime}}^{+}$(for $\left.m^{\prime}=1,2, \ldots, M\right)$, and integrating the result over 
the cross-section $S_{1}$, we can obtain a set of $M$ equations:

$$
\begin{gathered}
\sum_{m=1}^{M} \iint_{S_{1}}\left[a_{1, m}^{+} e^{i\left(k_{1 z, m}+k_{1 z, m^{\prime}}\right) \zeta(\rho, \phi)} \mathbf{H}_{1, m}^{+} \times \mathbf{E}_{1, m^{\prime}}^{+}\right. \\
\left.+a_{1, m}^{-} e^{-i\left(k_{1 z, m}-k_{1 z, m^{\prime}}\right) \zeta(\rho, \phi)} \mathbf{H}_{1, m}^{-} \times \mathbf{E}_{1, m^{\prime}}^{+}\right] \cdot \hat{n} \rho d \rho d \phi= \\
\sum_{n=1}^{N} \iint_{S_{1}}\left[a_{2, n}^{+} e^{i\left(k_{2 z, n}+k_{1 z, m^{\prime}}\right) \zeta(\rho, \phi)} \mathbf{H}_{2, n}^{+} \times \mathbf{E}_{1, m^{\prime}}^{+}\right. \\
\left.+a_{2, n}^{-} e^{-i\left(k_{2 z, n}-k_{1 z, m^{\prime}}\right) \zeta(\rho, \phi)} \mathbf{H}_{2, n}^{-} \times \mathbf{E}_{1, m^{\prime}}^{+}\right] \cdot \hat{n} \rho d \rho d \phi
\end{gathered}
$$

The above set of equations can be written in a matrix form as

$$
\overline{\bar{Y}}_{1,1}^{+} \bar{a}_{1}^{+}+\overline{\bar{Y}}_{1,1}^{-} \bar{a}_{1}^{-}=\overline{\bar{Y}}_{1,2}^{+} \bar{a}_{2}^{+}+\overline{\bar{Y}}_{1,2}^{-} \bar{a}_{2}^{-},
$$

where the reaction integrals

$$
Y_{i(m), j(n)}^{ \pm}=\iint_{S_{i}} e^{i\left(+k_{i z, m} \pm k_{j z, n}\right) \zeta(\rho, \phi)} \mathbf{E}_{i, m}^{+} \times \mathbf{H}_{j, n}^{ \pm} \cdot \hat{n} \rho d \rho d \phi
$$

were written as the matrix

$$
\left.\overline{\bar{Y}}_{i, j}^{ \pm}\right|_{m, n}=Y_{i(m), j(n)}^{ \pm}
$$

Note that $\left[\overline{\bar{Y}}_{i, j}^{+}\right]^{t}=\overline{\bar{X}}_{i, j}^{+}$, and the above coupling matrices have the following dimensions (rows, columns):

$$
\begin{aligned}
\operatorname{dim}\left(\overline{\bar{X}}_{1,2}^{ \pm}\right) & =(N, M), \\
\operatorname{dim}\left(\overline{\bar{X}}_{2,2}^{ \pm}\right) & =(N, N), \\
\operatorname{dim}\left(\overline{\bar{Y}}_{1,2}^{ \pm}\right) & =(M, N), \\
\operatorname{dim}\left(\overline{\bar{Y}}_{1,1}^{ \pm}\right) & =(M, M) .
\end{aligned}
$$

Combining the (5-13) and (5-19) we can relate the forward and backward modal amplitudes using a generalized scatering matrix:

$$
\left[\begin{array}{c}
\bar{a}_{1}^{-} \\
\bar{a}_{2}^{+}
\end{array}\right]=\left[\begin{array}{cc}
\overline{\bar{R}}_{12}^{(n)} & \overline{\bar{T}}_{21}^{(n)} \\
\overline{\bar{T}}_{12}^{(n)} & \overline{\bar{R}}_{21}^{(n)}
\end{array}\right]\left[\begin{array}{c}
\bar{a}_{1}^{+} \\
\bar{a}_{2}^{-}
\end{array}\right],
$$


where

$$
\begin{aligned}
& \overline{\bar{R}}_{12}^{(n)}=-\left[\overline{\bar{Y}}_{11}^{-}-\overline{\bar{Y}}_{12}^{+}\left(\overline{\bar{X}}_{22}^{+}\right)^{-1} \overline{\bar{X}}_{12}^{-}\right]^{-1}\left[\overline{\bar{Y}}_{11}^{+}-\overline{\bar{Y}}_{12}^{+}\left(\overline{\bar{X}}_{22}^{+}\right)^{-1} \overline{\bar{X}}_{12}^{+}\right], \\
& \overline{\bar{T}}_{21}^{(n)}=\left[\overline{\bar{Y}}_{11}^{-}-\overline{\bar{Y}}_{12}^{+}\left(\overline{\bar{X}}_{22}^{+}\right)^{-1} \overline{\bar{X}}_{12}^{-}\right]^{-1}\left[\overline{\bar{Y}}_{12}^{-}-\overline{\bar{Y}}_{12}^{+}\left(\overline{\bar{X}}_{22}^{+}\right)^{-1} \overline{\bar{X}}_{22}^{-}\right], \\
& \overline{\bar{T}}_{12}^{(n)}=\left[\overline{\bar{X}}_{22}^{+}-\overline{\bar{X}}_{12}^{-}\left(\overline{\bar{Y}}_{11}^{-}\right)^{-1} \overline{\bar{Y}}_{12}^{+}\right]^{-1}\left[\overline{\bar{X}}_{12}^{+}-\overline{\bar{X}}_{12}^{-}\left(\overline{\bar{Y}}_{11}^{-}\right)^{-1} \overline{\bar{Y}}_{11}^{+}\right] \text {and }, \\
& \overline{\bar{R}}_{21}^{(n)}=-\left[\overline{\bar{X}}_{22}^{+}-\overline{\bar{X}}_{12}^{-}\left(\overline{\bar{Y}}_{11}^{-}\right)^{-1} \overline{\bar{Y}}_{12}^{+}\right]^{-1}\left[\overline{\bar{X}}_{22}^{-}-\overline{\bar{X}}_{12}^{-}\left(\overline{\bar{Y}}_{11}^{-}\right)^{-1} \overline{\bar{Y}}_{12}^{-}\right] .
\end{aligned}
$$

The coupling integrals $X_{i(m), j(n)}^{ \pm}$and $Y_{i(m), j(n)}^{ \pm}$could be generalized by the form

$$
C_{i(m), j(n)}^{s_{i}, s_{j}}=\iint_{S_{i}} e^{i\left(s_{i} k_{i z, m}+s_{j} k_{j z, n}\right) \zeta(\rho, \phi)} \mathbf{E}_{i, m}^{s_{i}} \times \mathbf{H}_{j, n}^{s_{j}} \cdot \hat{n} \rho d \rho d \phi,
$$

where $s_{i}$ and $s_{j}$ independently assume the signs + or - . Since the fields in region $j$ are express as a double sum in the form $\sum_{n} \sum_{p} \mathbf{G}_{j, n p}^{ \pm}(\rho, \phi)$, we must redefine the reaction integrals to be:

$$
C_{i(n p), j\left(n^{\prime} p^{\prime}\right)}^{s_{i}, s_{j}}=\iint_{S_{i}} e^{i\left(s_{i} k_{i z, m}+s_{j} k_{j z, n}\right) \zeta(\rho, \phi)}\left(\mathbf{E}_{i, n p}^{s_{i}} \times \mathbf{H}_{j, n^{\prime} p^{\prime}}^{s_{j}}\right) \cdot \hat{n} \rho d \rho d \phi .
$$

Solving the scalar triple product in the above equation, we can derive

$$
\begin{aligned}
\left(\mathbf{e}_{i}^{s_{i}} \times \mathbf{h}_{j}^{s_{j}}\right) \cdot \hat{n}=e^{i\left(n+n^{\prime}\right) \phi}\left[\left(e_{i \phi} h_{j z}-s_{i} s_{j} e_{i z} h_{j \phi}\right) \sin \theta_{i} \cos \left(\phi-\phi_{i}\right)\right. & \\
-\left(s_{i} s_{j} e_{i z} h_{j \rho}-e_{i \rho} h_{j z}\right) & \sin \theta_{i} \sin \left(\phi-\phi_{i}\right) \\
& \left.+s_{j}\left(e_{i \rho} h_{j \phi}-e_{i \phi} h_{j \rho}\right) \cos \theta_{i}\right]
\end{aligned}
$$

where the field in the shape $g_{q \alpha}$ are $\rho$-dependent functions, and: $g=e$ or $h$, $\alpha=\rho, \phi$ or $z$, and $q=i$ or $j$.

Introducing

$$
\kappa_{i j}=\left(s_{i} k_{i z, n p}+s_{j} k_{j z, n^{\prime} p^{\prime}}\right) \rho \tan \theta_{i},
$$

we can write the reaction modal reaction between regions 1 and 2 as

$$
\begin{aligned}
& C_{1(n p), 2\left(n^{\prime} p^{\prime}\right)}^{s_{1}, s_{2}}=e^{i\left(s_{1} k_{1 z, n p}+s_{2} k_{2 z, n^{\prime} p^{\prime}}\right) z_{1}} \int_{\rho}\left[\left(e_{1 \phi} h_{2 z}+s_{1} s_{2} e_{1 z} h_{2 \phi}\right) \sin \theta_{1} I_{\phi 1}\right. \\
& \left.-\left(s_{1} s_{2} e_{1 z} h_{2 \rho}-e_{1 \rho} h_{2 z}\right) \sin \theta_{1} I_{\phi 2}+s_{2}\left(e_{1 \rho} h_{2 \phi}-e_{1 \phi} h_{2 \rho}\right) \cos \theta_{1} I_{\phi 3}\right] \rho d \rho .
\end{aligned}
$$

where

$$
\begin{gathered}
I_{\phi 1}=\int_{-\pi}^{\pi} e^{i\left(n+n^{\prime}\right) \phi} e^{-i \kappa_{12} \cos \left(\phi-\phi_{1}\right)} \cos \left(\phi-\phi_{i}\right), \\
I_{\phi 2}=\int_{-\pi}^{\pi} e^{i\left(n+n^{\prime}\right) \phi} e^{-i \kappa_{12} \cos \left(\phi-\phi_{1}\right)} \sin \left(\phi-\phi_{i}\right), \\
I_{\phi 3}=\int_{-\pi}^{\pi} e^{i\left(n+n^{\prime}\right) \phi} e^{-i \kappa_{12} \cos \left(\phi-\phi_{1}\right)} .
\end{gathered}
$$


Changing the integration to be over $\tilde{\phi}=\phi-\phi_{1}, d \tilde{\phi}=d \phi$, we can write the integrals $I_{\phi 1}, I_{\phi 2}$ and $I_{\phi 3}$ in a more compact fashion as

$$
\left\{\begin{array}{c}
I_{\phi 1} \\
I_{\phi 2} \\
I_{\phi 3}
\end{array}\right\}=e^{i\left(n+n^{\prime}\right) \phi_{1}} \int_{-\pi-\phi_{1}}^{\pi-\phi_{1}} e^{-i \kappa_{12} \cos \tilde{\phi}} e^{i\left(n+n^{\prime}\right) \tilde{\phi}}\left\{\begin{array}{c}
\cos \tilde{\phi} \\
\sin \tilde{\phi} \\
1
\end{array}\right\} d \tilde{\phi}
$$

The above integrals are over a full period in $\tilde{\phi}$, which allow us to solve the integral over the half period of the even functions, i.e.,

$$
\left\{\begin{array}{c}
I_{\phi 1} \\
I_{\phi 2} \\
I_{\phi 3}
\end{array}\right\}=2 e^{i\left(n+n^{\prime}\right) \phi_{1}} \int_{0}^{\pi} e^{-i \kappa_{12} \cos \phi}\left\{\begin{array}{c}
\cos \left(n+n^{\prime}\right) \phi \cos \phi \\
i \sin \left(n+n^{\prime}\right) \phi \sin \phi \\
\cos \left(n+n^{\prime}\right) \phi
\end{array}\right\} d \phi
$$

or in a more suitable form as

$$
\left\{\begin{array}{c}
I_{\phi 1} \\
I_{\phi 2} \\
I_{\phi 3}
\end{array}\right\}=e^{i\left(n+n^{\prime}\right) \phi_{1}} \int_{0}^{\pi} e^{-i \kappa_{12} \cos \phi}\left\{\begin{array}{c}
\cos \left(n+n^{\prime}-1\right) \phi+\cos \left(n+n^{\prime}+1\right) \phi \\
i \cos \left(n+n^{\prime}-1\right) \phi-i \cos \left(n+n^{\prime}+1\right) \phi \\
2 \cos \left(n+n^{\prime}\right) \phi
\end{array}\right\} d \phi .
$$

Now we can use the integral form of the Bessel function shown in (3-250) to derive

$$
\left\{\begin{array}{c}
I_{\phi 1} \\
I_{\phi 2} \\
I_{\phi 3}
\end{array}\right\}=\pi e^{i\left(n+n^{\prime}\right) \phi_{1}}\left\{\begin{array}{c}
i^{n+n^{\prime}-1} J_{n+n^{\prime}-1}\left(-\kappa_{12}\right)+i^{n+n^{\prime}+1} J_{n+n^{\prime}+1}\left(-\kappa_{12}\right) \\
i i^{n+n^{\prime}-1} J_{n+n^{\prime}-1}\left(-\kappa_{12}\right)-i i^{n+n^{\prime}+1} J_{n+n^{\prime}+1}\left(-\kappa_{12}\right) \\
2 i^{n+n^{\prime}} J_{n+n^{\prime}}\left(-\kappa_{12}\right)
\end{array}\right\}
$$

which can be readily written as

$$
\begin{gathered}
I_{\phi 1}=-2 \pi(-i)^{n+n^{\prime}} e^{i\left(n+n^{\prime}\right) \phi_{1}} i J_{n+n^{\prime}}^{\prime}\left(\kappa_{12}\right), \\
I_{\phi 2}=-2 \pi(-i)^{n+n^{\prime}} e^{i\left(n+n^{\prime}\right) \phi_{1}} \frac{n+n^{\prime}}{\kappa_{12}} J_{n+n^{\prime}}\left(\kappa_{12}\right), \\
I_{\phi 3}=2 \pi(-i)^{n+n^{\prime}} e^{i\left(n+n^{\prime}\right) \phi_{1}} J_{n+n^{\prime}}\left(\kappa_{12}\right) .
\end{gathered}
$$

Without loss of generality, assuming that the waveguide junction shown in Fig. 5.2 is at $z_{1}=0$, we can find

$$
\begin{aligned}
C_{1(n p), 2\left(n^{\prime} p^{\prime}\right)}^{s_{1}, s_{2}}= & 2 \pi(-i)^{n+n^{\prime}} e^{i\left(n+n^{\prime}\right) \phi_{1}} \\
& \times \int\left[-\left(e_{1 \phi} h_{2 z}-s_{1} s_{2} e_{1 z} h_{2 \phi}\right) i J_{n+n^{\prime}}^{\prime}\left(\kappa_{12}\right) \sin \theta_{1}\right. \\
& +\left(s_{1} s_{2} e_{1 z} h_{2 \rho}-e_{1 \rho} h_{2 z}\right) \frac{n+n^{\prime}}{\kappa_{12}} J_{n+n^{\prime}}\left(\kappa_{12}\right) \sin \theta_{1} \\
& \left.\quad+s_{2}\left(e_{1 \rho} h_{2 \phi}-e_{1 \phi} h_{2 \rho}\right) J_{n+n^{\prime}}\left(\kappa_{12}\right) \cos \theta_{1}\right] \rho d \rho .
\end{aligned}
$$




\subsection{1}

\section{Special Case of a Horizontal Bed}

For small tilt deviations, i.e., $\theta_{1} \rightarrow 0$, the two first and second terms in the RHS of (5-46) become zero:

$$
\begin{aligned}
\left.C_{1(n p), 2\left(n^{\prime} p^{\prime}\right)}^{s_{1}, s_{2}}\right|_{\theta_{1} \rightarrow 0}=2 & (-i)^{n+n^{\prime}} e^{i\left(n+n^{\prime}\right) \phi_{1}} \\
& \times\left.\int\left[s_{2}\left(e_{1 \rho} h_{2 \phi}-e_{1 \phi} h_{2 \rho}\right) J_{n+n^{\prime}}\left(\kappa_{12}\right)\right] \rho d \rho\right|_{\theta_{1} \rightarrow 0} .
\end{aligned}
$$

The parameter $\kappa_{12}$ defined in (5-34) vanish, and we can now use

$$
J_{n+n^{\prime}}\left(\kappa_{12}\right) \rightarrow \delta_{n,-n^{\prime}}, \quad \text { for } \quad \kappa_{12} \rightarrow 0,
$$

to further simplify (5-47):

$$
\left.C_{1(n p), 2\left(n^{\prime} p^{\prime}\right)}^{s_{1}, s_{2}}\right|_{\theta_{1} \rightarrow 0}=s_{2} 2 \pi \int\left(e_{1 \rho, n p} h_{2 \phi,-n p^{\prime}}-e_{1 \phi, n p} h_{2 \rho,-n p^{\prime}}\right) \rho d \rho
$$

where we have recovered the modal indices $n p$ (for fields in region 1) and $n^{\prime} p^{\prime}$ (for fields in region 2).

$$
C_{1(n p), 2\left(n^{\prime} p^{\prime}\right)}^{\left.s_{1}\right)\left.\right|_{\theta_{1} \rightarrow 0}}= \begin{cases}s_{2} 2 \pi \int\left(e_{1 \rho, n p} h_{2 \phi,-n p^{\prime}}-e_{1 \phi, n p} h_{2 \rho,-n p^{\prime}}\right) \rho d \rho, & \text { for } n=-n^{\prime} \\ 0, & \text { else }\end{cases}
$$

The above equation shows that fields are azimuthally orthogonal over the coupling cross-section as the tilt angle $\theta_{1} \rightarrow 0$. Further, by using the azimuthal symmetries in (3-129), we can immediately obtain

$$
\left.C_{1(n p), 2\left(-n p^{\prime}\right)}^{s_{1}, s_{2}}\right|_{\theta_{1} \rightarrow 0}=-s_{2} 2 \pi(-1)^{n} \int\left(s_{n} e_{1 \rho, n p} h_{2 \phi, n p^{\prime}}+e_{1 \phi, n p} h_{2 \rho, n p^{\prime}}\right) \rho d \rho,
$$

where $s_{n}=1-2 \delta_{0, n}$. The above coupling integral is invariant in terms of $s_{1}(=$ $\pm)$, and over the sign of $s_{2}(= \pm)$ we can derive $C_{1(n p), 2\left(-n p^{\prime}\right)}^{s_{1},-}=-C_{1(n p), 2\left(-n p^{\prime}\right)}^{s_{1},+}$ for $\theta_{1}=0$.

Should be observed that for $s_{2}=+(5-49)$ is equal to the definition of the reaction in (3-165) that we have derived for the case $\theta_{1}=0$, as expected. Also, the following relations can be obtained for the coupling integrals (5-14) and $(5-20)$ :

$$
\begin{aligned}
X_{i(m), j(n)}^{s_{1},+} & =Y_{i(m), j(n)}^{s_{1},+}, \\
X_{i(m), j(n)}^{s_{1},-} & =X_{i(m), j(n)}^{s_{1},+}, \\
Y_{i(m), j(n)}^{s_{1},-} & =-Y_{i(m), j(n)}^{s_{1},+},
\end{aligned}
$$


where $s_{1}$ can independently assume any value in LHS and RHS.

Case the matrices $\overline{\bar{X}}_{i, j}^{ \pm}$and $\overline{\bar{Y}}_{i, j}^{ \pm}$are mounted in the same shape of (4-315) (by using sub-matrices to relating the azimuthal dependences in region 1 and 2 ), for the special case in which $\theta_{1}=0$, we derive the following relations:

$$
\begin{aligned}
\left.\overline{\bar{X}}_{2,2}^{ \pm}\right|_{+n,-n} & =\overline{\bar{Q}}_{2}, \\
\left.\overline{\bar{X}}_{1,2}^{ \pm}\right|_{+n,-n} & =\overline{\bar{X}}_{1,2}, \\
\left.\overline{\bar{Y}}_{1,1}^{ \pm}\right|_{+n,-n} & = \pm \overline{\bar{Q}}_{1}, \\
\left.\overline{\bar{Y}}_{1,2}^{+}\right|_{+n,-n} & =\overline{\bar{X}}_{1,2}^{t}, \\
\left.\overline{\bar{Y}}_{1,2}^{-}\right|_{+n,-n} & =-\overline{\bar{X}}_{1,2},
\end{aligned}
$$

where $\overline{\bar{Q}}_{1}, \overline{\bar{Q}}_{2}$ and $\overline{\bar{X}}_{1,2}$ are the reaction matrices we have derived in Chapter 3 . All others off-diagonal sub-matrices are mull; as a consequence of the modal orthogonality in terms of the azimuthal harmonics. We can finally verify that under the above relations, the GSM sub-matrices in (5-27) will reduce exactly to their counterparts presented in (3-151) (for a PEC boundary condition over $S_{2}-S_{1}$, i.e., $\left.\overline{\bar{L}}_{2}=\overline{\overline{0}}\right)$.

\section{2 .2}

\section{Symmetry Relations for the Coupling Integrals}

Using the symmetry relations in (3-129), the coupling integral involving the modal fields with azimuthal indices $-n$ (in region 1 ) and $-n^{\prime}$ (in region 2 ) are given by

$$
\begin{aligned}
C_{1(-n p), 2\left(-n^{\prime} p^{\prime}\right)}^{s_{1}, s_{2}} & =2 \pi i^{n+n^{\prime}} e^{-i\left(n+n^{\prime}\right) \phi_{1}} \\
\times & \times \int\left[-\left(e_{1 \phi} h_{2 z}-s_{1} s_{2} e_{1 z} h_{2 \phi}\right) i J_{n+n^{\prime}}^{\prime}\left(\kappa_{12}\right) \sin \theta_{1}\right. \\
+ & +\left(s_{1} s_{2} e_{1 z} h_{2 \rho}-e_{1 \rho} h_{2 z}\right) \frac{n+n^{\prime}}{\kappa_{12}} J_{n+n^{\prime}}\left(\kappa_{12}\right) \sin \theta_{1} \\
& \left.\quad+s_{2}\left(e_{1 \rho} h_{2 \phi}-e_{1 \phi} h_{2 \rho}\right) J_{n+n^{\prime}}\left(\kappa_{12}\right) \cos \theta_{1}\right] \rho d \rho .
\end{aligned}
$$

Comparing the last two equation, we clearly see a symmetrical relationship of the azimuthal indices in relation to angle $\phi_{1}$ :

$$
C_{1(-n p), 2\left(-n^{\prime} p^{\prime}\right)}^{s_{1}, s_{2}}=(-1)^{n+n^{\prime}} e^{-2 i\left(n+n^{\prime}\right) \phi_{1}} C_{1(n p), 2\left(n^{\prime} p^{\prime}\right)}^{s_{1}, s_{2}} .
$$




\section{3}

\section{Preliminary Conclusion}

In this chapter, we have explored the mode-matching technique along oblique surfaces. The preliminary mathematical description of this problem show that we can reuse all closed-form well-known expressions for modeling radially-stratified media in conjunction of the new generalized scattering matrices (GSMs) derived here to proper model dipping-bed formations.

An important result derived here is about the modal orthogonality: the modal fields are no longer orthogonal over the oblique matching surface. In this way, the new GSM matrices require the inclusion of fields associated to several azimuthal index. In contrast, the axial mode-matching along a cylindrical cross-section (presented in Chapter 3) can use the modal orthogonality to simplify the problem to be over fields of same azimuthal index.

Further investigations are required to verify the efficacy of the approach presented herein. 


\section{6}

\section{Conclusions and Suggestions for Future Research}

Accurate electromagnetic propagation analysis of complex geophysical formations enables the modeling of well-logging tools as an alternative to costly experimental tests. The modeling of logging-while-drilling (LWD) and measurement-while-drilling (MWD) tools used in hydrocarbons exploration have been successfully handled by means of a 3D-FD, 3D-FE or 3D-FV methods. However, the CPU time and the computer memory required by these methods can be prohibitive. In addition, these techniques require fine spatial discretization to accurately model complex geophysical formations. An alternative to improve the efficiency rely on the mode-matching technique: we can employ a $2 \mathrm{D}-\mathrm{FD}$ or $2 \mathrm{D}-\mathrm{FE}$ formulation to solve the fields along the well logging cross-section regions and then match the field continuities along each junction. In this way, the discretization of the axial space is no longer needed, reducing one dimension from the initial problem. Going further, we can use the azimuthal symmetry of the problem to match fields with the same azimuthal harmonic dependence, such just a 1D-FD or 1D-FE formulation can handle the radial variations of the fields. This last approach combined with the modematching technique is known as NMM. Remarkably, or initial 3D problem was now transformed to an equivalent form where the discretization of two spatial coordinates was circumvented.

In Chapter 3, we proposed a method to model well-logging tools based on an eigenmode expansion that does not rely on spatial discretization. The advantage of this approach is that the fields can be represented by a sum of a relatively small number of eigenmodes compared to the number of grid points or discrete mesh required to discretize the space. The main scientific contribution of this work was the introduction of a new pseudo-analytical technique that allows the accurate representation the electromagnetic response of LWD sensors using a restrict numerical effort. As a consequence, a number of remarkable features are allowed in the modeling of stratified cylindrical structures: (1) The eigenfields are orthogonal both in terms of azimuthal as well as axial harmonics, which simplify substantially our analysis; (2) The linear combination of Bessel and Hankel functions naturally satisfies the boundary conditions of the radially-stratified problem; (3) As we know the analytical 
solution for the fields, we can use the invariance of Maxwell's equations under the complex stretching mapping of the PML in order to archive closed form solutions to mimic unbounded regions. Note that we do not need to employ radially-dependent biaxial media as well as we can avoid the staircase approximation for the PML profile; (4) The aforementioned eigenfields allow an analytical mode-matching along the axial direction. Again, the inclusion of the PML is very easy, requiring just a modification on the reaction integral's upper bound; (5) We were able to solve both the source excitation as well as the received voltage of TCA antennas in closed-forms. In addition, a further generalization allow us to proper model TCAs crossing axial regions. In Chapter 3, we showed numerical results that validate our method, and we also presented numerical simulations of TCAs placed within grooves into a metallic mandrel, and its implications to the design of novel directional welllogging sensors.

In Chapter 4, we developed a new analytical formulation to modeling the electromagnetic propagation along radially-stratified and axially-bent structures. Validation and preliminary results were presented, and we have derived a generalization of the axial mode-matching to analyze the fields in curved wells. This technique can be extended in the future to analyze more complex LWD tools inside directional wells with axial stratifications.

The initial results showed in Chapter 3 brought to light some inquisitive questions. The effectiveness of the complex stretching of the PML, for example, appear not be a critical issue for lossy media at typical LWD operation frequencies. We believed this happen due the prevalence of evanescent modal fields, but we consider that this issue need be studied with more detail. Another aspect we must draw attention is that our numerical algorithm becomes illconditioned for high conductivity media. This problem comes from the complex large argument of the cylindrical functions. In that situations, we could consider approximated impedance boundary condition (as those proposed in Section 3.3.3.1) and also the rescaling of Bessel functions (recently proposed in $[50,52,53])$ to prevent this numerical problem.

As a future work, we plan to extend our studies of novel well-logging tools in dipping bed formations. In Chapter 5, we have shown an elementary application of the mode-matching technique along oblique surfaces. This initiative was mathematically described as a combination of radial and axial mode-matching techniques and allow us to depict the fields of a LWD tools in a dipping-bed layer. We observe that the new modal scattering matrices derived in this approach can be easily combined with the closed-form results for fields and sources inside cylindrically layered media. 
Preliminary results shown in Chapter 5 can be naturally generalized in future to model more complex structures where the axial bending is present. Another similar formulation can also be of great interest: the directional modematching could instead be formulated in terms of a surface normal to a misaligned borehole, as illustrated in Fig. 6.1. In this case, we believe that new radial scattering matrices can be derived to relate the vertical field expansions used in $[8,9]$.

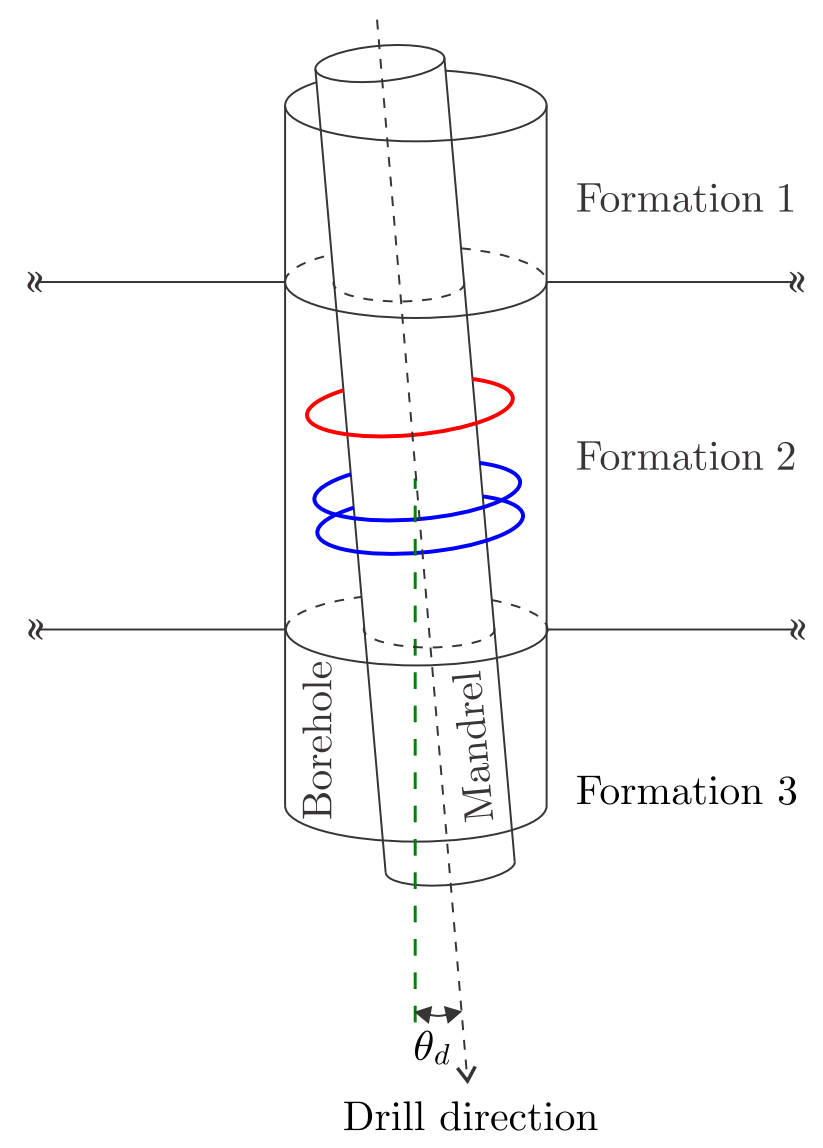

Figure 6.1: Geometry of LWD sensor inside a misaligned borehole. 


\section{Bibliography}

[1] G. Minerbo, A. Davydychev, and K. Sun, "Determining anisotropic resistivity," Oct. 15 2013. U.S. Patent 8558548 B2.

[2] M. Bittar and R. Chemali, "Apparatus and methods of determining formation resistivity," Feb. 28 2013. U.S. Patent 20130054145 A1.

[3] T. Burgess et al., "Horizontal drilling comes of age," Oil Field Review, vol. 2, no. 3, pp. 22-33, 1991.

[4] R. C. Smith, L. A. Hayes, and J. F. Wilkin, "The lateral tie-back system: The ability to drill and case multiple laterals," in SPE IADC Drilling Conference, (Dallas, TX, USA), pp. 55-64, 15-18 Feb. 1994.

[5] Y.-K. Hue and F. L. Teixeira, "FDTD simulation of MWD electromagnetic tools in large-contrast geophysical formations," IEEE Trans. Magn., vol. 40, pp. 1456-1459, Mar. 2004.

[6] Y.-K. Hue, Analysis of electromagnetic well-logging tools. PhD thesis, Dept. Elect. Eng., The Ohio State University, Columbus, OH, USA, 2006.

[7] M. S. Novo, Numerical analysis of electromagnetic well-logging tools by using finite volume methods. PhD thesis, Dept. Elect. Eng., Pontifical Catholic University of Rio de Janeiro, Rio de Janeiro, RJ, Brazil, 2007. in Portuguese.

[8] Y.-K. Hue and F. L. Teixeira, "Numerical mode-matching method for tilted-coil antennas in cylindrically layered anisotropic media with multiple horizontal beds," IEEE Trans. Geosci. Remote Sens., vol. 45, pp. 2451-2462, 2007.

[9] M. dos Santos Canabarro, Application of Numerical Mode-Matching Method in the study of well logging oil with multiple knots. PhD thesis, Dept. Elect. Eng., Pontifical Catholic University of Rio de Janeiro, Rio de Janeiro, RJ, Brazil, 2016. in Portuguese.

[10] W. C. Chew, S. Barone, B. Anderson, and C. Hennessy, "Diffraction of axisymmetric waves in a borehole by bed boundary discontinuities," Geophysics, vol. 49, pp. 1586-1595, Oct. 1984. 
[11] D. G. Dudley, "Wireless propagation in circular tunnels," IEEE Trans. Antennas Propag., vol. 53, pp. 435-441, Jan. 2005.

[12] D. G. Dudley and S. F. Mahmoud, "Linear source in a circular tunnel," IEEE Trans. Antennas Propag., vol. 54, pp. 2034-2047, Jul. 2006.

[13] P. Moon and D. Spencer, Field theory handbook: including coordinate systems, differential equations, and their solutions. New York, NY, USA: Springer-Verlag, 2 ed., 1988.

[14] J. Lovell and W. Chew, "Response of a point source in a multicylindrically layered medium," IEEE Trans. Geosci. Remote Sens., vol. 25, pp. 850-858, Nov. 1987.

[15] W. C. Chew, Waves and Fields in Inhomogeneous Media. New York, NY, USA: John Wiley \& Sons, 1995.

[16] A. Q. Howard, "Petrophysics of magnetic dipole fields in an anisotropic earth," IEEE Trans. Antennas Propag., vol. 48, pp. 1376-1383, Sep. 2000.

[17] H. O. Lee and F. L. Teixeira, "Cylindrical FDTD analysis of LWD tools through anisotropic dipping-layered earth media," IEEE Trans. Geosci. Remote Sens., vol. 45, pp. 383-388, Feb. 2007.

[18] M. S. Novo, L. C. da Silva, and F. L. Teixeira, "A comparative analysis of krylov solvers for three-dimensional simulations of borehole sensors," IEEE Geosci. Remote Sens. Lett., vol. 8, pp. 98-102, Jan. 2011.

[19] H. O. Lee, F. L. Teixeira, L. E. S. Martin, and M. S. Bittar, "Numerical modeling of eccentered LWD borehole sensors in dipping and fully anisotropic earth formations," IEEE Trans. Geosci. Remote Sens., vol. 50, pp. 727-735, Mar. 2012.

[20] L. Hui, S. Yi-ze, and Z. Xi-fang, "Numerical simulation of resistivity LWD tool based on higher-order vector finite element," J. Petrol. Explor. Prod. Technol., vol. 6, pp. 533-543, Sep. 2015.

[21] K. Yee, "Numerical solution of initial boundary value problems involving maxwell's equations in isotropic media," IEEE Trans. Antennas Propag., vol. 14, pp. 302-307, May 1966.

[22] D. M. Pai and M. Huang, "A generalized haskell matrix method for borehole electromagnetics; theory and applications," Geophysics, vol. 53, no. 12, pp. 1577-1586, 1988. 
[23] Q. H. Liu and W. C. Chew, "Numerical mode-matching method for the multiregion vertically stratified media," IEEE Trans. Antennas Propag., vol. 38, pp. 498-506, Apr. 1990.

[24] W. C. Chew, Z. Nie, Q. H. Liu, and B. Anderson, "An efficient solution for the response of electrical well logging tools in a complex environment," IEEE Trans. Geosci. Remote Sens., vol. 29, pp. 308-313, Mar. 1991.

[25] Q.-H. Liu and W. C. Chew, "Diffraction of nonaxisymmetric waves in cylindrically layered media by horizontal discontinuities," Radio Science, vol. 27, no. 5, pp. 569-581, 1992.

[26] Q.-H. Liu, "Electromagnetic field generated by an off-axis source in a cylindrically layered medium with an arbitrary number of horizontal discontinuities," Geophysics, vol. 58, pp. 616-625, May 1993.

[27] D. M. Pai, J. Ahmad, and W. D. Kennedy, "Two-dimensional induction log modeling using a coupled-mode, multiple-reflection series method," Geophysics, vol. 58, pp. 466-474, Apr. 1993.

[28] J. Li and L. C. Shen, "Vertical eigenstate method for simulation of induction and MWD resistivity sensors," IEEE Trans. Geosci. Remote Sens., vol. 31, pp. 399-406, Mar. 1993.

[29] H. Wang, P. So, S. Yang, W. Hoefer, and H. Du, "Numerical modeling of multicomponent induction well-logging tools in the cylindrically stratified anisotropic media," IEEE Trans. Geosci. Remote Sens., vol. 46, pp. 11341147, Apr. 2008.

[30] W. Li, Z. Nie, X. Sun, and Y. Chen, "Numerical modeling for excitation and coupling transmission of near field around the metal drilling pipe in lossy formation," IEEE Trans. Geosci. Remote Sens., vol. 52, pp. 3862-3871, Jul. 2014.

[31] H. Wang, H. Tao, J. Yao, and Y. Zhang, "Efficient and reliable simulation of multicomponent induction logging response in horizontally stratified inhomogeneous $\mathrm{TI}$ formations by numerical mode matching method," IEEE Trans. Geosci. Remote Sens., vol. 50, pp. 3383-3395, Sep. 2012.

[32] J. Dai and Q. H. Liu, "Efficient computation of electromagnetic waves in anisotropic orthogonal-plano-cylindrically layered media using the improved numerical mode matching (NMM) method," IEEE Trans. Antennas Propag., vol. 63, pp. 3569-3578, Aug. 2015. 
[33] H. Liu, Principles and Applications of Well Logging. Springer Mineralogy, Berlin, Germany: Springer, 2017.

[34] B. Clark et al., "Electromagnetic propagation logging while drilling: Theory and experiment," SPE Formation Evaluation, pp. 263-271, Sep. 1990.

[35] P. T. Wu, J. R. Lovell, B. Clark, S. D. Bonner, and J. R. Tabanou, "Dielectric-independent 2-MHz propagation resistivities," in SPE Annual Technical Conference and Exhibition, (Houston, TX, USA), 3-6 Oct. 1999.

[36] N. R. Council, Drilling and Excavation Technologies for the Future. Washington, DC, USA: The National Academies Press, 1994.

[37] R. Nurmi et al., "Horizontal highlights," Middle East Well Evaluation Review, no. 16, pp. 7-25, 1995.

[38] T. Ma, P. Chen, and J. Zhao, "Overview on vertical and directional drilling technologies for the exploration and exploitation of deep petroleum resources," Geomechanics and Geophysics for Geo-Energy and Geo-Resources, vol. 2, no. 4, pp. 365-395, 2016.

[39] "ADR azimuthal deep resistivity sensor datasheet." Halliburton Sperry Drilling, Houston, TX, USA, 2016.

[40] "PeriScope datasheet." Schlumberger, Houston, TX, USA, 2008.

[41] V. H. Rumsey, "Reaction concept in electromagnetic theory," Phys. Rev., vol. 94, pp. 1483-1491, Jun. 1954.

[42] J. Van Bladel, Electromagnetic Fields. IEEE Press Series on Electromagnetic Wave Theory, New York, NY, USA: IEEE Press, 2 ed., 2007.

[43] W. C. Chew and B. Anderson, "Propagation of electromagnetic waves through geological beds in a geophysical probing environment," Radio Science, vol. 20, pp. 611-621, May 1985.

[44] Y.-K. Hue, F. L. Teixeira, L. S. Martin, and M. S. Bittar, "Three-dimensional simulation of eccentric LWD tool response in boreholes through dipping formations," IEEE Trans. Geosci. Remote Sens., vol. 43, pp. 257-268, Feb. 2005.

[45] M. S. Novo, L. C. da Silva, and F. L. Teixeira, "Three-dimensional finitevolume analysis of directional resistivity logging sensors," IEEE Trans. Geosci. Remote Sens., vol. 48, pp. 1151-1158, Mar. 2010. 
[46] D. M. Pai, "Induction log modeling using vertical eigenstates," IEEE Trans. Geosci. Remote Sens., vol. 29, pp. 209-213, Mar. 1991.

[47] T. Hagiwara, E. J. Banning, R. M. Ostermeier, and S. M. Haugland, "Effects of mandrel, borehole, and invasion for tilt-coil antennas," in SPE 78th Ann. Tech. Conf. Exhibit., (Denver, CO, USA), 5-8 Oct. 2003.

[48] G.-S. Liu, F. L. Teixeira, and G.-J. Zhang, "Analysis of directional logging tools in anisotropic and multieccentric cylindrically-layered earth formations," IEEE Trans. Antennas Propag., vol. 60, pp. 318-327, Jan. 2012.

[49] Y.-K. Hue and F. L. Teixeira, "Analysis of tilted-coil eccentric borehole antennas in cylindrical multilayered formations for well-logging applications," IEEE Trans. Antennas Propag., vol. 54, pp. 1058-1064, 2006.

[50] H. Moon, F. L. Teixeira, and B. Donderici, "Stable pseudoanalytical computation of electromagnetic fields from arbitrarily-oriented dipoles in cylindrically stratified media," J. Comp. Phys., vol. 273, pp. 118-142, Sep. 2014.

[51] L. Lewin, D. Chang, and E. Kuester, Electromagnetic waves and curved structures. London, UK: Peter Peregrinus, 1977.

[52] K. Sainath, F. L. Teixeira, and B. Donderici, "Robust computation of dipole electromagnetic fields in arbitrarily anisotropic, planar-stratified environments," Phys. Rev. E, vol. 89, p. 013312, Jan. 2014.

[53] D. Hong, W. F. Huang, and Q. H. Liu, "Radiation of arbitrary magnetic dipoles in a cylindrically layered anisotropic medium for well-logging applications," IEEE Trans. Geosci. Remote Sens., vol. 54, pp. 6362-6370, Nov. 2016.

[54] J. Kong, Electromagnetic Wave Theory. New York, NY, USA: Wiley, 1986.

[55] R. F. Harrington, Time-harmonic electromagnetic fields. New York, NY, USA: McGraw-Hill, 1961.

[56] H. Moon, B. Donderici, and F. L. Teixeira, "Stable evaluation of Green's functions in cylindrically stratified regions with uniaxial anisotropic layers," J. Comp. Phys., vol. 325, pp. 174-200, 2016.

[57] P. Bienstman et al., "Analysis of cylindrical waveguide discontinuities using vectorial eigenmodes and perfectly matched layers," IEEE Trans. Microw. Theory Techn., vol. 49, pp. 349-354, Feb. 2001. 
[58] L. M. Delves and J. N. Lyness, "A numerical method for locating the zeros of an analytic function," Mathematics of Computation, vol. 21, pp. 543-560, Oct. 1967.

[59] J. W. Brown and R. V. Churchill, Complex Variables and Applications. New York, NY, USA: McGrawHill, 7 ed., 2004.

[60] G. S. Rosa and J. R. Bergmann, "Pseudo-analytical modeling for the electromagnetic propagation in stratified cylindrical structures," IEEE Antennas Wireless Propag. Lett., vol. 15, pp. 344-347, 2016.

[61] W. C. Chew and W. H. Weedon, "A 3D perfectly matched medium from modified Maxwell's equations with stretched coordinates," Microw. Opt. Tech. Lett., vol. 7, pp. 599-604, 1994.

[62] W. C. Chew, J. M. Jin, and E. Michielssen, "Complex coordinate stretching as a generalized absorbing boundary condition," Microw. Opt. Tech. Lett., vol. 15, pp. 363-369, Aug. 1997.

[63] F. L. Teixeira and W. C. Chew, "Complex space approach to perfectly matched layers: a review and some new developments," Int. J. Num. Model., vol. 13, pp. 441-455, 2000.

[64] P. Bienstman and R. Baets, "Advanced boundary conditions for eigenmode expansion models," Optical and Quantum Electronics, vol. 34, no. 5, pp. 523-540, 2002.

[65] F. L. Teixeira and W. C. Chew, "Finite-difference computation of transient electromagnetic waves for cylindrical geometries in complex media," IEEE Trans. Geosci. Remote Sens., vol. 38, pp. 1530-1543, Jul. 2000.

[66] H. Rogier and D. D. Zutter, "Berenger and leaky modes in optical fibers terminated with a perfectly matched layer," J. Lightw. Technol., vol. 20, pp. 1141-1148, Jul. 2002.

[67] L. Aiyong, N. Zaiping, and Z. Yanwen, "Numerical mode matching method with perfectly matching layer," in 2005 IEEE Antennas and Propagation Society International Symposium, vol. 4B, pp. 372-375 vol. 4B, Jul. 2005.

[68] H. O. Lee, Numerical Modeling of Electromagnetic Well-Logging Sensors. $\mathrm{PhD}$ thesis, Dept. Elect. Eng., The Ohio State University, Columbus, $\mathrm{OH}$, USA, 2010. 
[69] Z. S. Sacks, D. M. Kingsland, R. Lee, and J.-F. Lee, "A perfectly matched anisotropic absorber for use as an absorbing boundary condition," IEEE Trans. Antennas Propag., vol. 43, pp. 1460-1463, Dec. 1995.

[70] F. L. Teixeira and W. C. Chew, "Systematic derivation of anisotropic PML absorbing media in cylindrical and spherical coordinates," IEEE Microw. Guided Wave Lett., vol. 7, pp. 371-373, Nov. 1997.

[71] H. Massoudi, N. J. Damskos, and P. L. E. Uslenghi, "Scattering by a composite and anisotropic circular cylindrical structure: Exact solution," Electromagnetics, vol. 8, no. 1, pp. 71-83, 1988.

[72] D. Pratap, S. A. Ramakrishna, J. G. Pollock, and A. K. Iyer, "Anisotropic metamaterial optical fibers," Opt. Express, vol. 23, pp. 9074-9085, Apr. 2015.

[73] J. G. Pollock, A. K. Iyer, D. Pratap, and S. A. Ramakrishna, "A class of circular waveguiding structures containing cylindrically anisotropic metamaterials: Applications from radio frequency/microwave to optical frequencies," J. Appl. Phys., vol. 119, no. 8, p. 083103, 2016.

[74] T. B. A. Senior, "Approximate boundary conditions, part 1," Tech. Rep. Radiation Laboratory Report RL-861, University of Michigan, Ann Arbor, MI, Jun. 1990.

[75] T. B. A. Senior and J. L. Volakis, Approximate Boundary Conditions in Electromagnetics. IEE Electromagnetic Waves Series 41, London, UK: IEE Press, 1995.

[76] H. Rogier and D. D. Zutter, "Convergence behavior and acceleration of the berenger and leaky modes series composing the 2-D Green's function for the microstrip substrate," IEEE Trans. Microw. Theory Techn., vol. 50, pp. 1696-1704, Jul. 2002.

[77] J. Zhu and Y. Y. Lu, "Leaky modes of slab waveguides-asymptotic solutions," J. Lightw. Technol., vol. 24, pp. 1619-1623, Mar. 2006.

[78] D. G. Dudley, M. Lienard, S. F. Mahmoud, and P. Degauque, "Wireless propagation in tunnels," IEEE Antennas Propag. Mag., vol. 49, pp. 11-26, Apr. 2007.

[79] H. Rogier and D. V. V. Ginste, "A fast procedure to accurately determine leaky modes in multilayered planar dielectric substrates," IEEE Trans. Microw. Theory Techn., vol. 56, pp. 1413-1422, Jun. 2008. 
[80] C. Liu, H. X. Zhou, Q. Tang, Z. Song, and W. Hong, "A homotopy method for locating all the poles of a parallel plate waveguide with the PML," IEEE Trans. Antennas Propag., vol. 61, pp. 4685-4694, Sep. 2013.

[81] P. J. B. Clarricoats and B. C. Taylor, "Evanescent and propagating modes of dielectric-loaded circular waveguide," Proceedings of the Institution of Electrical Engineers, vol. 111, pp. 1951-1956, Dec. 1964.

[82] K. Zaki and C. Chen, "Complex modes in dielectric loaded waveguides," in Antennas and Propagation Society International Symposium, 1987, vol. 25, pp. 8-11, Jun. 1987.

[83] K. Zaki, S.-W. Chen, and C. Chen, "Modeling discontinuities in dielectricloaded waveguides," IEEE Trans. Microw. Theory Techn., vol. 36, pp. 18041810, Dec. 1988.

[84] K. Y. Kim, H.-S. Tae, and J.-H. Lee, "Application of Davidenko's method to rigorous analysis of leaky modes in circular dielectric rod waveguides," KIEE Int. Trans. EA, vol. 3, no. 5, pp. 199-206, 2003.

[85] J. Boyd, Solving Transcendental Equations: The Chebyshev Polynomial Proxy and Other Numerical Rootfinders, Perturbation Series, and Oracles. Other titles in applied mathematics, Society for Industrial and Applied Mathematics, 2014.

[86] S. H. Talisa, "Application of Davidenko's method to the solution of dispersion relations in lossy waveguiding systems," IEEE Trans. Microw. Theory Techn., vol. 33, pp. 967-971, Oct. 1985.

[87] H. Antia, Numerical Methods for Scientists and Engineers. Basel: Birkhauser, 2 ed., 2002.

[88] K. Y. Kim, H.-S. Tae, and J.-H. Lee, "Leaky dispersion characteristics in circular dielectric rod using Davidenko's method," J. of the Korea Electromagnetic Engineering Society, vol. 5, pp. 72-79, Jun. 2005.

[89] H. A. N. Hejase, "On the use of Davidenko's method in complex root search," IEEE Trans. Microw. Theory Techn., vol. 41, pp. 141-143, Jan. 1993.

[90] M. M. Shabat, D. Jager, M. A. Abd-El naby, and N. M. Barakat, "Numerical and analytical solutions of dispersion equation in lossy nonlinear waveguiding system," Microw. Opt. Tech. Lett., vol. 22, pp. 273-278, Aug. 1999. 
[91] W. H. Press, S. A. Teukolsky, W. T. Vetterling, and B. P. Flannery, Numerical recipes: the art of scientific computing. New York, NY, USA: Cambridge University Press, 3 ed., 2007.

[92] L. C. da Silva, "Determination of the roots of the characteristic equation for corrugated and dielectric loaded circular waveguides," IEEE Trans. Microw. Theory Techn., vol. 45, pp. 298-301, Feb. 1997.

[93] C. S. Lee, S.-W. Lee, and S.-L. Chuang, "Normal modes in an overmoded circular waveguide coated with lossy material," IEEE Trans. Microw. Theory Techn., vol. 34, pp. 773-785, Jul. 1986.

[94] R. C. Chou and S. W. Lee, "Modal attenuation in multilayered coated waveguides," IEEE Trans. Microw. Theory Techn., vol. 36, pp. 1167-1176, Jul. 1988.

[95] K. W. Whites, "Electromagnetic wave propagation through circular waveguides containing radially inhomogeneous lossy media," Tech. Rep. USACERL TM M-89/11, U.S. Army Construction Engineering Research Laboratory, Champaign, IL, USA, Sep. 1989.

[96] R. G. Fehlen, "Air gap error compensation for coaxial transmission line method of electromagnetic material characterization," Master's thesis, School of Engineering and Management, Air Force Institute of Technology, WrightPatterson Air Force Base, OH, USA, Mar. 2006.

[97] N. I. loakimidis and E. G. Anastasselou, "A modification of the DelvesLyness method for locating the zeros of analytic functions," J. Comput. Phys., vol. 59, no. 3, pp. 490-492, 1985.

[98] B. Davies, "Locating the zeros of an analytic function," J. Comput. Phys., vol. 66, no. 1 , pp. 36-49, 1986.

[99] E. Anemogiannis and E. N. Glytsis, "Multilayer waveguides: efficient numerical analysis of general structures," J. Lightw. Technol., vol. 10, pp. 13441351, Oct. 1992.

[100] P. Kravanja and M. Van Barel, "A derivative-free algorithm for computing zeros of analytic functions," Computing, vol. 63, no. 1, pp. 69-91, 1999.

[101] P. Kravanja, T. Sakurai, and M. Van Barel, "On locating clusters of zeros of analytic functions," BIT Numerical Mathematics, vol. 39, no. 4, pp. 646682, 1999. 
[102] C. Chen, P. Berini, D. Feng, S. Tanev, and V. P. Tzolov, "Efficient and accurate numerical analysis of multilayer planar optical waveguides in lossy anisotropic media," Opt. Express, vol. 7, pp. 260-272, Oct. 2000.

[103] C. L. Holloway, D. A. Hill, R. A. Dalke, and G. A. Hufford, "Radio wave propagation characteristics in lossy circular waveguides such as tunnels, mine shafts, and boreholes," IEEE Trans. Antennas Propag., vol. 48, pp. 13541366, Sep. 2000.

[104] M. Dellnitz, O. Schutze, and Q. Zheng, "Locating all the zeros of an analytic function in one complex variable," J. Comput. Appl. Math., vol. 138, no. 2, pp. 325-333, 2002.

[105] S. B. Gaal, H. J. W. M. Hoekstra, and P. V. Lambeck, "Determining PML modes in 2-D stratified media," J. Lightw. Technol., vol. 21, pp. 293-298, Jan. 2003.

[106] C. J. Gillan, A. Schuchinsky, and I. Spence, "Computing zeros of analytic functions in the complex plane without using derivatives," Computer Physics Communications, vol. 175, no. 4, pp. 304-313, 2006.

[107] T. Johnson and W. Tucker, "Enclosing all zeros of an analytic function - A rigorous approach," J. Comput. Appl. Math., vol. 228, no. 1, pp. 418-423, 2009.

[108] R. W. Moses and D. M. Cai, "Efficient numerical modal solutions for RF propagation in lossy circular waveguides," IEEE Trans. Antennas Propag., vol. 58, pp. 525-530, Feb. 2010.

[109] G. S. Rosa and J. R. Bergmann, "Electromagnetic propagation along lossy anisotropic and radially stratified cylindrical structures," in Loughborough Antennas and Propagation Conference (LAPC), (Loughborough, UK), pp. 155-159, Nov. 2014.

[110] G. S. Rosa, J. R. Bergmann, and S. R. Zang, "Improvements on the search modal propagation constants in lossy circular waveguides," IEEE Antennas Wireless Propag. Lett., vol. 13, pp. 754-757, 2014.

[111] S. Gustafsson, T. Biro, G. Cinar, M. Gustafsson, A. Karlsson, B. Nilsson, S. Nordebo, and M. Sjöberg, "Electromagnetic dispersion modeling and measurements for HVDC power cables," IEEE Trans. Power Del., vol. 29, pp. 2439-2447, Dec. 2014. 
[112] W. C. Chew, "The singularities of a fourier-type integral in a multicylindrical layer problem," IEEE Trans. Antennas Propag., vol. 31, pp. 653-655, Jul. 1983.

[113] D. G. Dudley, Mathematical Foundations for Electromagnetic Theory. New York, NY, USA: IEEE Press, 1994.

[114] J. D. Wade and R. H. MacPhie, "Conservation of complex power technique for waveguide junctions with finite wall conductivity," IEEE Trans. Microw. Theory Techn., vol. 38, pp. 373-378, Apr. 1990.

[115] G. V. Eleftheriades, A. S. Omar, L. P. B. Katehi, and G. M. Rebeiz, "Some important properties of waveguide junction generalized scattering matrices in the context of the mode matching technique," IEEE Trans. Microw. Theory Techn., vol. 42, pp. 1896-1903, Oct. 1994.

[116] M. Abramowitz and I. A. Stegun, Handbook of Mathematical Functions: With Formulas, Graphs, and Mathematical Tables. New York, NY, USA: Dover Publications, 1964.

[117] G. Watson, A Treatise on the Theory of Bessel Functions. London, UK: Cambridge University Press, 1922.

[118] D. M. Pozar, Microwave Engineering. Hoboken, NJ, USA: John Wiley \& Sons, Inc., 3 ed., 2005.

[119] G. S. Rosa, "Electromagnetics wave propagation in coaxial guides with nonhomogeneous load excited by the TEM Mode.," Master's thesis, Dept. Elect. Eng., Pontifical Catholic University of Rio de Janeiro, Rio de Janeiro, RJ, Brazil, 2013. in Portuguese.

[120] G. S. Rosa, J. R. Bergmann, and S. R. Zang, "Electromagnetic analysis of wireless telemetry for oil fields," in 2013 SBMO/IEEE MTT-S International Microwave Optoelectronics Conference (IMOC), (Rio de Janeiro, Brazil), pp. 1-4, Jul. 4-7 2013.

[121] K. A. Safinya et al., "System and method for communicating signals in a cased borehole having tubing," Jul. 13 1989. U.S. Patent 4839644.

[122] H. J. Vinegar et al., "Permanent downhole, wireless, two-way telemetry backbone using redundant repeaters," Oct. 25 2005. U.S. Patent 6958704 B2. 
[123] S. Y. Chen, W. C. Chew, V. R. N. Santos, K. Sainath, and F. L. Teixeira, Electromagnetic Subsurface Remote Sensing, pp. 1-28. Wiley Encyclopedia of Electrical and Electronics Engineering, 2016.

[124] C. S. Lavranos and G. A. Kyriacou, "Eigenvalue analysis of curved waveguides employing an orthogonal curvilinear frequency-domain finite-difference method," IEEE Trans. Microw. Theory Techn., vol. 57, pp. 594-611, Mar. 2009.

[125] C.-H. Lai, T. Chang, and Y.-S. Yeh, "Characteristics of bent terahertz antiresonant reflecting pipe waveguides," Opt. Express, vol. 22, pp. 84608472, Apr. 2014.

[126] J. Ma, J. M. Jin, and Z. Nie, "A nonconformal FEM-DDM with treecotree splitting and improved transmission condition for modeling subsurface detection problems," IEEE Trans. Geosci. Remote Sens., vol. 52, pp. 355364, Jan. 2014.

[127] M. Y. Xia and Z. Y. Chen, "Attenuation predictions at extremely low frequencies for measurement-while-drilling electromagnetic telemetry system," IEEE Trans. Geosci. Remote Sens., vol. 31, pp. 1222-1228, Nov. 1993.

[128] M. Heiblum and J. Harris, "Analysis of curved optical waveguides by conformal transformation," IEEE J. Quantum Electron., vol. 11, pp. 75-83, Feb. 1975.

[129] S. J. Garth, "Modes on a bent optical waveguide," IEE Proc. J - Optoelectronics, vol. 134, pp. 221-229, Aug. 1987.

[130] B. Donderici and F. L. Teixeira, "Metamaterial blueprints for reflectionless waveguide bends," IEEE Microw. Compon. Lett., vol. 18, pp. 233-235, Apr. 2008.

[131] M. S. Janaki and B. Dasgupta, "Eigenmodes for electromagnetic waves propagating in a toroidal cavity," IEEE Trans. Plasma Sci., vol. 18, pp. 7885, Feb. 1990.

[132] E. Bahar and G. Govindarajan, "Rectangular and annular modal analyses of multimode waveguide bends," IEEE Trans. Microw. Theory Techn., vol. 21, pp. 819-824, Dec. 1973.

[133] H. Li and M. Thumm, "Mode conversion due to curvature in corrugated waveguides," Int. J. Electron., vol. 71, no. 2, pp. 333-347, 1991. 
[134] E. Butkov, Mathematical Physics. Addison-Wesley, 1973.

[135] W. Sollfrey, "Wave propagation on helical wires," J. Appl. Phys., vol. 22, no. 7, pp. 905-910, 1951.

[136] C. Y. H. Tsao and W. A. Gambling, "Curvilinear optical fibre waveguide: Characterization of bound modes and radiative field," Proc. R. Soc. Lond. $A$, vol. 425, no. 1868, pp. 1-16, 1989.

[137] J. J. Krempasky, "Analysis of TEM mode on a curved coaxial transmission line," IEEE Trans. Microw. Theory Techn., vol. 38, pp. 739-747, Jun. 1990.

[138] K. W. Kark, "Perturbation analysis of electromagnetic eigenmodes in toroidal waveguides," IEEE Trans. Microw. Theory Techn., vol. 39, pp. 631-637, Apr. 1991.

[139] N. Schupfer, "Axisymmetric electromagnetic eigenmodes of plasma-filled toroidal resonators," IEEE Trans. Plasma Sci., vol. 19, pp. 906-911, Oct. 1991.

[140] F. M. Aghamir, H. Zakeri-Khatir, and M. K. Salem, "Dispersion characteristics of a plasma column in a toroidal waveguide," IEEE Trans. Plasma Sci., vol. 39, pp. 1204-1212, May 2011.

[141] Z. Menachem and S. Tapuchi, "Wave propagation in a helical waveguide with slab and rectangular dielectric profiles, and applications," Progress In Electromagnetics Research B, vol. 26, pp. 115-147, Sep. 2010.

[142] Z. Menachem and S. Tapuchi, "Wave propagation in a helical waveguide with slab and rectangular dielectric profiles, and applications," Progress In Electromagnetics Research B, vol. 34, pp. 77-102, Sep. 2011.

[143] J. Stratton, Electromagnetic Theory. An IEEE Press classic reissue, Hoboken, NJ, USA: Wiley, 2007.

[144] L. Lewin, "A decoupled formulation of the vector wave equation in orthogonal curvilinear coordinates, with application to ferrite-filled and curved waveguides of general cross section," IEEE Trans. Microw. Theory Techn., vol. 20, pp. 338-342, May 1972.

[145] C. Tang, "An orthogonal coordinate system for curved pipes (correspondence)," IEEE Trans. Microw. Theory Techn., vol. 18, pp. 69-69, Jan 1970.

[146] CST AG, CST Studio Suite 2016, Darmstadt, Germany, 2016. 
[147] G. S. Rosa, J. R. Bergmann, and F. L. Teixeira, "Axial mode-matching technique for analysis of directional well-logging sensor tools," in IEEE International Symposium on Antennas and Propagation/USNC-URSI National Radio Science, (Fajardo, Puerto Rico), pp. 2041-2042, Jun. 26-Jul. 1st 2016.

[148] G. S. Rosa, J. R. Bergmann, and F. L. Teixeira, "Pseudo-analytical modeling of tilted-coil antennas in anisotropic geophysical formations," in $17^{\circ}$ SBMO - Simpósio Brasileiro de Micro-ondas e Optoeletrônica - $12^{\circ}$ CBMag Congresso Brasileiro de Eletromagnetismo, (Porto Alegre, Brazil), Jul. 25-29 2016.

[149] G. S. Rosa, J. R. Bergmann, and F. L. Teixeira, "A robust mode-matching algorithm for the analysis of triaxial well-logging tools in anisotropic geophysical formations," IEEE Trans. Geosci. Remote Sens., vol. 55, pp. 2534-2545, May 2017.

[150] J. Happel and H. Brenner, Low Reynolds number hydrodynamics: with special applications to particulate media. The Hague, Netherlands: Martinus Nijhoff, 2 ed., 1983. 
A

\section{Wave Equation in Cylindrical Coordinates}

In this appendix we shown the solution for the wave equation in anisotropic media using the cylindrical coordinate system. The derivations presented here are heavily based on the work shown in [55, Ch. 5], where the problem is solved for isotropic media.

According the formulation in Chapter 3, the scalar Helmholtz equation for axial fields in cylindrical coordinates is given by

$$
\frac{1}{\rho} \frac{\partial}{\partial \rho}\left(\rho \frac{\partial \psi}{\partial \rho}\right)+\frac{1}{\rho^{2}} \frac{\partial^{2} \psi}{\partial \phi^{2}}+\frac{p_{z}}{p_{s}} \frac{\partial^{2} \psi}{\partial z^{2}}+\omega^{2} p_{z} \tilde{p}_{s} \psi=0
$$

Following the method of separation of variables, we seek to find solutions of the form

$$
\psi=R(\rho) \Phi(\phi) Z(z) .
$$

Substitution of (A-2) into (A-1), division by $\psi$, and noting that the partial derivatives now becomes total derivatives, allow us to derive

$$
\frac{1}{\rho R} \frac{d}{d \rho}\left(\rho \frac{d R}{d \rho}\right)+\frac{1}{\rho^{2} \Phi} \frac{d^{2} \Phi}{d \phi^{2}}+\frac{p_{z}}{p_{s}} \frac{1}{Z} \frac{d^{2} \psi}{d z^{2}}+\omega^{2} p_{z} \tilde{p}_{s}=0
$$

where the third term is explicitly independent of $\rho$ and $\phi$. This term must also be independent of $z$ because the equation is to sum to zero for all $\rho, \phi, z$. Hence, we can define

$$
\frac{1}{Z} \frac{d^{2} Z}{d z^{2}}=-k_{z}^{2}
$$

where $k_{z}$ is a constant. Substitution of the above into (A-3) multiplied by $\rho^{2}$ gives

$$
\frac{\rho}{R} \frac{d}{d \rho}\left(\rho \frac{d R}{d \rho}\right)+\frac{1}{\Phi} \frac{d^{2} \Phi}{d \phi^{2}}+\left(\omega^{2} p_{z} \tilde{p}_{s}-\frac{p_{z}}{p_{s}} k_{z}^{2}\right) \rho^{2}=0 .
$$

Now, the second term of the above is independent of $\rho$ and $z$, and the remaining terms are independents of $\phi$. Hence, we can properly define

$$
\frac{1}{\Phi} \frac{d^{2} \Phi}{d \phi^{2}}=-n^{2}
$$

where $n$ is a constant. Now, the preceding equation gives rise to

$$
\frac{\rho}{R} \frac{d}{d \rho}\left(\rho \frac{d R}{d \rho}\right)-n^{2}+\left(\omega^{2} p_{z} \tilde{p}_{s}-\frac{p_{z}}{p_{s}} k_{z}^{2}\right) \rho^{2}=0 .
$$

The wave equation is now separated by means of (A-4), (A-6) and (A-7). To summarize, we can introduce 


$$
k_{\rho}^{2}=\omega^{2} p_{s} \tilde{p}_{s}-k_{z}^{2},
$$

and also the wavenumber related to the transversal anisotropy given by

$$
k_{s}^{2}=\omega^{2} p_{s} \tilde{p}_{s}=\omega^{2} \epsilon_{s} \mu_{s} .
$$

We can write the separated equations as

$$
\begin{aligned}
\rho \frac{d}{d \rho}\left(\rho \frac{d R}{d \rho}\right)+\left[\left(\sqrt{\frac{p_{z}}{p_{s}}} k_{\rho} \rho\right)^{2}-n^{2}\right] R & =0, \\
\frac{d^{2} \Phi}{d \phi^{2}}+n^{2} \Phi & =0 \\
\frac{d^{2} Z}{d z^{2}}+k_{z}^{2} Z & =0 .
\end{aligned}
$$

The expression in (A-10) is a Bessel differential equation of order $n[116$, p. 358], whose general solution is given by

$$
R_{n}\left(\sqrt{\frac{p_{z}}{p_{s}}} k_{\rho} \rho\right)=A J_{n}\left(\sqrt{\frac{p_{z}}{p_{s}}} k_{\rho} \rho\right)+B Y_{n}\left(\sqrt{\frac{p_{z}}{p_{s}}} k_{\rho} \rho\right),
$$

where $J_{n}$ and $Y_{n}$ are fisrt and second kind Bessel functions, respectively. The constants $A$ and $B$ are determined by the boundary conditions. Another commonly used solution to Bessel's equation employ the first and second kind Hankel functions of order $n$ :

$$
R_{n}\left(\sqrt{\frac{p_{z}}{p_{s}}} k_{\rho} \rho\right)=A H_{n}^{(1)}\left(\sqrt{\frac{p_{z}}{p_{s}}} k_{\rho} \rho\right)+B H_{n}^{(2)}\left(\sqrt{\frac{p_{z}}{p_{s}}} k_{\rho} \rho\right),
$$

where

$$
H_{n}^{(1)}(x)=J_{n}(x)+i Y_{n}(x), \quad H_{n}^{(2)}(x)=J_{n}(x)-i Y_{n}(x) .
$$

In general, any two of the functions $J_{n}, Y_{n}, H_{n}^{(1)}$ and $H_{n}^{(2)}$ are linearly independent and can be used as a fundamental of solutions of (A-10). Note that only the function $J_{n}$ is nonsingular at $\rho=0$. Hence, if a field is to be finite at the radial origin, we have $R_{n}(\cdot)=A J_{n}(\cdot)$. In contrast, for an unbounded radial domain, we must select $R_{n}(\cdot)=A H_{n}^{(1)}(\cdot)$ in order to satisfy the Sommerfeld radiation condition.

Equations (A-11) and (A-12) are one-dimensional non-homogeneous Laplace's equations whose solutions are given by harmonic functions such as

$$
\begin{gathered}
\Phi(n \phi)=C \cos (n \phi)+D \sin (n \phi) \quad \text { or } \quad C e^{i n \phi}+D e^{-i n \phi}, \\
Z\left(k_{z} z\right)=E \cos \left(k_{z} z\right)+F \sin \left(k_{z} z\right) \quad \text { or } \quad E e^{i k_{z} z}+F e^{-i k_{z} z},
\end{gathered}
$$


where $C, D, E$ and $F$ are constants to be determined by enforcing the boundary conditions. The azimuthal coordinate is an angle, and for this reason, we need to restrict the choice of $\Phi(n \phi)$. In order to achieve single-valued fields, it is required that $\psi(\phi)=\psi(\phi+2 \pi p), p=0,1,2, \ldots$ Thus, we have immediately that $n$ must be an integer. Finally, the elementary solution for $(\mathrm{A}-1)$ is given by

$$
\psi=R_{n}\left(\sqrt{\frac{p_{z}}{p_{s}}} k_{\rho} \rho\right) \Phi(n \phi) Z\left(k_{z} z\right) .
$$


B

\section{Modal Amplitudes from the Transmitter to the Receiver}

The axial mode-matching at the junction between each pair of waveguides allows to get a generalized scattering matrix. We denote the associated matrices as local reflection and transmission operators. In this appendix, we will combine these local operators in order to proper describe the fields at observation point on the plane $z=z_{R}$ due to a source placed on the plane $z=z_{T}$.

The electromagnetic field components in a radially-stratified, axiallyinfinity cylindrical structure can be write as

$$
G_{\alpha}(\rho, \phi, z)=\sum_{n=-\infty}^{\infty} \sum_{p=1}^{\infty} A_{T, n p}^{ \pm} g_{j \alpha, n p}^{ \pm}\left(k_{j \rho, n p} \rho\right) e^{ \pm i k_{z, n p}\left(z-z_{T}\right)+i n \phi} \quad \text { for } z_{T} \lessgtr z,
$$

where $G_{\alpha}=\left\{E_{\alpha}, H_{\alpha}\right\}$ and $\alpha=\{\rho, \phi, z\}$. The $g_{j \alpha, n p}^{ \pm}=\left\{e_{j \alpha, n p}^{ \pm}, h_{j \alpha, n p}^{ \pm}\right\}$is the proper electric and magnetic cylindrical function for the radial layer $j$. Based on the azimuthal orthogonality of the fields, we can rewrite our fields as

$$
G_{\alpha}(\rho, \phi, z)=\sum_{n=-\infty}^{\infty} G_{n \alpha}(\rho, z) e^{i n \phi}
$$

where $G_{n \alpha}$ can be found comparing the last two equations. Truncating the sum over $p$ in order to include $M$ modes, we can also write $G_{n \alpha}$ in a compact matrix form as

$$
G_{n \alpha}(\rho, z)=\left[\bar{g}_{j \alpha}^{ \pm}(\rho)\right]^{t} \overline{\bar{P}}^{ \pm}\left(z_{T}, z\right) \bar{A}_{T}^{ \pm} \quad \text { for } z_{T} \lessgtr z,
$$

where $\bar{g}_{j \alpha}^{ \pm}(\rho)$ is a $M \times 1$ column vector containing the entries $g_{j \alpha, n p}^{ \pm}$for the proper radial position, $\overline{\bar{P}}^{ \pm}\left(z_{T}, z\right)$ is a $M \times M$ diagonal matrix with the elements $e^{ \pm i k_{z, n p}\left(z-z_{T}\right)}$ and $\bar{A}_{T}^{ \pm}$is a $M \times 1$ column vector with the modal source amplitudes.

\section{B.1}

\section{Fields Matching Approach}

We use here an algorithm similar to that in $[25,26,29]$ and $[15$, pp. 360 $365]$ to compute the fields along the axial stratifications. We should mention that this technique was used before in the NMM, where the eigenmodes of an infinitely long cylindrical layered medium are solved by using a onedimensional finite-element method. Here, instead, we will employ our closed 
form eigenfunction expansion for the fields in cylindrical coordinates.

Consider now an axially-stratified structure composed by $N$ regions, as illustrated in Fig. B.1. Each region $j$ comprises $z_{j-1}<z<z_{j}, j=$ $1,2,3, \ldots, N$, for $0<\phi<2 \pi$. Note that these regions can also be radiallystratified. It is clear that fields in a multi-stratified medium can no longer be represented just by one forward or one backward wave as in (B-3). In general, we need a combination of two waves propagating to $+z$ and to $-z$ to proper express our field solutions. To proceed our analysis, we will consider just our transversal (to $z$ ) electric field due the axial symmetry of its forward and backward components ${ }^{1}$.

Suppose that an source is placed in the region $m$ and we are interested in the fields in the region $n$. The transversal electric field components in a region $n$ above the source, $m<n$, can be written as ${ }^{2}$

$$
E_{n}(\rho, z)=\bar{e}_{n}^{t}(\rho)\left[\overline{\bar{P}}_{n}^{+}\left(z_{n}, z\right)+\overline{\bar{P}}_{n}^{-}\left(z_{n}, z\right) \tilde{\overline{\bar{R}}}_{n, n+1}\right] \bar{A}_{n} .
$$

Here $\bar{e}_{n}^{t}(\rho)$ is a $1 \times M_{n}$ matrix with the eigenmodes of the region $n, \overline{\bar{P}}_{n}^{+}\left(z_{n}, z\right)$ is a $M_{n} \times M_{n}$ diagonal matrix that propagates a forward wave from $z_{n}$ to $z$ such as

$$
\left.\overline{\bar{P}}_{j}^{ \pm}\left(z^{\prime}, z\right)\right|_{p p}=e^{ \pm i k_{p z}\left(z-z^{\prime}\right)}
$$

We also have introduced the global reflection operator for the up-going waves in region $n$ given by the $M_{n} \times M_{n}$ matrix $\tilde{\bar{R}}_{n, n+1}$ and $\bar{A}_{n}$; a $M_{n} \times 1$ column vector for the up-going waves in region $n$ at $z=z_{n}$. Notice that the number of modes considered in the axial layer is $j$ given by $M_{j}$.

Recursive relations for the global reflection operator $\tilde{\overline{\vec{R}}}_{n, n+1}$ and for the up-going wave expansion vector $\bar{A}_{n}$ can be derived by considering the local reflection and transmission matrices derived by the mode-matching.

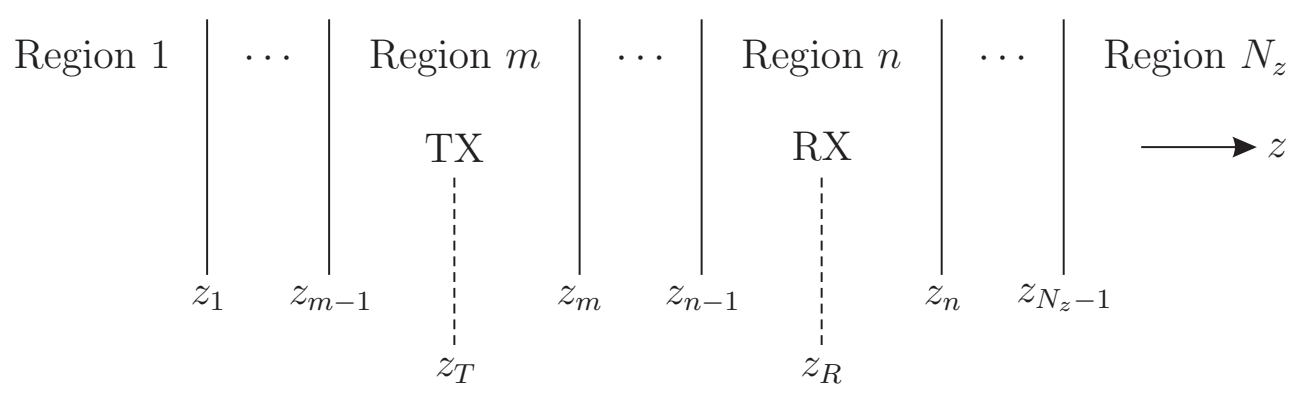

Figure B.1: The axially-stratified structure under consideration.

\footnotetext{
${ }^{1}$ See (3-128) for more details. Notice that the axial magnetic field presents the symmetry in respect to axis $z$. If we had used the axial electric or the transversal magnetic fields the sign of the backward wave must be negative instead of positive in (B-4).

${ }^{2}$ For the sake of simplicity the superscripts in the scattering matrices omitted, but take in mind that it could assume the quantity $(z)$.
} 
Following the formulation proposed in [26], At the boundary $z=z_{n}$, the down-going waves consist of two contribution: (1) the transmission of the down-going waves from region $n+1$ (characterized by $\overline{\bar{T}}_{n+1, n}$ ), and $(2)$ the reflection of the up-going waves in region $n+1$ (characterized by $\overline{\bar{R}}_{n+1, n}$ ). Using the appropriated expressions of the down-going and up-going waves in (B-4), we can write

$$
\tilde{\overline{\bar{R}}}_{n, n+1} \bar{A}_{n}=\overline{\bar{T}}_{n+1, n} \overline{\bar{P}}_{n+1} \tilde{\overline{\bar{R}}}_{n+1, n+2} \bar{A}_{n+1}+\overline{\bar{R}}_{n, n+1} \bar{A}_{n},
$$

where

$$
\overline{\bar{P}}_{m}=\overline{\bar{P}}_{m}^{+}\left(z_{m-1}, z_{m}\right)=\overline{\bar{P}}_{m}^{-}\left(z_{m}, z_{m-1}\right) .
$$

Similarly, at the boundary $z=z_{n}$, the up-going waves in region $n$ consist of two contributions: (1) the transmission of the up-going waves from region $n-1$ (characterized by $\overline{\bar{T}}_{n-1, n}$ ) and (2) the reflection of the of the down-going waves in region $n-1$ (characterized by $\overline{\bar{R}}_{n-1, n}$ ). Using (B-4), we can write

$$
\bar{A}_{n}=\overline{\bar{P}}_{n} \overline{\bar{T}}_{n-1, n} \bar{A}_{n-1}+\overline{\bar{P}}_{n} \overline{\bar{R}}_{n, n-1} \overline{\bar{P}}_{n} \tilde{\bar{R}}_{n, n+1} \bar{A}_{n} .
$$

From (B-8), we can find a recursive relation for the up-going vector $\bar{A}_{n}$ given by

$$
\bar{A}_{n}=\overline{\bar{P}}_{n} \overline{\bar{M}}_{n}^{+} \overline{\bar{T}}_{n-1, n} \bar{A}_{n-1}
$$

where

$$
\overline{\bar{M}}_{n}^{+}=\left(\overline{\bar{I}}-\overline{\bar{R}}_{n, n-1} \overline{\bar{P}}_{n} \tilde{\overline{\bar{R}}}_{n, n+1} \overline{\bar{P}}_{n}\right)^{-1}
$$

Substituting (B-9) into (B-6) allows us to find a recursive relation for the global reflection matrices as

$$
\tilde{\overline{\bar{R}}}_{n, n+1}=\overline{\bar{R}}_{n, n+1}+\overline{\bar{T}}_{n+1, n} \overline{\bar{P}}_{n+1} \tilde{\overline{\bar{R}}}_{n+1, n+2} \overline{\bar{P}}_{n+1} \overline{\bar{M}}_{n+1}^{+} \overline{\bar{T}}_{n, n+1} .
$$

Notice that $\tilde{\overline{\bar{R}}}_{N-1, N}=\overline{\bar{R}}_{N-1, N}$ at the last boundary $z=z_{N-1}$, and all global reflection matrices for the up-going waves can be found recursively from (B-11).

Suppose now we are interested in the fields in the region $n$ below the source, i.e., $m>n$. Similar to (B-4), the transversal electric field components in region $n$ can be written as

$$
E_{n}(\rho, z)=\bar{e}_{n}^{t}(\rho)\left[\overline{\bar{P}}_{n}^{-}\left(z_{n-1}, z\right)+\overline{\bar{P}}_{n}^{+}\left(z_{n-1}, z\right) \tilde{\overline{\bar{R}}}_{n, n-1}\right] \bar{B}_{n},
$$

where the global reflection operator for the down-going waves in region $n$ is given by the $M_{n} \times M_{n}$ matrix $\tilde{\overline{\bar{R}}}_{n, n-1}$ and $\bar{B}_{n}$; a $M_{n} \times 1$ column vector for the down-going waves in region $n$ at $z=z_{n-1}$. We can derive recursive relations for $\bar{B}_{n}$ and $\tilde{\overline{\bar{R}}}_{n, n-1}$ using the same argument leading to (B-6) and (B-8) but now employing the fields in the shape of those in (B-12). For example, at $z=z_{n-1}$, the up-going waves in region $n$ satisfy

$$
\tilde{\overline{\bar{R}}}_{n, n-1} \bar{B}_{n}=\overline{\bar{T}}_{n-1, n} \overline{\bar{P}}_{n-1} \tilde{\overline{\bar{R}}}_{n-1, n-2} \bar{B}_{n-1}+\overline{\bar{R}}_{n, n-1} \bar{B}_{n} .
$$


The down-going waves also at $z=z_{n-1}$ in region $n$ must satisfy

$$
\bar{B}_{n}=\overline{\bar{P}}_{n} \overline{\bar{T}}_{n+1, n} \bar{B}_{n+1}+\overline{\bar{P}}_{n} \overline{\bar{R}}_{n, n+1} \overline{\bar{P}}_{n} \tilde{\bar{R}}_{n, n-1} \bar{B}_{n} .
$$

From (B-14), we can find a recursive relation for the up-going vector $\bar{B}_{n}$ given by

$$
\bar{B}_{n}=\overline{\bar{P}}_{n} \overline{\bar{M}}_{n}^{-} \overline{\bar{T}}_{n+1, n} \bar{B}_{n+1}
$$

where

$$
\overline{\bar{M}}_{n}^{-}=\left(\overline{\bar{I}}-\overline{\bar{R}}_{n, n+1} \overline{\bar{P}}_{n} \tilde{\overline{\bar{R}}}_{n, n-1} \overline{\bar{P}}_{n}\right)^{-1} \text {. }
$$

Substituting (B-15) into (B-13) allows us to find a recursive relation for the global reflection matrices as

$$
\tilde{\overline{\bar{R}}}_{n, n-1}=\overline{\bar{R}}_{n, n-1}+\overline{\bar{T}}_{n-1, n} \overline{\bar{P}}_{n-1} \tilde{\overline{\bar{R}}}_{n-1, n-2} \overline{\bar{P}}_{n-1} \overline{\bar{M}}_{n-1}^{-} \overline{\bar{T}}_{n, n-1} .
$$

Notice that $\tilde{\overline{\bar{R}}}_{2,1}=\overline{\bar{R}}_{2,1}$ at the first boundary $z=z_{1}$, and all global reflection matrices for the down-going waves can be found recursively from (B-17).

Suppose now we are looking for the fields in the region of the source. In this region the fields can be written in three parts [26]: (1) the direct field from the source in the absence of the axial discontinuities at $z=z_{m}$ and $z=z_{m-1}$ (the regions $m$ is considered as an infinite-long and axialhomogeneous medium), (2) the up-going waves due to multiple reflections, and (3) the down-going waves due to multiple reflections. Thus, the field in region $m$ becomes [26], [15, p. 363]

$$
E_{m}(\rho, z)=\bar{e}_{m}^{t}(\rho)\left[\overline{\bar{P}}_{m}^{ \pm}\left(z_{T}, z\right) \bar{A}_{T}^{ \pm}+\overline{\bar{P}}_{m}^{+}(0, z) \bar{C}_{m}+\overline{\bar{P}}_{m}^{-}(0, z) \bar{D}_{m}\right]
$$

for $z_{T} \lessgtr z$, where we have assumed that the source may radiate differentially for forward and backward directions as $\bar{A}_{T}^{ \pm 3}$, and the $M_{m} \times 1$ column vectors $\bar{C}_{m}$ and $\bar{D}_{m}$ are the up-going and down-going amplitudes yet to be determined.

At $z=z_{m-1}$, the up-going waves result from the multiple reflections (characterized by $\widetilde{\overline{\bar{R}}}_{m, m-1}$ ) of the down-going waves, i.e.,

$$
\overline{\bar{P}}_{m}^{+}\left(0, z_{m-1}\right) \bar{C}_{m}=\tilde{\overline{\bar{R}}}_{m, m-1}\left[\overline{\bar{P}}_{m}^{-}\left(z_{T}, z_{m-1}\right) \bar{A}_{T}^{-}+\overline{\bar{P}}_{m}^{-}\left(0, z_{m-1}\right) \bar{D}_{m}\right] .
$$

Similarly, at $z=z_{m}$, we must satisfy

${ }^{3}$ To the best of our knowledge, the application of axial mode-matching (or its NMM version, i.e., the $\mathrm{A}-\mathrm{NMM}$ ) appear restrict to model point sources or horizontal coils $[10,15,23-26,29-32,60]$. This happen because both source and the axial discontinuities are matched over a constant axial plane. For sources with non-zero span along the axial direction (such as tilted-coil antennas that are described in Section 3.5.4), vertical mode expansion described in $[6,8,27,46]$ (where both sources and radial discontinuities are matched over a constant radial plane) appears an appropriate way to model the problem. In [147-149] we demonstrate a way to compute the contribution of general and non-zero span sources using the axial mode-matching. To use this method; that is described in detail in Section 3.5.6; we need to consider the existence of general modal excitation coefficients, which may leads to $\bar{A}_{T}^{+} \neq \bar{A}_{T}^{-}$. 


$$
\overline{\bar{P}}_{m}^{-}\left(0, z_{m}\right) \bar{D}_{m}=\tilde{\overline{\bar{R}}}_{m, m+1}\left[\overline{\bar{P}}_{m}^{+}\left(z_{T}, z_{m}\right) \bar{A}_{T}^{+}+\overline{\bar{P}}_{m}^{+}\left(0, z_{m}\right) \bar{C}_{m}\right]
$$

Solving the coupled equations (B-19) and (B-20) yields the vectors $\bar{C}_{m}$ and $\bar{D}_{m}$ as

$$
\begin{aligned}
& \bar{C}_{m}=\overline{\bar{P}}_{m}^{+}\left(z_{m-1}, 0\right) \tilde{\bar{M}}_{m}^{+} \tilde{\overline{\bar{R}}}_{m, m-1}\left[\overline{\bar{P}}_{m}^{-}\left(z_{T}, z_{m-1}\right) \bar{A}_{T}^{-}+\overline{\bar{P}}_{m} \tilde{\overline{\bar{R}}}_{m, m+1} \overline{\bar{P}}_{m}^{+}\left(z_{T}, z_{m}\right) \bar{A}_{T}^{+}\right] \\
& \bar{D}_{m}=\overline{\bar{P}}_{m}^{-}\left(z_{m}, 0\right) \tilde{\bar{M}}_{m}^{-} \tilde{\overline{\bar{R}}}_{m, m+1}\left[\overline{\bar{P}}_{m}^{+}\left(z_{T}, z_{m}\right) \bar{A}_{T}^{+}+\overline{\bar{P}}_{m} \tilde{\overline{\bar{R}}}_{m, m-1} \overline{\bar{P}}_{m}^{-}\left(z_{T}, z_{m-1}\right) \bar{A}_{T}^{-}\right]
\end{aligned}
$$

where we have introduced the multiple reflection matrices $\tilde{\bar{M}}_{m}^{ \pm}$given by

$$
\tilde{\bar{M}}_{m}^{ \pm}=\left(\overline{\bar{I}}-\tilde{\overline{\bar{R}}}_{m, m \mp 1} \overline{\bar{P}}_{m} \tilde{\overline{\bar{R}}}_{m, m \pm 1} \overline{\bar{P}}_{m}\right)^{-1} .
$$

It should be observed that the fields in (B-18), (B-21) and (B-22) involve propagation matrices similar to $\overline{\bar{P}}_{m}^{ \pm}\left(0, z_{a}\right)$ or $\overline{\bar{P}}_{m}^{\mp}\left(0, z_{a}\right)$. For an arbitrary position $z=z_{a}$ (mainly large positive or negative $z_{a}$ ), these matrices might require very large exponents [31]. In order to prevent the overflow during the numerical computing of the fields, it is better to rewrite (B-18) as

$$
E_{m}(\rho, z)=\bar{e}_{m}^{t}(\rho)\left[\overline{\bar{P}}_{m}^{ \pm}\left(z_{T}, z\right) \bar{A}_{T}^{ \pm}+\overline{\bar{P}}_{m}^{+}\left(z_{m-1}, z\right) \hat{\bar{C}}_{m}+\overline{\bar{P}}_{m}^{-}\left(z_{m}, z\right) \hat{\bar{D}}_{m}\right],
$$

where the forward reflected waves at $z=z_{m-1}$ and the backward reflected waves at $z=z_{m}$ are given by

$$
\begin{gathered}
\hat{\bar{C}}_{m}=\tilde{\overline{\bar{M}}}_{m}^{+} \tilde{\overline{\bar{R}}}_{m, m-1}\left[\overline{\bar{P}}_{m}^{-}\left(z_{T}, z_{m-1}\right) \bar{A}_{T}^{-}+\overline{\bar{P}}_{m} \tilde{\overline{\bar{R}}}_{m, m+1} \overline{\bar{P}}_{m}^{+}\left(z_{T}, z_{m}\right) \bar{A}_{T}^{+}\right], \text {and } \\
\hat{\bar{D}}_{m}=\tilde{\overline{\bar{M}}}_{m}^{-} \tilde{\overline{\bar{R}}}_{m, m+1}\left[\overline{\bar{P}}_{m}^{+}\left(z_{T}, z_{m}\right) \bar{A}_{T}^{+}+\overline{\bar{P}}_{m} \tilde{\overline{\bar{R}}}_{m, m-1} \overline{\bar{P}}_{m}^{-}\left(z_{T}, z_{m-1}\right) \bar{A}_{T}^{-}\right],
\end{gathered}
$$

respectively.

Now, the fields into region $m$ can be completely determined by means of (B-24). Therefore, the expansion vector $\bar{A}_{m}$ of the up-going waves at $z=z_{m}$ is given by

$$
\bar{A}_{m}=\overline{\bar{P}}_{m}^{+}\left(z_{T}, z_{m}\right) \bar{A}_{T}^{+}+\overline{\bar{P}}_{m} \hat{\bar{C}}_{m}
$$

and the expansion vector $\bar{B}_{m}$ of the down-going waves at $z=z_{m-1}$ is given by

$$
\bar{B}_{m}=\overline{\bar{P}}_{m}^{-}\left(z_{T}, z_{m-1}\right) \bar{A}_{T}^{-}+\overline{\bar{P}}_{m} \hat{\bar{D}}_{m}
$$

The expansion vectors for other regions can be derived recursively from (B-9) and (B-15).

The above results are derived for the transversal electric fields, however, we can use them to find all electromagnetic field components. In general, we can write the fields at an observation plane $z=z_{R}$ (characterized by $\bar{A}_{R}^{ \pm}$) due 
to a source located at $z=z_{T}$ (characterized by $\left.\bar{A}_{T}^{ \pm}\right)$as

$$
G_{\alpha}\left(\rho, \phi, z_{R}\right)=\sum_{n=-\infty}^{\infty} \sum_{p=1}^{M_{R}(n)}\left(A_{R, n p}^{+} \pm A_{R, n p}^{-}\right) g_{j \alpha, n p}\left(k_{j \rho, n p} \rho\right) e^{i n \phi},
$$

where $G_{\alpha}=\left\{E_{\alpha}, H_{\alpha}\right\}, \alpha=\{\rho, \phi, z\}$ and the non-harmonic portion of the fields in radial layer $j$ is given by $g_{j \alpha, n p}=\left\{e_{j \alpha, n p}, h_{j \alpha, n p}\right\}$. In the above summation $M_{R}(n)$ is the number of modes considered in the axial region of the receiver point, and the sign \pm must be selected such as: $(+)$ for the transversal electric and the axial magnetic fields, and $(-)$ for the axial electric and transversal magnetic fields.

The proper values of $\bar{A}_{R}^{ \pm}$can be extracted from the forward and backward contribution of the fields in (B-4), (B-12) and (B-24) by using the algorithms shown in Fig. B.2 to Fig. B.6.

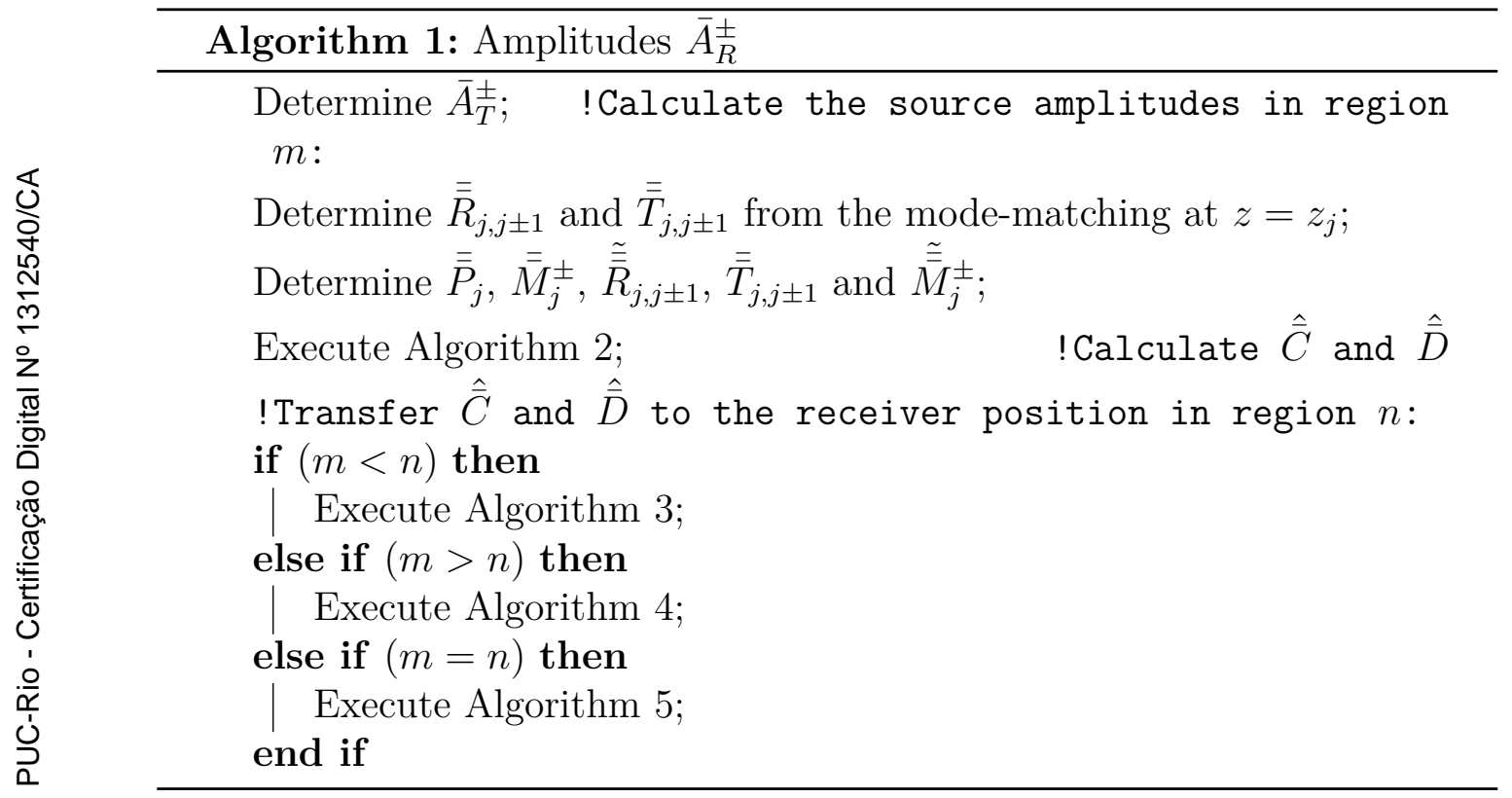

Figure B.2: Description of the algorithm used to calculate the amplitudes of the forward and backward waves at the observation point. 


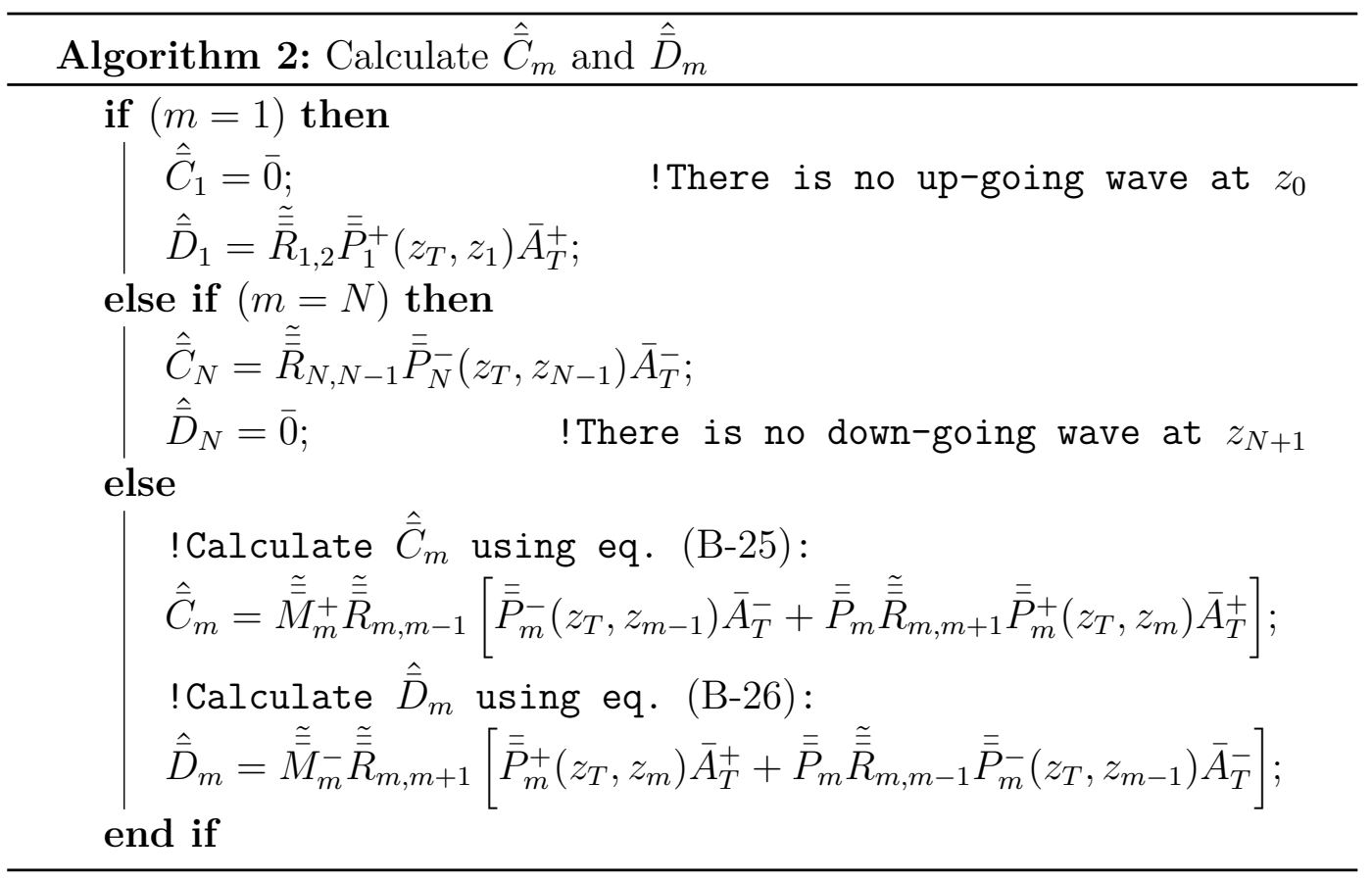

Figure B.3: Algorithm used to calculate $\hat{\bar{C}}_{m}$ and $\hat{\bar{D}}_{m}$.

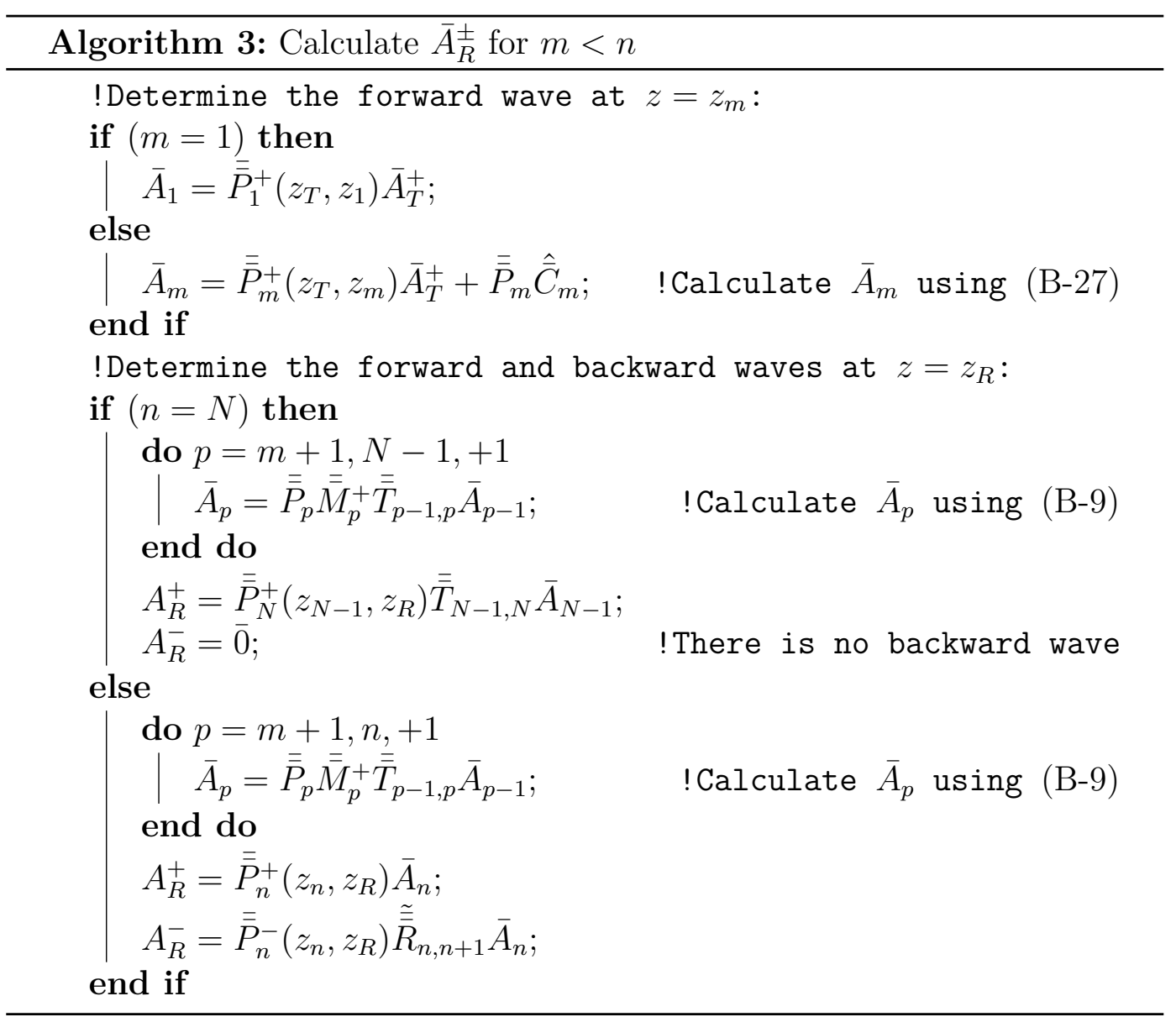

Figure B.4: Algorithm used to calculate the amplitudes of the forward and backward waves at $z=z_{R}$ (in region $n$ ) due to a source at $z=z_{T}$ (in region $m)$, for $m<n$. 


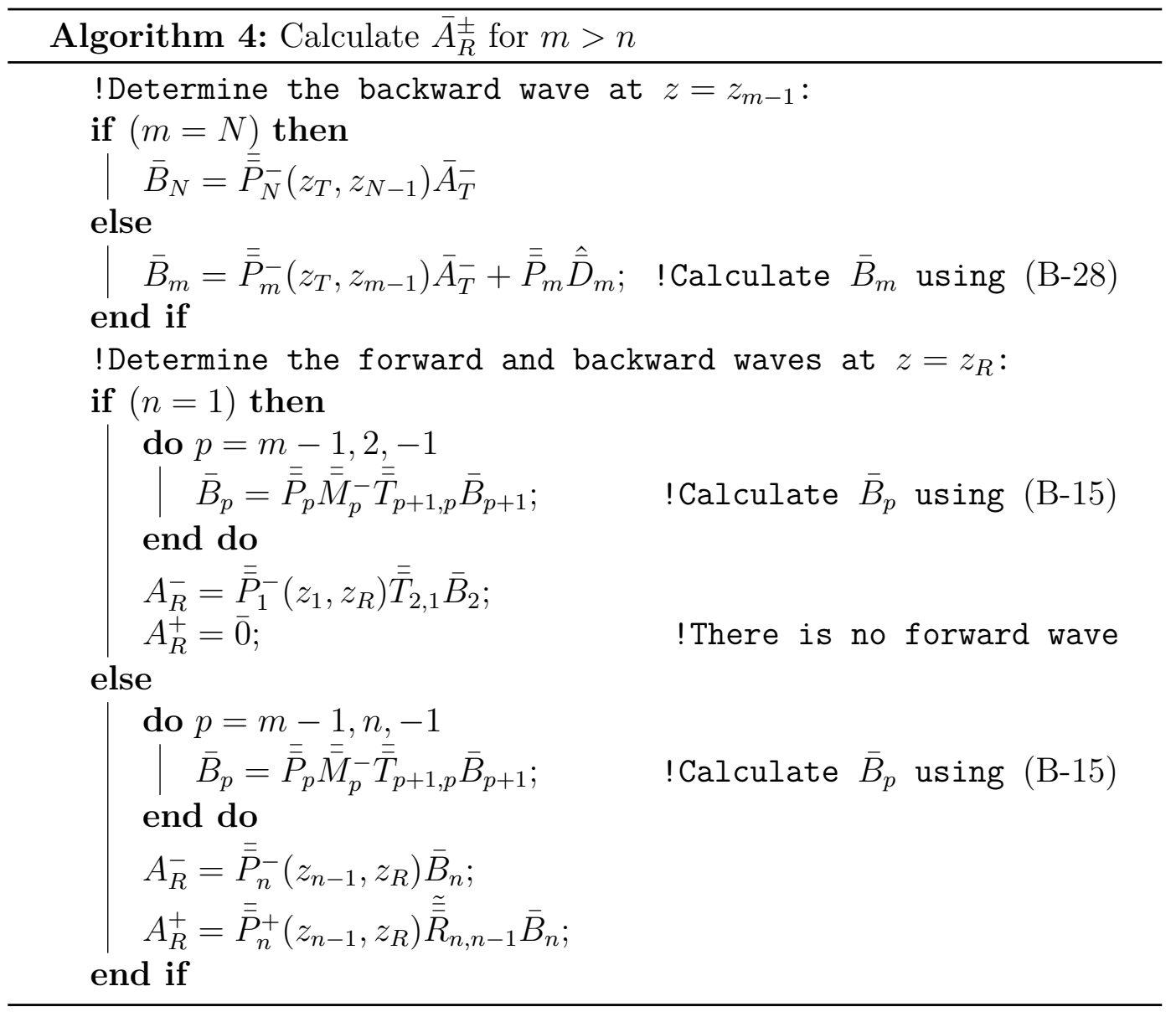

Figure B.5: Algorithm used to calculate the amplitudes of the forward and backward waves at $z=z_{R}$ (in region $n$ ) due to a source at $z=z_{T}$ (in region $m)$, for $m>n$. 


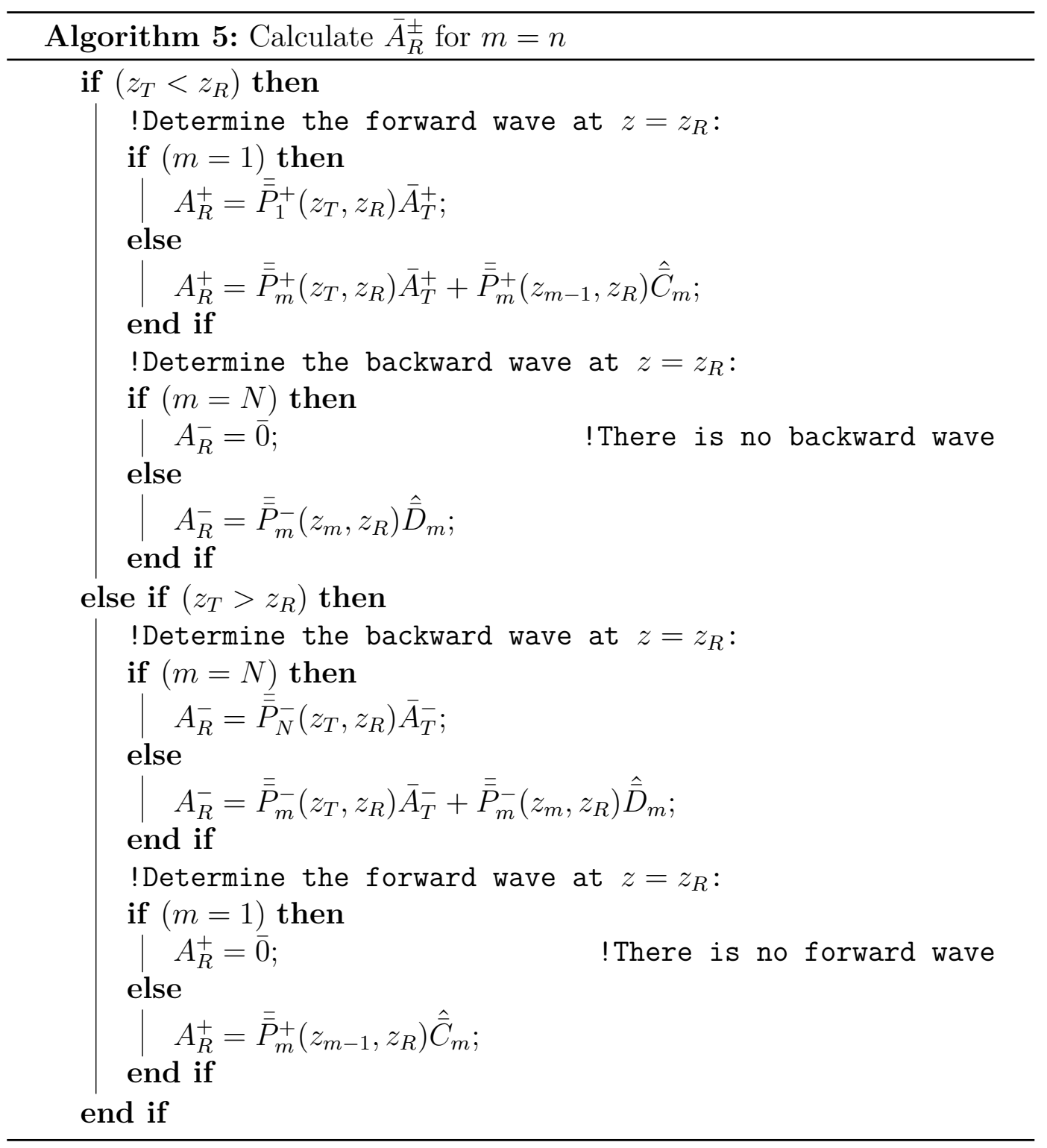

Figure B.6: Algorithm used to calculate the amplitudes of the forward and backward waves at $z=z_{R}$ due to a source at $z=z_{T}$ when these positions are in region $m=n$. 


\section{B.2}

\section{Geometrical Optics Approach}

In order to proper express the fields that a transmitter antenna at the plane $z=z_{T}$ generates at a receiver antenna at the plane $z=z_{R}$, here we use a geometrical optics approach to proper transfer the amplitudes $\overline{\bar{A}}_{T}^{ \pm}$of a source embedded in the axial region $m$ to an observation point placed at region $n$. In this section, we combine the GSM matrix derived at each axial junction to express the fields as a superposition of direct and multiple reflected rays by means of a convergent geometric series. We consider the same axially-layered structure used in last section (see Fig. B.1) and the appropriated modal field expansion in terms of $\exp \left( \pm i k_{z} z\right)$.

In the following analysis, we first consider that the observation point is at the same axial region of the source $(m=n)$ and also we assume that $z_{T}<z_{R}$, as illustrated in Fig. B.7. In our analysis, we will decompose the electromagnetic fields into forward and backward propagating waves in relation to the axial axis.

Initially, consider only the forward amplitudes of the source $\bar{A}_{T}^{+}$, and for simplicity, we will seek just to the forward amplitudes at the receiver position on the plane $z=z_{T}$. For this situation, the fields excited by the source amplitude $\bar{A}_{T}^{+}$will propagate from $z=z_{T}$ to $z=z_{R}$, as shown in Fig. B.8. Additionally, the associated field will also propagates forwardly, and will experience a reflection at $z=z_{m}$, and will propagates backwardly from $z=z_{m}$ to $z=z_{m-1}$. At this step, this field will suffer a new reflection at $z=z_{m-1}$, changing its propagating direction again, becoming a forward wave. This wave propagates from $z=z_{m-1}$ to $z=z_{R}$. This propagation process continues endlessly. In a compact manner, we can mathematically describes the forward amplitude at $z=z_{R}$ due $\bar{A}_{T}^{+}$as

$$
\bar{A}_{R(T+)}^{+}=\bar{a}+\overline{\bar{b}} \bar{a}+\overline{\bar{b}} \overline{\bar{b}} \bar{a}+\overline{\bar{b}} \overline{\bar{b}} \overline{\bar{b}} \bar{a}+\ldots,
$$
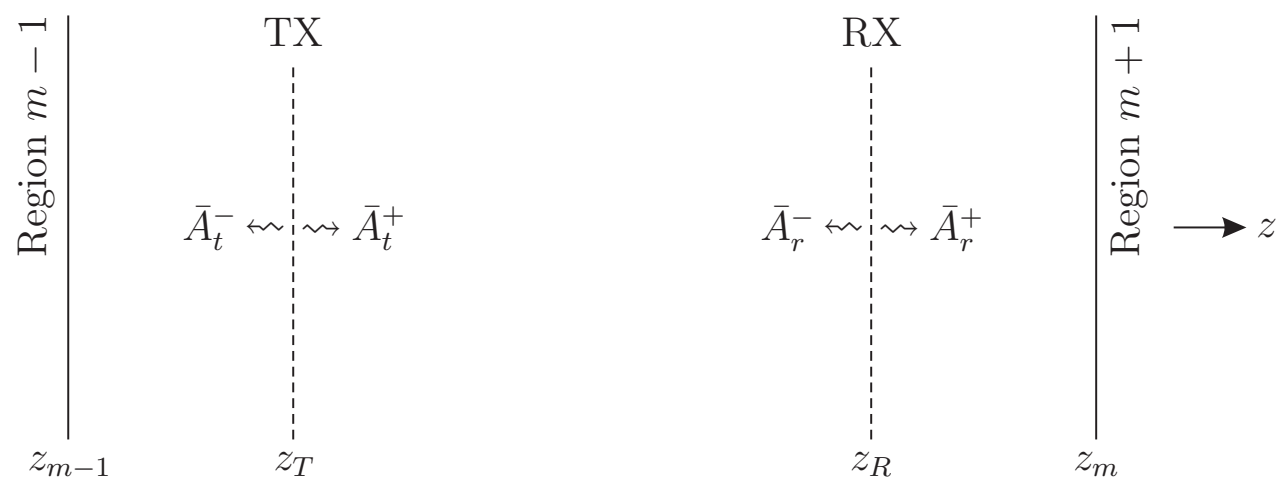

Figure B.7: The source and the observation point in the region $m=n$. 


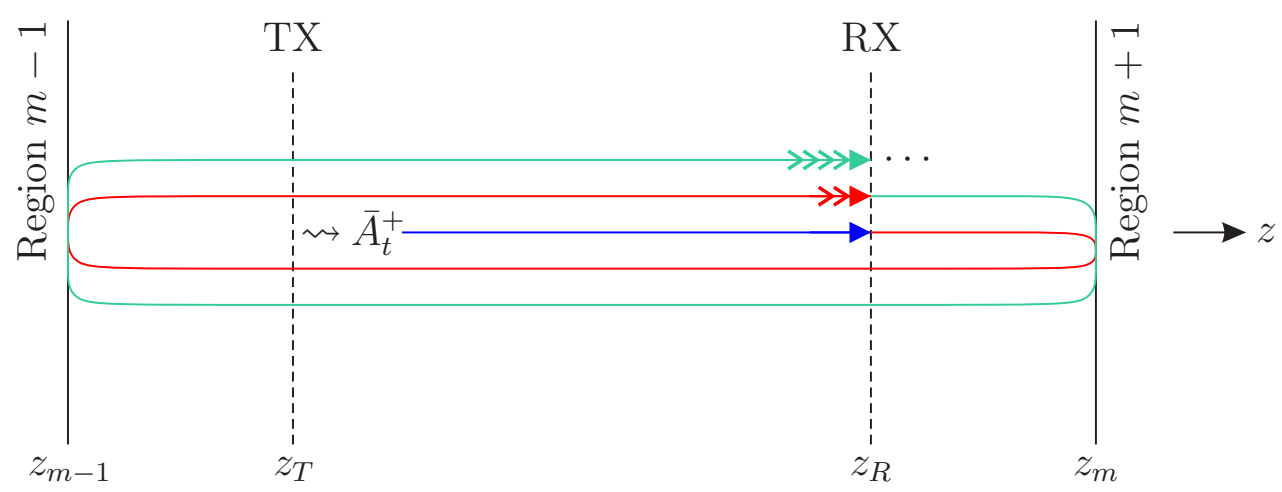

Figure B.8: The contribution of the forward source amplitudes to the received fields in the forward direction.

where

$$
\begin{gathered}
\bar{a}=\overline{\bar{P}}_{m}^{+}\left(z_{T}, z_{R}\right) \bar{A}_{T}^{+} \\
\overline{\bar{b}}=\overline{\bar{P}}_{m}^{+}\left(z_{m-1}, z_{R}\right) \tilde{\overline{\bar{R}}}_{m, m-1} \overline{\bar{P}}_{m}^{-}\left(z_{m}, z_{m-1}\right) \tilde{\overline{\bar{R}}}_{m, m+1} \overline{\bar{P}}_{m}^{+}\left(z_{R}, z_{m}\right) .
\end{gathered}
$$

We have introduced the diagonal propagation matrices $\overline{\bar{P}}_{j}^{ \pm}\left(z^{\prime}, z\right)$, that relates the axial propagation along the axial positions $z^{\prime}$ to $z$ on the region $m$. The elements for a mode $p$ is given by

$$
\left.\overline{\bar{P}}_{j}^{ \pm}\left(z^{\prime}, z\right)\right|_{p p}=e^{ \pm i k_{p z}\left(z-z^{\prime}\right)}
$$

where $k_{p z}$ is the axial wavenumber of the $p$ th mode of the region $j$. Note that $\overline{\bar{P}}_{j}^{+}\left(z^{\prime}, z\right)=\overline{\bar{P}}_{j}^{-}\left(z, z^{\prime}\right)^{4}$. The generalized reflections matrices $\tilde{\overline{\bar{R}}}_{m, \pm m}$ comprise all the reflection that the fields are subject in the forward/backward direction.

From the definitions in (B-33), it is clear that amplitude entries of $\overline{\bar{P}}_{j}^{ \pm}\left(z^{\prime}, z\right)$ are limited to 1 for $z^{\prime} \lessgtr z$. From this, we can verify that $\lim _{j \rightarrow \infty} \overline{\bar{b}}^{j}=$ $\overline{\overline{0}}$, and the geometrical series $\overline{\bar{I}}+\overline{\bar{b}}+\overline{\bar{b}}^{2}+\overline{\bar{b}}^{3}+\ldots$ converges to $(\overline{\bar{I}}-\overline{\bar{b}})^{-1}[116$, p. 15$]$. Then,

$$
\begin{array}{r}
\bar{A}_{R(T+)}^{+}=\left[\overline{\bar{I}}-\overline{\bar{P}}_{m}^{+}\left(z_{m-1}, z_{R}\right) \tilde{\overline{\bar{R}}}_{m, m-1} \overline{\bar{P}}_{m}^{-}\left(z_{m}, z_{m-1}\right) \tilde{\overline{\bar{R}}}_{m, m+1} \overline{\bar{P}}_{m}^{+}\left(z_{R}, z_{m}\right)\right]^{-1} \\
\times \overline{\bar{P}}_{m}^{+}\left(z_{T}, z_{R}\right) \bar{A}_{T}^{+} .
\end{array}
$$

In order to simplify the notation, we will introduce the generalized multiple reflection matrix $\tilde{\bar{M}}_{j}^{+}(z)$ at the axial position $z$, given by

$$
\tilde{\overline{\bar{M}}}_{j}^{+}(z)=\left[\overline{\bar{I}}-\tilde{\overline{\bar{D}}}_{j}^{+}(z, z)\right]^{-1}
$$

where

$$
\tilde{\overline{\bar{D}}}_{j}^{+}\left(z^{\prime}, z\right)=\overline{\bar{P}}_{j}^{+}\left(z_{j-1}, z\right) \tilde{\overline{\bar{R}}}_{j, j-1} \overline{\bar{P}}_{j}^{-}\left(z_{j}, z_{j-1}\right) \tilde{\overline{\bar{R}}}_{j, j+1} \overline{\bar{P}}_{j}^{+}\left(z^{\prime}, z_{j}\right) .
$$

${ }^{4}$ At first glance, this may seem like a redundant notation. However, we prefer to use this form because it preserves the sense of forward/backward $( \pm)$ propagation. 
The matrix $\tilde{\overline{\bar{D}}}_{j}^{+}\left(z^{\prime}, z\right)$ can be understood as an operation that transfer a forward propagating wave from $z^{\prime}$ to $z$ experiencing a double reflection in the borders of region $j$. The matrix $\stackrel{\bar{M}}{j}_{j}^{+}(z)$, on the other hand, can be interpreted as the sum of all multiple reflections that a forward field shows in the region $j$, with reference to the axial position $z$.

Using the above definitions, we can rewrite the contribution of the forward source amplitudes to the received fields in the forward direction as

$$
\bar{A}_{R(T+)}^{+}=\tilde{\bar{M}}_{m}^{+}\left(z_{R}\right) \overline{\bar{P}}_{m}^{+}\left(z_{T}, z_{R}\right) \bar{A}_{T}^{+} .
$$

The physical interpretation of the above equation can be done from right to left: the forward source fields propagate to the receiver position, but it are subject to the multiple reflections at the junctions of regions $m \rightarrow m+1$ and $m \rightarrow m-1$.

Now, we consider the excitation of the backward source amplitude $\bar{A}_{T}^{-}$. The down-going associated fields will also generate a forward field at $z=z_{R}$. A sketch of this situation is shown in Fig. B.9. For this situation, the fields excited by $\bar{A}_{T}^{-}$will propagate in the backward direction from $z_{T}$ to $z_{m-1}$. This field suffer a reflection at $z_{m-1}$, change its propagation direction, becoming a forward wave that propagates from $z_{m-1}$ to the $z_{R}$. At this point, this forward wave will experience multiple reflections in the region $j$. In a compact fashion, we can write the forward amplitude at $z_{R}$ due $\bar{A}_{T}^{-}$as

$$
\bar{A}_{R(T-)}^{+}=\tilde{\overline{\bar{M}}}_{m}^{+}\left(z_{R}\right) \tilde{\overline{\bar{U}}}_{m}^{-}\left(z_{T}, z_{R}\right) \bar{A}_{T}^{-},
$$

in which we define a matrix that describes a unique reflection of the backward fields given by

$$
\tilde{\overline{\bar{U}}}_{j}^{-}\left(z^{\prime}, z\right)=\overline{\bar{P}}_{j}^{+}\left(z_{j-1}, z\right) \tilde{\overline{\bar{R}}}_{j, j-1} \overline{\bar{P}}_{j}^{-}\left(z^{\prime}, z_{j-1}\right) .
$$

The combination of (B-37) and (B-38) gives us the complete forward

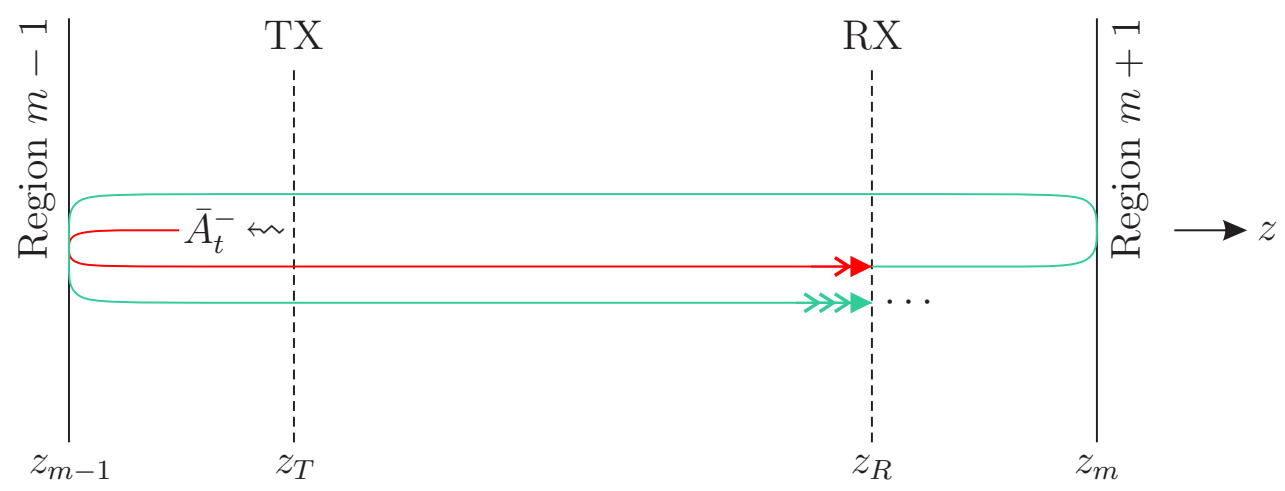

Figure B.9: The contribution of the backward source amplitudes to the received fields in the forward direction. 
received field amplitudes at $z_{R}$, i.e.,

$$
\bar{A}_{R}^{+}=\tilde{\bar{M}}_{m}^{+}\left(z_{R}\right)\left[\overline{\bar{P}}_{m}^{+}\left(z_{T}, z_{R}\right) \bar{A}_{T}^{+}+\tilde{\bar{U}}_{m}^{-}\left(z_{T}, z_{R}\right) \bar{A}_{T}^{-}\right], \text {for } m=n \text { and } z_{T}<z_{R}
$$

In order to find the backward fields at the receiver position $z_{R}$, we first consider the contribution of the forward source amplitudes to the received fields in the backward direction, as illustrated in Fig. B.10. By going through an analysis similar that to used above, we can write the backward amplitude at $z_{R}$ due $\bar{A}_{T}^{+}$as

$$
\bar{A}_{R(T+)}^{-}=\tilde{\overline{\bar{M}}}_{m}^{-}\left(z_{R}\right) \tilde{\overline{\bar{U}}}_{m}^{+}\left(z_{T}, z_{R}\right) \bar{A}_{T}^{+},
$$

where the generalized multiple reflection matrix $\tilde{\bar{M}}_{j}^{-}(z)$ defined at the axial position $z$ is given by

$$
\tilde{\bar{M}}_{j}^{-}(z)=\left[\overline{\bar{I}}-\tilde{\overline{\bar{D}}}_{j}^{-}(z, z)\right]^{-1}
$$

The matrix $\tilde{\overline{\bar{D}}}_{j}^{-}\left(z^{\prime}, z\right)$ can be interpreted similarly to $\tilde{\overline{\bar{D}}}_{j}^{+}\left(z^{\prime}, z\right)$, but operating in the opposite direction. Now, $\tilde{\overline{\bar{D}}}_{j}^{-}\left(z^{\prime}, z\right)$ transfers a backward propagating wave from $z^{\prime}$ to $z$ experiencing a double reflection in the borders of region $j$, i.e.,

$$
\tilde{\overline{\bar{D}}}_{j}^{-}\left(z^{\prime}, z\right)=\overline{\bar{P}}_{j}^{-}\left(z_{j}, z\right) \tilde{\overline{\bar{R}}}_{j, j+1} \overline{\bar{P}}_{j}^{+}\left(z_{j-1}, z_{j}\right) \tilde{\overline{\bar{R}}}_{j, j-1} \overline{\bar{P}}_{j}^{-}\left(z^{\prime}, z_{j-1}\right),
$$

The matrix $\tilde{\overline{\bar{U}}}_{j}^{+}\left(z^{\prime}, z\right)$ is similar to $\tilde{\overline{\bar{U}}}_{j}^{-}\left(z^{\prime}, z\right)$, but, operate in the opposite direction, i.e.,

$$
\tilde{\overline{\bar{U}}}_{j}^{+}\left(z^{\prime}, z\right)=\overline{\bar{P}}_{j}^{-}\left(z_{j}, z\right) \tilde{\bar{R}}_{j, j+1} \overline{\bar{P}}_{j}^{+}\left(z^{\prime}, z_{j}\right) .
$$

Finally, we can write the contribution of the backward source amplitudes to the received fields in the backward direction (see the illustration shown in Fig. B.11) in the fashion of

$$
\bar{A}_{R(T-)}^{-}=\tilde{\overline{\bar{M}}}_{m}^{-}\left(z_{R}\right) \tilde{\overline{\bar{D}}}_{m}^{-}\left(z_{T}, z_{R}\right) \bar{A}_{T}^{-} .
$$

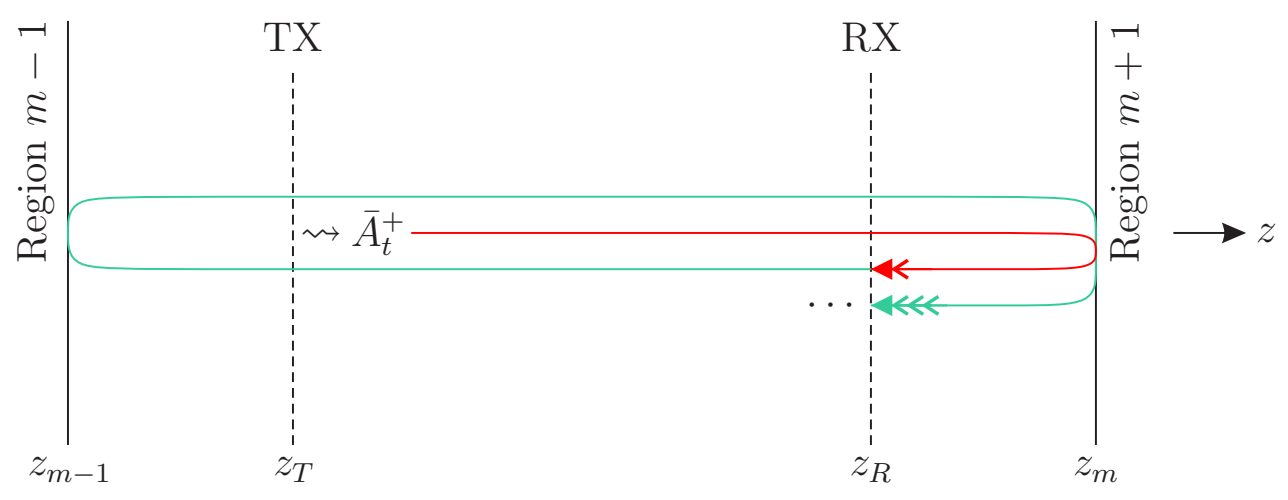

Figure B.10: The contribution of the forward source amplitudes to the received fields in the backward direction. 


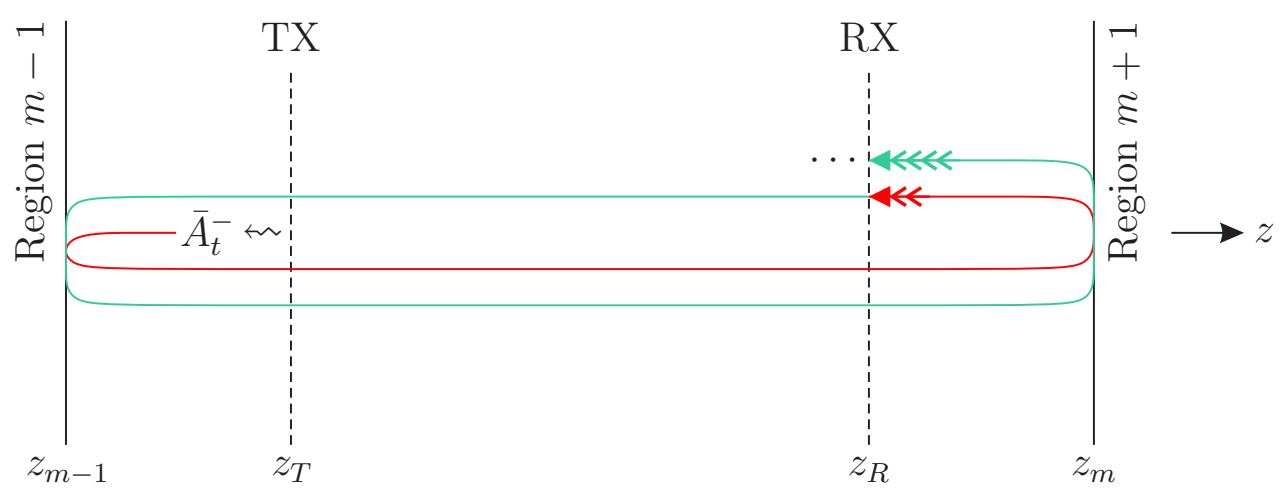

Figure B.11: The contribution of the backward source amplitudes to the received fields in the backward direction.

The combination of (B-41) and (B-45) give us the complete backward received field amplitudes at at $z_{R}$, i.e.,

$$
\bar{A}_{R}^{-}=\tilde{\bar{M}}_{m}^{-}\left(z_{R}\right)\left[\tilde{\overline{\bar{U}}}_{m}^{+}\left(z_{T}, z_{R}\right) \bar{A}_{T}^{+}+\tilde{\overline{\bar{D}}}_{m}^{-}\left(z_{T}, z_{R}\right) \bar{A}_{T}^{-}\right], \text {for } m=n \text { and } z_{T}<z_{R}
$$

Now we consider the case in which the source is at an axial region $m$, and the source is at a region $n$, for $m<n$ (and $z_{T}<z_{R}$ ). The first step is to transfer the source amplitudes $\bar{A}_{T}^{ \pm}$from $z=z_{T}$ to $z=z_{m}$, i.e.,

$$
\left.\bar{A}_{m}^{+}\right|_{z=z_{m}}=\tilde{\bar{M}}_{m}^{+}\left(z_{m}\right)\left[\overline{\bar{P}}_{m}^{+}\left(z_{T}, z_{m}\right) \bar{A}_{T}^{+}+\tilde{\overline{\bar{U}}}_{m}^{-}\left(z_{T}, z_{m}\right) \bar{A}_{T}^{-}\right] .
$$

Note that we have used (B-40) and (B-46), but we replaced the receiver position by $z_{m}$. Next, we can use the generalized transmission matrix from the regions $m$ to $n$, denoted by $\tilde{\overline{\bar{T}}}_{m n}$, to get the forward fields in region $n$ at $z=z_{n-1}$ as

$$
\left.\bar{A}_{n}^{+}\right|_{z=z_{n-1}}=\left.\stackrel{\overline{\bar{T}}}{m n}_{\bar{A}}^{+}\right|_{z=z_{m}} .
$$

Now, we need to transfer $\left.\bar{A}_{n}^{+}\right|_{z=z_{n-1}}$ to the plane of the receiver at $z_{R}$ using

$$
\begin{aligned}
& \bar{A}_{R}^{+}=\left.\overline{\bar{P}}_{n}^{+}\left(z_{n-1}, z_{R}\right) \bar{A}_{n}^{+}\right|_{z=z_{n-1}}, \\
& \bar{A}_{R}^{-}=\left.\tilde{\bar{U}}_{n}^{+}\left(z_{n-1}, z_{R}\right) \bar{A}_{n}^{+}\right|_{z=z_{n-1}},
\end{aligned}
$$

or, combining the preceding results, we have

$$
\begin{aligned}
& \bar{A}_{R}^{+}=\overline{\bar{P}}_{n}^{+}\left(z_{n-1}, z_{R}\right) \tilde{\overline{\bar{T}}}_{m n} \tilde{\overline{\bar{M}}}_{m}^{+}\left(z_{m}\right)\left[\overline{\bar{P}}_{m}^{+}\left(z_{T}, z_{m}\right) \bar{A}_{T}^{+}+\tilde{\overline{\bar{U}}}_{m}^{-}\left(z_{T}, z_{m}\right) \bar{A}_{T}^{-}\right], \\
& \bar{A}_{R}^{-}=\tilde{\overline{\bar{U}}}_{n}^{+}\left(z_{n-1}, z_{R}\right) \tilde{\overline{\bar{T}}}_{m n} \tilde{\bar{M}}_{m}^{+}\left(z_{m}\right)\left[\overline{\bar{P}}_{m}^{+}\left(z_{T}, z_{m}\right) \bar{A}_{T}^{+}+\tilde{\overline{\bar{U}}}_{m}^{-}\left(z_{T}, z_{m}\right) \bar{A}_{T}^{-}\right],
\end{aligned}
$$

valid for $m<n$.

The above formulation consider that the $z_{T}<z_{R}$ and for that reason $m<n$. Case the source in an axial position greater than the observation point, 
we can analyze the problem considering the inversion of the $z$-axis direction. With the mapping $\hat{z} \rightarrow-\hat{z}$, we can preserve the above definitions just replacing $z_{m} \rightarrow z_{m-1}, z_{n-1} \rightarrow z_{n}$, and all superscripts $\pm \rightarrow \mp$. Performing these changes in (B-40), (B-46), (B-51) and (B-52) we can derive general expressions for $\bar{A}_{R}^{ \pm}$ as

$$
\begin{aligned}
& \bar{A}_{R}^{ \pm}=\overline{\bar{P}}_{n}^{ \pm}\left(z_{n}^{\mp}, z_{R}\right) \tilde{\overline{\bar{T}}}_{m n} \tilde{\overline{\bar{M}}}_{m}^{ \pm}\left(z_{m}^{ \pm}\right)\left[\overline{\bar{P}}_{m}^{ \pm}\left(z_{T}, z_{m}^{ \pm}\right) \bar{A}_{T}^{ \pm}+\tilde{\overline{\bar{U}}}_{m}^{\mp}\left(z_{T}, z_{m}^{ \pm}\right) \bar{A}_{T}^{\mp}\right], \\
& \bar{A}_{R}^{\mp}=\tilde{\overline{\bar{U}}}_{n}^{ \pm}\left(z_{n}^{\mp}, z_{R}\right) \tilde{\overline{\bar{T}}}_{m n} \tilde{\overline{\bar{M}}}_{m}^{ \pm}\left(z_{m}^{ \pm}\right)\left[\overline{\bar{P}}_{m}^{ \pm}\left(z_{T}, z_{m}^{ \pm}\right) \bar{A}_{T}^{ \pm}+\tilde{\overline{\bar{U}}}_{m}^{\mp}\left(z_{T}, z_{m}^{ \pm}\right) \bar{A}_{T}^{\mp}\right],
\end{aligned}
$$

valid for $m \lessgtr n$, and

$$
\begin{aligned}
& \bar{A}_{R}^{ \pm}=\tilde{\overline{\bar{M}}}_{m}^{ \pm}\left(z_{R}\right)\left[\overline{\bar{P}}_{m}^{ \pm}\left(z_{T}, z_{R}\right) \bar{A}_{T}^{ \pm}+\tilde{\overline{\bar{U}}}_{m}^{\mp}\left(z_{T}, z_{R}\right) \bar{A}_{T}^{\mp}\right], \\
& \bar{A}_{R}^{\mp}=\tilde{\bar{M}}_{m}^{\mp}\left(z_{R}\right)\left[\tilde{\overline{\bar{U}}}_{m}^{ \pm}\left(z_{T}, z_{R}\right) \bar{A}_{T}^{ \pm}+\tilde{\overline{\bar{D}}}_{m}^{\mp}\left(z_{T}, z_{R}\right) \bar{A}_{T}^{\mp}\right],
\end{aligned}
$$

for $m=n$ and $z_{T} \lessgtr z_{R}$. In order to improve the symmetry of our equations, notice that in the above we have introduced $z_{j}^{ \pm}$such as

$$
\begin{aligned}
& z_{j}^{+}=z_{j}, \\
& z_{j}^{-}=z_{j-1} .
\end{aligned}
$$

It is important to be noted that the field matching between the source and the observation point is done apart from the source excitation. This allow us to reuse all transfer matrices in (B-53), (B-54), (B-55) and (B-56) case we want to analyze the response of different types of sources, i.e., we can store the local and global scattering matrices of the structure and the field for many transmitters and receivers can be computed efficiently [26].

\section{B.2.1}

\section{Generalized Reflection Matrices}

A geometrical optics interpretation is evident in both equations (B-11) and (B-17). For example, the generalized reflection of the up-going wave at $z=z_{n}^{+}$comes from the local reflection $\overline{\bar{R}}_{n, n+1}$ plus a term accounting the multiple reflections in all regions above $n$. This second term can be written as the transmission from region $n \rightarrow n+1$, the forward propagation from $z_{n+1}^{-} \rightarrow z_{n+1}^{+}$, and the generalized reflection from region $n+1 \rightarrow n+2$, the backward propagation from $z_{n+1}^{+} \rightarrow z_{n+1}^{-}$. Further, we need to consider all 
multiple reflections in region $n+1$, by using $\overline{\bar{M}}_{n+1}^{+}(z)^{5}$. Finally, we need to transfer the wave back from region $n+1 \rightarrow n$ using $\overline{\bar{T}}_{n+1, n}$. We can write the above mathematically as

$$
\tilde{\overline{\bar{R}}}_{n, n+1}=\overline{\bar{R}}_{n, n+1}+\overline{\bar{T}}_{n+1, n} \overline{\bar{P}}_{n+1} \tilde{\overline{\bar{R}}}_{n+1, n+2} \overline{\bar{P}}_{n+1} \overline{\bar{M}}_{n+1}^{+}\left(z_{n}^{+}\right) \overline{\bar{T}}_{n, n+1}
$$

where the forward multiple reflection matrix $\overline{\bar{M}}_{j}^{+}(z)$ satisfies

$$
\begin{gathered}
\overline{\bar{M}}_{j}^{+}(z)=\left[\overline{\bar{I}}-\overline{\bar{D}}_{j}^{+}(z, z)\right]^{-1}, \text { and } \\
\overline{\bar{D}}_{j}^{+}\left(z^{\prime}, z\right)=\overline{\bar{P}}_{j}^{+}\left(z_{j}^{-}, z\right) \overline{\bar{R}}_{j, j-1} \overline{\bar{P}}_{j}^{-}\left(z_{j}^{+}, z_{j}^{-}\right) \tilde{\overline{\bar{R}}}_{j, j+1} \overline{\bar{P}}_{j}^{+}\left(z^{\prime}, z_{j}^{+}\right) .
\end{gathered}
$$

Noting that $\overline{\bar{M}}_{n+1}^{+}\left(z_{n}^{+}\right)=\overline{\bar{M}}_{n+1}^{+}$, comparing (B-59) with (B-11) we can see that both equations are identical.

It is interesting to be noted that in the above analysis we have included the multiple reflection term just before the down-going wave be transmitted from region $n+1 \rightarrow n$ at $z=z_{n}^{+}$. Generally speaking, the inclusion of the multiple reflection term is not strictest to this position, and could be consider at any point inside region $n+1$. In this way, any of the above forms are equivalent to (B-59):

$$
\begin{aligned}
\tilde{\overline{\bar{R}}}_{n, n+1} & =\overline{\bar{R}}_{n, n+1}+\overline{\bar{T}}_{n+1, n} \overline{\bar{M}}_{n+1}^{+}\left(z_{n}^{+}\right) \overline{\bar{P}}_{n+1} \tilde{\overline{\bar{R}}}_{n+1, n+2} \overline{\bar{P}}_{n+1} \overline{\bar{T}}_{n, n+1}, \\
& =\overline{\bar{R}}_{n, n+1}+\overline{\bar{T}}_{n+1, n} \overline{\bar{P}}_{n+1} \overline{\bar{M}}_{n+1}^{+}\left(z_{n+1}^{+}\right) \tilde{\overline{\bar{R}}}_{n+1, n+2} \overline{\bar{P}}_{n+1} \overline{\bar{T}}_{n, n+1}, \\
& =\overline{\bar{R}}_{n, n+1}+\overline{\bar{T}}_{n+1, n} \overline{\bar{P}}_{n+1} \tilde{\overline{\bar{R}}}_{n+1, n+2} \overline{\bar{M}}_{n+1}^{+}\left(z_{n+1}^{+}\right) \overline{\bar{P}}_{n+1} \overline{\bar{T}}_{n, n+1} .
\end{aligned}
$$

Similar results hold also for $\tilde{\overline{\bar{R}}}_{n, n-1}$, and we can be easily verify that the changing the subscripts and superscripts $+\rightarrow-$ in (B-59) results in (B-17), where

$$
\begin{gathered}
\overline{\bar{M}}_{j}^{-}(z)=\left[\overline{\bar{I}}-\overline{\bar{D}}_{j}^{-}(z, z)\right]^{-1} \text {, and } \\
\overline{\bar{D}}_{j}^{-}\left(z^{\prime}, z\right)=\overline{\bar{P}}_{j}^{-}\left(z_{j}^{+}, z\right) \overline{\bar{R}}_{j, j+1} \overline{\bar{P}}_{j}^{+}\left(z_{j}^{-}, z_{j}^{+}\right) \tilde{\bar{R}}_{j, j-1} \overline{\bar{P}}_{j}^{-}\left(z^{\prime}, z_{j}^{-}\right) .
\end{gathered}
$$

The same is true for the equivalent forms shown in (B-62).

\section{B.2.2}

\section{Generalized Transmission Matrices}

The forward propagating amplitude in region $j+1$ at $z_{j}^{+}$can be written as

${ }^{5}$ Note that we could instead employ the generalized multiple reflection matrix $\tilde{\bar{M}}_{n+1}^{+}(z)$. However, in this case we will couple the generalized forward and backward reflections, i.e., $\tilde{\overline{\bar{R}}}_{n, n+1}$ and $\tilde{\overline{\bar{R}}}_{n, n-1}$. Here we prefer to employ $\overline{\bar{M}}_{n+1}^{+}(z)$ and fields in the shape of those in (B-4) in order to express $\tilde{\overline{\bar{R}}}_{n, n+1}$ in terms of $\tilde{\overline{\bar{R}}}_{n+1, n+2}$. 


$$
\bar{A}_{j+1,-}^{+}=\overline{\bar{M}}_{j+1}^{+}\left(z_{j}^{+}\right) \overline{\bar{T}}_{j, j+1} \bar{A}_{j,+}^{+},
$$

in which allow us to define the generalized transmission matrix between the region $j$ to $j+1$ as

$$
\tilde{\overline{\bar{T}}}_{j, j+1}=\overline{\bar{M}}_{j+1}^{+}\left(z_{j}^{+}\right) \overline{\bar{T}}_{j, j+1}
$$

At $z=z_{j+1}^{+}$, the forward amplitude in region $j+2$ can be written as the forward amplitude in region $j+1$ at $z_{j+1}^{-}$propagated up to $z_{j+1}^{+}$and transmitted from region $j+1$ to $j+2$, i.e.,

$$
\begin{aligned}
\bar{A}_{j+2,-}^{+} & =\tilde{\overline{\bar{T}}}_{j+1, j+2} \overline{\bar{P}}_{j+1}^{+}\left(z_{j+1}^{-}, z_{j+1}^{+}\right) \bar{A}_{j+1,-}^{+} \\
& =\tilde{\overline{\bar{T}}}_{j+1, j+2} \overline{\bar{P}}_{j+1}^{+}\left(z_{j+1}^{-}, z_{j+1}^{+}\right) \tilde{\overline{\bar{T}}}_{j, j+1} \bar{A}_{j,+}^{+} \\
& =\tilde{\overline{\bar{T}}}_{j, j+2} \bar{A}_{j,+}^{+}
\end{aligned}
$$

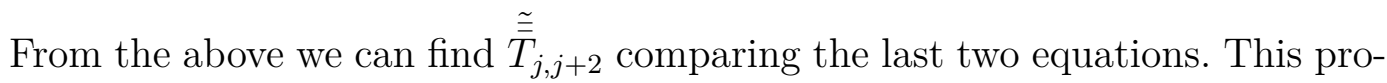
cedure can be used to derive the remaining generalized transmission matrices

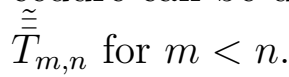

The backward propagating amplitude in region $j-1$ at $z_{j}^{-}$can be written as

$$
\bar{A}_{j-1,+}^{-}=\overline{\bar{M}}_{j-1}^{-}\left(z_{j}^{-}\right) \overline{\bar{T}}_{j, j-1} \bar{A}_{j,-}^{-},
$$

in which allow us to define the generalized transmission matrix between the region $j$ to $j+1$ as

$$
\stackrel{\overline{\bar{T}}}{j, j-1}=\overline{\bar{M}}_{j-1}^{-}\left(z_{j}^{-}\right) \overline{\bar{T}}_{j, j-1} .
$$

At $z=z_{j-1}^{-}$, the backward amplitude in region $j-2$ can be written as the backward amplitude in region $j-1$ at $z_{j-1}^{+}$propagated up to $z_{j-1}^{-}$and transmitted from region $j-1$ to $j-2$, i.e.,

$$
\begin{aligned}
\bar{A}_{j-2,+}^{-} & =\stackrel{\overline{\bar{T}}}{j-1, j-2} \overline{\bar{P}}_{j-1}^{-}\left(z_{j-1}^{+}, z_{j-1}^{-}\right) \bar{A}_{j-1,+}^{-} \\
& =\tilde{\overline{\bar{T}}}_{j-1, j-2} \overline{\bar{P}}_{j-1}^{-}\left(z_{j-1}^{+}, z_{j-1}^{-}\right) \tilde{\overline{\bar{T}}}_{j, j-1} \bar{A}_{j,-}^{-} \\
& =\tilde{\overline{\bar{T}}}_{j, j-2} \bar{A}_{j,-}^{-}
\end{aligned}
$$

From the above we can find $\tilde{\overline{\bar{T}}}_{j, j-2}$ comparing the last two equations. This procedure can be used to derive the remaining generalized transmission matrices $\tilde{\overline{\bar{T}}}_{m, n}$ for $m>n$.

The above recursive formulas can be generalized as

$$
\begin{gathered}
\tilde{\overline{\bar{T}}}_{j, j \pm 1}=\overline{\bar{M}}_{j \pm 1}^{ \pm} \overline{\bar{T}}_{j, j \pm 1}, \text { and } \\
\tilde{\bar{T}}_{m, n}=\left(\prod_{j=\mp n}^{m \pm 2} \tilde{\overline{\bar{T}}}_{\mp j \mp 1, \mp j} \overline{\bar{P}}_{\mp j \mp 1}\right) \tilde{\overline{\bar{T}}}_{m, m \pm 1},
\end{gathered}
$$


for $m \lessgtr n$, where $\prod_{j=1}^{J} \overline{\bar{A}}_{j}$ means $\overline{\bar{A}}_{1} \overline{\bar{A}}_{2} \ldots \overline{\bar{A}}_{J}$.

\section{B.2.3}

\section{Further Remarks}

We can use the above expressions for sources and observation points at any positions. However, we can simplify (B-51) and (B-52) for spacial case. First, we can use the following helpful definitions for regions 1 and $N_{z}$ :

$$
\begin{gathered}
\tilde{\overline{\bar{U}}}_{1}^{-}\left(z^{\prime}, z\right)=\tilde{\overline{\bar{D}}}_{1}^{ \pm}\left(z^{\prime}, z\right)=\overline{\overline{0}}, \\
\tilde{\overline{\bar{U}}}_{N}^{+}\left(z^{\prime}, z\right)=\tilde{\overline{\bar{D}}}_{N}^{ \pm}\left(z^{\prime}, z\right)=\overline{\overline{0}}, \\
\tilde{\overline{\bar{M}}}_{1}^{ \pm}(z)=\overline{\bar{M}}_{1}^{ \pm}(z)=\overline{\bar{I}} \\
\tilde{\overline{\bar{M}}}_{N}^{ \pm}(z)=\overline{\bar{M}}_{N}^{ \pm}(z)=\overline{\bar{I}} .
\end{gathered}
$$

If The GSM matrix is normalized such as $\overline{\bar{S}} \overline{\bar{S}}=\overline{\bar{I}}$, it allow us to write

$$
\begin{gathered}
\overline{\bar{R}}_{j, j \pm 1}=\left[\overline{\bar{R}}_{j, j \pm 1}\right]^{t}, \\
\overline{\bar{T}}_{j, j+1}=\left[\overline{\bar{T}}_{j+1, j}\right]^{t} .
\end{gathered}
$$

Additionally, we can easy verify that

$$
\tilde{\overline{\bar{R}}}_{j, j \pm 1}=\left[\tilde{\overline{\bar{R}}}_{j, j \pm 1}\right]^{t} \text {. }
$$

\section{B.3}

\section{Comparison of Formulations}

The matrices in (B-7) are related to the ones in (B-33) via

$$
\overline{\bar{P}}_{j}^{ \pm}\left(z_{j}^{\mp}, z_{j}^{ \pm}\right)=\overline{\bar{P}}_{j} .
$$

Notice also that the generalized multiple reflections matrices in (B-35) and (B-42) are closely related to (B-10) and (B-16) such as

$$
\tilde{\overline{\bar{M}}}_{j}^{ \pm}\left(z_{j}^{\mp}\right)=\tilde{\overline{\bar{M}}}_{j}^{ \pm}\left(z_{j \mp 1}^{ \pm}\right)=\tilde{\overline{\bar{M}}}_{j}^{ \pm} .
$$

Similar relations also hold for $\overline{\bar{M}}_{j}^{ \pm}(z)$ (without the tilde). Consequently, after a few simplifications, we can show (B-55) and (B-56) can be represented in the same manner as described in the algorithm shown in Fig. B.6 for $m=n$.

For $m<n$ we can verify that the forward propagation of $\bar{A}_{j+1,-}^{+}$from $z_{j+1}^{-}$to $z_{j+1}^{+}$in (B-65) results in (B-9), i.e., $\bar{A}_{j+1}=\overline{\bar{P}}_{j+1} \bar{A}_{j+1,-}^{+}$. Similarly, for $m>n$ we can verify that the backward propagation of $\bar{A}_{j-1,+}^{-}$from $z_{j-1}^{+}$to $z_{j-1}^{-}$in (B-70) results in (B-15), i.e., $\bar{B}_{j-1}=\overline{\bar{P}}_{j-1} \bar{A}_{j-1,+}^{-}$. 
Accordingly, we can conclude that (B-53) and (B-54) are the same as those calculated by the a $\underset{\sim}{\operatorname{T}}$ gorithms shown in Fig. B.4 and Fig. B.5 under the

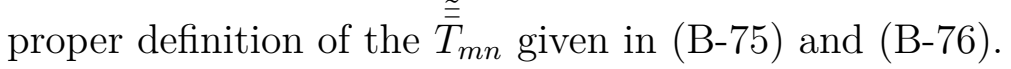

In our numerical calculations we have verified the above reported equivalence between the modal received amplitudes derived using the algorithm described in Fig B.2 and the amplitudes derived by means of (B-54), (B-53), (B-55) and (B-56). By this reason, all results shown in this Dissertation where calculated by using the second formulation due to its symmetry that enable us to relate the source to the observation point using one simple step. 


\section{C \\ Local Toroidal Coordinate System}

Consider the curved, circular cross section waveguide shown in Fig. C.1. This structure is centered at the origin of the Cartesian coordinates $(X, Y, Z)$, and has a constant curvature $R^{-1}$. A local toroidal coordinate system is defined through the transversal polar parameters $\rho$ and $\phi$, and by the bend axial coordinate $\zeta$. We will assume that the curvature radius $R$ is larger than the maximum required $\rho$. Denoting the toroidal angle as $\Phi=\zeta / R$, and the poloidal angle such as $\phi+\psi$, as shown in Fig. C.1, the torus transformation of the coordinates is given by

$$
\begin{aligned}
& X=(R-\rho \cos (\phi+\psi)) \cos \left(\frac{\zeta}{R}\right), \\
& Y=(R-\rho \cos (\phi+\psi)) \sin \left(\frac{\zeta}{R}\right), \\
& Z=\rho \sin (\phi+\psi) .
\end{aligned}
$$

As the coordinate system $(\rho, \phi, \zeta)$ is clearly orthogonal, the metric coefficients can be calculated by using the formulas in [143, pp. 47-50], such

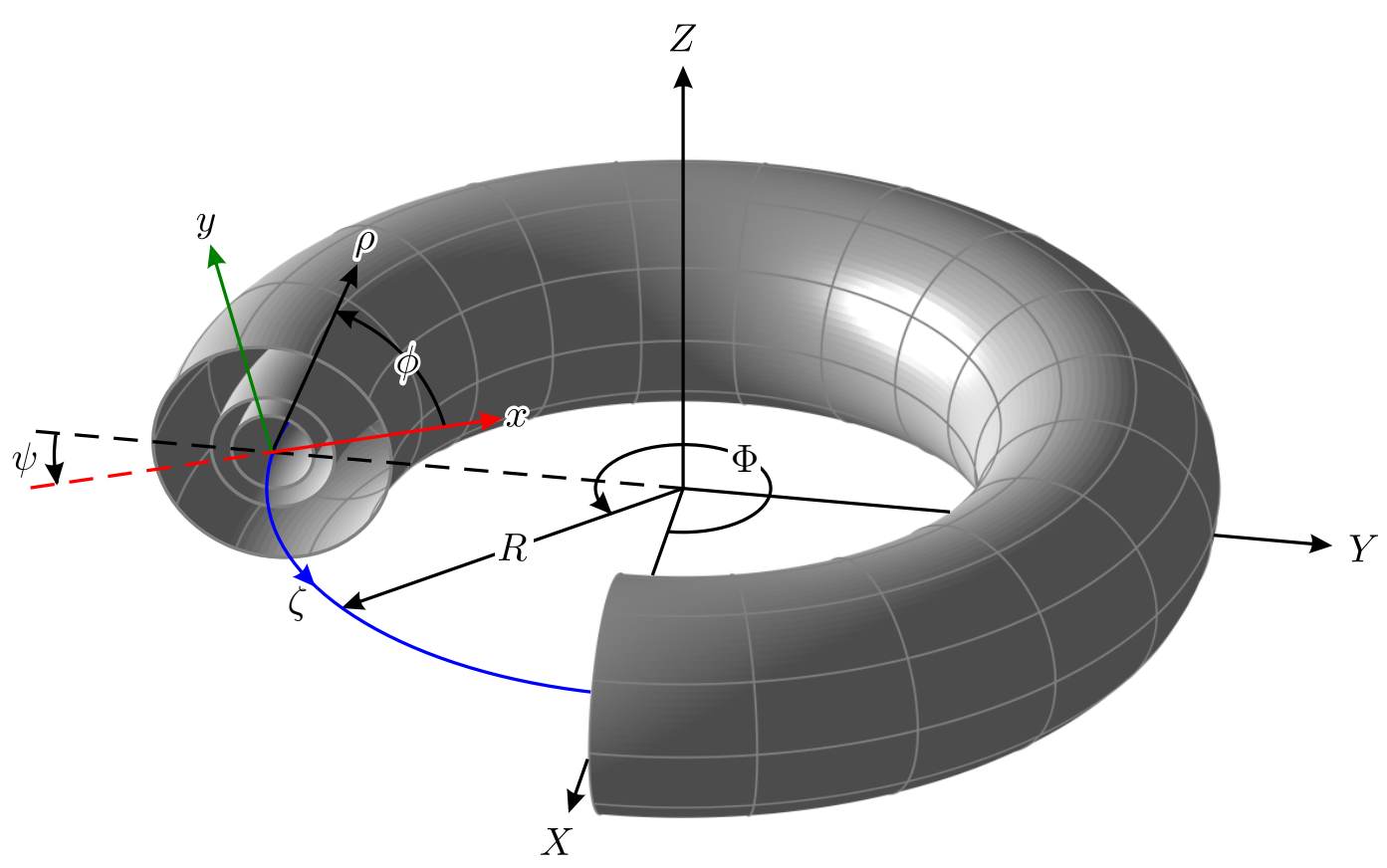

Figure C.1: Geometry of a Torus and its coordinate systems. 
as

$$
\begin{gathered}
h_{\rho}=\sqrt{\left(\frac{\partial X}{\partial \rho}\right)^{2}+\left(\frac{\partial Y}{\partial \rho}\right)^{2}+\left(\frac{\partial Z}{\partial \rho}\right)^{2}}=1, \\
h_{\phi}=\sqrt{\left(\frac{\partial X}{\partial \phi}\right)^{2}+\left(\frac{\partial Y}{\partial \phi}\right)^{2}+\left(\frac{\partial Z}{\partial \phi}\right)^{2}}=\rho, \\
h_{\zeta}=\sqrt{\left(\frac{\partial X}{\partial \zeta}\right)^{2}+\left(\frac{\partial Y}{\partial \zeta}\right)^{2}+\left(\frac{\partial Z}{\partial \zeta}\right)^{2}}=1-\frac{\rho}{R} \cos (\phi+\psi) .
\end{gathered}
$$

Let $\mathbf{r}$ denote the vector from the origin to a variable point $P(X, Y, Z)$. We can write the position vector as

$$
\mathbf{r}=X \hat{X}+Y \hat{Y}+Z \hat{Z}
$$

Now, we can compute the unit vectors in our toroidal coordinate system as

$$
\begin{aligned}
& \hat{\rho}=\frac{1}{h_{\rho}} \frac{\partial \mathbf{r}}{\partial \rho}=-\cos (\phi+\psi)\left[\cos \left(\frac{\zeta}{R}\right) \hat{X}+\sin \left(\frac{\zeta}{R}\right) \hat{Y}\right]+\sin (\phi+\psi) \hat{Z}, \\
& \hat{\phi}=\frac{1}{h_{\phi}} \frac{\partial \mathbf{r}}{\partial \rho}=\sin (\phi+\psi)\left[\cos \left(\frac{\zeta}{R}\right) \hat{X}+\sin \left(\frac{\zeta}{R}\right) \hat{Y}\right]+\cos (\phi+\psi) \hat{Z}, \\
& \hat{\zeta}=\frac{1}{h_{\zeta}} \frac{\partial \mathbf{r}}{\partial \zeta}=-\sin \left(\frac{\zeta}{R}\right) \hat{X}+\cos \left(\frac{\zeta}{R}\right) \hat{Y} .
\end{aligned}
$$

Using the above equations, we can transform the Cartesian unit vectors $\hat{X}, \hat{Y}$ and $\hat{Z}$ into our unit toroidal vectors $\hat{\rho}, \hat{\phi}$ and $\hat{\zeta}$. Similarly, we can derive the reverse transformation, given by

$$
\begin{aligned}
& \hat{X}=[-\cos (\phi+\psi) \hat{\rho}+\sin (\phi+\psi) \hat{\phi}] \cos \left(\frac{\zeta}{R}\right)-\sin \left(\frac{\zeta}{R}\right) \hat{\zeta} \\
& \hat{Y}=[-\cos (\phi+\psi) \hat{\rho}+\sin (\phi+\psi) \hat{\phi}] \sin \left(\frac{\zeta}{R}\right)+\cos \left(\frac{\zeta}{R}\right) \hat{\zeta} \\
& \hat{Z}=\sin (\phi+\psi) \hat{\rho}+\cos (\phi+\psi) \hat{\phi}
\end{aligned}
$$

In addition to the above formulas, we can express the local polar vectors $\hat{\rho}$ and $\hat{\phi}$ according to their associated local Cartesian coordinates $\hat{x}$ and $\hat{y}$ :

$$
\begin{aligned}
& \hat{\rho}=\hat{x} \cos \phi+\hat{y} \sin \phi, \\
& \hat{\phi}=-\hat{x} \sin \phi+\hat{y} \cos \phi .
\end{aligned}
$$

From the above results, we can verify that our toroidal coordinate system preserves the well-know relations of polar coordinates, as expected. 
The differentiation of the unit vectors in (C-8) yields [150, Appendix A]:

$$
\begin{aligned}
& \frac{\partial \hat{\rho}}{\partial \rho}=\mathbf{0}, \\
& \frac{\partial \hat{\rho}}{\partial \phi}=\hat{\phi}, \\
& \frac{\partial \hat{\rho}}{\partial \zeta}=\hat{\zeta} \frac{1}{h_{\rho}} \frac{\partial h_{\zeta}}{\partial \rho}=-\hat{\zeta} R^{-1} \cos (\phi+\psi),
\end{aligned}
$$

$$
\begin{aligned}
& \frac{\partial \hat{\phi}}{\partial \rho}=\mathbf{0}, \\
& \frac{\partial \hat{\phi}}{\partial \phi}=-\hat{\rho}, \\
& \frac{\partial \hat{\phi}}{\partial \zeta}=\hat{\zeta} \frac{1}{h_{\phi}} \frac{\partial h_{\zeta}}{\partial \phi}=\hat{\zeta} R^{-1} \sin (\phi+\psi),
\end{aligned}
$$

$\frac{\partial \hat{\zeta}}{\partial \rho}=\mathbf{0}$

$\frac{\partial \hat{\zeta}}{\partial \phi}=\mathbf{0}$

$\frac{\partial \hat{\zeta}}{\partial \zeta}=-\hat{\rho} \frac{1}{h_{\rho}} \frac{\partial h_{\zeta}}{\partial \rho}-\hat{\phi} \frac{1}{h_{\phi}} \frac{\partial h_{\zeta}}{\partial \phi}=\hat{\rho} R^{-1} \cos (\phi+\psi)-\hat{\phi} R^{-1} \sin (\phi+\psi)$.

The metric coefficient defined in [150, p. 477] differs from ours, introduced in (C-4), (C-5) and (C-6). We can show that the coefficients in [150] are equal to $1 / h_{\rho}, 1 / h_{\phi}$ and $1 / h_{\zeta}$ in our notation. 